\title{
BIOKINETIC STUDY FOR SRC-I WASTEWATER
}

Final Technical Report

Work Performed Under Contract No. AC05-780R03054

Catalytic, Inc.

Philadelphia, Pennsylvania

Technical Information Center

Office of Scientific and Technical Information United States Department of Energy 


\section{DISCLAIMER}

This report was prepared as an account of work sponsored by an agency of the United States Government. Neither the United States Government nor any agency Thereof, nor any of their employees, makes any warranty, express or implied, or assumes any legal liability or responsibility for the accuracy, completeness, or usefulness of any information, apparatus, product, or process disclosed, or represents that its use would not infringe privately owned rights. Reference herein to any specific commercial product, process, or service by trade name, trademark, manufacturer, or otherwise does not necessarily constitute or imply its endorsement, recommendation, or favoring by the United States Government or any agency thereof. The views and opinions of authors expressed herein do not necessarily state or reflect those of the United States Government or any agency thereof. 


\section{DISCLAIMER}

Portions of this document may be illegible in electronic image products. Images are produced from the best available original document. 


\title{
DISCLAIMER
}

\begin{abstract}
This report was prepared as an account of work sponsored by an agency of the United States Government. Neither the United States Government nor any agency thereuf, nur any of their employees, makes any warranty, express or implied, or assumes any legal liability or responsibility for the accuracy, completeness, or usefulness of any information, apparatus, product, or process disclosed, or represents that its use wüld nut infringe privately owned rights. Keference herein to any specific commercial product, process, or service by trade name, trademark, manufacturer, or otherwise does not necessarily constitute or imply its endorsement, recommendation, or favoring by the United States Government or any agency thereof. The views and opinions of authors expressed herein do not necessarily state or reflect those of the United States Government or any agency thereof.
\end{abstract}

This report has been reproduced directly from the best available copy.

Available from the National Technical Information Service, U. S. Department of Commerce, Springfield, Virginia 22161.

Price: Printed Copy A08

Microfiche AnI

Codes are used for pricing all publications. The code is determined by the number of pages in the publication. Information pertaining to the pricing codes can be found in the current issues of the following publications, which are generally available in most libraries: Energy Research Abstracts (ERA); Government Reports Announcements and Index (GRA and I); Scientific and Technical Abstract Reports (STAR); and publication NTIS-PR-360 available from NTIS at the above address. 
DOE/OR/03054-107

(DE84013780)

Distribution Category UC-89

\section{BIOKINETIC STUDY FOR SRC-I WASTEWATER}

Final Technical Report

Catalytic, Inc.

Philadelphia, Pennsylvania 
PAGE

I. SUMMARY 1

II. INTRODUCTION 2

III. BACKGROUND 3

IV. SPECIFIC AREAS OF EVALUATION 5

V. INVESTIGATIVE PROGRAM 7

Feed Storage and Preparation 7

Phenol Extraction 9

Ammonia and Sulfide Stripping 9

Concentration Adjusliment and Nẹtral1zatioñ 10

Bioreactor Operation $\quad 10$

Equipment $\quad 10$

System Operation 13

Monitoring 14

Sampling and Analysis 15

Summary Description of System Operations 17

VI. RESULTS AND DISCUSSION 30

Steady-State Conditions $\quad 30$

Apilicabilily of Kinetic Model 33

Evaluation of Kinetic Coefficients $\quad 35$

BOD Basis 36

COD Basis $\quad 36$

TOC Bas is 39

Comparison of Kinetic Coefficients 39

Oxygen Utilization $\quad 43$

Nitrification $\quad 45$

Mixed Liquor Settling Characterislics 46

$\begin{array}{llr}\text { VII. CONCLUSIONS } & 49\end{array}$

VIII. REFERENCES 51

APPENDIX A - TABLES AND FIGURES 52

$\begin{array}{ll}\text { APPENDIX } 8 \text { - ANALYTICAL METHODS } & 161\end{array}$ 


\section{SUMMARY}

Biooxidation is an important part of the overall wastewater treatment system for the proposed SRC-I Demonstration Plant in Newman, Kentucky. After the completion of a Baseline Design for the plant in April 1982, various refinements were evaluated. One of these was the inclusion of a process for phenol recovery from four sour-water streams. As part of this evaluation, an extensive laboratory treatability study (1) was conducted on wastewaters both with and without this phenol recovery step. The purpose of the study was to compare the results of different treatment schemes on different wastewaters and did not include the development of the kinetic coefficients that govern the biooxidation process. As an extension to that study, additional treatability testing was performed and is documented herein. The purpose of this additional work is to establish the kinetic coefficients for biooxidation of the SRC-I wastewaters. This will provide a rational basis for any subsequent changes in design.

The wastewater feed used in this study was process recycle water from the Fort Lewis, Washington, pilot plant. Prior to biooxidation, it was pretreated by solvent extraction for removal of phenolics and by steam stripping for removal of ammonia and hydrogen sulfide. Two, 2-stage bench scale bioreactors were operated for approximately eight months, during which time they were stabilized at various steady-state conditions. Kinetic coefficients were evaluated according to the Lawrence and McCarty model, based on BOD, COD, and TOC. Nitrification, oxygen utilization, and solids settling velocitles were also studied.

The results showed that $B O D$ is a poor basis for the model mainly because almost all $\mathrm{BOO}_{5}$ was removed at all steady-state conditions. Also, the model was more accurate for steady-state conditions of lower solids retention times. As was noted in the previous study, the bulk of both urganic and ammonia reductions occurred in the first stage reactors. 


\section{INTRODUCTION}

To reduce America's dependence on imported petroleum, the U.S. Department of Energy (DOE) initiated the Solvent-Refined COal (SRC-I) Direct Coal Liquefaction Project to demonstrate the technical and economic feasibility of coal liquefaction. A prime factor in establishing this feasibility was the overall environmental acceptability of the process, including control of gaseous, liquid, and solid wastes. Under its prime contract (No. DE-AC05-78-ORO-3054) with DOE, the International Coal Refining Company (ICRC) completed the baseline design of a 6000-TPD demnnstration plant in April 1982.

DOE has subsequently decided to indefinitely postpone construction of the demonstration plant. This postponement has provided an opportunity to upgrade the baseline design, and a major effort has since been made to generate additional design information for the wastewater treatment facilities. This post-baseline work included extensive laboratory treatability studies. The purposes were to evaluate alternate treatment schemes and to establish design criteria for individual unit processes. Two major focuses were the effects of phenul exlraction as a pretreatment for SRC-I sour water and the use of reverse osmosis as a means of achieving zero discharge. This report is an extension of the phenol extraction study. Its relationship to that work is explained in lie next section. 
III. BACKGROUNO

One primary objective of 'ICRC's post-baseline environmental program was to compare two alternative wastewater treatment schemes. One included phenol extraction before biological treatment, and the other did not. Under a previous work agreement, Catalytic, Inc., a subcontractor to International Coal Refining Company (ICRC), operated a number of biological reactors utilizing a strong SRC-I sour water, collected from the Fort Lewis, Washington, pilot plant. The impacts of phenol extraction on secondary (biooxidation) and tertiary (coagulation, filtration, carbon adsorption, and ozonation) treatment processes were assessed. Also under that work agreement, samples were produced for toxicological testing at SRI International. All results are documented in a report (1) issued in September 1983.

In that report, it was recommended that phenol extraction be employed. Phenol extraction has been incorporated in the current design of the waste treatment system, as specified in a document prepared by ICRC (2). Effluent limitations are also listed therein. The actual system design was by Catalytic and is documented in another report (3).

Because the primary objective in the previous study was to generate data. for comparison of the two alternative treatment schemes, the bioreactors were operated primarily under a single set of conditions. Also, a necessity to pronuce large volumes of treated wastewater for the tertiary treatment studies and for toxicological testing precluded a systematic biokinetic study (i.e., varying steady-state conditions).

The purpose of this study is to evaluate bioreactor performance as a function of operating conditions. Two of the biological systems from the previous study will be utilized. Both of these are two-stage systems, treating dephenolated wastewater. (They had been maintained at the completion of that work.) These systems will be stabilized at various steady-state 
conditions, thereby determining the biosystem performance over a range of operating conditions. The first stage reactors are primarily intended for removal of organics, and the second stages for nitrification. During the previous study work, however, it was found that both ammonia and carbonaceous BOD were removed in each reactor. It was also found that these removals occurred mostly in the first stage reactors. This will be investigated for each steady-state condition.

The purpose of this study is not to establish any optimum set of operating conditions, such as retention time, mixed liquor solids, or sludge age. There is no target concentration of $B O D$ or $C O D$ that the bioreactor effluent must meet. Rather. this work will estahlish the basic relationships between operating conditions and system performance. It will then be possible to design the system for any given effluent limitations. Also, because this study has been designed to cover a range of conditions, it will serve to establish the limits of treatability that may be expected from a secondary system. 


\section{SPECIFIC AREAS OF EVALUATION}

The first stage biological reactors can be modeled mathematically, based on empirical relationships. There are many such models available. This report will use the Lawrence and McCarty method, which describes mean cell retention time (SRT) as the primary independent variable in system control. The model is based on the following equations:

$$
\begin{aligned}
& I / S R T=Y(F / M)-k_{d} \\
& F / M=\frac{k S}{K_{S}+S}=\frac{Q}{V} \frac{(S 0-S)}{X} \\
& S=\frac{K_{S}}{S R T\left(1+k_{d}\right.} \frac{(S R T)]}{\left(Y K-k_{d}\right)-1}
\end{aligned}
$$

Where SRT = Solids residence time, days

$Y=$ Yield Coefficient, lbs. biological solids produced/ 1b. substrate removed

$k_{d}=$ Decay Constant, 1/days

$\mathbf{k}=$ Maximum specific substrate utilization rate, lbs. removed per day/1b. MLVSS

$S=$ Substrate concentration in the bioreactor

$S_{0}=$ Substrate concentration in the feed to the bioreactor

$K_{s}=$ Substrate concentation at removal rate of $k / 2$

$Q=$ Volumetric flow rate to the reactor

$V=$ Volume of the reactor

$X=$ Mixed liquor volatile suspended solids (MLVSS) concentration

$F / M=$ Substrate to bio. mass ratio, lbs. removed per day/lb. MLVSS.

The Lawrence and McCarty procedure allows a graphical determination of $k$, $K_{s}, k_{d}$, and $Y$. Once the kinetic parameters are determined, Equation 3 can be used to predict system performance for selected values of SRT. In 
addition, oxygen requirements can be calculated, based on measured oxygen uptake rates and mixed liquor solids.

As noted in the previous section, nitrification will also be evaluated. A nitrogen balance will be established for each bioreactor. Ammonia, cyanide, thiocyanate, nitrite, nitrate, and organic nitrogen levels will be monitored. In addition, the nitrification data will be applied to a first-order kinetic mode 1 , as follows:

$$
c / C_{0}=c^{-k_{n} O X}
$$

Where

$$
\begin{aligned}
\mathrm{C} / \mathrm{Co}_{0} & =\text { Influent ammonia concentration, } \mathrm{mg} / 1 \\
\mathrm{c} & =\text { Effluent ammonia concentration, mg/l } \\
k_{n} & =\text { Rate coefficient, } 1 / \mathrm{mg}-\text { day } \\
0 & =\text { Hydraulic retention time, days } \\
\mathrm{x} & =\text { MLVSS, } \mathrm{mg} / 1 .
\end{aligned}
$$

One final area of interest is system stability. The fluctuations of key parameters (COO, BOO, TOC, dild $\mathrm{NH}_{3}$ ) will be presented in the form of time-series plots. This will allow a subjective comparison between different steady-state conditions. 


\section{INVESTIGATIVE PROGRAM}

\section{FEED STORAGE AND PREPARATION}

The raw wastewater used for this study was obtained from the DOE-owned, 50-TPO coal liquefaction pilot plant at Fort Lewis, Washington. Forty-five (45) 55-gallon drums of Process Recycle Water (PRW) were collected from August 12 through August 14, 1980, at which time the plant was running in the SRC-I mode. (The plant was designed to run primarily in the SRC-II mode.)

The Fort Lewis wastewater was thoroughly characterized in an earlier study (4), in which the wastewater was compared with samples obtained from another SRC pilot plant, located in Wilsonville, Alabama. The design of the Wilsonville plant more closely resembles the proposed SRC-I Demonstration Plant, but it is too small to produce the quantities of wastewater required for continuous treatability testing. However, a comparison of these two pilot plant waste streams shows that they have similar characteristics. The fort Lewis samples are, therefore, representative of the wastewater anticipated from the demonstration plant and provide a valid basis for treatability testing.

The Fort Lewis facility does not include certain equipment and pretreatment steps that will be provided in the demonstration plant. Therefore, these pretreatment steps were simulated in the laboratory. As shown on Figure 1, this included phenol extraction, ammonia and sulfide stripping. concentration adjustment, and neutralization. Data was collected on these pretreatment steps in an earlier study (1). The laboratory equipment and procedures described below are based on that previous work. 
Figure 1

Pretreatment Before Biooxidation

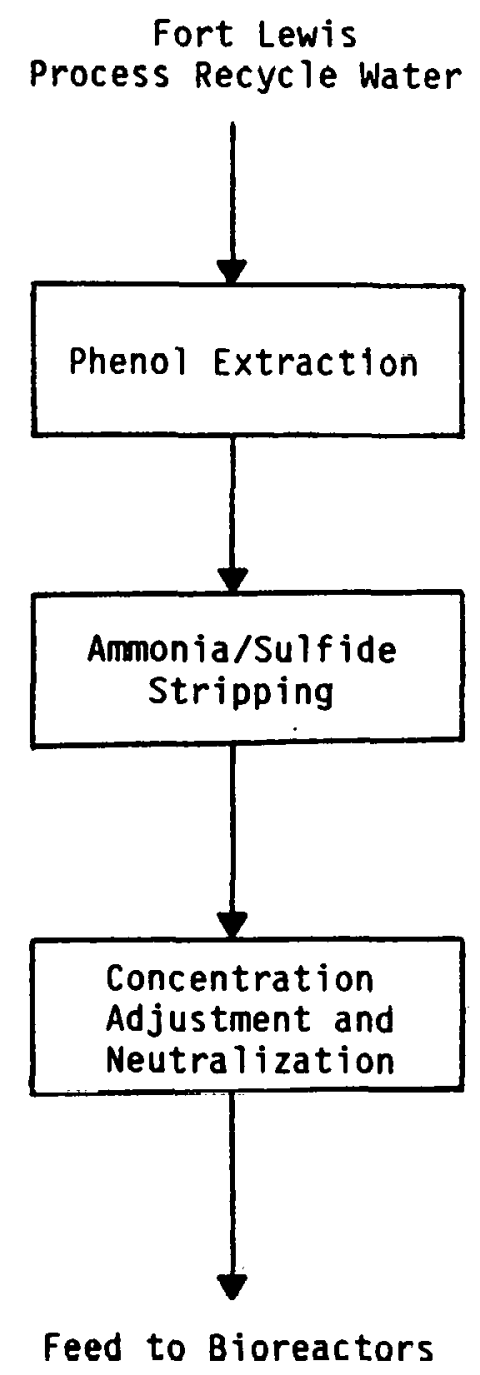

$-8-$ 


\section{Phenol Extraction}

Phenol extraction, which is the first pretreatment step, was provided by two different laboratories. Because the project was an extension of a previous study, the feed source from that study, was continued. This water had been pretreated by a vendor (Chem Pro Corporation), using pilot scale equipment. Operated on a continuous flow basis, the pilot facilities provided phenol removal via solvent extraction, followed by steam stripping of sulfide and ammonia. When this wastewater was exhausted, additional feed was prepared in the Catalytic laboratory. The Chem Pro feed was simulated as closely as possible. For solvent extraction, this was accomplished in a two-stage, batch procedure. Measured quantities of solvent and raw wastewater were agitated in a glass vessel for 15 minutes, settled for 1 hour, and decanted. Fresh solvent was then added, and the steps repeated. The effluent phenolics ranged from 7 to $14 \mathrm{mg} / 1$, versus 0.3 to $5 \mathrm{mg} / 1$ when treated by the continuous flow pilot facilities.

\section{Ammonia and Sulfide Stripping}

Steam stripping was used for removal of ammonia and sulfide. As noted in the preceding paragraph, the initial feed was pretreated by the chem Pro Corporation in their pilot-scale facilities. In addition to removing phenolics, this pilot equipment also provided continuous steam stripping of both sulfide and ammonia. Sulfide was stripped at an initial wastewater pH of about 9-10. Caustic soda was then used to raise the pH for ammonia removal. A detailed description of the Chem Pro apparatus and procedures, for both extraction and stripping, is available in a previous report (4).

When the stripping was conducted in Catalytic's laboratory, it was accomplished in two passes through continuously operating, packed glass columns. The first pass, primarily for removal of hydrogen sulfide, was conducted without pH adjustment. (The pH was generally between 9 and 10.) Approximately $90 \%$ of the ammonia was also removed. For the second pass, caustic soda was used to raise the $\mathrm{pH}$ to 11.5 . This allowed further removal of ammonia, producing an effluent concentration of approximately $200 \mathrm{mg} / 1$. 


\section{Concentration Adjustment and Neutralization}

Table 1 shows the targeted feed specifications. After the stripping steps, the wastewater was diluted and the $\mathrm{pH}$ adjusted to about 7 , using concentrated (98\%) sulfuric acid. Supplementary chemicals were added at this point, based on the demonstration plant material balance (3) and the refinements of earlier characterization and study work. Table 2 lists the measured parametric concentrations for each feed batch.

\section{BIOREACTOR OPERATION}

Two bench-scale, biological treatment systems were operated in parallel. Each system was comprised of two complete-mix, activated sludge reactors. connected in series. All reactors were equipped with internal baffles to prevent washout of suspended solids. The reactors in system 1 are designated as Units $1 A$ and 1B; likewise, those in System 2 are $2 A$ and $2 B$. The influent COD target concentration to each system was kept at $2000 \mathrm{mg} / 1$ throughout the study, and a 2-day hydraulic residence time was maintained in each stage. sludge age (SRT) was varied to provide different rates of biomass growth and substrate utilization.

\section{Equipment}

All equipment in contact with wastewater and treated effluent was glass, Type 316 stainless steel or plastic having excellent resistance to adsorption and leaching. Each bioreactor was an independent physical unit consisting of feed pumps, an agitator, and feed/effluent handling containers.

Bioreactors. Two sizes were used. primarily to maintain the same hydraulic loading in both stages of the two-stage systems. System losses resulting from sampling and evaporation are not negligible for hench scale units. These effects result in a reduction in flow from the first to the second stage for each system. To compensate for these losses, the second stage reactors were smaller, thereby maintaining the specified 2-day hydraulic

residence time. The large first stage reactor was constructed of stainless 
Table 1

Feed Composition

\section{Component}

COD

$\mathrm{PO}_{4}^{3-}$

$\mathrm{NH}_{3}-\mathrm{N}$

$\mathrm{SCN}^{-}$(Sodium Form)

$\mathrm{CN}^{-}$

*Ca ${ }^{2+}$

$\mathrm{Fe}^{3+}$

$\mathrm{Mg}^{2+}$

*Phenolics

*TDS

*TOC

*Sodium

\section{Concentration (mg/1)}

$$
2,000 \pm 200
$$

$75^{\star \star}$

$$
\begin{gathered}
200 \pm 20 \star * \\
200 \pm 20 \\
10 \pm 2 \\
600 \\
7 \\
14 \\
1-25 \\
4,000-6,000 \\
500-600 \\
800-1,000
\end{gathered}
$$

* These constituents are NOT adjusted. Values are the resultant expected range. **or a concentration for biological growth and reproduction, whichever is greater. 
DEPHENOLATED TABLE 2 FHARACTERISTICS

(ALL PARQKETERS IN ES/I EICDPT PH)

\begin{tabular}{|c|c|c|c|c|c|c|c|c|c|c|c|c|c|c|c|c|c|c|c|c|c|}
\hline $\begin{array}{c}\text { FEDD } \\
\text { BATCH NUEER }\end{array}$ & $\begin{array}{l}\text { FERD } \\
\text { SIPRT } \\
\text { DATE }\end{array}$ & $\begin{array}{l}\text { ORIGINAL } \\
\text { VaLuKE } \\
\text { (LITER) }\end{array}$ & 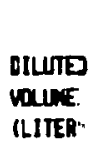 & $\begin{array}{l}1 \\
1 \\
1\end{array}$ & 000 & BD & 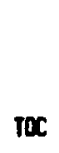 & 山 & TSS & TDS & $n+3-N$ & TOTA & our & 500 & $m e-n$ & $n 03-1$ & PHENOIC & CACIUA & Irom & MEESIIU & sobius \\
\hline 27. & $24-\Omega l-83$ & و & 176 & I & 20185 & - & 363 & 58 & - & 4080 & 194 & 60.2 & - & 54.1 & (10.25 & 5.5 & 1.59 & 635 & 6.17 & 17 & 800 \\
\hline 284 & $11-A L G-\infty]$ & 100 & 175 & i & 2036 & - & $5 \pi$ & 120 & - & 488 & 29 & 46.7 & 2.81 & 19 & a.25 & 30 & 1.56 & $6+0$ & 6.96 & 18 & 860 \\
\hline 294 & $27-8,6-23$ & 10 & 170 & i & 2550 & $14 \pi$ & 59 & 91 & - & 4394 & 186 & 50.5 & 1.78 & 19 & 0.25 & 2.0 & 1.59 & 52 & 7.1 & $\mathbf{a}$ & 230 \\
\hline 3 & $12-500-17$ & 101 & 170 & $i$ & 2395 & 1411 & 697 & 9.7 & - & 4502 & 19 & B1.7 & 2.24 & 191 & 0.25 & 2. & 1.72 & 559 & 6.6 & $\boldsymbol{a}$ & 870 \\
\hline 314 & $28-5[5-83$ & 100 & 175 & i & 2210 & - & $x$ & 11.3 & - & $46+5$ & 199 & 13.3 & 1.08 & 192 & (1.25 & (1.26 & 0.37 & 632 & 5.26 & 21.3 & 59 \\
\hline 2 & $14-\infty<1-\infty]$ & 10 & 175 & 1 & 2123 & - & 681 & 11.1 & - & 460 & 194 & S4.1 & 1.79 & 198 & 11.25 & 11.25 & 0.33 & 570 & 1. 32 & 20.2 & 739 \\
\hline 334 & $4-100-83$ & $: 10$ & 175 & 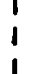 & 2104 & - & Es8 & 11.5 & - & 4000 & 178 & 5.7 & 0.97 & 174 & 2.12 & (1.25 & 5.1 & 510 & i.22 & 498 & 810 \\
\hline 34t & $10-104-83$ & 100 & 176 & i & 2098 & 1060 & $\$$ & 11.i. & - & 4199 & 182 & 1.1 & 11.1 & 162 & 0.61 & 11.25 & 0.55 & 378 & 2.0 & 1.32 & 840 \\
\hline 354 & $24-\mathrm{HOV}-83$ & BS. & 160 & I & 2662 & 1110 & 7.15 & 11.6 & 788 & 4300 & 182 & 69.2 & 12.4 & 170 & 0.33 & (1.26 & 0.33 & 632 & $=5$ & 19 & 878 \\
\hline $36 \mathrm{H}$ & $24+104-83$ & 73 & 145 & i & 2242 & $\Delta 4$ & 60 & 11..! & 874 & 3985 & 179 & 60.1 & 0.84 & 2018 & 10.26 & (1.25 & 9.2 & 587 & 57 & 21.4 & 780 \\
\hline $37+4$ & $24-D E C-83$ & B & 170 & i & 2098 & 1040 & 69 & 9.6. & 676 & 3855 & 220 & 60.4 & 2.4 & $a$ & 10.25 & 1.7 & 13.8 & 339 & 4.73 & 19.1 & 654 \\
\hline $30 \mathrm{H}$ & BQ-JAH-BA & 73 & 167 & i & 2055 & 1060 & SE & 10.6 & 74 & 1255 & 206 & 45.7 & 0.12日 & ผ9 & 0.62 & 2.98 & 8.5 & 548 & 51 & 15.1 & 168 \\
\hline 394 & $25-\mathrm{J} A \mathrm{AOA}$ & is & 243 & i & 2170 & 960 & 78 & 7.1 & 208 & 5660 & 192 & 495 & 4.45 & 199 & 10.25 & 11.25 & 9.97 & 551 & 7.1 & 15.5 & 910 \\
\hline 404 & $07+$ EO-84 & 69.5 & 121.5 & 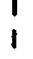 & 224 & 980 & 700 & Q.2 & 86 & 5270 & 191 & 2.26 & 12.7 & IJI & 10.28 & 3.9 & 9.6 & 651 & 52 & 16.0 & 830 \\
\hline $41 \mathrm{H}$ & $17+[28-84$ & $\pi$ & 150 & i & 1939 & - & 611 & 11.9 & - & 4050 & 188 & - & 11.1 & 15 & 0.35 & 3.4 & 7.5 & 565 & 4.1 & 17.5 & 800 \\
\hline 424 & $20-\operatorname{AR}-A$ & 76 & 180 & 1 & 2080 & - & $61 !$ & 12.0 & - & 4875 & 199 & - & 7.5 & 10 & 0. 35 & 11.25 & 9.7 & 554 & 4.7 & 15.7 & 20 \\
\hline
\end{tabular}

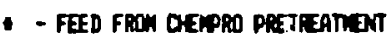

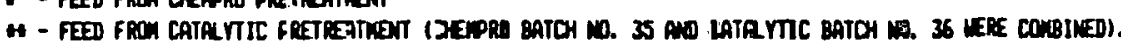


stee1. Its total capacity was 12-L, and it was approximately $127 \mathrm{~mm}$ wide, 254 $\mathrm{mm}$ long, and $405 \mathrm{~mm}$ high (up to the outlet nozzle invert). The reactor was divided into two sections by a removable baffle: a 9.3-L aeration zone and a 2.7-L clarification zone. The baffle was adjustable for clearance between it and the sloped bottom in the clarifier section. The smaller reactor was constructed of polymethyl methacrylate. Its total volume was $8.6-\mathrm{L}$ and it was approximately $114 \mathrm{~mm}$ wide, $240 \mathrm{~mm}$ long, and $340 \mathrm{~mm}$ high (to the overflow nozzle). The design was the same as the larger reactor, and it provided a 7.0-L aeration zone and $1.6-\mathrm{L}$ clarification zone.

Pumps. Feed was pumped from individual glass bottles holding a 24-hour supply by a peristaltic pump with variable-speed drive. Silicone tubing. connected to tefion tubing on the suction and discharge ends, was used in the pump head. To achieve the low flow rates necessary, all pumps were electrically controlled by a timer, which ran for 20 seconds in a 2-minute cycle. Each pump had a separate speed controller for individual adjustment.

Agitators. Electrical drive agitators with two speed ranges and individual variable-speed controllers were used. These were equipped with standard three-blade stainless steel propellers.

\section{System Operation}

System monitoring was initiated on July 26, 1983 (reference point Day 0 ). A11 systems were operated continuously, 24 hours/day, 7 days/week. Monitoring was performed during one shift, 7 days/week. All systems were fed from a container holding a 24-hour feed supply. The feed to each second stage unit was the effluent from the corresponding first stage unit collected over the previous 24 hours. Both units in two-stage systems were operated at the same hydraulic retention time (HRT). As noted previously, the second stage reactors were chiefly intended for nitrification, which characteristically produces a small. slow-settling floc. Powdered activated carbon (PAC) was added to the second stage units in small amounts (nominally $500 \mathrm{mg} / 1$ ) in order to improve solids settling. 
Systems 1 and 2 were operated under the following specifications:

$\begin{array}{lcc} & \begin{array}{c}\text { Unit 1A/2A } \\ \text { (First Stage) }\end{array} & \begin{array}{c}\text { Unit 1B/2B } \\ \text { (Second Stage) }\end{array} \\ \text { Aerated Volume (1) } & 9.3 & 7.0 \\ \text { SRT (days) } & \text { Varied } & \text { Varied } \\ \text { HRT (days) } & 2 & 2 \\ \text { F/M (g COO/g MLVSS) } & <0.5 & <0.2 \\ \text { Mixed Liquor PAC Conc. (mg/1) } & 0 & 500 \\ \text { PH (reactor) } & 6.75-8.0 & 7.0-8.5 \\ \text { Temperature }\left({ }^{\circ} \mathrm{C} \text { ) }\right. & 18-26 & 18-26 \\ \text { Dissolved Oxygen (mg/1) } & >2.0 & >2.0 \\ \text { Feed } & \text { Dephenolated } & \text { EFPluent from }\end{array}$

1 MLVSS, Mixed Liquor Volatile Suspended Solids, with correction for PAC.

\section{Monitoring}

Selected data were analyzed daily to monitor operational parameters and adjust the system. In addition to maintaining $\mathrm{pH}$, temperature, and U.0. specifications for each system, total suspended solids (TSS) in the basin and effluent were measured in order to calculate daily sludge wasting and, thus, to control SRT. Volatile (biological) suspended solids (VSS) were measured twice a week, and the daily value required for $F / M$ calculations was calculated by applying the ratio (VSS/TSS) of the previous data set. All solids data for a system containing PAC were further adjusted to shuw concentrations without PAC.

Feed and effluent rates were established for each reactor by daily volumetric measurements. This data was required for SRT, HRT, F/M, and sludge wasting calculations. 
Data were recorded in several documents, e.g., the operator's log hook, laboratory notebooks, and analysis report forms. These were compiled into summary sheets which are included in the Appendix, Tables A-1 to A-4.

Sampling and Analys is

Other than $\mathrm{pH}, \mathrm{COD}$ was the only effluent parameter analyzed on a daily basis. Other pollutant parameters were analyzed from composite samples in accordance with the schedules on Table 3 .

Feeds and effluents were sampled daily and refrigerated. Composite samples were prepared by mixing equal portions of daily samples from the interval needed. For example, once-a-week analyses were performed on a sample containing equal volumes of daily samples collected during the preceding 7 days. 
Table 3

Number of Weekly Analyses Required

Starting October 31, 1983

No. of Sample Pts.

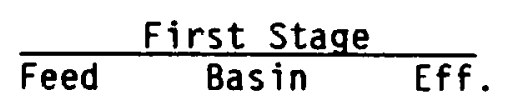

$\frac{\text { Second Stage }}{\text { Basin Eff. }}$

\begin{tabular}{|c|c|c|c|c|c|}
\hline Parameter & 1 & 2 & 2 & 2 & 2 \\
\hline COD & 2 & 0 & 7 & 0 & 7 \\
\hline TOC & 2 & 0 & 2 & 0 & 2 \\
\hline$B 00$ & 2 & 0 & 2 & 0 & 2 \\
\hline $\mathrm{NH}_{3}$ & 2 & 0 & 2 & 0 & 2 \\
\hline TKN & $1 / 2^{*}$ & 0 & $1 / 2^{\pi}$ & $\dot{0}$ & $1 / 2^{\star}$ \\
\hline $\mathrm{CN}^{-}$ & $1 / 2$ & 0 & $1 / 2$ & 0 & $1 / 2$ \\
\hline $\mathrm{SCN}^{-}$ & $1 / 2$ & 0 & $1 / 2$ & 0 & $1 / 2$ \\
\hline $\mathrm{NO}_{2}^{-}$ & 0 & 0 & $1 / 2$ & 0 & $1 / 2$ \\
\hline $\mathrm{NO}_{3}^{-}$ & 0 & 0 & $1 / 2$ & 0 & $1 / 2$ \\
\hline TSS & 1 & $j \star \pi$ & 7 & $7 * \star$ & 7 \\
\hline VSS & 1 & $2 * *$ & 2 & $2^{* \star}$ & 2 \\
\hline Tempèra lure & 0 & 7 & 0 & I & 0 \\
\hline $\mathrm{pH}$ & 7 & 7 & 7 & 7 & 0 \\
\hline DO & 0 & 7 & 0 & 7 & 0 \\
\hline $\mathrm{O}_{2}$ Uptake Rate & 0 & 3 & 0 & 3 & 0 \\
\hline Color & 1 & 0 & 1 & 0 & 1 \\
\hline Phenolics & 1 & 0 & 1 & 0 & ? \\
\hline Ortho-PO & 0 & 0 & 2 & 0 & 2 \\
\hline Settling Test & 0 & $1 / 2$ & 0 & $1 / 2$ & 0 \\
\hline TDS & 0 & 0 & 1 & 0 & 1 \\
\hline
\end{tabular}

$\star 1 / 2=$ Once in two weeks (every other week).

**Sample to be taken with the baffle removed. 
Summary Description of System Operations

As noted previously, this report includes data collected during a time period prior to the formal initiation of this study. Systems' operations during that period were as described in the preceding section although the data collection was not always as extensive. The data presented in this report cover the period from July 26, 1983, (Day 0) to March 16, 1984 (Day 234). At Day 0, all four units were operating at a 30-day SRT and had been for about 1 year.

The overall effectiveness of Systems 1 and 2 is shown graphically on Figures 2 and 3 . These plots show influent and effluent (both stages) COD concentrations from Day 0 to the completion of this study (Day 236). The plots show that system COD removals were generally stable, with efficiencies of about 90\%. Figures 4 and 5 show the effluent COD levels in greater detail and also include the effluent $\mathrm{TOC}$ and first stage effluent $\mathrm{BOD}_{5}$ concentrations. System 2, except for the initial 30-day SRT period, produced effluent COD concentrations from the first stage (Unit 2A) between 120 and $220 \mathrm{mg} / 1$ (Figure 5). For System 1, the first stage effluent COD was also generally between 120 and $220 \mathrm{mg} / 1$ when operating under a 30- or 15-day SRT. Shorter SRT periods of 12 and 9 days, however, caused an increase to about $250 \mathrm{mg} / 1$.

TOC effluent concentrations generally followed the COD trends but to a lesser magnitude of variability and with no large isolated spikes. Effluent $\mathrm{BOD}_{5}$ concentrations were usually very low (less than $10 \mathrm{mg} / 1$ ), even at the lower sludge ages. Overall, the second stages removed relatively small additional quantities of $\operatorname{COD}$ and TOC. However, high concentrations, or spikes, from the first stage reactors were dampened considerably by the second stages.

The prime purpose of the second stage reactors was to achieve ammonia removal through nitrification. Figures 6 and 7 show influent and effluent ammonia concentrations during the study. Although not shown on the graphs, increased nitrite and nitrate production was observed to follow increased 
FIGURE 2

SYSTEM 1

INF + EFF SOD

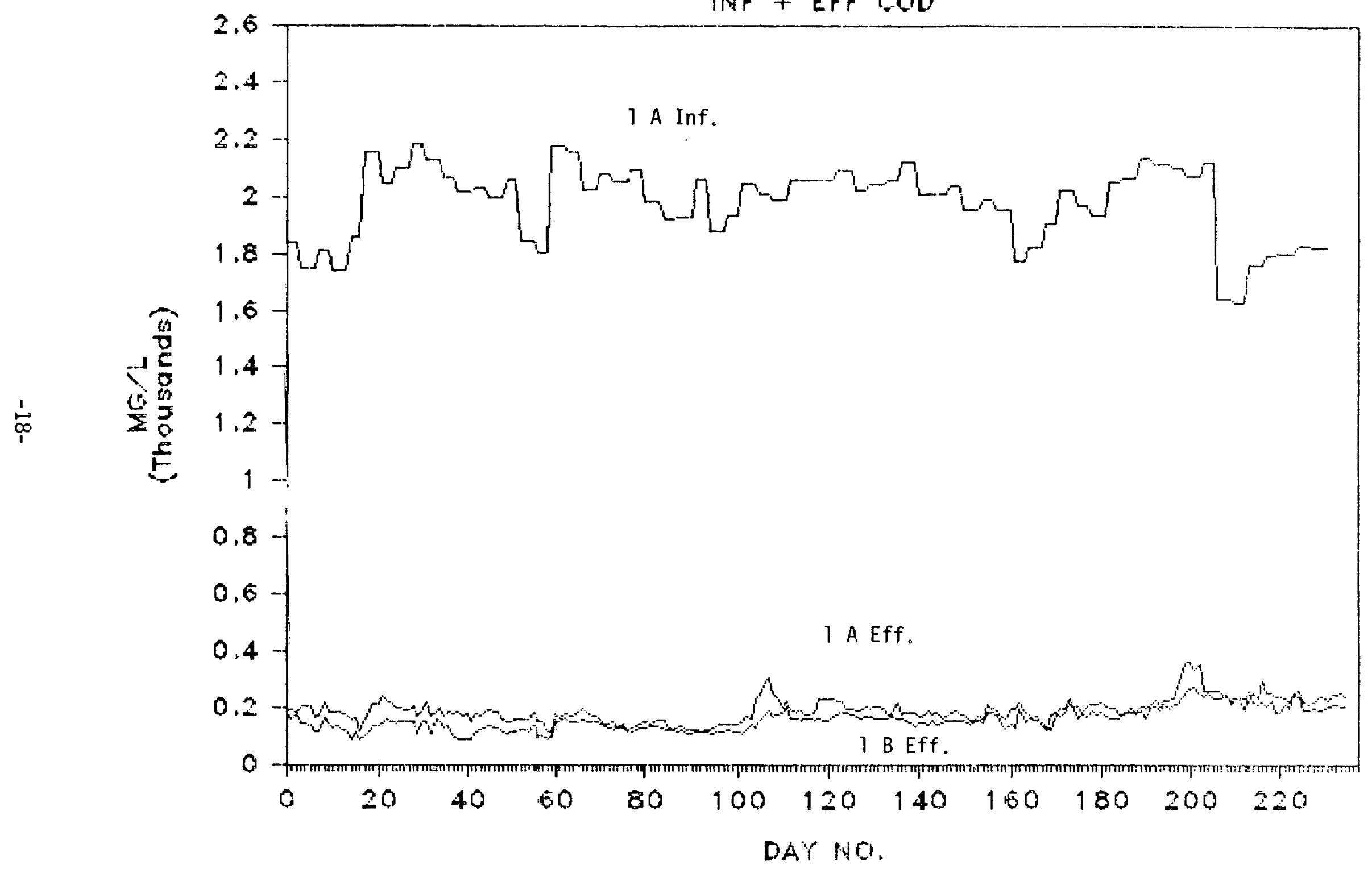


FIGURE 3

SYSTEM R

INF + EFF COD

岕

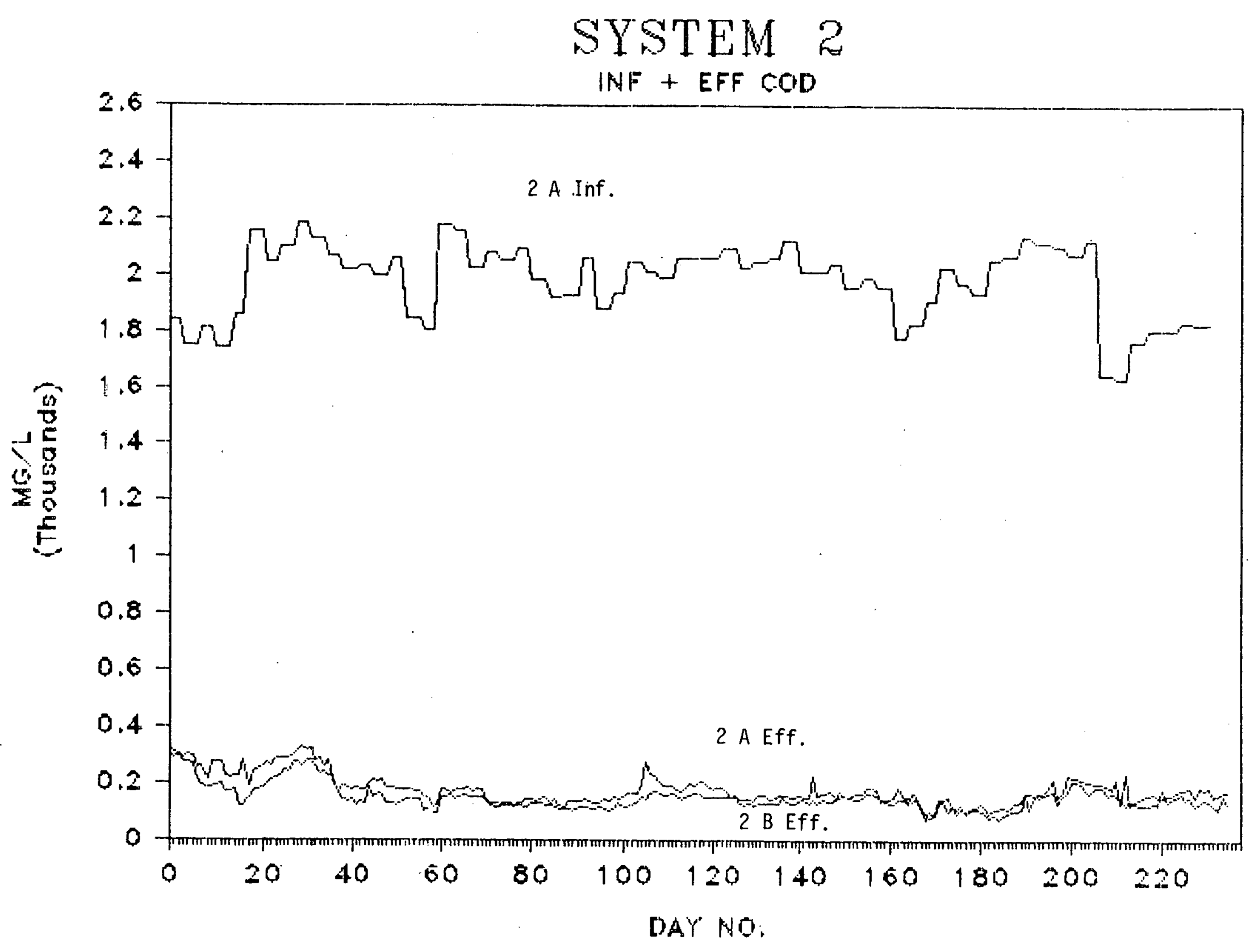


FIGURE 4

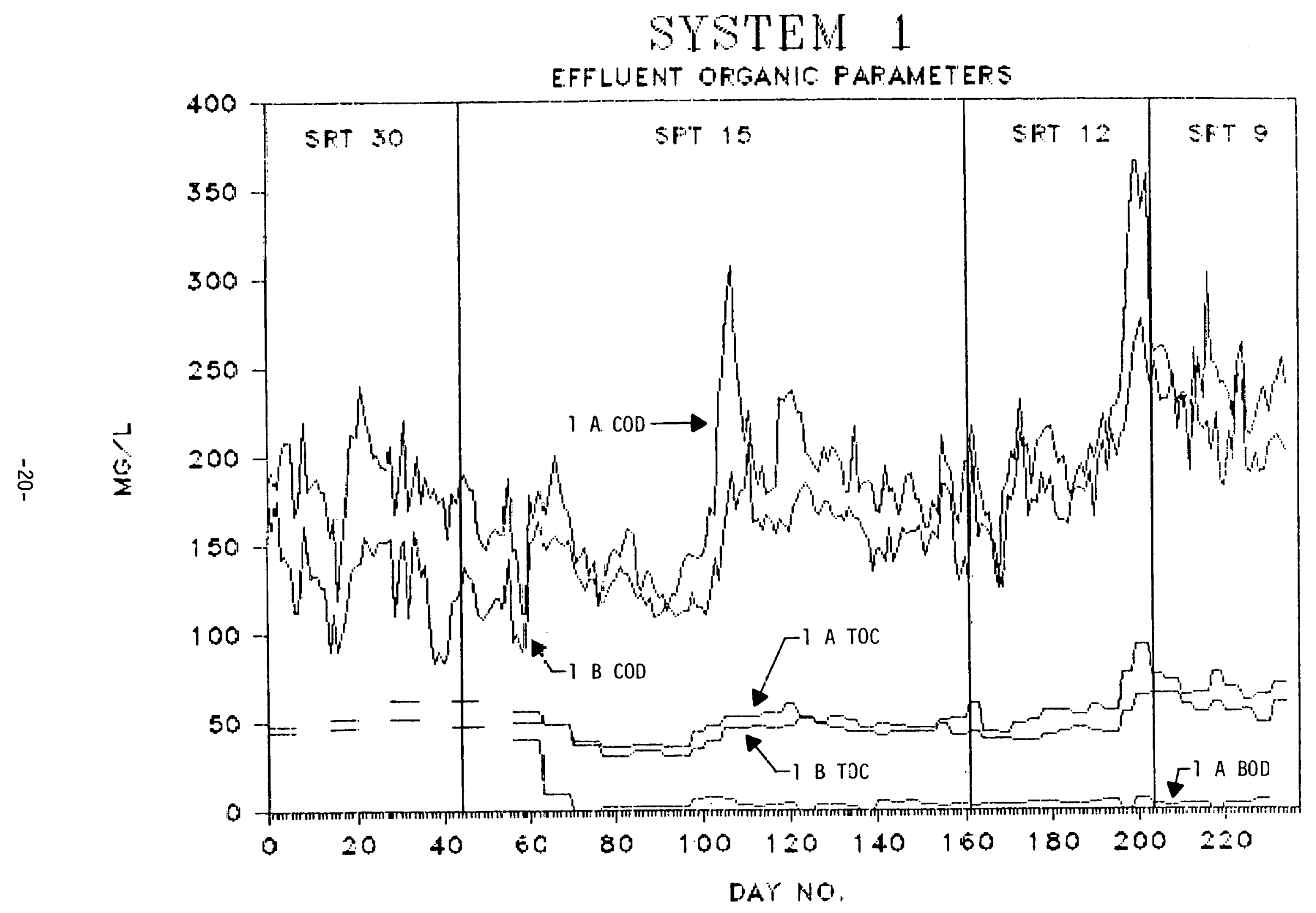


FIGURE 5

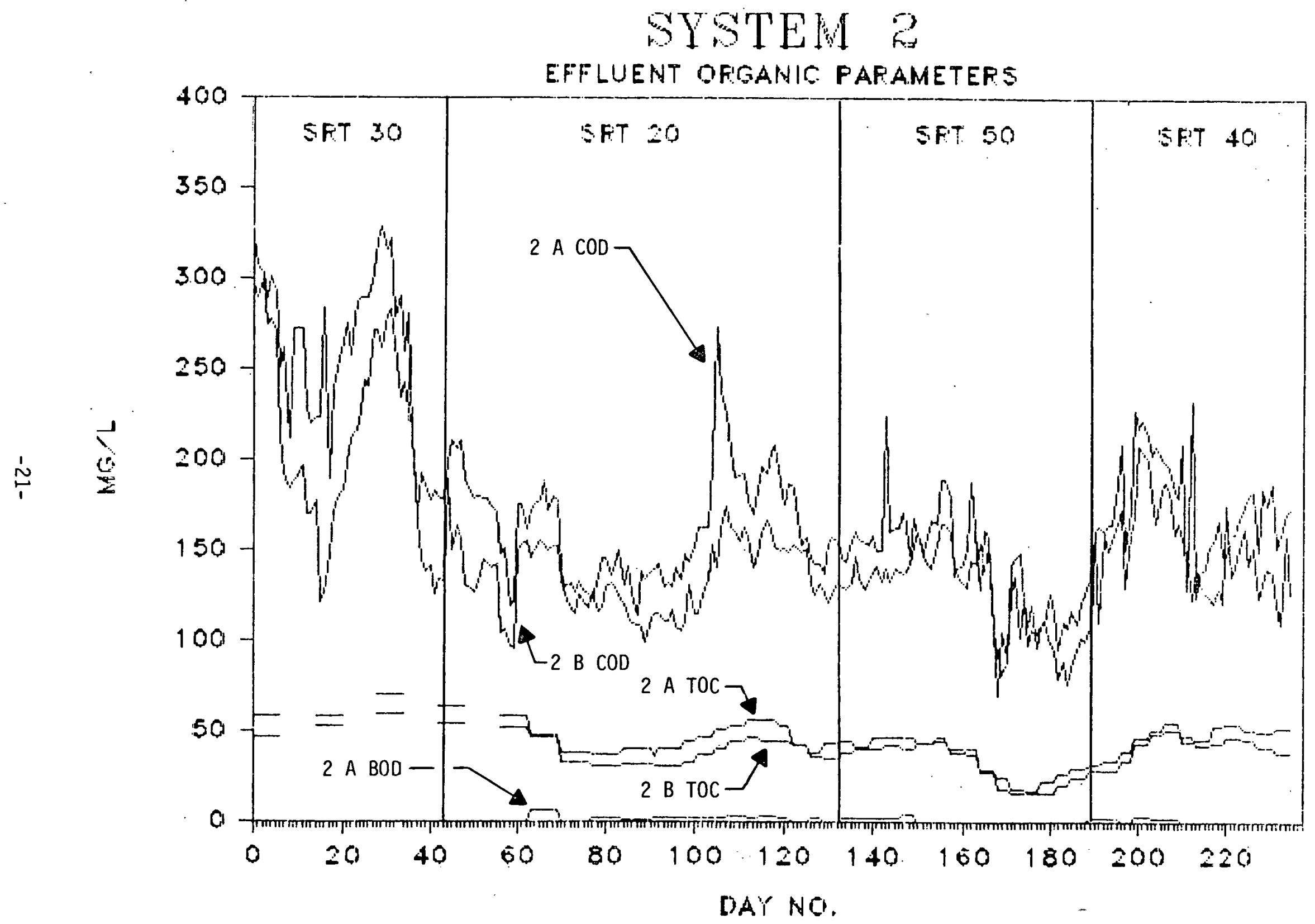


FIGIJRE 6

\section{SYSTEM 1}

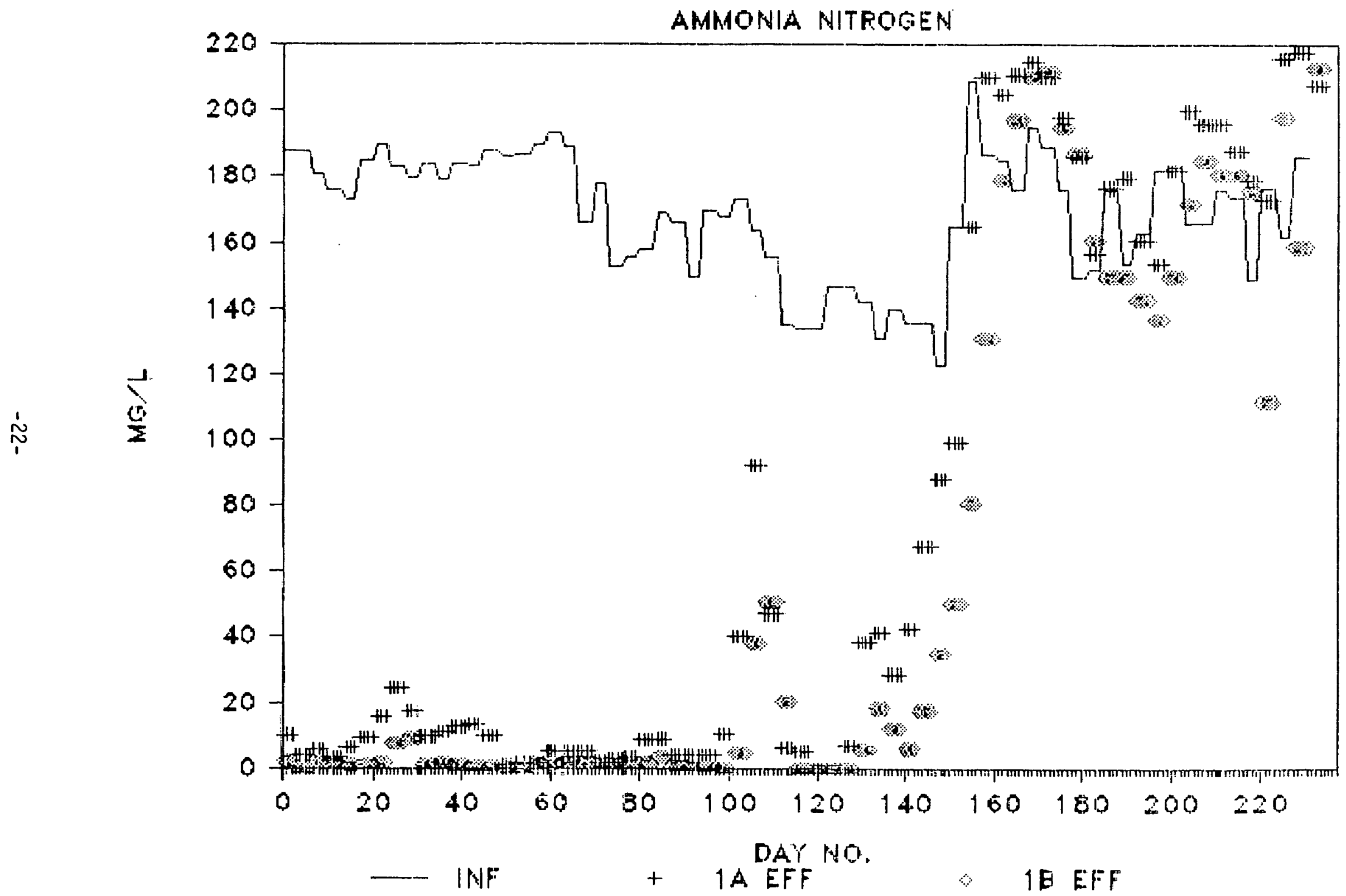


FIGURE 7

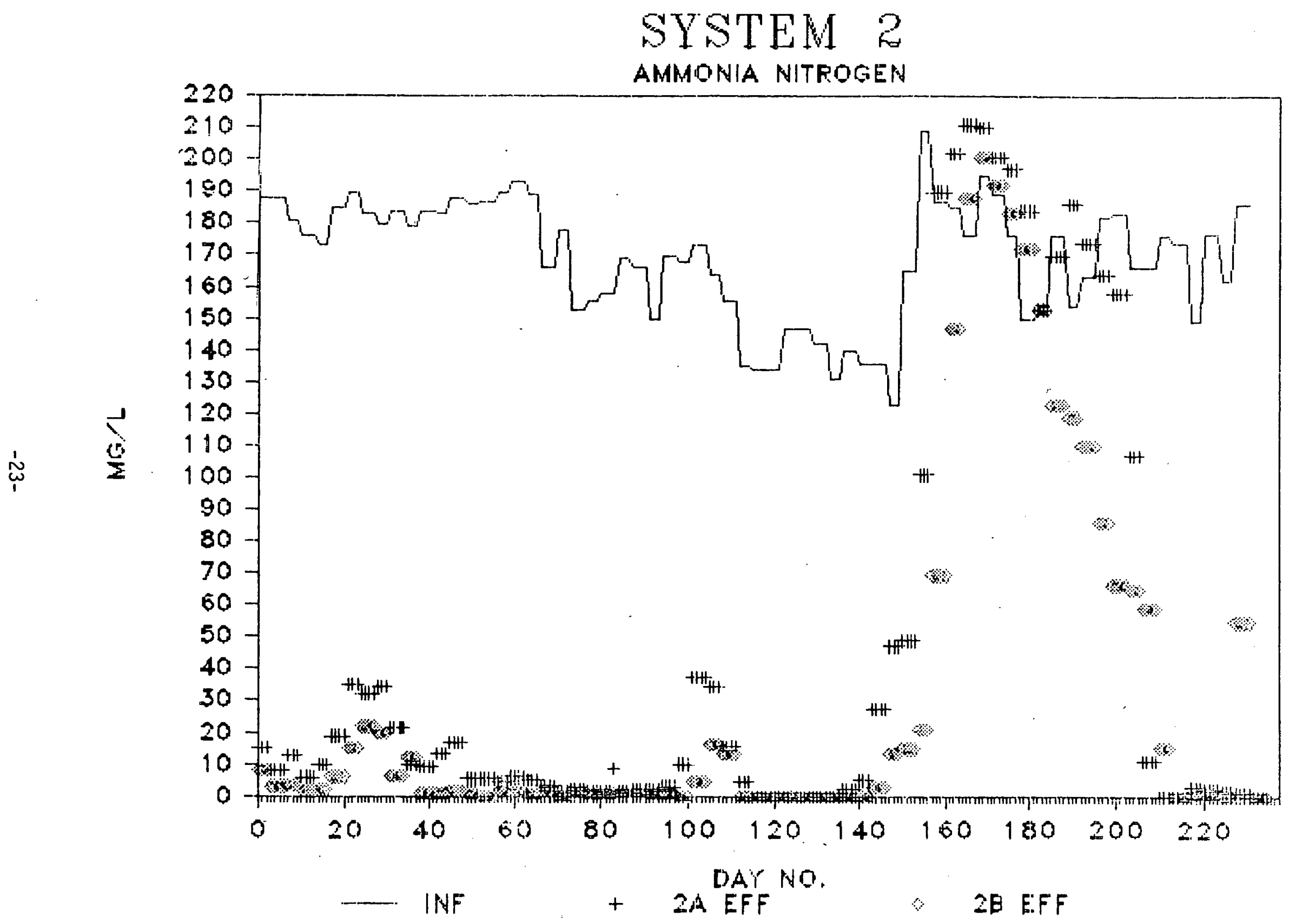


ammonia removal, which is indicative of nitrification. It is evident that most of the nitrification occurred in the first stages. The second stages provided only minimal additional removals and were not more resistant to upset conditions. Note that effluent concentrations are sometimes higher than influent concentrations as a result of the biooxidation of thiocyanate (SCN). The breakdown of this compound in the bioreactors forms ammonia as a by-product. The nitrogen balance for the system will be discussed later.

The loss of nitrification beginning on Day 120 for System 1 (15-day SRT) and Day 140 for System 2 (50-day SRT) coincided with the change from Chem Pro-prepared feed to Catalytic-prepared feed. A feed batch consisting nf one part Chem Pro-prepared feed and one part Catalytic-prepared feed was begun on Day 121 for both systems. Full strength Catalytic-prepared feed was started on Day 151. System 2 had a longer sludge age and, as would be expected, took longer to lose nitrification and recovered more quickly than system 1 . This feed change produced no discernible $i 11$ effects on the removal of any other parameters.

Figures 8 and 9 show the oxygen uptake rates for Systems 1 and 2 , respectively. They are generally in the $20-50 \mathrm{mg} / 1 / \mathrm{hr}$. range in the first stage reactors, when nitrification is occurring, and drop to about 10 to $20 \mathrm{mg} / 1 / \mathrm{hr}$. after nitrification is inhibited. The initial 30-day SRT periods show a greater variability than later operaliuns.

Figures 10 and 11 show weekly averages of the mixed liquor volatile and suspended solids concentrations. The trends are consistent with the sludge ages imposed. Note the increase in the nonvolatlle (primarily inorganic) fraction over the course of the study. (The SS/VSS ratio increased from 0.5 to 0.9$)$. Previous to this study, nutrient phosphorus had been added during the feed preparation, resulting in the formation of a precipitate that was settled and removed prior to use of the feed. (For more detalis, see reference 1.) Direct application of that mode of operations would result in the generation of a separate sludge. This would require additional sludge 


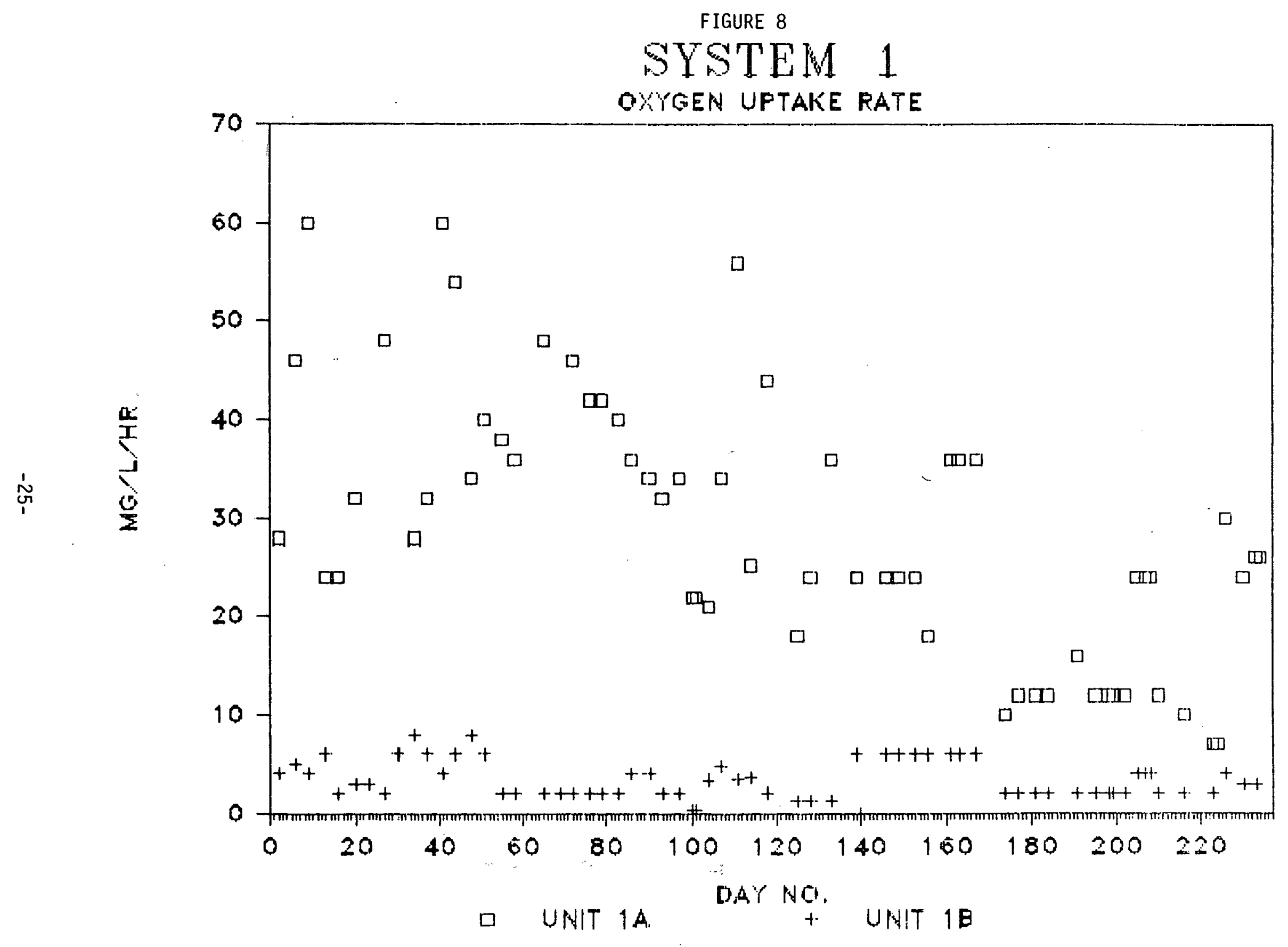


FIGURE 9

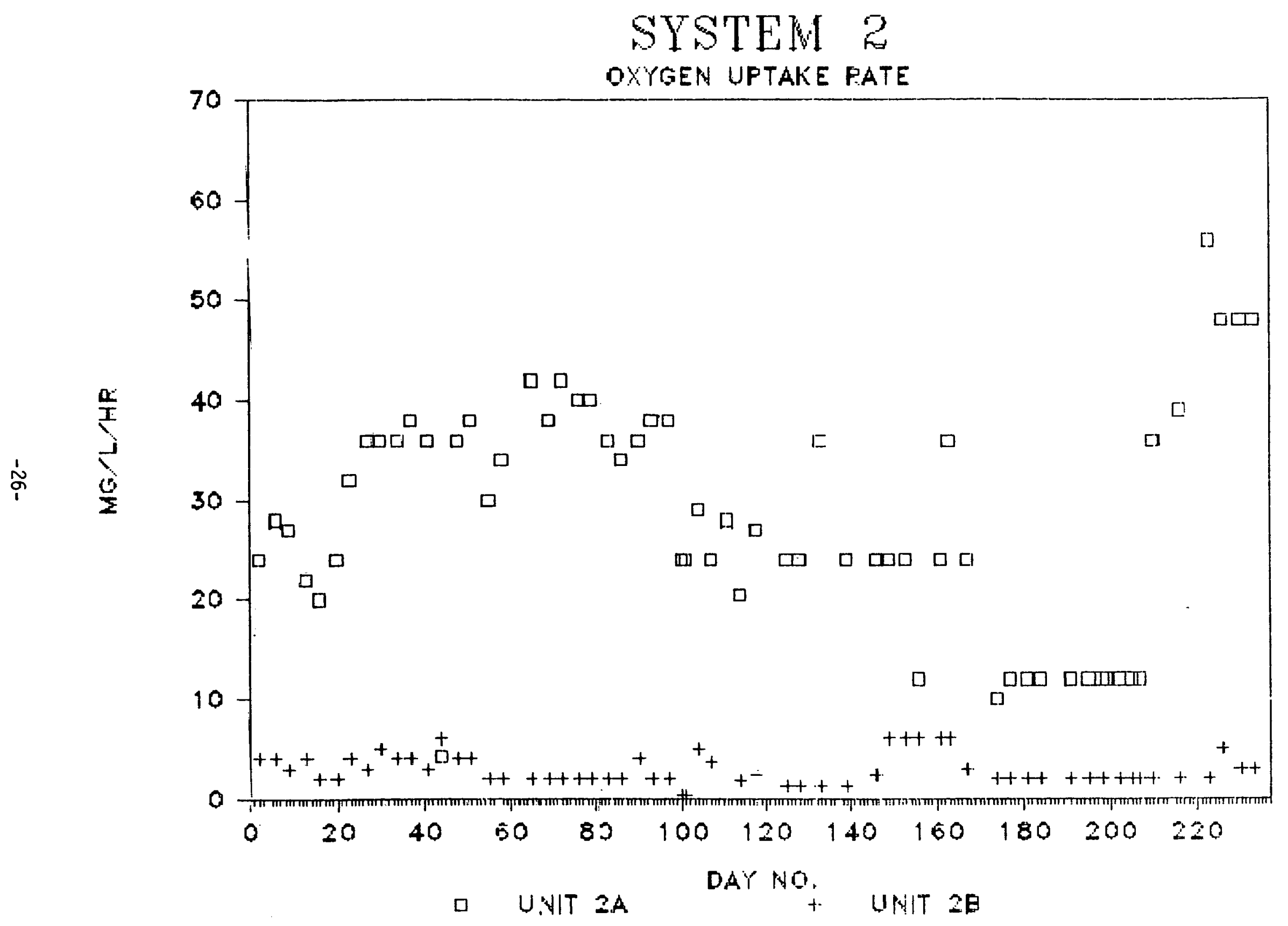




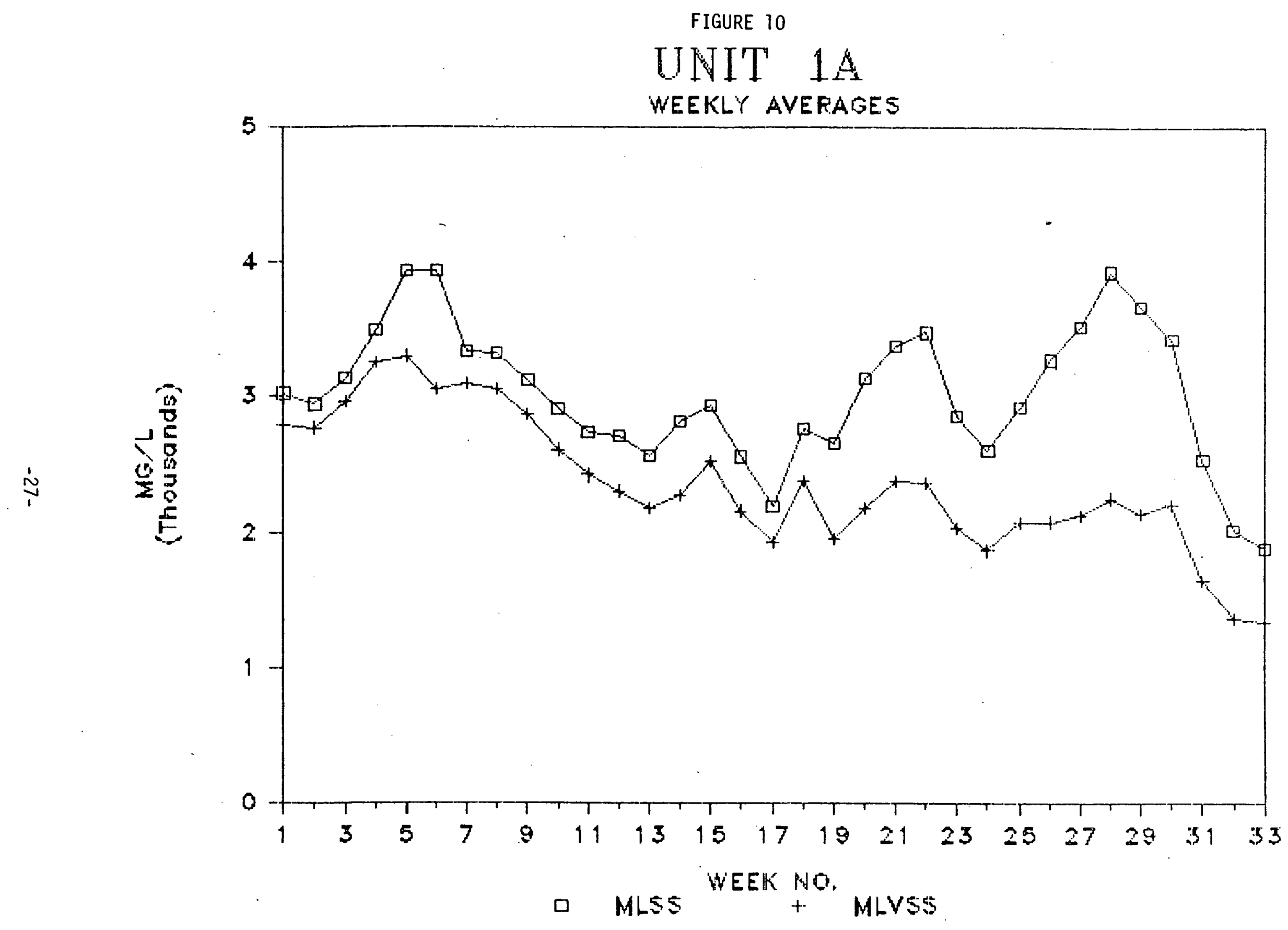




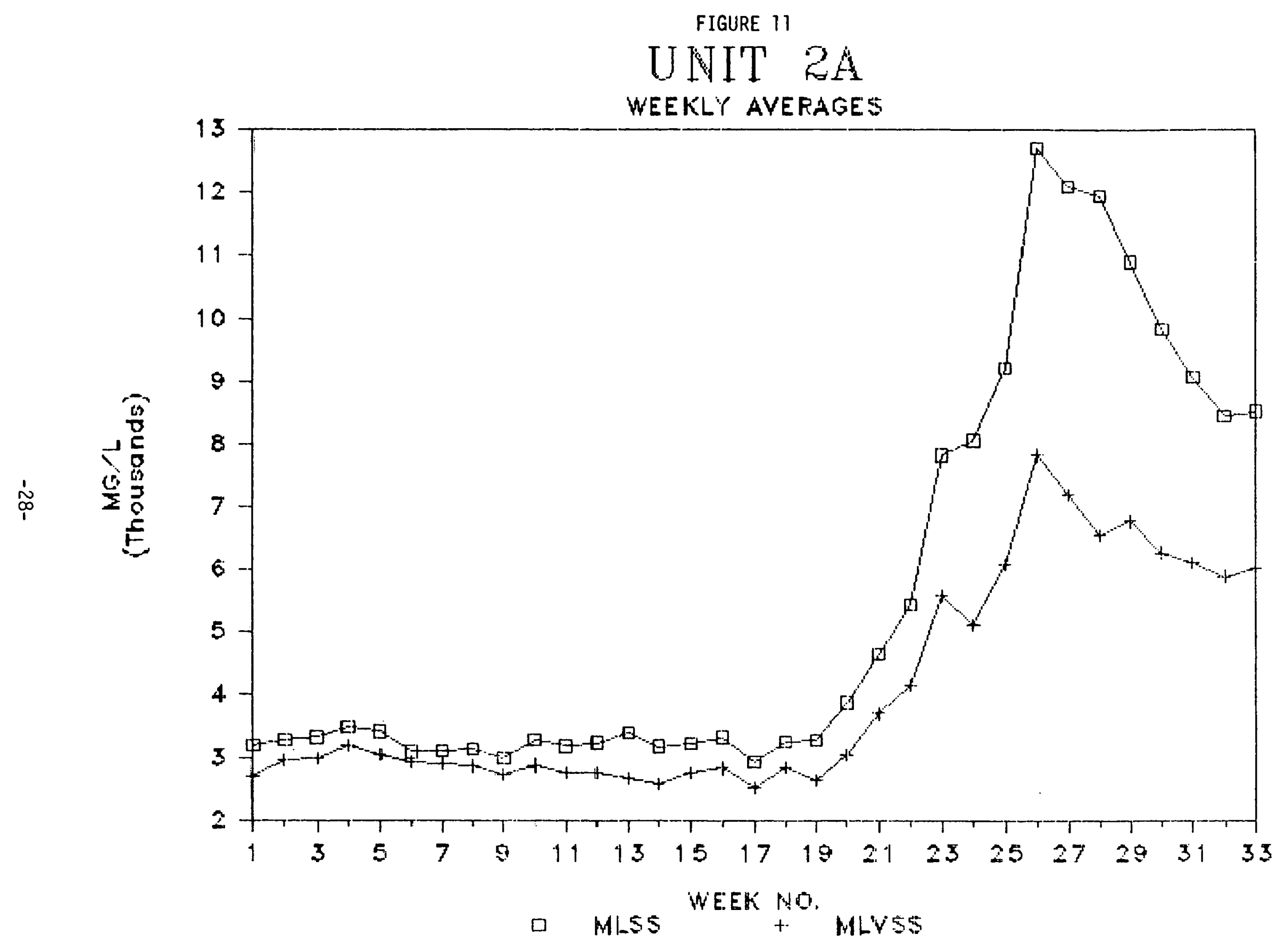


handling equipment, not included in the current design. To more closely simulate the design conditions, the nutrient phosphate for this study was added directly to the aeration basin. This did not adversely affect the operation of the bioreactors but did form an inorganic precipitate that gradually increased the SS/VSS ratio, as shown. 


\section{RESULTS AND DISCUSSION}

The reactors were operated from July 26, 1983, to March 16, 1984. The feed and effluent streams to and from each reactor were analyzed daily. In addition, standard operating parameters were monitored to define the overall operating conditions. Ali of this data is included in the Appendix, Tables $A-1$ to $A-4$.

\section{STEADY-STATE CONOITIONS}

The first step in evaluating the treatability data is to determine the periods of operation that are considered steady-state. For this report. steady-state was defined as period of at least two weeks duration, during which the sludge age, mixed liquor volatile solids, and organic removal efficiencies were relatively constant. Figures 12 and 13 are time series plots of these parameters for the first stage reactors (1A and $2 A$ ). Based on the above criteria, eight steady-state periods were selected, as shown on Table 4 .

Table 4

Stcady State Operating Period

\begin{tabular}{ccccc} 
Reactor & $\begin{array}{c}\text { SRT } \\
\text { (Days) }\end{array}$ & $\begin{array}{c}\text { Duration } \\
\text { (Days) }\end{array}$ & $\begin{array}{c}\text { MLVSS } \\
(\mathrm{mg} / 1)\end{array}$ & $\begin{array}{c}\text { COD Removal } \\
(\boldsymbol{\alpha})\end{array}$ \\
\cline { 1 - 2 } & 9 & $217-234$ & 1,493 & 87.5 \\
IA & 12 & $174-195$ & 2,100 & 90.6 \\
IA & 15 & $72-103$ & 2,338 & 93.4 \\
IA & 15 & $124-161$ & 2,229 & 91.5 \\
IA & 20 & $70-100$ & 2,687 & 93.5 \\
IA & 30 & $1-45$ & 3,026 & 91.7 \\
$2 A$ & 40 & $213-226$ & 6,163 & 91.4 \\
$2 A$ & 50 & $169-189$ & 6,354 & 94.6
\end{tabular}


FIGURE 12

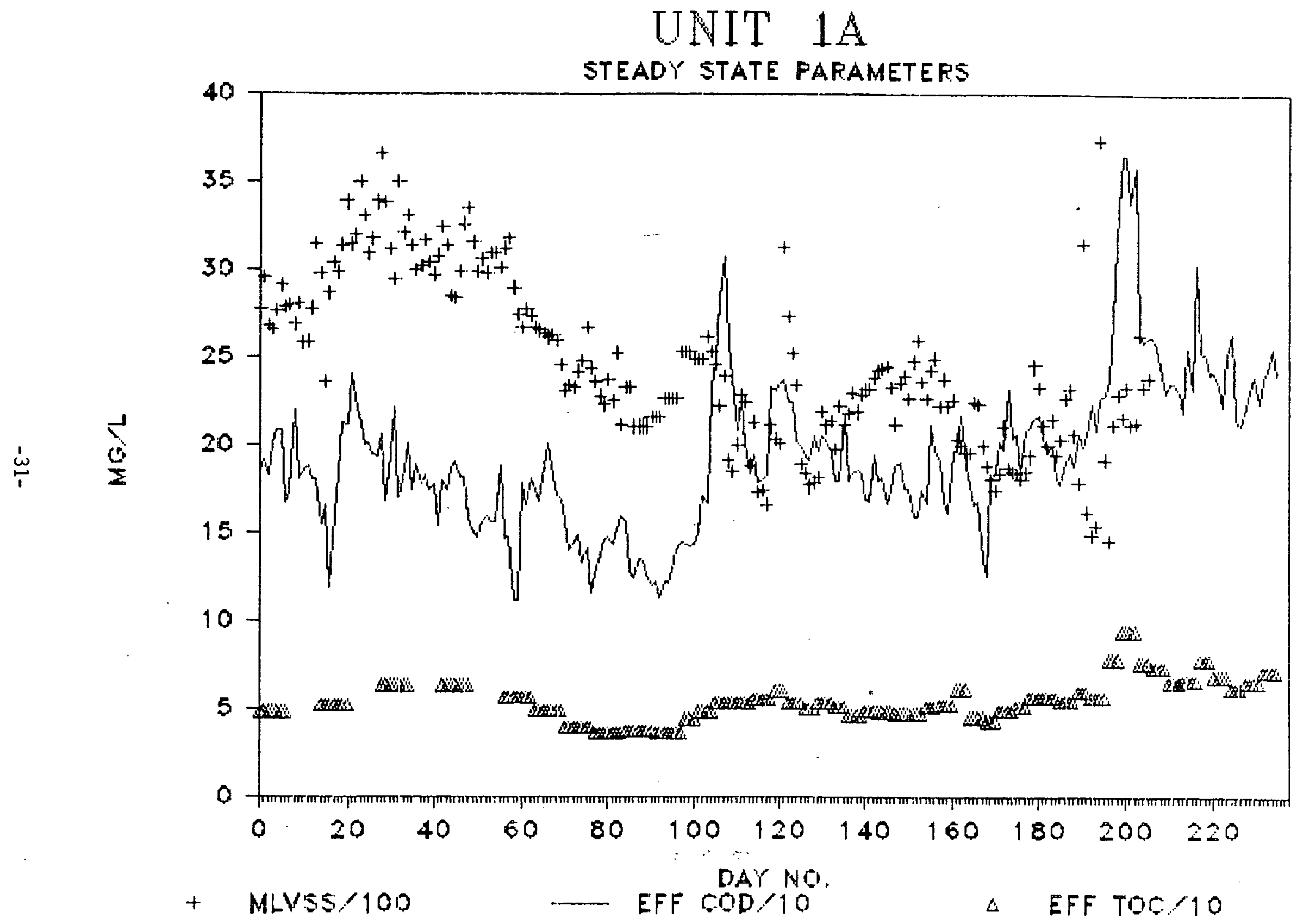


FIGURE 13

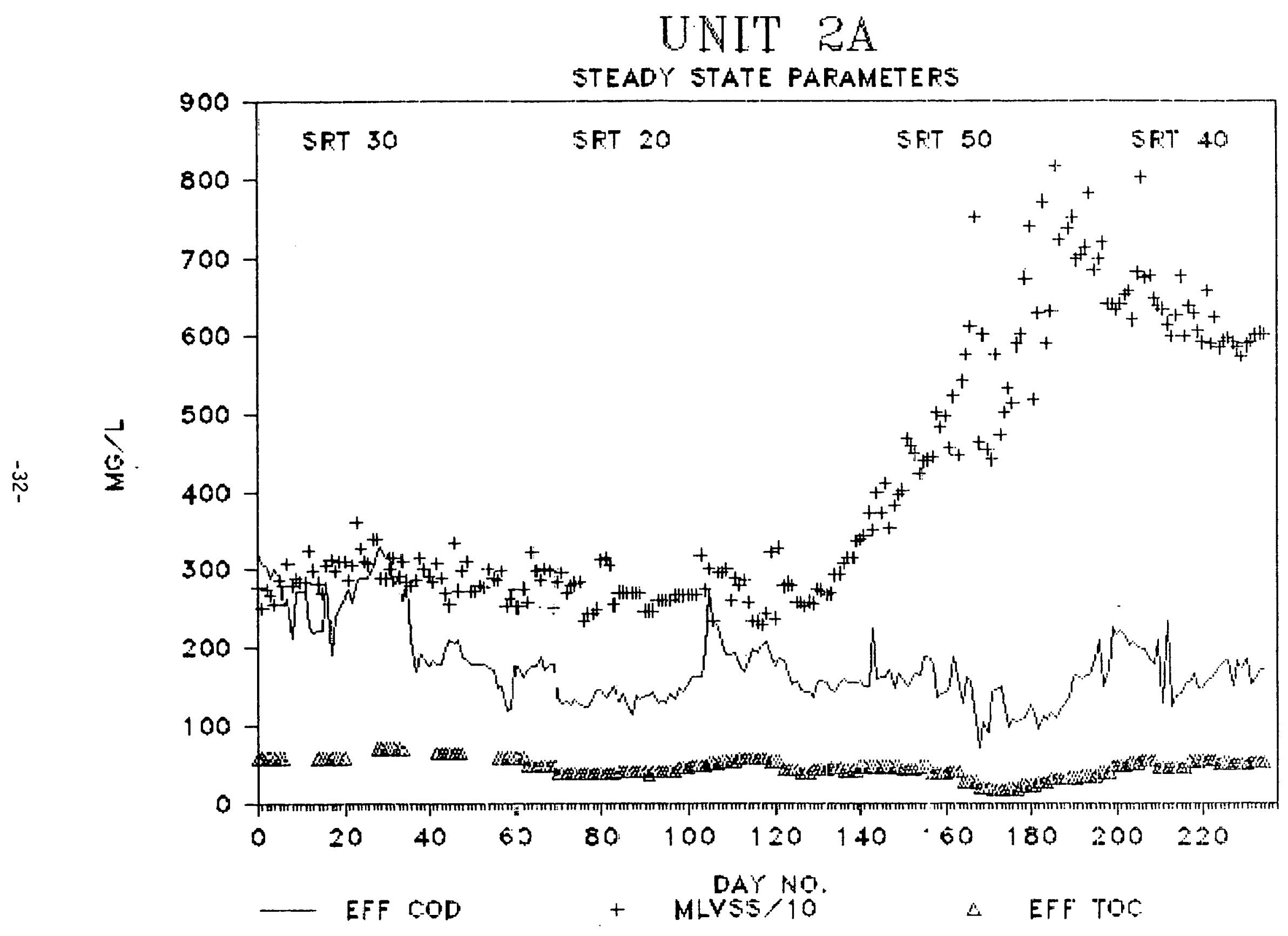


As shown on Table 4, there are two steady-state conditions listed for the 15-day SRT. The second one occurred after an upset in the reactor, and although stabilized, the unit did not achieve the performance levels of the earlier period. Complete treatability data for the steady-state periods are summarized in the Appendix, Table A-5.

\section{APPLICABILITY OF KINETIC MODEL}

Each bench-scale system consisted of two reactors, or units, connected in series. The first unit in each system provided the bulk of the organic removal. The data generated by these reactors (Units $1 A$ and $2 A$ ) are the basis of the kinetic model.

The equations for the Lawrence and McCarty model are straightforward. However, one parameter, substrate, requires clarification. Substrate is the food (energy) source required to sustain the biochemical reactions in the bioreactors. It is classically measured by the BOD test, itself dependent on biological activity. Use of $\mathrm{BOO}_{5}$ measurements can pose a problem because the difference between influent and effluent concentrations may not provide an accurate indication of the actual substrate utilization. As found in an earlier study on SRC-I wastewater (1), significant drops in COD can occur in a biological system without an apparent change in 800 because so-called refractory (non-biodegradable) compounds can be gradually broken down in the reactors, thereby reducing $\mathrm{COD}$. This biodegradation does not occur in the $B 00$ sample bottle, however. meaning the measured influent substrate level may be artificially low.

Use of $C O D$ or TOC in place of $B O D$ also presents a problem. Some of the organics in the SRC-I wastewater are truly refractory and simply not amenable to biological treatment. As shown on Figure 14, a graph of $F / M$ versus effluent $\operatorname{COD}$ for the steady-state periods does not pass through the origin. Rather, it intercepts the $x$-axis in the range of 60 to $130 \mathrm{mg} / 1$. The 
FIGURE 14

Refractory COD

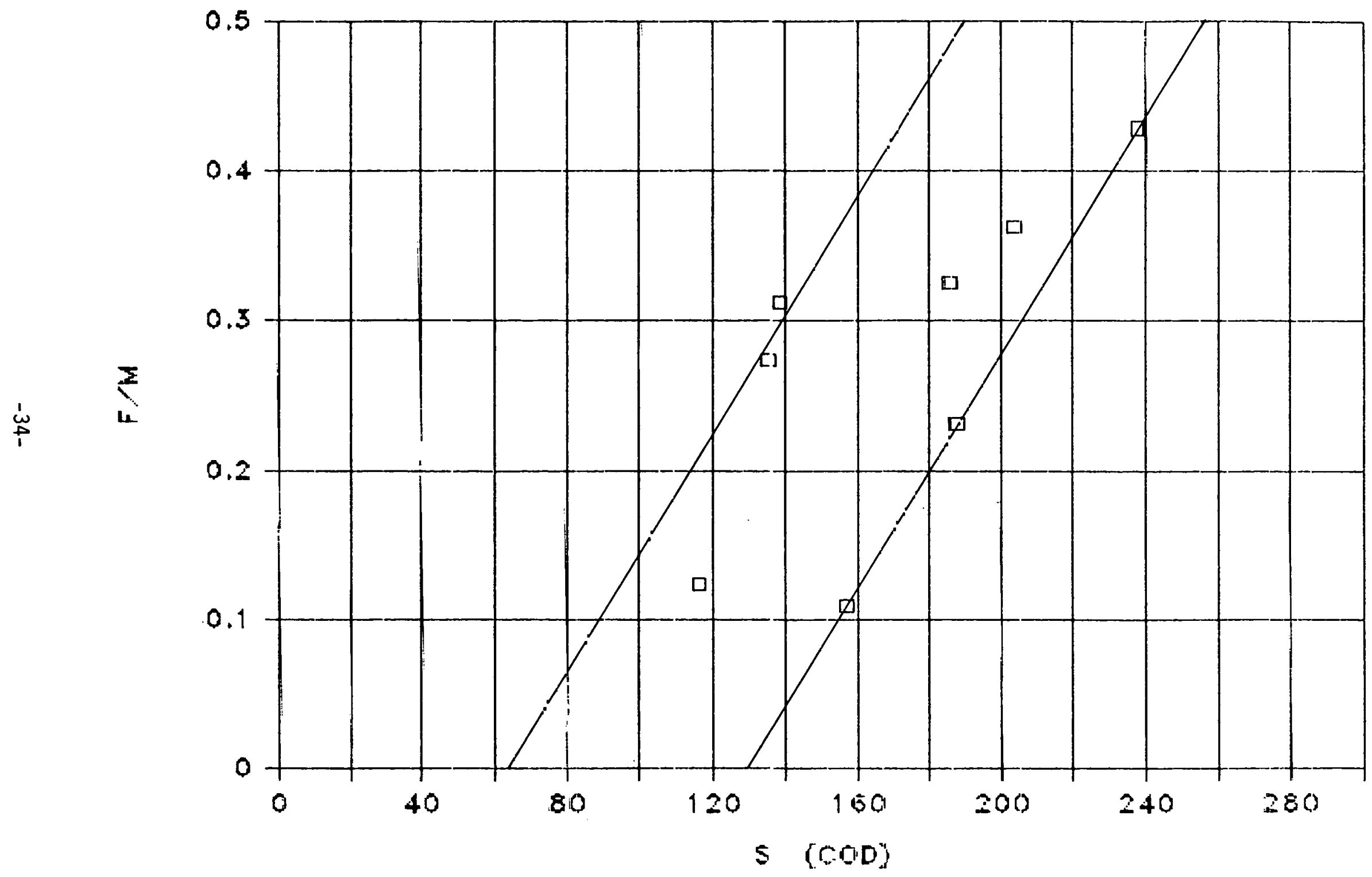


intercept value represents the refractory organic concentration, in that this quantity remains even at the lowest possible loading. Similar effects have been noted by others (6). The Lawrence and McCarty model does not account for this and assumes the curve would pass through the origin.

In this study, the kinetic coefficients are developed in terms of $B O D$, COD, and TOC, in order to see which parameter best fits the model.

\section{EVALUATION OF KINETIC COEFFICIENTS}

The kinetic parameters to be evaluated are the following: yield coefficient $Y$, endogenous respiration coefficient $k_{d}$, maximum substrate removal $k$, and the substrate concentration $k_{s}$, corresponding to a removal rate of one-half the ultimate rate. For a given waste stream, these parameters are constants and can be used to define the performance of a biological treatment system.

To determine these constants, bench-scale reactors were operated over a range of SRT values. The feed streams to the reactors were held as nearly constant as practical throughout the study, and the SRT's. were varied by controlled wastage of the mixed liquor solids. Values of $Y$ and $k_{d}$ are calculated from a straight line plot of $1 / S R T$ versus $F / M$, as was presented in Equation 1 (Section IV). Values of $k$ and $k_{s}$ are defined by Equation 2 . This equation can be linearized in the following form:

$$
\frac{1}{F / M}=\frac{K}{k} \frac{1}{S}+\frac{1}{k}
$$

This shows a straight line relationship between the reciprocals of $F / M$ and effluent substrate $(S)$, with a slope of $K_{s} / k$ and an intercept of $1 / k$. 
BOD Basis

Figure 15 is a graph of Equation 5 where the substrate (S) is the effluent $\mathrm{BOD}_{5}$ concentration. Equilibrium $\mathrm{BOO}_{5}$ values for all steady-state periods studied during this investigation were typically very low. Effluent concentrations for the 40- and 50-day SRT periods were too low to be quantified via the 800 test and are not included in Figure 15 . From this graph, the maximum substrate removal rate, $k$, is 0.26 pounds $\mathrm{BOD}_{5}$ per day per pound MLVSS, and the half-rate constant, $K_{5}$, is $0.5 \mathrm{mg} / 180 \mathrm{O}_{5}$.

A plot of Equation 1 for $\mathrm{BOO}_{5}$ is shown on Figure 16. All eight steady-state periods fit the model reasonably well. However, the 40 - and 50-day SRT periods were discounted from impacting the kinetic coefficients $Y$ and $k_{d}$ since these periods were not used to determine the other coefficients, $k$ and $k_{s}$. The yield coefficient, as determined from figure 16 (excluding 40- and 50-day SRT results), is 0.41 pounds biological solids produced per pound $\mathrm{BOD}_{5}$ removed, and the decay constant is 0.01 days ${ }^{-1}$.

\section{COD Basis}

Equation 5 for $C O D$ is presented as Figure 17. It is apparent that two data points, corresponding to the 40- and 50-day SRT periods, do not correlate to a linear fit. The reason these data points do not fit the model is unknown. However, the remaining six steady-state periods fit reasonably well and were used to evaluate the kinetic parameters. As determined from Figure 17. the maximum COD removal rate, $k$, is 0.56 pounds per day per pound MLVSS, and the half-rate constant, $K_{s}$, is $140 \mathrm{mg} / 1 \mathrm{COD}$.

Figure 18 is a plot of Equation 1 for COD. Although all eight periods appear usable, the two data points corresponding to the 40- and 50-day SRT were discounted because of the discrepancy noted in Figure 17. Values for $Y$ and $k_{d}$ from Figure 18 are 0.37 pounds biological solids produced per pound COD removed and 0.05 days $^{-1}$, respectively. 
FIGURE 15

Determination of $\mathrm{K}_{\mathrm{s}}$ and $\mathrm{k}, \mathrm{BOD}_{5}$ Basis

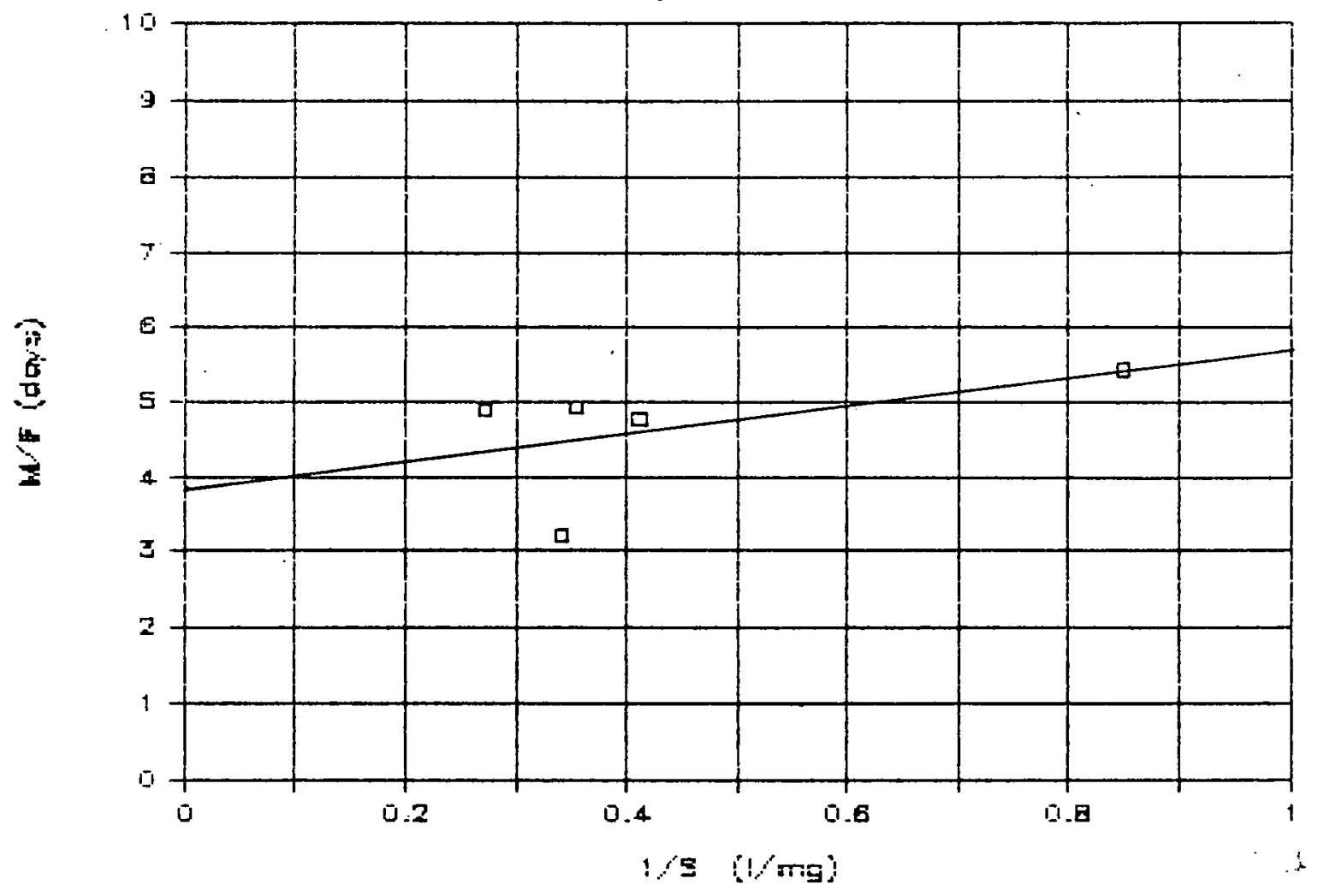

FIGURE 16

Determination of $Y$ and $k_{d}, 80 D_{5}$ Basis

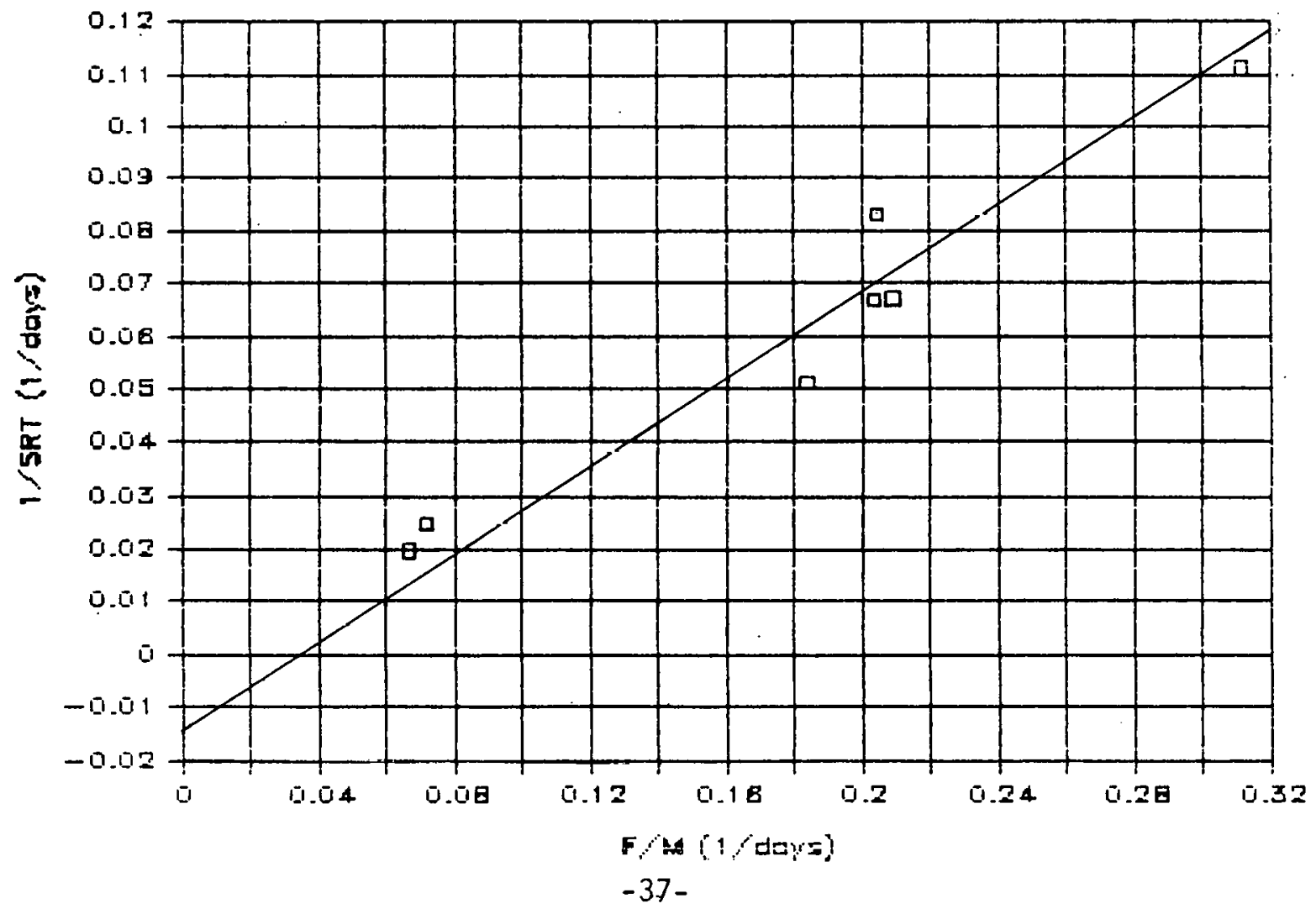


FIGURE 17

Determination of $k_{S}$ and $k$, COD Basis

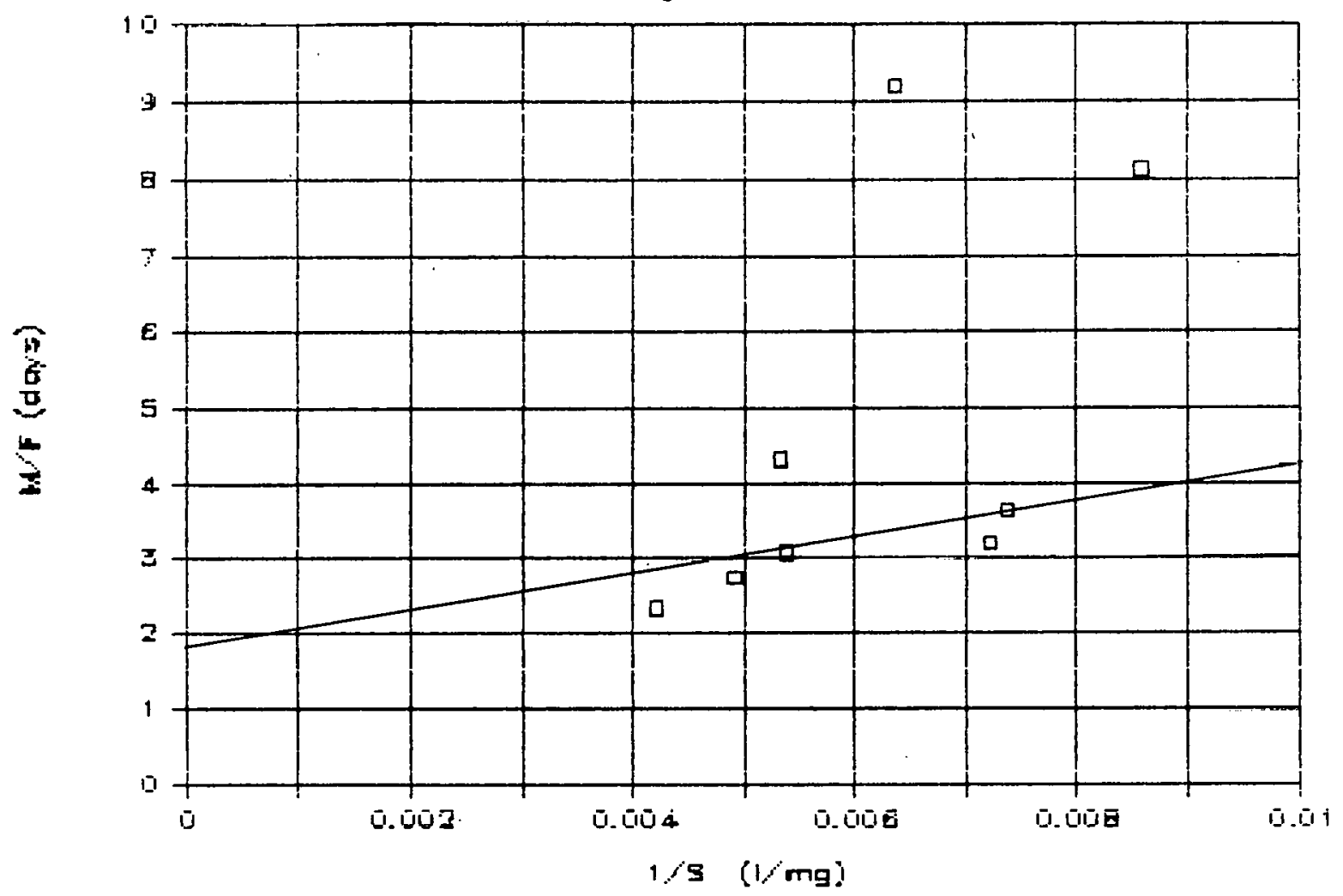

FIGURE 18

Determination of $Y$ and $k_{d}$, COD Basis

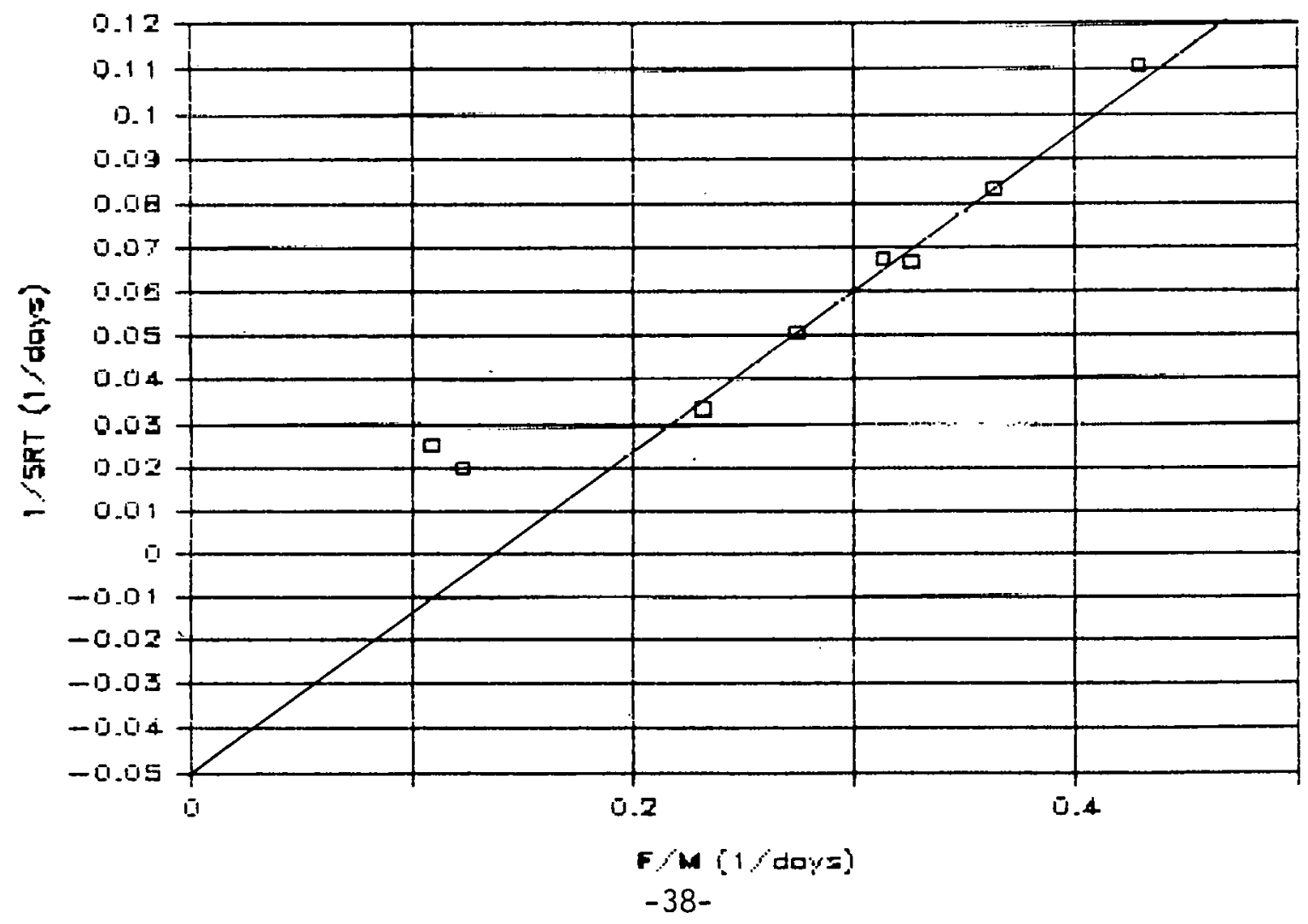




\section{TOC Basis}

Graphs for TOC as the measured substrate are shown on Figures 19, and 20 .

In Figure 19, two data points which appear as obvious outliers again correspond to the 40- and 50-day SRT periods and were not considered in evaluating any of the kinetic parameters for TOC. The four kinetic constants, $k, K_{s}, Y$, and $k_{d}$, as determined from Figures 5 and 6 are, respectively. 0.15 pounds TOC per day per pound MLVSS, $27 \mathrm{mg} / 1$ TOC, 1.22 pounds biomass produced per pound TOC removed, and 0.06 days $^{-1}$.

\section{COMPARISON OF KINETIC COEFFICIENTS}

The kinetic constants developed by applying the Lawrence and McCarty model to $800, C O D$, and TOC data are summarized in Table 5. These values can be utilized in Equation 3 (previously presented) to predict achievable effluent concentrations at any selected SRT. Figures 21,22 and 23 show the relationship between SRT and effluent substrate concentrations under steady-state performance for $800, C O D$, and $T O C$, respectively. In these figures, the data points plotted are the mean effluent values observed during each SRT period investigated. The lines in Figures 22 and 23 for COO and TOC represent the effluent values predicted by the model (Equation 3 ). The line for BOD could not be evaluated, as is discussed below.

\section{Table 5}

Summary of Lawrence and McCarty Kinetic Coefficients

\begin{tabular}{|c|c|c|c|c|}
\hline Basis & $\begin{array}{l}\text { Yield Coeff. } \\
Y(1 \mathrm{~b} . / 1 \mathrm{~b} .)\end{array}$ & $\begin{array}{c}\text { Endogenous Respir. Rate } \\
k_{d}(\text { day }-1) \\
\end{array}$ & $\begin{array}{l}\text { Max. Rem. Rate } \\
k \quad(1 b \cdot / 1 b .- \text { day })\end{array}$ & $\begin{array}{r}s(\mathrm{k} / 2 \\
\underline{K}_{\mathrm{s}}(\mathrm{mg} / 1)\end{array}$ \\
\hline${ }^{*} \mathrm{COO}$ & 0.37 & 0.05 & 0.56 & 140 \\
\hline${ }^{*} \mathrm{BOD}$ & 0.41 & 0.01 & 0.26 & 0.5 \\
\hline *TOC & 1.22 & 0.06 & 0.15 & 27 \\
\hline
\end{tabular}

*Excluding 40- and 50-day SRT periods. 
FIGURE 19

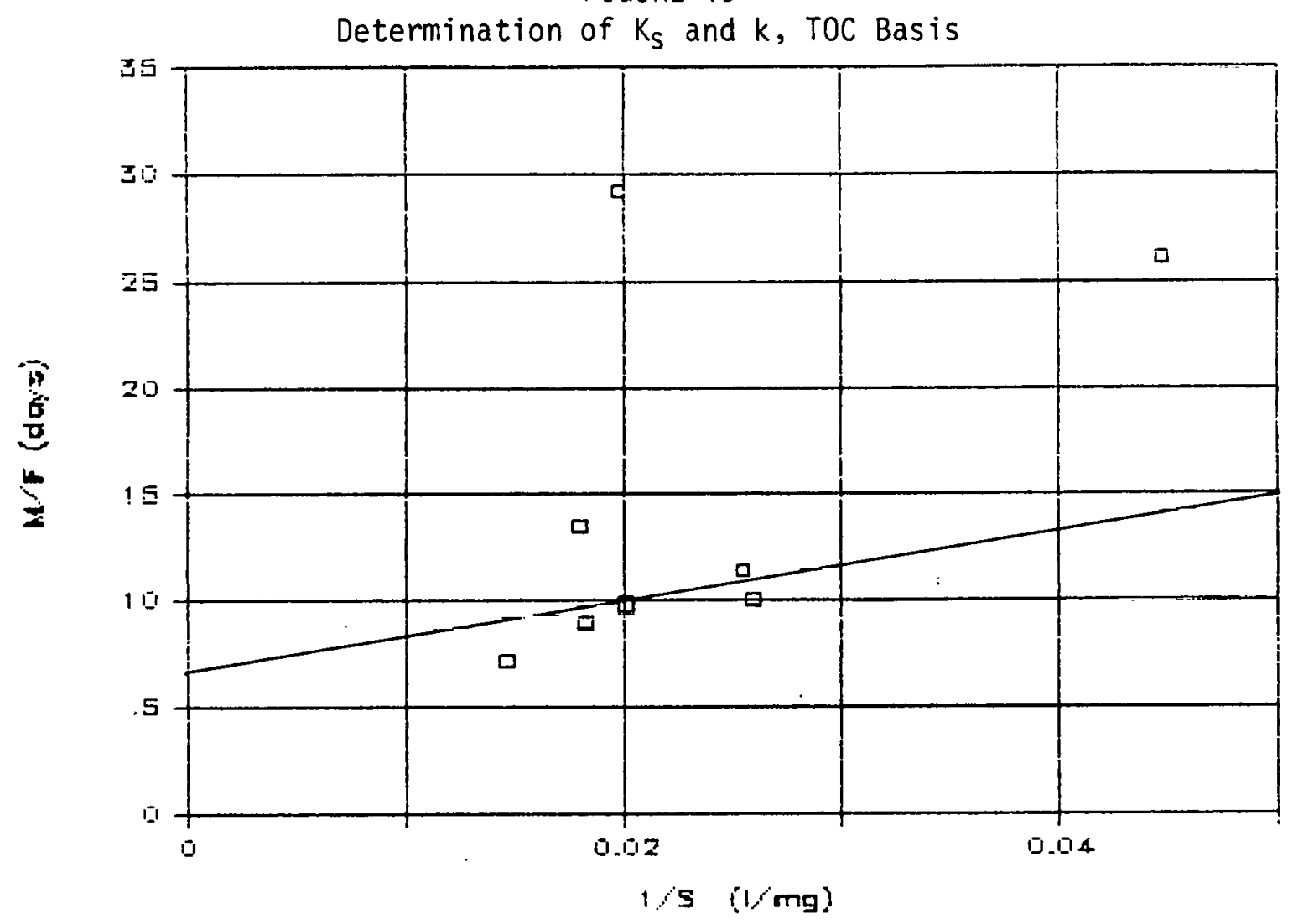

FIGURE 20

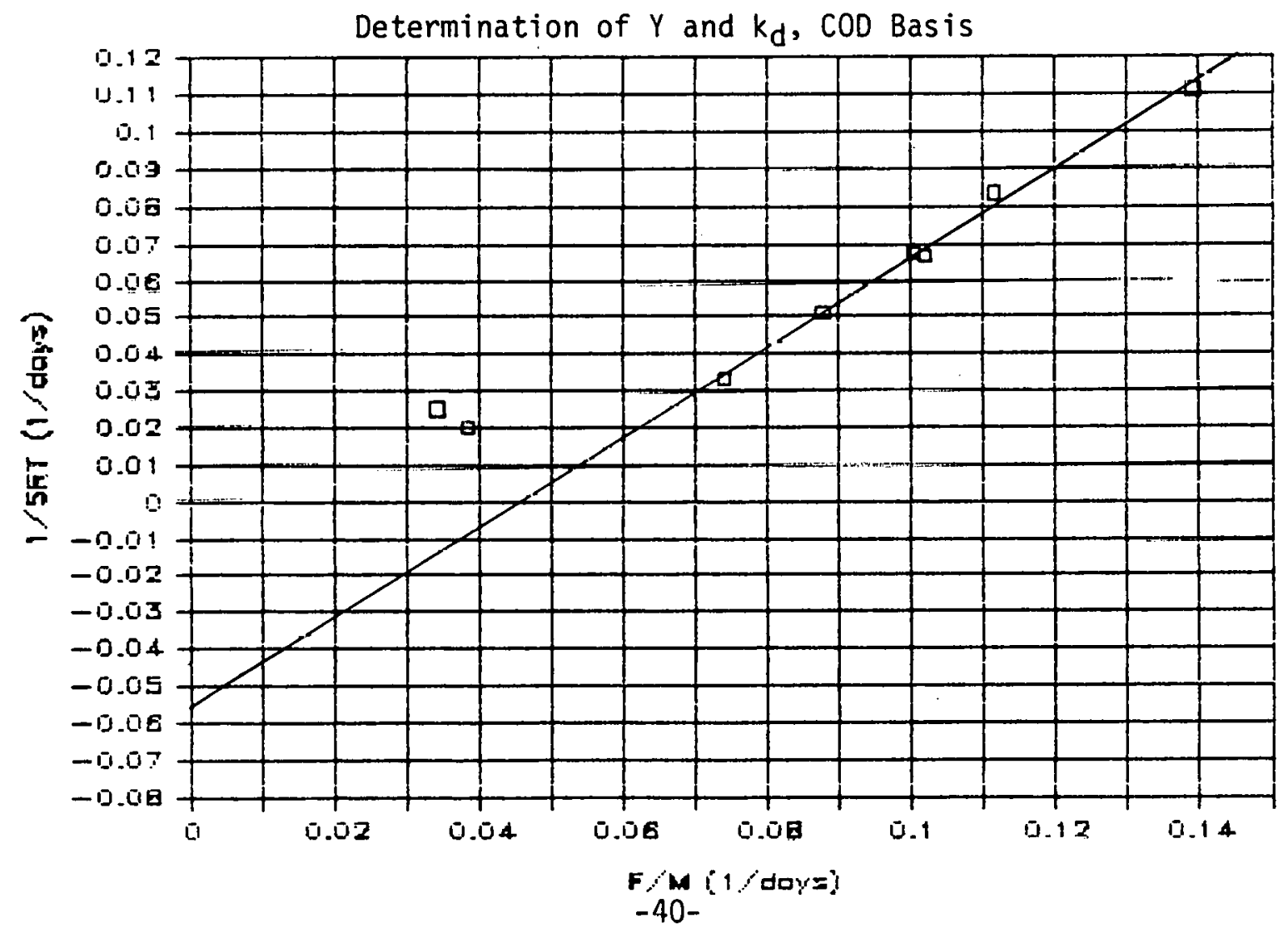


FIGURE 21

Effluent $\mathrm{BOO}_{5}$ as a Function of SRT

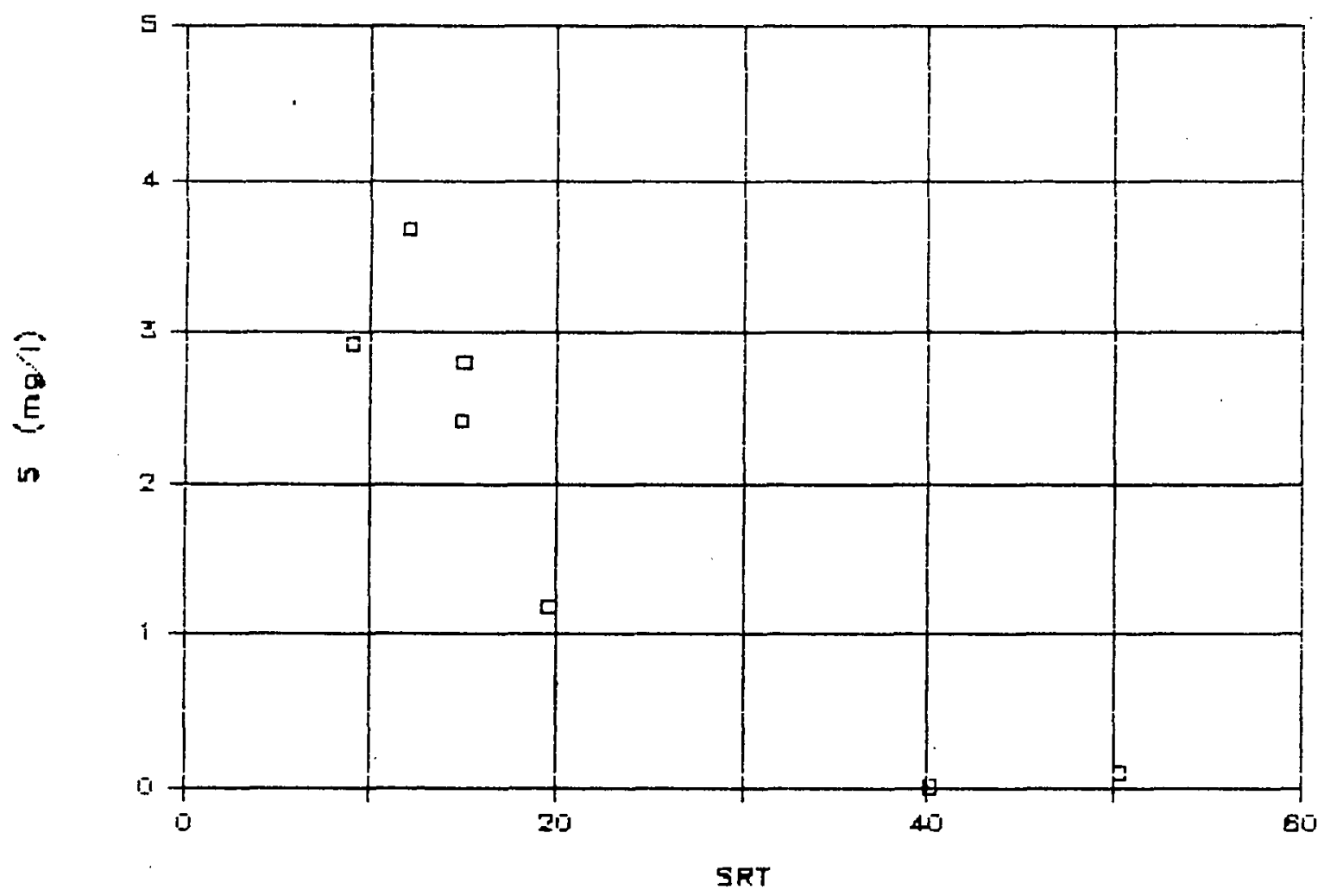

FIGURE 22

Effluent COD as a Function of SRT

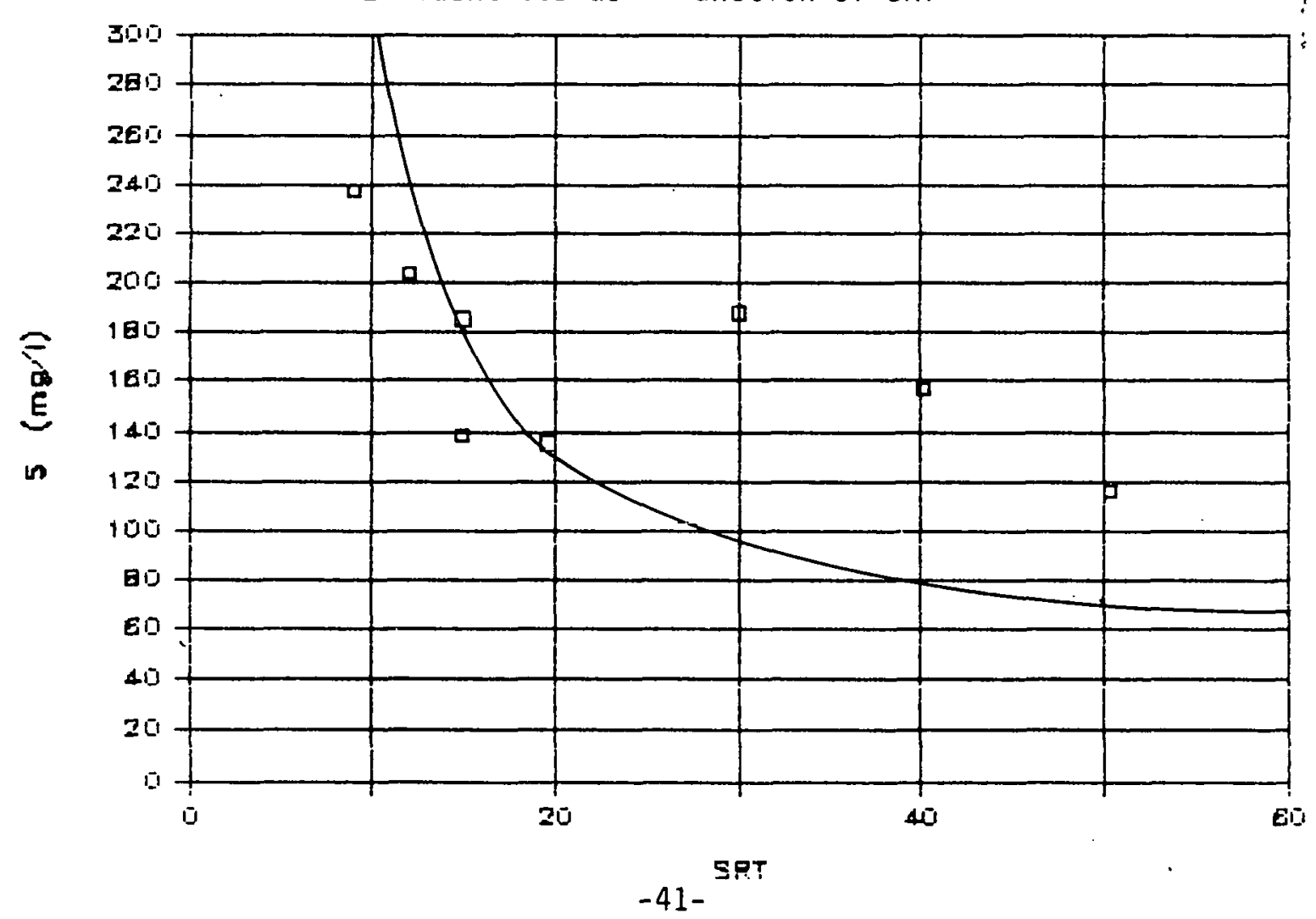


FIGURE 23

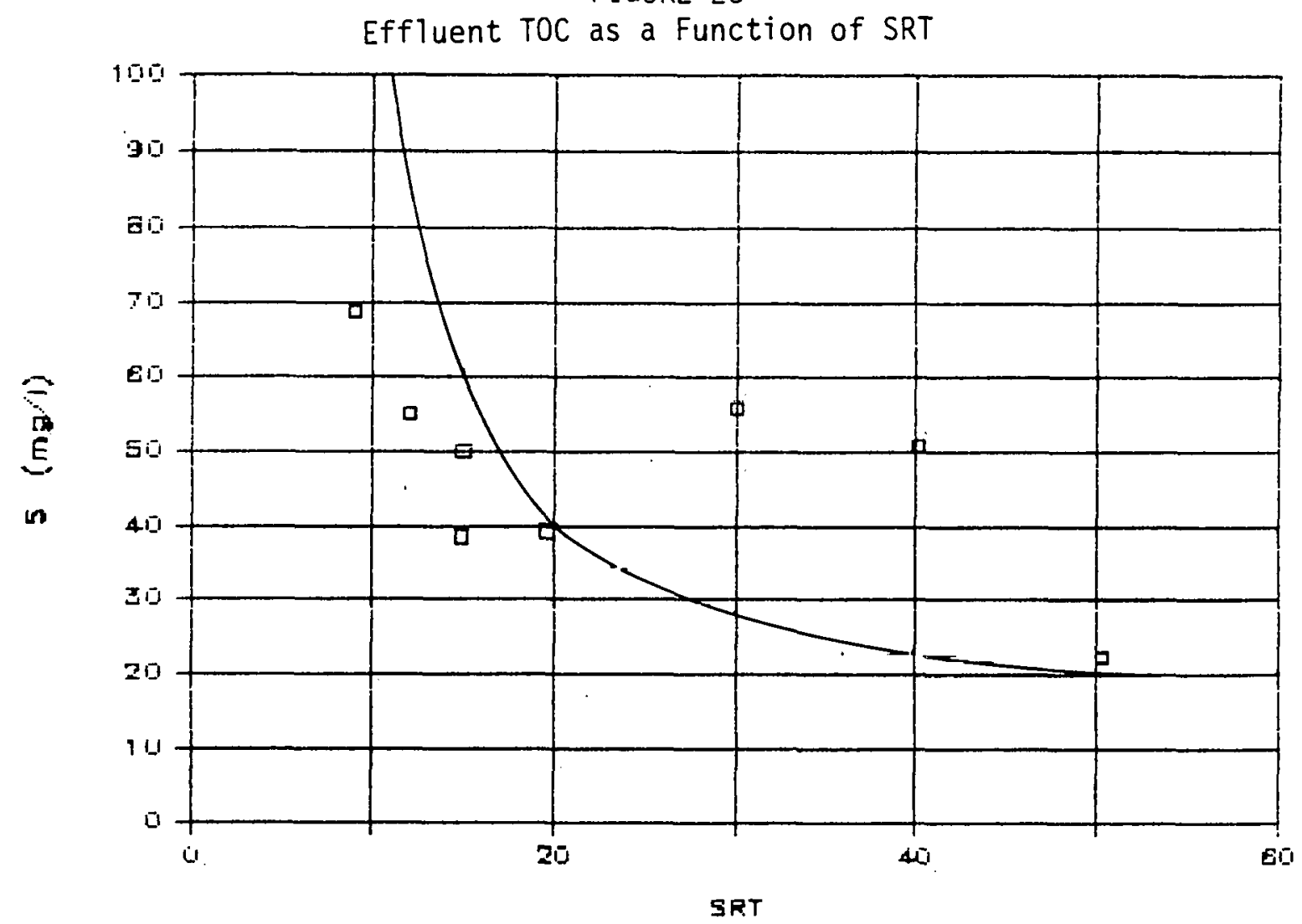


For all SRT's studied, the resulting effluent BOD values were very low. Reference to Figure 21 indicates that the maximum effluent mean observed was less than $4 \mathrm{mg} / 1$ and occurred during the 12-day SRT period. However, by applying the kinetic parameters developed for BOD in Equation 3, the resulting effluent values predicted are negative for any SRT less than 10 days. Since the kinetic parameters should be valid at all SRT's, the model is inappropriate for the 800 data derived from this study. Nevertheless, the actual data points on Figure 21 did exhibit the same pattern as shown in Figures 22 and 23 (i.e., the effluent substrate concentration increases with a decreasing SRT).

Alternately, COD and TOC data could be compared with values predicted by the model. In Figures 22 and 23 , the model predicts rapidly improving effluent values as the SRT is increased from 10 to 20 days. Within this operating range, the measured effluent $C O D$ and TOC data were significantly better than predicted by the model at lower SRT's and agreed very we 11 with predicted values as the SRT is increased to 20 days. Beyond 20-day SRT, the model predicted better performance than was observed for both COD and TOC. In this region, the predicted values for TOC fit the observed data better than coo.

\section{OXYGEN UTILIZATION}

oxygen uptake rates were measured every few days for both systems. These uptake rates allow a graphical determination of the oxygen requirements for. substrate utilization and cell maintenance. This method (6) requires a graph of $F / M$ (Ibs. COD removed/day per Ib. MLVSS) versus oxygen used per unit weight of solids (lbs. $\mathrm{O}_{2}$ consumed/day per 1b. MLVSS). This data is plotted on Figure 24 for the eight steady-state periods. It should be noted that the values of F/M and MLVSS are not the average values summarized in Table A-5 in the Appendix. Rather, the ratios required for this plot were calculated separately for each day on which the oxygen uptake rate had been measured. All of the data for a given steady-state period were then averaged and plotted on Figure 22. 
FIGURE 24

OXYGEN UTILIZATION COEFFICIENTS

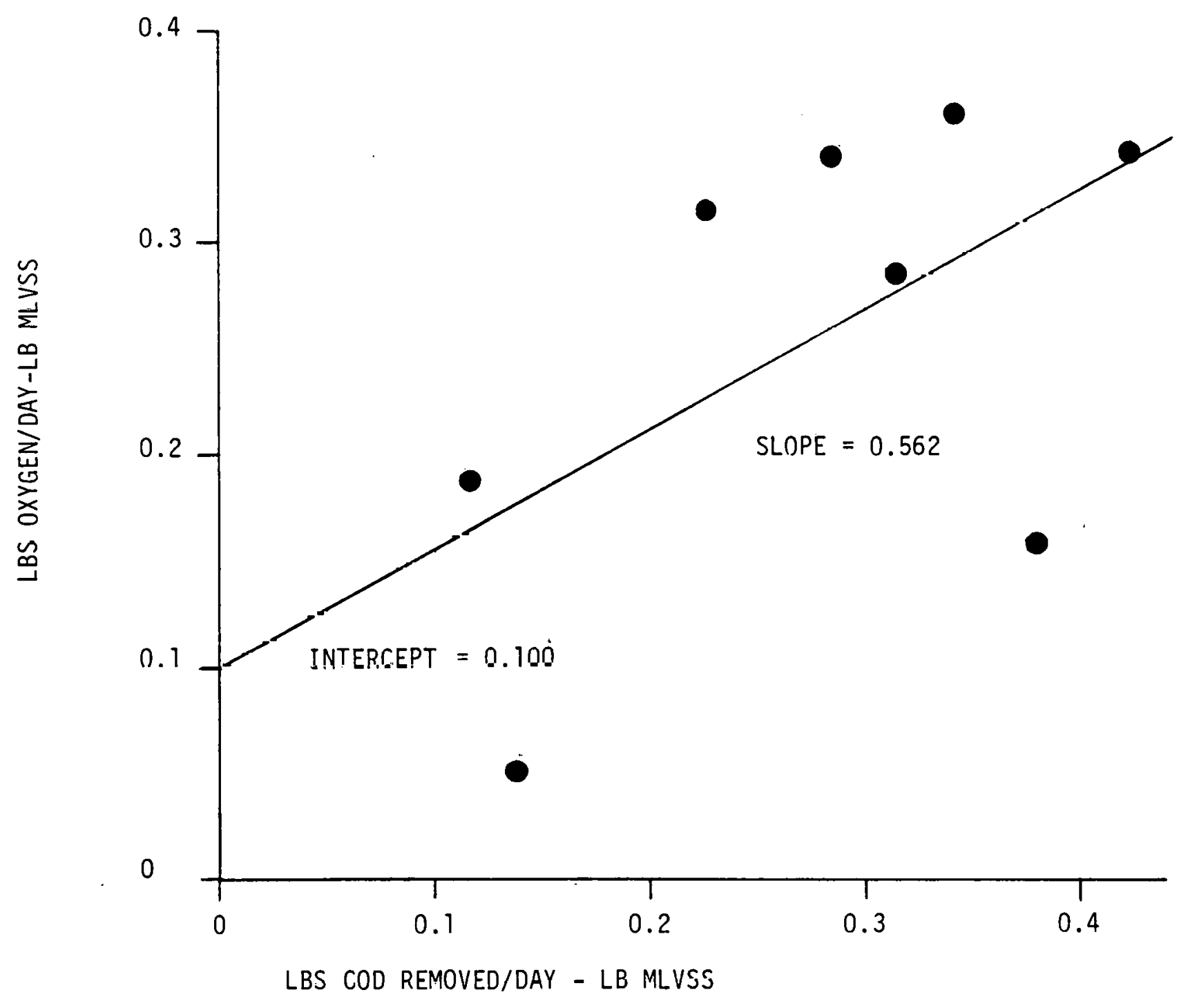


The intercept of this graph is the oxygen required to maintain the cells, without any removal of $\mathrm{COD}$. As shown on Figure 24, it is 0.1 . pounds of oxygen per day per pound of volatile solids under aeration. The slope of the line shows the oxygen required for substrate utilization, which is 0.562 pounds of oxygen per pound of COD removed.

\section{NITRIFICATION}

Reduction of ammonia nitrogen was observed in both the first and second stage units, with the gross removal occurring in the initial stages. Figures 6 and 7 show influent and effluent nitrogen concentrations for systems 1 and 2, respectively. For System 1, a relatively steady-state period was observed between days 44 and 97 . In System 2, days 48 to 97 were selected as steady-state.

Kinetic coefficients were evaluated for both stages of each system by applying a first-order model previously defined as Equation 4 . This model predicts that nitrification efficiency varies as an exponential function of the product of a rate constant, the MLVSS concentration, and the hydraulic retention time. For system 1 , the rate constant was determined to be $7.37 \times$ $10^{-4}$ for Unit $1 A$ and $1.15 \times 10^{-3}$ for second stage Unit.1B. For System 2 , the first stage (Unit $2 \mathrm{~A}$ ) rate constant is $7.64 \times 10^{-4}$. For Unit $2 \mathrm{~B}$ it is $7.43 \times 10^{-4}$. Values for the rate constant are summarized in Table 6 .

Included in Table 6 is a mass balance for nitrogen for each steady-state period. All influent and effluent streams were analyzed for ammonia, nitrite, nitrate, cyanide, thiocyanate, and organic nitrogen. Also, wasted sludge was assumed to be 12 percent nitrogen on a dry-weight basis. As shown on the table, the influent and effluent nitrogen summations all balanced to within 10 percent. For the first stage reactors, where most of the ammonia removal occurred, the balance was even better (within approximately 4 percent). 
Table 6

Kinetic Constant for Nitrification

\begin{tabular}{lllll} 
& \multicolumn{1}{c}{ Unit 1A } & $\underline{\text { Unit 1B }}$ & $\underline{\text { Unit 2A }}$ & $\underline{\text { Unit 2B }}$ \\
$K_{n}$ & $7.37 \times 10^{-4}$ & $1.15 \times 10^{-3}$ & $7.64 \times 10^{-4}$ & $7.43 \times 10^{-4}$ \\
$\begin{array}{l}\text { Nitrogen 8alance } \\
\text { (In/Out) }\end{array}$ & 0.958 & 0.93 & 0.979 & 0.90
\end{tabular}

The range of kinetic data reported in Table 6 is from $7.37 \times 10^{-4}$ to $1.15 \times 10^{-3}$. All values were consistent to within one-half order of magnitude. The mean $K_{n}$ value determined using steady-state data is $8.48 \times$ $10^{-4}$. In an earlier study (1), the kinetic constant for nitrification was found to range from $5.3 \times 10^{.4}$ to $8.7 \times 10^{-4}$. Although the range of values determined during this investigation was spread slightly, there is substantial agreement between the two studies, and the first-order model can be considered valid.

\section{MIXED LIQUOR SETTLING CHARACTERISTICS}

Batch settling tests were periodically run on samples of the mixed liquors from all reactors at each SRT studied. These were 60-minute tests in 1-liter graduated cylinders. Settling curves for the first stage reactors of Systems 1 and 2 are included in the Appendix, Figures $A-1$ to $A-20$. No data is included for the second stage reactors (Units $1 B$ and $2 B$ ). There was limited microbiological growth in these units, and flocculant settling did not occur. This same problem was noted in the previous biooxidation study ( 1 ).

A summary of the zone settling velocity determined from each test appears in Table 7. Mean settling velocities ranged from $0.03 \mathrm{ft} . / \mathrm{min} .$, corresponding to an average MLSS concentration of $10,500 \mathrm{mg} / 1$ for a 40 -day SRT, to a maximum 
mean value of $0.43 \mathrm{ft} . / \mathrm{min}$. for a $2,944 \mathrm{mg} / 1 \mathrm{MLSS}$ at a 15-day SRT. A general trend of decreasing zone settling velocity with increasing MLSS concentration was observed.

The settling velocities varied by more than an order of magnitude over the range of MLSS concentrations studied. However, the mean velocity varied by less than a factor of 2.5 for MLSS concentrations below $4,000 \mathrm{mg} / 1$. Mixed liquor solids levels above this level corresponded to the 40 - and 50-day SRT periods. 
Table 7

Summation of Batch Settling Tests

\begin{tabular}{|c|c|c|c|}
\hline $\begin{array}{l}\text { SRT } \\
\text { (Days) }\end{array}$ & $\begin{array}{l}\text { MLSS } \\
(\mathrm{mg} / 1)\end{array}$ & Reactor & $\begin{array}{l}\text { Zone Settling Velocity } \\
\text { (Ft./Min.) }\end{array}$ \\
\hline 50 & 5,875 & $2 A$ & 0.038 \\
\hline 50 & 5,170 & $2 A$ & 0.056 \\
\hline 50 & 4,335 & $2 A$ & 0.077 \\
\hline 50 & 3,980 & $2 A$ & 0.085 \\
\hline 40 & 11,880 & $2 A$ & 0.014 \\
\hline 40 & 10,095 & $2 A$ & 0.017 \\
\hline 40 & 9,525 & $2 A$ & 0.052 \\
\hline 20 & 3,223 & $2 A$ & 0.187 \\
\hline 20 & 2,910 & $2 A$ & 0.239 \\
\hline 15 & 3,697 & $1 A$ & 0.525 \\
\hline 15 & 3,470 & $1 A$ & 0.292 \\
\hline 15 & 3,230 & $1 A$ & 0.262 \\
\hline 15 & 3,045 & $1 A$ & 0.292 \\
\hline 15 & 2,880 & $1 \mathrm{~A}$ & 0.328 \\
\hline 15 & 2,735 & $I A$ & 0.398 \\
\hline 15 & 2,335 & $1 \mathrm{~A}$ & 0.656 \\
\hline 15 & 2,133 & $1 A$ & 0.656 \\
\hline 12 & 2,482 & IA & 0.875 \\
\hline 9 & 4,002 & IA & 0.525 \\
\hline 9 & 2,665 & $1 A$ & 0.375 \\
\hline
\end{tabular}




\section{CONCLUSIONS}

1. Kinetic parameters according the Lawrence and McCarty model were developed for gross organic reduction in terms of $B 00, C O O$, and TOC. A summary of the rate constants is presented in the summary table, below. The kinetic coefficients evaluated in terms of TOC provided the best fit of the data derived from this study.

Summary of Lawrence and McCarty Xinetic Coefficients

\begin{tabular}{|c|c|c|c|c|}
\hline Basis & $\begin{array}{l}\text { Yield Coeff. } \\
\text { y (lb./1b.) }\end{array}$ & $\begin{array}{c}\text { Endogenous Respir. Rate } \\
k_{d}(\text { day }-1)\end{array}$ & $\begin{array}{l}\text { Max. Rem. Rate } \\
k(1 b . / 1 b .- \text { day })\end{array}$ & $\begin{array}{r}S \propto k / 2 \\
\underline{K}_{S}(\mathrm{mg} / 1)\end{array}$ \\
\hline $\mathrm{COO}$ & 0.37 & 0.05 & 0.56 & 140 \\
\hline$B 00$ & 0.41 & 0.01 & 0.26 & 0.5 \\
\hline TOC & 1.22 & 0.06 & 0.15 & 27 \\
\hline
\end{tabular}

2. The treatability results expressed on a $B O D$ basis do not fit the Lawrence and McCarty model. This is due to the very low average levels of effluent 800 (less than $4 \mathrm{mg} / 1$ ) for all eight steady-state conditions.

3. A kinetic rate constant for a first-order relationship for nitrification was determined to be $8.48 \times 10^{-4}$ liters per milligram-day. The range of values determined for each reactor of parallel two-stage systems was $7.37 \times 10^{-4}$ to $1.15 \times 10^{-3} \mathrm{l} / \mathrm{mg}$-day. This range is consistent with values determined in a previous study.(1).

4. 0xygen requirements for substrate utilization were determined to 0.562 pounds of oxygen per pound of COD removed. The respiration coefficient was 0.1 pounds of oxygen per day per pound of MLVSS. These coefficients include oxygen requirements for nitrification. 
5. Zone settling velocities of the mixed liquor solids varied widely, ranging from 0.014 to $0.875 \mathrm{ft} . / \mathrm{min}$. for the first stage reactors. The lowest settling rates correspond to the highest mixed liquor concentrations, indicative of hindered settling. For mixed liquor concentrations less than $4,000 \mathrm{mg} / 1$, the velocities were mostly in the range of 0.2 to $0.7 \mathrm{ft} . / \mathrm{min}$. Flocculant settling did not occur for the second stage mixed liquors, and zone settling velocities could not be determined. 


\section{REFERENCES}

1. Mitche11, 3. W., et a1. 1983. Final.Technical Report: Evaluation of Effects of Phenol Recovery on Biooxidation and Tertiary Treatment of SRC-I Wastewater. DOE/OR/03054-49. International Coal Refining Company, Allentown, PA.

2. International Coal Refining Company. 1983. Design Basis Memorandum, Section 8, covering Area 17. Waste Treatment and Disposal Section Discharge Mode (Rev. C). International Coal Refining Company, Allentown, PA.

3. Wastewater Treatment System and Solid Wastes Landfill for 6000 TPD SRC-I Demonstration Plant. DOE/OR/03054-71. Prepared for International Coal Refining Company by Catalytic, Inc., September 1983.

4. Final Report: Wilsonville Wastewater Sampling Program. DOE/OR/03054-79. Prepared for International Coal Refining Company by Catalytic, Inc., October 1983.

5. Laboratory Tests of Phenol Removal, and Ammonia and Hydrogen Sulfide Stripping from SRC-I Wastewater. DOE/OR/03054-87. Prepared for International Coal Refining Company by Chem-Pro Corporation, September 1983.

6. Luthy, et al. Biological Treatment of Synthetic Fuel Wastewater, Journal of the American Society of Civil Engineers, Environmental Engineering Division, June 1980 . 
APPENDIX A

TABLES ANO FIGURES

$-52-$ 


\section{REFERENCES}

1. Mitche11, J.W., et a1. 1983. Final Technical Report: Evaluation of Effects of Phenol Recovery on Biooxidation and Tertiary Treatment of SRC-I Wastewater. DOE/OR/03054-49. International Coal Refining Company, Allentown, PA.

2. International Coal Refining Company, 1983. Design Basis Memorandum, Section 8, covering Area 17. Waste Treatment and Disposal Section Discharge Mode (Rev. C). International Coal Refining Company, Allentown, PA.

3. Wastewater Treatment System and Solid Wastes Landfill for 6000 TPO SRC-I Demonstration Plant. OOE/OR/03054-71. Prepared for International Coal Refining Company by Catalytic, Inc., September 1983.

4. Final Report: Wilsonville Wastewater Sampling Program. DOE/OR/03054-79. Prepared for International Coal Refining Company by Catalytic, Inc., October 1983.

5. Laboratory Tests of Phenol Removal, and Ammonia and Hydrogen Sulfide Stripping from SRC-I Wastewater. DOE/OR/03054-87. Prepared for International Coal Refining Company by Chem-Pro Corporation, September 1983.

6. Luthy, et al. Biological Treatment of Synthetic Fuel Wastewater, Journal of the American Society of Civil Engineers, Environmental Engineering Division, June 1980 . 
APPENDIX A

TABLES AND FIGURES

$-52-$ 


\begin{tabular}{|c|c|c|c|c|c|c|c|c|c|}
\hline XENTH & JLLYY & JULY & JULY & JULY & JULY & JULY & RLIGLST & RUGUST & AUGUST \\
\hline SAY & 36 & 27 & 28 & 29 & 30 & 31 & 1 & 2 & 3 \\
\hline כăY : 10. & 0 & 1 & 2 & 3 & 4 & 5 & 6 & 7 & a \\
\hline \multicolumn{10}{|l|}{ SIS:EM PARAMETERS } \\
\hline ×Sludgoe Age & 30 & 30 & 30 & 38 & 30 & 30 & 30 & 30 & 30 \\
\hline$*(F / M) C O D$ & 0.24 & 0.25 & 0.25 & 0.27 & 8.24 & 0.25 & 0.25 & 0.10 & 0.28 \\
\hline *iRT & 2.2 & 1.7 & 2.2 & 1.9 & 2.1 & 1.9 & 1.3 & 5.2 & 1.9 \\
\hline FEED AARAMETERS (BATCH \#)(BATCH & 27 & 27 & 27 & 27 & 27 & 27 & 27 & 27 & 27 \\
\hline Volune (liters) & 4.3 & 4.85 & 4. 32 & 5 & 4.5 & 4.3 & 4.85 & 1.8 & 4.9 \\
\hline $\mathrm{pH}$ (std units) & \multicolumn{3}{|c|}{$9.7 / 10.23 .8 /: 0.39 .8 / 10.3$} & \multicolumn{3}{|c|}{$9.79 .7 / 10.19 .7 / 10.1$} & \multicolumn{2}{|c|}{$109.8 / 10.3$} & 9.8 \\
\hline $\cos (\operatorname{sig} / 1)$ & 1840 & 1840 & 1848 & 1750 & 1750 & 1758 & 1758 & 1810 & 1810 \\
\hline NHIJN $(n g / 1)$ & 188 & 188 & 188 & 188 & 188 & 188 & 188 & 181 & 181 \\
\hline SS $\mathrm{ing} / \mathrm{I})$ & 24 & 24 & 24 & 24 & 24 & 24 & 24 & 44 & 44 \\
\hline USS $\mathrm{img} / \mathrm{ll}$ & 24 & 24 & 24 & 24 & 24 & 24 & 24 & 44 & 44 \\
\hline$C N(m g / 1)$ & 0.159 & 0.159 & 0.159 & 0.159 & Q. 159 & 0.159 & 0.159 & 0.217 & 0.217 \\
\hline SCN $i m g / 1)$ & 54.1 & 54.1 & 54.1 & 54.1 & 54.1 & 54.1 & 54.1 & 63.6 & 69.6 \\
\hline \multicolumn{10}{|l|}{ suto 5 -day (no/:i) } \\
\hline TCC (mq/1) & 584 & 584 & 584 & 584 & 584 & 584 & 584 & & \\
\hline \multicolumn{10}{|l|}{ Shenolics (ng/l) } \\
\hline \multicolumn{10}{|l|}{$504-p$ (ng/l) } \\
\hline \multicolumn{10}{|l|}{$\operatorname{Brg}-x(\operatorname{gg} / 1)$} \\
\hline \multicolumn{10}{|l|}{ vo $(m 0 / 1)$} \\
\hline \multicolumn{10}{|l|}{$\operatorname{VCJ}(m g / 1)$} \\
\hline \multicolumn{10}{|l|}{$T D S(\mathrm{mg} / 1)$} \\
\hline \multicolumn{10}{|l|}{ Color (APHA Units) } \\
\hline \multicolumn{10}{|l|}{ SASIN FASEMETERS } \\
\hline Vol Hasted (m) & 366 & 332 & 336 & 341 & 357 & 375 & 351 & 390 & 354 \\
\hline D.0. $(m g / i)$ & 7.4 & 7.4 & 6.7 & 6.5 & 7.2 & 6.7 & 6.3 & 6.4 & 6.1 \\
\hline SS $(\operatorname{tag} / 1)$ & 3898 & 3280 & 2880 & 2860 & 2970 & 3130 & 2970 & 2980 & 2860 \\
\hline \multicolumn{10}{|l|}{ *SS (PAC corrected) } \\
\hline Temo (C) & 24 & 24 & 24.5 & 25 & 25 & 25 & 25 & 25 & 25 \\
\hline DH (std units) & 6.6 & 6.7 & 7.5 & 7.1 & 6.9 & 7.2 & 7.4 & 7 & 7.3 \\
\hline OUR $(\mathrm{mg} / \mathrm{l} / \mathrm{hr})$ & & & 28 & & & & 46 & & \\
\hline VSS (mog/l) & 2783 & 2954 & 2580 & 2661 & 2764 & 2913 & 2790 & 2799 & 2687 \\
\hline \#USS (PAC corrected) & 2783 & 2354 & 2630 & 2661 & 2764 & 2913 & 2790 & 2799 & 2687 \\
\hline YSS/SS & & & 0.93 & & & & 0.94 & & \\
\hline \multicolumn{10}{|l|}{ tPAC Added (ng) } \\
\hline \multicolumn{10}{|l|}{ Inventory (mg/l basin) } \\
\hline EFFLLUENT PARAMETERS & & & & & & & & & \\
\hline Voline (liters) & 3.75 & 4.27 & 3.85 & 4.45 & 4 & 4.4 & 4.31 & 0.9 & 4.35 \\
\hline $\operatorname{COD}(n g / !)$ & 185 & 191 & 183 & 200 & 208 & 208 & 167 & 173 & 220 \\
\hline SS $(\operatorname{mg} / 1)$ & 28 & 52 & 48 & 38 & 32 & 18 & 34 & 34 & 30 \\
\hline APAC corrected & & & & & & & & & \\
\hline $\mathrm{OH}$ (std units) & 6.9 & 7.1 & 7.5 & 7.2 & 7.1 & 7.2 & 7.4 & 7.5 & 7.4 \\
\hline VSS (mg/1) & & & 48 & & & & 34 & & \\
\hline *PAC currected & & & & & & & & & \\
\hline NHSN (mg/l) & 18 & 10 & 10 & 4 & 4 & 4 & 4 & 5.8 & 5.8 \\
\hline C.N $(\mathrm{gg} / 1)$ & 8.4 & 8.4 & 8.4 & 8.4 & 8.4 & 8.4 & 8.4 & 1.32 & 1.32 \\
\hline $\operatorname{SCN}(\mathrm{mg} / 1)$ & 10.1 & 10.1 & 10.1 & 10.1 & 18.1 & 10.1 & 10.1 & 10.3 & 10.3 \\
\hline NC2 (mg/l) & 8.79 & 8.79 & 8.79 & 8.79 & 8.79 & 8.79 & 8.79 & 8.7 & 8.7 \\
\hline $\mathrm{NO3}(\mathrm{mg} / 1)$ & 240 & 240 & 240 & 240 & 240 & 240 & 240 & 150 & 150 \\
\hline Phenclics $(\mathrm{mg} / 1)$ & & & & & & & & 1.025 & 1.025 \\
\hline$f 04-P(\mathrm{mg} / 1)$ & & & & & & & & 1.3 & 1.3 \\
\hline $\begin{array}{l}\text { Org-N }(m g / 1) \\
\operatorname{Tos}(m g / 1)\end{array}$ & & & & 7 & 7 & 7 & & & \\
\hline Color (APHA units) & 250 & 250 & 250 & 250 & 250 & 250 & 250 & & \\
\hline TOC $(\log / 1)$ & 48 & 48 & 48 & 48 & 48 & 48 & 48 & & \\
\hline Bü 5-day $(m g / 1)$ & & & $-53-$ & & & & & & \\
\hline
\end{tabular}


UNIT IA TABLE AI

\begin{tabular}{|c|c|c|c|c|c|c|c|c|c|c|}
\hline MONTH & RUGUST & ALGUST & RUGUST & AUGUST & AUGUST & AUGUST & AUGUST & AUGLST & AUGUST & AUELST \\
\hline DAY & 4 & 5 & 6 & 7 & 8 & 9 & 10 & 11 & 12 & 13 \\
\hline DAY NO. & 9 & 18 & 11 & 12 & 13 & 14 & 15 & 16 & 17 & 18 \\
\hline \multicolumn{11}{|l|}{ SYSTEY FGRAMETERS } \\
\hline tSludge Age & 38 & J0 & 30 & 30 & 30 & 30 & 30 & 30 & 38 & 30 \\
\hline$+(F / M) C O D$ & 0.26 & 0.26 & 0.27 & 0.26 & 0.21 & 0.23 & 0.25 & 0.21 & 0.24 & 0.24 \\
\hline *HRT & 1.9 & 2 & 1.9 & 1.9 & 2.1 & 2.1 & 2.4 & 2.4 & 2.3 & 2.4 \\
\hline FEED FRRAMETERS (BATCH \#) & 27 & 27 & 27 & 27 & 27 & 27 & 27 & 28 & 28 & 28 \\
\hline Voluwe (liters) & 4.93 & 4.7 & 4.85 & 4.9 & 4.5 & 4.40 & 3.85 & 3.93 & 4.00 & 3.95 \\
\hline pH (std units) & $9.7 / 10.69$ & $8 / 18.7$ & 9.8 & 9.8 & 9.8 & 9.8 & 9.81 & 110.39 & $9.9 / 10.49$ & $.7 / 10.2$ \\
\hline COD (mg/l) & 1810 & 1745 & 1745 & 1745 & 1745 & 1858 & 1858 & 1858 & 2159 & 2159 \\
\hline$N$ NhōN (ạ/l) & 181 & 176 & 176 & 176 & 176 & 173 & 173 & 173 & 185 & 185 \\
\hline SS img/1) & 44 & 44 & 44 & 44 & 44 & 100 & 108 & 180 & 108 & 100 \\
\hline USS $(m g / 1)$ & 44 & 44 & 44 & 44 & 44 & 32 & 32 & 32 & 32 & 32 \\
\hline CN (ng/l) & 0.217 & 0.217 & 0.217 & 0.217 & 0.217 & 0.481 & 0.481 & 0.481 & 0.481 & 0.481 \\
\hline SCN (mg/i) & 69.6 & 69.6 & 69.6 & 69.6 & 69.6 & 149 & 149 & 149 & 149 & 149 \\
\hline 800 J-day (mg/l) & & & . & & & & & & & \\
\hline TOC (mg/1) & & & & & & 632 & 632 & 632 & 632 & 632 \\
\hline \multicolumn{11}{|l|}{ Phenolics (mg/l) } \\
\hline \multicolumn{11}{|l|}{$904-p(\mathrm{mg} / 1)$} \\
\hline \multicolumn{11}{|l|}{ Org-N (mg/l) } \\
\hline \multicolumn{11}{|l|}{ 102 (mg/l) } \\
\hline \multicolumn{11}{|l|}{$103\left(r_{i} / 1\right)$} \\
\hline \multicolumn{11}{|l|}{ TDS (mg/1) } \\
\hline \multicolumn{11}{|l|}{ Color (APHA Units) } \\
\hline \multicolumn{11}{|l|}{ BASIN SAREMETERS } \\
\hline Visl Hasted (al) & 361 & 354 & 364 & 342 & 358 & 349 & 373 & 376 & 388 & 375 \\
\hline D.0. $(\mathrm{mg} / 1)$ & 6.7 & 7 & 6.8 & 7 & 6.1 & 6.0 & 6.6 & 6.8 & 6.3 & 7.0 \\
\hline SS $(m g / 1)$ & 2350 & $27: 0$ & 2728 & 2920 & 3480 & 3290 & 2610 & 2948 & $3120^{\circ}$ & 3050 \\
\hline t55 (PAC corrected) & & & & & & 3290 & 2610 & 2940 & 3120 & 3050 \\
\hline Teray $\quad(C)$ & 25.5 & 25.5 & 25 & 25 & 26 & 27 & 25 & 25 & 24 & 23 \\
\hline ph (std units) & 6.9 & 7.3 & 7.3 & 7.3 & 7 & 6.8 & 6.8 & 6.6 & $6 . \overline{8}$ & 7.0 \\
\hline OLR (mg/:/hr) & 60 & & & & 24 & & & 24 & & \\
\hline VSS $(\operatorname{mg} / 1)$ & 2810 & 2581 & 2521 & 2781 & 3150 & 2978 & 2362 & 2878 & 3046 & 2987 \\
\hline *USS (PAC corrected) & 2810 & 2581 & 3591 & 2781 & 3150 & 2978 & 2362 & 2879 & .9046 & 2987 \\
\hline VSS/SS & 0.35 & & & & 0.91 & & & 0.98 & & \\
\hline PAC Added (mg) & & & & & & b & 8. & 8 & 8 & 0 \\
\hline +lnventory (mg/l basin) & & & & & & 0 & 8 & 8 & 8 & 0 \\
\hline \multicolumn{11}{|l|}{ EFFLUENT PARAME IERS } \\
\hline Volume (1:ters) & 4.41 & 4.14 & 4.13 & 4.45 & 3.85 & 3.88 & 3.25 & 3.25 & 3.45 & 3.50 \\
\hline $\operatorname{COD} \operatorname{lng} g / 1)$ & 182 & 284 & IRA & 181 & 181 & 155 & 166 & 119 & 161 & 184 \\
\hline SS $(m g / 1)$ & 26 & 30 & 24 & 38 & 38 & 44 & 22 & 22 & 18 & 22 \\
\hline *PAC corrected & & & & & & 44 & 22 & 22 & 18 & 22 \\
\hline oH (std units) & 7.3 & 7.2 & 7.7 & 7.5 & 7.4 & 7.2 & 7.8 & 6.6 & 6.8 & 7.0 \\
\hline VSS (mg/1) & 26 & & & & 28 & & & 18 & & \\
\hline aidic corrected & & & & & & & & 18 & & \\
\hline NHSN $(m g / 1)$ & 5.8 & 3.5 & 3.5 & 3.5 & 3.5 & 6.3 & $6 . \overline{3}$ & 6.3 & 9.4 & $\bar{y} .4$ \\
\hline CN $(n g / 1)$ & 1.32 & 1.32 & 1.32 & 1.32 & 1.32 & 1.006 & 1.086 & 1.006 & 1.006 & 1.006 \\
\hline $\mathrm{SCN}(\mathrm{mg} / 1)$ & $10: 3$ & 10.3 & 10.3 & 18.3 & 10.3 & 7.4 & 7.4 & 7.4 & 7.4 & 7.4 \\
\hline $\mathrm{NO2}(\mathrm{mg} / \mathrm{l})$ & 8.7 & 8.7 & 8.7 & 8.7 & 8.7 & 17.6 & 17.6 & 17.6 & 17.6 & 17.6 \\
\hline NO3 ing / 11$)$ & 150 & 150 & 150 & 150 & 150 & 171 & 171 & 171 & 171 & $17 !$ \\
\hline Phenolics $(\mathrm{mg} / \mathrm{l})$ & $<.025$ & 1.0 .25 & 1.025 & 1.025 & 1.025 & & & & & \\
\hline$P 04-p(m q / 1)$ & 1.3 & 1.3 & 1.3 & 1.3 & 1.3 & & & & & \\
\hline $\begin{array}{l}\text { Org-N (mg/1) } \\
\text { TOS (mg/1) }\end{array}$ & \multicolumn{10}{|c|}{ iús $(m \underline{q} / 1)$} \\
\hline Color (APHA Inits) & & & & & & 250 & 250 & 250 & 250 & 250 \\
\hline $\operatorname{TOC}(\mathrm{mg} / 1)$ & & & & & & 52 & 52 & 52 & 52 & 52 \\
\hline
\end{tabular}


UNIT IA TABLE AI

\begin{tabular}{|c|c|c|c|c|c|c|c|c|c|c|}
\hline YONTH & AUGUST & RUGUST & RUSLLST & ÂLGUST & AUGUST & ÂUGUST & ALGUST & RUGUST & AUGUST & RUGUST \\
\hline SAYY & 14 & 15 & 16 & 17 & 18 & 19 & 20 & 21 & 22 & 23 \\
\hline DAY NO. & 19 & $\hat{\varepsilon}$ & $2 !$ & 22 & 23 & 24 & 25 & 25 & 27 & 28 \\
\hline \multicolumn{11}{|l|}{ SYSTEY PARFYYETERS } \\
\hline FSiudợe Açe & 30 & 30 & 30 & 30 & 30 & 30 & 30 & 30 & 30 & 30 \\
\hline$+(F / M) C O D$ & 0.27 & 0.24 & 0.27 & 0.26 & 0.23 & 0.26 & 0.28 & 0.28 & 0.24 & 8.24 \\
\hline *HRT & 2 & 2 & 1.9 & 1.9 & 1.9 & 1.9 & 1.9 & 1.9 & 2 & 1.9 \\
\hline FEED FARAMETERS (BRTCH ) & 28 & 28 & 28 & 28 & 28 & 28 & 28 & 28 & $2 B$ & 28 \\
\hline Volune (liters) & 4.70 & 4.57 & 4.95 & 4.86 & 4.89 & 5.20 & 5.00 & 5.80 & 4.71 & 4.90 \\
\hline $2 H$ (std units) & 9.7 & 9.8 & 10 & 9.8 & 9.9 & 9.7 & 9.8 & \multicolumn{3}{|c|}{$9.83 .9 / 10.39 .9 / 10.5$} \\
\hline $\operatorname{coD}(\mathrm{ng} / \mathrm{l})$ & 2159 & 2159 & 20151 & 2051 & 2051 & 2105 & 2105 & 2105 & 2185 & 2185 \\
\hline$N H \bar{N} N(\mathrm{mg} / 1)$ & 185 & 185 & 190 & 190 & 190 & 183 & 183 & 183 & 183 & 180 \\
\hline SS $(m g / 1)$ & 100 & 108 & & & & & & & & 48 \\
\hline USS $(m g / 1)$ & 32 & 32 & & & & & & & & 10 \\
\hline CN $(\mathrm{mg} / 1)$ & 0.481 & 0.481 & & & & & & & & 0.739 \\
\hline SC.N $(\mathrm{mg} / \mathrm{l})$ & 149 & 149 & & & & & & & & $1 \pi$ \\
\hline $8 \pi 05$-day (n:q/1) & & & & & & & & & & 1230 \\
\hline TOC $(0 \mathrm{~g} / 1)$ & 632 & 632 & & & & & & & & 645 \\
\hline Fhenolics (mg/l) & & & 1.56 & 1.56 & 1.56 & 1.56 & 1.56 & 1.56 & 1.56 & \\
\hline \multicolumn{11}{|l|}{$P O 4-P(\mathrm{mg} / 1)$} \\
\hline \multicolumn{11}{|l|}{$\begin{array}{l}\text { Jrg-N }(m g / 1) \\
\text { NO2 }(\mathrm{mg} / 1)\end{array}$} \\
\hline \multicolumn{11}{|l|}{ V03 (mg/1) } \\
\hline \multicolumn{11}{|l|}{$\operatorname{TDS}(\log / 1)$} \\
\hline \multicolumn{11}{|l|}{ Color (APHA Units) } \\
\hline \multicolumn{11}{|l|}{ BRSIN FARAMETERS } \\
\hline Vol Wasted (ml) & 364 & 358 & 326 & 336 & 358 & 348 & 370 & 364 & 346 & 337 \\
\hline D.0. $(\mathrm{mg} / 1)$ & 6.9 & 6.9 & 7.0 & 7.3 & 6.8 & 7.2 & 7.0 & 7.1 & 6.8 & 7.3 \\
\hline SS (ing/1) & 3228 & 3760 & 3490 & 3550 & 3708 & 3500 & 3270 & 3360 & 3650 & 3940 \\
\hline ISS (PRC corrected) & 3220 & 3760 & 3490 & 3550 & 3780 & 3500 & 3270 & 3360 & 3650 & 3940 \\
\hline Temp (C) & 22 & २2 & 23 & 24 & 25 & 24 & 25 & 24 & 24 & 24 \\
\hline oH (std units) & 7.3 & 7.1 & 7.1 & 7.0 & 6.3 & 6.9 & 6.8 & 6.9 & 6.9 & 6.8 \\
\hline OUR (mg/l/hr) & & 32 & & & & & & & 48 & \\
\hline USS (mog/1) & 3143 & 3398 & 3147 & 3281 & 3500 & 3311 & 3093 & 3178 & 3390 & 3659 \\
\hline *USS (FAC corrected) & $314 \hat{\jmath}$ & 3358 & 3147 & 3201 & 3500 & 3311 & 3093 & 3178 & 3390 & 3659 \\
\hline USS/SS & & 0.90 & & & 0.95 & & & & 0.93 & \\
\hline$₫ F A C$ Added (mg) & 0 & 8 & 0 & 8 & 0 & 8 & 0 & 8 & 8 & 0 \\
\hline Inventory (mạ/! basin) & 8 & 0 & $\theta$ & 0 & 0 & 0 & 0 & 0 & 0 & 8 \\
\hline \multicolumn{11}{|l|}{ EFFLUENT PARAMETERS } \\
\hline Volume (1iters) & 4.13 & 4. 11 & 4.48 & 4.38 & 4.32 & 4.57 & 4.53 & 4.35 & 4.69 & 4.25 \\
\hline $\operatorname{COD}(\mathrm{mg} / \mathrm{l})$ & 213 & 212 & 240 & 227 & $2 ! 2$ & 280 & 201 & 195 & 194 & 286 \\
\hline SS $(\mathrm{mg} / 1)$ & 28 & 38 & 58 & 16 & 36 & 40 & 22 & $2 B$ & 48 & 58 \\
\hline ^PAC corrected & 28 & 38 & 58 & 16 & 36 & 40 & 22 & 28 & 48 & 58 \\
\hline $\mathrm{pH}$ (std units) & 7.2 & 7.3 & 7.1 & 7.9 & 7.2 & 7.0 & 7.0 & 7.1 & 7.1 & 6.9 \\
\hline VSS (mg/1) & & 26 & & & 28 & & & & 32 & \\
\hline - NAC corrected & $\cdot$ & 26 & & & 28 & & & & 32 & \\
\hline NHiZN (og/l) & 9.4 & 9.4 & 15.8 & 15.8 & 15.8 & 24.3 & 24.3 & 24.3 & 24.3 & 17.5 \\
\hline CN (mg/l) & 1.806 & 1.806 & 3.02 & 3.02 & 3.02 & $3.0 \mathrm{C}$ & 3.02 & 3.02 & 3.82 & 1.88 \\
\hline $\mathrm{SCN}$ (mg/l) & 7.4 & 7.4 & 6.3 & 6.3 & 6.3 & 6.3 & 6.3 & 6.3 & 6.3 & 5.8 \\
\hline N02 (ng/l) & 17.6 & 17.6 & 22.7 & 22.7 & 22.7 & 22.7 & 22.7 & 22.7 & 22.7 & 13.7 \\
\hline NO3 $(-g / 1)$ & 171 & 171 & 167 & 167 & 167 & 167 & 167 & 167 & 167 & 171 \\
\hline Phenolics $(\mathrm{mg} / \mathrm{l})$ & & & 1.025 & 1.025 & 1. 025 & 1.025 & 1.025 & 1.825 & 1.025 & \\
\hline$P 04-P(m 0 / 1)$ & & & 3.9 & 3.9 & 3.9 & 3.9 & 3.9 & 3.9 & 3.9 & \\
\hline \multicolumn{11}{|l|}{$\operatorname{TDS}(\mathrm{mg} / 1)$} \\
\hline Color (APHA units) & 250 & 250 & & & & & & & & 500 \\
\hline $\begin{array}{l}\text { TOC (mo/l) } \\
\text { BOD j-day (ma/l) }\end{array}$ & 5 కू? & 52 & & $55-$ & & & & & & 63 \\
\hline
\end{tabular}


UNIT IA TAELE AI

\begin{tabular}{|c|c|c|c|c|c|c|c|c|c|c|}
\hline MONTH & AUGUST & AUGUST & AUGUST & AUGUST & RUGUST & RLGUST & AUGLST & AUGUST & SEPT & SEPT \\
\hline SAY & 24 & 25 & 26 & 27 & 28 & 29 & jo & 31 & 1 & 2 \\
\hline DAY NO. & 39 & 30 & 31 & 32 & 33 & 34 & 35 & 36 & 37 & 38 \\
\hline \multicolumn{11}{|l|}{ SYSTEM FARFHETERS } \\
\hline \$5l jodge Hge & 30 & 30 & 30 & 30 & 30 & 30 & 30 & 30 & 38 & 38 \\
\hline$+(F / M) C O D$ & 0.26 & 0.29 & 0.30 & 0.25 & 0.27 & 0.23 & 0.23 & 0.22 & 0.29 & 8.26 \\
\hline AHRT & 1.9 & 1.9 & 1.9 & 1.9 & 1.9 & 2.2 & 2.2 & 2.4 & 1.9 & 1.9 \\
\hline FEED FARGMETERS (BATCH & 28 & 28 & 28 & 28 & 29 & 29 & 29 & 29 & 29 & 29 \\
\hline Volume (liters) & 4.85 & 5.80 & 5.00 & 4,85 & 4.85 & 4.28 & 4.15 & 3.90 & 5.98 & 4.90 \\
\hline oH (std units) & $9.8 / 10.29$. & $7 / 10.39$ & $7 / 10.2$ & 9.79 & $7 / 10.49$ & $7 / 10.49$ & $7 / 10.79$ & $5 / 10.9$ & 9.6 & 9.7 \\
\hline $\cos (\mathrm{mg} / 1)$ & 2185 & 2185 & 2130 & 2130 & 2130 & 2130 & 2070 & 2070 & 2078 & ç022 \\
\hline NH3̄N (mg/!!) & 180 & 180 & 184 & 184 & $、 184$ & 184 & 179 & 179 & 179 & 184 \\
\hline SS (ang/1) & 40 & 40 & 40 & 40 & 40 & 40 & 26 & 26 & 26 & 26 \\
\hline 'SSS · (mg/1) & 10 & 10 & 18 & 10 & 10 & 10 & 14 & 14 & 14 & 14 \\
\hline CN $(m g / 1)$ & 8.739 & 0.739 & 0.739 & Q. 739 & 0.739 & 0.739 & 0.25 & 0.25 & 8.25 & 0.25 \\
\hline $\operatorname{SCN}(\pi g / 1)$ & 177 & 177 & 177 & 177 & 177 & 177 & 182 & 182 & 182 & 182 \\
\hline Evo 5-day $(n \underline{n} / 1)$ & 12990 & 1290 & 1290 & 1290 & 1470 & 1470 & 1470 & 1470 & 1470 & 1470 \\
\hline TOC $(00 / 1)$ & 645 & 645 & 645 & 645 & 645 & 645 & & & & \\
\hline Phenolics (mg/l) & & & & & & & 1.48 & 1.48 & 1.48 & 1.48 \\
\hline \multicolumn{11}{|l|}{$F(04-P(0 g / 1)$} \\
\hline \multicolumn{11}{|l|}{ Orgọ $-N$ (ong/l) } \\
\hline \multicolumn{11}{|l|}{$102(\pi g / 1)$} \\
\hline \multicolumn{11}{|l|}{$: 103(\mathrm{mg} / 1)$} \\
\hline \multicolumn{11}{|l|}{$\operatorname{TDS} \mathrm{img} / 11$} \\
\hline \multicolumn{11}{|l|}{ Color (APHA Units) } \\
\hline \multicolumn{11}{|l|}{ BASIN FRRAMETERS } \\
\hline Vol Wasted (ml) & 355 & 394 & 364 & 367 & 343 & 389 & 396 & 377 & 389 & 354 \\
\hline $0.0 .(\mathrm{mg} / 1)$ & 7.0 & 5.8 & 7.2 & 6.8 & 6.9 & 6.8 & 6.8 & 6.6 & 6.6 & 6.6 \\
\hline SS (ng/l) & 3640 & 3580 & 3310 & 3930 & 3600 & 3510 & 3330 & 3180 & 3220 & 3380 \\
\hline ISS (PAC corrected) & 3640 & 3500 & 3310 & 3930 & 3680 & 35ֵ & 3330 & 3180 & 3220 & 3380 \\
\hline Temo (C) & 24 & 23.5 & 24 & 25 & 24 & 25 & 24 & 24 & 24 & 24 \\
\hline pH (std units) & 7.0 & 7.0 & 7.2 & 7.2 & 7.1 & 6.9 & 7.8 & 6.9 & 7.4 & 7.8 \\
\hline OUR (mg/1/hr) & & & & & & 28 & & & 32 & \\
\hline USS $(\mathrm{mg} / 1)$ & 3381 & 3120 & 29.51 & 3503 & 3209 & 3318 & 3148 & 2999 & 3050 & 3170 \\
\hline *USS (PAC corrected) & 3381 & 3120 & 2951 & 3503 & 3209 & 3318 & 3140 & 2799 & 3028 & 3170 \\
\hline VSS/SS & & 8.89 & & & & 0.84 & & & 0.77 & \\
\hline †PAC Added (mg) & 0 & 0 & 0 & 0 & 0 & 8 & $\theta$ & 0 & 0 & $a$ \\
\hline Inventory (mg/l basin) & 0 & 8 & 8 & 8 & 0 & 0 & $\theta$ & 8 & 0 & 0 \\
\hline \multicolumn{11}{|l|}{ EFFLLUENT PARFMIETETSS } \\
\hline Volume (lizers) & 4.26 & 4.78 & 4.50 & 4.39 & 4.39 & 3.70 & 3.50 & 3.35 & 4.56 & 4.89 \\
\hline $\cos (\operatorname{mg} / 1)$ & 168 & 195 & 221 & 170 & 186 & 201 & 174 & 189 & 177 & 183 \\
\hline SS $(n g / 1)$ & 38 & 4 & 26 & 30 & 48 & 10 & 4 & בृ & 8 & 32 \\
\hline PAC corrected & 38 & 4 & 26 & 30 & 48 & 10 & 4 & 22 & 8 & 32 \\
\hline oH (std units) & 6.9 & 7.8 & 7.2 & 7.3 & 7.3 & 7.2 & 6.8. & 6.8 & 7.2 & 7.2 \\
\hline USS (qg/I) & & 4 & & & & 19 & & & 8 & \\
\hline ¿PAC currected & & 4 & & & & 10 & & & 8 & \\
\hline NHJ N $(m g / 1)$ & 17.5 & 17.5 & 9.5 & 9.5 & 9.5 & 9.5 & 11 & 11 & 11 & 12.5 \\
\hline$C N(m g / 1)$ & 1.88 & 1.88 & 1.88 & 1.88 & 1.88 & 1.88 & 1.88 & 1.88 & 1.88 & 1.88 \\
\hline $\operatorname{SCN}(m g / 1)$ & 5.8 & 5.8 & 5.8 & 5.8 & 5.8 & 5.8 & 6.2 & 6.2 & 6.2 & 6.2 \\
\hline N02 (mg/l) & 13.7 & 13.7 & 13.7 & 13.7 & 13.7 & 13.7 & 10.2 & 10.2 & 10.2 & 18.2 \\
\hline $\mathrm{N03}(\mathrm{mg} / 1)$ & 171 & 171 & 171 & 171 & 171 & 171 & 199 & 199 & 199 & 199. \\
\hline Whenolics $(\mathrm{mg} / \mathrm{l})$ & & & & & & & ८.025 & 1.025 & 1.025 & 1.025 \\
\hline$F 04-D(m 0 / 1)$ & & & & & & & 8.1 & 8.1 & 8.1 & 8.1 \\
\hline Org-N (mg/1) & & & 2.8 & 2.8 & 2.8 & 2.8 & & & & 1.8 \\
\hline \multicolumn{11}{|l|}{$\operatorname{TDS}(m g / 1)$} \\
\hline Color (APUA units) & 500 & 520 & 500 & 500 & 500 & 500 & & & & \\
\hline TOC $(m g / 1)$ & 63 & 63 & 63 & 63 & 63 & 63 & & & & \\
\hline ACD 5-day (isg/l) & & & & & & & & & & \\
\hline
\end{tabular}




\begin{tabular}{|c|c|c|c|c|c|c|c|c|c|c|}
\hline MINTH & SEPT & SEPT & SEPT & SEPT & SEPT & SEPT & SEPT & SEPT & SEPT & SEPT \\
\hline EAY & 3 & 4 & 5 & 6 & 7 & 8 & 9 & 10 & 11 & 12 \\
\hline EAY NO. & 39 & 40 & 41 & 42 & 43 & 44 & 45 & 46 & 47 & 48 \\
\hline \multicolumn{11}{|l|}{ SYSTEM PARAMETERS } \\
\hline *Sludge Ag̣e & 38 & 38 & 30 & 30 & 30 & 30 & 30 & 25 & 25 & 25 \\
\hline+ F/M)COD & 0.26 & 0.27 & 0.27 & 0.24 & 0.27 & 0.23 & 0.28 & 0.26 & 0.24 & 0.22 \\
\hline EHRT & 1.9 & 1.9 & 1.9 & 2.0 & 1.9 & 1.9 & 2.0 & 2.0 & 2.8 & 2.1 \\
\hline FEED FARAMETERS (BATCH *) & 29 & 29 & 29 & 29 & 29 & 29 & 29 & 29 & 29 & 30 \\
\hline Volune (liters) & 4.79 & 4.83 & 4.30 & 4.68 & 5.00 & 4.90 & 4.78 & 4.70 & 4.78 & 4.47 \\
\hline $\mathrm{pH}$ (std units) & \multicolumn{3}{|c|}{$9.7 / 10.23 .7 / 10.29 .7 / 10.2$} & \multicolumn{2}{|c|}{$9.79 .7 / 10.5$} & 3.7 & \multicolumn{4}{|c|}{$9.89 .7 / 11.89 .8 / 11.19 .0 / 10.1$} \\
\hline $\cos (\mathrm{mg} / 1)$ & 2022 & 2002 & 2822 & 2033 & 2033 & 2033 & 2000 & 2000 & 2000 & 2880 \\
\hline NHSN (ag/l) & 184 & 184 & 184 & 183 & 183 & 183 & 188 & 188 & 188 & 188 \\
\hline SS (mg/l) & 26 & 26 & 26 & 106 & 106 & 106 & 106 & 186 & 106 & 186 \\
\hline VSS $(2 g / 1)$ & 14 & 14 & 14 & 44 & 44 & 44 & 44 & 44 & 44 & 44 \\
\hline CN (mg/1) & 0.25 & 0.25 & 0.25 & 0.93 & 0.93 & 0.93 & 0.93 & 8.93 & 8.93 & 0.93 \\
\hline SCN (mg/l) & 102 & 182 & 182 & 183 & 183 & 183 & 183 & 183 & 193 & 283 \\
\hline 8005 -day (xg/1) & 1470 & 1470 & 1478 & 1470 & 1470 & 1470 & 1478 & 1470 & 1470 & 1418 \\
\hline TOC $(\operatorname{mg} / 3)$ & & & & 683 & 683 & 683 & 683 & 683 & 683 & 683 \\
\hline Phenolics $(\mathrm{mg} / \mathrm{l}$ ) & 1.48 & 1.48 & 1.48 & & & & & & & \\
\hline \multicolumn{11}{|l|}{$F 04-p$ (mg/1) } \\
\hline \multicolumn{11}{|l|}{$\operatorname{Trg} g-N(m g / 1)$} \\
\hline \multicolumn{11}{|l|}{ W2 (mg/1) } \\
\hline \multirow{2}{*}{\multicolumn{11}{|c|}{$\begin{array}{l}: 03(\mathrm{mg} / \mathrm{l}) \\
\text { TDS (mg/l) }\end{array}$}} \\
\hline DS (mg/1) & & & & & & & & & & \\
\hline \multicolumn{11}{|l|}{ Color (AFHA Units) } \\
\hline \multicolumn{11}{|l|}{ BASIN PARAMETERS } \\
\hline Yol dasted (ml) & 349 & 348 & 389 & 365 & 343 & 394 & 257 & 298 & 328 & 316 \\
\hline D.0. $(m g / 1)$ & 6.7 & 6.8 & 6.9 & 7.1 & 6.8 & 6.9 & 7.8 & 7.0 & 6.8 & 7.7 \\
\hline SS $(m g / 1)$ & 3250 & 3170 & 3260 & 3440 & 3320 & 3850 & 3040 & 3200 & 3430 & 3820 \\
\hline *SS (FAC corrected) & 3250 & 3170 & 3260 & 3440 & 3320 & 3850 & 3040 & 3280 & 3490 & 3820 \\
\hline Tespo (C) & 24 & 24 & 24 & 24 & 26 & 25 & 24 & 25 & 26 & 26.5 \\
\hline $\mathrm{pH}$ (std units) & 6.9 & 7.8 & 6.9 & 7.0 & 6.7 & 7.0 & 6.8 & 6.7 & 7.2 & 7.2 \\
\hline OuR (ing $/ ! / \mathrm{hr}$ ) & & & 60 & & & 54 & & & & 34 \\
\hline VSS (mg/l) & 3848 & 2973 & 3080 & 3250 & 3137 & 2850 & 2841 & 2990 & 3261 & 3358 \\
\hline *USS (PAC corrected) & 3048 & 2973 & 3080 & 3250 & 3137 & 2850 & 2841 & 2990 & 3261 & 3350 \\
\hline 'VSS/SS & & & 0.78 & & & 0.93 & & & & 0.88 \\
\hline IPAC Added (mg) & 0 & 0 & D & 8 & D & e & 0 & B & 0 & e \\
\hline Inventory (mg/l basin) & 0 & 0 & $\theta$ & 0 & 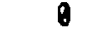 & 0 & a & e & $\theta$ & e \\
\hline \multicolumn{11}{|l|}{ EFLLUENT PARAMETERS } \\
\hline Volume (liters) & 4.35 & 4.32 & 4.45 & 4.08 & 4.33 & 4.17 & 4.18 & 4.16 & 4.22 & 4.11 \\
\hline $\operatorname{coD}(\operatorname{mg} / 1)$ & 174 & 177 & 154 & 180 & 174 & 186 & 190 & 182 & 182 & 158 \\
\hline SS (ng/l) & 38 & 38 & 8 & 38 & 44 & 4 & 104 & 148 & 132 & 152 \\
\hline *PAC corrected & 38 & 38 & 8 & .30 & 44 & 4 & 104 & 140 & 132 & 152 \\
\hline ôH (std units) & 7.8 & 7.1 & 7.8 & 6.8 & 6.8 & 6.9 & 6.9 & 6.7 & 6.8 & 7.2 \\
\hline USS (ma/1) & & & 8 & & & 4 & & & & 188 \\
\hline \#HAC corrected & & & 8 & & & 4 & & & & 108 \\
\hline NH3N (mo/l) & 12.5 & 12.5 & 12.5 & 13.3 & 13.3 & 13.3 & 9.8 & 9.8 & 9.8 & 9.8 \\
\hline CN $(m g / 1)$ & 1.88 & 1.88 & 1.88 & 1.66 & 1.66 & 1.66 & 1.66 & 1.65 & 1.66 & 1.66 \\
\hline $\operatorname{SCN}$ (mg/l) & 6.2 & 6.2 & 6.2 & 6.9 & 6.9 & 6.9 & 6.9 & 6.9 & 6.9 & 6.9 \\
\hline N02 (mg/l) & 10.2 & 10.2 & 10.2 & 2.2 & 2.2 & 2.2 & 2.2 & 2.2 & 2.2 & 2.2 \\
\hline N03 (ag/1) & 199 & 199 & 199 & 197 & 197 & 197 & 197 & 197 & 197 & 197 \\
\hline Phenolics (mg/l) & 4.825 & 1.025 & 1.225 & & & & & & & \\
\hline PO4-P (ngill) & 8.1 & 8.1 & 8.1 & & & & & & & \\
\hline $\operatorname{Org}-\mathrm{N}(\mathrm{mg} / \mathrm{l})$ & 1.8 & 1.8 & 1.9 & & & & 2.3 & 2.3 & 2.3 & 2.3 \\
\hline \multicolumn{11}{|l|}{ TDS (ng/1) } \\
\hline Color (APHA units) & & & & 875 & 875 & 875 & 875 & 875 & 875 & 875 \\
\hline $\begin{array}{l}\text { TOC (mg/1) } \\
\text { BCD 5-day (me/1) }\end{array}$ & & & $-57-$ & 63 & 63 & 63 & 63 & 63 & 63 & 63 \\
\hline
\end{tabular}


LiVIT IA TABLE AI

\begin{tabular}{|c|c|c|c|c|c|c|c|c|c|c|}
\hline MONTH & SEPT & SEPT & SEPT & ȘEPT & SEPT & SEPT & SEPT & SEPT & SEPT & SEPT \\
\hline DAY & 13 & 14 & 15 & 16 & 17 & 18 & 19 & 20 & 21 & 22 \\
\hline DaY NO. & 49 & 50 & 51 & 52 & .53 & 54 & 55 & 56 & 57 & 58 \\
\hline \multicolumn{11}{|l|}{ SYSTFM PARAYYFIFAS } \\
\hline \&Sludgae Age & 20 & 20 & 20 & 20 & 20 & 20 & 20 & 15 & 15 & 15 \\
\hline$\star(F / M) C O D$ & 0.27 & 0.27 & 0.28 & 0.24 & 0.25 & 0.25 & 0.24 & 0.23 & 0.24 & 0.25 \\
\hline \#HRT & 1.9 & 2.8 & 1.3 & 2.0 & 1.9 & 1.9 & 2.8 & 1.9 & 1.9 & 1.9 \\
\hline FEED PARAMETERS (BATCH & 30 & 30 & 30 & 30 & 30 & 30 & 30 & 30 & 30 & 30 \\
\hline Volume (1iters) & 5 & 4.75 & 5.80 & 4.70 & 5.80 & 5.00 & 4.70 & 4.80 & 5.00 & 4.98 \\
\hline pH (std units) & $9.5 / 10.4$ & 9.69 & $.5 / 10.5$ & $10 / 10.8$ & 9.6 & 9.79 & $8 / 10.63$ & $1 / 10.39$ & 110.49 & $1 / 10.4$ \\
\hline $\cos (n g / 1)$ & 2063 & 2063 & 2063 & 1846 & 1846 & 1846 & 1846 & 1902 & 1802 & 1882 \\
\hline $\mathrm{NH} 3 \mathrm{~N}(\mathrm{mg} / 1)$ & 186 & 186 & 186 & 187 & 187 & 187 & 187 & 190 & 190 & 190 \\
\hline SS $(\pi \mathrm{g} / 1)$ & 66 & 66 & 66 & 66 & 66 & 66 & 66 & 126 & 126 & 126 \\
\hline USS (mg/1) & 32 & 32 & 32 & 32 & 32 & 32 & 32 & 46 & 46 & 46 \\
\hline CN (mg/1) & 8.33 & 0.53 & 0.33 & 0.33 & 0.33 & 0.33 & 0.33 & & & \\
\hline $\operatorname{SCN}(\mathrm{mg} / 1)$ & 193 & 193 & 193 & 193 & 193 & 193 & 193 & & & \\
\hline 800 5-day (mg/1) & 1410 & 1410 & 1410 & 1410 & 1410 & 1410 & 1410 & 1620 & 1620 & 1620 \\
\hline TOC ( $\mathrm{ng} / 1)$ & & & & & & & & 689 & 689 & 689 \\
\hline Phenolics (mg/l) & 1.54 & 1.54 & 1.54 & 1.54 & 1.54 & 1.54 & 1.54 & & & \\
\hline \multicolumn{11}{|l|}{$P D 4-P(x y / 1)$} \\
\hline \multicolumn{11}{|l|}{$D r g-N(n g / 1)$} \\
\hline \multicolumn{11}{|l|}{ 1 } \\
\hline \multicolumn{11}{|l|}{ N03 :ng/ll } \\
\hline & & & & & & & & & & \\
\hline \multicolumn{11}{|l|}{ Color (APHA Units) } \\
\hline \multicolumn{11}{|l|}{ BASIN PARAMETERS } \\
\hline Vol Wasted (al) & 434 & 426 & 478 & 577 & 580 & 560 & 560 & 778 & 758 & 752 \\
\hline D.0. $(\mathrm{mg} / 1)$ & 7.4 & 7.6 & 8.1 & 8.0 & 8.0 & 7.7 & 6.6 & 6.8 & 6.7 & 6.8 \\
\hline SS (mg/1) & 3600 & 3410 & 3270 & 31900 & 3310 & $3 \overline{3} 10$ & 3280 & 3320 & 3380 & 3190 \\
\hline SSS (PAC. mrrerted) & 3680 & 3410 & 3270 & 3190 & 3310 & 3310 & jov & 3320 & 3380 & 3130 \\
\hline Tenp (C) & 25 & 23 & 22 & 22 & 23 & 23 & 23 & 24 & 25 & 24 \\
\hline pH (std units) & 6.8 & 7.8 & 6.5 & 7.1 & 7.4 & 6.8 & 6.5 & 6.6 & 6.6 & 6.5 \\
\hline OUR (ag/l/hr) & & & $4 \mathbf{d}$ & & & & 38 & & & 36 \\
\hline VSS (ixy/l) & 3151 & 2990 & 3060 & 2985 & 3097 & 3897 & 3010 & 3123 & 3179 & 2900 \\
\hline \&VSS (PAC corrected) & 3157 & 2990 & 3068 & 2985 & 3097 & 3097 & 3010 & 3123 & 3179 & 2300 \\
\hline USS/SS & & & 0.94 & & & & 0.94 & & & 8.91 \\
\hline IPAC Added (ag) & 0 & 0 & 0 & 0 & 0 & 0 & 0 & 0 & 0 & 0 \\
\hline * Inventory (mg/l basin) & $\theta$ & 0 & a & 0 & 0 & $\boldsymbol{\theta}$ & 0 & 0 & $\theta$ & - \\
\hline \multicolumn{11}{|l|}{ EFFLUENT PRRAMETERS } \\
\hline Volume (liters) & 4.46 & 4.13 & 4.25 & 4.06 & 4.60 & 4.10 & 4.90 & 4.50 & 4.70 & 4.50 \\
\hline $\operatorname{COD}(\mathrm{mg} / 1)$ & 150 & 148 & 156 & 159 & 155 & 156 & IRB & 148 & 148 & 112 \\
\hline $5 s(m g / 1)$ & 134 & 144 & 94 & 18 & 14 & 32 & 26 & 16 & 30 & 34 \\
\hline +PRC corrected & 134 & 144 & 94 & 18 & 14 & 32 & 26 & 16 & 39 & .34 \\
\hline$O H$ (slu units) & 6.9 & 6.8 & 6.8 & 6.7 & 7.5 & 7.7 & 6.5 & 6.3 & 6.9 & 7.0 \\
\hline VSS $(m o / 1)$ & - & & 90 & & & & 22 & & & 26 \\
\hline APAC corrected & & & 90 & & & & 22 & & & 26 \\
\hline NHZN $(m g / 1)$ & 0.9 & 0.9 & 0.9 & 1.7 & 1.7 & 1.7 & 1.7 & 1.7 & 1.7 & 1.7 \\
\hline CN $(\mathrm{mg} / 1)$ & 1.19 & 1.19 & 1.19 & 1.19 & 1.19 & 1.19 & 1.19 & & & \\
\hline $\operatorname{SCN}(m g / 1)$ & 5.1 & 5.1 & 5.1 & 5.1 & 5.1 & 5.1 & 5.1 & & & \\
\hline NO2 (mg/l) & 4.84 & 4.04 & 4.84 & 4.04 & 4.04 & 4.04 & 4.84 & & & \\
\hline N03 $(n g / 1)$ & 195 & 195 & 195 & 195 & 195 & 195 & 195 & & & \\
\hline Phenolics $(\mathrm{ag} / \mathrm{l})$ & 10.025 & 10.025 & 18.025 & (0.0.025 & 10.025 & 10.025 & $<0.025$ & & & \\
\hline$P 04-P(\log / 1)$ & 11.1 & 11.1 & 11.1 & 11.1 & 11.1 & 11.1 & 11.1 & & & \\
\hline Org-N $(m g / 1)$ & & & & 2.9 & 2.9 & 2.9 & 2.9 & & & \\
\hline \multicolumn{11}{|l|}{ TLS $(\operatorname{mg} / 1)$} \\
\hline Color (APHA units) & & & & & & & & 750 & 750 & 750 \\
\hline TOC $(\pi g / 1)$ & & & & & & & & 56 & 56 & 56 \\
\hline BCD 5-day $(\mathrm{mg} / 1)$ & & & & $-58-$ & & & & 48 & 40 & 40 \\
\hline
\end{tabular}


UNIT IA TABLE AI

\begin{tabular}{|c|c|c|c|c|c|c|c|c|c|c|}
\hline MONTH & SEPT & 5EPT & SEPT & SEPT & SEPT & SEPT & SEPT & SEPT & $\mathrm{OCT}$ & OCT \\
\hline JAY & 23 & 24 & 25 & 26 & 27 & 28 & 29 & 30 & 1 & 2 \\
\hline DAY NO. & 59 & 68 & 61 & 62 & 63 & 64 & 65 & 66 & 67 & 68 \\
\hline \multicolumn{11}{|l|}{ SYSTEM FAKAMETERS } \\
\hline ^Sludge Age & 15 & 15 & 15 & 15 & 15 & 15 & 15 & 15 & 15 & 15 \\
\hline$F(F / M) C O D$ & 0.33 & 0.34 & 0.33 & 0.27 & 0.30 & 0.32 & 0.33 & 0.32 & 0.30 & 0.31 \\
\hline *HRT & 1.9 & 1.9 & 1.9 & 2.2 & 2.1 & 1.9 & 1.9 & 1.9 & 2.0 & 1.9 \\
\hline FEED PARAMETERS (BATCH & 38 & 30 & 38 & 30 & 30 & 31 & 31 & 31 & 31 & 31 \\
\hline Volume (liters) & 5.00 & 5.60 & 5.80 & 4.10 & 4.4 & 4.70 & 4.80 & 5.80 & 4.60 & 4.80 \\
\hline $\mathrm{pH}$ (std units) & 9.5 & 9.49. & $5 / 10.39$ & $5 / 10.43$ & $5 / 18.3$ & 18.1 & 18.1 & 11.3 & 11.4 & 11.4 \\
\hline COD (mg/1) & 2178 & 2178 & 2178 & 2178 & 2161 & 2161 & 2161 & 2028 & 2028 & 2028 \\
\hline NH3N (Gg/I) & 193 & 193 & 193 & 193 & 189 & 189 & 189 & 166 & 166 & 166 \\
\hline SS $(\mathrm{mg} / \mathrm{ll})$ & 126 & 126 & 126 & 126 & 244 & 244 & 244 & 244 & 244 & 244 \\
\hline VSS (*g/1) & 46 & 46 & 46 & 46 & 60 & 60 & 68 & 60 & 60 & 60 \\
\hline \multicolumn{11}{|l|}{ CN (ng/l) } \\
\hline 800 5-day $(\mathrm{mg} / 1)$ & 1628 & 1620 & 1620 & \multicolumn{7}{|c|}{ SCN $(n g / 1)$} \\
\hline TOC (ag/ $/ 1)$ & 689 & 689 & 689 & 689 & 660 & 660 & 668 & 660 & 650 & 660 \\
\hline Phenolics (ng/l) & & & & & 0.67 & 8.67 & 0.67 & 0.67 & 0.67 & 0.67 \\
\hline \multicolumn{11}{|l|}{ PO4-P (mg/1) } \\
\hline \multicolumn{11}{|l|}{$\operatorname{Org}-N(\operatorname{agg} / 1)$} \\
\hline \multicolumn{11}{|l|}{ NO2 (mg/l) } \\
\hline \multicolumn{11}{|l|}{$x 03(n g / 1)$} \\
\hline \multicolumn{6}{|l|}{$\operatorname{TDS}(\mathrm{gg} / \mathrm{l})$} & & & & & \\
\hline \multicolumn{11}{|l|}{ BASIN PARAMETERS } \\
\hline Vol Wasted (al) & 763 & 787 & 785 & 766 & 768 & 748 & 731 & 726 & 732 & 745 \\
\hline D.0. $(\operatorname{mg} / 1)$ & 7.0 & 7.0 & 7.8 & 7.4 & 7.6 & 7.5 & 7.6 & 7.6 & 7.6 & 7.2 \\
\hline SS (agg/l) & 3220 & 2940 & 3855 & 2960 & 2890 & 2870 & 2980 & 2960 & 2970 & 2900 \\
\hline ISS (PAC corrected) & 3028 & 2940 & 3055 & 2968 & 2890 & 2870 & 2980 & 2960 & 2970 & 2900 \\
\hline Temo (C) & 23 & 21 & 21 & 21 & 21 & 22 & 21 & 21 & 21 & 21 \\
\hline $\mathrm{pH}$ (std units) & 7.3 & 6.7 & 5.7 & 6.5 & 6.3 & 7.1 & 7.4 & 7.5 & 7.7 & 7.8 \\
\hline QUR (ng/!/hr) & & & & & & & 48 & & & \\
\hline VSS (ng/1) & 2745 & 2673 & 2777 & 2740 & 2675 & 2657 & 2640 & 2522 & 2631 & 2596 \\
\hline WSS (PAC corrected) & 2745 & 2673 & 2777 & 2740 & 2675 & 2657 & 2640 & 2622 & 2631 & 2596 \\
\hline VSSiSS & & & & 0.93 & & & 0.89 & & & \\
\hline PPAC Added (mg) & 8 & 8 & 0 & 8 & 0 & - & 8 & 8 & 0 & 8 \\
\hline +Inventory (mg/l basin) & 8 & 0 & 0 & 8 & 8 & 0 & 0 & 8 & 8 & 0 \\
\hline \multicolumn{11}{|l|}{ EFFLUENT FARAMETERS } \\
\hline Volune (liters) & 4.60 & 4.78 & 4.60 & 3.60 & 4.20 & 4.30 & 4.45 & 4.55 & 4.30 & 4.00 \\
\hline $\operatorname{COD}(\operatorname{mg} / 1)$ & 112 & 179 & 166 & 181 & 174 & 168 & 187 & 201 & 188 & 171 \\
\hline SS $(m g / 1)$ & 24 & 8 & 18 & 28 & 22 & 48 & 46 & 48 & 48 & 48 \\
\hline AFAC corrected & 24 & 8 & 10 & 28 & 22 & 48 & 46 & 48 & 48 & 40 \\
\hline pH (std units) & 7.2 & 7.1 & 6.2 & 6.7 & 6.8 & 6.9 & 7.4 & 7.1 & 7.6 & 7.8 \\
\hline USS (og/1) & & & & 16 & & & 40 & & & \\
\hline FPAC corrected & & & & 16 & & & 40 & & & \\
\hline NHJN $(\operatorname{mg} / 1)$ & 5.2 & 5.2 & 5.2 & 5.2 & 4.9 & 4.9 & 4.9 & 5.2 & 5.2 & 5.2 \\
\hline \multicolumn{11}{|l|}{ CN $(\mathrm{mg} / 1)$} \\
\hline \multicolumn{11}{|l|}{$\operatorname{SCN}(m / 1)$} \\
\hline \multirow{2}{*}{\multicolumn{8}{|c|}{$\mathrm{NO3}(\operatorname{mg} / 1)$}} & & & \\
\hline & & & & & 10.025 & 10.025 & & & & \\
\hline $\begin{array}{l}\text { Dhenolics }(\pi g / 1) \\
\text { PC4-D }(\pi g / 1)\end{array}$ & & & & & 12.3 & 12.3 & $\begin{array}{r}12.3 \\
12.303\end{array}$ & $\begin{array}{r}10.0 \mathrm{Cl} \\
15.3\end{array}$ & $\begin{array}{r}10.865 \\
12.3\end{array}$ & $\begin{array}{l}10.025 \\
123\end{array}$ \\
\hline \multicolumn{11}{|l|}{$\operatorname{Org}-N(\mathrm{mg} / \mathrm{l})$} \\
\hline $\operatorname{DDS}(\mathrm{ng} / 1)$ & & & & & & & & & & \\
\hline Color (APHA units) & 750 & 750 & 750 & 750 & & & & & & \\
\hline TOC $(\mathrm{mg} / \mathrm{l})$ & 56 & 56 & 55 & 56 & 49 & 49 & 49 & 49 & 49 & 49 \\
\hline BūD Juday (nig/1) & 40 & 40 & 40 & 48 & 9 & 3 & 9 & 9 & 9 & 9 \\
\hline
\end{tabular}


UNIT IA TABLE AI

\begin{tabular}{|c|c|c|c|c|c|c|c|c|c|c|}
\hline MONTH & OCT & OCT & $\mathrm{OCT}$ & OCT & OCT & OCT & OCT & OCT & OCT & OCT \\
\hline CAY & 3 & 4 & 5 & 6 & 7 & 8 & 9 & 10 & 11 & 12 \\
\hline DGY No. & 69 & 70 & 71 & 72 & 73 & 74 & 75 & 76 & 77 & 78 \\
\hline \multicolumn{11}{|l|}{ SYSTEM PAREMETERS } \\
\hline Sludge Age & 15 & 15 & 15 & 15 & 15 & 15 & 15 & 15 & 15 & 15 \\
\hline$+(F / M) C O D$ & 0.32 & 0.33 & 0.30 & 0.34 & 0.34 & 0.31 & 0.23 & 0.29 & 0.30 & 0.35 \\
\hline HHRT & 2.0 & 2.1 & 2.3 & 2.0 & 2.0 & 2.0 & 2.6 & 2.2 & 2.3 & 2.0 \\
\hline FEED PARGMETERS (BATCH ह) & 31 & 31 & 31 & $3 !$ & 31 & 31 & 31 & 31 & $3 !$ & 31 \\
\hline Volume (liters) & 4.60 & 4.45 & 4.88 & 4.60 & 4.75 & 4.55 & 3.55 & 4.18 & 4.1 & 4.63 \\
\hline$O H$ (std units) & 11.5 & $1.5 / 10.1$ & $11.5 / 10$ & 11.6 & 11.3 & $.5 / 10.1$ & 11.5 & 11.5 & 11.3 & 11.2 \\
\hline $\operatorname{COD}(\mathrm{ng} / 1)$ & 2028 & 2085 & 2085 & 2085 & 2052 & 2052 & 2052 & 2052 & 2098 & 2898 \\
\hline NHJN $($ ngg $/ 1)$ & 166 & 178 & 178 & 178 & 153 & 153 & 153 & 153 & 156 & 156 \\
\hline $55(\mathrm{mg} / \mathrm{l})$ & 244 & 436 & 436 & 436 & 436 & 436 & 436 & 436 & & \\
\hline USS (mg/1) & 60 & 92 & 92 & 92 & 92 & 92 & 92 & 92 & & \\
\hline \multicolumn{11}{|l|}{ CN (ng)/1) } \\
\hline \multicolumn{11}{|l|}{$\operatorname{SCN}(m g / 1)$} \\
\hline BOD 5-day (xg/1) & 1470 & 1400 & 1400 & 1400 & 1400 & 1400 & 1408 & 1400 & IJEO & 1360 \\
\hline TOC $(\mathrm{mg} / \mathrm{l})$ & 660 & 646 & 646 & 646 & 646 & 646 & 646 & 646 & 649 & 649 \\
\hline Phenolics (mg/l) & 0.67 . & & & & & & & & & \\
\hline \multicolumn{11}{|l|}{$204-P($ mga/1) } \\
\hline \multicolumn{11}{|l|}{$\operatorname{Org}-N(a g / 1)$} \\
\hline \multicolumn{11}{|l|}{$102(\mathrm{mg} / 1)$} \\
\hline \multicolumn{11}{|l|}{$403(\mathrm{mg} / \mathrm{ll})$} \\
\hline \multicolumn{11}{|l|}{ TDS $(\mathrm{mg} / \mathrm{ll}$} \\
\hline \multirow{2}{*}{\multicolumn{11}{|c|}{ Color (APHA Units) }} \\
\hline \multicolumn{2}{|l|}{ BASIN PARRMETERS } & & & & & & & & & \\
\hline Vol Wasted (o1) & 759 & 772 & 753 & 732 & 758 & 730 & 708 & 723 & 753 & 756 \\
\hline 0.0. $(\mathrm{mg} / \mathrm{l})$ & 7.4 & 7.1 & 7.2 & 7.4 & 7.2 & 7.3 & 7.1 & 7.2 & 7.4 & 7.3 \\
\hline SS $(m g / 1)$ & 2840 & 2670 & 2700 & 2590 & 2690 & 2755 & 2970 & 2780 & 2690 & 2680 \\
\hline ISS (PAC corrected) & 8840 & 2670 & 2700 & 2590 & 2690 & 8755 & 2970 & 2780 & 2698 & 2600 \\
\hline Temo (C) & 22 & 24 & 24 & 23 & 23 & 21 & 22 & 23 & 22 & 23 \\
\hline $\mathrm{pH}$ (std units) & 7.9 & 8.0 & 7.9 & 7.8 & 7.8 & 8.0 & 7.9 & 7.8 & 8.1 & 7.9 \\
\hline DUR (mg/1/hr) & & & & 46 & & & & 42 & & \\
\hline VSS $(\mathrm{mg} / 1)$ & 2460 & 2313 & 2339 & 2330 & 2420 & 2478 & 2672 & 2440 & 2361 & 2282 \\
\hline 4USS (PAC corrected) & 2468 & 2313 & 2339 & 2330 & 2420 & 2478 & 2672 & 2448 & 2351 & 2282 \\
\hline USS/SS & 0.87 & & & 0.90 & & & & 0.88 & & \\
\hline spaC Added (mg) & $\theta$ & ( & 0 & 0 & 0 & 0 & $\theta$ & a & 0 & 8 \\
\hline Inventory (mg/l basin) & D & 0 & 0 & 0 & 8 & 9 & 0 & 8 & 0 & 0 \\
\hline \multicolumn{11}{|l|}{ EEFIUUENT FARATETERS } \\
\hline Volune (literg) & 4.85 & 4.10 & 3.70 & 4.35 & 4.40 & 4.20 & 2.55 & 4.45 & 3.75 & 4.45 \\
\hline $\operatorname{coD}(\operatorname{mg} / 1)$ & 169 & 157 & 140 & 145 & 149 & 133 & 141 & 116 & 127 & 137 \\
\hline $55(\mathrm{igg} / \mathrm{i})$ & 24 & 18 & 34 & 40 & 26 & 46 & 116 & 48 & 34 & 26 \\
\hline APAC corrected & 24 & 18 & 34 & 40 & 26 & 46 & 116 & 48 & 34 & 26 \\
\hline PH (std units) & 7.9 & 8.0 & 8.0 & 8.1 & 8.0 & 8.0 & 8 & 8.0 & 8.2 & 8.8 \\
\hline USS (mg/1) & 24 & & & 34 & & & & 42 & & \\
\hline APAC corrected & 24 & & & 34 & & & & 42 & & \\
\hline NHBN $(\mathrm{mg} / 1)$ & 5.2 & 1.8 & 1.8 & 1.8 & 2.9 & 2.9 & 2.9 & 2.9 & 3.5 & 3.5 \\
\hline \multicolumn{11}{|l|}{ CN (mg/l) } \\
\hline \multicolumn{11}{|l|}{$\operatorname{SCN}(m g / 1)$} \\
\hline \multicolumn{11}{|l|}{ No2 $(\mathrm{mg} / \mathrm{l})$} \\
\hline \multicolumn{11}{|l|}{$: 00.3(\infty g / 1)$} \\
\hline Fhenolics $(\mathrm{mg} / \mathrm{l})$ & 10.025 & & & & & & & & & \\
\hline$P O 4-P(n g / 1)$ & 12.3 & & & & & & & & 1.3 & 1.3 \\
\hline $\begin{array}{l}O r g-N(m g / 1) \\
T D S(n g / 1)\end{array}$ & & & & & & & & & & \\
\hline Co:or (APHA units) & & 625 & 625 & 625 & 625 & 625 & 625 & 625 & & \\
\hline $\operatorname{TOC}(\mathrm{mo} / 1)$ & 49 & 39 & 39 & 39 & 39 & 39 & 39 & 39 & 36 & 36 \\
\hline BOD 5-day $(m \mathrm{~m} / 1)$ & 9 & 13.4 & 13.4 & 13.4 & $(3.4$ & (3.4 & 13.4 & 13.4 & 1.8 & 1.8 \\
\hline & & & & $-60-$ & & & & & & \\
\hline
\end{tabular}


UNIT IA TABLE AI

\begin{tabular}{|c|c|c|c|c|c|c|c|c|c|c|}
\hline MONTH & OCT & OCT & OCT & OCT & OCT & OCT & OCT & OCT & OCT & OCT \\
\hline DAY & 13 & 14 & 15 & 16 & 17 & 18 & 19 & 20 & 21 & 22 \\
\hline DAY NO. & 79 & 88 & 81 & 82 & 83 & 84 & 85 & 86 & 97 & 88 \\
\hline \multicolumn{11}{|l|}{ SYSTEM FGRAMETERS } \\
\hline ISludge Age & 15 & 15 & 15 & 15 & 15 & 15.80 & 15.01 & 15.03 & 15.80 & 15.00 \\
\hline$\star(F / M) C O D$ & 0.18 & 0.30 & 0. 32 & 0.30 & 0.39 & 0.34 & 0.34 & 0.38 & 0.38 & 0.38 \\
\hline +HRT & 4.1 & $2 . \hat{1}$ & 2.1 & 2.8 & 2.1 & 1.86 & 1.86 & 1.96 & 1.36 & 1.86 \\
\hline FEED PARAMETERS (BATCH & 31 & 31 & 31 & 31 & 31 & 32 & 32 & 32 & 32 & 32 \\
\hline Volume (liters) & 2.25 & 4.35 & 4.40 & 4.62 & 5.80 & 5 & 5 & 5 & 5 & 5 \\
\hline $\mathrm{pH}$ (std units) & 11.8 & 10.9 & 10.9 & 11.71 & $5 / 9.8$ & 11.5 & 11.4 & 11.5 & 11.5 & 11.5 \\
\hline $\cos (\mathrm{ng} / 1)$ & 2098 & 1983 & 1983 & 1983 & 1983 & 1925 & 1925 & 1925 & 1926 & 1926 \\
\hline NHBN $(m g / 1)$ & 156 & 158 & 158 & 158 & 158 & 169 & 169 & 169 & 166 & 166 \\
\hline SS (mg/1) & & & & & & 140 & 140 & 148 & 140 & 140 \\
\hline VSS · (mฐ $/ 1)$ & & & & & & 46 & 46 & 46 & 46 & 46 \\
\hline \multicolumn{11}{|l|}{ CN $(m g / 1)$} \\
\hline \multicolumn{11}{|l|}{$\operatorname{SCN}(\mathrm{mg} / 1)$} \\
\hline 800 5-day (mg/1) & 1360 & 1360 & 1360 & 1360 & 1368 & 1110 & 1110 & 1110 & 1110 & 1110 \\
\hline TOC $(m g / 1)$ & 649 & 649 & 649 & 649 & 649 & 642 & 642 & 642 & 642 & 642 \\
\hline \multicolumn{11}{|l|}{ Phenolics $(m g / 1)$} \\
\hline \multicolumn{11}{|l|}{$P 04-P$ (ng/1) } \\
\hline \multicolumn{11}{|l|}{ Org-N (mg/1) } \\
\hline \multicolumn{11}{|l|}{$1402(\mathrm{mg} / 1)$} \\
\hline \multicolumn{11}{|l|}{$: 03$ (mg/1) } \\
\hline \multicolumn{11}{|l|}{$\operatorname{TDS}(\mathrm{mg} / 1)$} \\
\hline \multicolumn{11}{|l|}{ Color (APHA Units) } \\
\hline \multicolumn{11}{|l|}{ BCSIN PARAMETERS } \\
\hline Vol Hasted (mI) & 761 & 703 & 714 & 718 & 748 & 708 & 719 & 720 & 466 & 722 \\
\hline $0.0 .(\mathrm{mg} / 1)$ & 7.4 & 7.5 & 7.5 & 7.3 & 7.2 & 7.2 & 6.8 & 6.4 & 6.2 & 6.2 \\
\hline SS $(m g / 1)$ & 2650 & 2820 & $2 \in 75$ & 3000 & 2578 & 2580 & 2660 & 2590 & 2550 & 2518 \\
\hline ISS (PAC corrected) & 2650 & 2820 & 2675 & 3080 & 2570 & 2580 & 2668 & 2530 & 2550 & 2510 \\
\hline Tenp (C) & 23 & ฉ2 & 22 & 21 & 22 & 22 & 22 & 20 & 20 & 21 \\
\hline ọ (std units) & 8.? & 7.9 & 8.8 & 8.8 & 8.8 & 7.9 & 7.7 & 8.1 & 8 & 7.8 \\
\hline OUR $(\mathrm{mg} / \mathrm{l} / \mathrm{hr})$ & 42 & & & & 40 & & & 36 & & \\
\hline USS $(m g / 1)$ & 2230 & 2373 & 2251 & $\hat{2525}$ & 2120 & 2330 & 2330 & 2110 & 2110 & 2110 \\
\hline IVSS (PAC corrected) & 2230 & 2373 & 2251 & 2525 & 2120 & 2330 & 2330 & 2110 & 2110 & 2110 \\
\hline USS/SS & 0.84 & & & & 0.82 & & & 0.81 & & \\
\hline FAC Added (mg) & 0 & 8 & 0 & 8 & 0 & 8 & 0 & 0 & 0 & 0 \\
\hline Inventory (mg/l basin) & 0 & 8 & e & 0 & 8 & 8 & 0 & 0 & 0 & 0 \\
\hline \multicolumn{11}{|l|}{ EFFLUENT FARAMETERS } \\
\hline Voiune (liters) & 2.85 & 4.02 & 4.80 & 4.26 & 4.72 & 4.75 & 4.71 & 4.73 & 4.73 & 4.75 \\
\hline $\operatorname{COD}(\pi g / 1)$ & 146 & 148 & 144 & 152 & 168 & 156 & 129 & 124 & 135 & 134 \\
\hline SS (ng/1) & 50 & 68 & 48 & 58 & 28 & 50 & 46 & 44 & 180 & 42 \\
\hline \#PAC corrected & 50 & 68 & 48 & 58 & 28 & 50 & 46 & 44 & 180 & 42 \\
\hline oH (std units) & 8.2 & 8.0 & 8.1 & 8.1 & 8.1 & 8.2 & 9.0 & 8.2 & 8.2 & 8.0 \\
\hline USS (ag/1) & 36 & & & & 26 & 34 & 34 & 34 & 34 & 34 \\
\hline *AAC corrected & 36 & & & & 26 & 34 & 34 & 34 & 34 & 34 \\
\hline NHIN $(a g / 1)$ & 3.5 & 8.7 & 8.7 & 8.7 & 8.7 & 0.9 & 8.9 & 8.9 & 3.8 & 3.8 \\
\hline \multicolumn{11}{|l|}{ CN $(\pi g / 1)$} \\
\hline \multicolumn{11}{|l|}{$\operatorname{SCN}(\operatorname{mg} / 1)$} \\
\hline \multicolumn{11}{|l|}{ NO2 (mg/l) } \\
\hline $\mathrm{N03}(\mathrm{gg} / 1)$ & & & & & & & & & & \\
\hline Penolics (ag/l) & & & & & & & & & & \\
\hline$=04-\rho(m g / 1)$ & 1.3 & 1.3 & 1.3 & 1.3 & 1.3 & & & & & \\
\hline $\begin{array}{l}\text { Drg } N(\mathrm{mg} / 1) \\
\operatorname{TDS}(\mathrm{mg} / \mathrm{l})\end{array}$ & & & & & & & & & & \\
\hline Colur (APHA units) & & & & & & 508 & 508 & 500 & 580 & 500 \\
\hline TOC (mg//1) & 36 & 36 & 36 & 36 & 36 & 37 & 37 & 37 & 37 & 37 \\
\hline BOD 5-cay (ng/1) & 1.8 & 1.8 & 1.8 & 1.8 & 1.8 & 1.5 & 1.5 & 1.5 & 1.5 & 1.5 \\
\hline
\end{tabular}


UNIIT IA TABLE A1

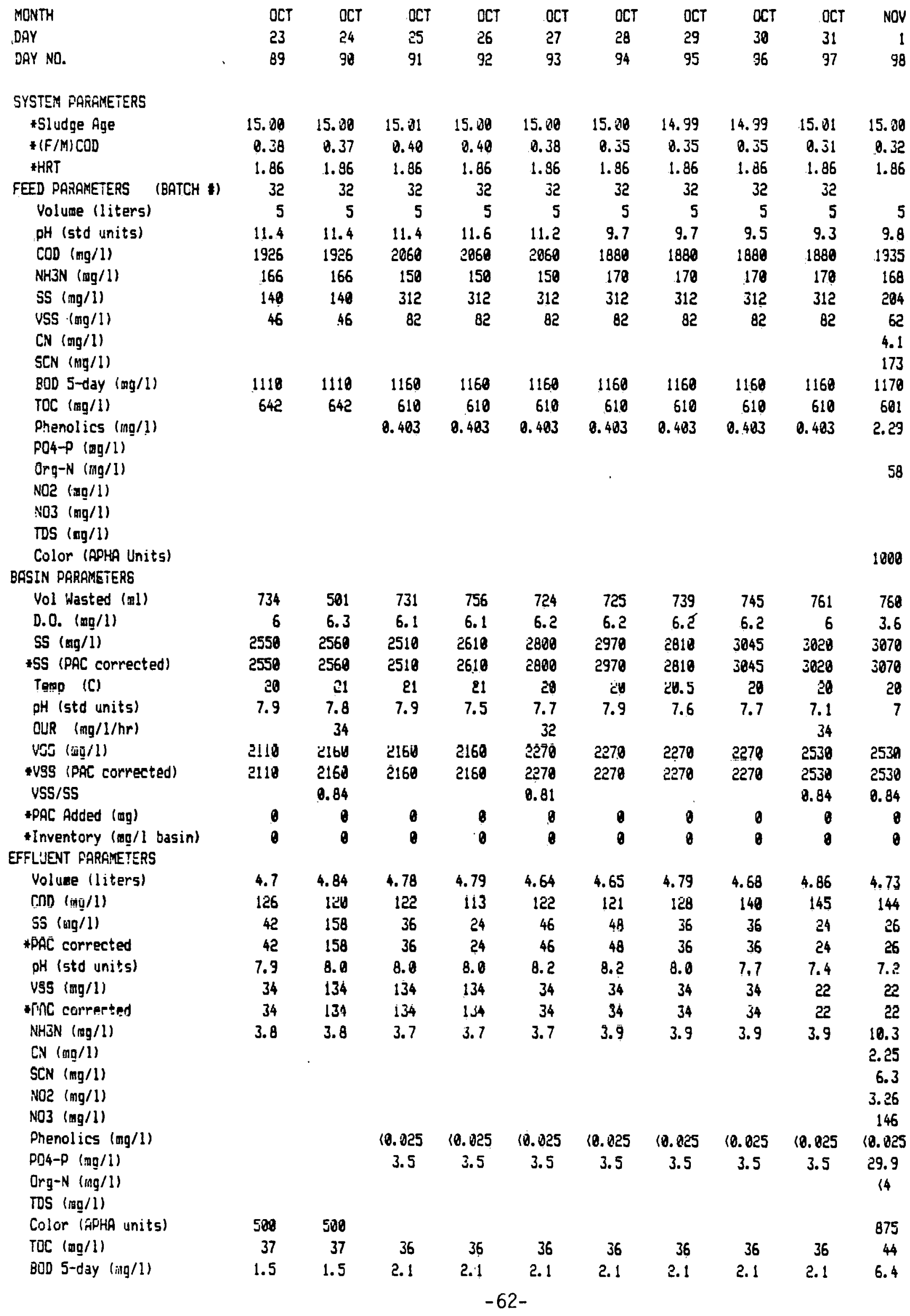


UNIT IA TABLE AI

\begin{tabular}{|c|c|c|c|c|c|c|c|c|c|c|}
\hline MONTH & NOV & NOV & NOV & NOV & NOV & NOV & NOW & NOV & NOV & NOV \\
\hline DAY & 2 & 3 & 4 & 5 & 6 & 7 & 8 & 9 & 10 & 11 \\
\hline DAY NO. & 99 & 188 & 101 & 102 & 103 & 104 & 105 & 106 & 107 & 108 \\
\hline \multicolumn{11}{|l|}{ SYSTEM FGRAMETERS } \\
\hline tSludge न̃ge & 15.80 & 14.99 & 11.07 & 14.39 & 15.01 & 15.00 & 14.99 & 15.09 & 15.12 & 15.01 \\
\hline$+(F / M) C O D$ & 0.32 & 0.32 & 0.34 & 0.34 & 0.33 & 0.34 & 0.37 & 0.34 & 0.31 & 0.38 \\
\hline *HRT & 1.86 & 1.86 & 1.86 & 1.86 & 1.86 & 1.86 & 1.69 & 2.87 & 2.10 & 2.10 \\
\hline \multicolumn{11}{|c|}{ FEED PARAMETERS (BATCH } \\
\hline Volume (liters) & 5 & 5 & 5 & 5 & 5 & 5 & 5.5 & 4.5 & 4.42 & 4.42 \\
\hline pH (std units) & 9.8 & 9.7 & 9.6 & 12.2 & 12.2 & 12.2 & 11.2 & 12.4 & 11.9 & 10.6 \\
\hline $\operatorname{COD}(\mathrm{mg} / 1)$ & 1935 & 1935 & 2050 & 2050 & 2050 & 2858 & 2011 & 2011 & 2011 & 1992 \\
\hline NHJN $(\pi g / 1)$ & 168 & 168 & 173 & 173 & 173 & 173 & 164 & 164 & 164 & 156 \\
\hline SS $(m g / 1)$ & 284 & 204 & 204 & 204 & 294 & 204 & 338 & 338 & 338 & 338 \\
\hline USS $\cdot(\mathrm{mg} / 1)$ & 62 & 62 & 62 & 62 & 62 & 62 & 90 & 90 & 90 & 90 \\
\hline CN (mq/1) & 4.1 & 4.1 & -4.1 & 4.1 & 4.1 & 4.1 & & & & \\
\hline SCN (mq/1) & $\$ 73$ & 173 & 173 & 173 & 173 & 173 & & & & \\
\hline 800 5-day $(\mathrm{mg} / 1)$ & 1170 & 1178 & 1330 & 1330 & 1330 & 1330 & 1218 & 1218 & 1210 & 1290 \\
\hline TOC (mg/l) & 601 & 601 & 629 & 629 & 629 & 623 & 626 & 626 & 626 & 643 \\
\hline Ohenolics (mg/l) & 2.29 & 2.27 & 2.29 & 2.29 & 2.29 & 2.29 & 5.24 & 5.24 & 5.24 & $5 . \hat{c} 4$ \\
\hline$P 04 \rightarrow$ (mg/l) & & & & & & & & & & \\
\hline $\mathrm{Crg}-\mathrm{H}(\mathrm{ng} / \mathrm{l})$ & 58 & 58 & 58 & 58 & 58 & 58 & & & & \\
\hline
\end{tabular}

NO2 (ng/1)

Y03 $(\mathrm{ng} / \mathrm{ll})$

TDS (mg/1)

Color (APHA Units)

$\begin{array}{llllllllll}1000 & 1000 & 1080 & 1000 & 1000 & 1000 & 750 & 750 & 750 & 750\end{array}$

BASIN FARAMETERS

Vol Hasted (al)

D.0. $(\operatorname{mg} / 1)$

SS (mo/l)

*SS (PAC corrected)

Teno (C)

of (sto units)

OUR (mg/l/hr)

VSS (mg/l)

*USS (PAC corrected)

USS/SS

IPAC Added (og)

*Inventory (ag/l basin)

EFFLUENT FARAYETERS

Volune (liters)

COD $(\operatorname{mg} / 1)$

$55(\mathrm{mg} / 1)$

APAC corrected

oH (std units)

VSS (ng/l)

PAC corrected

NHAN (ag/l)

CN (mg/1)

SCN (ng/1)

NO2 (mg/l)

$\mathrm{NO} 3$ (周/1)

Phenolics (mg/l)

PO4-P (mg/l)

Org-N (mg/1)

TOS (mg/l)

Color (AD̈HA units)

TOC (mo/l)

aOD g-day $(m y / 1)$

\section{5}

3.

2880

2880

28

6.46 .6

253

2530

0.84

0.87
0

748

8.5

2860

2860

19.5

6.6

22

2490

2490

0. 87

5

2671

2671

19.5

6.4

32

22

90

2490

2498

0.87

753

5.8

2810

2810

19

6.3

685

5.6

3018

3018

28

8.4

2621

2521

0.87

0

4.77

4.69

4.86

4.75

142

50

50

7.3

22

22

10.3

2.25

144

150

590

171

4.66

4.74

167

74

590

28

74

32
6.9

6.8

6.7

8. 2

24

24

24

24

24

24

24

40.2

40.2

40.2

2. 25

2.25

2. 25

2. 25

6.3

6.3

3.26

3.26

6.3

3.36

3.26

$146 \quad 146$

10.025

10.025

146

18. 825

146

10.025

146

21. 5

10. 025

21.5

4

$<4$

875

$44 \quad 44$

6.46 .4

875

48

875

48

6.9

$-63-$
733

3268

3260

19

9.4

21

2530

2530

0. 78

0

$\begin{array}{rrrr}700 & 725 & 746 & 765 \\ 8.2 & 8.1 & 8.6 & 8.6 \\ 3170 & 2860 & 2735 & 2180 \\ 3170 & 2860 & 2735 & 2180 \\ 19 & 19.5 & 19.5 & 19 \\ 8.6 & 7.9 & 7.9 & 7.2 \\ & & 34 & \\ 2460 & 2220 & 2395 & 1989 \\ 2460 & 2220 & 2395 & 1909 \\ 0.78 & 0.78 & 0.88 & 0.88 \\ 0 & 0 & 0 & 0 \\ 0 & 0 & 0 & 0\end{array}$

5.49

4.31

284

$4.33 \quad 4.2$

248

58

50

308

34

257

50

8.8

$34 \quad 18$

8.8

8.5

38

$8.0 \quad 7.6$

38

40.2

2. 25

38

38

$38 \quad 38$

92.4

92.4

92.4

47.1

6.3

3.26

146

10.025

3.6

3.6

3.6

10.8

1 
UNIT IA TABLE A!

\begin{tabular}{|c|c|c|c|c|c|c|c|c|c|c|}
\hline MONTH & NOV & NOV & NOV & NOV & NOV & NOV & NOV & NOV & NOV & NOV \\
\hline OAY & 12 & 13 & 14 & 15 & 16 & 17 & 18 & 19 & 20 & 21 \\
\hline DAY NO. & 109 & 110 & 111 & 112 & 113 & 114 & 115 & iis & 117 & 118 \\
\hline \multicolumn{11}{|l|}{ SYSTEM FAR:AMETERS } \\
\hline tSludg̣e Age & 15.00 & 15.80 & 15.87 & 15.00 & 15. 02 & 15.80 & 15.87 & 14.21 & 14.99 & 15.16 \\
\hline$+(F / M) C O D$ & 0.44 & 0.37 & 0.34 & 0.34 & 0.40 & 0.37 & 0.43 & 0.49 & 0.43 & 0.38 \\
\hline *HRT & 1.70 & 2.18 & 1.36 & 2.07 & 2.09 & 2.00 & 2.16 & 1.85 & 2.24 & 1.96 \\
\hline \multicolumn{11}{|c|}{ FEED PARAMETERS (BATCH } \\
\hline Voluae (1iters) & 4.9 & 4.425 & 4.75 & 4.5 & 4.45 & 4.64 & 4.3 & 5.83 & 4.15 & $4.7 \overline{4}$ \\
\hline oH (std units) & 11.8 & 11.7 & 11.8 & 11.9 & 12 & 11.8 & 11.2 & 11.8 & 11.8 & 11.9 \\
\hline $\operatorname{COD}(n g / 1)$ & igg̀ & 1992 & 1992 & 2859 & 2059 & 2059 & 2859 & 28359 & $2859^{\circ}$ & 2059 \\
\hline NHZ3N $(\mathrm{mg} / 1)$ & 156 & 156 & 156 & 135 & 135 & 135 & 134 & 134 & 134 & 134 \\
\hline SS $(\infty \mathrm{mg} / 1)$ & $338^{\circ}$ & 338 & 338 & 700 & 700 & 700 & 700 & $780^{\circ}$ & 708 & 700 \\
\hline VSS. (mg/1) & 90 & 98 & 99 & 122 & 122 & 122 & 122 & 122 & 122 & 122 \\
\hline$C N(\operatorname{lgg} / 1)$ & & & & 3.99 & 3.99 & 3.99 & 3.99 & 3.99 & 3.99 & 3.99 \\
\hline $\operatorname{SCN}(\operatorname{mg} / 1)$ & & & & 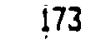 & 173 & 173 & 173 & 173 & 173 & 173 \\
\hline BOD 5-day (mg/1) & $1280^{\circ}$ & 1280 & 1280 & 1850 & 1050 & $1850^{\circ}$ & 1328 & 1320 & 1320 & 1320 \\
\hline $\operatorname{Toc}(\mathrm{og} / 1)$ & 643 & 643 & 643 & 637 & 637 & 637 & 653 & 653 & 653 & 653 \\
\hline Phenolics (ag/li) & 5.24 & 5.24 & 5.24 & 3.33 & 3.33 & 3.33 & 3.33 & 3.33 & 3.33 & 3.33 \\
\hline \multicolumn{11}{|l|}{$P O 4-P(m g / 1)$} \\
\hline Org-H (mg/1) & & & & 60 & 60 & 60 & 68 & 60 & 5n & 60 \\
\hline
\end{tabular}

No2 (mg/l)

$\mathrm{NO3}(\mathrm{mg} / 1)$

TOS (mg/l)

$\begin{array}{lllllllllll}\text { Color (APHA Units) } & 750 & 750 & 750 & 1250 & 1250 & 1250 & 1250 & 1250^{\circ} & 1250 & 1250\end{array}$

BASIN PARAMETERS

Vol Wasted (ml)

D.0. (mg/1)

SS (mg/1)

ISS (PAC corrected)

Temo (C)

oH (std units)

OUR (mg $/ 1 / \mathrm{hr}$ )

VSS (ag/1)

-VSS (PAC corrected)

VSS/SS

:PAC Aoded (ig)

-Inventory (mg/l basin)

EFFLUENT FARARETERS

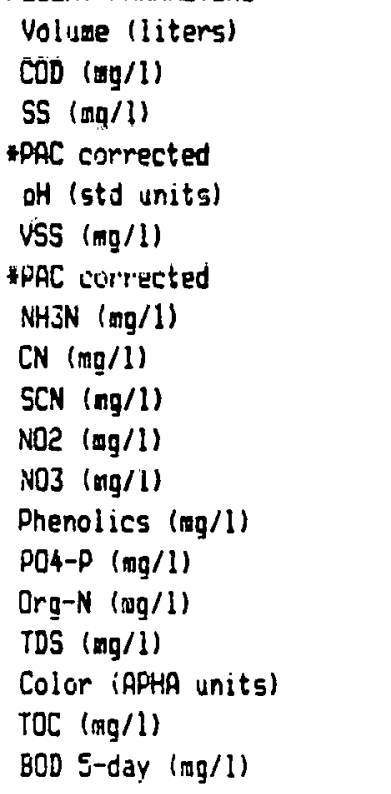

$\begin{array}{rrrrrrrrrrr}762 & 595 & 760 & 770 & 770 & 710 & 760 & 260 & 587 & 660 \\ 8.6 & 8.2 & 8.6 & 8.2 & 6.8 & 8.6 & 8 & 8 & 7.9 & 8.2 \\ 2110 & 2298 & 2590 & 2538 & 2135 & 2443 & 1983 & 1998 & 1985 & 2345 \\ 2110 & 2290 & 2590 & 2538 & 2135 & 2443 & 1983 & 1998 & 1995 & 2345 \\ 19 & 19 & 18.5 & 18 & 19.5 & 19 & 18 & 19 & 19 & 19 \\ 7.7 & 7.7 & 7.3 & 8 & 6.8 & 7.9 & 7.5 & 7.6 & 7.9 & 8.2 \\ 18.9 & 2085 & 2290 & 2244 & 1889 & 2133 & 1731 & 1744 & 1663 & 2113 \\ 1848 & 2085 & 2290 & 2244 & 1888 & 2133 & 1731 & 1744 & 1663 & 2113 \\ 1848 & 0.88 & 0.88 & 0.88 & 0.88 & 0.88 & 0.87 & 0.87 & 0.87 & 0.87 & 0.90 \\ 0 & 0 & 0 & 0 & 0 & 0 & 0 & 0 & 0 & 0 \\ 0 & 0 & 0 & 0 & 0 & 0 & 0 & 0 & 0 & 0\end{array}$

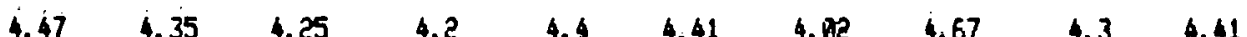

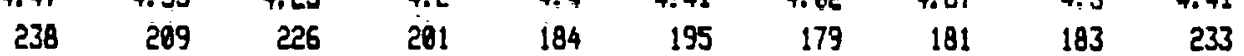

$\begin{array}{llllllllll}18 & 108 & 22 & 18 & 14 & 50 & 18 & 250 & 130 & 70\end{array}$

$18 \quad 180 \quad 22$

$7,3 \quad 7.3 \quad 7.8$

$38 \quad 38 \quad 28$

$\begin{array}{lll}38 & 38 & 20\end{array}$

$47.1 \quad 47.1 \quad 47.1$

$\begin{array}{lllllll}8.2 & 7.9 & 7.6 & 7.5 & 7.7 & 8.1 & 8.4\end{array}$

49

$\begin{array}{lllllll}6.3 & 6.3 & 6.3 & 4.9 & 4.9 & 4.9 & 4.9\end{array}$

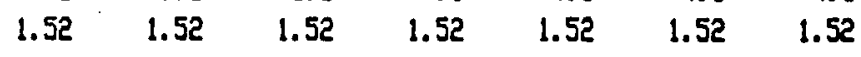

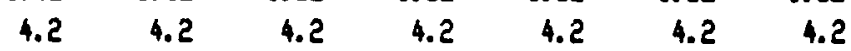

$\begin{array}{lllllll}17.3 & 17.3 & 17.3 & 17.3 & 17.3 & 17.3 & 17.3\end{array}$

$\begin{array}{lllllll}148 & 148 & 148 & 148 & 148 & 148 & 148\end{array}$

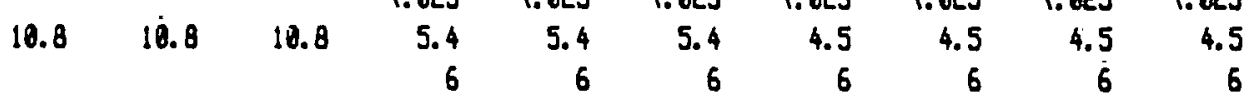

$\begin{array}{llllllllll}875 & 875 & 875 & 750 & 758 & 750 & 750 & 750 & 750 & 750\end{array}$

$\begin{array}{llllllllll}53 & 53 & 53 & 53 & 53 & 55 & 55 & 55 & 55 & 55\end{array}$

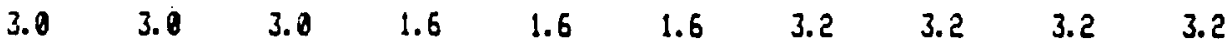


UNIT IA TABLE AI

\begin{tabular}{|c|c|c|c|c|c|c|c|c|c|c|}
\hline MONTH & NOV & NDV & NON & NOV & NOV & NOV & NOV & NOV & NOV & DEC \\
\hline DAY & 22 & 23 & 24 & 25 & 36 & 27 & 28 & 29 & 38 & 1 \\
\hline DAY NO. & 119 & 128 & 121 & 122 & 123 & 124 & 125 & $\$ 26$ & 127 & 128 \\
\hline \multicolumn{11}{|l|}{ SYSTEM PARAGMETERS } \\
\hline *Sludge Âge & 15.07 & 15.21 & 15.81 & 14.93 & 14.99 & 15.00 & 15.00 & 15.00 & 15.00 & 15.20 \\
\hline$\pm(F / M) C O D$ & 0.38 & 0.37 & 0.27 & 0.01 & 0.24 & 0.23 & 0.35 & 0.37 & 0.49 & 8.45 \\
\hline HRT & 2.84 & 2.12 & 1.39 & +66.42857 & 2.67 & 2.43 & 2.48 & 2.33 & 1.80 & 1.94 \\
\hline \multicolumn{11}{|l|}{ FEED FARAMETERS (BATCH } \\
\hline Volune (liters) & 4.55 & 4.39 & 4.93 & 0.14 & 3.46 & 3.825 & 3.75 & 4 & 5.17 & 4.8 \\
\hline $\mathrm{pH}$ (std units) & 11.9 & 11 & 12 & 10 & 12 & 11.5 & 11.9 & 11.8 & 11.8 & 11.7 \\
\hline $\operatorname{COD}(\mathrm{mg} / 1)$ & 2059 & 2059 & 2059 & 2098 & 2098 & 2098 & 2098 & 2027 & 2027 & 2027 \\
\hline NH3N $(\operatorname{mg} / 1)$ & 134 & 134 & 134 & 147 & 147 & 147 & 147 & 147 & 147 & 147 \\
\hline SS (ng/1) & 795 & 795 & 795 & 795 & 795 & 795 & 795 & 395 & 395 & 395 \\
\hline VSS $(\log / 1)$ & 170 & 170 & 170 & 170 & 170 & 170 & 178 & 85 & 85 & 85 \\
\hline CN (ang/l) & 1.24 & 1.24 & 1.24 & 1.24 & 1.24 & 1.24 & 1.24 & 1.24 & 1.24 & 1.24 \\
\hline $5 C N(m g / 1)$ & 188 & 188 & 188 & 188 & 180 & 188 & 188 & 188 & 188 & 188 \\
\hline 800 5-day (mo/d) & 1248 & 1240 & 1248 & 1220 & 1220 & 1228 & 1220 & 1200 & 1200 & 1200 \\
\hline TOC $(m g / 1)$ & 650 & 650 & 650 & 660 & 660 & 660 & 660 & 647 & 647 & 647 \\
\hline Phenolics (Gg/l) & 5.11 & 5.11 & 5.11 & 5.11 & 5.11 & 5.11 & 5.11 & 5.89 & 5.09 & 5.09 \\
\hline \multicolumn{11}{|l|}{$P 04-P(\mathrm{mg} / \mathrm{l})$} \\
\hline Org-N (mg/l) & 73 & 73 & 73 & 73 & 73 & 73 & 73 & 73 & 73 & 73 \\
\hline \multicolumn{11}{|l|}{ NOP $(m g / 1)$} \\
\hline \multicolumn{11}{|l|}{$\mathrm{NO3}(\mathrm{mg} / 1)$} \\
\hline \multicolumn{11}{|l|}{ TOS $(\operatorname{mg} / 1)$} \\
\hline Color (APHA Units) & 1560 & 1500 & 1500 & 1580 & 1580 & 1500 & 1500 & 1500 & 1580 & 1500 \\
\hline \multicolumn{11}{|l|}{ BASIN PARAMETERS } \\
\hline Vol Wasted (ml) & 680 & 720 & 640 & 710 & 169 & 525 & 470 & 531 & 502 & 647 \\
\hline $0.0 .(\mathrm{gg} / 1)$ & 8.6 & 7.8 & 7.8 & 7.8 & 8 & 8 & 8 & 8 & 8 & 8 \\
\hline SS (mg/l) & 2260 & 2285 & 3555 & 3108 & $\hat{c} 870$ & 2663 & 2617 & 2545 & 2445 & 2425 \\
\hline ISS (PAC corrected) & 2260 & 2285 & 3555 & 3108 & 2870 & 2663 & 2617 & 2545 & 2445 & 2425 \\
\hline Temp (C) & 19 & 19 & 20 & 19 & 19 & 19 & 19 & 28 & 19 & 19 \\
\hline $\mathrm{pH}$ (std units) & 8 & 7.6 & 8 & 7.9 & 8 & 7.8 & 8 & 8.2 & 8.3 & 8 \\
\hline OUR (mg/I/hr) & & & & & & & 19 & & & 24 \\
\hline USS $(n g / 1)$ & 2036 & 2010 & 3127 & 2734 & 2525 & 2343 & 1895 & 1843 & 1770 & 1790 \\
\hline * VSS (PAC corrected) & 2036 & 2018 & 3127 & 2734 & 2525 & 2343 & 1895 & 1843 & 1778 & 1790 \\
\hline USS/SS & 0.90 & 0.88 & 0.88 & 0.98 & 0.88 & 0.88 & 0.72 & 0.72 & 0.72 & 0.74 \\
\hline sPAC Added (mg) & 8 & 6 & 8 & 0 & 8 & 0 & 0 & 0 & 0 & 0 \\
\hline Inventory (ag/l basin) & 8 & 0 & 8 & 0 & 0 & 0 & 0 & 0 & $\theta$ & 8 \\
\hline \multicolumn{11}{|l|}{ EFFLUENT PARAMETERS } \\
\hline Volume (liters) & 4.38 & 4.16 & 4.64 & 8.29 & 3 & 3.7 & 3.375 & 3.6 & 4.73 & 4.32 \\
\hline $\operatorname{COD}(m g / 1)$ & 231 & 235 & 237 & 225 & 225 & 202 & 199 & 194 & 192 & 205 \\
\hline SS (ng/1) & 68 & 38 & 122 & 1004 & 684 & 198 & 256 & 190 & 154 & 86 \\
\hline \multicolumn{11}{|l|}{ APAC corrected } \\
\hline oH (std anits) & 8.5 & 8.3 & 7.7 & 8.2 & 7.9 & 7.8 & 7.8 & 8.0 & 8.1 & 8.0 \\
\hline USS (mg/l) & & & 38 & & & & 116 & & & 46 \\
\hline \multicolumn{11}{|l|}{ *AC corrected } \\
\hline NHSNV (ng/1) & 14 & $<4$ & $<4$ & 14 & $<4$ & $<4$ & 14 & 7.1 & 7.1 & 7.1 \\
\hline$C N(x g / 1)$ & & & & & & & & 1.41 & 1.41 & 1,41 \\
\hline $\operatorname{SCN}(\mathrm{mg} / 1)$ & & & & & & & & 3.7 & 3.7 & 3.7 \\
\hline NO2 (mg/l) & & & & & & & & 15.5 & 15.5 & 15.5 \\
\hline $\mathrm{NO} 3$ (mg/l) & & & & & & & & 131 & 131 & 131 \\
\hline Phenolics $(m g / 1)$ & 1.025 & 1.025 & 1.025 & 1.025 & 1.025 & 1.025 & 1.025 & 1.025 & 1.025 & 1.025 \\
\hline $\mathrm{PO4}-\mathrm{P}(\mathrm{mg} / \mathrm{l})$ & 3.6 & 3.6 & 3.6 & 4.4 & 4.4 & 4.4 & 4.4 & 3.6 & 3.6 & 3.6 \\
\hline $\operatorname{Org}-N(m g / 1)$ & & & & & & & & 5.5 & 5.5 & 5.5 \\
\hline $\operatorname{TDS}(\log / 1)$ & & & & & & & & 4780 & 4780 & 4780 \\
\hline Color (AFHA units) & 750 & 750 & 750 & 758 & 750 & 758 & 750 & 750 & 758 & 758 \\
\hline $\operatorname{TDC}(\operatorname{mg} / 1)$ & 60 & 60 & 68 & 53 & 53 & 53 & 53 & 58 & 50 & 58 \\
\hline EOD S-day ing/1) & 3.7 & 3.7 & 3.1 & $-65-^{13}$ & 13 & 13 & 13 & 3.4 & 3.4 & 3.4 \\
\hline
\end{tabular}


UNIT IA TABLE AI

\begin{tabular}{|c|c|c|c|c|c|c|c|c|c|c|}
\hline MONTH & DEC & DEC & DEC & DEC & $\mathrm{DEC}$ & DEC & DEC & DEC & DEC & DEC \\
\hline DAY & 2 & 3 & 4 & 5 & 6 & 7 & 8 & 9 & 10 & 11 \\
\hline DAY NO. & 129 & 130 & 131 & 132 & 133 & 134 & 135 & 136 & 137 & 138 \\
\hline \multicolumn{11}{|l|}{ SYSTEX PORGMETERS } \\
\hline *5lidge Age & 14.99 & 15.81 & 15.81 & 15.01 & 15.80 & 14.96 & 15.20 & 15.08 & 14.98 & $\$ 5.01$ \\
\hline$(F / M) C O D$ & 0.47 & 0.43 & 0.36 & 0.39 & 0.35 & 0.40 & 0.40 & 0.40 & 0.42 & 0.37 \\
\hline +HRT & 1.86 & 1.69 & 2.87 & 1.88 & 2.27 & 1.82 & 1.98 & 1.90 & 1.69 & 2.04 \\
\hline \multicolumn{11}{|l|}{ FEED PARAMETERS (BATCH } \\
\hline Volume (liters) & 4.99 & 5.5 & 4.5 & 4.95 & 4.1 & 5.12 & 4.95 & 4.9 & 5.5 & 4.55 \\
\hline pH (std units) & 11.7 & 11.7 & 11.6 & 11.5 & 11 & 11.6 & 11.8 & 11.8 & 11.8 & 11.5 \\
\hline $\operatorname{COD}(\mathrm{ag} / 1)$ & 2046 & 2046 & 2046 & 2046 & 2860 & 2060 & 2060 & 2122 & 2122 & 2122 \\
\hline$N H 3 N(\mathrm{mg} / \mathrm{l})$ & 142 & 142 & 142 & 142 & 131 & 131 & 131 & 140 & 140 & 148 \\
\hline SS $(\mathrm{mg} / 1)$ & 395 & 395 & 395 & 395 & 522 & 522 & 522 & 522 & 522 & 522 \\
\hline VSS $(m g / 1)$ & 85 & 85 & 85 & 85 & 114 & 114 & 114 & 114 & 114 & 114 \\
\hline CN (iag/l) & 1.24 & 1.24 & 1.24 & 1.24 & & & & & & \\
\hline $\operatorname{SCN}(\operatorname{mg} / 1)$ & 188 & 188 & 188 & 188 & & & & & & \\
\hline 800 5-day (ag/1! & 1258 & 1250 & 1250 & 1250 & 1210 & 1218 & 1218 & 1260 & 1260 & 1260 \\
\hline TOC $(m g / 1)$ & 638 & 638 & 638 & 638 & 637 & 637 & 637 & 664 & 664 & 664 \\
\hline Phenolics (mg/l) & 5.89 & 5.09 & 5.09 & 5.09 & 5.61 & 5.61 & 5.61 & 5.61 & 5.61 & 5.61 \\
\hline \multicolumn{11}{|l|}{ P04-P (ogg/1) } \\
\hline $\operatorname{Org}-\mathrm{N}(\mathrm{mg} / 1)$ & 73 & 73 & 73 & 73 & & & & & & \\
\hline \multicolumn{11}{|l|}{ No? $(m 0 / 1)$} \\
\hline \multicolumn{11}{|l|}{$\mathrm{NO3}(\mathrm{mg} / 1)$} \\
\hline \multicolumn{11}{|l|}{$\operatorname{TDS}(\mathrm{mg} / 1)$} \\
\hline Color (GPHA Units) & 1500 & 1500 & 1560 & 1500 & 1580 & 1500 & 1500 & 1500 & 1580 & 1580 \\
\hline \multicolumn{11}{|l|}{ BASIN PARAMETERS } \\
\hline Vol Wasted (al) & 638 & 680 & 709 & 729 & 608 & 740 & 627 & 694 & 690 & 559 \\
\hline D.0. $(\mathrm{mg} / 1)$ & 8 & 8 & 8.1 & 7.9 & 8 & 7.2 & 7.1 & 7 & 7 & 7 \\
\hline $5 S(n g / 1)$ & 2460 & 2965 & 2862 & 2895 & 2858 & 3198 & 3845 & 3138 & 3312 & 3167 \\
\hline ASS (PAR: conrected) & 2,60 & 2368 & 2002 & 2095 & 2858 & $3 i 98$ & 3045 & 3138 & SSIL & 3167 \\
\hline Temp (C) & 18 & 18 & 18 & 19 & 19 & 18.5 & 19 & 19 & 19 & 19 \\
\hline DH (sto units) & 8 & 8 & 8 & 7.3 & 8.2 & 8.3 & 8.2 & 8 & 7.8 & 7.8 \\
\hline OUR $(\mathrm{hg} / \mathrm{l} / \mathrm{h} / \mathrm{r})$ & & & & & 36 & & & & & \\
\hline VSS $(\mathrm{sag} / \mathrm{l})$ & 1816 & 2189 & 2113 & 2140 & 1983 & २225 & 2112 & 2177 & 2297 & 2197 \\
\hline USS (PAC corrected) & 1816 & 2189 & 2113 & 2140 & 1983 & 2225 & 2112 & 2177 & 2297 & 2197 \\
\hline VSS/SS & 0.74 & 0.74 & 0.74 & 0.74 & 0.78 & 0.70 & 0.69 & 0.69 & 0.69 & 8.69 \\
\hline \&PAC Added (rg) & 0 & B & 0 & 8 & 8 & 0 & 0 & 0 & 0 & 0 \\
\hline Inventory (ug/l basin) & 8 & $\theta$ & $\theta$ & 9 & 0 & 0 & 8 & $\theta$ & 0 & 0 \\
\hline \multicolumn{11}{|l|}{ EFFLUENT PARAMETERS } \\
\hline Vollune (liters) & 4.5 & 5.67 & 4. 3E & 4.45 & 3.7 & 4.73 & 4.46 & 4.5 & 5.4 & 4.33 \\
\hline $\operatorname{cod}(m g / 1)$ & 197 & 205 & 203 & 196 & 180 & 189 & 217 & 180 & 184 & 186 \\
\hline SS $(\infty g / 1)$ & 56 & 70 & 68 & 46 & 148 & 42 & 118 & 74 & 68 & 176 \\
\hline \multicolumn{11}{|l|}{ IPAC corrected } \\
\hline $\mathrm{pH}$ (std units) & B. 8 & 8.1 & 8.3 & 8.1 & 8.1 & 8.3 & 8.1 & 8.1 & 7.9 & 7.8 \\
\hline USS (ng/l) & & & & $3: 2$ & 18 & & 58 & & & \\
\hline \multicolumn{11}{|l|}{ FAC corrected } \\
\hline NHJN (ng/1) & 38.6 & 38.6 & 39.6 & 38.6 & 41.4 & 41.4 & 41.4 & 28.8 & 28.8 & 28.8 \\
\hline CN $(n g / 1)$ & 1.41 & 1.41 & 1.41 & 1.41 & & & & & & \\
\hline $\operatorname{SCN}(n g / 1)$ & 3.7 & 3.7 & 3.7 & 3.7 & & & & & & \\
\hline NO2 $(m g / 1)$ & 15.5 & 15.5 & 15.5 & 19.5 & & & & & & \\
\hline $\mathrm{NO3}(\mathrm{mg} / 1)$ & 131 & 131 & 131 & 131 & & & & & & \\
\hline Phenolics (og/l) & 1.825 & 1.025 & (.825 & 1.025 & 1.025 & 1.025 & 1.825 & 1.025 & 1.825 & 1.025 \\
\hline$P C 4-P(m g / 1)$ & 3.6 & 3.6 & 3.6 & 3.6 & 4.6 & 4.6 & 4.6 & 6.1 & $6: 1$ & 6.1 \\
\hline Org-N $(q g / 1)$ & 5.5 & 5.5 & 5.5 & 5.5 & & & & & & \\
\hline TDS (ng/1) & 4780 & 4780 & 4780 & 4780 & 4505 & 4505 & 4505 & 4585 & 4505 & 4585 \\
\hline Color (APHA units) & 750 & 750 & 750 & 758 & 750 & 750 & 750 & 750 & 750 & 750 \\
\hline TOC (mg/1) & 53 & 53 & 53 & 53 & 51 & 51 & 51 & 46 & 46 & 46 \\
\hline BCD 5-day (me/1) & 3 & 3 & 3 & $\begin{array}{l}3 \\
-6\end{array}$ & ? & 2 & 2 & 12 & 12 & 12 \\
\hline
\end{tabular}


UNIT IA TABLE AI

\begin{tabular}{|c|c|c|c|c|c|c|c|c|c|c|}
\hline MCNTH & DEC & DEC & DEC & DEC & DEC & DEC & DEC & DEC & DEC & $D E C$ \\
\hline DAY & 12 & 13 & 14 & 15 & 16 & 17 & 18 & 19 & 20 & 21 \\
\hline DAY NO. & 139 & 140 & 191 & 142 & 143 & 144 & 145 & 146 & 147 & 148 \\
\hline \multicolumn{11}{|l|}{ SYSTEM FARAMETERS } \\
\hline tSludge Ag̣e & 14.99 & 14.99 & 15.80 & 15.81 & 14.99 & 15.01 & 14.99 & 15.80 & 15.80 & 14.99 \\
\hline$F(F / M) C O D$ & 0.38 & 0.36 & 0.36 & 0.36 & 0.37 & 0.34 & 0.33 & 0.32 & 0.33 & 0.31 \\
\hline *HRT & 1.89 & 1.86 & 1.86 & 1.81 & 1.72 & 1.86 & 1.92 & 2.07 & 2.27 & 2.16 \\
\hline \multicolumn{11}{|c|}{ FEED FARAMETERS (BATCH } \\
\hline Volune (liters) & 4.95 & 5 & 5 & 5.15 & 5.4 & 4.99 & 4.85 & 4.5 & 4.1 & 4.3 \\
\hline DH (std units) & 11.5 & 11.6 & 11.5 & 11.6 & 11.5 & 11.5 & 11.6 & 11.3 & 10.5 & 9.8 \\
\hline $\operatorname{coD}(\mathrm{mg} / \mathrm{l})$ & 2122 & 2011 & 2011 & 2011 & $\hat{\varepsilon} 016$ & 2016 & 2016 & $c 016$ & 2037 & 2037 \\
\hline NH3N $(m g / 1)$ & 140 & 136 & 136 & 136 & 136 & 136 & 136 & 136 & 123 & 123 \\
\hline SS (mg/1) & 522 & 482 & 482 & 482 & 482 & 482 & 482 & 482 & 568 & 500 \\
\hline VSS $(\mathrm{mg} / \mathrm{l})$ & 114 & 94 & 94 & 94 & 94 & 94 & 94 & 94 & 188 & 108 \\
\hline CN $(m g / 1)$ & & 1.88 & 1.88 & 1.88 & 1.88 & 1.88 & 1.08 & 1.08 & & \\
\hline SCN (-og/1) & & 184 & 184 & 184 & 184 & 184 & 184 & 194 & & \\
\hline 800 5-day (ag/1) & 1268 & 1200 & 1280 & 1200 & 1228 & 1220 & 1228 & 1220 & 1168 & 1160 \\
\hline TOC $(a g / l)$ & 664 & 648 & 640 & 640 & 651 & 651 & 651 & 651 & 658 & 650 \\
\hline Phenolics (mo/l) & 5.61 & 5.43 & 5.43 & 5.43 & 5.43 & 5.43 & 5.43 & 5.43 & 6.96 & 6.96 \\
\hline
\end{tabular}

P04-P (ag/1)

Org-N (mg/1)

$\begin{array}{llll}78 & 78 & 78 & 78\end{array}$

NO2 (mg/l)

io3 (ng/1)

TDS (ng/l)

Color (APHA Units)

EASIN PRRAMETERS

1500

1580

$1580 \quad 1500$

1500

1500

1500

2590

2500

Vol Wasted (al)

D.0. $\log / 1)$

SS (mg/1)

+SS (PAC corrected)

Temp (C)

DH (std units)

OUR (mg/l/hr)

USS (ng/l)

+USS (PAC corrected)

VSS/SS

tPAC Added (og)

Inventory (mg/l basin)

743

7.2

3230

3230

19

7.8

24

2292

2392

0.71

0

EFFLUENT PARAMETERS

Volume (liters)

COD (mg/l)

SS $(\mathrm{mg} / \mathrm{l})$

IPAC corrected

pH (std units)

USS (mig/l)

¿PAC corrected

NHJN (ng/l)

CN (mg/l)

SCN $(\mathrm{mg} / \mathrm{H})$

NO2 (mg/l)

NO3 (mg/l)

Phenolics (mg/l)

p04-p (mg/1)

Org-N (mg/l)

TDS (mg/1)

Color (APHA urits)

TOC (mg/1)

BOD 5-day $(m n g / 1)$

184

32

28.

(I)
$729 \quad 754 \quad 740$

7.2

3262

3262

7.2

3267

3267

7.1

3355

3355

19
8

7.9

2315

2315

2318

2318

0.71

2387

2387

0.71

8.71

619

7
3415

3415

19

7.7

2430

2430

0.71

0

694

74

723

717

7.2

3470

3425

3440

3440

3470

8.2

8.2

19

8.5

2437

2437

0.71

2447

2447

0.71

0

$4.63 \quad 4.65$

$4.7 \quad 4.75$

5.25

4.64

4. 75

168

195

180
118

$7.9 \quad 7.9$

8.0

8.8

7.8

8.8

42.8

42.8

42.8

2.41

2.41

2.41

67.6

2.41

67.6

2.41

3.9

9.05

9.85

9.05

3.9

9.85

3.9

9.05

95

1.025 $\quad 1.825$

(. . 025

95

1.025

1.825

95

1.025

3.4

3.4

$\begin{array}{rr}6.1 & 4.3 \\ & 12\end{array}$

4.3

$4505 \quad 4345$

12
4345

12

12

12
4345

875

4345
875

4345

875

$46 \quad 49$

5.3

5.3

49

48

9.67

$2.41 \quad 2.41$

$3.9 \quad 3.9$

$9.85 \quad 9.85$

$95 \quad 95$

1.825

1.825

$3.4 \quad 6.2$

4345

12
4345

5.3

$563 \quad 737$

$7.2 \quad 7.2$

$3157 \quad 3495$

$3157 \quad 3495$

$19 \quad 18$

$0.1 \quad 8$

$2122 \quad 2349$

$2122 \quad 2349$

$0.67 \quad 0.67$

80

$3.6 \quad 3.95$

$188 \quad 190$

$208 \quad 56$

$\begin{array}{rrrr}8.3 & 8.5 & 8.3 & 8.2 \\ 45 & 48 & 126 & 34\end{array}$

$\begin{array}{llll}67.6 & 67.6 & 88.2 & 88.2\end{array}$

$1.825 \quad 1.025$

6.26 .2

$4375 \quad 4375$

$1000 \quad 1000$

$-67-$ 
UNIT IA TABLE A1

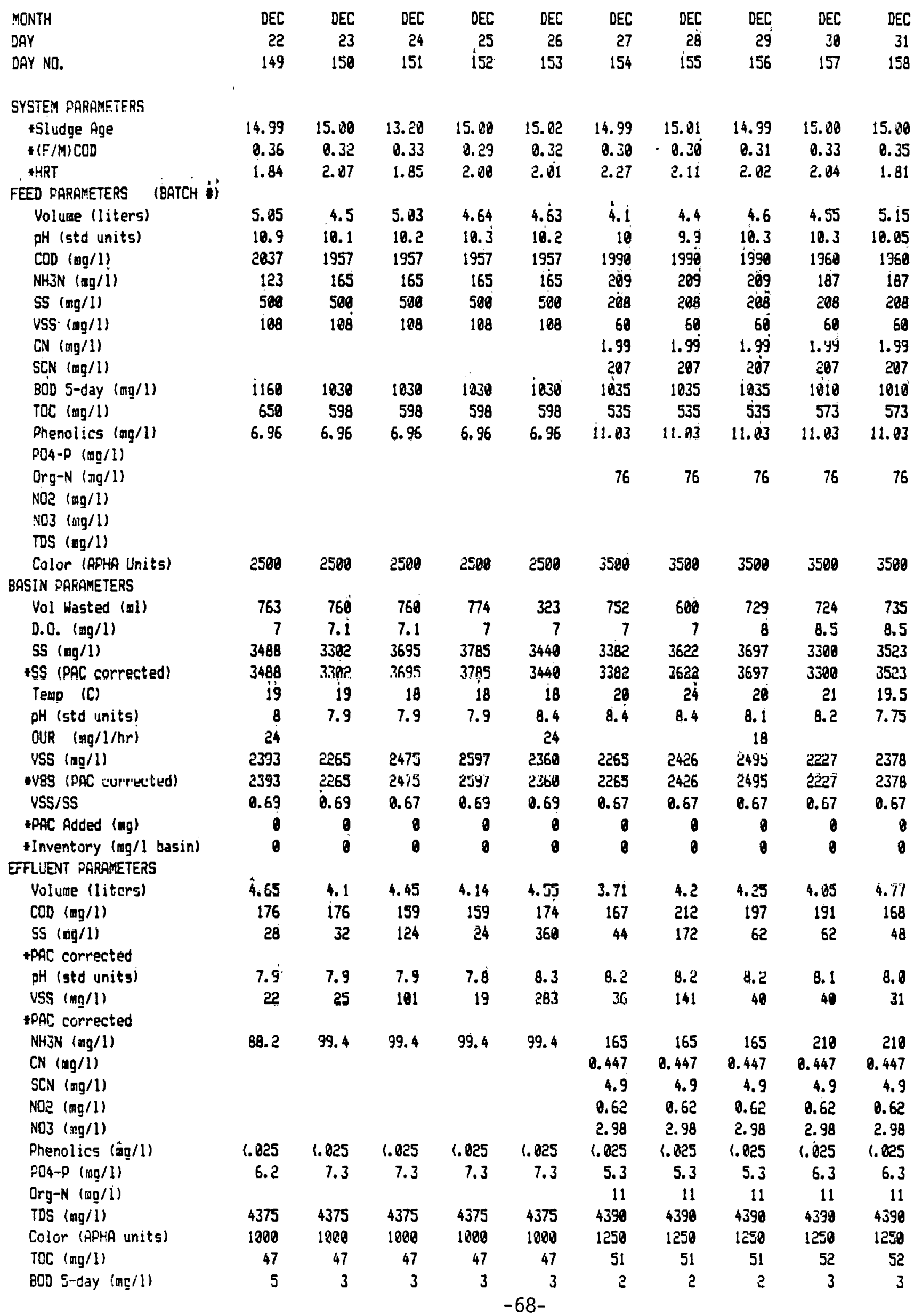




\begin{tabular}{|c|c|c|c|c|c|c|c|c|c|c|}
\hline YCENTH & JAN & JAN & JAN & JAN & JAN & JAN & JAN & JAN & JHN & JAN \\
\hline DRY : & 1 & 2 & 3 & 4 & 5 & 6 & 7 & 8 & 9 & 10 \\
\hline DAY NO. & 159 & 160 & 161 & 162 & 163 & 164 & 165 & $\$ 66$ & 167 & 168 \\
\hline \multicolumn{11}{|l|}{ SYSTEM PARAMETERS } \\
\hline *Sludge Age & 15.00 & 15.00 & 15.81 & 12.00 & 12.80 & 12.80 & 12.80 & 12.08 & 12.01 & 12.88 \\
\hline$+(F / M) C O D$ & 0.39 & 0.31 & 0.30 & 0.36 & 0.37 & 0.36 & 0.35 & 0.32 & 0.35 & 0.39 \\
\hline AHRT & 1.75 & 2.19 & 2. 27 & 1.92 & 1.92 & 2.80 & 1.78 & 1.36 & 2.04 & 2.02 \\
\hline \multicolumn{11}{|l|}{ FEED FARRRMETERS (BRTCH } \\
\hline Volume (liters) & 5.3 & 4.25 & 4.1 & 4.65 & 4.85 & 4.65 & 5.23 & 4.75 & 4.55 & 4.6 \\
\hline pH (std units) & 18.5 & 10.6 & 10.5 & 11.5 & 9.8 & 11.4 & 11.5 & 10.5 & 18.2 & 10.3 \\
\hline $\cos (n g / 1)$ & 1960 & 1960 & 1780 & 1780 & 1780 & 1823 & 1823 & 1823 & 1823 & 1910 \\
\hline NH3N $(\mathrm{mg} / 1)$ & 187 & 187 & 185 & 185 & 185 & 176 & 176 & 176 & 176 & 195 \\
\hline SS (ng/1) & 208 & 208 & 474 & 474 & 474 & 474 & 474 & 474 & 474 & 344 \\
\hline VSS $\cdot(\mathrm{mg} / 1)$ & 60 & 60 & 94 & 94 & 94 & 94 & 94 & 94 & 94 & 102 \\
\hline CN $(m g / 1)$ & 1.99 & 1.99 & & & & & & & & 0.203 \\
\hline $\operatorname{SCN}(m g / 1)$ & 287 & 207 & & & & & & & & 155 \\
\hline BOD 5-day (mg/1) & 1818 & 1018 & 1258 & 1850 & 1850 & 1030 & 1030 & 1030 & 1030 & 1820 \\
\hline TOC $(m g / 1)$ & 573 & 573 & 560 & 560 & 560 & 568 & 560 & 560 & 560 & 603 \\
\hline Phenolics (mg/l) & 11.83 & 11.83 & 10.3 & 10.3 & 10.3 & 18.3 & 10.3 & 10.3 & 10.3 & 9.3 \\
\hline \multicolumn{11}{|l|}{ f04-P (mg/l) } \\
\hline Crg-N (mg/1) & 76 & 76 & & & & & & & & 66 \\
\hline \multicolumn{11}{|l|}{ HO2 (며닉) } \\
\hline \multicolumn{11}{|l|}{$\mathrm{v03}(\mathrm{sig} / 1)$} \\
\hline \multicolumn{11}{|l|}{ TDS (mg/l) } \\
\hline Color (APHA Units) & 3500 & 3500 & 1750 & 1750 & 1750 & 1750 & 1750 & 1750 & 1750 & 2500 \\
\hline \multicolumn{11}{|l|}{ BASIN PARGMETERS } \\
\hline Vol Nasted (ol) & 756 & 691 & 719 & 931 & 678 & 930 & 981 & 963 & 810 & 865 \\
\hline D.0. (ng/1) & 8.5 & 8.5 & 8.4 & 8.4 & 8 & 8 & 8 & 8 & 8 & 7.1 \\
\hline SS $(m g / 1)$ & 3292 & 3350 & 2920 & 2862 & 2732 & 2735 & 3133 & 3118 & 2793 & 2635 \\
\hline ISS (PAC corrected) & 3292 & 3350 & 2920 & 2862 & 2732 & 2735 & 3133 & 3118 & 2793 & 2635 \\
\hline Tend (C) & 19 & 20 & 20 & 20 & 19 & 21 & 19 & 18 & 19 & 20 \\
\hline oH (std units) & 7.7 & 8.45 & 8.4 & 8.5 & 8.4 & 8.3 & 7.8 & 7.5 & 7.5 & 7.8 \\
\hline DUR (ng/1/hr) & & & 36 & & 36 & & & & 36 & \\
\hline USS (agg/1) & २2२2 & 2261 & 2035 & 1995 & 1955 & 1957 & 2242 & 2231 & 1995 & 1882 \\
\hline *USS (AFC corrected) & २२२2 & 2261 & 2035 & 1975 & 1955 & 1957 & 2242 & 2231 & 1995 & 1882 \\
\hline USS/SS & 0.67 & 0.67 & 0.70 & 0.70 & 0.72 & 0.72 & 0.72 & 0.72 & 0.71 & 0.71 \\
\hline FPAC Added (ag) & o & 6 & 8 & 0 & 0 & 8 & 0 & 0 & 0 & 0 \\
\hline Inventory (䀦/l basin) & 8 & 8 & 0 & 0 & 8 & 0 & 8 & $B$ & 8 & 8 \\
\hline \multicolumn{11}{|l|}{ EFFLLENT PARAMETERS } \\
\hline Volume (liters) & 4.83 & 4.25 & 3.8 & 4.5 & 4.15 & 4.35 & 4.97 & 4.42 & 4.2 & 4.35 \\
\hline $\operatorname{COD}(\mathrm{mg} / 1)$ & 162 & 197 & 197 & 217 & 188 & 177 & 166 & 168 & 135 & 125 \\
\hline $55(\mathrm{ng} / 1)$ & 38 & 86 & 62 & 44 & 212 & 44 & 12 & 25 & 126 & 82 \\
\hline \multicolumn{11}{|l|}{ PPAC corrected } \\
\hline गुH (std units) & 7.8 & 8.4 & 8.2 & 8.5 & B. 3 & 8.4 & 8.8 & 7.8 & 7.5 & 7.7 \\
\hline VSS (mg/l) & 19 & 55 & 48 & 28 & 142 & 29 & 8 & 17 & 70 & 46 \\
\hline \multicolumn{11}{|l|}{ *FAC corrected } \\
\hline :HHSN (mg/l) & 210 & 210 & 205 & 205 & 385 & 211 & 211 & 211 & 211 & 215 \\
\hline CN (F⿻コ一 $/ 1)$ & 0.447 & 0.447 & & & & & & & & 0.516 \\
\hline SCN (ng/1) & 4.9 & 4.9 & & & & & & & & 4.9 \\
\hline NO2 $(\mathrm{mg} / 1)$ & 0.62 & 0.62 & & . & & & & & & 18.25 \\
\hline $\mathrm{NO3}$ (mg/l) & 2.98 & 2.98 & & & & & & & & 12.5 \\
\hline Phenolics (mg/l) & 1.825 & 1.825 & 1.025 & 1.025 & 1.025 & 1.025 & 1.825 & 1.025 & 1.025 & 1.025 \\
\hline PC4-P $(\mathrm{sg} / 1)$ & 6.3 & 6.3 & 3.6 & 3.6 & 3.6 & 13.5 & 13.5 & 13.5 & 13.5 & 13.1 \\
\hline $\operatorname{Org}-\mathrm{N}(\mathrm{gg} / 1)$ & 11 & 11 & & & & & & & & 5 \\
\hline TDS (mg/1) & 4390 & 4398 & 4766 & 4766 & 4766 & 4766 & 4766 & 4766 & 4766 & 5128 \\
\hline Color (AFHA units) & 1550 & 1250 & 1080 & 1808 & 1000 & 1000 & 1000 & 1000 & 1000 & 750 \\
\hline TOC $(m g / 1)$ & 52 & 52 & 61 & 61 & 61 & 45 & 45 & 45 & 45 & 43 \\
\hline BOD 5-day $(\mathrm{mg} / 1)$ & 3 & 3 & 2 & 2 & 2 & 3 & 3 & 3 & 3 & 3 \\
\hline
\end{tabular}


UNIT IA TAELÈ Ài

\begin{tabular}{|c|c|c|c|c|c|c|c|c|c|c|}
\hline MONTH & JAN & JAN & JAN & JAN & JGN & JAN & JAN & JAN & JAN & JAM \\
\hline DẢY & 11 & 12 & 13 & 14 & 15 & 16 & i7 & 18 & 19 & 28 \\
\hline DAY vó. & 169 & 170 & 171 & 172 & 173 & 174 & $i 75$ & 176 & 177 & 178 \\
\hline \multicolumn{11}{|l|}{ SYSTEM PARAMETERS } \\
\hline *Sludge Age & $12.8 ?$ & 12.00 & 12.88 & 12.00 & 9.74 & 12.00 & 12.20 & 12.80 & 12.00 & 12.20 \\
\hline$+(F / M) C O D$ & 0.39 & 0.42 & 0.44 & 0.37 & 0.45 & 0.37 & 0.41 & 0.42 & 0.43 & 0.40 \\
\hline \#HRT & 2.89 & 2.82 & 1.94 & 2.02 & 1.86 & 2.30 & 2.84 & 2.80 & 1.94 & 1.92 \\
\hline \multicolumn{11}{|c|}{ FEED PARAMETERS (BATCH } \\
\hline Volume (liters) & 4.45 & 4.6 & 4.8 & 4.6 & 5 & 4.85 & 4.55 & 4.65 & 4.8 & 4.85 \\
\hline pH (std units) & $1 \hat{0} . !$ & 18.5 & 10.9 & 10.2 & 11 & 10.7 & 18.1 & 18.4 & 9.9 & 9.9 \\
\hline $\operatorname{COD}(\operatorname{sg} / \mathrm{i})$ & $1910^{\circ}$ & 1910 & 2030 & $203 \theta^{\circ}$ & 2030 & 2038 & 1975 & 1975 & 1975 & 1936 \\
\hline NHZ3N $(\mathrm{ag} / \mathrm{l})$ & 195 & 195 & 189 & 189 & 189 & 189 & 176 & 176 & 176 & 150 \\
\hline SS (mg/1) & 344 & 344 & 344 & 344 & 344 & 344 & 684 & 684 & 684 & 684 \\
\hline USS - (ag/1) & 102 & 102 & 102 & 102 & 102 & 102 & 148 & 148 & 148 & 148 \\
\hline$C N(m g / l)$ & 8.203 & 8.203 & 0.203 & 0.203 & ด. 29.3 & 1. 2013 & & & & \\
\hline $\operatorname{SCN}(\mathrm{mg} / \mathrm{l})$ & 155 & 155 & 155 & 155 & 155 & 155 & & & & \\
\hline BŌo 5-dày $(a g / 1)$ & 1020 & $10<0$ & 980 & 988 & $988^{\circ}$ & 988 & 960 & $9 \in 0$ & 360 & 1120 \\
\hline TOC (mg/l) & 603 & 603 & 587 & 587 & 587 & 587 & 606 & $60 \overline{6}$ & 686 & 608 \\
\hline Phenolics (mg/1) & 9.3 & 9.3 & 9.3 & 9.3 & 9.3 & 9.3 & 9.59 & 9.59 & 9.59 & 9.59 \\
\hline
\end{tabular}

$\operatorname{Org}-\mathrm{N}(\operatorname{mg} / 1)$

NOS $(\mathrm{mg} / \mathrm{l})$

NO3 $(\mathrm{gg} / \mathrm{ll})$

TDS $(\mathrm{mg} / 1)$

Color (APHA Units)

$\begin{array}{llllll}66 & 66 & 66 & 66 & 66 & 66\end{array}$

BASIN PARAMETERS

Vol Wasted (ml)

D.0. (mg/l)

SS (mg/1)

*SS (PAC corrected)

remo (C)

oH (std units).

OUR $(\mathrm{mg} / 1 / \mathrm{hr})$

USS $(\operatorname{mg} / 1)$

* USS (AAC corrected)

USS/SS

*PAC Added (ng)

+Inventory (mg/l basin)

EFFLUENT BAROMETERS

volume (liters)

COD (ng/l)

is $(\mathrm{mg} / \mathrm{ll})$

APRC corrected

$\mathrm{oH}$ (std units)

USS (an/l)

* FAC corrected

NHİN $(\mathrm{mg} / \mathrm{l})$

CN $i m g / 11$

SCN (rig/1)

NO2 (ng/1)

$\mathrm{NO3}$ (mg/1)

Phenolics (mg/i)

ró-p (mg/i)

Org-N (mg/l)

TDS (mg/1)

Color (APHA units)

TOC (mig/l)

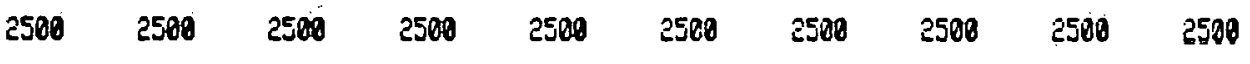

$\begin{array}{rrrrrrrrrr}800 & 800 & 717 & 807 & 800 & 785 & 818 & 827 & 885 & 833 \\ 8 & 8 & 7.1 & 8 & 8 & 7.4 & 7.5 & 7.3 & 7 & 7 \\ 2530 & 2442 & 2580 & 2955 & 2618 & 2590 & 2560 & 2512 & 2597 & 2725 \\ 2530 & 2442 & 2580 & 2955 & 2618 & 2590 & 2560 & 2512 & 2597 & 2725 \\ 20 & 20 & 20 & 19 & 18 & 18 & 21 & 19 & 19 & 19 \\ 7.7 & 8 & 8 & 8 & 8 & 8.3 & 8.3 & 0.3 & 8.1 & 8 \\ & & & & & 10 & & & 12 & \\ 1807 & 1744 & 1843 & 2111 & 1870 & 1855 & 1838 & 1804 & 1850 & 1941 \\ 1807 & 1744 & 1843 & 2111 & 1870 & 1955 & 1838 & 1804 & 1850 & 1941 \\ 0.71 & 0.71 & 0.71 & 0.71 & 0.71 & 0.72 & 0.72 & 0.72 & 0.71 & 0.71 \\ 0 & 0 & 0 & 0 & 0 & 0 & 0 & 0 & 0 & 0 \\ 0 & 0 & 0 & 0 & 0 & 0 & 0 & 0 & 0 & 0\end{array}$

$\begin{array}{llllllllll}4.1 & 4.3 & 4.45 & 4.92 & 4.64 & 4.22 & 4.48 & 4.35 & 4.53 & 4.55\end{array}$

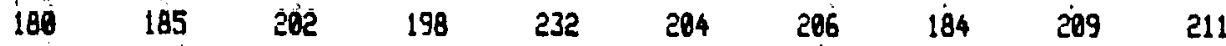

$\begin{array}{llllll}84 & 68 & 164 & 116 & 244 & 132\end{array}$

$\begin{array}{llllllllll}7.7 & 8.0 & 8.1 & 8.1 & 8.1 & 7.9 & 8.2 & 8.2 & 8.1 & 0.6\end{array}$

$\begin{array}{llllllllll}47 & 38 & 91 & 64 & 136 & 78 & 61 & 59 & 80 & 71\end{array}$

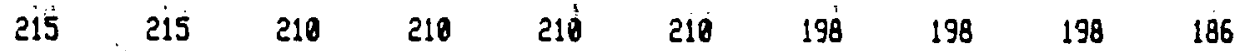

$\begin{array}{llllll}0.616 & 0.616 & 0.616 & 0.616 & 0.616 & 0.616\end{array}$

$\begin{array}{llllll}4.9 & 4.9 & 4.9 & 4.9 & 4.9 & 4.9\end{array}$

$\begin{array}{llllll}10.25 & 10.25 & 10.25 & 10.25 & 10.25 & 10.25\end{array}$

$\begin{array}{llllll}12.5 & 12.5 & 12.5 & 12.5 & 12.5 & 12.5\end{array}$

$\begin{array}{llllll}1.825 & 1.025 & 1.025 & 1.025 & 1.025 & 1.025\end{array}$

$\begin{array}{rrrrrr}13.1 & 13.1 & 6.3 & 6.3 & 6.3 & 6.3\end{array}$

$\begin{array}{llll}1.825 & 1.825 & 1.825 & 1.025\end{array}$

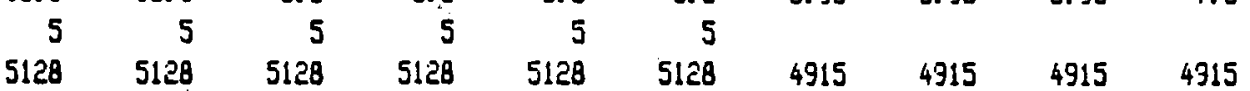

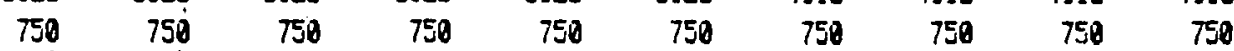

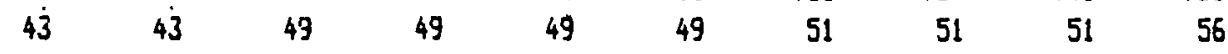

BOD 5-day (mg/l)

$\begin{array}{llll}3 & 3 & 3 & 3\end{array}$ 
UNIT IA TABLE AI

\begin{tabular}{|c|c|c|c|c|c|c|c|c|c|c|}
\hline MONTH & JAN & JAN & JAN & JAN & JAN & JAN & JAN & JAN & JAN & JAN \\
\hline DAY & 21 & 22 & 23 & 24 & 25 & 26 & 27 & 28 & 29 & 30 \\
\hline DAY NO. & 179 & 180 & 181 & 182 & 183 & 184 & 185 & 186 & 187 & 188 \\
\hline \multicolumn{11}{|l|}{ SYSTEM FARAMETERS } \\
\hline *Sludge Age & 12.88 & 12.80 & 12.88 & 12.08 & 12.00 & 11.99 & 12.20 & 12.80 & 11.99 & 12.00 \\
\hline$\pm(F / M) C O D$ & 0.34 & 0.34 & 0.35 & 0.46 & 0.37 & 0.39 & 0.38 & 0.42 & 0.41 & 0.39 \\
\hline$*$ HRT & 1.79 & 1.89 & 2. 12 & 1.72 & 2.02 & 2.09 & 2.89 & 1.69 & 1.69 & 2.02 \\
\hline \multicolumn{11}{|l|}{ FEED ORRAMETERS (BATCH \#) } \\
\hline Volune (liters) & 5.2 & 4.93 & 4.6 & 5.4 & 4.6 & 4.45 & 4.45 & 5.5 & 5.5 & 4.6 \\
\hline pit (std units) & 10.25 & 10.05 & 9.8 & 10.2 & 10.1 & 18.5 & 10.4 & 11.4 & 11.3 & 10.6 \\
\hline $\cos (\mathrm{mg} / 1)$ & 1936 & 1936 & 1936 & 2056 & 2056 & 2056 & 2068 & 2068 & 2068 & $\hat{c} 868$ \\
\hline NH3N $(\mathrm{mg} / 1)$ & 150 & 158 & 150 & 152 & 152 & 152 & 176 & 176 & 176 & 176 \\
\hline SS $(\mathrm{mg} / \mathrm{ll})$ & 684 & 684 & 684 & 480 & 488 & 480 & 480 & 480 & 488 & 480 \\
\hline VSS (mg/1) & 148 & 148 & 148 & 154 & 154 & 154 & 154 & 154 & 154 & 154 \\
\hline $\mathrm{CN}(\mathrm{mg} / 1)$ & & & & 1.58 & 1.58 & 1.58 & 1.58 & 1.58 & 1.58 & 1.58 \\
\hline $\mathrm{SCN}(m g / 1)$ & & & & 192 & 192 & 192 & 192 & 192 & 192 & 192 \\
\hline g00 5-day (mg/1) & 1120 & 1120 & 1120 & 1840 & 1040 & 1840 & 1050 & 1050 & 1860 & 1060 \\
\hline TOC (mg/I) & 688 & 628 & 688 & 600 & 600 & 580 & 661 & 661 & 661 & 661 \\
\hline \multicolumn{11}{|l|}{$P O 4-P(m g / 1)$} \\
\hline \multicolumn{10}{|l|}{ NOE (mo/i) } & 98 \\
\hline \multicolumn{11}{|l|}{$\mathrm{NO3}(\mathrm{mg} / 1)$} \\
\hline \multicolumn{11}{|l|}{$\operatorname{TDS}(\mathrm{Rg} / \mathrm{l})$} \\
\hline Color (APHA Units) & 2500 & 2500 & 2500 & 2580 & 2500 & 2500 & 2500 & 2500 & 2500 & 2500 \\
\hline \multicolumn{11}{|l|}{ BASIN PARAMETERS } \\
\hline Vol Wasted (nl) & 895 & 660 & 729 & 805 & 755 & 750 & 885 & 886 & 844 & 937 \\
\hline D. O. $(\mathrm{mg} / 1)$ & 7 & 7 & 7 & 7.1 & 7 & 6.5 & 6.4 & 6.4 & 6.5 & 6.5 \\
\hline SS $(\pi g / 1)$ & 3445 & 3275 & 3060 & 2888 & 3093 & 3048 & 3182 & 3553 & 3622 & 3458 \\
\hline +SS (PAC corrected) & 3445 & 3275 & 3060 & 2888 & 3093 & 3048 & 3182 & 3553 & 3622 & 3458 \\
\hline Temp (C) & 18 & 18 & 19 & 19 & 19 & 32 & 29 & 20 & 20 & 20 \\
\hline oH (std units) & 8.1 & 8.85 & 8 & 8 & 8 & 8 & 8 & 8.1 & 8.1 & 8.1 \\
\hline OUR $(n g / 1 / h r)$ & & & 12 & & & 12 & & & & \\
\hline VSS $(\mathrm{mg} / 1)$ & 2454 & 2333 & 2120 & 2001 & 2143 & 1948 & 2034 & 2271 & 2315 & 2058 \\
\hline *USS (PAC corrected) & 2454 & 2333 & 2120 & 2001 & 2143 & 1948 & 2034 & 2271 & 2315 & 2058 \\
\hline VSS/SS & 0.71 & 0.71 & 0.69 & 0.69 & 0.69 & 0.64 & 0.64 & 0.64 & 0.64 & 0.60 \\
\hline †PQC Added (ag) & 0 & 8 & 8 & 8 & 0 & 0 & 0 & 0 & 8 & 8 \\
\hline Inventory (mg/l basin) & 8 & 0 & 8 & 8 & 0 & 0 & $\theta$ & 0 & 0 & 8 \\
\hline \multicolumn{11}{|l|}{ EFFLUUENT PARRMETEAS } \\
\hline Volume (1iters) & 4.9 & 4.6 & 4.15 & 4.7 & 4.3 & 4.15 & 4.15 & 5.85 & 5.45 & 4.2 \\
\hline $\operatorname{coD}(\mathrm{ag} / 1)$ & 215 & 217 & 295 & 196 & 208 & 181 & 178 & 190 & 196 & 188 \\
\hline SS (mg/1) & 74 & ç42 & 200 & 120 & 176 & 184 & 88 & 80 & 104 & 52 \\
\hline \multicolumn{11}{|l|}{ \#PAC corrected } \\
\hline $\mathrm{pH}$ (std units) & 8.0 & 8.0 & 8.1 & 8.1 & 8.0 & 8.0 & 8.0 & 8.1 & 8.0 & 8.2 \\
\hline USS $(\mathrm{mg} / 1)$ & 53 & 172 & 139 & 83 & 122 & 118 & 56 & 51 & 66 & 31 \\
\hline \multicolumn{11}{|l|}{-PAC corrected } \\
\hline NH3̈N (ng/l) & 186 & 186 & 186 & 157 & 157 & 157 & 177 & 177 & 17 & 177 \\
\hline C.N $(\operatorname{mg} / 1)$ & , & & & 0.447 & 0.447 & 0.447 & 0.447 & 0.447 & 0.447 & 8.447 \\
\hline $\operatorname{SCN}(n g / 1)$ & & & & 5 & 5 & 5 & 5 & 5 & 5 & 5 \\
\hline NO2 (ng/l) & & & & 0.88 & 0.88 & 0.88 & 0.88 & 0.88 & 0.88 & 0.88 \\
\hline :N03 (mg/1) & & & & 2.2 & 2.2 & 2.2 & 2.2 & 2.2 & 2.2 & $2 . ?$ \\
\hline Phenolics $(a g / 1)$ & 1.025 & <.025 & 1.825 & 10.25 & $<0.25$ & 10.25 & 10.25 & .10 .25 & 10.25 & 10.25 \\
\hline$P 04-p(m g / 1)$ & 7.5 & 7.5 & 7.5 & 18.5 & 18.5 & 10.5 & 8 & 8 & 8 & 8 \\
\hline$O r g-N(\mathrm{~kg} / 1)$ & & & & 37 & 37 & 37 & 37 & 37 & 37 & 37 \\
\hline TDS $(m g / 1)$ & 4915 & 4915 & 4915 & 5458 & 5458 & 5458 & 5458 & 5458 & 5458 & 5458 \\
\hline Color (APhiA units) & 750 & 750 & 750 & 750 & 758 & 750 & 750 & 758 & 750 & 750 \\
\hline TOC (mg/1) & 56 & 56 & 56 & 56 & 56 & . 56 & 54 & 54 & 54 & 54 \\
\hline BCD 5-day (mg/l) & 4 & 4 & 4 & 3 & 3 & 3 & 3 & 3 & 3 & 3 \\
\hline
\end{tabular}


UNIT IA TABLE AI

\begin{tabular}{|c|c|c|c|c|c|c|c|c|c|c|}
\hline MONTH & JAN & FEB & FEB & FEB & FEB & FEB & FEB & FEB & FEB & FEB \\
\hline IAY & 31 & 1 & 2 & 3 & 4 & 5 & 6 & 7 & 8 & 9 \\
\hline DAY NO. & 189 & 190 & 191 & 192 & 193 & 194 & 195 & 136 & 197 & 198 \\
\hline \multicolumn{11}{|l|}{ SYSTEMY FARGMETERS } \\
\hline *Sludge Age & 12.20 & 12.80 & 12.00 & 11.99 & 12.88 & 12.80 & 12.80 & 12.81 & 12.80 & 12.80 \\
\hline$+(F / M) C O D$ & 0.43 & 0.31 & 0.60 & 0.64 & 0.63 & 0.24 & 8.48 & 0.66 & 0.45 & 0.35 \\
\hline *HRT & 2.14 & 1.69 & 1.69 & 1.72 & 1.69 & 1.87 & 1.79 & 1.69 & 1.69 & 2.02 \\
\hline \multicolumn{11}{|l|}{ FEED PARAMETERS (BATCH \#) } \\
\hline Volure (liters) & 4.35 & 5.5 & 5.5 & 5.4 & 5.5 & 4.38 & 5.2 & 5.5 & 5.5 & 4.6 \\
\hline $\mathrm{pH}$ (std units) & 18.3 & 10.4 & 11.3 & 11 & 10.9 & 10.9 & 11.5 & 11.5 & 11.2 & 10.4 \\
\hline $\operatorname{COD}(\mathrm{mg} / 1)$ & 2137 & 2137 & 2137 & 2118 & 2118 & 2118 & 2118 & 2100 & 2100 & 2180 \\
\hline NHJN $(\log / 1)$ & 154 & 154 & 154 & 163 & 163 & 163 & 163 & 182 & 182 & 182 \\
\hline SS (ng/1) & 296 & 296 & 296 & 296 & 296 & 296 & 296 & 336 & 336 & 336 \\
\hline USS (ngo/l) & 106 & 186 & 106 & 106 & 186 & 106 & 186 & 154 & 154 & 154 \\
\hline CN (mg/l) & & & & & & & & 9.59 & 9.59 & 9.59 \\
\hline $\operatorname{SCN}(m g / 1)$ & & & & & & & & 184 & 184 & 184 \\
\hline 3005 -day (mg/1) & 1830 & 1830 & 1030 & 1860 & 1060 & 1860 & 1860 & 930 & 930 & 930 \\
\hline TOC (xgl1) & 648 & 648 & 648 & 610 & 610 & 610 & 610 & 645 & 645 & 645 \\
\hline Phenolics (mg/l) & 9.7 & 9.7 & 9.7 & 9.7 & 9.7 & 9.7 & 9.7 & 8.5 & 8.5 & 8.5 \\
\hline \multicolumn{11}{|l|}{$\mathrm{PO} 4-\mathrm{P}(\mathrm{ag} / 1)$} \\
\hline Drg-N $(m g / 1)$ & & & & & & & & 80 & 80 & 80 \\
\hline \multicolumn{11}{|l|}{ NOS (mg/1) } \\
\hline \multicolumn{11}{|l|}{$\mathrm{N} 03$ (mg/1) } \\
\hline \multicolumn{11}{|l|}{ TDS (ng/l) } \\
\hline Color (APHA Units) & 2508 & 2500 & 2500 & 2500 & 2500 & 2508 & 2500 & 2500 & 2500 & 2500 \\
\hline \multicolumn{11}{|l|}{ BASIN PARRAETERS } \\
\hline Vol Wasted (ni) & 965 & 946 & 886 & 918 & 859 & 942 & 912 & 986 & 913 & 804 \\
\hline D. O. (mg/1) & 6.4 & 6.4 & 6.2 & 6.2 & 6.2 & 5.6 & 6.2 & 6.2 & 6.2 & 6.2 \\
\hline SS (mg/1) & 3885 & 5295 & 2702 & 2482 & 2568 & 6235 & 3080 & 2335 & 3398 & 4070 \\
\hline IGE (MnC eorreeted) & 3085 & 5295 & 2702 & 2482 & 8568 & 6235 & 3080 & 2335 & 3398 & 4070 \\
\hline Temp (C) & 28 & 20 & 20 & 20 & 20 & 20 & 28 & 28 & 20 & 28 \\
\hline ôH (std units) & 8.3 & 8.3 & 8.4 & 8.3 & 8.4 & 8.3 & 8.2 & 8.7 & 8.5 & 8.5 \\
\hline OUR (ag/1/hr) & & & 16 & & & & 12 & & & IE \\
\hline VSS (mg/1) & 1788 & 3151 & 1620 & 1488 & 1548 & 3738 & 1920 & 1456 & 2118 & 2288 \\
\hline *VS̈S (AAC sorrected) & 1788 & $315 !$ & 1620 & 1488 & 1548 & 3738 & 1920 & 1456 & 2118 & 2088 \\
\hline USS/S5 & 8.60 & 0.60 & 0.60 & 0.60 & 0.60 & 0.60 & 8.62 & 0.62 & 0.62 & 0.56 \\
\hline APAC ACded (ag) & 8 & 8 & 0 & 0 & 8 & 0 & 0 & $\theta$ & 0 & 0 \\
\hline +Inventory (ag/l basin) & 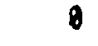 & 8 & 0 & 8 & 8 & 8 & 0 & e & 0 & 0 \\
\hline \multicolumn{11}{|l|}{ EFFLUENT PARAMETERS } \\
\hline Volutee (11ters) & 4 & 5.26 & 5.5 & 4.65 & 5.5 & 5.15 & 4.85 & 5. 2 & J. 1 & 4.25 \\
\hline $\operatorname{ClD}(\operatorname{mg} / 1)$ & 286 & 199 & 206 & 223 & 208 & 227 & 229 & 237 & 274 & 335 \\
\hline $55(\mathrm{mg} / \mathrm{l})$ & 26 & 54 & 56 & 44 & 66 & 70 & 56 & 42 & 58 & 188 \\
\hline \multicolumn{11}{|l|}{ +PAC corrected } \\
\hline DH (std units) & 8.3 & 8.3 & 8.4 & 8.4 & 8.4 & 8.3 & 8.4 & 8.6 & 8.5 & 8.5 \\
\hline VSS (ng/l) & 15 & 32 & 34 & 26 & 48 & 42 & 35 & 26 & 35 & 185 \\
\hline \multicolumn{11}{|l|}{ *PAC corrected } \\
\hline NH3N (mrg/1) & 180 & 180 & 180 & 161 & 161 & 161 & 161 & 154 & 154 & 154 \\
\hline CN $(n g / l)$ & & & & & & & & 0.716 & 0.716 & 0.716 \\
\hline SCN (ng/1) & & & & & & & & 43.9 & 43.9 & 43.9 \\
\hline NOS $(0 \mathrm{~g} / 1)$ & & & & & & & & 0.92 & 0.92 & 0.92 \\
\hline N03 (mg/1) & & & & & & & & 11.25 & 11.25 & 14.25 \\
\hline Phenolics (mg/1) & 18.25 & 10.825 & 18.825 & 18.025 & 10.825 & $(0.025$ & 10.025 & 10.825 & 18.025 & 10.025 \\
\hline$P 04-P$ (ng/l) & 3.3 & 3.3 & 3.3 & 1.9 & 1.9 & 1.9 & 1.9 & 3.6 & 3.6 & 3.6 \\
\hline $\operatorname{Org}-N(\operatorname{mg} / 1)$ & & & & & & & & 27 & 27 & 27 \\
\hline TUS $(\operatorname{mog} / 1)$ & 4532 & 4532 & 4592 & 4532 & 4592 & 4592 & 4592 & 4832 & 4892 & 4892 \\
\hline Color (APHA units) & 1400 & 1480 & 1480 & 1480 & 1400 & 1480 & 1480 & 1500 & 1500 & 1580 \\
\hline TOC $(m g / 1)$ & 59 & 59 & 59 & 56 & 56 & 56 & 56 & 78 & 78 & 78 \\
\hline \multirow[t]{2}{*}{ BOD 5-day (mg/l) } & 3.5 & 3.5 & 3.5 & 5 & 5 & 5 & 5 & 16.5 & 16.5 & 16.5 \\
\hline & \multicolumn{10}{|c|}{$-72-$} \\
\hline
\end{tabular}


UNIT IA TABLE A!

\begin{tabular}{|c|c|c|c|c|c|c|c|c|c|c|}
\hline MCNTH & FEB & FEB & FEB & FEB & FEB & FEB & FEB & FEB & FEB & FEB \\
\hline CAY & 10 & 11 & 12 & 13 & 14 & 15 & 16 & 17 & 18 & 19 \\
\hline EAY NO. & 199 & 200 & 291 & 202 & 203 & 204 & 205 & 206 & 207 & 208 \\
\hline \multicolumn{11}{|l|}{ SYSTEM FRRAYETERS } \\
\hline tSludge Fige & 12.30 & 12.00 & 12.80 & 12.30 & 12.80 & 9.00 & 3.20 & 9.30 & 9.20 & 9.80 \\
\hline$H(F / M) C O D$ & 0.37 & 0.34 & 0.40 & 0.41 & 0.32 & 0.36 & 0.32 & 0.32 & 0.31 & 0.35 \\
\hline FHRT & 2.02 & 2.04 & 1.89 & 1.82 & 1.94 & 1.36 & 2.14 & 1.75 & 1.88 & 1.95 \\
\hline \multicolumn{11}{|l|}{ FEED PARAMETERS (BATCH } \\
\hline Volune (liters) & 4.6 & 4.55 & 4.92 & 5.1 & 4.8 & 4.75 & 4.35 & 5.3 & 4.94 & 4.78 \\
\hline pH (std units) & 18.4 & 18.4 & 10 & 10 & 10.1 & 9.6 & 9.6 & 11.3 & 10.6 & 10.3 \\
\hline $\operatorname{COD}(\mathrm{ng} / 1)$ & 2076 & 2076 & 2976 & 2076 & 2124 & 2124 & 2124 & 1649 & 1649 & 1649 \\
\hline NH3N $(\mathrm{gg} / 1)$ & 183 & 183 & 183 & 183 & 166 & 166 & 166 & 166 & 166 & 166 \\
\hline SS (mg/l) & 336 & 336 & 336 & 336 & 254 & 254 & 254 & 254 & 254 & 254 \\
\hline VSS. $( \pm g / 1)$ & 154 & 154 & 154 & 154 & 124 & 124 & 124 & 124 & 124 & 124 \\
\hline $\mathrm{CN}$ (mg/1) & 9.59 & 9.59 & 9.59 & 9.59 & & & & & & \\
\hline $\operatorname{SCN}(m g / 1)$ & 184 & 184 & 184 & 184 & & & & & & \\
\hline BOD 5-day (ng/l) & 1130 & 1130 & 1130 & 1130 & 1080 & 1080 & 1080 & 1030 & 1030 & 1030 \\
\hline TOC $(\mathrm{mg} / 1)$ & 711 & 711 & 711 & 711 & 698 & 698 & 698 & 575 & 575 & 575 \\
\hline Phenolics (ng/l) & 8.5 & 8.5 & 8.5 & B. 5 & 9.8 & 9.8 & 9.8 & 9.8 & 9.8 & 9.8 \\
\hline \multicolumn{11}{|l|}{$P 04-P(\operatorname{Pg} / 1)$} \\
\hline \multicolumn{11}{|l|}{ NOS $(\mathrm{mg} / \mathrm{l})$} \\
\hline N03 (mg/1) & . & & & & & & & & & \\
\hline \multicolumn{11}{|l|}{ TDS ing/1) } \\
\hline Color (APHA Units) & 2500 & 2500 & 2500 & 2500 & 2500 & 2500 & 2508 & 2500 & 2580 & ; 2500 \\
\hline \multicolumn{11}{|l|}{ EASIN PARAMETERS } \\
\hline Vol Wasted (w) & 884 & 842 & 466 & 369 & 480 & 856 & 1041 & 978 & 1294 & 1230 \\
\hline D.0. $(\operatorname{mg} / 1)$ & 6.2 & 6.2 & 6.2 & 6.2 & 6.2 & 6.2 & 6.2 & 6.2 & 6.2 & 6.2 \\
\hline SS (ng/l) & 3855 & 4145 & 3760 & 3718 & 4588 & 4860 & 4855 & 3908 & 3708 & 3185 \\
\hline *SS (PRC corrected) & 3855 & 4145 & 3760 & 3710 & 4580 & 4868 & 4055 & 3500 & 3700 & 3185 \\
\hline Temp $(C)$ & 19 & 20 & 20 & 20 & 20 & 20 & 20 & 20 & 19 & 19 \\
\hline pH (std units) & 8.3 & 8.4 & 8.1 & 8.2 & 8 & 8.1 & 8.2 & 8.2 & 8.3 & 8.1 \\
\hline OUR (ng/1/hr) & 12 & & & 12 & & & 24 & & 24 & 24 \\
\hline VSS (mg/1) & 2159 & 2330 & 2114 & 2128 & 2627 & 2329 & 2385 & 2294 & 2176 & $\$ 873$ \\
\hline *USS (FAC corrected) & 2159 & 2338 & 2114 & 2128 & 2627 & 2329 & 2385 & 2294 & 2175 & 1873 \\
\hline USS/SS & 0.56 & 0.56 & 0.56 & 0.57 & 0.57 & 0.57 & 0.59 & 0.59 & 0.59 & 0.59 \\
\hline FPAC Added (ng) & 0 & 8 & $\theta$ & 8 & 0 & 0 & 0 & 8 & 0 & 0 \\
\hline *Inventory (mg/l basin) & $\theta$ & 8 & $\theta$ & 0 & 0 & 8 & 0 & 0 & 0 & 0 \\
\hline \multicolumn{11}{|l|}{ EFFLIENT PARGMETERS } \\
\hline Voluse (Iiters) & 4.25 & 4.25 & 4.65 & 4.8 & 5.2 & 4.45 & 4.15 & 5.1 & 4.8 & 4.58 \\
\hline $\operatorname{coD}(\operatorname{ag} / 1)$ & 365 & 365 & 338 & 359 & 265 & 259 & $\hat{c} 62$ & 262 & 256 & 240 \\
\hline SS $(m g / 1)$ & 188 & 154 & 432 & 488 & 458 & 436 & 286 & 272 & 30 & 72 \\
\hline \multicolumn{11}{|l|}{ APAC corrected } \\
\hline $\mathrm{pH}$ (std units) & 8.5 & 8.4 & 8.2 & 8.2 & 8.0 & 8.1 & 8.2 & 8.2 & 8.4 & 8.3 \\
\hline VSS (mq/1) & 106 & 87 & 243 & 280 & 263 & 250 & 168 & 160 & 18 & 42 \\
\hline \multicolumn{11}{|l|}{ IPAC correeted } \\
\hline NH3̄N (mg/l) & 182 & 182 & 182 & 182 & 290 & 200 & 200 & 196 & 196 & 196 \\
\hline$[N(\mathrm{mg} / 1)$ & 0.716 & 0.716 & 0.716 & 9. 716 & & & & & & \\
\hline SCN (ng/1) & 43.9 & 43.9 & 43.9 & 43.9 & & & & & & . \\
\hline N02 (-7g/l) & 0.92 & 0.92 & 0.92 & 0.92 & & & & & & \\
\hline $\mathrm{NOJ}(\mathrm{ng} / \mathrm{l})$ & 11.25 & 11.25 & 11.25 & 11.25 & & & & & & \\
\hline Phenolics (mg/l) & 18.825 & 10.025 & 10.825 & 10.025 & 18.025 & 18.025 & 10.025 & 18.825 & 10.025 & 10.025 \\
\hline$P 04-P(m g / 1)$ & 2.9 & 2.9 & 2.9 & 2.9 & 3.9 & 3.9 & 3.9 & 3.7 & 3.7 & 3.7 \\
\hline Org-N $(m g / 1)$ & 27 & 27 & 27 & 27 & & & & & & \\
\hline TDS (ng/l) & 4892 & 4892 & 4892 & 4892 & 5352 & 5352 & 5352 & 5352 & 5352 & 5352 \\
\hline Color (APHA units) & 1580 & 1500 & 1500 & 1500 & 1500 & 1500 & 1500 & 1500 & 1500 & 1500 \\
\hline TOC (mg/l) & 94 & 94 & 94 & 94 & 76 & 76 & 76 & 73 & 73 & 73 \\
\hline BOD S-day (mg/1) & 6 & 6 & 6 & $73^{6}$ & 3 & 3 & 3 & 2 & 2 & 2 \\
\hline
\end{tabular}


UNIT IA TABLE A1

\begin{tabular}{|c|c|c|c|c|c|c|c|c|c|c|}
\hline FONTH & FEB & FEB & FEB & FE,B & FEB & FEB & FEB & FEB & FEB & FEB \\
\hline ¿îYY & 28 & 21 & 22 & 23 & 24 & 25 & 26 & 27 & 28 & 29 \\
\hline CAY NO. & 209 & $2 ! 0$ & 211 & 212 & 213 & 214 & 215 & 216 & 217 & 218 \\
\hline \multicolumn{11}{|l|}{ SYSTEM MREMMETLRG } \\
\hline +Sludge Age & 8. 97 & 9.00 & 9.80 & 9.20 & 3.00 & 9.80 & 9.00 & 9.80 & 9.80 & 9.88 \\
\hline$t(F / M) C O D$ & 0.29 & 0.31 & 0.30 & 0.26 & 0.29 & 0.34 & 0.36 & 0.35 & 0.44 & 0.39 \\
\hline *HRT & 2.72 & 1.79 & 1.86 & 1.86 & 1.88 & 1.80 & 1.80 & 2.14 & 1.82 & 2.11 \\
\hline \multicolumn{11}{|c|}{ FEED PARAMETERS (BATCH I) } \\
\hline Volume (liters) & 3.42 & 5.2 & 5 & 5 & 4.95 & 5.18 & 5.18 & 4.35 & 5.12 & 4.4 \\
\hline phH (std units) & 11 & 11 & 18.6 & 11 & 10.3 & 11 & 18.7 & 9.8 & 18.8 & 9.9 \\
\hline $\operatorname{coD}(\operatorname{mg} / 1)$ & 1649 & 1630 & 1630 & 1630 & 1766 & 1766 & 1766 & 1766 & 1795 & 1795 \\
\hline NH3N $(g / 1)$ & 166 & 176 & 176 & 176 & 174 & 174 & 174 & 174 & 149 & 149 \\
\hline SS $(n g / 1)$ & 254 & & & & & & & & 188 & 188 \\
\hline VSS. (mg/l) & 124 & & & & & & & & 89 & 80 \\
\hline CN $(\operatorname{lng} / 1)$ & & 2.55 & 2.55 & 2.55 & 2.55 & 2.55 & 2.55 & 2.55 & & \\
\hline $\operatorname{SCN}(x g / 1)$ & & 174 & 174 & 174 & 174 & 174 & 174 & 174 & & \\
\hline BOD 5-day $(5 g / 1)$ & 1030 & 900 & 900 & 980 & 985 & 985 & 985 & 985 & 1090 & 1890 \\
\hline TOC $(\arg / 1)$ & 575 & 566 & 566 & 566 & 564 & 564 & 564 & 564 & 572 & 572 \\
\hline Phenolics ( $/ 1)$ & 9.8 & 6.6 & 6.6 & 6.6 & 6.6 & 6.6 & 6.6 & 6.6 & & \\
\hline
\end{tabular}

P04-P (gg/1)

Org-N (mg/l)

NO2 (mo/1)

NO3 (ng/1)

TDS (mg/l)

Color (APHA Units)

BASIN PARAMETERS

Vol Hasted (al)

D. O. (no/l)

SS (Eng/1)

- GS (PAC Eorrected)

Teno (C)

$\mathrm{pH}$ (std units)

OUR (ng/l/hr)

VSS (itig $/ 1$ )

+USS (PAC corrected)

USS/SS

tPAC Added (mg)

Inventory (mg/l basin)

EFFLUENT PARGMETSRS

Volune (11ters)

Cod (og/I)

SS (mg/l)

-PAC corrected

oH (std units)

USS (ma/1)

*AAC corrected

NHIN (mg/l)

CN ( $m g / 1)$

SCN (ng/l)

: $102(\mathrm{mg} / 1)$

N03 (ng/l)

Phenolics (mg/l)

POA-P (mg/l)

Org-N (og/l)

TDS (mg/l)

Color (AFHA units)

TOC (mg/l)

$\begin{array}{lllllll}71 & 71 & 71 & 71 & 71 & 71 & 71\end{array}$

BOD 5-day (mg/1)

\begin{tabular}{|c|c|c|c|c|c|c|c|c|c|}
\hline 2560 & 3000 & 3008 & 3008 & 3000 & 3000 & 3808 & 3000 & & \\
\hline 1380 & 1304 & 1182 & 1178 & 1111 & 1276 & 1180 & 1294 & 662 & 1233 \\
\hline 6.4 & 6.2 & 6.2 & 6.2 & 6.2 & 6.2 & 6.2 & 8.7 & 8.7 & 8.7 \\
\hline 2768 & 4802 & 3968 & 3992 & 3835 & 3415 & 3165 & 2898 & 2765 & 2665 \\
\hline 2760 & 4002 & 2968 & 3992 & 3839 & 3415 & 3165 & 2898 & 2765 & 26665 \\
\hline 18 & 19 & 20 & 19 & 19 & 19 & 19 & 18 & 19 & 19 \\
\hline \multirow[t]{2}{*}{8.3} & 7.8 & 7.9 & 7.9 & 8 & 7.7 & 8 & 7.9 & 7.7 & 7.5 \\
\hline & 12 & & & & & & 10 & & \\
\hline 1598 & 2310 & 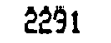 & 2660 & 2555 & 2276 & 2109 & 1822 & 1738 & 1676 \\
\hline 1598 & 2310 & 2291 & 2660 & 2555 & 2276 & 2109 & 1822 & 1738 & 1676 \\
\hline 0.58 & 0.58 & 0.58 & 0.67 & 0.67 & 0.67 & 0.67 & 0.63 & 0.63 & 0.63 \\
\hline 0 & 0 & 0 & 0 & 8 & 0 & 0 & 8 & 8 & 0 \\
\hline 0 & 0 & 0 & 0 & $\theta$ & 0 & 0 & 0 & 0 & 8 \\
\hline 3.5 & 4.95 & 4.68 & 4.63 & 4. 95 & 5.4 & 4.85 & 4.43 & 5.1: & 4.6 \\
\hline 230 & 235 & 235 & 231 & 219 & 255 & 232 & 383 & 253 & 251 \\
\hline 30 & 24 & 128 & 134 & 172 & 36 & 100 & 26 & 364 & 58 \\
\hline 8.2 & 8.0 & 8.1 & 8.0 & 8.1 & 7.9 & 8.8 & 7.7 & 8.0 & 7.7 \\
\hline 17 & 14 & 74 & 89 & 115 & 24 & 87 & 16 & 229 & 36 \\
\hline \multirow[t]{5}{*}{136} & 136 & 196 & 196 & 198 & 188 & 188 & 188 & 179 & 179 \\
\hline & 0.451 & 0.451 & 0.451 & 0.451 & 0.451 & 0.451 & $8.45 !$ & & \\
\hline & 5.3 & 5.3 & 5.3 & 5.3 & 5.3 & 5.3 & 5.3 & & \\
\hline & 0.36 & 0. 56 & 0.36 & 0.36 & 0.36 & 0.36 & 8.36 & & \\
\hline & 4 & 4 & 4 & 4 & 4 & 4 & 4 & & \\
\hline$<0.825$ & 10.025 & 10.025 & 10.825 & 10.025 & 10.025 & 10.025 & 10.025 & & \\
\hline \multirow[t]{2}{*}{3.7} & 8.4 & 8.4 & 8.4 & 13.5 & 13.5 & 13.5 & 13.5 & & \\
\hline & 12 & 12 & 12 & 12 & 12 & 12 & 12 & & \\
\hline 5352 & 5714 & 5714 & 5714 & 5714 & 5714 & 5714 & 5714 & & \\
\hline 1580 & 1500 & 1508 & 1500 & 1500 & 1580 & 1580 & 1500 & & \\
\hline 73 & 64.5 & 64.5 & 64.5 & 65.3 & 65.3 & 55.3 & 65.3 & 77.2 & 77.2 \\
\hline \multirow[t]{2}{*}{2} & 3 & 3 & 3 & 3 & 3 & 3 & 3 & 17.4 & 17.4 \\
\hline & & & -74 & & & & & ' & \\
\hline
\end{tabular}


UNIT IA TABLE AI

\begin{tabular}{|c|c|c|c|c|c|c|c|c|c|c|}
\hline MONTH & MAR & MAR & MAR & MAR & MAR & MAR & YAR & MAR & HARR & MAR \\
\hline DAY & 1 & 2 & 3 & 4 & 5 & 6 & 7 & 8 & $\exists$ & 10 \\
\hline DAY NO. & 219 & 220 & 221 & 222 & 223 & 224 & 225 & 226 & 227 & 228 \\
\hline \multicolumn{11}{|l|}{ SYSTEY PARAMETERS } \\
\hline Sludge Age & 9.20 & 9.00 & 9.80 & 9.80 & 9.20 & 9.20 & 9.80 & 9.80 & 3.80 & 8.93 \\
\hline$+(F / M) C O D$ & 8.41 & 0.44 & $0.5 !$ & 0.46 & 0.37 & 0. 44 & 0.55 & 0.56 & 0.53 & 0.64 \\
\hline HRT & 1.91 & 2.02 & 1.69 & 1.81 & 2.22 & 2.00 & 1.71 & 1.82 & 1.96 & 1.74 \\
\hline \multicolumn{11}{|l|}{ FEED FRRAMETERS (BATCH } \\
\hline Volume (liters) & 4.86 & 4.6 & 5.5 & 5.14 & 4.18 & 4.65 & 5.45 & 5.1 & 5 & 5.35 \\
\hline $\mathrm{pH}$ (std units) & 10.5 & 12 & 11.7 & 11.8 & 11.8 & 11.7 & 11.7 & 11.8 & 11.7 & 11.8 \\
\hline $\operatorname{coD}(\operatorname{mg} / 1)$ & 1795 & 1893 & 1803 & 1803 & 1803 & 1834 & 1834 & 1834 & 1828 & 1828 \\
\hline NHJ̈N (mg/l) & 149 & 177 & 177 & 177 & 177 & 162 & 162 & 162 & 185 & 186 \\
\hline SS $(a g / 1)$ & 188 & 188 & 188 & 188 & 188 & & & & 143 & 143 \\
\hline VSS. (mg/1) & 80 & 80 & 80 & 68 & 80 & & & & 66 & 66 \\
\hline \multicolumn{11}{|l|}{$\mathrm{CN}(\mathrm{mg} / 1)$} \\
\hline \multicolumn{11}{|l|}{$\operatorname{SCN}(n g / 1)$} \\
\hline BOD 5-day (mg/1) & 1030 & 1090 & 1030 & 1090 & 1090 & 1200 & 1200 & 1280 & 1170 & 1178 \\
\hline TOC $(n g / 1)$ & 572 & 569 & 569 & 569 & 569 & 570 & 570 & 578 & 578 & 578 \\
\hline \multicolumn{11}{|l|}{ Phenolics (mg/l) } \\
\hline \multicolumn{11}{|l|}{$P Q 4-D(m g / 1)$} \\
\hline \multicolumn{11}{|l|}{$\operatorname{Org}-N(\mathrm{mg} / 1)$} \\
\hline NO2 (mg/l) & & & . & & & & & & & \\
\hline \multicolumn{11}{|l|}{$\mathrm{NO3}(\mathrm{mg} / 1)$} \\
\hline \multicolumn{11}{|l|}{$\operatorname{TDS}(\operatorname{mg} / 1)$} \\
\hline \multicolumn{11}{|l|}{ Color (AFHA Unit5) } \\
\hline \multicolumn{11}{|l|}{ BRSIN FARAMEIERS } \\
\hline Vol Wasted (m!) & 1131 & 1118 & 914 & 1238 & 1102 & 852 & 578 & 520 & 759 & 40 \\
\hline D.0. $(\mathrm{gg} / 1)$ & 8.7 & 0.7 & 8.6 & 8.6 & 8.4 & 8.3 & 7.8 & 8.2 & 8.2 & 9.1 \\
\hline SS $(\mathrm{mg} / 1)$ & 2718 & 2330 & 2515 & 2555 & 2468 & 2468 & 2323 & 2020 & 2090 & 1855 \\
\hline \&SS (PAC corrected) & 2718 & 2390 & 2515 & 2555 & 2460 & 2460 & 2323 & 2020 & 2090 & 1855 \\
\hline Temp (C) & 19 & 18 & 18 & 19 & 18 & 19 & 19 & 18 & 18 & 18 \\
\hline pH (std units) & 7.3 & 8.1 & 8 & 7.25 & 7.2 & 7.7 & 7.7 & 7.3 & 7.4 & 7.2 \\
\hline OUR (ng/1/hr) & & & & & 7 & 7 & & 30 & & \\
\hline VSS $(m g / 1)$ & 1768 & 1555 & 1636 & 1662 & 1600 & 1599 & 1511 & 1385 & 1433 & 1272 \\
\hline WSS (PAC corrected) & 1768 & 1555 & 1636 & IE62 & 1520 & 1599 & 1511 & 1385 & 1433 & 1272 \\
\hline USS/SS & 0.65 & 0.65 & 0.63 & 0.55 & 0.65 & 0.65 & 0.65 & 0.69 & 0.69 & 0.69 \\
\hline PAC Added (mg) & 0 & 8 & 0 & 0 & 0 & 8 & 0 & 0 & 0 & 0 \\
\hline Inventory (og/l basin) & 8 & $\theta$ & 8 & b & 8 & 0 & 0 & 0 & 8 & 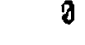 \\
\hline \multicolumn{11}{|l|}{ EFFLUEYT PARAMETERS } \\
\hline Voluwe (Iiters) & 4.79 & 4.6 & 5.4 & 4.85 & 3.9 & 4.45 & 5.1 & 4.95 & 4.88 & 5.1 \\
\hline $\operatorname{coD}(\operatorname{mg} / 1)$ & 240 & 242 & 234 & 222 & 252 & 264 & 216 & 212 & 222 & 231 \\
\hline SS (ng/1) & $\$ 16$ & 116 & 242 & 50 & 146 & 146 & 344 & 332 & 246 & 480 \\
\hline \multicolumn{11}{|l|}{ APAC corrected } \\
\hline $\mathrm{pH}$ (std units) & 7.6 & 7.9 & 8.0 & 7.9 & 7.6 & 7.3 & 7.7 & 7.6 & 7.5 & 7.3 \\
\hline VSS $(\mathrm{ng} / 1)$ & 75 & 75 & 157 & 33 & 35 & 95 & 224 & 228 & 169 & 329 \\
\hline \multicolumn{11}{|l|}{ APAC corrected } \\
\hline NH.je (agdl) & 179 & 173 & 173 & 173 & 173 & 216 & 216 & 216 & 219 & 218 \\
\hline \multicolumn{11}{|l|}{ CN (og/1) } \\
\hline $\operatorname{SCN}(\operatorname{mg} / 1)$ & & & & & & & & & & \\
\hline NO2 (mg/1) & & & & & & & & & & \\
\hline N03 (ng/1) & & & & & & & & & & \\
\hline Phenolics (og/l) & & & & & & & & & & \\
\hline $\begin{array}{l}\mathrm{PO4-P}(\mathrm{mg} / 1) \\
\operatorname{Crg}-\mathrm{N}(\mathrm{aga} / 1)\end{array}$ & & & & & & & & & & \\
\hline TDS (ag/1) & & & & & & & & & & \\
\hline Color (APHA units) & & & & & & & & & & \\
\hline TOC $\operatorname{lng} / 1)$ & 77.2 & 68.6 & 68.6 & 68.6 & 68.6 & 61.6 & 61.6 & 61.6 & 64.7 & 64.7 \\
\hline 800 5-day (mg/!) & 17.4 & 3 & 3 & 3 & 3 & 3 & 3 & 3 & 5 & 5 \\
\hline & & & & -75 & & & & & & \\
\hline
\end{tabular}




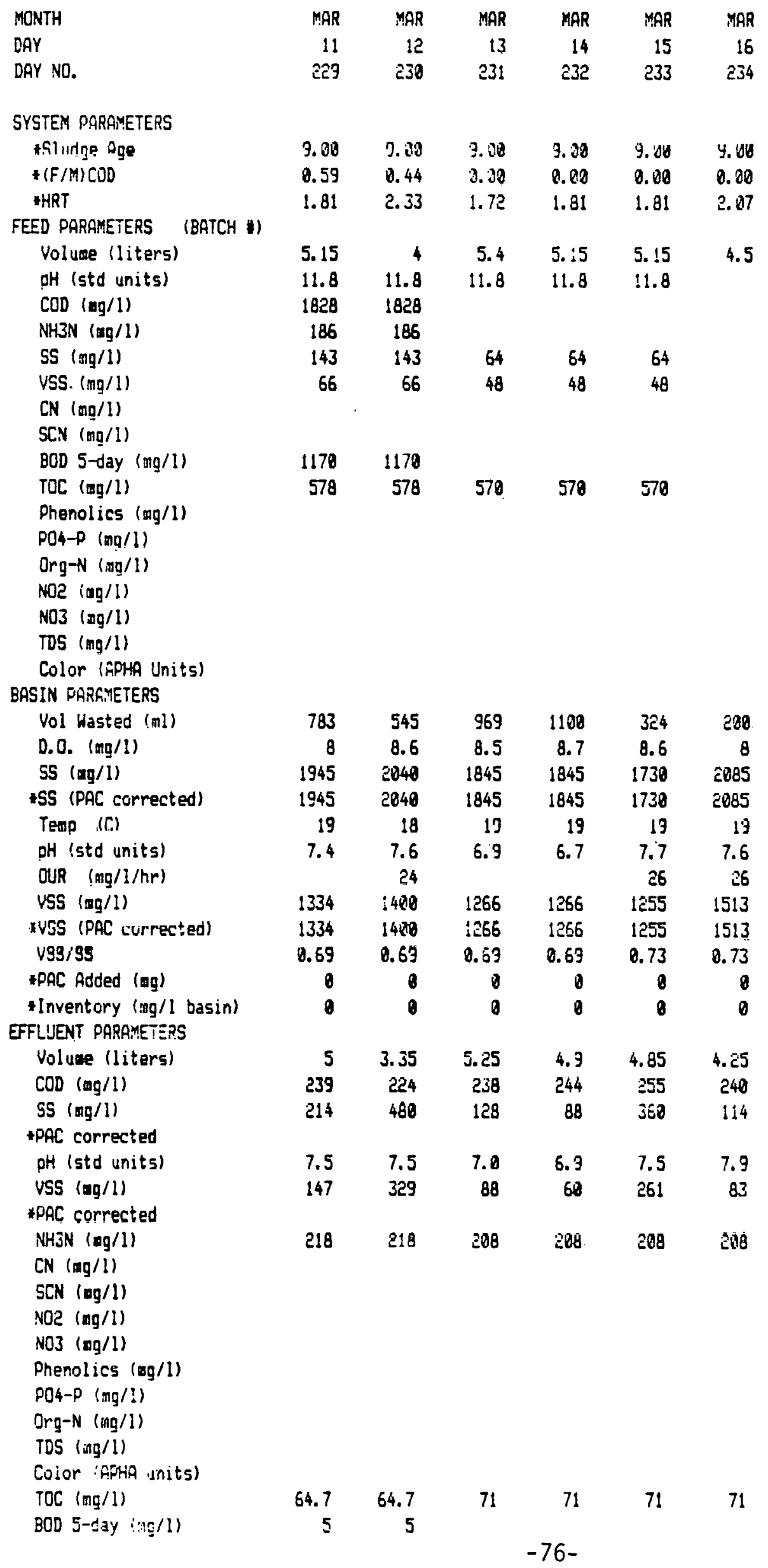


UNIT IB TABLE A2

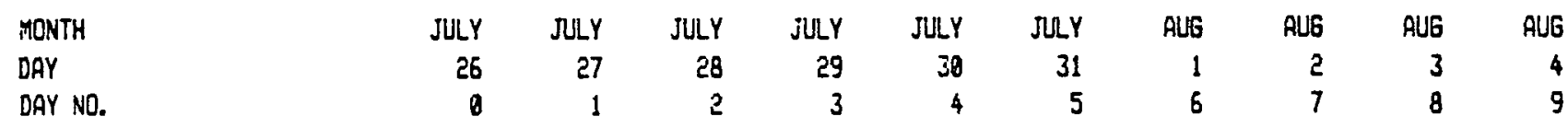

\section{SYSTEM PARAMETERS}

s Sludge Age

$*$ (F/M) COD

$\begin{array}{rr}38 & 30 \\ 0.85 & 0.85\end{array}$

30
0.86

30
0.08

30
0.07

30
0.86

30
0.07

30
0.86

30
0.86

30
0.98

*HRT

2.2

2.1

2. 2

2.2

$2 \quad 2.2$

2.1

2

2.2

2.2

$3.22 \quad 3.41 \quad 3.2$

3.15

$3.45 \quad 3.23$

3.27

3.46

27

3.14

of (std units)

$6.3 / 9.7 \quad 7.1 / 9.0$

$7.47 .1 / 8.8 \quad 7.2 / 9.2$

7.4

7.5

3.2

$\operatorname{coD}(\mathrm{mg} / 1)$

NH3̄N (mg/l)

$\begin{array}{rr}217 & 185 \\ 12 & 10\end{array}$

191

183

208
4

167

$7.4 \quad 7.3$

SS (ng/1)

VSS (mo/l)

CN (mg/l)

SCN (mo/l)

$2.2 \quad 0.84$

0.84

0.84

8.84

0.84

0.84

8.84

1.32

1.32

800 5-day (mg/l)

6.410 .1

10.1

10.1

10.1

10.1

10.1

10.1

10.3

10.3

TOC (ang/1)

Phenolics (mo/l)

$48 \quad 48$

$$
48
$$

$$
48
$$

48

1.025

P04-p (mg/l)

4.2

Org-N (mg/1)

ND2 (ag/l)

$\mathrm{No3}(\mathrm{mg} / 1)$

TDS (mg/l)

Color (APHA Unit5)

BASIN PARAMETERS

Vol Wasted (all)

D. O. (mg/1)

SS (mg/1)

*SS (PAC corrected)

Temp (C)

oH (std units)

OUR (ag/l/hr)

USS (mg/l)

* USS (FAC corrected)

VSS/SS

tPAC Added (ng)

* Inventory (wo/l basin)

8
59.8

$8.79 \quad 8.79$

$\begin{array}{rrrrr} & 7 & 7 & 7 & 7 \\ 0.79 & 8.79 & 8.79 & 9.79 & 8.79\end{array}$

$1.825 \quad 1.025$

$1.3 \quad 1.3$

152

240

$240 \quad 240$

240

240

240

$8.7 \quad 8.7$

$\begin{array}{lllllll}250 & 250 & 250 & 250 & 250 & 250 & 250\end{array}$

$$
7.7
$$

7.7

2270

177

25

7.5

1903

$1484 \quad 1383$

63

$$
138
$$

138
7.6

$\begin{array}{rrrrrrr}175 & 188 & 227 & 180 & 222 & 182 & 157 \\ 7.6 & 7.8 & 7.2 & 7.2 & 7.4 & 7.3 & 7.3 \\ 1660 & 1950 & 2190 & 1930 & 1930 & 1830 & 1668 \\ 1160 & 1450 & 1690 & 1430 & 1430 & 1330 & 1160 \\ 26 & 27 & 27 & 27 & 27 & 27 & 27 \\ 7.7 & 7.6 & 8 & 8 & 7.9 & 7.8 & 7.5 \\ 1262 & 1482 & 1664 & 1520 & 1520 & 1441 & 1380 \\ 882 & 1102 & 1284 & 1126 & 1126 & 1047 & 964 \\ 143 & 143 & 143 & 143 & 143 & 144 & 143 \\ 580 & 500 & 500 & 508 & 500 & 500 & 500\end{array}$

EFLUENT PARAMETERS

Volume (1iters)

COD (mg/l)

SS (ing/1)

PPAC corrected

pH (std units)

USS (mg/1)

-PAC corrected

NHISN (mg/1)

CN (ag/1)

SCN (mg/l)

NO2 (tog/1)

NO3 (ag/l)

Phenolics (ag/l)

2000

1508

26

8.1

4

1500

1148

143

$\theta 0$

3.00

2. 85

2. 85

3.18

2. 83

2. 75

177

78

7.3

3.28
160
168

$143 \quad 145 \quad 138$

113

113

16

143

po4-p (ng/1)

Org-N (mg/1)

TDS (ng/1)

Color (APHA units)

TOC (mg/1)

8. 10

$7.80 \quad 7.68$

7.98

7.90

8.08

7.98

7.70

1.48
5.
5.7
217

2

5.2

2
1.48

1. 48

12
1.48

$1 ?$
1.48

12

48

5.25 .2

5.2

5.2

5.2

5.7

5.7

5.7

217

217

217

3

3

3

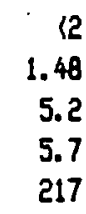

2.3
1.37

2.3
1.37

2. 3

5.2

3.

176

3.

1.825

176

1.825

0.90 .9

1.02

$\begin{array}{rrrrrrr}1008 & 1080 & 1800 & 1000 & 1008 & 1000 & 1000 \\ 45 & 45 & 45 & 45 & 45 & 45 & 45\end{array}$

BOD 5-day $(\mathrm{mg} / 1)$ 


\begin{tabular}{|c|c|c|c|c|c|c|c|c|c|c|}
\hline MONTH & AUG & ALG & AUG & AUG & AUG & AUG & AUG & AUG & AUG & AUG \\
\hline DAY & 5 & 6 & 7 & 8 & 9 & 10 & 11 & 12 & 13 & 14 \\
\hline DAY NO. & 18 & 11 & 12 & 13 & 14 & 15 & 16 & 17 & 18 & 19 \\
\hline \multicolumn{11}{|l|}{ SYSTEM PGRAMETERS } \\
\hline *Sludue Rye & 30 & 30 & 30 & 30 & 30 & 30 & 36 & 30 & 30 & 38 \\
\hline$*(F / M) C O D$ & 0.89 & 0.87 & 0.05 & 0.06 & 0.06 & 0.07 & 0.05 & 0.83 & 0.07 & 0.08 \\
\hline *HRT & 2.2 & 2 & 2.2 & 2.2 & 2.4 & 2 & 2.4 & 2.4 & 2.3 & 2.3 \\
\hline FEED PRRAMETERS (BATCH \#) & 27 & 27 & 27 & 27 & 27 & 27 & 27 & 28 & 28 & 28 \\
\hline Volune (liters) & 3.2 & 3.5 & 3.2 & 3.25 & 2.95 & 3.55 & 2.95 & 2. 95 & 3.10 & 3.18 \\
\hline oH (std units) & $7.3 / 9.9$ & 7.7 & 7.5 & 7.4 & 7.2 & 7.06 & $9 / 9.1$ & $7.3 / 9.0$ & 7.8 & 7.2 \\
\hline $\operatorname{CCD}(\mathrm{mg} / \mathrm{l})$ & 182 & 184 & 188 & 181 & 181 & 155 & 166 & 119 & 161 & 184 \\
\hline NH3N $(m g / 1)$ & 5.8 & 3.5 & 3.5 & 3.5 & 3.5 & 6.3 & 6.3 & 6.3 & 9.4 & 9.4 \\
\hline \multicolumn{11}{|l|}{$\begin{array}{l}\text { SS }(\operatorname{mog} / 1) \\
\text { vSS }(\operatorname{mg} / 1)\end{array}$} \\
\hline CN (mg/l) & 1.32 & 1.32 & 1.32 & 1.32 & 1.32 & 1.806 & 1.806 & 1.806 & 1.006 & 1.006 \\
\hline $\begin{array}{l}\text { SCN (mg/1) } \\
\text { EOD 5-day (mg/1) }\end{array}$ & 10.3 & 10.3 & 10.3 & 10.3 & 18.3 & 7.4 & 7.4 & 7.4 & 7.4 & 7.4 \\
\hline TnR: $(\mathrm{mn} / !)$ & & & & & & 52 & 52 & 52 & 52 & 52 \\
\hline Phenolics (mo/l) & 1.025 & 1.025 & (. 825 & 1.025 & & 1.025 & & & & \\
\hline $\mathrm{PO}_{4}-\mathrm{P}$ (ing/1) & 1.3 & 1.3 & 1.3 & 1.3 & & 1.3 & & & & \\
\hline $\operatorname{Org}-\mathrm{N}(\mathrm{mg} / \mathrm{l})$ & & & 8.9 & 8.9 & & 8.9 & & & 5.7 & 5.7 \\
\hline 102 (mp/1) & 8.7 & 8.7 & 8.7 & 8.7 & & 8.7 & 17.6 & 17.6 & 17.6 & 17.6 \\
\hline $\mathrm{NO3}(\mathrm{nog} / \mathrm{l})$ & 158 & 158 & 150 & 150 & & 150 & 171 & 171 & 171 & 171 \\
\hline \multicolumn{11}{|l|}{ IUS (mg/1) } \\
\hline Color (APHA Units) & & & & & & & 250 & 258 & 250 & 250 \\
\hline \multicolumn{11}{|l|}{ BASIN PARAMETERS } \\
\hline Vol Hasted (ml) & 126 & 198 & 163 & 149 & 165 & 151 & 138 & 155 & 187 & 162 \\
\hline D. $0 . \quad(\mathrm{mg} / 1)$ & 7.5 & 7.3 & 7.3 & 7.4 & 7.2 & 7.7 & 8.2 & 7.5 & 8.8 & 8.8 \\
\hline SS $(\mathrm{mg} / 1)$ & 1450 & 1818 & 2120 & 1990 & 1790 & 1690 & 1938 & ટ220 & 1530 & 1448 \\
\hline *SS (PAC corrected) & 958 & 1310 & 1620 & 1490 & 1290 & 1198 & 1438 & 1720 & 1030 & 940 \\
\hline Temp (C) & 27 & 27 & 26 & 27 & 28 & 37 & 35 & 25.5 & 25 & 24 \\
\hline $\begin{array}{l}\text { pH (std units) } \\
\text { OUR (nq/1/hr) }\end{array}$ & 7.3 & 8 & 7.9 & 7.8 & 7.8 & 7.7 & $\begin{array}{r}7.3 \\
2\end{array}$ & 7.7 & 7.9 & 7.5 \\
\hline Vis (mg/l) & 1205 & 1903 & 1762 & 1560 & 1333 & 1329 & 1630 & 1875 & IEפL & ILIC \\
\hline $\begin{array}{l}\text { *VSS (PAC corrected) } \\
\text { USS/SS }\end{array}$ & 790 & 1089 & 1347 & $\begin{array}{l}1168 \\
0.78\end{array}$ & 1003 & 933 & $\begin{array}{l}1308 \\
0.84\end{array}$ & 1453 & 870 & 794 \\
\hline *PAC Added (mg) & 144 & 144 & 144 & 143 & 143 & 143 & 143 & 143 & 143 & 143 \\
\hline Inventory (mg/1 basin) & 500 & 580 & 500 & 500 & 500 & 580 & 500 & 500 & 580 & 580 \\
\hline \multicolumn{11}{|l|}{ EFFLUENT PARAMETERS } \\
\hline Volume (liters) & 2.79 & 3.10 & 2. 80 & 2.85 & 2.70 & 3.10 & 2.65 & 2.65 & 2.80 & 2.98 \\
\hline $\operatorname{cod}(\mathrm{mg} / 1)$ & 152 & 134 & 126 & 126 & 98 & 112 & 98 & 103 & 116 & 135 \\
\hline SS $(m g / 1)$ & 84 & 52 & 94 & 96 & 80 & 74 & 108 & 118 & 98 & 62 \\
\hline \multicolumn{11}{|l|}{ \#PAC corrected } \\
\hline $\begin{array}{l}\text { pH (sto units) } \\
\text { USS (ng/1) }\end{array}$ & 7.48 & B. 20 & 8.08 & $\begin{array}{r}7.90 \\
74\end{array}$ & 7.9 & 7.8 & $\begin{array}{r}7.3 \\
82\end{array}$ & 7.6 & 7.8 & 7.7 \\
\hline \multicolumn{11}{|l|}{ *PAC corrected } \\
\hline NH3N $(\mathrm{mg} / 1)$ & 1.2 & 1.2 & 1.2 & 1.2 & 0.6 & 0.6 & 0.6 & 0.9 & 0.9 & 0.9 \\
\hline CN (ogg/l) & 1.37 & 1.37 & 1.37 & 1.37 & 1.886 & 1.886 & 1.806 & 1.886 & 1.886 & 1.006 \\
\hline $\mathrm{SCN}$ (ag/1) & 4 & 4 & 4 & 4 & 4.8 & 4.8 & 4.8 & 4.8 & 4.8 & 4.8 \\
\hline NO2 (mg/l) & 3.4 & 3.4 & 3.4 & 3.4 & 1.9 & 1.9 & 1.9 & 1.9 & 1.9 & 1.9 \\
\hline NO3 (ng/l) & 176 & 176 & 176 & 176 & 194 & 194 & 194 & 194 & 194 & 194 \\
\hline Phenolics (mg/1) & 1.825 & <. 025 & 1.825 & 1.025 & & & & & & \\
\hline$P 04-P(m g / 1)$ & 0.9 & 0.9 & 0.9 & 0.9 & & & & & & \\
\hline $\begin{array}{l}\text { Org-N (mg/1) } \\
\text { TDS (mo/1) }\end{array}$ & 3.9 & 3.9 & 3.9 & \\
\hline Color (APHA units) & & & & & 1080 & 1080 & 1008 & 1080 & 1080 & 1880 \\
\hline $\begin{array}{l}\text { TOC (mg/1) } \\
\text { BOD 5-day (mg/1) }\end{array}$ & & & & & 46 & 46 & 46 & 46 & 46 & 46 \\
\hline
\end{tabular}


UNIT 18 TABLE A2

\begin{tabular}{|c|c|c|c|c|c|c|c|c|c|c|}
\hline MONTH & RUG & RUG & AU6 & AUG & RUG & AUG & AUG & AU6 & AUG & AU6 \\
\hline DAY & 15 & 16 & 17 & 18 & 19 & 20 & 21 & 22 & 23 & 24 \\
\hline DAY NO. & 20 & 21 & 22 & 23 & 24 & 25 & 26 & 27 & 28 & 29 \\
\hline SYSTEY PARAMETERS & & & & & & & & & & \\
\hline *Sludge Age & 30 & 30 & 30 & 30 & 30 & 30 & 30 & 30 & 30 & 30 \\
\hline$(F / M) C O D$ & 0.07 & 0.98 & 0.89 & 0.11 & 0.08 & 0.07 & 0.08 & 0.07 & 0.09 & 0.06 \\
\hline HHRT & 2 & 1.9 & 2 & 2.1 & 1.9 & 1.9 & 1.9 & 2.1 & 2.8 & 2.1 \\
\hline FEED PARAMETERS (BATCH \#) & 28 & 28 & 28 & 28 & 28 & 28 & 28 & 28 & 28 & 28 \\
\hline Volure (liters) & 3.59 & 3.65 & 3.46 & 3.36 & 3.70 & 3.70 & 3.70 & 3.33 & 3.52 & 3.48 \\
\hline $\mathrm{pH}$ (std units) & $7.3 / 8.8$ & 7.1 & 7.3 & 7.2 & 7.3 & 7.0 & \multicolumn{2}{|c|}{$7.17 .1 / 8.8$} & $6.9 / 9.1$ & $6.9 / 9.0$ \\
\hline $\operatorname{CSD}$ (mg/l) & 213 & 212 & 248 & 227 & 212 & 200 & 201 & 195 & 194 & 206 \\
\hline NH3N $(m g / 1)$ & 9.4 & 9.4 & 15.8 & 15.8 & 15.8 & 24.3 & 24.3 & 24.3 & 24.3 & 17.5 \\
\hline \multicolumn{11}{|l|}{$\begin{array}{l}\text { SS }(\mathrm{gg} / 1) \\
\text { YSS }(\mathrm{mg} / \mathrm{l})\end{array}$} \\
\hline $\mathrm{CN}(\mathrm{gg} / 1)$ & ২.006 & 1.006 & 3.02 & 3.02 & 3.02 & 3.02 & 3.82 & 3.02 & 3.02 & 3.0 .2 \\
\hline $\operatorname{SCN}(\operatorname{tg} / 1)$ & 7.4 & 7.4 & 6.3 & 6.3 & 6.3 & 6.3 & 6.3 & 6.3 & 6.3 & \\
\hline \multicolumn{11}{|l|}{ BOD 5-day $(m o / 1)$} \\
\hline TOC $(m g / 1)$ & 52 & 52 & & & & & & & & 63 \\
\hline Phenolics (Bg/l) & & & 1.025 & 1.025 & 1.025 & 1.025 & (.025 & 1.825 & 1.825 & 1.025 \\
\hline $\mathrm{PO4}-\mathrm{P}$ (mg/1) & & & 3.9 & 3.9 & 3.3 & 3.9 & 3.9 & 3.9 & 3.9 & \\
\hline Org-N $(a q / 1)$ & 5.7 & 5.7 & & & & 1.5 & 1.5 & 1.5 & 1.5 & \\
\hline NO2 (mg/l) & 17.6 & 17.6 & & & & & & & 22.7 & 13.7 \\
\hline No3 (mg/l) & 171 & 171 & & & & & & & 167 & 171 \\
\hline \multicolumn{11}{|l|}{ TDS $(\mathrm{mg} / 1)$} \\
\hline Color (APHA Units) & 250 & 250 & & & & & & & & 508 \\
\hline \multicolumn{11}{|l|}{ BASIN PARAMETERS } \\
\hline Vol Hasted (घl) & 117 & 113 & 91 & 145 & 166 & 195 & 223 & 168 & 144 & 215 \\
\hline D.0. (go/l) & 7.8 & 7.6 & 7.8 & 7.4 & 7.6 & 7.5 & 7.5 & 8.0 & 8.4 & 8.1 \\
\hline SS $(\mathrm{ag} / 1)$ & 2120 & 1858 & 1770 & 1400 & 1880 & 1840 & 1720 & 1750 & 1540 & 2150 \\
\hline \SS (PAC corrected) & 1620 & 1350 & 1270 & 900 & 1380 & 1340 & 1220 & $1 \hat{c} 50$ & 1040 & 1650 \\
\hline Temo (C) & 24 & 25 & 25.5 & 26 & 25 & 26 & 25 & 25 & 25 & 24 \\
\hline $\mathrm{pH}$ (std units) & 7.1 & 7.9 & 7.5 & 7.1 & 6.9 & 6.9 & 7.8 & 6.8 & 7.4 & 7.3 \\
\hline OUR (mg/l/hr) & 3 & & & 3 & & & & 2 & & $\therefore$ \\
\hline VSS (mo/1) & 1710 & 1492 & 1428 & 1220 & 1638 & 1603 & 1499 & 1538 & 1346 & 1880 \\
\hline *USS (PAC corrected) & 1307 & 1089 & 1024 & 784 & 1203 & 1168 & 1063 & 1093 & 909 & 1443 \\
\hline USS/SS & 0.81 & & & 0.87 & & & & 0.87 & & \\
\hline *PAC Added (ag) & 143 & 144 & 143 & 143 & 143 & 143 & 143 & 143 & 143 & 144 \\
\hline Inventory (ag/l basin) & 500 & 508 & 500 & 508 & 500 & 508 & 580 & 580 & 500 & 580 \\
\hline \multicolumn{11}{|l|}{ EFFLIJENT PARAMETERS } \\
\hline Volume (liters) & 3.18 & 3.50 & 3.20 & 3.10 & 3.43 & 3.25 & 3.23 & 2. 88 & 3.13 & 3.10 \\
\hline $\cos (\mathrm{mg} / 1)$ & 139 & 140 & 155 & 148 & 145 & 151 & 152 & 152 & 154 & 111 \\
\hline SS (mg/1) & 116 & 92 & 108 & 64 & 66 & 52 & 34 & 72 & 70 & 50 \\
\hline \multicolumn{11}{|l|}{ APAC corrected } \\
\hline pH (std units) & 7.3 & 7.6 & 7.8 & 7.7 & 7.0 & 7.8 & 6.9 & 6.9 & 7.1 & 7.1 \\
\hline USS $(a g / 1)$ & 78 & & & 48 & & & & 58 & & \\
\hline \multicolumn{11}{|l|}{ PAC corrected } \\
\hline NH3N $(n g / 1)$ & 0.9 & 1.5 & 1.5 & 1.5 & 7.6 & 7.6 & 7.6 & 7.6 & 8.9 & 8.9 \\
\hline CN $(a g / 1)$ & 1.006 & 2.18 & 2.18 & 2.18 & 2.18 & 2.18 & 2.18 & 2.18 & 0.373 & 8.373 \\
\hline SCN (bg/l) & 4.8 & & & & & & & & 4.2 & 4.2 \\
\hline NO2 (mg/1) & 1.9 & & & & & & & & 1.2 & 1.2 \\
\hline $\mathrm{ND3}(\mathrm{ag} / 1)$ & 194 & & & & & & & & 202 & 202 \\
\hline Phenolics (mo/l) & & 1.025 & 1.025 & 1.025 & 1.025 & ८.025 & ८.025 & 1.025 & & \\
\hline$P 04-P(\mathrm{ag} / 1)$ & & 4.2 & 4.2 & 4.2 & 4.2 & 4.2 & 4.2 & 4.2 & & \\
\hline \multicolumn{10}{|l|}{$\operatorname{TOS}(n g / 1)$} & \\
\hline Color (APHA units) & 1000 & & & & & & & & 758 & 758 \\
\hline TOC $(m g / I)$ & 46 & & & & & & & & 52 & 52 \\
\hline BOD 5-day (mog/1) & & & & -79 & & & & & & \\
\hline
\end{tabular}


UNIT IS TABLE A2

MONTH

DAY

DAY NO.

$\begin{array}{rrrrrrrrrr}\text { SEPT } & \text { SEPT } & \text { SEPT } & \text { SEPT } & \text { SEPT } & \text { SEPT } & \text { SEPT } & \text { SEPT } & \text { SEPT } & \text { SEPT } \\ 4 & 5 & 6 & 7 & 8 & 9 & 10 & 11 & 12 & 13 \\ 40 & 41 & 42 & 43 & 44 & 45 & 46 & 47 & 48 & 49\end{array}$

\section{SISTEM PARAMETERS}

*Sludge Age

$*(F / M) C O D$

*HRT

$\begin{array}{rrrr}30 & 30 & 30 & 30 \\ 0.05 & 0.07 & 0.04 & 0.06\end{array}$

30.1
0.07

19.6

26.8

17.2

0.07

0.09

42.5

0.87

44.1

$\begin{array}{llll}2.8 & 2.0 & 2.2 & 1.9\end{array}$

2.0

2.0

2.1

2.8

2.0

0.87

FEED PARGMETERS (BRTCH \#)

Volume (Iiters)

oH (std units)

$\operatorname{COD}(\mathrm{mg} / 1)$

NHIN (mog/l)

5S (mgo/l)

USS $(m 0 / 1)$

CN (mg/1)

SCN (mg/1)

$\begin{array}{llll}3.48 & 3.45 & 3.25 & 3.62\end{array}$

3.56

29
3.49

29

$29 \quad 29$

1.9

$7.1 / 8.9 \quad 7.8 / 9.8 \quad 6.8 / 7.8$

$7.9 \quad 7.3$

$3.49 \quad 3.38$

$3.44 \quad 3.44$

3.7

174

$\begin{array}{llll}12.5 & 12.5 & 12.5 & 13.3\end{array}$

174

186

$7 / 9.6$

$6 / 8 / 8.8 \quad 7.2 / 8.5$

7.5

13.3

13.3

9.8

9.8

9.8

158

BOD 5-day (mg/l)

TOC (mg/1)

Phenolics (mg/l)

P04-P (mg/1)

Org-N (mg/1)

Noe $(\mathrm{mg} / \mathrm{l})$

$\therefore 03(\mathrm{mg} / \mathrm{ll}$

TDS (mg/1)

Color (APHA Units)

BASIN FARAMETERS

Vol Wasted (al)

D. O. (ag/l)

SS (mg/1)

*SS (PAC corrected)

Teap (C)

PH (std units)

DUR $(\mathrm{mg} / \mathrm{l} / \mathrm{hr})$

USS $(m g / 1)$

* VSS (PAC corrected)

USS/SS

*PAC Added (mg)

Inventory (rag/l basin)

SFFLUENT PARAMETERS

Volume (Iiters)
COD (mg/l)
SS $(\mathrm{mg} / 1)$
IPAC corrected

$\mathrm{pH}$ (std units)

USS (mg/1)

*PAC corrected

NH3N $(m g / 1)$

CN (mg/l)

SCN (mg/l)

NO2 $(\operatorname{lng} / 1)$

NO3 (ng/1)

Phenolics (mg/l)

PO4-P (mg/1)

Org-N (ng/l)

TDS (og/l)

Color (9PHA units)

TOC (rgg/1)

$\begin{array}{lll}1.88 & 1.88 \quad 1.88\end{array}$

1.66

1.66

1.66

1.66

1.66

1.66

1.66

6.2

$1.025 \quad 1.825 \quad 10.025$

$\begin{array}{rrr}8.1 & 8.1 & 8.1 \\ 1.8 & 1.8 & 1.8 \\ 10.2 & 10.2 & 10.2 \\ 199 & 199 & 199\end{array}$

$$
63
$$

63

63

63

53

63

0.1

.8

0.2

$\begin{array}{lllll}2.3 & 2.3 & 2.3 & 2.3\end{array}$

$\begin{array}{lllllll}2.2 & 2.2 & 2.2 & 2.2 & 2.2 & 2.2 & 2.2\end{array}$

19.

$$
16
$$

167

7.7

172

$$
7.4
$$

7.4

1975

153

143

$$
143
$$

50

$\begin{array}{rrrrrrrrrr}3.10 & 3.12 & 2.85 & 3.15 & 3.89 & 3.89 & 2.90 & 3.35 & 3.30 & 3.52 \\ 84 & 98 & 118 & 128 & 127 & 138 & 132 & 129 & 112 & 188 \\ 90 & 32 & 2 & 152 & 72 & 222 & 182 & 214 & 104 & 94\end{array}$

$\begin{array}{llllllllll}7.5 & 7.6 & 7.4 & 8.0 & 8.3 & 7.7 & 7.3 & 7.7 & 8.0 & 7.9\end{array}$

$\begin{array}{llllllllll}0.8 & 0.8 & 0.3 & 0.3 & 0.3 & 0.3 & 0.3 & 0.3 & 0.3 & 10.1\end{array}$

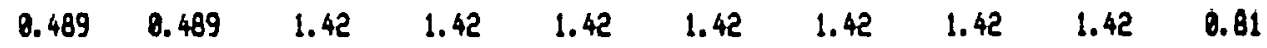

$\begin{array}{llllllllll}3.1 & 3.1 & 3.6 & 3.6 & 3.6 & 3.6 & 3.6 & 3.6 & 3.6 & 3.3\end{array}$

$\begin{array}{llllllllll}0.41 & 0.41 & 0.63 & 0.63 & 0.63 & 0.63 & 0.63 & 0.63 & 0.63 & 0.65\end{array}$

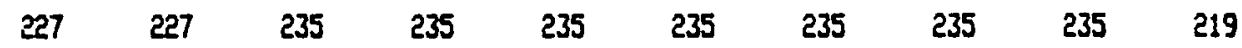

$\begin{array}{llll}1.025 & 1.025 & 10.025\end{array}$

$5.3 \quad 5.3$

$6.4 \quad 6.4$
7.4

$\begin{array}{rrrrrrrr}1250 & 1250 & 1250 & 1250 & 1250 & 1250 & 1250 & 1250 \\ 48 & 48 & 48 & 48 & 48 & 48 & 48 & 48\end{array}$

BOD 5-day (mg/1) 


\begin{tabular}{|c|c|c|c|c|c|c|c|c|c|c|}
\hline MONTH & SEPT & SEPT & SEPT & SEPT & SEPT & SEPT & SEPT & SEPT & SEPT & SEPT \\
\hline DAY & 14 & 15 & 16 & 17 & 18 & 19 & 20 & 21 & 22 & 23 \\
\hline DAY NO. & 58 & 51 & 52 & 53 & 54 & 55 & 56 & 57 & 58 & 59 \\
\hline \multicolumn{11}{|l|}{ SYSTEM FARAMETERS } \\
\hline *Sludge Age & 30.1 & 17.5 & 30 & 30 & 30 & 30 & 30 & 30 & 30 & 30 \\
\hline$+(F / M) C O D$ & 0.85 & 8.86 & 0.26 & 0.06 & 8.86 & 0.07 & 8.08 & 0.09 & 0.86 & 8.26 \\
\hline *HRT & 1.9 & 1.9 & 2.0 & 1.9 & 1.9 & 1.9 & 1.9 & 1.9 & 1.9 & 1.9 \\
\hline FEED PARAMETERS (BRTCH \#) & 30 & 30 & 30 & 30 & 30 & 30 & 30 & 30 & 30 & 30 \\
\hline Volure (liters) & 3.70 & 3.70 & 3.50 & 3.70 & 3.70 & 3.70 & 3.70 & 3.70 & 3.70 & 3.70 \\
\hline $\mathrm{DH}$ (std units) & 7.4 & $6.9 / 8.8$ & $6.7 / 9.3$ & 7.7 & 7.7 & 6.8 & 6.5 & 6.9 & $7.1 / 8.8$ & 7.3 \\
\hline $\cot (\mathrm{mg} / 1)$ & 150 & 148 & 156 & 159 & 156 & 156 & 188 & 148 & 148 & 112 \\
\hline NHZN $(a g / 1)$ & 0.9 & 0.9 & 0.9 & 1.7 & 1.7 & 1.7 & 1.7 & 1.7 & 1.7 & 1.7 \\
\hline $5 S(\mathrm{mg} / 1)$ & & & & & & & 26 & & & \\
\hline VSS (mo/l) & & & & & & & 己 & & & \\
\hline CN (ng/1) & 1.19 & 1.19 & 1.19 & 1.19 & 1.19 & 1.19 & 1.19 & & & \\
\hline $\operatorname{SCN}(n g / 1)$ & 5.1 & 5.1 & 5.1 & 5.1 & 5.1 & 5.1 & 5.1 & & & \\
\hline BOD 5-day (mg/1) & & & & & & & 48 & 40 & 40 & 40 \\
\hline TOC $(m g / 1)$ & & & & & & & 56 & 56 & 56 & 56 \\
\hline Phenolics (mg/s) & 10.025 & 10.025 & 30.025 & 10.025 & 10.025 & $>0.825$ & 10.025 & & & \\
\hline $\mathrm{PD4}-\mathrm{P}(\mathrm{mg} / 1)$ & 11.1 & 11.1 & 11.1 & 11.1 & 11.1 & 11.1 & 11.1 & & & \\
\hline $\operatorname{Org}-N(\mathrm{mg} / 1)$ & & & & 2.9 & 2.9 & 2.9 & 2.9 & & & \\
\hline $\operatorname{No2}(\mathrm{ag} / 1)$ & 4.04 & 4.04 & 4.04 & 4.04 & 4.84 & 4.84 & 4.84 & & & \\
\hline $\mathrm{NO3}(\mathrm{mg} / 1)$ & 195 & 195 & 195 & 195 & 195 & 195 & 195 & & & \\
\hline \multicolumn{11}{|l|}{$\operatorname{TDS}(\operatorname{mg} / 1)$} \\
\hline Color (APHA Units) & & & . & & & & & 758 & 750 & 750 \\
\hline \multicolumn{11}{|l|}{ BASIN PARAMETERS } \\
\hline Vol Hasted (nl) & 187 & 10 & 146 & 119 & 61 & 54 & 163 & 132 & 241 & 207 \\
\hline D.0. $(\operatorname{ag} / 1)$ & 8.2 & 8.8 & 8.7 & 8.6 & 8.1 & 7.5 & 7.6 & 7.6 & 7.8 & 7.8 \\
\hline SS $(n g / 1)$ & 2070 & 1710 & 1680 & 1820 & 1770 & 1580 & 1730 & 1330 & 1840 & 1428 \\
\hline *SS (PAC corrected) & 1570 & 1210 & 1180 & 1320 & 1270 & 1080 & 1230 & 830 & 1340 & 920 \\
\hline Fewp (C) & Es & 'בי & 23 & 23 & 24 & 23 & 24 & 25 & 24 & 23 \\
\hline $\mathrm{pH}$ (std units) & 7.7 & 7.4 & 8.8 & 8.3 & 8.1 & 7.8 & 7.4 & 7.2 & 7.1 & 8.1 \\
\hline OUR (ng/1/hr) & & 6 & & & & 2 & & & $?$ & \\
\hline USS (mg/1) & 1663 & 1470 & 1444 & 1565 & 1522 & 1369 & 1489 & 1145 & 1520 & 1173 \\
\hline *USS (PRC corrected) & 1261 & 1048 & 1014 & 1135 & 1092 & 989 & 1059 & 714 & 1107 & 760 \\
\hline USS/SS & & 0.86 & & & & & & & 0.83 & \\
\hline *PAC Added (mg) & 143 & 246 & 143 & 143 & 143 & 143 & 143 & 143 & 144 & 143 \\
\hline IInventory (ag/l basin) & 500 & 508 & 508 & 508 & 500 & 500 & 580 & 500 & 508 & 500 \\
\hline \multicolumn{11}{|l|}{ EFRLUENT PARAHLTERS } \\
\hline Volunte (Iiters) & 3.50 & 3.49 & 3.30 & 3.68 & 3.70 & 3.68 & 3.50 & 3. fh & 3.50 & 3.60 \\
\hline $\cos (n g / 1)$ & 112 & 115 & 120 & 120 & 113 & 143 & 97 & 108 & 90 & 92 \\
\hline SS $(m q / 1)$ & 106 & P.Th & 72 & 86 & 108 & 102 & 62 & 50 & 24 & 32 \\
\hline \multicolumn{11}{|l|}{ *PAC corrected } \\
\hline pH (std units) & 7.7 & 7.5 & 7.7 & 8.1 & 8. 1 & 8. 8 & 7.4 & 7.4 & 7.2 & 7.5 \\
\hline VSS (mg/l) & & 196 & & & & 88 & & & 16 & \\
\hline \multicolumn{11}{|l|}{ ¿PAC corrected } \\
\hline NH3N $(\operatorname{mg} / 1)$ & $<0.1$ & 10.1 & 10.1 & 10.1 & 18.1 & (0.1 & 1.2 & 1.2 & 1.2 & 1.4 \\
\hline$C N(n g / 1)$ & 0.81 & 0.81 & 0.81 & 0.81 & 0.81 & 0.81 & & & & \\
\hline SCN (ng/1) & 3.3 & 3.3 & 3.3 & 3.3 & 3.3 & 3.3 & & & & \\
\hline NO2 (6g $/ 1)$ & 0.65 & 8.65 & 8.65 & 8.65 & 8.65 & 8.65 & & & & \\
\hline N03 (rg/l) & 219 & 219 & 219 & 219 & 219 & 219 & & & & \\
\hline Phenolics (mg/l) & 18.025 & 10.025 & 10.025 & 10.025 & 18.025 & 18.825 & & & & \\
\hline$P 04-P(G g / 1)$ & 7.4 & 7.4 & 7.4 & 7.4 & 7.4 & 7.4 & & & & \\
\hline $\begin{array}{l}\text { Org-N (mg/l) } \\
\operatorname{TDS}(\mathrm{mg} / 1)\end{array}$ & & & 4.3 & 4.3 & 4.3 & 4.3 & & & & \\
\hline Color (APHA units) & & & & & & & 1080 & 1800 & 1000 & 1080 \\
\hline TOC $(m g / l)$ & & & & & & & 59 & 50 & 50 & 50 \\
\hline BOD 5-day (mg/1) & & & & -82 & & & 110 & 110 & 110 & 118 \\
\hline
\end{tabular}


LNIT IB TABLE A2

\begin{tabular}{|c|c|c|c|c|c|c|c|c|c|c|}
\hline MONTH & SEPT & SEPT & SEPT & SEPT & SEPT & SEPT & SEPT & OCT & UCT & OCT \\
\hline DRY & 24 & 25 & 26 & 27 & 28 & 29 & 30 & 1 & 2 & 3 \\
\hline DAY NO. & 60 & 61 & 62 & 63 & 64 & 65 & 66 & 67 & 68 & 69 \\
\hline \multicolumn{11}{|l|}{ SYSTEM PARAMETERS } \\
\hline *Sludge Age & 30 & 30 & 30 & 30 & 30 & 38 & 30 & 30 & 30 & 30 \\
\hline$*(F / M) C C D$ & ERR & 0.14 & 0.20 & 0.10 & 0.29 & 0.12 & 0.14 & 0.12 & 0.11 & 0.10 \\
\hline *HRT & 1.9 & 1.9 & 2.2 & 1.9 & 1.9 & 1.3 & 1.3 & 1.9 & 1.9 & 1.9 \\
\hline FEED PARAYYETERS (BATCH ) & 38 & 30 & 38 & 38 & $3 !$ & 31 & 31 & 31 & 31 & 31 \\
\hline Volume (1iters) & 3.78 & 3.70 & 3.26 & 3.7 & 3.7 & 3.7 & 3.7 & 3.7 & 3.7 & 3.7 \\
\hline oH (std units) & 7.5 & 6.3 & 7.0 & $7.1 / 8.9$ & 7.1 & 7.5 & 7.7 & 7.7 & 7.8 & 8.0 \\
\hline $\cos (m g / 1)$ & 112 & 179 & 166 & 181 & 174 & 168 & 187 & 201 & 198 & 171 \\
\hline NH3N $(m g / 1)$ & 5.2 & 5.2 & 5.2 & 5.2 & & & & & & \\
\hline SS $(m g / 1)$ & & & & 28 & & & & & & \\
\hline VSS (ogg/l) & & & & 16 & & & & & & \\
\hline \multicolumn{11}{|l|}{ CN (ng/l) } \\
\hline \multicolumn{11}{|l|}{$\operatorname{SCN}(00 / 1)$} \\
\hline EOD 5-day $(\operatorname{mg} / 1)$ & 40 & 40 & 48 & 40 & & & & & & \\
\hline TOC (mg/1) & 56 & 56 & 56 & 56 & & & & & & \\
\hline \multicolumn{11}{|l|}{ Phenolics (mg/1) } \\
\hline \multicolumn{11}{|l|}{ PO4-P (ng/1) } \\
\hline \multicolumn{11}{|l|}{ Jrg-N (mg/1) } \\
\hline \multicolumn{11}{|l|}{$102($ mog/1) } \\
\hline \multicolumn{11}{|l|}{ V03 (mg/1) } \\
\hline \multicolumn{11}{|l|}{ TDS (mg/1) } \\
\hline Color (APHA Units) & 750 & 750 & 750 & 750 & & & & & & \\
\hline \multicolumn{11}{|l|}{ BRSIN PARAMETERS } \\
\hline Vol Wasted (nl) & 10 & 238 & 186 & 246 & 198 & 216 & 96 & 88 & 143 & 198 \\
\hline $0.0 . \quad(\mathrm{mg} / \mathrm{l})$ & 7.8 & 7.8 & 8.1 & 8.6 & 8.4 & 8.6 & 8.8 & 8.8 & 8.1 & 8.3 \\
\hline SS $(n g / 1)$ & & 1178 & 890 & 1410 & 818 & 1200 & 1160 & 1310 & 1350 & 1358 \\
\hline ISS (PAC corrected) & & 678 & 390 & 910 & 310 & 700 & 660 & 810 & 850 & 850 \\
\hline Teap (C) & 21 & 21 & 21 & 21 & 22 & 21 & 21 & 21 & 20 & 22 \\
\hline $\mathrm{pH}$ (sto units) & 7.7 & 7.4 & 7.4 & 6.9 & 7.8 & 7.5 & 7.5 & 7.6 & 7.8 & 8.0 \\
\hline OUR (ag/l/hr) & & & & & & 2 & & & & 2 \\
\hline USS (mg/1) & & 967 & 730 & 1157 & 664 & 1048 & 1005 & 1135 & 1178 & 1150 \\
\hline NUSS (PAC corrected) & & 553 & 328 & 746 & 254 & 607 & 572 & 702 & 737 & 724 \\
\hline VSS/SS & & & 0.82 & & & 0.87 & & & & 0.85 \\
\hline APAC Added (⿴囗十) & 143 & 143 & 143 & 143 & 144 & 143 & 143 & 143 & 143 & 143 \\
\hline IInventory (mg/l basin) & 500 & 500 & 500 & 580 & 500 & 500 & 500 & 500 & 500 & 580 \\
\hline \multicolumn{11}{|l|}{ EFFLUENT PARAMETERS } \\
\hline Volume (liters) & 3.60 & 3.60 & 2.98 & 3.28 & 3.55 & 3.55 & 3.45 & 3.40 & 3.35 & 3.30 \\
\hline $\operatorname{coD}(m g / 1)$ & 151 & 153 & 164 & 153 & 148 & 153 & 155 & 152 & 158 & 153 \\
\hline SS $(m g / 1)$ & 12 & 16 & 30 & 18 & 22 & 24 & 64 & 76 & 58 & 36 \\
\hline \multicolumn{11}{|l|}{ *PAC corrected } \\
\hline pH (std units) & 7.8 & 7.6 & 7.4 & 7.2 & 7.4 & 7.7 & 7.4 & 7.4 & 7.7 & 7.8 \\
\hline VSS (mg/1) & & & 14 & & & 22 & & & & 34 \\
\hline \multicolumn{11}{|l|}{ APAC corrected } \\
\hline$N H 3 N(m g / 1)$ & 1.4 & 1.4 & 1.4 & 2.4 & 2.4 & 2.4 & 1.8 & 1.8 & 1.8 & 1.8 \\
\hline \multicolumn{11}{|l|}{ CN $(\operatorname{mg} / 1)$} \\
\hline SCN (mg/1) & & & & & & & & & & \\
\hline $\mathrm{NO2}(\mathrm{ag} / 1)$ & & & & & & & & & & \\
\hline N03 (mg/l) & & & & & & & & & & \\
\hline Thenolics (mg/J) & & & & 18.025 & $<0.025$ & 10.025 & 10.825 & 10.025 & 10.025 & 10.825 \\
\hline$P 04-D(m g / 1)$ & & & & 12.9 & 12.9 & 12.9 & 12.9 & 12.9 & 12.9 & 12.9 \\
\hline $\operatorname{Org}-\mathrm{N}(\mathrm{mg} / \mathrm{l})$ & & & & & & & & & & \\
\hline TDS (ag $/ 1)$ & & & & & & & & & & \\
\hline Color (APHA units) & 1808 & 1860 & 1008 & & & & & & & \\
\hline $\operatorname{TOC}(\mathrm{mg} / \mathrm{l})$ & 50 & 50 & 50 & 49 & 49 & 49 & 49 & 49 & 49 & 49 \\
\hline BOD 5-day $(\mathrm{mg} / \mathrm{l})$ & 110 & $(10$ & 118 & 5.4 & 5.4 & 5.4 & 5.4 & 5.4 & 5.4 & 5.4 \\
\hline & & & & $-83-$ & & & & & & \\
\hline
\end{tabular}


UNIT IB TABLE A2

\begin{tabular}{|c|c|c|c|c|c|c|c|c|c|c|}
\hline MONTH & OCT & OCT & OCT & OCT & OCT & OCT & OCT & OCT & OCT & OCT \\
\hline DAY & 4 & 5 & 6 & 7 & 8 & 9 & 10 & 11 & 12 & 13 \\
\hline DRY NO. & 70 & 71 & 72 & 73 & 74 & 75 & 76 & 77 & 78 & 79 \\
\hline \multicolumn{11}{|l|}{ SYSTEM FARAMETERS } \\
\hline +Sludge Ag̣e & 30 & 38 & 30 & 38 & 30 & 30 & 30 & 30 & 30 & 30 \\
\hline$F(F / M) C O D$ & 0.14 & 0.17 & 0.17 & 0.03 & 0.07 & 0.08 & 9.09 & 0.07 & 0.87 & 8.13 \\
\hline *HRT & 1.9 & 1.9 & 1.9 & 1.9 & 1.3 & 1.9 & 1.9 & 1.9 & 1.9 & 1.9 \\
\hline FEED PARAMETERS (BATCH \#) & 31 & 31 & 31 & 31 & 31 & 31 & 31 & 31 & 31 & 31 \\
\hline Volume (1iters) & 3.78 & 3.70 & 3.70 & 3.70 & 3.78 & 3.70 & 3.70 & 3.7 & 3.7 & 3.7 \\
\hline DH (std units) & $8.2 / 7.4$ & 8.2/6.4 & 8.2 & 7.8 & $8.1 / 6.7$ & 8.0 & 8.2 & 8.3 & 8.8 & 8.3 \\
\hline $\operatorname{coD}(\mathrm{mg} / 1)$ & 169 & 157 & 148 & 145 & 149 & 133 & 141 & 116 & 127 & 137 \\
\hline NH3N (mg/l) & 5.2 & 1.8 & 1.8 & 1.8 & 2.9 & 2.9 & 2.9 & 2.9 & 3.5 & 3.5 \\
\hline SS $(\mathrm{mg} / \mathrm{l})$ & 24 & & & 40 & & & & 48 & & \\
\hline VSS (mg/l) & 24 & & & 34 & & & & 42 & & \\
\hline \multicolumn{11}{|l|}{ CN $(m g / 1)$} \\
\hline \multicolumn{11}{|l|}{ SCN ( $\pi \mathrm{gg} / 1)$} \\
\hline 900 5-day $(\mathrm{mg} / \mathrm{l})$ & 9 & ८3.4 & 〈3.4 & 13.4 & 13.4 & 13.4 & 13.4 & 13.4 & 1.8 & 1.8 \\
\hline$T O C(\operatorname{mg} / 1)$ & 49 & 39 & 39 & 39 & 39 & 39 & 39 & 39 & 36 & 36 \\
\hline Phenolics $(\mathrm{mg} / 1)$ & (0.825 & & & & & & & & & \\
\hline PC4-P (mg/1) & 12.3 & & & & & & & & & \\
\hline \multicolumn{11}{|l|}{ Org $g-N(\operatorname{sig} / 1)$} \\
\hline \multicolumn{11}{|l|}{ :02 (ntg/1) } \\
\hline \multirow{2}{*}{\multicolumn{11}{|c|}{$\begin{array}{l}: 103 \text { (kng/l) } \\
\text { TDS (ng/l) }\end{array}$}} \\
\hline & & & & & & & & & & \\
\hline Color (APHA Units) & & 625 & 625 & 625 & 625 & 625 & 625 & 625 & & \\
\hline \multicolumn{11}{|l|}{ BASIN PARAMETERS } \\
\hline Vol Wasted (al) & 113 & 146 & 179 & 234 & 209 & 140 & 226 & 226 & 248 & 183 \\
\hline D.0. $(20 / 1)$ & 8.0 & 8.0 & 8.1 & 8.8 & 7.8 & 7.8 & 8.0 & 8.2 & 8.0 & 8.0 \\
\hline SS (ag/l) & 1138 & 968 & 920 & 1338 & 1628 & 1330 & 1350 & 1390 & 1470 & 1850 \\
\hline *SS (PAC corrected) & 630 & 460 & 420 & 830 & 1120 & 830 & 850 & 890 & 970 & 560 \\
\hline (emö (L) & 24 & 24 & 23 & $\not 2$ & 20 & 22 & 21 & 22 & 23 & 23 \\
\hline pH (std units) & 8.2 & 8.2 & 8.0 & 7.9 & 8.1 & 8.0 & 8.2 & 8.2 & 8.2 & 8.3 \\
\hline OUR (mg/1/hr) & & & ᄅ & & & & 2 & & & 2 \\
\hline VSS (ag/l) & 363 & 818 & 7AQ & 1128 & 1373 & 1128 & 1110 & 1143 & 1289 & 850 \\
\hline *VSS (PAC corrected) & 537 & 392 & 356 & 704 & 958 & 704 & 699 & 732 & 798 & 449 \\
\hline USS/SS & & & 0.85 & & & & 0.82 & & & 0.80 \\
\hline *PAC Added (mg) & 143 & 143 & 144 & 143 & 143 & $\$ 43$ & 143 & 143 & 143 & 143 \\
\hline * Inventory (mg/l basin) & 500 & 500 & 580 & 500 & 500 & 500 & 500 & 560 & 500 & 500 \\
\hline \multicolumn{11}{|l|}{ EFFLUENT PARAMETERS } \\
\hline Voluwe (liters) & 3.50 & 3.55 & 3.59 & 3.50 & 3.50 & 3.25 & 3.73 & 3.58 & 3.54 & 3.65 \\
\hline $\operatorname{COD}(\operatorname{mg} / 1)$ & 144 & 134 & 130 & 124 & 131 & 131 & 129 & 110 & 123 & 128 \\
\hline SS (ng/1) & 56 & 38 & 28 & 20 & 36 & 60 & 22 & 24 & 16 & 30 \\
\hline \multicolumn{11}{|l|}{ APAC corrected } \\
\hline PH (std units) & 8.1 & 8.1 & 8.8 & 8.0 & 8.0 & 8.0 & 8.2 & 8.2 & 8.2 & 8.3 \\
\hline VSS (mg/1) & & & 26 & & & & $2 n$ & & & 28 \\
\hline \multicolumn{11}{|l|}{ APAC corrected } \\
\hline NHJN (ng/1) & 1.5 & 1.5 & 1.5 & 1.3 & 1.3 & 1.3 & 1.3 & 0.9 & 0.9 & 0.9 \\
\hline \multicolumn{11}{|l|}{ CN (mg/I) } \\
\hline \multicolumn{11}{|l|}{$\operatorname{SCN}(\mathrm{mg} / 1)$} \\
\hline \multicolumn{11}{|l|}{$\begin{array}{l}\mathrm{NO2}(\mathrm{mg} / 1) \\
\mathrm{NO3}(\mathrm{mg} / 1)\end{array}$} \\
\hline $\mathrm{NO3}(\mathrm{ng} / 1)$ & & & & & & & & & & \\
\hline \multicolumn{11}{|l|}{ Phenolics $(\mathrm{mg} / \mathrm{l})$} \\
\hline PO4-P (mg/1) & & & & & & & & 1.9 & 1.9 & 1.9 \\
\hline Org $-\mathrm{N}(\operatorname{mg} / \mathrm{l})$ & & & & & & & & & & \\
\hline TDS (ng/1) & & & & & & & & & & \\
\hline Color (APHA units) & 750 & 750 & 750 & 750 & 750 & 750 & 750 & & & \\
\hline TOC (هog/1) & 37 & 37 & 37 & 37 & 37 & 37 & 37 & 31 & 31 & 31 \\
\hline 800 5-day (mg/1) & 22.6 & (2.6 & ८2.6 & ८2.6 & «2.6 & 12.6 & $\langle 2.6$ & 41.8 & $\langle 1.8$ & $\$ 1.8$ \\
\hline & & & & & & & & & & \\
\hline
\end{tabular}




\begin{tabular}{|c|c|c|c|c|c|c|c|c|c|c|}
\hline MONTH & OCT & OCT & OCT & OCT & OCT & OCT & OCT & OCT & UCT & OCT \\
\hline DAY & 14 & 15 & 16 & 17 & 18 & 19 & 28 & 21 & 22 & 23 \\
\hline DAY NO. & 88 & 81 & 82 & 83 & 84 & 85 & 86 & 87 & 88 & 89 \\
\hline \multicolumn{11}{|l|}{ SYSTEM FARRMETERS } \\
\hline \&Sludge Age & 30 & 30 & 30 & 30 & 30.84 & 30.00 & 26.55 & 30.30 & 30.08 & 30.80 \\
\hline$+(F / M) C O D$ & 0.07 & 0.11 & 0.86 & 0.09 & 0.00 & 0.18 & 0.08 & 0.07 & 0.07 & 0.89 \\
\hline *HRT & 1.9 & 1.3 & 2.2 & 1.9 & 1.89 & 1.38 & 1.89 & 1.89 & 1.89 & 1.95 \\
\hline FEED PARAMETERS (BATCH \#) & 31 & 31 & 31 & 31 & & & & & & \\
\hline Voluxe (liters) & 3.7 & 3.7 & 3.2 & 3.7 & 3.7 & 3.53 & 3.7 & 3.7 & 3.7 & 3.59 \\
\hline OH (std units) & 8.0 & 8.1 & 8.3 & $8.4 / 5.8$ & 8.3 & 8.1 & 8.3 & 8.3 & 8.2 & 7.9 \\
\hline $\operatorname{COD}(\operatorname{ag} / 1)$ & 146 & 148 & 144 & 152 & & 156 & 139 & 124 & 135 & 134 \\
\hline NHoN $(m g / 1)$ & 3.5 & 8.7 & 8.7 & 8.7 & & 8.9 & 8.9 & 8.9 & 3.8 & 3.8 \\
\hline SS (mg/1) & & & & 28 & & 50 & 46 & 44 & 180 & 42 \\
\hline VSS (mg/1) & & & & 26 & & 34 & 34 & 34 & 34 & 34 \\
\hline \multirow{2}{*}{\multicolumn{11}{|c|}{$\begin{array}{l}\text { CN }(\pi g / 1) \\
\operatorname{sen}(\pi 0 / 1)\end{array}$}} \\
\hline \multicolumn{9}{|l|}{$\operatorname{SCN}(\pi g / 1)$} & & \\
\hline 800 5-day (mg/l) & 1.8 & 1.8 & 1.8 & 1.8 & & & & & & \\
\hline TOC $(m g / 1)$ & 36 & 36 & 36 & 36 & & & & & & \\
\hline \multicolumn{11}{|l|}{ Phenolics (mg/1) } \\
\hline \multicolumn{11}{|l|}{ PJ4-P (mg/1) } \\
\hline \multicolumn{11}{|l|}{$\operatorname{Org}-N(a 1 g / 1)$} \\
\hline \multicolumn{11}{|l|}{ Yo2 $(\mathrm{mg} / 1)$} \\
\hline \multicolumn{11}{|l|}{$: N 03(m g / 1)$} \\
\hline \multicolumn{11}{|l|}{ TOS (mg/l) } \\
\hline \multirow{2}{*}{\multicolumn{11}{|c|}{$\begin{array}{l}\text { Color (APHA Units) } \\
\text { BASIN PARAMETERS }\end{array}$}} \\
\hline & & & & & & & & & & \\
\hline Vol Wasted (ml) & 252 & 245 & 225 & 255 & 246 & 262 & 225 & 199 & 109 & 42 \\
\hline $0.0 .(\mathrm{mg} / 1)$ & 8.2 & 8.2 & 8.8 & 8.0 & 8 & 8.3 & 9.5 & 8.4 & 8.4 & 8.2 \\
\hline S5 (mg/1) & 1570 & 1240 & 1660 & 1358 & 1430 & 1360 & 1430 & 1500 & 1610 & 1428 \\
\hline *SS (PAC corrected) & 1078 & 740 & 1160 & 850 & 930 & 860 & 930 & 1802 & 1112 & 922 \\
\hline Temp (C) & 22 & 21 & 21 & 22 & 22 & 22 & 28 & 20 & 18 & 20 \\
\hline pH (std units) & 8.3. & 8.3 & 8.3 & 8.4 & 8.2 & 8.1 & 8.3 & 8.4 & 8.1 & 7.8 \\
\hline CUR (mg/1/hr) & & & & 2 & & & 4 & & & \\
\hline VSS (mg/1) & 1259 & 994 & 1331 & 1130 & 1212 & 1153 & 1160 & 1217 & 1306 & 1152 \\
\hline IVSS (PAC corrected) & 858 & 593 & 930 & 711 & 1212 & 653 & 660 & 719 & 808 & 654 \\
\hline VSS/SS & & & & 0.84 & 0.85 & 0.85 & 0.81 & 0.81 & 0.81 & 0.81 \\
\hline *PAC Added (mg) & 143 & 143 & 143 & 143 & 143 & 143 & 143 & 143 & 143 & 143 \\
\hline *Inventory (mo/l basin) & 580 & 500 & 580 & 500 & 560 & 500 & 500 & 498 & 498 & 498 \\
\hline \multicolumn{11}{|l|}{ EFFLUENT PARAMETERS } \\
\hline Volume (liters) & 3.45 & 3.68 & 3.00 & 3.59 & 3.6 & 3.35 & 3.69 & 3.45 & 3.4 & 3.4 \\
\hline $\operatorname{COD}(\operatorname{mg} / 1)$ & 130 & 138 & 134 & 134 & 124 & 128 & 122 & 116 & 120 & 109 \\
\hline SS (mg/11) & 16 & 14 & 34 & 12 & 16 & 10 & 24 & 38 & 84 & 102 \\
\hline \multicolumn{11}{|l|}{ *PAC corrected } \\
\hline OH (std units) & 8.3 & 8.4 & 8.3 & 8.3 & 8.3 & 8.2 & 8.3 & 8.4 & 8.2 & . \\
\hline USS (ng/l) & & & & 10 & 26 & 26 & 18 & 18 & 18 & 18 \\
\hline \multicolumn{11}{|l|}{ «PAC corrected } \\
\hline NH.3N (mg/1) & 1.2 & 1.2 & 1.2 & 1.2 & 3.4 & 3.4 & 3.4 & 0.6 & 0.6 & 0.6 \\
\hline \multicolumn{11}{|l|}{ CN (mg/1) } \\
\hline SCN (ag/1) & & & & & & & & & & \\
\hline NO2 $(-g / 1)$ & & & & & & & & & & \\
\hline $\mathrm{NDO3}(\mathrm{ng} / \mathrm{ll})$ & & & & & & & & & & \\
\hline Phenolics (ng/l) & & & & & & & & & & \\
\hline $\mathrm{PO4}-\mathrm{P}(\mathrm{mg} / 1)$ & 1.9 & 1.9 & 1.9 & 1.9 & & & & & & \\
\hline $\operatorname{Org}-\mathrm{N}(\mathrm{mg} / \mathrm{l})$ & & & & & & & & & & \\
\hline TDS (四/1) & & & & & & & & & & \\
\hline Color (APHA units) & & & & & 580 & 500 & 580 & 580 & 580 & 580 \\
\hline $\operatorname{TOC}(m g / 1)$ & 31 & 31 & 31 & 31 & 34 & 34 & 34 & 34 & 34 & 34 \\
\hline BOD 5-day (mg/l) & 11.8 & 11.8 & 11.8 & 11.8 & 0.9 & 8.9 & 0.9 & 0.9 & 0.9 & 0.9 \\
\hline & & & & 35 & & & & & & \\
\hline
\end{tabular}




\begin{tabular}{|c|c|c|c|c|c|c|c|c|c|c|}
\hline MONTH & OCT & OCT & OCT & OCT & OCT & OCT & OCT & OCT & NOV & NOV \\
\hline DAY & 24 & 25 & 26 & 27 & 28 & 29 & 30 & 31 & 1 & 2 \\
\hline DAY NO. & 90 & 91 & 92 & 93 & 94 & 95 & 96 & 97 & 98 & 99 \\
\hline \multicolumn{11}{|l|}{ SYSTEM PARAMETERS } \\
\hline *Sludge Age & 30.88 & 29.93 & 29.97 & 29.36 & 29.36 & 23.39 & 29.35 & 29.38 & 38.67 & 29.35 \\
\hline$*(F / M) C C D$ & 0.27 & 0.85 & 0.08 & 0.85 & 0.12 & 0.10 & 0.05 & 0.07 & 0.07 & 0.07 \\
\hline *HRT & 1.89 & 1.89 & 1.89 & 1.89 & 1.89 & 1.89 & 1.99 & 1.89 & 1.95 & 1.89 \\
\hline \multicolumn{11}{|l|}{ FEED PARAMETERS (BATCH *) } \\
\hline Volume (liters) & 3.7 & 3.7 & 3.7 & 3.7 & 3.7 & 3.7 & $3.5 !$ & 3.7 & 3.59 & 3.7 \\
\hline ôH (std units) & 8.1 & 8.1 & 8 & 8.3 & 8.3 & 8 & 8 & 7.6 & 7.5 & 7.3 \\
\hline $\cos (\operatorname{mg} / 1)$ & 126 & 128 & 122 & 113 & 122 & 121 & 128 & 140 & 145 & 144 \\
\hline NHi3N (mg/1) & 3.8 & 3.8 & 3.7 & 3.7 & 3.7 & 3.9 & 3.9 & 3.9 & & 10.3 \\
\hline SS (mg/l) & 42 & 158 & 36 & 24 & 46 & 48 & 36 & 36 & & 26 \\
\hline VSS (mg/1) & 34 & 134 & 134 & 134 & 34 & 34 & 34 & 34 & & 22 \\
\hline CN (ag/l) & & & & & & & & & & 2.25 \\
\hline ECN $(\mathrm{mgg} / \mathrm{ll})$ & & & & & & & & & & 6.3 \\
\hline BOD 5-day (mg/1) & & & & & & & & & & 6.4 \\
\hline TOC $(\pi g / 1)$ & & 610 & 610 & 610 & 610 & 610 & 610 & 610 & & 44 \\
\hline Phenolics (mg/l) & & 0.483 & 0.483 & 0.403 & 0.403 & 0.403 & 0.403 & 0.403 & & 10.025 \\
\hline$p 04-p(a g / 1)$ & & & & & & & & & & 29.9 \\
\hline $\operatorname{Org}-\mathrm{N}(\mathrm{mg} / \mathrm{l})$ & & & & & & & & & & 14 \\
\hline No2 img/1) & & & & & & & & & & 3.26 \\
\hline $\operatorname{NDO3}(\mathrm{mg} / 1)$ & & & & & & & & & & 146 \\
\hline \multicolumn{11}{|l|}{ TDS (mg/1) } \\
\hline Color (APitA Units) & & & & & & & & & & 875 \\
\hline \multicolumn{11}{|l|}{ BRSIN PARAMETERS } \\
\hline Vol Hasted (m1) & 125 & 177 & 142 & 189 & 125 & 145 & 189 & 231 & 186 & 185 \\
\hline $0.0 .(\mathrm{gg} / \mathrm{l})$ & 0.6 & 0.6 & 8.6 & 8.8 & 8.8 & 8.8 & 8.8 & 8.7 & 8.2 & 9.1 \\
\hline SS (ag $/ 1)$ & 1540 & 1760 & 1420 & 1750 & 1160 & 1240 & 1880 & 1630 & 1560 & 850 \\
\hline *SS (PAC corrected) & 1042 & 1262 & 922 & 1252 & 662 & 742 & 1382 & 1132 & 1062 & 352 \\
\hline Tenp (C) & 19 & 19 & 18 & 18 & 10 & 19 & 18 & 18 & 18 & 18 \\
\hline $\mathrm{pH}$ (std units) & 0.3 & 8.4 & 8.2 & 8.3 & 8.3 & 8.3 & 8.4 & 8.1 & 8.1 & 7.8 \\
\hline CUR $(m g / 1 / h r)$ & 4 & & & 2 & & & & 2 & & \\
\hline VSS (mg/1) & 1268 & 1440 & 1162 & 1430 & 948 & 1013 & 1536 & 1380 & 1380 & 1380 \\
\hline WSS (PAC corrected) & 762 & 942 & 664 & 932 & 450 & 515 & 1038 & 882 & 882 & 892 \\
\hline VS9/S8 & 0.82 & 0.82 & 9.82 & 8.82 & 0.82 & 0.82 & 0.82 & 0.85 & 0.85 & 0.85 \\
\hline FFAC Added (ng) & 143 & 143 & 143 & 143 & 143 & 143 & 143 & 143 & 143 & 143 \\
\hline +Inventory (mg/1 basin) & 498 & 498 & 498 & 498 & 498 & 498 & 498 & 498 & 498 & 498 \\
\hline \multicolumn{11}{|l|}{ EFFLUENT PARAMETERS } \\
\hline Volume (Iiters) & 3.45 & 3.45 & 3.43 & 3.3 & 3.52 & 3.38 & 3.15 & 3.5 & 3.56 & 3.5 \\
\hline $\operatorname{coD}(\mathrm{mg} / \mathrm{l})$ & 111 & 113 & $\$ 17$ & 113 & $1 \mathbb{6}^{\mathrm{j}}$ & 111 & 113 & $11 \overline{3}$ & 123 & iis \\
\hline SS (mg/1) & 72 & 56 & 68 & 52 & 60 & 52 & 58 & 26 & 44 & 48 \\
\hline \multicolumn{11}{|l|}{ \#PAC corrected } \\
\hline pH (std units) & 8.1 & 8.5 & 8.25 & 8.4 & 8.4 & 8.3 & 8.3 & 8.2 & 8.1 & 8.0 \\
\hline .VSS (mg/1) & 46 & 46 & 46 & 34 & 34 & 34 & 34 & 24 & 24 & 24 \\
\hline \multicolumn{11}{|l|}{ *PAC corrected } \\
\hline NH3N (mg/1) & 0.6 & 0.3 & 0.3 & 0.3 & 0.3 & 0.3 & 0.3 & 8.3 & 14.0 & $<4.0$ \\
\hline CN $(\pi g /])$ & & & & & & & & & 0.65 & 0.65 \\
\hline $\operatorname{SCN}(\operatorname{mg} / 1)$ & & & & & & & & & 2.7 & 2.7 \\
\hline ND2 (mg/1) & & & & & & & & & 0.19 & 0.19 \\
\hline $\mathrm{NO3}$ (ng/1) & & & & & & & & & 179 & 179 \\
\hline Fhenolics (mg/1) & & 10.025 & 10.025 & 10.025 & 10.025 & 10.025 & 10.025 & 10.025 & (0.025 & 10.025 \\
\hline$P O 4-P(n g / l)$ & & 2.5 & 2.5 & 2.5 & 2.5 & 2.5 & 2.5 & 2.5 & 8.46 & 8.46 \\
\hline $\operatorname{Org}-N(\omega g / 1)$ & & & & & & & & & 14.8 & 14.0 \\
\hline \multicolumn{11}{|l|}{ TDS (ng/l) } \\
\hline Color (APHA units) & 508 & & & & & & & & 750 & 750 \\
\hline TOC $(m g / 1)$ & 34 & 31 & 31 & 31 & 31 & 31 & 31 & 31 & 35 & 35 \\
\hline 800 5-day (mg/1) & 0.9 & 0.8 & 0.8 & 8.8 & 0.8 & 0.8 & 0.8 & 8.8 & 2.5 & 2.5 \\
\hline & & & & -86 & & & & & & \\
\hline
\end{tabular}


UNIT 18 TABLE RC

\begin{tabular}{|c|c|c|c|c|c|c|c|c|c|c|}
\hline MCNTH & NOV & NOV & NOV & NOV & NOV & NOV & NOV & NOV & NOV & NOV \\
\hline DAY & 3 & 4 & 5 & 6 & 7 & 8 & 9 & 10 & 11 & 12 \\
\hline DAY NO. & 180 & 101 & 102 & 103 & 104 & 105 & 106 & 107 & 108 & 109 \\
\hline \multicolumn{11}{|l|}{ SYSTEM PARAMETERS } \\
\hline *Sludge Agge & 30.00 & 23.76 & 29.96 & 29.98 & 38.02 & 29.95 & 30.36 & 29.96 & 29.95 & 30.01 \\
\hline$F(F / M) C O D$ & 0.43 & 0.44 & 0.44 & 8.09 & 0.08 & 0.11 & 0.10 & 0.19 & 0.32 & 0.16 \\
\hline *HRT & 1.89 & 1.89 & 1.89 & 1.89 & 1.89 & 1.89 & 2.00 & 2.83 & 2.86 & 1.92 \\
\hline \multicolumn{11}{|l|}{ FEED FARAMETERS (BATCH \$) } \\
\hline Volune (1iters) & 3.7 & 3.7 & 3.7 & 3.7 & 3.7 & 3.7 & 3.5 & 3.45 & 3.4 & 3.64 \\
\hline$\rho H$ (std units) & 6.9 & 6.8 & 6.8 & 8.2 & 8.2 & 8.5 & 8.8 & 8 & 7.5 & 7.5 \\
\hline $\cos (\mathrm{mg} / 1)$ & 142 & 144 & 150 & 171 & 167 & 233 & 248 & 284 & 308 & 257 \\
\hline NH3N $(\mathrm{mg} / 1)$ & 10.3 & 10.3 & 48.2 & 40.2 & 40.2 & 40.2 & 92.4 & 92.4 & 92.4 & 47.1 \\
\hline SS $(\operatorname{mg} / 1)$ & 58 & 32 & 590 & 28 & 74 & 46 & 58 & 50 & 34 & 18 \\
\hline VSS $(\mathrm{gg} / 1)$ & 22 & 24 & 24 & 24 & 24 & 38 & 38 & 38 & 38 & 38 \\
\hline CN (mg/l) & 2. 25 & 2.25 & 2.25 & 2.25 & 2. 25 & 2.25 & & & & \\
\hline $\operatorname{SCN}(m g / 1)$ & 6.3 & 6.3 & 6.3 & 6.3 & 6.3 & 6.3 & & & & \\
\hline BOD 5-day (mg/1) & 6.4 & 6.4 & 6.9 & 6.9 & 6.9 & 6.9 & 6.6 & 6.6 & 6.6 & 3.8 \\
\hline TOC (mg/1) & 44 & 44 & 48 & 48 & 48 & 48 & 53 & 53 & 53 & 53 \\
\hline Phenolics (mg/1) & 10.025 & 18.025 & 10.025 & 18.025 & 10.025 & 18.025 & & & & \\
\hline $\mathrm{PO4}-\mathrm{P}(\mathrm{mg} / \mathrm{l})$ & 29.9 & 29.9 & 21.5 & 21.5 & 21.5 & 21.5 & 3.6 & 3.6 & 3.6 & 10.8 \\
\hline $\operatorname{Org}-N(n g / 1)$ & 14 & $\langle 4$ & $\langle 4$ & $\ll 4$ & 14 & 14 & & & & \\
\hline NO2 (ag/l) & 3.26 & 3.26 & 3.26 & 3.26 & 3.26 & 3.26 & & & & \\
\hline $\mathrm{N03}(\mathrm{mg} / !)$ & 146 & 146 & 146 & 146 & 146 & 146 & & & & \\
\hline \multicolumn{11}{|l|}{$\operatorname{TDS}(\mathrm{mg} / 1)$} \\
\hline Color (APHA Units) & 875 & 875 & 875 & 875 & 875 & 875 & 875 & 875 & 875 & 875 \\
\hline \multicolumn{11}{|l|}{ BASIN PARAMETERS } \\
\hline Vol Wasted (田) & 108 & 10 & 225 & 65 & 180 & 216 & 223 & 220 & 208 & 223 \\
\hline D.0. $(\mathrm{gg} / 1)$ & 8.8 & 9.1 & 9.2 & 8.3 & 9.6 & 9.5 & 9.4 & 9.2 & 9.2 & 9.2 \\
\hline SS $(\operatorname{ag} / 1)$ & 750 & 540 & 1780 & 1540 & 1620 & 1630 & 1800 & 1260 & 1080 & 1378 \\
\hline ISS (PAC corrected) & 252 & 42 & 1286 & 1846 & 1126 & 1136 & 1305 & 765 & 505 & 875 \\
\hline Temp (C) & 18.5 & 18.5 & 18 & 18 & 17.5 & 18 & 18 & 18.5 & 18 & 18 \\
\hline pH (std units) & 8 & 7.4 & 6.7 & 6.6 & 8.1 & 8.4 & 7.9 & 7.9 & 7 & 6.8 \\
\hline OUR (mg/1/hr) & 0.25 & 0.25 & & & 3.4 & & & 4.8 & & \\
\hline VSS $(m g / 1)$ & 640 & 640 & 648 & 1314 & 1370 & 1378 & 1522 & 1100 & 873 & 1196 \\
\hline *VSS (PAC corrected) & 142 & 142 & 146 & 820 & 876 & 884 & 1028 & 605 & 378 & 701 \\
\hline USS/SS & 0.85 & 0.85 & 0.85 & 0.85 & 0.85 & 0.85 & 0.85 & 0.87 & 0.87 & 0.87 \\
\hline tFAC Added (mg) & 143 & 143 & 143 & 143 & 143 & 143 & 143 & 143 & 143 & 143 \\
\hline Inventory (mg/l basin) & 498 & 498 & 494 & 494 & 494 & 494 & 495 & 495 & 495 & 495 \\
\hline \multicolumn{11}{|l|}{ EFFLUJENT PARAMETERS } \\
\hline Volume (liters) & 3.35 & 3.52 & 3.45 & 3.35 & 3.45 & 3.41 & 3.35 & 3.25 & 3.35 & 3.35 \\
\hline $\operatorname{COD}(\operatorname{ag} / 1)$ & 115 & 111 & 121 & 142 & 130 & 155 & 170 & 192 & 170 & 180 \\
\hline SS $(m g / 1)$ & 40 & 54 & 32 & 102 & 50 & .34 & 34 & 26 & 26 & 26 \\
\hline \multicolumn{11}{|l|}{ \&PAC corrected } \\
\hline pH (std units) & 7.7 & 7.3 & 6.9 & 6.6 & 7.0 & 8.8 & 8.1 & 7.7 & 7.4 & 6.7 \\
\hline VSS (mg/l) & 26 & 26 & 26 & 26 & 32 & 32 & 32 & 32 & 32 & 32 \\
\hline \multicolumn{11}{|l|}{ EPAC corrected } \\
\hline NA3N (ag/1) & 14.0 & 4.4 & 4.4 & 4.4 & 4.4 & 38.2 & 38.2 & 38.2 & 50.5 & 58.5 \\
\hline$C N(\pi g / 1)$ & 0.65 & 0.65 & 0.65 & 0.65 & 0.65 & & & & & \\
\hline $\operatorname{SCH}(\operatorname{mg} / 1)$ & 2.7 & 2.7 & 2.7 & 2.7 & 2.7 & & & & & \\
\hline No2 (ag/l) & 0.19 & 0.19 & 0.19 & 0.19 & 0.19 & & & & & \\
\hline $\mathrm{N03}(\mathrm{mg} / 1)$ & 179 & 179 & 179 & 179 & 179 & & & & & \\
\hline Phenolics $(a g / 1)$ & 10.025 & 10.025 & 10.025 & 10.025 & 10. 025 & $\cdot$ & & & & \\
\hline POA-P (mg/l) & 8.46 & 26.8 & 26.8 & 26.8 & 26.8 & 11.3 & 11.3 & 11.3 & 14.3 & 14.3 \\
\hline $\begin{array}{l}\operatorname{Org}-N(\mathrm{ng} / 1) \\
\operatorname{Tng}(\mathrm{gg} / 1)\end{array}$ & 14.0 & 14.0 & 14.0 & 14.0 & $(4.0$ & & & & & \\
\hline Color (APHA units) & 750 & 750 & 758 & 750 & 758 & 875 & 875 & 875 & 875 & 875 \\
\hline TOC $(m g / 1)$ & 35 & 39 & 39 & 39 & 39 & 46 & 46 & 46 & 46 & 46 \\
\hline 800 5-day (mg/1) & 2.5 & 1.1 & 1.1 & 1.1 & 1.1 & 1.6 & 1.6 & 1.6 & 2.8 & 2.8 \\
\hline
\end{tabular}




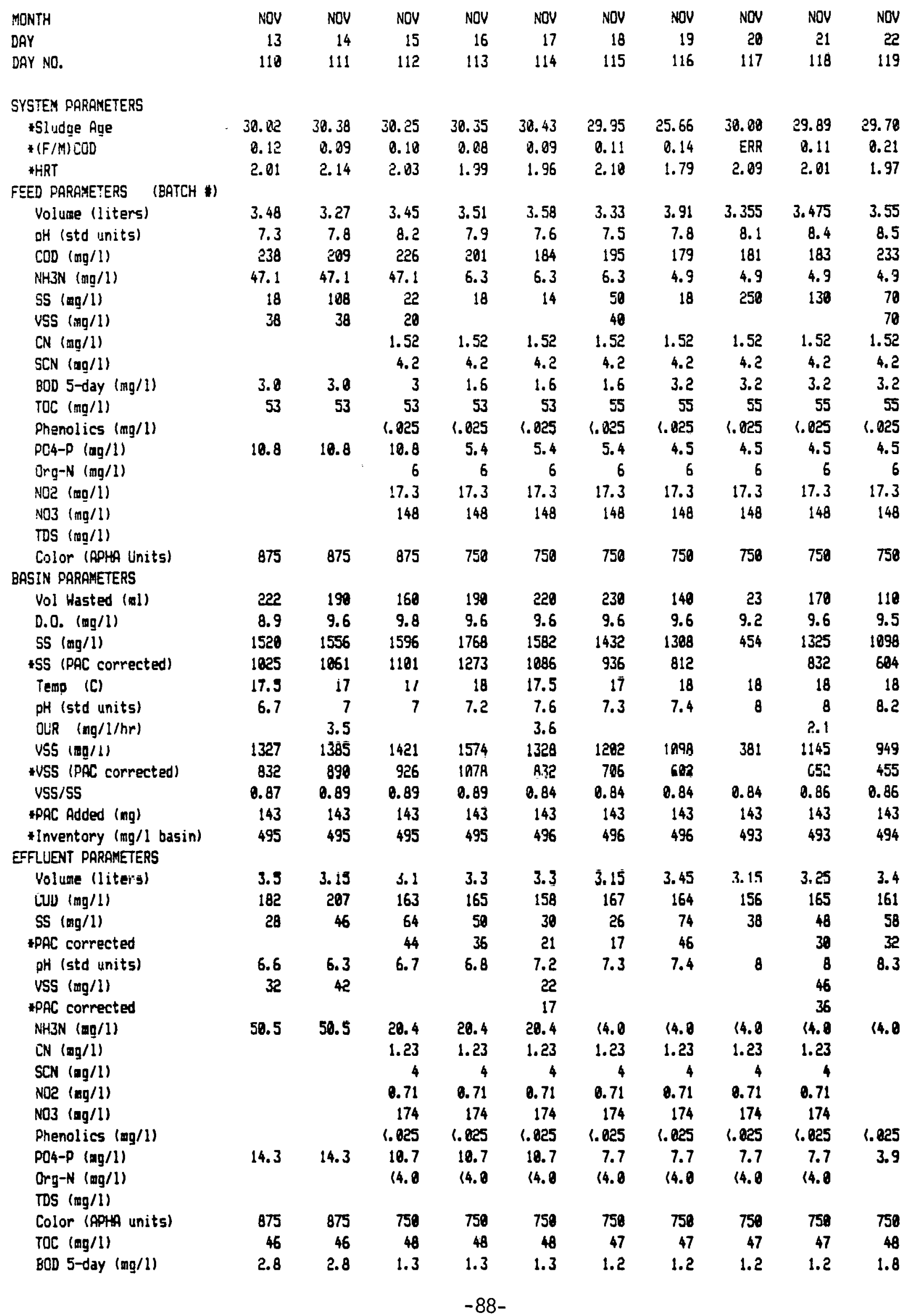




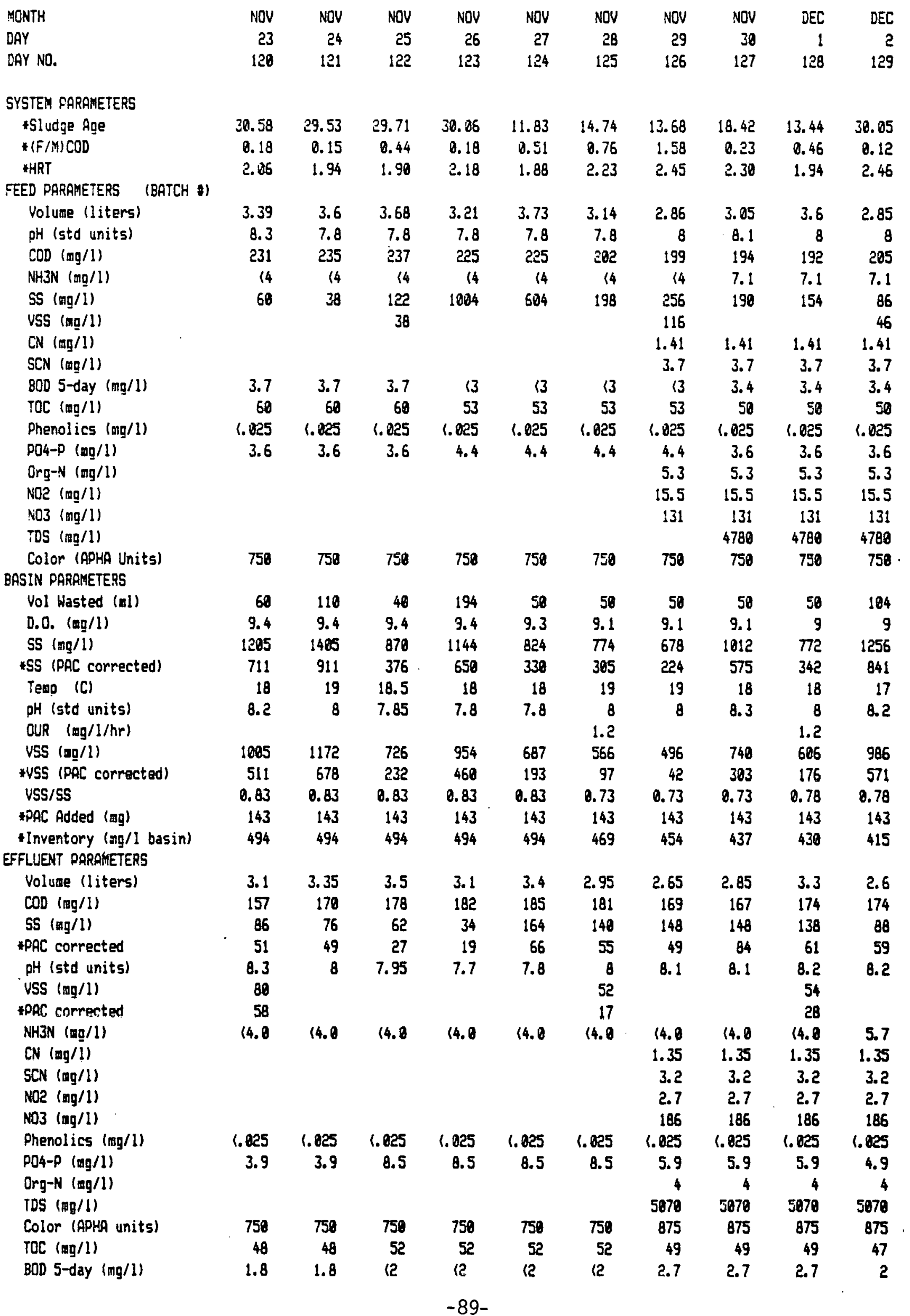


UNIT 18 TABLE A2

\begin{tabular}{|c|c|c|c|c|c|c|c|c|c|c|}
\hline MONTH &.$D E C$ & DEC & DEC & DEC & DEC & DEC & DEC & DEC & DEC & DEC \\
\hline DAY & 3 & 4 & 5 & 6 & 7 & 8 & 9 & 10 & 11 & 12 \\
\hline DAY NO. & 130 & 131 & 132 & 133 & 134 & 135 & 136 & 137 & 138 & 139 \\
\hline \multicolumn{11}{|l|}{ SYSTEM PARAMETERS } \\
\hline *Sludge Age & 31.89 & 36.28 & 38.42 & 5.99 & 30.27 & 28.74 & 53.80 & 23.64 & 33.73 & 57.14 \\
\hline$\because F / M) C O D$ & 0.10 & 0.11 & 0.10 & ERR & 0.14 & 0.08 & 0.89 & 0.26 & 0.15 & 0.12 \\
\hline \#HRT & 1.73 & 1.80 & 1.83 & 2.04 & 1.82 & 1.84 & 1.82 & 1.75 & 1.89 & 1.79 \\
\hline \multicolumn{11}{|l|}{ FEED FARAMETERS (EATCH \#) } \\
\hline Volume (liters) & 4.85 & 3.89 & 3.83 & 3.43 & 3.85 & 3.8 & 3.85 & 4 & 3.7 & 3.9 \\
\hline $\mathrm{OH}$ (std units) & 8.1 & 8.3 & 8.1 & 8.1 & 8.3 & 8.1 & 8.1 & 7.9 & 7.8 & 7.9 \\
\hline $\operatorname{COD}(n g / 1)$ & 197 & 205 & 203 & 196 & 180 & 180 & 217 & 180 & 184 & 186 \\
\hline NHBN $(\mathrm{mg} / 1)$ & 38.6 & 38.6 & 38.6 & 38.6 & 41.4 & 41.4 & 41.4 & 28.8 & 28.8 & 28.8 \\
\hline $55(\mathrm{mg} / 1)$ & 56 & 70 & 60 & 46 & 148 & 42 & 118 & 74 & 68 & 176 \\
\hline VSS $(\mathrm{mg} / 1)$ & & & & 32 & 78 & & 58 & & & \\
\hline$C N(m g / l)$ & 1.41 & 1.41 & 1.41 & 1.41 & & & & & & \\
\hline $\operatorname{SCN}(\mathrm{mg} / 1)$ & 3.7 & 3.7 & 3.7 & 3.7 & & & & & & \\
\hline BOD 5-day (mg/l) & 3 & 3 & 3 & 3 & 2 & 2 & 2 & 12 & 12 & 12 \\
\hline $\mathrm{TOC}(\mathrm{mg} / \mathrm{l})$ & 53 & 53 & 53 & 53 & 51 & 51 & 51 & 46 & 46 & 46 \\
\hline Phenolics (ng/l) & 1.025 & 1.025 & 1.025 & 1.025 & 1.025 & 1.025 & 1.025 & 6.025 & 1.025 & 1.025 \\
\hline PnA-P (䐓/ $/ 1)$ & 3.6 & 3.6 & 3.6 & 3.6 & 4.6 & 4,6 & 4:G & G. 1 & 6.1 & 6.1 \\
\hline Org-N (mg/1) & 5.3 & 5.3 & 5.3 & 5.3 & & & & & & \\
\hline $\mathrm{ND2}(\mathrm{mg} / 1)$ & 15.5 & 15.5 & 15.5 & $: 5.5$ & & & & & & \\
\hline v03 $(\operatorname{mg} / 1)$ & 131 & 131 & 131 & 131 & & & & & & \\
\hline $\operatorname{TDS}(\pi g / 1)$ & 4780 & 4780 & 4780 & 4780 & 4585 & 4585 & 4585 & 4585 & 4505 & 4505 \\
\hline Color (APHA Units) & 750 & 750 & 759 & 750 & 750 & 750 & 758 & 750 & 750 & 758 \\
\hline \multicolumn{11}{|l|}{ BASIN PARAMETERS } \\
\hline Vol Wasted (al) & 0 & 50 & 50 & 58 & 150 & 100 & 50 & 50 & 50 & 50 \\
\hline D.0. $(m g / 1)$ & 9 & 9 & 8. 4 & 9 & 8.5 & 8.8 & 8.8 & 8.8 & 8.4 & 8.6 \\
\hline SS $(a g / 1)$ & 1770 & 1600 & 1624 & 636 & 1435 & 1638 & 1784 & 872 & 1144 & 1380 \\
\hline *SS (PAC corrected) & 1352 & 1179 & 1198 & 204 & 1059 & 1257 & 1400 & 479 & 75 & 982 \\
\hline Temp $(C)$ & 19 & 18 & 18 & 18 & 17.5 & 18 & 18 & 18 & 18 & 18 \\
\hline pH (sto units) & 8.2 & 8.4 & 7.8 & 8.1 & 7.8 & 8 & 7.9 & 7.5 & 7.6 & 7.6 \\
\hline OUR (ng/l/hr) & & & & 1.2 & & & & & & 6 \\
\hline USS $(\mathrm{mg} / 1)$ & 1389 & 1256 & 1390 & 422 & 952 & 1338 & 1457 & 712 & 334 & 1128 \\
\hline * USS (PAC corrected) & 972 & 835 & 874 & & 576 & 957 & 1073 & 319 & 541 & 730 \\
\hline VSS/SS & 0.78 & 0.78 & 0.80 & 0.66 & 0.66 & 0.82 & 0.82 & 0.82 & 0.82 & 8.82 \\
\hline \#PAC Added (mg) & 143 & 143 & 143 & 143 & 143 & 143 & 143 & 143 & 143 & 143 \\
\hline -Inventory (mg/l basin) & 418 & 421 & 426 & 432 & 376 & 381 & 384 & 393 & 393 & 398 \\
\hline \multicolumn{11}{|l|}{ EFFLUENT PARAMETERS } \\
\hline Volume (Iiters) & 3.85 & 3.65 & 3.62 & 2.9 & 3.7 & 3.4 & 3.5 & 3.8 & 3.35 & 3.65 \\
\hline $\cos (\log / 1)$ & 167 & 165 & 167 & 163 & 171 & 165 & 165 & 158 & 153 & 135 \\
\hline S5 $(m g / 1)$ & 124 & 82 & 78 & 304 & 52 & 96 & 56 & 72 & 70 & 38 \\
\hline *PAC corrected & 95 & 60 & 58 & 98 & 38 & 74 & 44 & 40 & 46 & 27 \\
\hline oH (sto units) & 8.2 & 8.1 & 8 & 7.8 & 7.6 & 7.8 & 7.9 & 7.6 & 7.5 & 7.6 \\
\hline VSS (ag/l) & & & 52 & 186 & & 66 & & & & 28 \\
\hline \#AC corrected & & & 38 & & & 58 & & & & 21 \\
\hline NH3N $(\square g / 1)$ & 5.7 & 5.7 & 5.7 & 18.3 & 18.3 & 18.3 & 12.2 & 12.2 & 12.2 & 12.2 \\
\hline CN $(a g / 1)$ & 1.35 & 1.35 & 1.35 & & & & & & & \\
\hline $\operatorname{SCN}(m g / 1)$ & 3.2 & 3.2 & 3.2 & & & & & & & \\
\hline NO2 $(\mathrm{mg} / 1)$ & 2.7 & 2.7 & 2.7 & & & & & & & \\
\hline $\mathrm{NO} 3(\mathrm{ag} / 1)$ & 186 & 186 & 185 & & & & & & & \\
\hline Phenolics (ng/1) & 1.025 & 1.825 & 1.025 & 1.025 & 1.825 & 1.025 & 1.025 & 1.025 & 1.025 & 1.025 \\
\hline$P 04-P(N Q / 1)$ & 4.9 & 4.9 & 4.9 & 7.9 & 7.9 & 7.9 & 8.6 & 8.6 & 8.6 & 8.6 \\
\hline Org-N (mg/l) & 4 & 4 & 4 & & & & & & & \\
\hline $\operatorname{TDS}(\mathrm{mg} / 1)$ & 5070 & 5970 & 5070 & 4855 & 4855 & 4855 & 4855 & 4855 & 4855 & 4855 \\
\hline Color (APHA units) & 875 & 875 & 875 & 1000 & 1080 & 1080 & 1080 & 1080 & 1000 & 1000 \\
\hline $\operatorname{TOC}(m g / 1)$ & 47 & 47 & 47 & 44 & 44 & 44 & 45 & 45 & 45 & 45 \\
\hline 80D 5-day (mg/l) & 2 & 2 & ? & 3 & 3 & 3 & 2.3 & 2.3 & 2.3 & 2.3 \\
\hline
\end{tabular}


LNIT IB TAELE AC

\begin{tabular}{|c|c|c|c|c|c|c|c|c|c|c|}
\hline MONTH & DEC & DEC & DEC & $D E C$ & $D E C$ & DEC & DEC & DEC & UEC & DEC \\
\hline DAY & 13 & 14 & 15 & 16 & 17 & 18 & 19 & 20 & 21 & 22 \\
\hline DAY NO. & 140 & 141 & 142 & 143 & 144 & 145 & 146 & 147 & 148 & 149 \\
\hline \multicolumn{11}{|l|}{ SYSTEM PARGMETERS } \\
\hline *Sludge Age & 34.57 & 47.50 & 28.48 & 55.23 & 82.61 & 39.76 & 46.91 & 31.39 & 22.66 & 43.68 \\
\hline$+(F / M) C O D$ & 0.21 & 0.20 & 0.62 & 0.75 & 0.42 & -1.65 & 0.15 & 0.22 & 0.43 & 0.18 \\
\hline HHRT & 1.92 & 1.79 & 1.79 & 1.83 & 1.52 & 1.78 & 1.94 & 1.79 & $1 . \pi$ & 1.84 \\
\hline \multicolumn{11}{|l|}{ FEED PARAMETERS (BRTCH *) } \\
\hline Voluwe (liters) & 3.65 & 3.9 & 3.9 & 3.82 & 4.6 & 3.93 & 3.61 & 3.9 & 3.95 & 3.8 \\
\hline ọh (std units) & 7.9 & 8 & 8 & 7.8 & 8.2 & 8.3 & 8.5 & 8.3 & 8.2 & 7.9 \\
\hline $\operatorname{coD}(\operatorname{mg} / 1)$ & 184 & 169 & 168 & 195 & 180 & 182 & 167 & 174 & 188 & 190 \\
\hline NH3N $(m g / 1)$ & 28.8 & 42.8 & 42.8 & 42.8 & 67.6 & 67.6 & 67.6 & 67.6 & 88.2 & 88.2 \\
\hline SS (mg/1) & 40 & 58 & 32 & 42 & 118 & 78 & 56 & 66 & 208 & 56 \\
\hline VSS (mg/l) & 32 & 8 & 26 & 34 & 36 & 63 & 45 & 40 & 126 & 3 \\
\hline CN (mg/1) & 0 & 2.41 & 2.41 & 2.41 & 2.41 & 2.41 & 2.41 & 2.41 & 0 & \\
\hline $\operatorname{SCN}(\operatorname{mog} / 1)$ & 0 & 3.9 & 3.9 & 3.9 & 3.9 & 3.9 & 3.9 & 3.9 & 0 & \\
\hline BOD 5-day $(\mathrm{mg} / \mathrm{l})$ & 12 & 5.3 & 5.3 & 5.3 & 4 & 4 & 4 & 4 & 5 & \\
\hline TOC (mo/l) & 46 & 49 & 49 & 49 & 48 & 48 & 48 & 48 & 47 & 4 \\
\hline Phenolics (mg/l) & 1.825 & 1.025 & 1.025 & 1.025 & 1.025 & 1.825 & 1.025 & 1.025 & 1.825 & 1.02 \\
\hline PO4-P (mg/1) & 6.1 & 4.3 & 4.3 & 4.3 & 3.4 & 3.4 & 3.4 & 0 & 0 & \\
\hline \multicolumn{11}{|l|}{$O r g-N(m g / l)$} \\
\hline NO2 (mg/l) & 0 & 9.85 & 9.85 & 9.05 & 9.05 & 9.05 & 9.85 & 9.85 & 0 & \\
\hline$N 03(00 / 1)$ & 0 & 95 & 95 & 95 & 95 & 95 & 35 & 95 & 0 & \\
\hline TDS $: \pi g / 1)$ & 4505 & 4345 & 4345 & 4345 & 4345 & 4345 & 4345 & 4345 & 8 & \\
\hline Color (APHA Units) & 750 & 875 & 875 & 875 & 875 & 875 & 875 & 875 & 1000 & 1000 \\
\hline \multicolumn{11}{|l|}{ BASIN PARAMETERS } \\
\hline Vol Wasted (ml) & 50 & 50 & 59 & 58 & 50 & 58 & 50 & 50 & 58 & 50 \\
\hline D. O. $(\operatorname{ag} / 1)$ & 8.6 & 8.8 & 8.8 & 8.4 & 8.4 & 8.6 & 8.5 & 8.6 & 8.6 & 8.4 \\
\hline SS (xg/1) & 950 & 968 & 1200 & 1190 & 1460 & 856 & 1188 & 1038 & 842 & 1096 \\
\hline +5S (PAC corrected) & 542 & 552 & 784 & 772 & 1033 & 427 & 744 & 587 & 388 & 646 \\
\hline Texp (C) & 18 & 18 & 18 & 19 & 17 & 17 & 18 & 18 & 18 & 18 \\
\hline $\begin{array}{l}\text { oH (std units) } \\
\text { OUR (ng/1/hr) }\end{array}$ & 8.1 & 7.7 & 7.6 & 7.5 & 7.8 & 8 & $\begin{array}{r}8.3 \\
6\end{array}$ & 7.9 & 7.7 & \\
\hline USS $(m g / 1)$ & 777 & 785 & 538 & 534 & 655 & 388 & 926 & 889 & 656 & 912 \\
\hline *USS (PAC corrected) & 368 & 377 & 122 & 115 & 227 & -50 & 482 & 358 & 203 & 462 \\
\hline USS/SS & 8.82 & 0.82 & 0.45 & 0.45 & 8.45 & 0.45 & 8.78 & 0.78 & 0.78 & 0.83 \\
\hline IPAC Added (ag) & 143 & 143 & 143 & 143 & 143 & 143 & 143 & 143 & 143 & 143 \\
\hline Inventory (mg/l basin) & 488 & 408 & 416 & 418 & 427 & 439 & 444 & 451 & 454 & 45 \\
\hline \multicolumn{11}{|l|}{ EFFLUENT PARAMETERS } \\
\hline Volume (liters) & 3.75 & 3.7 & 3.6 & 3.7 & 3.95 & 3.6 & 3.3 & 3.75 & 3.65 & 3.5 \\
\hline $\cos (\mathrm{mg} / \mathrm{l})$ & 147 & 148 & 140 & 159 & 140 & 150 & 159 & 156 & 157 & 157 \\
\hline SS $(m g / 1)$ & 76 & 34 & 84 & 34 & 20 & 40 & 48 & 62 & 76 & \\
\hline IPAC corrected & 43 & & & & & & & & & \\
\hline OH (std units) & 7.6 & 7.6 & 7.6 & 7.5 & 7.9 & 8.1 & 8.2 & 8.1 & 7.6 & 7.2 \\
\hline USS $(m g / 1)$ & & 25 & 48 & 19 & 11 & 23 & 32 & 41 & 51 & \\
\hline WPAC corrected & $\theta$ & & & & & & & & & \\
\hline NHב̄N (mg/l) & b & 6 & 6 & 17.7 & 17.7 & 17.7 & 17.7 & 34.8 & 34.0 & 34.8 \\
\hline CN (bog/1) & 1.81 & 1.81 & 1.81 & 1.81 & 1.81 & 1.81 & 1.81 & & & \\
\hline $\operatorname{SCN}(\operatorname{mg} / 1)$ & 3.3 & 3.3 & 3.3 & 3.3 & 3.3 & 3.3 & 3.3 & & & \\
\hline ND2 (ag/1) & 2.26 & 2.26 & 2.26 & 2.26 & 2.26 & 2.26 & 2. 26 & & & \\
\hline $\mathrm{N}[13$ (用/1) & 158 & 158 & 158 & 158 & 158 & 158 & 158 & & & \\
\hline Phenolics (mg/1) & ८.825 & $<.025$ & ८. 825 & 1.025 & <.825 & $<.825$ & $<.825$ & 1.825 & 1.025 & 1.025 \\
\hline$P 04-P(m g / 1)$ & 8.7 & 8.7 & B.7 & 5.4 & 5.4 & 5.4 & 5.4 & & & \\
\hline \multicolumn{11}{|l|}{ Org $-N(m g / 1)$} \\
\hline $\operatorname{TDS}(\mathrm{mg} / 1)$ & 4655 & 4655 & 4655 & 4655 & $465 \underline{5}$ & 4655 & 4655 & & & \\
\hline Color (APHA units) & 873 & 875 & 875 & 875 & 875 & 875 & 875 & 875 & 875 & 875 \\
\hline TOC (ng/1) & 42 & 42 & 42 & 45 & 45 & 45 & 45 & 44 & 44 & \\
\hline BOD 5-day (mg/1) & 2.8 & 2.8 & 2.8 & 4 & 4 & 4 & 4 & 4 & 4 & \\
\hline
\end{tabular}


ÚNIT IB TABLE A2

\begin{tabular}{|c|c|c|c|c|c|c|c|c|c|c|}
\hline MONTH & DEC & DEC & DEC & DEC & DEC & DEC & DEC & DEC & DEC & JAN \\
\hline DAY & 23 & 24 & 25 & 26 & 27 & $2 \theta$ & 29 & 30 & 31 & \\
\hline DAY NO. & 150 & 151 & ¿52 & 153 & 154 & 155 & 156 & 157 & 158 & 15 \\
\hline \multicolumn{11}{|l|}{ SYSTEM PARAMETERS } \\
\hline Sludge Age & 47.46 & 42.12 & 88.52 & 35.56 & 135.31 & 31.45 & 30.86 & 30.86 & 23.85 & 30.09 \\
\hline$*(F / M) C O D$ & 0.23 & 0.71 & 0.85 & 0.16 & 0.02 & 0.85 & 0.16 & 0.07 & 0.11 & 0.08 \\
\hline *HRT & 1.77 & 1.75 & 1.75 & 1.95 & 1.96 & 1.75 & 1.75 & 1.83 & 1.75 & 1.64 \\
\hline \multicolumn{11}{|l|}{ FEED PRRAMETERS (BATCH } \\
\hline Volume (1iters) & 3.95 & 4 & 4 & 3.59 & 3.57 & 4 & 4 & 3.7 & 4 & 4.28 \\
\hline oH (std units) & 7.9 & 7.9 & 7.9 & 8.3 & 8.2 & 8.2 & 8.2 & 8 & 8 & 7.8 \\
\hline $\operatorname{coD}(\operatorname{mg} / 1)$ & 176 & 176 & 159 & 159 & 174 & 167 & 212 & 197 & 191 & 168 \\
\hline NH3N $(00 / 1)$ & 88.2 & 99.4 & 99.4 & 99.4 & 99.4 & 165 & 165 & $i 65$ & 210 & 21 \\
\hline SS $(\lg / \overline{1})$ & 28 & 32 & 124 & 24 & 360 & 44 & 172 & $\dot{62}$ & 62 & \\
\hline US5 $(\mathrm{mg} / 1)$ & 22 & 25 & 101 & 19 & 283 & 36 & 141 & 48 & 40 & \\
\hline CN $(m g / 1)$ & 0 & 0 & 0 & 0 & 0 & 0.447 & 0.447 & 0.447 & 0.447 & 0.447 \\
\hline $\operatorname{SCN}(\operatorname{ag} / 1)$ & 8 & 0 & 8 & 0 & $\theta$ & 4. 9 & 4.9 & 4.9 & 4.9 & 4.9 \\
\hline 800 5-day $(\mathrm{mg} / \mathrm{l})$ & 5 & 3 & 3 & 3 & 3 & 2 & 2 & 2 & 3 & \\
\hline TOC (ag/1) & 47 & 47 & 47 & 47 & 47 & 51 & $5 t$ & 51 & 52 & \\
\hline Phenolics (oxg/1) & 1.625 & 1.025 & 1.025 & 1.025 & 1.025 & 1.025 & 1.025 & 1.025 & 1.025 & 1.025 \\
\hline $\mathrm{PO} 4-\mathrm{P}\langle\mathrm{GH} / 1)$ & n & 9 & 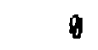 & 0 & 7.3 & 8.3 & 5.3 & 5.3 & 6.3 & 6.3 \\
\hline Org-N $(m g / 1)$ & & & & & 8 & 11 & 11 & 11 & 11 & 11 \\
\hline NO2 (mg/l) & $\theta$ & 0 & 0 & 0 & 0 & 0.62 & 0.62 & 0.62 & 0.62 & 0.62 \\
\hline N03 (mg/1) & 8 & 0 & 0 & 8 & 0 & 2.98 & 2.98 & 2.9月 & 2.98 & 2.38 \\
\hline $\operatorname{TDS}(\mathrm{ag} / 1)$ & 8 & 0 & 0 & 0 & 4375 & 4390 & 4390 & 4390 & 4390 & 4390 \\
\hline Color (APHA Units) & 1000 & 1000 & 1000 & 1080 & 1000 & 1250 & 1250 & 1250 & 1250 & 1250 \\
\hline \multicolumn{11}{|l|}{ BASIN PARAMETERS } \\
\hline Vol Wasted (al) & 50 & 58 & 58 & 58 & 58 & 58 & 50 & 58 & 50 & \\
\hline 0.0. $(\mathrm{mg} / 1)$ & 8.5 & 8.5 & 8.5 & 8.5 & 8.4 & 0.5 & 0.2 & 7 & 7 & \\
\hline SS (mg/li) & 974 & 728 & 2322 & 978 & 6840 & 2284 & 1412 & 2142 & 1628 & 2048 \\
\hline *5S (PAC corrected) & 517 & 256 & 1853 & 497 & 5546 & 1789 & 917 & 1646 & 1128 & 1548 \\
\hline Teup (C) & 18 & 17 & 17 & 17 & 18 & 25 & 22 & 22 & 22 & 22 \\
\hline $\begin{array}{l}\text { pH (std units) } \\
\text { OUR (ng/1/hr) }\end{array}$ & 7 & 6.65 & 6.9 & $\begin{array}{l}7 \\
6\end{array}$ & 7 & 6.9 & $\begin{array}{r}6.6 \\
6\end{array}$ & 6.5 & 6.2 & 6.7 \\
\hline VSS (mg/l) & 810 & 589 & 1932 & 814 & 4862 & 1839 & 976 & 1481 & 1120 & 1410 \\
\hline *VSS (PAC corrected) & 354 & 116 & 1463 & 414 & 4465 & 1440 & 634 & 1138 & 780 & 1070 \\
\hline USS/SS & 0.83 & 0.80 & 0.83 & 8.83 & 0.80 & 0.80 & 0.69 & 0.69 & 0.69 & 0.69 \\
\hline IPAC Added (mg) & 143 & 143 & 143 & 143 & 143 & 143 & 143 & 143 & 143 & 143 \\
\hline Inventory (mg/1 basin) & 457 & 464 & 469 & 481 & 494 & 495 & 495 & 496 & 492 & 492 \\
\hline \multicolumn{11}{|l|}{ EFFLUENT PARAMETERS } \\
\hline Volume (liters) & 3.55 & 3.7 & 3.65 & 3.35 & 3.15 & 4.05 & 3.75 & 3.45 & 3.7 & 3.7 \\
\hline $\cos (\operatorname{mog} / 1)$ & 161 & 144 & 151 & 157 & 153 & 184 & 184 & 178 & 142 & 130 \\
\hline SS $(a g / 1)$ & 36 & 30 & 30 & 56 & 26 & 126 & 126 & 142 & 136 & 130 \\
\hline PAC corrected & & & & 28 & 24 & 99 & 82 & 109 & 95 & 99 \\
\hline PH (std units) & 6.9 & 6.7 & 7 & 7 & 7 & 6.8 & 6.8 & 6.9 & 6.6 & 6.9 \\
\hline VSS (mg/1) & 34 & 25 & 29 & 54 & 18 & 87 & 62 & 70 & 82 & \\
\hline *PAC corrected & & & & 45 & 14 & 78 & 43 & 48 & 57 & \\
\hline NH3N $(\operatorname{mg} / 1)$ & 50.3 & 50.3 & 58.3 & 50.3 & 81.8 & 81.0 & 81.0 & 131 & 131 & 13! \\
\hline CN $(\mathrm{gg} / 1)$ & & & & & 1.0 & 1.0 & 1.0 & 1.0 & 1.0 & \\
\hline $\operatorname{SCN}(\mathrm{ag} / 1)$ & & & & & 3.6 & 3.6 & 3.6 & 3.6 & 3.6 & 3. \\
\hline NOS (mg/1) & & & & & 1.2 & 1.2 & 1.2 & 1.2 & 1.2 & 1. \\
\hline $\mathrm{NOS}(\mathrm{mg} / \mathrm{l})$ & & & & & 21 & 21 & 21 & 21 & 21 & 2 \\
\hline Phenolics $(m g / 1)$ & ८.025 & 1.825 & 1.025 & 1.825 & 1.025 & 1.825 & 1.025 & 1.025 & 1.825 & 1.025 \\
\hline$P 04-P(\operatorname{mg} / 1)$ & & & & 11.6 & 11.9 & 11.9 & 11.9 & 9.5 & 9.5 & 9.5 \\
\hline Org-N (mg/l) & & & & & 14 & 14 & 14 & 14 & 14 & \\
\hline TDS $(\mathrm{mg} / 1)$ & & & & 4880 & 4620 & 4628 & 4620 & 4620 & 4620 & 4628 \\
\hline Color (APHA units) & 875 & 875 & 875 & 975 & 1800 & 1800 & 1080 & 1080 & 1008 & 1080 \\
\hline TOC (mg/I) & 45 & 45 & 45 & 45 & 49 & 49 & 49 & 42 & 42 & 42 \\
\hline BUD 5-day $(\operatorname{mg} / 1)$ & 12 & «2 & 12 & 12 & 3 & 3 & 3 & 5 & 2 & \\
\hline
\end{tabular}




\begin{tabular}{|c|c|c|c|c|c|c|c|c|c|c|}
\hline MONTH & JAN & JAN & JAN & JAN & JAN & JAN & JAN & JAN & JḦN & JAN \\
\hline DAY & 2 & 3 & 4 & 5 & 6 & 7 & 8 & 9 & 10 & 11 \\
\hline JAY NO. & 160 & 161 & 162 & 163 & 164 & 165 & 166 & 167 & 168 & 169 \\
\hline \multicolumn{11}{|l|}{ SYSTEM PARAMETERS } \\
\hline *Sludge Age & 23.26 & 35.75 & 19.11 & 13.71 & 25.88 & 22.49 & 38.37 & 28.10 & 20.86 & 27.82 \\
\hline$*(F / A) C O D$ & 0.10 & 0.07 & 0.08 & 0.12 & 0.08 & 0.11 & 0.29 & 0.08 & 0.06 & 0.05 \\
\hline *HRT & 1.70 & 1.94 & 1.75 & 1.75 & 1.75 & 1.84 & 1.79 & 1.73 & 1.89 & 1.75 \\
\hline \multicolumn{11}{|l|}{ FEED PARAMETERS (BATCH } \\
\hline Volume (liters) & 4.12 & 3.61 & 4 & 4 & 4 & 3.8 & 3.91 & 4.04 & 3.7 & 4 \\
\hline pH (std units) & 8.3 & 6.2 & 8.5 & 8.3 & 8.4 & 8 & 7.8 & 7.5 & 7.7 & 7.7 \\
\hline $\operatorname{cod}(n g / 1)$ & 162 & 197 & 197 & 217 & 188 & 177 & 166 & 168 & 135 & 125 \\
\hline NH3N $(w g / 1)$ & 218 & 218 & 205 & 285 & 205 & 211 & 211 & 211 & 211 & 215 \\
\hline SS $(\mathrm{mg} / 1)$ & 30 & 86 & 62 & 44 & 212 & 44 & 12 & 26 & 126 & 82 \\
\hline USS (ng/1) & 19 & 55 & 40 & 28 & 142 & 29 & 8 & 17 & 70 & 46 \\
\hline$C N(m g / 1)$ & 0.447 & 0.447 & 0 & 8 & 0 & 0 & 0 & 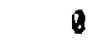 & 0 & 0.616 \\
\hline $\operatorname{SCN}(\pi g / 1)$ & 4.9 & 4.9 & 0 & 8 & 0 & 0 & 0 & $\theta$ & 0 & 4.9 \\
\hline BOD 5-day (mq/1) & 3 & 3 & 2 & 2 & 2 & 3 & 3 & 3 & 3 & 3 \\
\hline TOC $(m g / 1)$ & 52 & 52 & 61 & 61 & 61 & 45 & 45 & 45 & 45 & 43 \\
\hline Phenolics (mg/I) & $<.025$ & 1.025 & 1.025 & 1.025 & 1.025 & 1.025 & 1.025 & ८.025 & 1.025 & <.625 \\
\hline P04-P (mg/l) & 6.3 & 6.3 & 3.6 & 3.6 & 3.6 & 13.5 & 13.5 & 13.5 & 13.5 & 13.1 \\
\hline Org-N $(m g / 1)$ & 11 & 11 & 0 & 8 & 0 & 0 & 0 & $a$ & 8 & 0 \\
\hline NO2 (mg/1) & 0.62 & 0.62 & 0 & 0 & 8 & 0 & 0 & 0 & 10.25 & 10.25 \\
\hline N03 (mg/1) & 2.98 & 2.98 & 0 & $\theta$ & 8 & b & 0 & 0 & 12.5 & 〈2.5 \\
\hline TDS $(\mathrm{mg} / 1)$ & 4390 & 4390 & 4766 & 4766 & 4766 & 4766 & 4766 & 4766 & 4766 & 0 \\
\hline Color (APHA Units) & 1250 & 1258 & 1008 & 1000 & 1008 & 1000 & 1080 & 1000 & 1008 & 758 \\
\hline \multicolumn{11}{|l|}{ BASIN PARAMETERS } \\
\hline Vol Wasted (nl) & 58 & 58 & 50 & 50 & 50 & 20 & 20 & 50 & 50 & 50 \\
\hline D.0. $(\mathrm{gg} / 1)$ & 7 & 7 & 6.5 & 7 & 6.9 & 6.9 & 6.9 & 6.9 & 7.8 & 7 \\
\hline SS $(m g / 1)$ & 1570 & 2028 & 1886 & 1590 & 1696 & 1430 & 1595 & 1728 & 1594 & 1856 \\
\hline *SS (PAC corrected) & 1083 & 1538 & 1405 & 1116 & 1424 & 963 & 1123 & 1256 & 1129 & 1391 \\
\hline Temo (C) & 22 & 22 & 21 & 20 & 23 & 21 & 20 & 20 & 21 & 21 \\
\hline oH (std units) & 7.4 & 7.7 & 8 & 7.9 & 8.1 & 7.4 & 6.2 & 6.9 & 6.3 & 7.2 \\
\hline OUR $(\pi g / 1 / h r)$ & & 6 & & 6 & & & & 6 & & \\
\hline VSS (mg/l) & 1085 & 1568 & 1458 & 1230 & 1467 & 1186 & 1234 & 1374 & 1267 & 1476 \\
\hline \#USS (PAC corrected) & 748 & 1189 & 1086 & 864 & 1102 & 745 & 869 & 999 & 898 & 1106 \\
\hline VSS/SS & 8.69 & 0.77 & 0.77 & 0.77 & 0.77 & 0.77 & 0.77 & 0.80 & 0.80 & 0.80 \\
\hline FPAC Added (mg) & 143 & 143 & 143 & 143 & 143 & 143 & 143 & 143 & 143 & 143 \\
\hline * (nventory (Bg/l basin) & 487 & 490 & 481 & 474 & 472 & 467 & 472 & 472 & 465 & 465 \\
\hline \multicolumn{11}{|l|}{ EFFLUENT PARAMETERS } \\
\hline Voiuwe (literg) & 3.8 & 3.45 & 3.83 & 3.7 & 3.77 & 3.55 & 3.1 & 3.75 & 3.55 & 3.7 \\
\hline $\operatorname{coD}(\operatorname{lng} / 1)$ & 142 & 130 & 202 & 154 & 162 & 160 & 156 & 149 & 133 & 125 \\
\hline SS (ng/l) & 132 & 112 & 196 & 166 & 142 & 146 & 88 & 118 & 178 & 130 \\
\hline $P A C$ corrected & 91 & 85 & 146 & 117 & 107 & 98 & 62 & 86 & 120 & 97 \\
\hline oH (std units) & 7.7 & 7.7 & 8.1 & 8 & 8.1 & 7.7 & 7.3 & 6.7 & 6.7 & 7 \\
\hline VSS $(m g / 1)$ & 89 & 78 & 138 & 118 & 100 & 180 & 62 & 84 & 120 & 94 \\
\hline EPAC corrected & 55 & 60 & 107 & 91 & 77 & 77 & 48 & 67 & 95 & 75 \\
\hline NH3N $(\mathrm{gg} / \mathrm{l})$ & 131 & 179 & 179 & 179 & 197 & 197 & 197 & 197 & 218 & 210 \\
\hline CN $(n g / 1)$ & 1.0 & & & & & & & & 0.876 & 0.876 \\
\hline $\operatorname{SCN}(m g / 1)$ & 3.6 & & & & & & & & 3.2 & 3.2 \\
\hline NOR (ng/1) & 1.2 & & & & & & & & 10.25 & 10.25 \\
\hline $\mathrm{NO3}(\mathrm{mg} / 1)$ & 21 & & & & & & & & 7.2 & 7.2 \\
\hline Phenolics (agg/1) & 1.825 & 1.025 & 1.025 & 1.025 & 1.025 & 1.025 & 1.025 & 1.025 & <.025 & 1.825 \\
\hline$P 04-P(n g / 1)$ & 9.5 & 7.0 & 7.0 & 7.8 & 9.6 & 9.6 & 9.6 & 9.6 & 16.5 & 16.5 \\
\hline Org-N (mg/1) & 14 & & & & & & & & & \\
\hline $\operatorname{ToS}(\mathrm{mg} / 1)$ & 4620 & 4770 & 4770 & 4770 & 4770 & 4770 & 4770 & 4770 & & \\
\hline Color (APHA units) & 1009 & 1000 & 1000 & 1000 & 1080 & 1000 & 1000 & 1000 & 750 & 750 \\
\hline TOC $(\operatorname{sg} / 1)$ & 42 & 44 & 44 & 44 & 40 & 40 & 40 & 40 & 40 & 40 \\
\hline BOD 5-day (ตg/I) & 2 & 3 & 3 & 3 & 3 & 3 & 3 & 3 & (2 & 12 \\
\hline
\end{tabular}


UNIT IB TABLE A2

\begin{tabular}{|c|c|c|c|c|c|c|c|c|c|c|}
\hline MONTH & JAN & JAN & JAN & JAN & JAN & JAN & JAN & JAN & JAN & JAN \\
\hline DAY & 12 & 13 & 14 & 15 & 16 & 17 & 18 & 19 & 20 & 21 \\
\hline DAY NO. & 170 & 171 & 172 & 173 & 174 & 175 & 176 & 177 & 178 & 179 \\
\hline \multicolumn{11}{|l|}{ SYSTEM FARAMETERS } \\
\hline tSIudge Age & 46.56 & 23.73 & 33.95 & 38.66 & 49.98 & 23.59 & 30.77 & 35.28 & 20.83 & 23.68 \\
\hline$*(F / M) C O D^{\circ}$ & 8.88 & 0.09 & 0.14 & 0.15 & 0.06 & 0.08 & 0.08 & 0.08 & 0.12 & 0.20 \\
\hline$¥$ HRT & 1.75 & 1.75 & 1.79 & 1.75 & 1.84 & 1.77 & 1.80 & 1.77 & 1.75 & 1.77 \\
\hline \multicolumn{11}{|l|}{ FEED PARAMETERS (BATCH \#) } \\
\hline Volume (liters) & 4 & 4 & 3.9 & 4 & 3.81 & 3.95 & 3.89 & 3.95 & 4 & 3.95 \\
\hline $\mathrm{pH}$ (std units) & 8 & 8 & 8.1 & g. 1 & 7.9 & 8.2 & 8.2 & 8.1 & 8 & 8 \\
\hline $\operatorname{COD}(n g / 1)$ & 180 & 185 & 202 & 198 & 232 & 204 & 206 & 184 & 299 & 211 \\
\hline NHJN (mg/1) & 215 & 215 & 210 & 210 & 210 & 210 & 198 & 198 & 198 & 186 \\
\hline SS (mg/1) & 84 & 68 & 164 & 116 & 244 & 132 & 104 & 100 & 112 & 100 \\
\hline USS (mg/l) & 47 & 38 & 91 & 64 & 136 & 78 & 61 & 59 & 80 & 71 \\
\hline CN $(m g / 1)$ & 0.616 & 0.616 & 0.616 & 0.616 & 0.616 & 0.616 & 0 & 8 & 0 & 0 \\
\hline $\operatorname{SCN}(\operatorname{ag} / 1)$ & 4.9 & 4.9 & 4.9 & 4.3 & 4.9 & 4.9 & 0 & 0 & $\theta$ & 0 \\
\hline 800 5-day (ag/l) & 3 & 3 & 3 & 3 & 3 & 3 & 3.5 & 3.5 & 3.5 & 4 \\
\hline TOC (ag/l) & 43 & 43 & 47 & 49 & 49 & 49 & 51 & 51 & II & 56 \\
\hline Phenolics $(m \mathrm{~m} / 1)$ & <. 025 & 1.025 & 1.025 & $<.025$ & 1.825 & 1.025 & 1.025 & 1.025 & 1.825 & 1.025 \\
\hline$P 04 \sim P(x g / 1)$ & 13.1 & 13.1 & $\theta$ & $\theta$ & $\theta$ & 6.3 & 8.95 & 8.95 & 8.95 & 7.5 \\
\hline Org-N $(m g / 1)$ & 0 & 0 & 0 & 0 & 0 & 5 & 0 & 0 & 0 & 0 \\
\hline NO2 (mg/l) & 10.25 & 10.25 & 10.25 & $<8.25$ & 10.25 & 0 & 8 & 0 & 0 & 0 \\
\hline N03 (mg/l) & 〈2.5 & 亿2.5 & ২2. 5 & (2.5 & (2. 5 & 0 & 0 & 0 & 0 & 0 \\
\hline $\operatorname{TDS}(m g / 1)$ & 0 & 8 & 8 & 0 & 0 & 5128 & 4915 & 4915 & 4915 & 4915 \\
\hline Color (APHA Units) & 750 & 750 & 750 & 750 & 750 & 750 & 750 & 750 & 750 & 750 \\
\hline \multicolumn{11}{|l|}{ BASIN PARAMETERS } \\
\hline Vol Wasted (nl) & 58 & 58 & 20 & 58 & 62 & 50 & 50 & 58 & 50 & 20 \\
\hline $0.0 .(\mathrm{gg} / 1)$ & 7 & 7.8 & 7 & 7 & 7.4 & 7.4 & 7.4 & 7.5 & 7.5 & 7.5 \\
\hline SS $(m g / 1)$ & 1862 & 1654 & 1320 & 1220 & 2696 & 1934 & 1870 & 1736 & 1508 & 1085 \\
\hline \#SS (PAC corrected) & 1391 & 1186 & 849 & 748 & 2217 & 1458 & 1393 & 1256 & 1034 & 615 \\
\hline rêto (L) & 21 & 2! & 19 & 18 & 18 & 20 & 19 & 19 & 19 & 19 \\
\hline oH (std units) & 7.7 & 7.2 & 7.9 & 8 & $B$ & 8 & 7.8 & 7.9 & 7.7 & 7.6 \\
\hline OUR (mg/1/hr) & & $\cdot$ & & & 2 & & & 2 & & \\
\hline VSS $(m g / 1)$ & 1481 & 1315 & 1050 & 970 & 2130 & 1528 & 1477 & 1396 & 1213 & 873 \\
\hline * VSS (PAC corrected) & 1106 & 943 & 675 & 595 & 1751 & 1152 & 1101 & 1010 & 832 & 494 \\
\hline VSS/SS & 0.80 & 0.88 & 0.80 & 0.80 & 0.79 & 0.73 & 0.79 & 0.80 & 0.80 & 0.80 \\
\hline FPAC Added (mg) & 143 & 143 & 143 & 143 & 143 & 143 & 143 & 143 & 143 & 143 \\
\hline Inventory (ag/l basin) & 471 & 468 & 471 & 472 & 479 & 476 & 477 & 480 & 474 & 478 \\
\hline \multicolumn{11}{|l|}{ EFFLUENT PARAFETERS } \\
\hline Volume (liters) & 3.8 & 3.4 & 3.5 & 3.7 & 3.45 & 3.85 & 3.7 & 3.85 & 3.8 & 3.8 \\
\hline $\operatorname{coD}(m g / 1)$ & 178 & 174 & 183 & 213 & 202 & 166 & 175 & 173 & 198 & 179 \\
\hline SS $(m g / 1)$ & 66 & 152 & 88 & 76 & 86 & 158 & 116 & 88 & 144 & 88 \\
\hline APAC corrected & 49 & 109 & 57 & 47 & 71 & 119 & 86 & 64 & 99 & 50 \\
\hline $\mathrm{pH}$ (std units) & 7.6 & 7.4 & 7.9 & 7.9 & 7.9 & 8.1 & 7.8 & 7.8 & 7.6 & 7.7 \\
\hline USS $(m g / 1)$ & 46 & 106 & 60 & 60 & 62 & 120 & 52 & 52 & 60 & 38 \\
\hline AAC corrected & 37 & 84 & 48 & 48 & 49 & 95 & 41 & 42 & 48 & 31 \\
\hline NH3̈N $(\mathrm{mg} / 1)$ & 218 & 212 & 212 & 212 & 212 & 195 & 195 & 195 & 187 & 187 \\
\hline CN $(m g / 1)$ & 0.876 & 0.876 & 0.876 & 0.876 & 0.876 & & & & & \\
\hline $\operatorname{SCN}(\operatorname{ag} / 1)$ & 3.2 & 3.2 & 3.2 & 3.2 & 3.2 & & & & & \\
\hline NO2 $(\mathrm{mg} / 1)$ & 10.25 & 10.25 & $<0.25$ & 10.25 & 10.25 & & & & & \\
\hline $\mathrm{N03}(\mathrm{ag} / 1)$ & 7.2 & 7.2 & 7.2 & 7.2 & 7.2 & & & & & \\
\hline Phenolics (mg/1) & 1.025 & 1.025 & 1.025 & 1.005 & 1.025 & 10.25 & 10.25 & 10.25 & 18.25 & 10.25 \\
\hline P04-P (ng/l) & 16.5 & & & & & 8.79 & 8.79 & 8.79 & 9.19 & 9.19 \\
\hline \multicolumn{11}{|l|}{ Org-N (ng/1) } \\
\hline TDS $(\mathrm{mg} / \mathrm{l})$ & & & & & & 5215 & 5215 & 5215 & 5215 & 5215 \\
\hline Color (АРНA units) & 750 & 750 & 750 & 750 & 750 & 875 & 875 & 875 & 875 & 875 \\
\hline TOC $(\mathrm{mg} / \mathrm{l})$ & 40 & 39 & 39 & 39 & 39 & 39 & 39 & 39 & 42 & 42 \\
\hline BOD 5-day $(\operatorname{mg} / 1)$ & 12 & 3 & 3 & 3 & 3 & 3 & 3 & 3 & 3 & 3 \\
\hline
\end{tabular}


UNIT IB TABLE AC

\begin{tabular}{|c|c|c|c|c|c|c|c|c|c|c|}
\hline MONTH & JAN & JAN & JAN & JAN & JAN & JAN & JAN & JAN & JAN & JAN \\
\hline DAY & 22 & 23 & 24 & 25 & 26 & 27 & 28 & 29 & 30 & 31 \\
\hline DAY ND. & 180 & 181 & 182 & 183 & 184 & 185 & 186 & 187 & 198 & 189 \\
\hline \multicolumn{11}{|l|}{ SYSTEM FARRAMETERS } \\
\hline ISludg̣e Age & 25.32 & 20.94 & 18.19 & 24.10 & 30.88 & 18.30 & 16.77 & 28.90 & 24.83 & 29.35 \\
\hline$E(F / M) C O D$ & 0.16 & 0.20 & 0.13 & 0.10 & 0.12 & 0.88 & 0.11 & 0.99 & 0.10 & 0.13 \\
\hline *HRT & 1.77 & 1.82 & 1.90 & 1.77 & 1.77 & 1.79 & 1.77 & 1.77 & 1.80 & 1.77 \\
\hline \multicolumn{11}{|l|}{ FEED PARAMETERS (BATCH \#) } \\
\hline Volume (liters) & 3.95 & 3.85 & 3.68 & 3.95 & 3.95 & 3.92 & 3.95 & 3.95 & 3.89 & 3.95 \\
\hline $\mathrm{OH}$ (std units) & 7.95 & 8.1 & 8.1 & 8 & 8 & 8 & 8.1 & 8 & 8.2 & 8.2 \\
\hline $\operatorname{COD}(\mathrm{mg} / 1)$ & 215 & 217 & 205 & 196 & 200 & 181 & 178 & 190 & 136 & 188 \\
\hline NH3N (mg/1) & 186 & 186 & 186 & 157 & 157 & 157 & 177 & 177 & 177 & 177 \\
\hline SS (mg/l) & 74 & 242 & 200 & 120 & 176 & 184 & 88 & 80 & 104 & 52 \\
\hline VSS (mg/1) & 53 & 172 & 139 & 83 & 122 & $\$ 18$ & 56 & 51 & 66 & 31 \\
\hline CN (ag/l) & 0 & 0 & 0 & 0.447 & 0.447 & 0.447 & 0.447 & 0.447 & 0.447 & 0.447 \\
\hline $\operatorname{SCN}(m g / l)$ & 0 & 0 & 0 & 5 & 5 & 5 & 5 & 5 & 5 & 5 \\
\hline 80D 5-day (ag/1) & 4 & 4 & 4 & 3 & 3 & 3 & 3 & 3 & 3 & 3 \\
\hline TOC $(\mathrm{ag} / 1)$ & 56 & 56 & 56 & 56 & 56 & 56 & 54 & 54 & 54 & 54 \\
\hline Phenolics ( $\square / 1)$ & 1.025 & 1.825 & 1.025 & 1.025 & <.825 & 1.825 & 1.825 & 1.025 & 1.825 & 1.025 \\
\hline PC4-P (mg/1) & 7.5 & 7.5 & 7.5 & 10.5 & 10.5 & 10.5 & 8 & 8 & 8 & \\
\hline Org-N $(\mathrm{mg} / \mathrm{l})$ & 0 & 0 & 0 & 37 & 37 & 37 & 37 & 37 & 37 & 37 \\
\hline Noe (mg/l) & 0 & 0 & 0 & 0.88 & 0.88 & 0.88 & 0.88 & 8.88 & 0.88 & 0.38 \\
\hline$N 03$ (mg/1) & 0 & 0 & 0 & 2.2 & 2.2 & 2.2 & 2.2 & 2.2 & 2.2 & 2.2 \\
\hline TDS (at/1) & 4915 & 4915 & 4915 & 5458 & 5458 & 5458 & 5458 & 5458 & 5458 & 5458 \\
\hline Color (APHA Units) & 750 & 758 & 750 & 750 & 750 & 758 & 750 & 750 & 758 & 750 \\
\hline \multicolumn{11}{|l|}{ BASIN PARAMEIERS } \\
\hline Vol Wasted (ml) & 28 & 50 & 50 & 50 & 58 & 50 & 50 & 50 & 50 & 50 \\
\hline $0.0 .(\mathrm{mg} / \mathrm{l})$ & 7.5 & 7.4 & 7.4 & 7.4 & 6.2 & 6.8 & 6.8 & 6.8 & 6.7 & 6.8 \\
\hline SS $(m g / \bar{l})$ & 1260 & 1058 & 1312 & 1556 & 1450 & 1744 & 1330 & 1596 & 1568 & 1282 \\
\hline *SS (PAC corrected) & 792 & 595 & 858 & 1104 & 997 & 1299 & 895 & 1159 & 1124 & 845 \\
\hline Temp (C) & 19 & 19 & 19 & 19 & 22 & 21 & 20 & 20 & 20 & 20 \\
\hline pH (std units) & 7.6 & 7.9 & 8 & 7.9 & 7.8 & 7.9 & 7.2 & 7.6 & 7.8 & 7.7 \\
\hline OUR $(\mathrm{mg} / 1 / \mathrm{hr})$ & & 2 & & & 2 & & & & & \\
\hline USS (畞/I) & 1013 & 854 & 1059 & 1256 & 1156 & 1390 & 1060 & 1272 & 1286 & 991 \\
\hline *VSS (PAC corrected) & 637 & 461 & 693 & 892 & 795 & 1036 & 713 & 924 & 869 & 653 \\
\hline VSS/SS & 0.80 & 0.81 & 9.81 & 0.81 & 0.80 & 8.80 & 0.80 & 0.88 & 0.77 & 0.77 \\
\hline tPAC Added (mg) & 143 & 143 & 143 & 143 & 143 & 143 & 143 & 143 & 143 & 143 \\
\hline - Inventory (on/l basin) & 468 & 463 & 454 & 452 & 453 & 445 & 435 & 437 & 436 & 437 \\
\hline \multicolumn{11}{|l|}{ EFFLUENT PARAMETERS } \\
\hline Vólufe (11ters) & 3.8 & 3.6 & 3.65 & 3.85 & 3.8 & 3.7 & 3.8 & 3.8 & 3.67 & 3.8 \\
\hline $\operatorname{coD}(\operatorname{sig} / 1)$ & 188 & 169 & 164 & 164 & 162 & 178 & 182 & 182 & 180 & 192 \\
\hline SS $(n g / 1)$ & 186 & 186 & 152 & 124 & 90 & 198 & 162 & 104 & 126 & 82 \\
\hline IPAC corrected & 67 & 68 & 99 & 88 & 62 & 147 & 109 & 76 & 91 & 54 \\
\hline ọ (std units) & 7.75 & 7.9 & $B$ & 8 & 7.8 & 7.9 & 7.4 & 7.6 & 7.5 & 7.7 \\
\hline VSS (mg/1) & 78 & 86 & 123 & 108 & 72 & 158 & 129 & 83 & 97 & 63 \\
\hline APAC corrected & 63 & 69 & 99 & 81 & 57 & 126 & 103 & 66 & 75 & 49 \\
\hline NHIN $(\mathrm{gg} / 1)$ & 187 & 187 & 161 & 161 & 161 & 150 & 150 & 150 & 158 & 150 \\
\hline$C N(m / 1)$ & & & 0.799 & 0.799 & 0.799 & 8.799 & 8.799 & 0.799 & 0.799 & \\
\hline $\operatorname{SCN}(x g / 1)$ & & & 3.3 & 3.3 & 3.3 & 3.3 & 3.3 & 3.3 & 3.3 & \\
\hline NO2 (ng/l) & & & 7.4 & 7.4 & 7.4 & 7.4 & 7.4 & 7.4 & 7.4 & \\
\hline $\mathrm{NO3}$ (ag/1) & & & 14.4 & 14.4 & 14.4 & 14.4 & 14.4 & 14.4 & 14.4 & \\
\hline Phenolics (mg/l) & 10.25 & (8.25 & 10.25 & 10.25 & 10.25 & (0.25 & (0.25 & 10.25 & $<0.25$ & <0.25 \\
\hline PO4-P (ma/1) & 9.19 & 9.19 & 8.6 & 8.6 & 8.6 & 13 & 13 & 13 & 13 & 8.7 \\
\hline $\operatorname{Org}-\mathrm{N}(\mathrm{mg} / \mathrm{l})$ & & & 72 & 72 & 72 & 72 & 72 & 72 & 72 & \\
\hline TDS (ug/l) & 5215 & 5215 & 5418 & 5418 & 5418 & 5418 & 5418 & 5418 & 5418 & 3960 \\
\hline Color (APHA units) & 875 & 875 & 1800 & 1000 & 1880 & 1008 & 1000 & 1800 & 1080 & 1080 \\
\hline TOC $(m g / 1)$ & 42 & 42 & 44 & 44 & 44 & 46 & 46 & 46 & 46 & 45 \\
\hline BOD 5-day (mg/1) & 3 & 3 & 2 & 2 & 2 & 2 & 2 & 2 & 2 & 3.6 \\
\hline
\end{tabular}




\begin{tabular}{|c|c|c|c|c|c|c|c|c|c|c|}
\hline MONTH & FEB & FEB & FEB & FEB & FEB & FEB & FEB & FEB & FEB & FEB \\
\hline DAY & 1 & 2 & 3 & 4 & 5 & 6 & 7 & 8 & 9 & \\
\hline DAY NO. & 190 & 191 & 192 & 193 & 134 & 195 & 196 & 197 & 198 & 199 \\
\hline \multicolumn{11}{|l|}{ SYSTEM PARAMETERS } \\
\hline tSludge Age & 18.15 & 40.77 & 57.19 & 29,02 & 33.67 & 53.06 & 38.50 & 31.85 & 47.59 & 47. \\
\hline$*(F / M) C O D$ & 0.38 & 0.12 & 0.07 & 0.10 & 0.11 & 0.11 & 0.10 & 0.15 & 0.15 & 0.1 \\
\hline *HRT & 1.89 & 1.65 & 1.83 & 1.85 & 1.86 & 2.80 & 1.79 & 1.77 & 1.79 & 1.7 \\
\hline \multicolumn{11}{|l|}{ FEED PARAMETERS (BATCH \#) } \\
\hline Volume (liters) & 3.7 & 4.25 & 3.83 & 3.79 & 3.77 & 3.5 & 3.9 & 3.95 & 3.9 & \\
\hline DH (std units) & 8.3 & 8.4 & 8.4 & 8.4 & 8.4 & 8.4 & 8.6 & 8.5 & 8.5 & \\
\hline $\operatorname{coD}(\operatorname{sig} / 1)$ & 206 & 199 & 286 & 223 & 288 & 227 & 229 & 237 & 274 & \\
\hline NH3N (mg/l) & 180 & 180 & 180 & 161 & 161 & $16 !$ & 161 & 154 & 154 & \\
\hline SS (mg/1) & 26 & 54 & 56 & 44 & 66 & 70 & 56 & 42 & 58 & \\
\hline USS (mg/l) & 15 & 32 & 34 & 26 & 48 & 42 & 35 & 25 & 36 & \\
\hline CN $(\operatorname{mg} / 1)$ & 0 & 0 & 0 & 0 & 0 & 0 & 0 & 0.716 & 0.716 & D. 7 \\
\hline $\operatorname{SCN}(m g / 1)$ & 8 & 8 & 0 & 8 & 8 & $\theta$ & 8 & 43.9 & 43.9 & \\
\hline BOD 5-day (mg/1) & 3.5 & 3.5 & 3.5 & 5 & 5 & 5 & 5 & 16.5 & 16.5 & 16 \\
\hline TOC (mg/!) & 59 & 59 & 59 & 56 & 56 & 56 & 56 & 78 & 78 & \\
\hline Phenolics (mg/1) & 1.025 & 1.825 & 1. 0.25 & 1.025 & 1.025 & 1.825 & 1.025 & (.025 & 1.825 & 1.8 \\
\hline P04-P (mg/l) & 3.3 & 3.3 & 3.3 & 1.9 & 1.9 & 1.9 & 1.9 & 3.6 & 3.6 & \\
\hline $\operatorname{Org}-\mathrm{N}(\mathrm{mg} / \mathrm{l})$ & 8 & $B$ & 0 & 0 & 0 & 0 & 0 & 27 & 27 & \\
\hline NO2 (mg/!) & 0 & 0 & 0 & 0 & 0 & $\theta$ & 8 & 0.92 & 0.92 & 0.9 \\
\hline $\mathrm{N} 03(\mathrm{mg} / \mathrm{l})$ & $\theta$ & 0 & 0 & 0 & 0 & 0 & 0 & 41.25 & 11.25 & 11.2 \\
\hline TDS (mo/l) & 4592 & 4592 & 4592 & 4592 & 4592 & 4592 & 4592 & 4892 & 4892 & 489 \\
\hline Color (APHA Units) & 1488 & 1408 & 1400 & 1400 & 1400 & 1400 & 1480 & 1500 & 1580 & 150 \\
\hline \multicolumn{11}{|l|}{ BASIN PARAMETEHS } \\
\hline Vol Wasted (al) & 50 & 58 & 50 & 59 & 58 & 58 & 50 & 50 & 50 & \\
\hline D.0. (mg/l) & 6.6 & 6.7 & 6.8 & 6.8 & 6.6 & 6.8 & 6.8 & 6.8 & 6.8 & \\
\hline SS $(m g / 1)$ & 814 & 1454 & 2892 & 1686 & 1472 & 1590 & 1794 & 1446 & 1508 & 150 \\
\hline *SS (PAC corrected) & 384 & 1818 & 1647 & 1246 & 1829 & 1139 & 1338 & 988 & 1043 & 183 \\
\hline Teap (C) & 21 & 20 & 20 & 20 & 20 & 20 & $2 !$ & clu & 20 & \\
\hline pll (sld units) & 7.5 & 8 & 8 & 7.5 & 7.6 & 8.1 & 8.2 & 8.5 & 8.5 & \\
\hline$(\mathrm{mg} / \mathrm{l} / \mathrm{hr})$ & & 2 & & & & 2 & & & 2 & \\
\hline USS (ng/l) & 629 & 1128 & 1623 & 1308 & 1142 & 1204 & 1358 & 1095 & 1168 & 116 \\
\hline +USS (PAC corrected) & 297 & 790 & 1270 & 967 & 798 & 862 & 1013 & 748 & A9B & \\
\hline VSS/SS & 0.77 & 0.70 & 0.78 & 0.18 & 0.78 & 0.76 & 0.76 & 0.76 & 0.77 & 0.7 \\
\hline tPAC Added (ong) & 143 & 143 & 143 & 143 & 143 & 143 & 143 & 143 & 143 & \\
\hline +Inventory (mg/l basin) & 430 & 436 & 445 & 440 & 443 & $45 !$ & 456 & 458 & 465 & \\
\hline \multicolumn{11}{|l|}{ EFFLUENT PARAMETERS } \\
\hline Volune (liters) & 3.45 & 3.9 & 3,5 & 3.95 & 3.6 & 3.3 & 3.7 & 3.7 & 3.65 & \\
\hline $\operatorname{cod}(\operatorname{mg} / 1)$ & 166 & 196 & 208 & 192 & 215 & 280 & 213 & 214 & 243 & $x$ \\
\hline SS (mg/1) & 100 & 60 & 68 & 162 & 84 & 54 & 90 & 86 & 54 & \\
\hline APAC corrected & 47 & 42 & 47 & 120 & 59 & 39 & 67 & 59 & 37 & \\
\hline pH (std units) & 7.5 & 8 & 7.9 & 7.4 & 7.7 & 8.2 & 8.3 & 8.5 & 8.5 & \\
\hline USS (mg/l) & $\pi$ & 47 & 47 & 126 & 65 & 41 & 68 & 65 & 42 & \\
\hline APAC corrected & 60 & 36 & 36 & 97 & $5 !$ & 31 & 32 & 49 & 32 & \\
\hline NHIN $(\mathrm{Mg} / 1)$ & 150 & 150 & 143 & 143 & 143 & 143 & 137 & 137 & 137 & \\
\hline CN (ag/1) & & & & & & & 1.83 & 1.83 & 1.83 & 1.8 \\
\hline $\operatorname{seN}(\mathrm{mg} / 1)$ & & & & & & & 4.4 & 4.4 & 4.4 & \\
\hline NOE (ag/1) & & & & & & & 14.5 & 14.5 & 14.5 & 14.5 \\
\hline $\mathrm{NDO}$ (ag/1) & & & & & & & 6.1 & 6.7 & 6.7 & 6. \\
\hline Phenolics (ng/l) & $<0.025$ & $<0.025$ & 18.025 & 10.025 & 10.025 & (8. 825 & 10.825 & $<0.025$ & $<0.825$ & $<8.8$ \\
\hline PO4-P (mg/l) & 8.7 & 8.7 & 4.1 & 4.1 & 4.1 & 4.1 & 2.5 & 2.5 & 2.5 & 5. \\
\hline $\operatorname{Org}-\mathrm{N}(\mathrm{mg} / \mathrm{l})$ & & & & & & & 17 & 17 & 17 & \\
\hline TDS (ag/l) & 3960 & 3960 & 3960 & 3960 & 3960 & 3960 & 5880 & 5000 & 5800 & 500 \\
\hline Color (APHA units) & 1808 & 1000 & 1800 & 1020 & 1888 & 1000 & 1500 & 1580 & 1588 & 158 \\
\hline $\mathrm{TOC}(\mathrm{ag} / 1)$ & 45 & 45 & 43 & 43 & 43 & 43 & 55 & 55 & 55 & \\
\hline BOD 5-day (mg/1) & 3.6 & 3.6 & 3 & 3 & 3 & 3 & 3 & 3 & 3 & \\
\hline
\end{tabular}


UNIT 18 TABLE AR

\begin{tabular}{|c|c|c|c|c|c|c|c|c|c|c|}
\hline MONTH & FEB & FEB & FEB & FEB & FEB & FEB & FEB & FEB & FEB & FEB \\
\hline DAY & 11 & 12 & 13 & 14 & 15 & 16 & 17 & 18 & 19 & 20 \\
\hline DAY NO. . & 200 & 201 & 202 & 203 & 204 & 205 & 206 & 297 & 208 & 209 \\
\hline \multicolumn{11}{|l|}{ SYSTEM PARAMETERS } \\
\hline \&ludge Age & $\hat{c} 7.91$ & 20.91 & 11.97 & 23.88 & 22.95 & 55.76 & 24.53 & 8.26 & 17.19 & 25.27 \\
\hline$\mp(F / M) C O D$ & 0.29 & 0.28 & 0.20 & 0.22 & 0.15 & 0.23 & 0.25 & 0.36 & 8.32 & 0.29 \\
\hline tHRT & 1.82 & 1.75 & 1.75 & 1.79 & 1.87 & $1: 93$ & 1.81 & 1.75 & 1.75 & 1.90 \\
\hline \multicolumn{11}{|l|}{ FEED PARAMETERS (BATCH } \\
\hline Volume (liters) & 3.85 & 4 & 4 & 3.9 & 3.75 & 3.63 & 3.87 & 4 & 4 & 3.68 \\
\hline pH (std units) & 8.4 & 8.2 & 8.2 & $\theta$ & 8.1 & 8.2 & 8.2 & 8.4 & 8.3 & 8.2 \\
\hline $\operatorname{COD}(\mathrm{mg} / 1)$ & 365 & 365 & 338 & 359 & 265 & 259 & 262 & 262 & 256 & 240 \\
\hline NHBN $(m g / 1)$ & 182 & 182 & 182 & 182 & 280 & 200 & 200 & 196 & 196 & 196 \\
\hline SS $(\pi g / 1)$ & 188 & 154 & 432 & 488 & 458 & 436 & 286 & 272 & 38 & 72 \\
\hline USS (ag/1) & 106 & 87 & 243 & 280 & 263 & 250 & 168 & 160 & 18 & 42 \\
\hline CN (mg/1) & 0.716 & 0.716 & 0.716 & 0.716 & 0 & 0 & 8 & 0 & 0 & \\
\hline $\operatorname{SCN}(m g / 1)$ & 43.9 & 43.9 & 43.9 & 43.9 & $\theta$ & 0 & $\theta$ & 0 & 0 & \\
\hline BOD 5-day $(\mathrm{mg} / 1)$ & 6 & 6 & 6 & 6 & 3 & 3 & 3 & 2 & 2 & 2 \\
\hline TOC $(m g / 1)$ & 94 & 94 & 94 & 94 & 76 & 76 & 76 & 73 & 73 & 73 \\
\hline Phenolics (mg/l) & 1.025 & 1.825 & 1.025 & $<.025$ & <.025 & ८.825 & 1.025 & 4.825 & <.025 & 1.025 \\
\hline PO4-P (mg/1) & 2.9 & 2.9 & 2.9 & 2.9 & 3.9 & 3.9 & 3.9 & 3.7 & 3.7 & 3.7 \\
\hline $\operatorname{Org}-\mathrm{N}(\mathrm{mg} / 1)$ & 27 & 27 & 27 & 27 & & & & & & \\
\hline NO2 (10/1) & 0.92 & 0.92 & 0.92 & 0.32 & & & & & & \\
\hline $\mathrm{NO3}(\mathrm{mg} / \mathrm{I})$ & 11.25 & 11.25 & 11.25 & 41.25 & & & & & & \\
\hline TDS (aģ/l) & 4892 & 4892 & 4892 & 4892 & 5352 & 5352 & 5352 & 5352 & 5352 & 5352 \\
\hline Color (APHA Units) & 1500 & 1500 & 1500 & 1500 & 1500 & 1508 & 1508 & 1580 & 1588 & 1500 \\
\hline \multicolumn{11}{|l|}{ BASIN PARAMETERS } \\
\hline Vol Wasted (al) & 50 & 50 & 50 & 50 & 50 & 50 & 50 & 58 & 50 & 50 \\
\hline D.0. (ब6g/l) & 6.9 & 6.9 & 6.8 & 6.8 & 6.8 & 6.9 & 6.9 & 6.9 & 6.9 & 7 \\
\hline SS $(m g / 1)$ & 1204 & 1240 & 1460 & 1378 & 1386 & 1042 & 1034 & 828 & 856 & 832 \\
\hline *SS (PAC corrected) & 732 & 774 & 1017 & 929 & 947 & 594 & 588 & 419 & 465 & 430 \\
\hline Tesp $(C)$ & 28 & 28 & 20 & 20 & 20 & 20 & 20 & 19 & 19 & 19 \\
\hline $\mathrm{pH}$ (std units) & 8.3 & 7.7 & 8. 1 & 8 & 8 & 8.2 & 8.2 & 8 & 8.1 & 8 \\
\hline OUR (mg/l/hr) & & & 2 & & & 4 & & 4 & 4 & \\
\hline USS $(m g / 1)$ & 933 & 960 & 1152 & 1081 & 1094 & 830 & 824 & 660 & 690 & 674 \\
\hline WSS (PRC corrected) & 567 & 608 & 802 & 733 & 747 & 473 & 468 & 334 & 370 & 348 \\
\hline VSS/SS & 0.77 & 0.77 & 0.79 & 0.79 & 0.79 & 0.80 & 0.80 & 0.80 & 0.80 & 0.81 \\
\hline APRC Added (mg) & 143 & 143 & 143 & 143 & 143 & $\cdot 143$ & 143 & 143 & 143 & 143 \\
\hline *Inventory (mg/l basin) & 472 & 466 & 443 & 441 & 439 & 448 & 446 & 489 & 401 & 402 \\
\hline \multicolumn{11}{|l|}{ EFFLUENT PARAMETERS } \\
\hline Volume (liters) & 3.7 & 4 & 4 & 3.6 & 3.75 & 3.62 & 3.7 & 3.8 & 3.9 & 3.45 \\
\hline $\cos (\omega g / 1)$ & 269 & 278 & 249 & 240 & 253 & 231 & 232 & 232 & 246 & 215 \\
\hline SS $(m g / 1)$ & 84 & 112 & 244 & 118 & 120 & 30 & 84 & 216 & 100 & 70 \\
\hline \&PAC corrected & 51 & 70 & 170 & 80 & 82 & 17 & 48 & 109 & 54 & 36 \\
\hline ỌH (std units) & 8.3 & 7.8 & 8. 1 & 8 & 8.1 & 8.2 & 8.2 & 8 & 8.2 & 7.9 \\
\hline USS $(m g / 1)$ & 65 & 87 & 193 & 93 & 95 & 24 & 67 & 172 & 80 & 57 \\
\hline \&AC corrected & 50 & 67 & 152 & 73 & 75 & 19 & 53 & 137 & 63 & 46 \\
\hline NHZN $(n g / 1)$ & 150 & 150 & 150 & 172 & 172 & 172 & 185 & 185 & 185 & 185 \\
\hline CN $(\mathrm{ma} / 1)$ & 1.83 & 1.83 & 1.83 & & & & & & & \\
\hline $\operatorname{SCN}(\lg / 1)$ & 4.4 & 4.4 & 4.4 & & & & & & & \\
\hline No2 (mg/l) & 14.5 & 14.5 & 14.5 & & & & & & & \\
\hline N03 (ag/1) & 6.7 & 6.7 & 6.7 & & & & & & & \\
\hline Phenolics (mg/l) & 10.025 & 10.025 & 10.025 & $<0.025$ & 10.025 & 10.025 & 10.025 & 10.025 & 10.025 & $<0.025$ \\
\hline$P 04-P(m g / 1)$ & 5.8 & 5.8 & 5.8 & 5 & 5 & 5 & 3.6 & 3.6 & 3.6 & 3.6 \\
\hline Org-N (mg/1) & 17 & 17 & 17 & & & & & & & \\
\hline $\operatorname{TDS}(\operatorname{agg} / 1)$ & 5000 & 5080 & 5000 & 5648 & 5648 & 5648 & 5648 & 5648 & 5648 & 5648 \\
\hline Color (APHA units) & 1500 & 1500 & 1500 & 1250 & 1250 & 1258 & 1258 & 1250 & 1258 & 1250 \\
\hline TOC $(\mathrm{ag} / 1)$ & 65 & 65 & 65 & 66 & 66 & 66 & 66 & 66 & 66 & 66 \\
\hline BOD 5-day (mg/l) & 4 & 4 & 4 & 3 & 3 & 3 & 3 & 3 & 3 & 3 \\
\hline
\end{tabular}




\begin{tabular}{|c|c|c|c|c|c|c|c|c|c|c|}
\hline MONTH & FEB & FEB & FEB & FEB & FEB & FEB & FEB & FEB & FEB & MAR \\
\hline DAY & 21 & 22 & 23 & 24 & 25 & 26 & 27 & 28 & 29 & 1 \\
\hline DAY NO. & 210 & 211 & 212 & 213 & 214 & 215 & 216 & 217 & $2 ! 8$ & 219 \\
\hline \multicolumn{11}{|l|}{ SYSTEM FRRAMETERS } \\
\hline \$Sludge Age & 45.40 & 33.61 & 18.82 & 27.40 & 39.67 & 24.98 & 47.48 & 29.68 & 42.88 & 26.14 \\
\hline$*(F / M) C O D$ & 0.17 & 0.18 & 0.11 & 0.16 & 0.27 & 0.21 & 0.12 & 0.26 & 0.19 & 0.21 \\
\hline EHRT & 1.87 & 1.82 & 1.90 & 1.82 & 1.75 & 1.83 & 2.85 & 1.75 & 2.08 & 1.85 \\
\hline \multicolumn{11}{|l|}{ FEED PARAMETERS (BATCH } \\
\hline Volune (liters) & 3.75 & 3.85 & 3.68 & 3.85 & 4 & 3.83 & 3.42 & 4 & 3.5 & 3.79 \\
\hline $\mathrm{pH}$ (std units) & 8 & 8.1 & 8 & 8.1 & 7.9 & 8 & 7.7 & 8 & 7.8 & 7.6 \\
\hline $\operatorname{COD}(\mathrm{mg} / 1)$ & 230 & 235 & 235 & 231 & 219 & 255 & 232 & 303 & 253 & 251 \\
\hline NHZN $(2 g / 1)$ & 196 & 196 & 196 & 196 & 188 & 188 & 188 & 188 & 179 & 179 \\
\hline SS $(\mathrm{mg} / \mathrm{l})$ & 38 & 24 & 128 & 134 & 172 & 36 & 180 & 26 & 364 & 58 \\
\hline USS (no/l) & 17 & 14 & 74 & 89 & 115 & 24 & 67 & 16 & 229 & 36 \\
\hline CN $(\mathrm{mg} /])$ & & 0.451 & 0.451 & 0.451 & 8.451 & 0.451 & 0.451 & 0.451 & & \\
\hline $\operatorname{SCN}(\operatorname{mg} / 1)$ & & 5.3 & 5.3 & 5.3 & 5.3 & 5.3 & 5,3 & 5.3 & & \\
\hline zod 5-day (mg/l) & 2 & 3 & 3 & 3 & 3 & 3 & 3 & 3 & & \\
\hline TOC $(\mathrm{mg} / 1)$ & 73 & 64.5 & 64.5 & 64.5 & 65.3 & 65.3 & 65.3 & 65.3 & 77.2 & 77.2 \\
\hline Phenolics (mg/1) & 1.025 & 1.025 & <.025 & 1.825 & 1.825 & 1.825 & & & & \\
\hline P04-P (mg/1) & 3.7 & 8.4 & 8.4 & 8.4 & 13.5 & 13.5 & 13.5 & 13.5 & & \\
\hline $\operatorname{Grg}-N(\operatorname{mg} / 1)$ & & 12 & 12 & 12 & 12 & 12 & 12 & 12 & & \\
\hline NO2 (mg/l) & & 0.36 & 0.36 & 0.36 & 8.36 & 0.36 & 0.36 & 0.36 & & \\
\hline vo3 (ng/l) & & 4 & 4 & 4 & 4 & 4 & 4 & 4 & & \\
\hline TDS $(n \underline{g} / 1)$ & 5352 & 5714 & 5714 & 5714 & 5714 & 5714 & 5714 & 5714 & & \\
\hline Color (APHA Units) & 1500 & 1500 & 1500 & 1500 & 1500 & 1500 & 1500 & 1508 & & \\
\hline \multicolumn{11}{|l|}{ BASIN PARAMETERS } \\
\hline Vol Hasted (al) & 58 & 58 & 50 & 58 & 50 & 50 & 50 & 50 & 50 & 50 \\
\hline D.0. $(\mathrm{mg} / \mathrm{l})$ & 6.8 & 6.4 & 6.4 & 6.4 & 6.4 & 6.4 & 9.1 & 9 & 9 & 9 \\
\hline SS $(\mathrm{mg} / 1)$ & 1136 & 1138 & 1634 & 1228 & 900 & 1110 & 1388 & 1092 & 1896 & 1858 \\
\hline *SS (PAC corrected) & 726 & 724 & 1225 & 817 & 483 & 693 & 963 & 665 & Gh? & 526 \\
\hline Te»p $\quad(C)$ & 19 & 20 & 19 & 19 & 19 & 19 & 19 & 19 & 19 & 18 \\
\hline $\mathrm{pH}$ (std units) & 8.2 & 8 & 7.7 & 7.8 & 7.4 & 7.8 & 7.1 & 7.3 & 7.5 & 7.4 \\
\hline OUR (mg/l/hr) & 2 & & & & & & 2 & & & \\
\hline VSS (mg/l) & 920 & 922 & 1270 & 954 & 700 & 863 & 1150 & 905 & 988 & 888 \\
\hline IVSS (PAC correrted) & 588 & 586 & 952 & 635 & 375 & 539 & 798 & 931 & 549 & 524 \\
\hline VSS/SS & 0.81 & 0.81 & 0. 78 & 0.78 & 0.78 & 0.78 & 0.83 & 0.83 & 0.83 & 0.84 \\
\hline IPAC Added (mg) & 143 & 143 & 143 & 143 & 143 & 143 & 143 & 143 & 143 & 143 \\
\hline Inventory (mg/l basin) & 410 & 414 & 409 & 411 & 417 & 417 & 425 & 427 & 434 & 434 \\
\hline \multicolumn{11}{|l|}{ EFFLUENT PARAMETERS } \\
\hline Volume (liters) & 3.6 & 3.55 & 3.5 & 3.6 & 3.95 & 3.55 & 3.25 & 3.85 & 3.3 & 3.6 \\
\hline $\operatorname{COD}(n g / 1)$ & 235 & 233 & 192 & 261 & 219 & 215 & 219 & 203 & 224 & 187 \\
\hline SS $(m g / 1)$ & 44 & 65 & 190 & 98 & 38 & 92 & 56 & 68 & 58 & 82 \\
\hline PAC corrected & 28 & 42 & 142 & 60 & 20 & 57 & 39 & 41 & 30 & 48 \\
\hline $\mathrm{pH}$ (std units) & 8.2 & 8.3 & 8 & 8 & 7.8 & 7.9 & 7 & 7.5 & 7.5 & 7.5 \\
\hline vss (mg/l) & 36 & 53 & 148 & 70 & 39 & 72 & 46 & 55 & 41 & 69 \\
\hline *PAC corrected & 29 & 43 & 115 & 54 & 23 & 56 & 38 & 47 & 34 & 58 \\
\hline NHI3N $(\operatorname{mg} / 1)$ & 181 & 181 & 181 & 181 & 181 & 181 & 181 & 176 & 176 & 176 \\
\hline CN (mg/I) & 1.12 & 1.12 & 1.12 & 1.12 & 1.12 & 1.12 & 1.12 & & & \\
\hline $\operatorname{SCN}(\operatorname{ag} / 1)$ & 3.6 & 3.6 & 3.6 & 3.6 & 3.6 & 3.6 & 3.6 & & & \\
\hline Noe $(\mathrm{mg} / 1)$ & 5.42 & 5.42 & 5.42 & 5.42 & 5.42 & 5.42 & 5.42 & & & \\
\hline NO3 $(a g / 1)$ & 5.6 & 5.6 & 5.6 & 5.6 & 5.6 & 5.6 & 5.6 & & & \\
\hline Phenolics $(a g / 1)$ & 10.025 & 10.025 & 10.025 & 10.025 & 18.025 & 10.025 & 10.025 & & & \\
\hline PO4-P (ag / 1 ) & 5.2 & 5.2 & 5.2 & 12.4 & 12.4 & 12.4 & 12.4 & & & \\
\hline Org-N (mg/l) & 11 & 11 & 11 & 11 & 11 & 11 & 11 & & & \\
\hline $\begin{array}{l}\text { TDS (ug/l) } \\
\text { Color (APHA units) }\end{array}$ & 5472 & 5472 & 5472 & 5472 & 5472 & 5472 & 5472 & & & \\
\hline $\mathrm{TOC}(\mathrm{mg} / 1)$ & 59.1 & 59.1 & 59.1 & 54.6 & 54.6 & 54.6 & 54.6 & 60.4 & 68.4 & 60.4 \\
\hline BOD 5-day (mg/l) & 6 & 6 & 6 & 4 & 4 & 4 & 4 & & & \\
\hline & & & & -98 & & & & & & \\
\hline
\end{tabular}


UNIT IB TARLE A2

\begin{tabular}{|c|c|c|c|c|c|c|c|c|c|c|}
\hline MONTH & MAR & MAR & MAR & MAR & MAR & MAR & MAR & MAR & MAR & MAR \\
\hline DAY & 2 & 3 & 4 & 5 & 6 & 7 & 8 & 9 & 10 & 11 \\
\hline DAY NO. & 220 & 221 & 222 & 223 & 224 & 225 & 226 & 227 & 228 & 229 \\
\hline \multicolumn{11}{|l|}{ SYSTEM PARAMETERS } \\
\hline ×Sludge Age & 27.09 & 4.12 & 25.29 & 48.88 & 7.36 & 25.45 & 18.04 & 15.89 & 23.17 & 13.74 \\
\hline$+(F / F) C O D$ & 0.24 & 0.27 & 0.18 & 0.88 & 0.20 & 0.30 & 0.18 & 0.27 & 0.21 & 8.21 \\
\hline *HRT & 1.91 & 1.75 & 1.86 & 2.28 & 2.84 & -1.77 & 1.86 & 1.89 & 1.79 & 1.83 \\
\hline \multicolumn{11}{|l|}{ FEED DARAMETERS (BATCH } \\
\hline Volume (liters) & 3.67 & 4 & 3.77 & 3.07 & 3.43 & 3.95 & $3 . \pi$ & 3.7 & 3.9 & 3.82 \\
\hline oH (std units) & 7.9 & 7.9 & 7.8 & 7.4 & 7.8 & 7.8 & 7.6 & 7.5 & 7.3 & 7.5 \\
\hline $\operatorname{COD}(\mathrm{mg} / 1)$ & 240 & 242 & 234 & 222 & 252 & 264 & 216 & 212 & 222 & 231 \\
\hline NH3̈N $(m g / 1)$ & 179 & 173 & 173 & 173 & 173 & 216 & 216 & 216 & 218 & 218 \\
\hline SS $(\operatorname{lgg} / 1)$ & 116 & 116 & 242 & 58 & 146 & 146 & 344 & 332 & 246 & 480 \\
\hline USS (mg/1) & 75 & 75 & 157 & 33 & 95 & 95 & 224 & 228 & 169 & 329 \\
\hline \multicolumn{11}{|l|}{ CN (ag/l) } \\
\hline \multicolumn{11}{|l|}{$\operatorname{SCN}(\operatorname{mg} / 1)$} \\
\hline BOD 5-day (mg/1) & & 3 & 3 & 3 & 3 & 3 & 3 & 3 & 5 & 5 \\
\hline $\operatorname{TOC}(\operatorname{mog} / 1)$ & 77.2 & 68.6 & 68.6 & 68.6 & 68.6 & 61.6 & 61.6 & 61.6 & 64.7 & 64.7 \\
\hline \multicolumn{11}{|l|}{ Phenolics (mg/l) } \\
\hline \multicolumn{11}{|l|}{$P 04-P(a g / 1)$} \\
\hline \multicolumn{11}{|l|}{$\operatorname{Org}-\mathrm{N}(\mathrm{mg} / \mathrm{l})$} \\
\hline \multicolumn{11}{|l|}{ NO2 (mg/1) } \\
\hline \multicolumn{11}{|l|}{$\mathrm{NC3}(\mathrm{ng} / \mathrm{l})$} \\
\hline \multicolumn{11}{|l|}{ DS (mg/l) } \\
\hline Color (APHA Units) & & & & & & & & & & * \\
\hline \multicolumn{11}{|l|}{ BASIN FARP国ETERS } \\
\hline Vol Wasted (nl) & 50 & 50 & 50 & 50 & 58 & 58 & 50 & 50 & 50 & 58 \\
\hline D.0. $(\operatorname{ag} / 1)$ & 9 & 9.2 & 9.2 & 9 & 8.8 & 8.7 & 8.7 & 8.6 & 8.5 & 8.6 \\
\hline $55 \log / 1)$ & 942 & 836 & 1028 & 1780 & 1038 & 936 & 1248 & 784 & 970 & 976 \\
\hline *SS (PAC corrected) & 588 & 490 & 679 & 1422 & 712 & 606 & 720 & 460 & 643 & 655 \\
\hline Tean (C) & 18 & 18 & 19 & 18 & 19 & 19 & 18 & 18 & 18 & 19 \\
\hline of (std units) & 7.7 & 7.7 & 7.7 & 7.9 & 8 & 7.9 & 7.9 & 7.9 & 7.7 & 7.5 \\
\hline OUR (ng/1/hr) & & & & 2 & & & 4 & & & \\
\hline VSS (no/1) & 791 & 702 & 863 & 1284 & 716 & 633 & 774 & 579 & 716 & 721 \\
\hline IVSS (PAC corrected) & 426 & 412 & 570 & 962 & 491 & 410 & 532 & 340 & 475 & 485 \\
\hline USS/SS & 0.84 & 0.84 & 0.84 & 0.68 & 0.68 & 0.68 & 0.74 & 0.74 & 0.74 & 0.74 \\
\hline ×PAC Added (mg) & 143 & 143 & 143 & 143 & 143 & 143 & 143 & 143 & 143 & 143 \\
\hline +Inventory (ag /l basin) & 434 & 346 & 349 & 358 & 326 & 330 & 328 & 324 & 327 & 320 \\
\hline \multicolumn{11}{|l|}{ EFFLUENT PARAMETERS } \\
\hline Volune (liters) & 3.5 & 4 & 3.55 & 2.95 & 3.35 & 3.85 & 3.55 & 3.5 & 3.8 & 3.65 \\
\hline $\operatorname{COD}(\operatorname{mg} / 1)$ & 183 & 214 & 199 & 247 & 243 & 194 & 199 & 281 & 190 & 192 \\
\hline SS (mg/l) & 72 & 426 & 84 & 76 & 138 & 78 & 126 & 118 & 82 & 154 \\
\hline \&PAC corrected & 39 & 250 & 56 & 61 & 95 & 45 & 87 & 65 & 54 & 104 \\
\hline pH (std units) & 7.8 & 7.6 & 7.95 & 7.6 & 8 & 7.9 & 7.9 & 7.9 & 7.8 & 7.6 \\
\hline VSS (mg/l) & 68 & 358 & 71 & $5 !$ & 61 & 47 & 93 & 81 & 61 & 114 \\
\hline *PAC corrected & 51 & 300 & 59 & 35 & 42 & 32 & 69 & 68 & 45 & 84 \\
\hline NH3N (mg/l) & 112 & 112 & 112 & 112 & 198 & .198 & 198 & 159 & 159 & 159 \\
\hline \multicolumn{11}{|l|}{ CN $(m g / d)$} \\
\hline \multicolumn{11}{|l|}{$\operatorname{SCN}(1+\mathrm{g} / \mathrm{l})$} \\
\hline NO2 (mg/l) & & & & & & & & & & \\
\hline N03 $(\mathrm{sg} / 1)$ & & & & & & & & & & \\
\hline Phenolics (mg/1) & & & & & & & & & & \\
\hline$P 04-P(\log / 1)$ & & & & & & & & & & \\
\hline $\operatorname{Org}-\mathrm{N}(\mathrm{mg} / 1)$ & & & & & & & & & & \\
\hline TDE (mg/l) & & & & & & & & & & \\
\hline Color (APHA units) & & & & & & & & & & \\
\hline $\operatorname{TOC}(\log / 1)$ & 55.4 & 55.4 & 55.4 & 55.4 & 56.2 & 56.2 & 56.2 & 48.5 & 48.5 & 48.5 \\
\hline 800 5-day (mq/1) & & & & & & & & & & \\
\hline & & & & -99 & & & & & & \\
\hline
\end{tabular}


UNIT IB TABLE AR

\begin{tabular}{|c|c|c|c|c|c|}
\hline NONTH & MAR & MAR & MAR & MAR & MAR \\
\hline DAY & 12 & 13 & 14 & 15 & 16 \\
\hline DAY NO. & 230 & 231 & 232 & 233 & 234 \\
\hline \multicolumn{6}{|l|}{ SYSTFM PARAMETERS } \\
\hline *Sludge Rge & 16.15 & 16.65 & 17.54 & 17.65 & 13.73 \\
\hline$\$(F / M) C O D$ & 0.22 & 0.25 & 0.23 & 0.23 & 0.23 \\
\hline *HRT & 1.82 & 1.77 & 1.89 & 1.77 & 1.94 \\
\hline \multicolumn{6}{|l|}{ FEED PRRAMETERS (BATCH ) } \\
\hline Volume (liters) & 3.84 & 3.95 & 3.7 & 3.95 & 3.8 \\
\hline of (std units) & 7.7 & 7 & 6.9 & 7.5 & 7.9 \\
\hline $\operatorname{COD}(\mathrm{mg} / 1)$ & 239 & 224 & 238 & 244 & 255 \\
\hline NHZN $(m g / 1)$ & 218 & 218 & 208 & 208 & 208 \\
\hline SS $(m g / 1)$ & 214 & 480 & 128 & 88 & $360^{\circ}$ \\
\hline VSS $(a \underline{a g} / 1)$ & 147 & 329 & 88 & 68 & 261 \\
\hline \multicolumn{6}{|l|}{$C N(a g / 1)$} \\
\hline \multicolumn{6}{|l|}{$\operatorname{SCN}(\mathrm{mg} / 1)$} \\
\hline BOD 5-day (mg/1) & 5 & 5 & 0 & 0 & 0 \\
\hline TOC (用o $/ 1)$ & 64.7 & 64.7 & 71 & 71 & 71 \\
\hline \multicolumn{6}{|l|}{ Phenolics (mg/l) } \\
\hline \multicolumn{6}{|l|}{$P 04-P(a g / 1)$} \\
\hline \multicolumn{6}{|l|}{$\operatorname{Org}-N(\mathrm{mg} / 1)$} \\
\hline \multicolumn{6}{|l|}{ NO2 (ng/1) } \\
\hline \multicolumn{6}{|l|}{$\mathrm{NC} 3(\mathrm{mg} / \mathrm{l})$} \\
\hline \multicolumn{6}{|l|}{ TDS (mg/l) } \\
\hline Color (APHA Units) & & & & . & \\
\hline \multicolumn{6}{|l|}{ BASIN PARAMETERS } \\
\hline Vol Nasted (ml) & 58 & 58 & 50 & 58 & 58 \\
\hline D.0. $(\mathrm{gg} / 1)$ & 8.9 & 8.7 & 8.8 & 8.8 & 8.1 \\
\hline SS (mg/1) & 1026 & 912 & 954 & 986 & 978 \\
\hline ASS (PAC corrected) & 710 & 598 & 641 & 674 & 664 \\
\hline Tewp (C) & 18 & 19 & 19 & 19 & 19 \\
\hline $\mathrm{pH}$ (std units) & 7.5 & 7.4 & 7.3 & 7.3 & 7 \\
\hline OUR $(\mathrm{mg} / 1 / \mathrm{hr})$ & 3 & & & 3 & \\
\hline $\operatorname{vSg}(\mathrm{mo} / 1)$ & 708 & 629 & 658 & 702 & 691 \\
\hline *VSS (PAC ccirrected) & 490 & 413 & 448 & 480 & 473 \\
\hline VSS/SS & 0.69 & 0.69 & 0.69 & 8.71 & 0.71 \\
\hline WFAC Added (mg) & 143 & 143 & 143 & 143 & 143 \\
\hline traventory (wo/l basin) & 316 & 314 & 313 & 312 & 306 \\
\hline \multicolumn{6}{|l|}{ EFF_LENT PARAMETERS } \\
\hline Volume (1ittcrs) & 3.75 & 3.8 & 3.5 & 3.85 & 3.45 \\
\hline $\cos (n g / 1)$ & 287 & 209 & 212 & 207 & 203 \\
\hline SS $(\operatorname{mg} / 1)$ & 132 & 112 & 120 & 112 & 162 \\
\hline PAC corrected & 91 & 73 & 81 & 77 & 111 \\
\hline oH (std units) & 7.5 & 7.5 & 7.6 & 7.5 & 7.6 \\
\hline VSS $(a g / 1)$ & 91 & $\pi$ & 83 & 88 & 119 \\
\hline EPAC corrected & 63 & 53 & 57 & 57 & 82 \\
\hline NH3N (ag/l) & 159 & 213 & 213 & 213 & 213 \\
\hline \multicolumn{6}{|l|}{ CN $(m g / 1)$} \\
\hline \multicolumn{6}{|l|}{ SCN (ag/l) } \\
\hline \multicolumn{6}{|l|}{ NDD $(\mathrm{mg} / 1)$} \\
\hline \multicolumn{6}{|l|}{ NO3 $(\mathrm{mg} / 1)$} \\
\hline \multicolumn{6}{|l|}{ Phenolics $(\mathrm{mg} / \mathrm{l})$} \\
\hline $\mathrm{PO4-p}(\mathrm{mg} / 1)$ & & & & & \\
\hline Org-N (mg/1) & & & & & \\
\hline $\operatorname{DDS}(\mathrm{ng} / 1)$ & & & & & \\
\hline Color (APHA units) & & & & & \\
\hline TOC $(m g / 1)$ & 48.5 & 60 & 60 & 60 & 68 \\
\hline BOD 5-day $(\operatorname{mg} / 1)$ & & & & & \\
\hline & & & & $-100-$ & \\
\hline
\end{tabular}




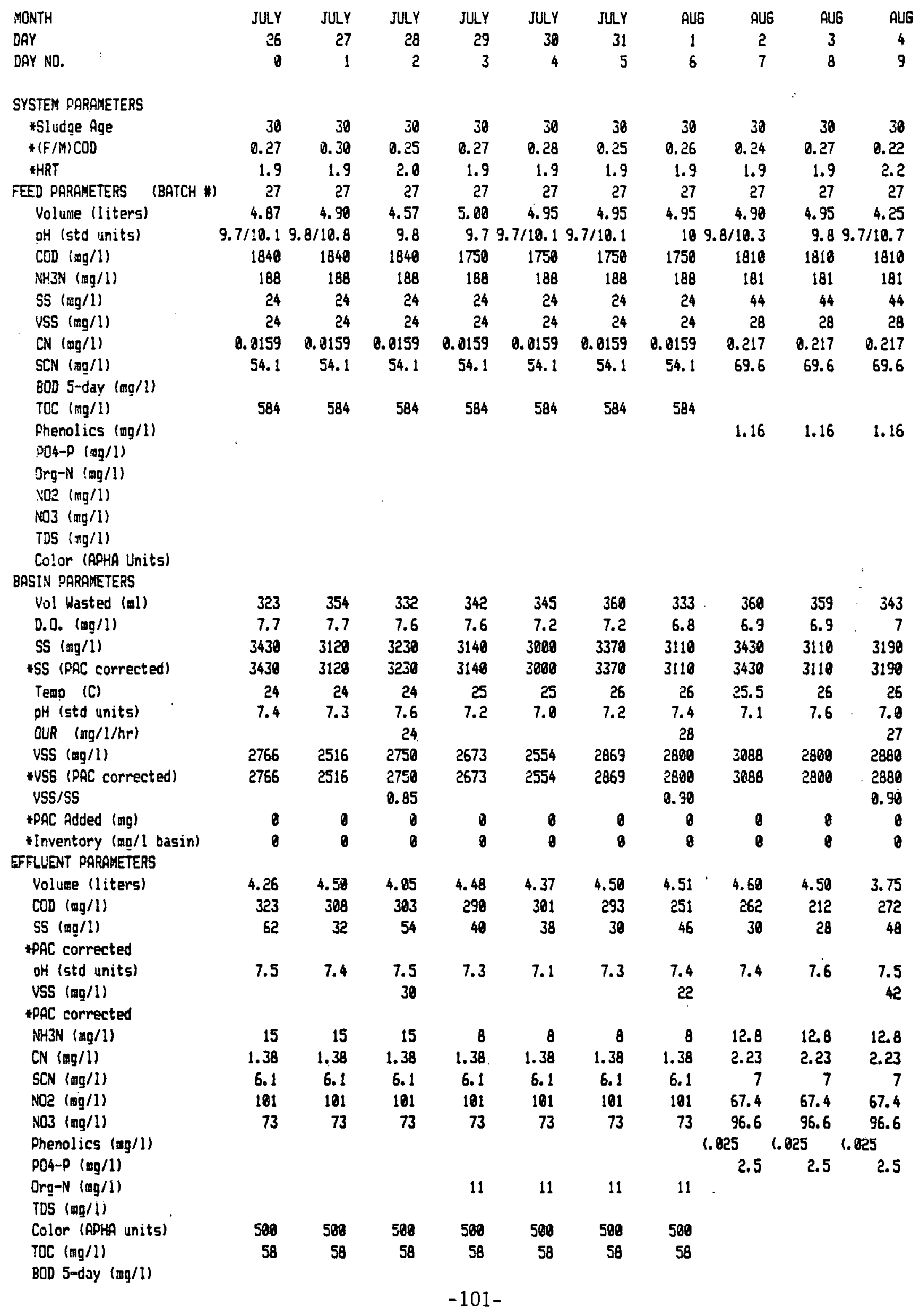




\begin{tabular}{|c|c|c|c|c|c|c|c|c|c|c|}
\hline MONTH & AUG & AUG & RUG & ALG & AUG & RUE & AUG & AUG & AUG & AUG \\
\hline DAY & 5 & 6 & 7 & 8 & 9 & 10 & 11 & 12 & 13 & 14 \\
\hline DAY NO. & 10 & 11 & 12 & 13 & 14 & 15 & 16 & 17 & 18 & 19 \\
\hline \multicolumn{11}{|l|}{ SYSTEM FARAMETERS } \\
\hline \$Sl udoge Age & 30 & 30 & 30 & 30 & 30 & 30 & 30 & 30 & 30 & 30 \\
\hline$*(F / M) C O D$ & 0.2 .2 & 0.24 & 0.20 & 0.23 & 0.22 & 0.25 & 0.23 & 0.25 & 0.26 & 0.28 \\
\hline *HRT & 2.2 & 2.0 & 2.1 & 2.0 & 2.3 & 2.1 & 2.0 & 2.1 & 2.2 & 1.9 \\
\hline FEED PARAMETERS (BATCH & 27 & 27 & 27 & 27 & 27 & 27 & 28 & 28 & 28 & 28 \\
\hline Volune (liters) & 4.23 & 4.62 & 4.47 & 4.70 & 4.80 & 4.45 & 4.60 & 4.35 & 4.30 & 4.90 \\
\hline $\mathrm{pH}$ (std units) & $9.8 / 10.8$ & 9.8 & 9.8 & 9.8 & 9.8 & 9.8 & $10 / 10.3$ & $9.9 / 10.4$ & 9.7 & 9.7 \\
\hline $\cos (\operatorname{mg} / 1)$ & 1745 & 1745 & 1745 & 1745 & 1858 & 1858 & 1858 & 2159 & 2159 & 2159 \\
\hline NHĒN $(0 g / 1)$ & 176 & 176 & 176 & 176 & 173 & 173 & 173 & 185 & 185 & 185 \\
\hline SS $(\mathrm{mg} / 1)$ & 44 & 44 & 44 & 44 & 100 & 180 & 100 & 100 & 100 & 100 \\
\hline VSS (mg/l) & 28 & 28 & 28 & 28 & 32 & 32 & 32 & 32 & 32 & 32 \\
\hline$C N(m g / 1)$ & 0.217 & 0.217 & 0.217 & 0.217 & 0.481 & 0.481 & 0.481 & 0.481 & 0.481 & 0.481 \\
\hline $\operatorname{SCN}(m g / 1)$ & 69.6 & 69.6 & 69.6 & 69.6 & 149 & 149 & 149 & 149 & 149 & 149 \\
\hline \multicolumn{11}{|l|}{ BOD 5-day $(\mathrm{mg} / \mathrm{l})$} \\
\hline$T E C$ ing/1) & & & & & 632 & $63 ?$ & $6.3 ?$ & 632 & 632 & 632 \\
\hline \multicolumn{11}{|l|}{ PCT-P (ang/1) } \\
\hline \multicolumn{11}{|l|}{$\operatorname{Srg}-\mathrm{N}(m \mathrm{~g} / 1)$} \\
\hline \multicolumn{11}{|l|}{ y0อ (ng/1) } \\
\hline \multicolumn{11}{|l|}{$: 403(\pi \underline{g} / 1)$} \\
\hline \multicolumn{11}{|l|}{ TOS $(\pi g / 1)$} \\
\hline \multicolumn{11}{|l|}{ Color (APHA Units) } \\
\hline \multicolumn{11}{|l|}{ BASIN JARAMETERS } \\
\hline Vol Hasted (al) & 352 & 330 & 353 & 350 & 363 & 374 & 378 & .384 & 367 & 355 \\
\hline D.0. $(\mathrm{mg} / 1)$ & 7.1 & 7.3 & 7.2 & 7.2 & 6.9 & 7.6 & 7.5 & 7.4 & 7.8 & 7.8 \\
\hline SS $(\mathrm{gg} / \mathrm{l})$ & 3168 & 3140 & 3600 & 3370 & 3180 & 3040 & 3398 & 3460 & 3300 & 3430 \\
\hline *SS (PAC corrected) & 3160 & 3140 & 3600 & 3370 & 3180 & 3040 & 3590 & 3468 & 3300 & 3430 \\
\hline Trapn $(r)$. & 26 & 36 & 86 & 27 & 27 & 25 & 25 & 24 & 23 & 22 \\
\hline pH (std units) & 7.2 & 7.3 & 7.4 & 7.0 & 7.0 & 7.7 & 7.0 & 7.4 & 7.5 & 7.5 \\
\hline OUR $(\mathrm{ng} / 1 / \mathrm{hr})$ & & & & 22 & & & 20 & & & \\
\hline VSS (mo/1) & 2853 & 2835 & 3250 & 3000 & 2831 & 2706 & 3070 & 3133 & 2988 & 3106 \\
\hline $\begin{array}{l}\text { *VS9 (PAC Lurrected) } \\
\text { VS5/S5 }\end{array}$ & 2853 & 2835 & 3050 & $\begin{array}{l}3000 \\
0.89\end{array}$ & 2931 & 2706 & $\begin{array}{l}3070 \\
0.91\end{array}$ & 3133 & 2988 & 3106 \\
\hline tPAC Added (mg) & 0 & 8 & 0 & 0 & 0 & 0 & 8 & 0 & 8 & 0 \\
\hline Inventory (mo/1 basin) & 0 & 0 & 0 & $\theta$ & 0 & 0 & 8 & 0 & 0 & 8 \\
\hline \multicolumn{11}{|l|}{ EFFLLENT PARAMETERS } \\
\hline Volume (liters) & 3.78 & 4.05 & 4.00 & 3.80 & 3.65 & 3.95 & 3.90 & 3.90 & 3.85 & 4.27 \\
\hline COD $($ mo/l) & 272 & 272 & 228 & 220 & 223 & 3 & 284 & 190 & 239 & 230 \\
\hline SS $(\operatorname{agg} / 1)$ & 40 & 54 & 42 & 44 & 32 & 20 & 26 & 14 & 28 & 36 \\
\hline FPAC corrected & & & & & 44 & 22 & 22 & 18 & 22 & 28 \\
\hline oH (std units) & 7.2 & 7.6 & 7.5 & 7.2 & 7.2 & 7.0 & 7.1 & 7.3 & 7.5 & 7.6 \\
\hline USS $(a g / 1)$ & & & & 40 & & & 16 & & & \\
\hline FPAC corrected & & & & & & & 18 & & & \\
\hline NHzN $(n g / 1)$ & 5.9 & 5.9 & 5.9 & 5.9 & 9.7 & 9.7 & 9.7 & 18.8 & 18.8 & 18.8 \\
\hline CN $(a g / 1)$ & 2.23 & 2.23 & 2.23 & 2.23 & 18.886 & 10.006 & 18.006 & 10.006 & 10.006 & 10.085 \\
\hline $\operatorname{scn}(a g / 1)$ & 7 & 7 & 7 & 7 & 7.5 & 7.5 & 7.5 & 7.5 & 7.5 & 7.5 \\
\hline NO2 (ng/1) & 67.4 & 67.4 & 67.4 & 67.4 & 43.8 & 43.8 & 43.8 & 43.8 & 43.8 & 43.8 \\
\hline$N 03(0 \mathrm{~g} / 1)$ & 96.6 & 96.6 & 96.6 & 96.6 & 116 & 116 & 116 & 116 & 116 & 116 \\
\hline Phenolics (ang/I) & 1.025 & 1.025 & 1.025 & ২.025 & & & & & & \\
\hline PO4-P (ag/1) & 2.5 & 2.5 & 2.5 & 2.5 & & & & & & \\
\hline $\begin{array}{l}\text { Org-N (mg/1) } \\
\text { TDS (qq/1) }\end{array}$ & 14.4 & 14.4 & 14.4 & 14.4 & & & & 18.7 & 18.7 & 18.7 \\
\hline Color (APHA units) & & & & & 580 & 580 & 500 & 500 & 500 & 500 \\
\hline TOC $(n g / 1)$ & & & & & 58 & 58 & 58 & 58 & 58 & 58 \\
\hline
\end{tabular}


UNIT $2 A$ TABLE A3

\begin{tabular}{|c|c|c|c|c|c|c|c|c|c|c|}
\hline MONTH & AUG & AUG & RUG & RUG & AUG & AUG & AUG & AUG & AULG & ALG \\
\hline DAY & 15 & 16 & 17 & 18 & 19 & 28 & 21 & 22 & 23 & 24 \\
\hline DAY NO. & 20 & 21 & 22 & 23 & 24 & 25 & 26 & 27 & 28 & 29 \\
\hline \multicolumn{11}{|l|}{ SYSTEM PARAMETERS } \\
\hline \&Sludge Age & 30 & 30 & 30 & 30 & 30 & 30 & 30 & 30 & 30 & 30 \\
\hline$\forall$ (F/M)COD & 0.26 & 0.29 & 0.27 & 0.23 & 0.27 & 0.28 & 0.29 & 8.24 & 8.26 & 0.28 \\
\hline *HRT & 2.0 & 1.9 & 1.9 & 1.9 & 1.9 & 1.9 & 1.9 & 2.0 & 1.9 & 2.1 \\
\hline FEED PARAPETERS (BATCH ) & 28 & 28 & 28 & 28 & 28 & 28 & 28 & 28 & 28 & 28 \\
\hline Volume (1iters) & 4.55 & 4.95 & 4.93 & 4.80 & 5.80 & 5.80 & 5.00 & 4.70 & 4.90 & 4.40 \\
\hline pH (std units) & 9.8 & 10 & 9.8 & 9.9 & 9.7 & 9.8 & 9.8 & \multicolumn{3}{|c|}{ 3.9/10.39.9/10.2 9.8/10.2 } \\
\hline $\operatorname{cod}(\operatorname{mg} / 1)$ & 2159 & 2051 & $285 !$ & 2051 & 2105 & 2105 & 2105 & 2105 & 2185 & 2185 \\
\hline NHS̄N $(m g / 1)$ & 185 & 190 & 190 & 190 & 183 & 183 & 183 & 183 & 180 & 180 \\
\hline SS $(\mathrm{mg} / \mathrm{ll})$ & 100 & 18 & 18 & 18 & 18 & 18 & 18 & 18 & 40 & 40 \\
\hline VSS (mg/1) & 32 & 18 & 18 & 18 & 18 & 18 & 18 & 18 & 18 & 10 \\
\hline CN (mg/1) & 0.481 & 0.559 & 0.559 & 0.559 & 0.559 & 0.559 & 0.559 & 0.559 & 0.739 & 0.739 \\
\hline $\operatorname{SCN}(\operatorname{mg} / 1)$ & 149 & 174 & 174 & 174 & 174 & 174 & 174 & 174 & 177 & 177 \\
\hline BOD 5-day (mg/1) & & & & & & & & & 1290 & 1290 \\
\hline TOC $(\operatorname{sg} / 1)$ & 632 & & & & & & & & 645 & 645 \\
\hline $\begin{array}{l}\text { Phenolics (ogg/l) } \\
\text { fo4-p (ag/l) }\end{array}$ & & 1.56 & 1.56 & 1.56 & 1.56 & 1.56 & 1.56 & 1.56 & & \\
\hline \multicolumn{11}{|l|}{$O r g-N(m g / 1)$} \\
\hline \multicolumn{11}{|l|}{ NOL $(\mathrm{mg} / 1)$} \\
\hline \multicolumn{11}{|l|}{$\mathrm{NO3}(\mathrm{mg} / 1)$} \\
\hline \multicolumn{11}{|l|}{$\operatorname{TDS}(\operatorname{mo} / 1)$} \\
\hline \multicolumn{11}{|l|}{ Color (APHA Units) } \\
\hline \multicolumn{11}{|l|}{ BASIN FARAMETERS } \\
\hline Vol Nasted (al) & 367 & 366 & 380 & 355 & 374 & 373 & 363 & 354 & 302 & 328 \\
\hline D.0. $(\operatorname{mog} / 1)$ & 7.8 & 7.7 & 7.9 & 7.3 & 7.6 & 7.4 & 7.5 & 6.7 & 7.1 & 7.3 \\
\hline SS (mg/1) & 3498 & 3220 & 3440 & 3840 & 3458 & 3300 & 3270 & 3798 & 3780 & 3230 \\
\hline *SS (PAC corrected) & 3498 & 3220 & 3440 & 3840 & 3490 & 3380 & 3278 & 3798 & 3780 & 3230 \\
\hline Tewp (C) & 22 & 23 & 23.5 & 25 & 24 & 25 & 25 & 24 & 25 & 24 \\
\hline pH (std units) & 7.6 & 7.7 & 7.8 & 7.6 & 7.5 & 7.4 & 7.6 & 7.4 & 7.1 & 7.1 \\
\hline OUR (ng/l/hr) & 24 & & & 32 & & & & 36 & & \\
\hline USS (ag/1) & 3118 & 2869 & 3065 & 3618 & 3281 & 3182 & 3074 & 3480 & 3391 & 2898 \\
\hline +USS (PAC corrected) & 3110 & 2869 & 32.55 & 3610 & 3281 & 3102 & 3074 & 3400 & 3391 & 2898 \\
\hline USS/SS & 0.89 & & & 0.34 & & & & 0.90 & & \\
\hline FPAC Added (mg) & 8 & 0 & 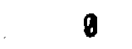 & 0 & e & 0 & $\theta$ & 0 & a & 0 \\
\hline Invẹntory (mg̣/l basin) & 0 & 8 & 8 & 0 & 0 & 8 & 8 & 0 & 0 & 8 \\
\hline \multicolumn{11}{|l|}{ EFFLUENT PARAMETERS } \\
\hline Volune (liters) & 4.10 & 4.58 & $4: 38$ & 4.28 & 4.52 & 4.46 & 4.30 & 4.12 & 4.42 & 4.00 \\
\hline $\operatorname{cod}(\operatorname{mg} / 1)$ & 265 & 276 & 258 & 287 & 290 & 290 & 289 & 303 & 320 & 329 \\
\hline SS (mg/1) & 28 & 24 & 16 & 40 & 20 & 20 & 28 & 42 & 84 & 58 \\
\hline PAC corrected & 38 & 58 & 16 & 36 & 40 & 22 & 28 & 48 & 58 & 38 \\
\hline $\mathrm{pH}$ (std units) & 7.5 & 7.8 & 7.9 & 7.7 & 7.6 & 7.7 & 7.6 & 7.5 & 7.3 & 7.4 \\
\hline VSS $(m g / 1)$ & 16 & & & 34 & & & & 30 & & \\
\hline \#PAC corrected & 26 & & & 28 & & & & 32 & & \\
\hline NHZN $(\mathrm{ag} / 1)$ & 18.8 & 34.8 & 34.8 & 34.8 & 32.2 & 32.2 & 32.2 & 32.2 & 34.2 & 34.2 \\
\hline CN $(a g / 1)$ & 18.006 & 3.28 & 3.28 & 3.28 & 3.28 & 3.28 & 3.28 & 3.28 & 2.37 & 2.37 \\
\hline $\operatorname{SCN}(\operatorname{ag} / 1)$ & 7.5 & 4.9 & 4.9 & 4.9 & 4.9 & 4.9 & 4.9 & 4.9 & 4.1 & 4.1 \\
\hline$N 02(8 g / 1)$ & 43.8 & 85.1 & 85.1 & 85.1 & 85.1 & 85.1 & 85.1 & 85.1 & 97.1 & 97.1 \\
\hline $\mathrm{NO3}(\mathrm{gg} / \mathrm{ll})$ & 116 & 96.9 & 96.9 & 96.9 & 96.9 & 96.9 & 96.9 & 96.9 & 83 & 83 \\
\hline Phenolics (mg/1) & & $<0.025$ & $<0.025$ & $<0.025$ & 10.825 & 10.025 & $<0.025$ & $<0.025$ & & \\
\hline$P O 4-P$ (ag/l) & & 4.2 & 4.2 & 4.2 & 4.2 & 4.2 & 4.2 & 4.2 & & \\
\hline $\begin{array}{l}\text { Org-N (ag/l) } \\
\text { TOS }(m g / 1)\end{array}$ & 10.7 & & & & 17.2 & 17.2 & 17.2 & 17.2 & & \\
\hline Color (ADHA units) & 500 & & & & & & & & 750 & 750 \\
\hline TOC $(\mathrm{mg} / 1)$ & 58 & & & & & & & & 69.6 & 69.6 \\
\hline BOD 5-day (mg/1) & & & & -103 & & & & & & \\
\hline
\end{tabular}


UNIT ZA TABLE A3

\begin{tabular}{|c|c|c|c|c|c|c|c|c|c|c|}
\hline MONTH & AU6 & AUG & AUG & RUG & AUG & AUE & AUG & SEPT & SEPT & SEPT \\
\hline DAY & 25 & 26 & 27 & 28 & 29 & 20 & 31 & 1 & 2 & 3 \\
\hline DAY NO. & 30 & 31 & 32 & 33 & 34 & 35 & 36 & 37 & 38 & 39 \\
\hline \multicolumn{11}{|l|}{ SYSTEM SRRAMETERS } \\
\hline †Sludge Age & 30 & 38 & 30 & 30 & 30 & 30 & 30 & 30 & 30 & 30 \\
\hline$F(F / M) C O D$ & 0.32 & 0.28 & 0.27 & 0.30 & 0.26 & 0.05 & 0.30 & 0.28 & 0.25 & 0.27 \\
\hline HRT & 1.9 & 1.9 & 1.9 & 1.9 & 2.0 & 18.9 & 1.9 & 2.8 & 2.8 & 1.9 \\
\hline FEED PARRMETERS (BRTCH \$) & 28 & 28 & 28 & 29 & 29 & 29 & 29 & 29 & 29 & 29 \\
\hline Volune (1 iters) & 5.80 & 4.83 & 4.85 & 4.88 & 4.56 & 0.85 & 4.83 & 4.62 & 4.70 & 4.88 \\
\hline oh (std units) & $9.7 / 10.49$ & $1.7 / 10.2$ & 9.79 & $7 / 10.49$ & $7 / 124$ & $8 / 10.43$ & $5 / 10.2$ & 9.5 & 9.75 & $7 / 18.2$ \\
\hline $\operatorname{COD}(\operatorname{mg} / 1)$ & 2185 & 2130 & 2130 & 2130 & 2130 & 2070 & 2070 & 2070 & 2022 & 2022 \\
\hline NH3N $(\mathrm{mg} / 1)$ & 180 & 184 & 184 & 184 & 184 & 179 & 179 & 179 & 184 & 184 \\
\hline SS (ag/l) & 40 & 40 & 40 & 40 & 40 & 26 & 26 & 26 & 26 & 26 \\
\hline USS (mg/1) & 10 & 10 & 10 & 10 & 10 & 14 & 14 & 14 & 14 & 14 \\
\hline$C N(m g / l)$ & 0.739 & 0.739 & 0.739 & 0.739 & 0.739 & 0.25 & 0.25 & 0.25 & 0.25 & 0.25 \\
\hline SCN $(m g / 1)$ & 177 & 177 & 177 & 177 & 177 & 182 & 182 & 182 & 192 & 182 \\
\hline 80D 5-day $(m g / 1)$ & 1299 & 1290 & 1290 & 1470 & 1478 & 1470 & 1470 & 1470 & 1470 & 1470 \\
\hline TOC $(m g / 1)$ & 645 & 645 & 645 & 645 & 645 & & & & & \\
\hline Phenolics (mg/1) & & & & & & 1.48 & 1.48 & 1.48 & 1.48 & 1.48 \\
\hline \multicolumn{11}{|l|}{$P 04-P(m g / 1)$} \\
\hline \multicolumn{11}{|l|}{ Jrq-N $(m q / 1)$} \\
\hline \multicolumn{11}{|l|}{ NO2 $(m g / 1)$} \\
\hline \multicolumn{11}{|l|}{$.603(\mathrm{mg} / 1)$} \\
\hline \multicolumn{11}{|l|}{ TDS $(3 g / 1)$} \\
\hline \multicolumn{11}{|l|}{ Color (APHA Units) } \\
\hline \multicolumn{11}{|l|}{ BASIN PARAMETERS } \\
\hline Vol idasted (mI) & 362 & 326 & 352 & 353 & 395 & 399 & 390 & 398 & 369 & 368 \\
\hline D.0. (mg/l) & 6.4 & 7.2 & 7.8 & 7.8 & 7.8 & 7.2 & 7.4 & 7.2 & 6.9 & 6.8 \\
\hline SS $(m g / 1)$ & 3260 & 3420 & 3570 & 3290 & 3350 & 3070 & 3000 & 3020 & 3320 & 3160 \\
\hline \SS (PAC corrected) & 3260 & 3420 & 3570 & 3290 & 3350 & 3078 & 3000 & 3020 & 3320 & 3160 \\
\hline Temp (C) & 24 & 24.5 & 25 & 24 & 25 & 24 & 24 & 24 & 24 & 24 \\
\hline $\mathrm{pH}$ (std units) & 6.9 & 7.3 & 7.4 & 6.9 & 7.1 & 7.2 & 7.2 & 7.4 & 7.1 & 7.3 \\
\hline OUR $(\mathrm{ng} / 1 / \mathrm{hr})$ & 36 & & & & 36 & & & 38 & & \\
\hline VSE $(n g / l)$ & 2880 & 3021 & 3154 & 2907 & 3120 & 2859 & 2794 & 2870 & 3155 & 3003 \\
\hline "YSS (PAC corrected) & 2000 & 3021 & 3154 & 2307 & $31 \varepsilon 0$ & $\varepsilon 053$ & $E 734$ & EB70 & 3155 & 3003 \\
\hline VSš/SS & 0.88 & & & & 0.93 & & & 0.95 & & \\
\hline *PAC Added (mg) & 0 & $\theta$ & 0 & 8 & 8 & 0 & 0 & 0 & 0 & $\theta$ \\
\hline \multicolumn{10}{|l|}{ EFFLUENT PARAMETERS } & 0 \\
\hline Volume (liters) & 4.73 & 4.34 & 4.32 & 4.33 & 4.10 & 0.63 & 3.90 & 4.22 & 4.62 & 4.27 \\
\hline $\operatorname{COD}(\mathrm{mg} / 1)$ & 316 & 322 & 279 & 291 & 260 & 281 & 213 & 178 & 193 & 186 \\
\hline SS $(\mathrm{mg} / \mathrm{l})$ & 26 & 58 & 40 & 36 & 4 & 2 & 8 & 2 & 22 & 24 \\
\hline \#PAC corrected & 4 & 26 & 30 & 48 & 18 & 4 & 22 & 8 & 32 & 38 \\
\hline $\mathrm{pH}$ (std units) & 7.2 & 7.4 & 7.5 & 7.2 & 7.3 & 7.2 & 7.2 & 7.4 & 7.4 & 7.4 \\
\hline VSS $(0 g / 1)$ & 22 & & & & 4 & & & 2 & & \\
\hline HPAC corrented & 4 & & & & 18 & & & 8 & & \\
\hline NH3N (mg/l) & 34.2 & 21.6 & 21.6 & 21.6 & 21.6 & 3.9 & 9.9 & 9.9 & 9.2 & 9.2 \\
\hline CN $(m g /)$ & 2.37 & 2.37 & 2.37 & 2.37 & 2.37 & 1.96 & 1.96 & 1.96 & 1.96 & 1.96 \\
\hline SCN $(n g / 1)$ & 4.1 & 4.1 & 4.1 & 4.1 & 4.1 & 5.9 & 5.9 & 5.9 & 5.9 & 5.9 \\
\hline NO2 (mg/l) & 97.1 & 97.1 & 97.1 & 97.1 & 97.1 & 22.9 & 22.9 & 22.9 & 22.9 & 22.9 \\
\hline $\mathrm{No3}(\mathrm{sg} / \mathrm{l})$ & 63 & 83 & 83 & 83 & 83 & 182 & 182 & 182 & 182 & 182 \\
\hline Phenolics (mg/1) & & & & & & 1.025 & 1.025 & 1.025 & 1.825 & 1.825 \\
\hline$P 04-P$ (ag/l) & & & & & & 3.9 & 3.9 & 3.9 & 3.9 & 3.9 \\
\hline $\begin{array}{l}\mathrm{Org}-\mathrm{N}(\mathrm{mg} / 1) \\
\operatorname{TDS}(\mathrm{mg} / 1)\end{array}$ & & 10.7 & 10.7 & 10.7 & 10.7 & & & & 3.5 & 3.5 \\
\hline Color (APHA units) & 750 & 750 & 750 & 750 & 750 & & & & & \\
\hline $\begin{array}{l}\text { TOC ( } \mathrm{wg} / 1) \\
800 \text { 5-day }(\mathrm{mg} / 1)\end{array}$ & 69.6 & 69.6 & 69.6 & 69.6 & 69.6 & & & & & \\
\hline
\end{tabular}




\begin{tabular}{|c|c|c|c|c|c|c|c|c|c|c|}
\hline MCNTH & SEPT & SEPT & SEPT & SEPT & SEPT & SEPT & SEPT & SEPT & SEPT & SEPT \\
\hline DAY & 4 & 5 & 6 & 7 & 8 & 9 & 10 & 11 & 12 & 13 \\
\hline DAY NO. & 40 & 41 & 42 & 43 & 44 & 45 & 46 & 47 & 48 & 49 \\
\hline \multicolumn{11}{|l|}{ SYSTE: PARAMETERS } \\
\hline tSludọe Age & 38 & 30 & 30 & 38 & 30.1 & 38 & 25 & 25 & 25 & 25 \\
\hline$\because(F / M) C O D$ & 0.28 & 0.27 & 0.25 & 8.29 & 0.31 & 8.33 & 0.24 & 0.23 & 0.27 & 0.28 \\
\hline IHRT & 1.9 & 1.9 & 2.0 & 1.9 & 1.9 & 1.9 & 1.9 & 1.9 & 1.9 & 1.9 \\
\hline FEED FARAMETERS (BATCH H) & 29 & 29 & 29 & 29 & 29 & 29 & 29 & 29 & 38 & 30 \\
\hline Volune (liters) & 4.82 & 4.95 & 4.55 & 5.80 & 5.80 & 5.80 & 4.87 & 4.80 & 4.88 & 5 \\
\hline $\mathrm{pH}$ (std units) & $9.7 / 18.29$ & $.7 / 10.2$ & 9.7 & 9.7 & 9.7 & 9.8 & $9.7 / 11.0$ & $9.8 / 11.1$ & $9.8 / 10.1$ & $9.5 / 10.4$ \\
\hline $\operatorname{COD}(\mathrm{mg} / 1)$ & 2022 & 2022 & 2033 & 2033 & 2033 & 2000 & 2000 & 2000 & 2008 & 2863 \\
\hline NHב̄N $(x g / 1)$ & 184 & 184 & 183 & 183 & 183 & 188 & 188 & 188 & 188 & 186 \\
\hline SS (mg/l) & 26 & 26 & 186 & 186 & 186 & 186 & 106 & 106 & 126 & 66 \\
\hline VSS (ag/1) & 14 & 14 & 44 & 44 & 44 & 44 & 44 & 44 & 44 & 32 \\
\hline CN $(m g / 1)$ & 8.25 & 0.25 & 0.93 & 0.93 & 0.93 & 0.93 & 0.93 & 0.93 & 0.93 & 0.33 \\
\hline $\operatorname{SCN}(x g / 1)$ & 182 & 182 & 183 & 183 & 183 & 183 & 183 & 183 & 183 & 193 \\
\hline BOD J-day $(m g / 1)$ & 1478 & 1470 & 1470 & 1470 & 1470 & 1470 & 1470 & 1470 & 1410 & 1410 \\
\hline TOC (ng/1) & & & 683 & 683 & 683 & 683 & 683 & 683 & 683 & \\
\hline Phenolics $(9 q / 1)$ & 1.48 & 1.48 & & & & & & & & 1.54 \\
\hline \multicolumn{11}{|l|}{$P 04-P(6 q / 1)$} \\
\hline \multicolumn{11}{|l|}{ Org-N $(m g / 1)$} \\
\hline \multicolumn{11}{|l|}{ NO2 $(m g / 1)$} \\
\hline \multicolumn{11}{|l|}{$\mathrm{N} 03(\mathrm{ng} / 1)$} \\
\hline \multicolumn{11}{|l|}{ TDS $(n g / 1)$} \\
\hline \multicolumn{11}{|l|}{ Color (APHA Units) } \\
\hline \multicolumn{11}{|l|}{ EASIN PARAMETERS } \\
\hline Vol dasted (ml) & 386 & 345 & 240 & 385 & 387 & 374 & 443 & 441 & 453 & 458 \\
\hline D.0. (mog/1) & 6.8 & 6.8 & 6.8 & 6.6 & 6.8 & 6.8 & 6.9 & 6.8 & 7.2 & 7.2 \\
\hline SS (ag/1) & 3070 & 3020 & 3270 & 3070 & 2850 & 2700 & 3550 & $\hat{\varepsilon} 300$ & 3360 & 3430 \\
\hline *SS (PAC corrected) & 3070 & 3020 & 3270 & 3070 & 2850 & 2700 & 3550 & 2900 & 3360 & 3490 \\
\hline Temo (C) & 24 & 24 & 24 & 26 & 25 & 24 & 25 & 25 & 25 & 25 \\
\hline oH (std units) & 7.3 & 7.2 & 7.3 & 7.3 & 7.2 & 7.1 & 6.9 & 7.1 & 7.5 & 6.9 \\
\hline OUR (mg/1/hr) & & 36 & & & 4.2 & & & & 36 & \\
\hline VSS (mg/1) & 2918 & 2850 & 3086 & 2897 & 2690 & 2548 & 3351 & 2737 & 2990 & 3106 \\
\hline IUSS (PAC corrected) & 2918 & 2850 & 3086 & 2897 & 2590 & 2548 & 3351 & 2737 & 2990 & 3106 \\
\hline VSS/SS & & 0.94 & & & 0.94 & & & & 0.89 & \\
\hline IPAC Added (ag) & 8 & 0 & 8 & 0 & 0 & 0 & 0 & 0 & 0 & 0 \\
\hline IInventory (mo/l basin) & 0 & 0 & 8 & 0 & 0 & 0 & 0 & 8 & 0 & 0 \\
\hline \multicolumn{11}{|l|}{ SFFLLIENT OARAMETERS } \\
\hline Volume (liters) & 4.28 & 4.40 & 4.10 & 4.58 & 4.36 & 4.42 & 4.35 & 4.35 & 4.11 & 4.25 \\
\hline $\operatorname{COD}(m g / 1)$ & 177 & 183 & 180 & 178 & 199 & 211 & 206 & 211 & 188 & 183 \\
\hline $5 S(\mathrm{mg} / 1)$ & 10 & 38 & 128 & 10 & 8 & 16 & 30 & 26 & 22 & 18 \\
\hline *PAC corrected & 38 & 8 & 30 & 44 & 4 & 104 & 140 & 132 & 152 & 134 \\
\hline pH (std units) & 7.3 & 7.3 & 7.4 & 8.0 & 8.3 & 7.7 & 7.3 & 7.7 & 8.0 & 7.9 \\
\hline VSS $(\operatorname{mg} / 1)$ & & 22 & & & 68 & & & & 62 & \\
\hline APAC corrected & & 8 & & & 4 & & & & 108 & \\
\hline NH3N (mg/l) & 9.2 & 9.2 & 13.3 & 13.3 & 13.3 & 16.7 & 16.7 & 16.7 & 16.7 & 5.5 \\
\hline$C N(m g / 1)$ & 1.76 & 1.96 & 2.02 & 2.02 & 2.82 & 2.02 & 2.02 & 2.02 & 2.82 & 1.62 \\
\hline SCN (ng/1) & 5.9 & 5.9 & 7.1 & 7.1 & 7.1 & 7.1 & 7.1 & 7.1 & 7.1 & 6.5 \\
\hline NO2 (mg/l) & 22.9 & 22.9 & 9.95 & 9.95 & 9.95 & 9.95 & 9.95 & 9.95 & 9.95 & 4.7 \\
\hline $\mathrm{NO3}(\mathrm{sg} / 1)$ & 182 & 182 & 160 & 188 & 180 & 188 & 180 & 188 & 180 & 196 \\
\hline Phenolics (mg/l) & $<.025$ & 1.025 & & & & & & & & 10.025 \\
\hline$P 04-P(0 g / 1)$ & 3.9 & 3.9 & & & & & & & & 4.3 \\
\hline \multicolumn{11}{|l|}{$\operatorname{TDS}(\log / 1)$} \\
\hline Color (APHA units) & & & 625 & 625 & 625 & 625 & 625 & 625 & 625 & \\
\hline $\operatorname{roc}(\log / 1)$ & & & 64 & 64 & 64 & 64 & 64 & 64 & 64 & \\
\hline BOD 5-day (mg/1) & & & & & & & & & & \\
\hline
\end{tabular}




\begin{tabular}{|c|c|c|c|c|c|c|c|c|c|c|}
\hline MONTH & SEPT & SEPT & SEPT & SETT & SEPT & SEOT & SEPT & SEPT & SEPT & SEPT \\
\hline DAY & 14 & 15 & 16 & 17 & 18 & 19 & 20 & 21 & 22 & 23 \\
\hline DAY NO. & 50 & 51 & 52 & 53 & 54 & 55 & 55 & 57 & 58 & 59 \\
\hline \multicolumn{11}{|l|}{ SYSTEM PARAMETERS } \\
\hline *Sludge Age & 25 & 25 & 25 & 25 & 25 & 25 & 20 & 20 & 14.2 & 20 \\
\hline$*(F / M) C C D$ & 0.32 & 0.31 & 0.26 & 0.28 & 0.26 & 0.27 & 0.26 & 0.25 & 0.30 & 0.34 \\
\hline *HRT & 1.9 & 1.9 & 1.9 & 1.9 & 1.9 & 1.9 & 1.9 & 1.9 & 1.9 & $! . \exists$ \\
\hline FEED PARA:ETERS (BRTCH & 30 & 30 & 30 & 30 & 30 & 38 & 30 & 30 & 38 & 20 \\
\hline Volume (1iters) & 5.80 & 5.00 & 4.80 & 5.00 & 5.20 & 5.00 & 5.08 & 5.60 & 5.00 & 5.80 \\
\hline $\mathrm{pH}$ (std units) & 9.6 & $9.5 / 10.4$ & $18 / 18.7$ & 9.6 & 3.7 & \multicolumn{2}{|c|}{$9.8 / 10.69 .6 / 10.3$} & $3.4 / 10.4$ & $3.5 /: 0.4$ & 9.5 \\
\hline $\cos (\mathrm{mg} / 1)$ & 2863 & 2063 & 1846 & 1846 & 1946 & 1846 & 1802 & 1902 & 1802 & 2178 \\
\hline NH3N $(\mathrm{mg} / 1)$ & 196 & 186 & 187 & 187 & 187 & 187 & 198 & 198 & 190 & 193 \\
\hline$S S(m g / 1)$ & 66 & 66 & 66 & 66 & 66 & 66 & 125 & 126 & 126 & 125 \\
\hline USS (ng/1) & 32 & 32 & 32 & 32 & 32 & 32 & 46 & 46 & 46 & 46 \\
\hline$C N(m g / 1)$ & 0.33 & 0.33 & 0.33 & 0.33 & 0. 33 & 0.33 & & & & \\
\hline SCN $(m g / 1)$ & 193 & 193 & 193 & 193 & 193 & 193 & & & & \\
\hline BOD 5-day (ng/1) & 1410 & 1410 & 1410 & 1410 & 1410 & 1410 & 160 & 1628 & 1600 & 1620 \\
\hline TOC $(\square 0 / 1)$ & & & & & & & 689 & 689 & 689 & 689 \\
\hline Phenolics (mg/l) & 1.54 & 1.54 & 1.54 & 1.54 & 1.54 & 1.54 & & & & \\
\hline
\end{tabular}

P04-p (ng/1)

Ora-N (mo/I)

NO2 (mg/l)

N03 (mg/1)

TDS (mg/1)

Color (APHA Units)

GASIN PGRAMETERS

Vol Hasted (ai)

D. J. $(\mathrm{ag} / 1)$

SS (mg/!)

*SS (PAC corrected)

Teap (C)

of (std units)

กII (mg///hr)

VSS (mo/l)

*iSS (InC corrected)

VSE/SS

*PAC Added (ma)

+Inventory (ma/l basin)

EFFLUENT PRRAMETERS

Ualuwe (1iters)

COD (mg/l)

SS $(\mathrm{mg} / 1)$

*PAC corrected

oH (std units)

vss (mg/l)

*PAC corrected

NH3N ( $\mathrm{kg} / 1)$

CN $(m g / 1)$

SCN (ag/1)

NO2 (ng/l)

$\mathrm{NO3}(\mathrm{mg} / \mathrm{ll})$

Thenolics (mg/l)

p04-p (mo/l)

Org-N (mg/l)

TDS $(\operatorname{rog} / 1)$

Color (APHA units)

TOC (mg/l)

$\begin{array}{rrrrrrrrrr}438 & 171 & 321 & 421 & 403 & 429 & 543 & 523 & 10 & 215 \\ 7.4 & 9.0 & 7.9 & 7.8 & 7.5 & 6.7 & 6.6 & 6.6 & 6.8 & 7.0 \\ 3060 & 2960 & 3030 & 3010 & 3260 & 3120 & 3110 & 3240 & 2910 & 2320 \\ 3060 & 2360 & 3030 & 3010 & 3260 & 3120 & 3110 & .7249 & 2419 & 2970 \\ 23 & 22 & 22 & 23 & 23 & 23 & 24 & 25 & 24 & 23 \\ 7.2 & 6.7 & 7.3 & 7.3 & 7.0 & 6.9 & 7.1 & 6.9 & 6.7 & 7.2 \\ & 28 & & & & 30 & & & 34 & \\ 2723 & 2730 & 2795 & 2770 & 3007 & 2980 & 2971 & 2991 & 2540 & 2539 \\ 2723 & 2730 & 2795 & 2776 & 3007 & 2800 & 2971 & 2991 & 2540 & 2639 \\ & 0.92 & & & & 0.92 & & & 0.50 & \\ 0 & 0 & 0 & 0 & 0 & 0 & 0 & 0 & 0 & 0 \\ 0 & 0 & 0 & 0 & 0 & 0 & 0 & 0 & 0 & 0\end{array}$

4. 3

4.35

4.30

4.78

179

180

179

112

144

$2: 0$

18

$\begin{array}{lll}7.7 & 7.5 & 7.7\end{array}$

196

30

$5.5 \quad 5.5$

$1.62 \quad 1.52$

5.5

1.62

6.5

$6.5 \quad 6.5$

$4.7 \quad 4.7$

4.7

$196 \quad 196$

10. 025

10. 225

196

(0. 025

4.3

2.6

8.1

1.62

4.7

196

10.025

4.3

2.6
4.50

$\lcm{48}$

8. 1

9. 0

88

르

5.5

5.5

5.5

1.62

1.62

6.5

4.7

4.7

196

10.025

196

4.3

<0. 025
38

16

6.8

4.70

151

54

30

7.1

4.60

$\begin{array}{lll}4.3 & 4.3 & 4.3\end{array}$

110

110
113

506

34

7.1

460

4.78

122

234

24

7.2

6.1

110

110 
UNIT $2 A$ TABLE A3

\begin{tabular}{|c|c|c|c|c|c|c|c|c|c|c|}
\hline MONTH & SEPT & SEPT & SEPT & SEPT & SEPT & SEPT & SEPT & OCT & DCT & OCT \\
\hline DRY & 24 & 25 & 26 & 27 & 28 & 29 & 30 & 1 & 2 & 3 \\
\hline DAY NO. & 50 & 61 & 82 & 63 & 54 & 65 & 65 & 57 & 68 & 89 \\
\hline
\end{tabular}

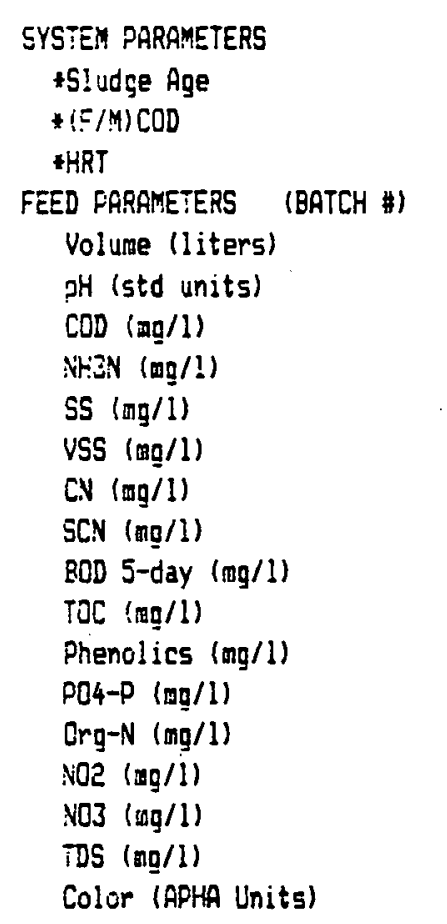

$\begin{array}{rrrrrrrrrr}20 & 20 & 20 & 16.3 & 20 & 20 & 20 & 20 & 20 & 18.7 \\ 0.33 & 0.36 & 0.30 & 0.35 & 0.28 & 0.30 & 0.29 & 0.28 & 0.22 & 0.29 \\ 1.9 & 1.9 & 2.0 & 1.9 & 1.9 & 1.9 & 1.9 & 1.9 & 2.4 & 2.2 \\ 30 & 30 & 30 & 30 & 31 & 3 ! & 31 & 31 & 31 & 31 \\ 5.00 & 5.00 & 4.60 & 5.20 & 5.80 & 5.80 & 5.00 & 5.80 & 3.85 & 4.30 \\ 9.4 & 9.5 / 10.3 & 9.5 / 10.4 & 3.5 / 10.3 & 10.1 & 10.1 & 11.3 & 11.4 & 11.4 & 11.5 \\ 2178 & 2178 & 2178 & 2161 & 2161 & 2161 & 2028 & 2028 & 2028 & 2028 \\ 193 & 193 & 193 & 189 & 189 & 189 & 166 & 166 & 166 & 166 \\ 126 & 126 & 126 & 244 & 244 & 244 & 244 & 244 & 244 & 244 \\ 46 & 46 & 46 & 60 & 60 & 60 & 60 & 60 & 60 & 60\end{array}$

$\begin{array}{rrrrrrrrrr}1620 & 1620 & 1620 & 1470 & 1470 & 1470 & 1470 & 1470 & 1470 & 1470 \\ 609 & 689 & 689 & 660 & 660 & 660 & 660 & 660 & 660 & 660 \\ & & & 0.67 & 0.67 & 0.67 & 0.67 & 0.67 & 0.67 & 0.67\end{array}$

BASIN PARAMETERS

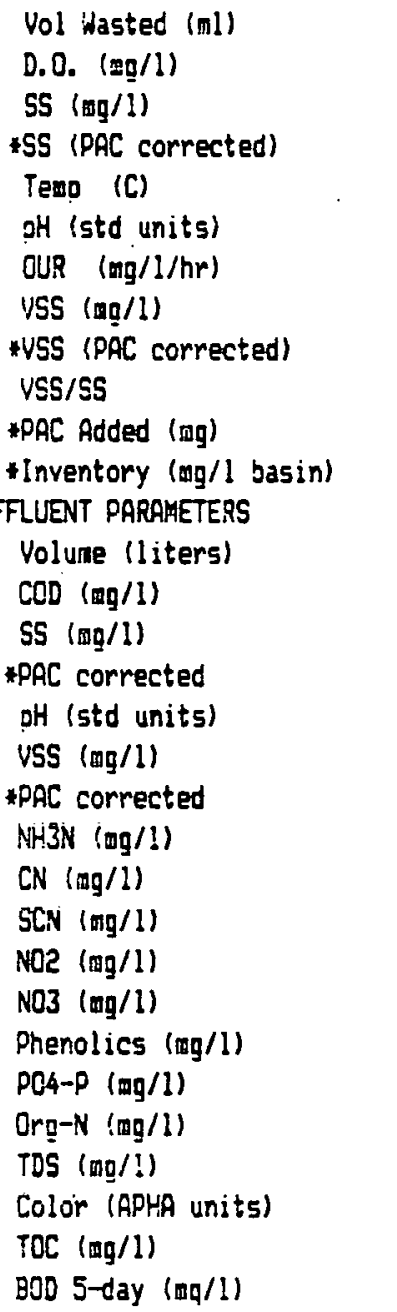

\begin{tabular}{|c|c|c|c|c|c|c|c|c|c|}
\hline 469 & 398 & 198 & 10 & 508 & 493 & 518 & 528 & $5 ! 2$ & 10 \\
\hline 7.0 & 7.8 & 7.3 & 7.4 & 7.4 & 7.6 & 7.7 & 7.8 & 7.2 & 7.6 \\
\hline 3030 & 2798 & 3030 & 2840 & 3540 & 3440 & 3300 & 3460 & 3440 & 2890 \\
\hline 3230 & 2750 & 3030 & 2840 & 3540 & 3440 & 3300 & 3460 & 3440 & 2890 \\
\hline 21 & 21 & 21 & 21 & 22 & 21 & 21 & 21 & 21 & 22 \\
\hline 6.9 & 6.2 & 6.7 & 7.4 & 7.2 & $\begin{array}{r}7.3 \\
42\end{array}$ & 7.3 & 7.6 & 7.8 & $\begin{array}{r}7.9 \\
38\end{array}$ \\
\hline 2739 & 2522 & $27 \in 0$ & 2587 & 32255 & 2990 & 2868 & 3007 & 2990 & 2520 \\
\hline 2739 & 2522 & $\begin{array}{l}2760 \\
0.91\end{array}$ & 2587 & 3225 & $\begin{array}{l}2990 \\
0.87\end{array}$ & 2868 & 3807 & 2990 & $\begin{array}{l}2520 \\
0.87\end{array}$ \\
\hline 0 & 8 & 0 & 0 & 8 & 0 & 0 & 0 & 8 & 0 \\
\hline 0 & 0 & 0 & 0 & $\theta$ & 8 & $\theta$ & 0 & 8 & 0 \\
\hline 4.80 & 4.68 & 4.25 & 4.70 & 5.60 & 4.60 & 4.50 & 4.50 & 3.15 & 4.28 \\
\hline 176 & 176 & 162 & 172 & 176 & 177 & 188 & 172 & 180 & 178 \\
\hline 86 & 124 & 294 & 422 & 66 & 80 & 60 & 54 & 96 & 450 \\
\hline 8 & 10 & 28 & 22 & 48 & 46 & 48 & 48 & 40 & 24 \\
\hline 7.4 & 6.7 & $\begin{array}{l}4.4 \\
290\end{array}$ & 7.0 & 7.2 & $\begin{array}{r}7.3 \\
52\end{array}$ & 7.4 & 7.6 & 7.8 & $\begin{array}{l}7.9 \\
308\end{array}$ \\
\hline & & 16 & & & 48 & & & & 24 \\
\hline
\end{tabular}

$\begin{array}{llllllllll}6.1 & 6.1 & 6.1 & 4.9 & 4.9 & 4.9 & 3.1 & 3.1 & 3.1 & 3.1\end{array}$

$\$ 18 \quad 188 \quad 110$
$\begin{array}{lllllll}10.025 & 10.025 & 10.025 & 10.025 & 10.025 & 10.025 & 10.025\end{array}$

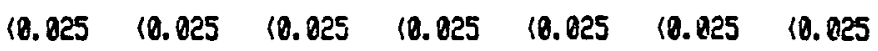

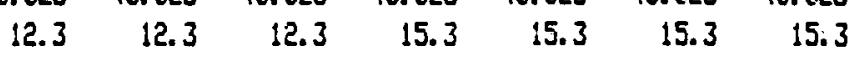

$\begin{array}{rrrrrrrrrr}500 & 500 & 500 & & & & & & & \\ 58 & 58 & 58 & 48 & 48 & 48 & 48 & 48 & 48 & 48 \\ 110 & 110 & 119 & 5.7 & 5.7 & 5.7 & 5.7 & 5.7 & 5.7 & 5.7\end{array}$




\begin{tabular}{|c|c|c|c|c|c|c|c|c|c|c|}
\hline MONTH & OCT & OCT & OCT & OCT & OCT & OCT & OCT & OCT & $\mathrm{OCT}$ & OCT \\
\hline DAY & 4 & 5 & 6 & 7 & 8 & 3 & 10 & 11 & 12 & 13 \\
\hline DAY NO. & 78 & 71 & 72 & 73 & 74 & 75 & 76 & 77 & 78 & 79 \\
\hline \multicolumn{11}{|l|}{ SYSTEM MNRIMETERS } \\
\hline *SI udge Age & 20 & 20 & 20 & 28 & 20 & 20 & 20 & 20 & 28 & 20 \\
\hline$*(F / M) C O D$ & 0.23 & 9.29 & 0.27 & 0.25 & 0.19 & 0.23 & 0.23 & 0.32 & 0.32 & 0.32 \\
\hline *HRT & 2.0 & 1.3 & 2.2 & 2.3 & 3.8 & 2.4 & 2.3 & ล. 1 & 2.1 & 2.0 \\
\hline FEED FARAXETERS (BRTCH & 31 & 31 & 31 & 31 & 31 & 31 & 31 & 31 & 31 & 31 \\
\hline Volume (liters) & 4.80 & 4.85 & 4.25 & 4.10 & 3.15 & 3.85 & 3.38 & 4.49 & 4.58 & 4.60 \\
\hline oH (std units) & $11.5 / 10.111$ & $.5 / 10.2$ & 11.6 & 11.3 & $11.5 / 9.8$ & 11.5 & 11.5 & 11.3 & 11.2 & 11.8 \\
\hline $\operatorname{COD}(\pi g / 1)$ & 2085 & 2885 & 2085 & 2052 & $\Sigma 252$ & 2052 & 2052 & 2098 & 2898 & 2298 \\
\hline$N H 3 N(m g / 1)$ & $\$ 78$ & 178 & 178 & 153 & 153 & 153 & 153 & 156 & $\$ 56$ & 156 \\
\hline SS $(m g / 1)$ & 436 & 436 & 4336 & 436 & 436 & 436 & 436 & & & \\
\hline VSS (mg/l) & 92 & 92 & 92 & 92 & 92 & 92 & 92 & & & \\
\hline \multicolumn{11}{|l|}{ CN (mg/1) } \\
\hline \multicolumn{11}{|l|}{$\operatorname{SCN}(m g / !)$} \\
\hline BOD 5-day (mg/1) & 1400 & 1420 & 1400 & 1480 & 1400 & 1480 & 1400 & 1368 & 1360 & 1360 \\
\hline TOC $(m g / 1)$ & 646 & 646 & 646 & 646 & 646 & 646 & 646 & 849 & 549 & 649 \\
\hline \multicolumn{11}{|l|}{ Phenolics $(\operatorname{mg} / 1)$} \\
\hline \multicolumn{11}{|l|}{$P 04-p(m g / 1)$} \\
\hline \multicolumn{11}{|l|}{ Org-N (mg/l) } \\
\hline \multicolumn{11}{|l|}{ NO2 (ag/l) } \\
\hline \multicolumn{11}{|l|}{ NO3 (mg/!) } \\
\hline \multicolumn{11}{|l|}{ TDS (ag/l) } \\
\hline \multicolumn{11}{|l|}{ Color (Frth 'mits) } \\
\hline \multicolumn{11}{|l|}{ BASIN PARAMETERS } \\
\hline Vol Wasted (nl) & 554 & 544 & 542 & 556. & 559 & 515 & 10 & 527 & 563 & 536 \\
\hline D.0. (mg/1) & 7.2 & 7.2 & 7.3 & 7.0 & 7.8 & 7.2 & 7.2 & 7.2 & 7.4 & 7.4 \\
\hline SS (mg/1) & 3270 & 3390 & 3130 & 3230 & 3270 & 3290 & 2700 & 2918 & 2810 & 2880 \\
\hline \#SS (PAC corrected) & .379 & 3390 & 3130 & 3230 & 3270 & 3298 & 2700 & 2810 & 2810 & 2880 \\
\hline Temo (C) & 23 & 24 & 23 & २2 & 20 & 22 & 22 & 23 & $2 \overline{3}$ & 23 \\
\hline oH (std units) & 8.0 & 7.9 & 7.8 & 7.9 & 8.8 & 8.8 & 7.7 & 7.9 & 7.9 & 8.2 \\
\hline OUR (ay/1/hn) & & & 18 & & & & 48 & & & 48 \\
\hline Vss $(a g / 1)$ & 2851 & 2956 & 2710 & 3797 & 2831 & 2849 & 2340 & 2435 & 2435 & 2580 \\
\hline tVSS (PAC corrected) & 2851 & ¿956 & 2718 & 2737 & 8931 & 2043 & 3340 & 2435 & 2435 & 2500 \\
\hline VSS/SS & & & 0.87 & & & & 0.87 & & & 0.87 \\
\hline PAC Added (mg) & 0 & 0 & 8 & 0 & 8 & 8 & 8 & 8 & 0 & 8 \\
\hline *Inventory (mg/l basin) & 8 & 0 & 0 & 8 & 0 & 8 & $\theta$ & 0 & 0 & 0 \\
\hline \multicolumn{11}{|l|}{ EFIUENT PARQMETERS } \\
\hline Volune (1iters) & 4.78 & 4.55 & 3.95 & 3.70 & 3.30 & 3.40 & 4.20 & 4.47 & 4.32 & 4.35 \\
\hline $\operatorname{COD}(\mathrm{mg} / 1)$ & 138 & 130 & 132 & 126 & 133 & 127 & lês & 123 & $1 \overline{35}$ & $\$ 46$ \\
\hline SS $(m g / 1)$ & 32 & 42 & 46 & 38 & 40 & 82 & 1696 & 46 & 24 & 42 \\
\hline APAC corrected & 18 & 34 & 40 & 26 & 46 & 116 & 48 & 34 & 26 & 50 \\
\hline $\mathrm{pH}$ (std units) & 8.8 & 8.0 & 8.8 & 8.0 & 8.0 & 8.8 & 8.8 & 8.1 & 8.3 & 8.1 \\
\hline VSS (mg/1) & & & 38 & & & & 1458 & & & 32 \\
\hline *PAC corrected & & & 34 & & & & 42 & & & 36 \\
\hline NH3N $(\mathrm{mg} / 1)$ & 1.5 & 1.5 & 1.5 & 1.8 & 1.8 & 1.8 & 1.8 & 1.4 & 1.4 & 1.4 \\
\hline \multicolumn{11}{|l|}{ CN $(\mathrm{ag} / 1)$} \\
\hline \multicolumn{11}{|l|}{$\operatorname{SCN}(\operatorname{gog} / 1)$} \\
\hline \multicolumn{11}{|l|}{$\operatorname{NDC}(\operatorname{mg} / 1)$} \\
\hline N03 ( $8 g / 1)$ & & & & & & & & & & \\
\hline Phenolics (ng/l) & & & & & & & & & & \\
\hline P04-P (mg/l) & & & & & & & & 2.2 & 2.2 & 2.2 \\
\hline $\begin{array}{l}\text { Org-N (mg/l) } \\
\text { TDS (mg/1) }\end{array}$ & & & & & & & & & & \\
\hline Color (APHA units) & 625 & 625 & 625 & 625 & 625 & 625 & 625 & & & \\
\hline TOC $(m g / 1)$ & 38 & 38 & 38 & 38 & 38 & 38 & 38 & 37 & 37 & 37 \\
\hline BOD 5-day (田/l) & 13.4 & 13.4 & 13.4 & 13.4 & 13.4 & 13.4 & 13.4 & 1.8 & 1.8 & 1.8 \\
\hline & & & & & & & & & & \\
\hline
\end{tabular}


UNIT $2 A$ TABLE A3

\begin{tabular}{|c|c|c|c|c|c|c|c|c|c|c|}
\hline MONTH & OCT & OCT & OCT & DCT & OCT & OCT & OCT & OCT & OCT & OCT \\
\hline DAY & 14 & 15 & 16 & 17 & 18 & 19 & 20 & 21 & 22 & 23 \\
\hline DAY NO. & 80 & 81 & 82 & 83 & 84 & 85 & 86 & 87 & 88 & 89 \\
\hline \multicolumn{11}{|l|}{ SYSTEM PARAMEIERS } \\
\hline tSludge Age & 20 & 28 & 20 & 20 & 19.98 & 19.93 & $\lfloor 4.27$ & 28.08 & 20.80 & 20.80 \\
\hline$*(F / M) C O D$ & 0.26 & 0.26 & 0.25 & 0.32 & 0.30 & 0.30 & 0.30 & 0.30 & 0.30 & 0.30 \\
\hline *HRT & 1.9 & 1.9 & 2.0 & 1.9 & 1.86 & 1.86 & 1.96 & 1.86 & 1.86 & 1.86 \\
\hline FEED PARAMETERS (BATCH & 31 & 31 & 31 & 31 & 32 & 32 & 32 & 32 & 32 & 32 \\
\hline Volune (1iters) & 5.08 & 5.80 & 4.55 & 5.88 & 5 & 5 & 5 & 5 & 5 & 5 \\
\hline $\mathrm{pH}$ (std units) & 18.9 & 18.9 & 11.7 & $5 / 9.8$ & 11.5 & 11.4 & 11.5 & 11.5 & 11.5 & 11.4 \\
\hline $\operatorname{COD}(\operatorname{sg} / 1)$ & 1983 & 1983 & 1983 & 1983 & 1925 & 1925 & 1925 & 1926 & 1926 & 1925 \\
\hline NH3N (mg/1) & 158 & 158 & 158 & 158 & 169 & 169 & 169 & 166 & $1 E 6$ & 166 \\
\hline SS (Eg/1) & & & & & 148 & 140 & 140 & 140 & 140 & 140 \\
\hline VSS $(\mathrm{mg} / 1)$ & & . & & & 46 & 46 & 46 & 46 & 46 & 46 \\
\hline \multicolumn{11}{|l|}{ CN (mg/I) } \\
\hline \multicolumn{11}{|l|}{$\mathrm{SCN}$ (ng/1) } \\
\hline 8005 -day (mg/1) & 1360 & 1360 & 1360 & 1360 & 1110 & 1110 & 1110 & $11: 0$ & 1110 & 1110 \\
\hline TOC (mg/l) & 649 & 649 & 649 & 649 & 642 & 642 & 642 & 642 & 642 & 642 \\
\hline \multicolumn{11}{|l|}{ Phenolics (mg/1) } \\
\hline \multirow{2}{*}{\multicolumn{11}{|c|}{$\begin{array}{l}0,4-P(m g / 1) \\
O r g-N(m g / 1)\end{array}$}} \\
\hline & & & & & & & & & & \\
\hline \multicolumn{11}{|l|}{ NO2 (田/1) } \\
\hline \multicolumn{11}{|l|}{$\mathrm{NO3}$ (ng/1) } \\
\hline \multicolumn{11}{|l|}{ TDS (mg/1) } \\
\hline \multirow{2}{*}{\multicolumn{11}{|c|}{$\begin{array}{l}\text { Color (APHA Units) } \\
\text { BASIN PARAMETERS }\end{array}$}} \\
\hline & & & & & & & & & & \\
\hline \multicolumn{11}{|l|}{ Vol Wasted (al) } \\
\hline D.0. $(m g / 1)$ & 7.6 & 7.6 & 7.6 & 7.4 & 7.4 & 7 & 7.2 & 7 & 7 & $\quad 7$ \\
\hline SS (mg/1) & 3600 & 3630 & 3520 & 3390 & 3360 & 3390 & 3460 & 3470 & 3420 & - 3540 \\
\hline *SS (PAC corrected) & 3620 & 3630 & 3520 & 3390 & 3360 & 3390 & 3460 & 3470 & 3428 & 3540 \\
\hline Tero (C) & 22 & 21.5 & 22 & 22 & 22 & 22 & 20 & 20 & 18 & 20 \\
\hline oH (std units) & 7.9 & 8.0 & 8.0 & 8.0 & 7.9 & 7.8 & 8.1 & 8.2 & 7.9 & 7.9 \\
\hline OUR (mģ/1/hr) & & & & 36. & & & 34 & & & \\
\hline VSS (mq/1) & 3125 & $315 !$ & 3856 & 2550 & 2710 & 2710 & 2788 & 2708 & 2700 & 2700 \\
\hline *USS (PAC corrected) & 3125 & $315 !$ & 3056 & 2550 & 2718 & 2710 & 2700 & 2700 & 2703 & 2700 \\
\hline VSS/SS & & & & 0.75 & 0.87 & 0.87 & 0.78 & 0.78 & 0.78 & 0.78 \\
\hline tPAC Added (mg) & 8 & 0 & 0 & 0 & 0 & 8 & 0 & 8 & 0 & 8 \\
\hline Inventory (mg/l basin) & 0 & - & 0 & 0 & 0 & 0 & 0 & 8 & 8 & 0 \\
\hline \multicolumn{11}{|l|}{ EFLUENT PARAMETERS } \\
\hline Voluare (1iters) & 4.93 & 4.58 & 4.45 & $4.7 \pi$ & 4.75 & 4.75 & 4.7 & 4.57 & 4.52 & 4.5 \\
\hline $\operatorname{COD}(\pi g / 1)$ & 146 & 136 & 142 & 150 & 131 & 141 & 126 & 114 & 1.38 & 134 \\
\hline SS (mg/1) & 80 & 192 & 123 & 66 & 64 & 36 & 214 & 64 & 64 & 82 \\
\hline *PAC corrected & 68 & 48 & 58 & 28 & 58 & 46 & 44 & 180 & 42 & 42 \\
\hline OH (std units) & 8.2 & 8.2 & 7.9 & 8.1 & 8.1 & 8 & 8.2 & 8.3 & 8.1 & 8.1 \\
\hline VSS (mg/1) & & & 42 & 26 & 38 & 38 & 128 & 128 & 128 & 128 \\
\hline IPAC corrected & & & & 26 & 34 & 34 & 34 & 34 & 34 & 34 \\
\hline NH3N (mg/1) & 1.2 & 1.2 & 1,2 & 8.7 & 1.4 & 1.4 & 1.4 & 2 & 2 & 2 \\
\hline$C N(\$ g / 1)$ & & & & & & & & & & \\
\hline \multicolumn{11}{|l|}{$\operatorname{SCN}(\operatorname{mg} / 1)$} \\
\hline \multicolumn{11}{|l|}{$\begin{array}{l}\text { ND2 }(\mathrm{mg} / 1) \\
\mathrm{ND3}(\mathrm{ng} / 1)\end{array}$} \\
\hline \multirow{2}{*}{\multicolumn{11}{|c|}{$\begin{array}{l}\text { NO3 (ng/l) } \\
\text { Phenolics (mg/l) }\end{array}$}} \\
\hline & & & & & & & & & & \\
\hline$P O 4-P(m g / d)$ & 2.2 & 2.2 & 2.2 & 2.2 & & & & & & \\
\hline \multicolumn{11}{|l|}{$\operatorname{Org}-\mathrm{N}(\mathrm{gg} / \mathrm{l})$} \\
\hline Color (APHA units) & & & & & G25 & 625 & 625 & 525 & 625 & \\
\hline $\operatorname{TOC}(\mathrm{mg} / 1)$ & 37 & 37 & 37 & 37 & 40 & 40 & 40 & 40 & 40 & 40 \\
\hline BOD 5-day (mg/l) & 1.8 & 1.8 & 1.8 & 1.8 & 0.9 & 0.9 & 0.9 & 0.9 & 0.9 & 0.9 \\
\hline & & & & 09- & & & & & & \\
\hline
\end{tabular}




\begin{tabular}{|c|c|c|c|c|c|c|c|c|c|c|}
\hline MONTH & OCT. & OCT & CCT & DCT & OCT & OCT & $\mathrm{OCT}$ & OCT & NDV & NOV \\
\hline DAY & 24 & 25 & 26 & 27 & 28 & 29 & 30 & 31 & 1 & 2 \\
\hline DAY NO. & 90 & 91 & 32 & 93 & 94 & 35 & 96 & 37 & 98 & 39 \\
\hline SYSTEM PARGAETERS & & & & & & & & & & \\
\hline *Sludgoe Age & 14.08 & 19.98 & 20.01 & 19.93 & 20.20 & 20.01 & 19.99 & 20.62 & 19.98 & 20.00 \\
\hline$*(F / M) C O D$ & 0.32 & 0.35 & 0.35 & 0.33 & V. 30 & 0.30 & 0.50 & 0.29 & 0.30 & 0.30 \\
\hline FHRT & 1.86 & 1.86 & 1.86 & 1.86 & 1.86 & 1.85 & 1.86 & 1.86 & 1.86 & 1.86 \\
\hline FEED FARAMETERS (BATCH है) & 32 & 32 & 32 & 32 & 32 & 32 & 32 & 32 & & \\
\hline Volume (liters) & 5 & 5 & 5 & 5 & 5 & 5 & 5 & 5 & 5 & 5 \\
\hline pH (std units) & 11.4 & 11.4 & 11.6 & 11.2 & 9.7 & 9.65 & 9.5 & 9.3 & 3.8 & 9.8 \\
\hline $\cos (\pi g / 1)$ & 1926 & 2060 & 2060 & 2068 & 1880 & 1880 & 1880 & 1880 & 1935 & 1935 \\
\hline NH3N $(\mathrm{mg} / 1)$ & 165 & 150 & 150 & 158 & 170 & 170 & 170 & 178 & 168 & 168 \\
\hline SS $(\operatorname{mg} / 1)$ & 140 & 312 & 312 & 312 & 312 & 312 & 312 & 312 & 204 & 284 \\
\hline USS $(\mathrm{mg} / 1)$ & 46 & 82 & 82 & 82 & 82 & 82 & 82 & 82 & 52 & 62 \\
\hline CN (mg/l) & & & & & & & & & 4.1 & 4.1 \\
\hline $\operatorname{SCN}(m g / 1)$ & & & & & & & & & 173 & 173 \\
\hline BOD 5-day (ong/1) & 1110 & 1160 & 1160 & 1160 & 1150 & 1160 & 1160 & $1: 60$ & 1170 & 1170 \\
\hline TOC $(\pi g / 1)$ & 642 & 610 & 610 & 610 & 610 & 610 & 610 & 510 & 601 & 601 \\
\hline $\begin{array}{l}\text { Phenolics (mg/l) } \\
\text { PO4-P (mg/l) }\end{array}$ & & 0.403 & 0.403 & 0.403 & 0.403 & 8.403 & 0.403 & 0.403 & 2.29 & 2. 29 \\
\hline $\begin{array}{l}\text { Org-N (mg/1) } \\
\text { NOS (mg/1) } \\
\text { NO3 (mg/1) } \\
\text { TOS (mg/1) }\end{array}$ & & & & & & & & & 58 & 58 \\
\hline Color (APHA Units) & & & & & & & & & 1000 & 1000 \\
\hline BASIN PARAMETERS & & & & & & & & & & \\
\hline Vol Wasted (ml) & 10 & 551 & 493 & 587 & 526 & 511 & 494 & 550 & 519 & 518 \\
\hline $0.0 .(\mathrm{sg} / 1)$ & 6.2 & 6 & 6 & 6 & 6.2 & 6.2 & 6.2 & 6.3 & 3.8 & 3.6 \\
\hline SS (mp/l) & 3120 & 3058 & 3360 & 3288 & 3320 & 3350 & 2610 & 3250 & 3258 & 3190 \\
\hline *SS (PAC corrected) & 3120 & 3060 & 3360 & 3280 & 3320 & 3350 & 2610 & 3258 & 3250 & 3130 \\
\hline Temp (C) & 20 & 20 & 21 & 20 & 20 & 21 & 20 & 20 & 20 & 28.5 \\
\hline $\mathrm{pH}$ (std units) & 7.9 & 7.8 & 7.75 & 7.7 & 7.6 & 1.6 & 7.3 & 6.9 & 7 & 6.4 \\
\hline OUR $(\mathrm{ag} / 1 / \mathrm{hr})$ & 36 & & & 38 & & & & 38 & & \\
\hline VSS (ag/1) & 2470 & 2470 & 2470 & 2600 & 2600 & 2600 & 2600 & 2670 & 2670 & 2670 \\
\hline *USS (PAC corrected) & 2470 & 2470 & 2470 & 2600 & 2600 & 2500 & 2600 & 2670 & 2670 & 2670 \\
\hline VSS/SS & 0.79 & v. 19 & 0.79 & 0.79 & 0.79 & 0.79 & ด. 79 & 0.82 & 0.82 & 0.08 \\
\hline rPAC AdJed (mg) & 0 & $\overline{0}$ & 0 & 8 & 0 & 8 & 0 & 0 & 0 & 0 \\
\hline +Inventory (ag/l basin) & 8 & 0 & 0 & 8 & 8 & $\theta$ & 0 & 8 & 0 & $\theta$ \\
\hline EFFLUENT PARAMETERS & & & & & & & & & & \\
\hline Volume (liters) & 4.28 & 4.95 & 4.72 & 4.78 & 4.73 & 4.79 & 4.79 & 5.03 & 4.85 & 4.85 \\
\hline $\operatorname{COD}(\operatorname{mg} / 1)$ & 138 & 139 & 142 & 130 & 132 & 130 & 140 & 134 & 149 & 144 \\
\hline SS (mg/l) & 614 & 30 & 76 & $6 \dot{4}$ & 52 & 62 & 58 & 32 & 54 & 52 \\
\hline APAC corrected & 158 & 36 & 24 & 46 & 48 & .36 & 36 & 23 & 26 & 50 \\
\hline$\mu H$ (std units) & 8.1 & 8.1 & 8 & 8.1 & 8 & 7.9 & 7.8 & 7.3 & 7.2 & 7.1 \\
\hline USS (ma/l) & 440 & 440 & 440 & 50 & 50 & 50 & 50 & 30 & 30 & 30 \\
\hline APAC corrected & 134 & 134 & 134 & 34 & 34 & 34 & 34 & 22 & 22 & 22 \\
\hline NH3N $(\mathrm{gg} / \mathrm{l})$ & 2 & 2.3 & 2.3 & 2.3 & 3.0 & 3.0 & 3.0 & 3.0 & 18.8 & 18. \\
\hline CN $(m g / 1)$ & & & & & & & & & 1.51 & 1.51 \\
\hline $\operatorname{SCN}(\mathrm{m} g / 1)$ & & & & & & & & & 3.1 & 3.1 \\
\hline NO2 $(\mathrm{mg} / 1)$ & & & & & & & & & 3.81 & 3.81 \\
\hline $\mathrm{NO3}(\mathrm{mg} / 11$ & & & & & & & & & 148 & 148 \\
\hline Phenolics (ng/l) & & <8. 025 & 10.025 & 10.025 & 10.025 & $<0.025$ & 10.025 & 10.025 & 18.025 & 10.025 \\
\hline$P 04-P(-g / 1)$ & & 3.5 & 4.9 & 4.9 & 4.9 & 4.9 & 4.9 & 4.9 & 34.6 & 34.6 \\
\hline $\begin{array}{l}\text { Org-N (mg/l) } \\
\text { TDS (mg/1) }\end{array}$ & & & & & & & & & $<4.0$ & 14.8 \\
\hline Color (APHA units) & 625 & & & & & & & & 875 & 875 \\
\hline TOC (mg/1) & 40 & 36 & 40 & 40 & 48 & 40 & 40 & 40 & 45 & 45 \\
\hline BOD 5-day (mg/1) & 0.9 & 1.7 & 1.7 & 1.7 & 1.7 & 1.7 & 1.7 & 1.7 & 1.9 & 1.9 \\
\hline
\end{tabular}




\begin{tabular}{|c|c|c|c|c|c|c|c|c|c|c|}
\hline MONTH & NOV & NOV & NOV & NOV & NOV & NOV & NOV & NOV & NOV & NOV \\
\hline DAY & 3 & . 4 & 5 & 6 & 7 & 8 & 3 & 10 & 11 & 12 \\
\hline ISYY NO. & 100 & 101 & 102 & 103 & 104 & 105 & 186 & 107 & 188 & 109 \\
\hline \multicolumn{11}{|l|}{ SYSTEM PARFMETERS } \\
\hline *5ludge Age & 19.39 & 19.36 & 20.22 & 29.01 & 20.54 & 19.38 & 20.21 & 19.95 & 20.01 & 28.01 \\
\hline$*(F / M) \operatorname{COD}$ & 0.30 & 0.32 & 0.32 & 0.27 & 0.31 & 0.31 & 0.31 & 0.24 & 0.24 & 0.26 \\
\hline$* H R T$ & 1.86 & 1.86 & 1.86 & 1.85 & 1.86 & 1.63 & 2.14 & 2.16 & 2.21 & 1.98 \\
\hline \multicolumn{11}{|l|}{ FEED PARAMETERS (BATCH \#) } \\
\hline Volume (liters) & 5 & 5 & 5 & 5 & 5 & 5.5 & 4.35 & 4.3 & 4.2 & 4.7 \\
\hline oH (std units) & 9.8 & 9.6 & 12.2 & 12.2 & 12.2 & 11.2 & 12.4 & 12 & 10.6 & 11.8 \\
\hline $\cos (\pi g / 1)$ & 1935 & 2050 & .2850 & 2050 & 3050 & 2011 & 2011 & 2011 & 1992 & 1992 \\
\hline NH3̈N (ag/l) & 168 & 173 & 173 & 173 & 173 & 164 & 164 & 164 & 156 & 156 \\
\hline SS (mg/1) & 284 & 204 & 294 & 204 & 284 & 338 & 338 & 338 & 338 & 338 \\
\hline USS (mg/1) & 62 & 62 & 62 & 62 & 62 & 90 & 98 & 98 & 90 & 90 \\
\hline$C N(\log / 1)$ & 4.1 & 4.1 & 4.1 & 4.1 & 4.1 & & & & & \\
\hline $\operatorname{SCN}(\operatorname{mog} / 1)$ & 173 & 173 & 173 & 173 & 173 & & & & & \\
\hline 80D 5-day (mg/1) & 1170 & 1330 & 1330 & 1330 & 1330 & 1210 & 1218 & 1210 & 1280 & 1280 \\
\hline $\operatorname{TOC}(\operatorname{mg} / \mathrm{l})$ & 601 & 629 & 629 & 629 & 623 & 626 & 626 & 626 & 643 & 643 \\
\hline Phenolics (mo/l) & 2.29 & 2.29 & 2.29 & 2.29 & 2.29 & 5.24 & 5.24 & 5.24 & 5.24 & 5.24 \\
\hline \multicolumn{11}{|l|}{$\mathrm{PO} 4-\mathrm{P}(\mathrm{gg} / \mathrm{H})^{-}$} \\
\hline$O r g-N(m g / 1)$ & 58 & 58 & 58 & 58 & 58 & & & & & \\
\hline \multicolumn{11}{|l|}{ NO2 (mo/1) } \\
\hline \multicolumn{11}{|l|}{$\mathrm{NO3}$ (mg/1) } \\
\hline \multicolumn{11}{|l|}{ TDS (mg/!) } \\
\hline Color (APPHA Units) & 1000 & 1800 & 1000 & 1000 & 1800 & 750 & 750 & 750 & 750 & 750 \\
\hline \multicolumn{11}{|l|}{ BRSIN PARAMETERS } \\
\hline Vol Wasted (mil) & 227 & 549 & 585 & 448 & 527 & 519 & 586 & 538 & 566 & 566 \\
\hline D.0. $(\operatorname{mog} / 1)$ & 8 & 7.6 & 7.4 & 7.2 & 5 & 5.2 & 4.8 & 5 & 5.8 & 5.8 \\
\hline SS $(m g / 1)$ & 3168 & 2380 & 3410 & 3760 & 3390 & 3730 & 2918 & 3420 & 3410 & 3460 \\
\hline *SS (PAC corrected) & 3168 & 2380 & 3410 & 3760 & 3390 & 3730 & 2910 & 3428 & 3410 & 3460 \\
\hline Temp (C) & 20 & 19.5 & 19 & 20 & 19 & 19 & 19 & 19 & 19 & 19 \\
\hline $\mathrm{DH}$ (std units) & 6.7 & 6.3 & 6.2 & 8.2 & 9 & 7.6 & 6.4 & 6.8 & 6.9 & 7.4 \\
\hline OUR (mg/1/hr) & 24 & 24 & & & 29 & & & 24 & $\because$ & \\
\hline VSS (mq/1) & 2688 & 2680 & 2680 & 3199 & 2740 & 3015 & 2352 & 2970 & 2961 & 3005 \\
\hline \#USS (PAC corrected) & 2688 & 2680 & 2680 & 3189 & 2740 & 3015 & 2352 & 2970 & 2961 & 3005 \\
\hline VSS/SS & 0.85 & 0.85 & 0.85 & 0.85 & 0.81 & 0.81 & 0.81 & 0.87 & 0.87 & 0.87 \\
\hline *PAC Added (ma) & 0 & 0 & 8 & 8 & 0 & 0 & 0 & 0 & 0 & 0 \\
\hline *inventory (mg/l basin) & 8 & 8 & 8 & 0 & 8 & 0 & 8 & $e$ & 0 & 0 \\
\hline \multicolumn{11}{|l|}{ EFFLUENT DARAMETERS } \\
\hline Volune (liters) & 4.68 & 5.68 & 4.65 & 4.6 & 4 & 5.24 & 4.26 & 4.85 & 4.11 & 4.18 \\
\hline $\operatorname{coD}(\operatorname{mo} / 1)$ & 152 & 163 & 163 & 163 & 197 & 273 & 237 & 226 & 205 & 190 \\
\hline SS $(\operatorname{mg} / 1)$ & 252 & 24 & 78 & 124 & 62 & 58 & 64 & 52 & 28 & 28 \\
\hline ¥PAC corrected & 32 & 598 & 28 & 74 & 46 & 58 & 58 & 34 & 18 & 18 \\
\hline $\mathrm{pH}$ (std units) & 6.9 & 6.6 & 6.5 & 8.3 & 8.6 & 8.1 & 7.4 & 7.1 & 7 & 7.2 \\
\hline USS $\left(\operatorname{sg}^{\prime} / 1\right)$ & 286 & 206 & 206 & 206 & 40 & 40 & 40 & 40 & 49 & 40 \\
\hline *PAC corrected & 24 & 24 & 24 & 24 & 38 & 38 & 38 & 38 & 38 & 38 \\
\hline NH3N $(\mathrm{gg} / 1)$ & 10.0 & 37.5 & 37.5 & 37.5 & 37.5 & 34.2 & 34.2 & 34.2 & 15.8 & 15.8 \\
\hline CN $(m g / 1)$ & 1.51 & 1.51 & $1.5 !$ & 1.51 & 1.51 & & & & & \\
\hline $\operatorname{SCN}(a g / 1)$ & 3.1 & 3.1 & 3.1 & 3.1 & 3.1 & & & & & \\
\hline NO2 (ag/1) & 3.81 & 3.81 & 3.81 & 3.81 & 3.81 & & & & & \\
\hline $\mathrm{NO3}(\operatorname{mg} / 1)$ & 148 & 148 & 148 & 148 & 148 & & & & & \\
\hline Phenolics (mg/1) & 10.025 & 10.025 & 18.025 & 10.025 & 10.025 & & & & & \\
\hline$P 04-P(\operatorname{mg} / 1)$ & 34.6 & 23.3 & 23.3 & 23.3 & 23.3 & 12.9 & 12.9 & 12.9 & 8.3 & 8.3 \\
\hline $\begin{array}{l}\text { Org-N (mg/l) } \\
\text { TDS (mo/l) }\end{array}$ & 14.0 & 14.0 & 14.0 & 44.0 & 14.0 & & & & & \\
\hline \multicolumn{10}{|l|}{ TDS $(m g / 1)$} & 875 \\
\hline $\begin{array}{l}\text { Color (APHA units) } \\
\text { TOC (mg/1) }\end{array}$ & $\begin{array}{r}875 \\
45\end{array}$ & $\begin{array}{r}875 \\
47\end{array}$ & $\begin{array}{r}875 \\
47\end{array}$ & $\begin{array}{r}875 \\
47\end{array}$ & $\begin{array}{r}815 \\
47\end{array}$ & $\begin{array}{r}815 \\
51\end{array}$ & $\begin{array}{r}875 \\
51\end{array}$ & $\begin{array}{r}875 \\
51\end{array}$ & $\begin{array}{r}875 \\
53\end{array}$ & $\begin{array}{r}815 \\
53\end{array}$ \\
\hline BOD 5-day (mg/1) & 1.9 & 2.3 & 2.3 & 2.3 & 2.3 & 1.6 & 1.6 & 1.6 & 3.3 & 3.3 \\
\hline & & & & $-111-$ & & & & & & \\
\hline
\end{tabular}




\begin{tabular}{|c|c|c|c|c|c|c|c|c|c|c|}
\hline YONTH & NOV & NOV & NOV & NOV & NOV & NOV & NOV & NOV & NOV & NOV \\
\hline DAY & 13 & 14 & 15 & 16 & 17 & 18 & 19 & 20 & 21 & 22 \\
\hline DAY NO. & 110 & 111 & 112 & 113 & 114 & 115 & 116 & 117 & 118 & 119 \\
\hline \multicolumn{11}{|l|}{ SYSTEM PARAMETERS } \\
\hline *Sludge Age & 19.99 & 28.17 & 19.94 & 20.18 & 20.13 & 20.12 & 19.22 & 19.93 & 19.94 & 20.85 \\
\hline$*(F / M) C O D$ & 0.26 & 0.25 & 0.25 & 0.27 & 0.30 & 0.31 & 8.38 & 0.29 & 0.32 & 0.23 \\
\hline *HRT & 2.27 & 2.13 & 2.24 & 2.89 & 2.82 & 2.21 & 1.79 & 2.42 & 2.82 & 2.18 \\
\hline \multicolumn{11}{|l|}{ FEED FARAMETERS (BATCH } \\
\hline Volume (liters) & 4.1 & 4.37 & 4.15 & 4.45 & 4.6 & 4.2 & 5.2 & 3.85 & 4.6 & 4.42 \\
\hline $\mathrm{pH}$ (std units) & 11.7 & 11.8 & 11.9 & i2 & 11.7 & 11.1 & 11.8 & 11.8 & 11.9 & 11.9 \\
\hline $\operatorname{coD}(\operatorname{mg} / 1)$ & 1992 & 1992 & 2259 & 2059 & 2059 & 2059 & 2059 & $2 \times 59$ & 2859 & $\hat{c} 059$ \\
\hline NHĴN (mo/l) & 156 & 156 & 135 & 135 & 135 & 134 & 134 & 134 & 134 & 134 \\
\hline $5 S(0 g / 1)$ & 338 & 338 & 700 & 708 & 700 & 738 & 700 & 700 & 700 & 795 \\
\hline VSS (mg/1) & 90 & 90 & 122 & 122 & 122 & 122 & 122 & 122 & 122 & 170 \\
\hline CN (mg/l) & & & 3.99 & 3.99 & 3.99 & 3.99 & 3.99 & 3.99 & 3.99 & 1.24 \\
\hline $\operatorname{SCN}(\pi g / 1)$ & & & 173 & 173 & 173 & 173 & 173 & 173 & 173 & 188 \\
\hline BOD 5-day $(\mathrm{mg} / 1)$ & 1288 & 1289 & 1050 & 1050 & 1250 & 1320 & 1320 & 1320 & 1320 & 12,48 \\
\hline TOC (ng/l) & 643 & 643 & 637 & 637 & 637 & 653 & 653 & 653 & 653 & 650 \\
\hline Phenolics (mg/1) & 5.24 & 5.24 & 3.33 & 3.33 & 3.33 & 3.33 & 3.33 & 3.33 & 3.33 & 5.11 \\
\hline \multicolumn{11}{|l|}{$P 04-P$ (mo/l) } \\
\hline Org-N (og/l) & & & 60 & 60 & 68 & 60 & 60 & 68 & 60 & 73 \\
\hline \multicolumn{11}{|l|}{ NDE (mp/l) } \\
\hline \multicolumn{11}{|l|}{$\mathrm{NO3}$ (ing/l) } \\
\hline \multicolumn{11}{|l|}{ TDS (mg/l) } \\
\hline Color (APHA Units) & 750 & 750 & 1258 & $\$ 250$ & 1250 & 1250 & 1250 & 1250 & 1250 & 1500 \\
\hline \multicolumn{11}{|l|}{ BASIN PARAMETERS } \\
\hline Vol Wasted (ml) & 487 & 550 & 530 & 530 & 530 & 530 & 410 & 552 & 520 & 550 \\
\hline D.0. (ag/1) & 6.1 & 4.5 & 8.2 & 7.4 & 8.7 & 8.2 & 8.2 & 8.1 & 8.4 & 8.6 \\
\hline SS $(\operatorname{mg} / 1)$ & 3888 & 3330 & 3223 & 3295 & 3030 & 2730 & 2725 & 2698 & 2755 & 3645 \\
\hline *SS (PAC corrected) & 3000 & 3330 & 3223 & 3295 & 3830 & 2730 & 2725 & 2630 & 2755 & 3645 \\
\hline Temo $(C)$ & 19 & 18.5 & 18 & 19.5 & 19.5 & 18 & 19 & 19 & วh & 19 \\
\hline aH (std units) & 7.6 & 7.8 & 7.8 & 7.7 & 7.8 & 7.2 & 7.5 & 7.9 & 8 & 7.8 \\
\hline OUR (ng/1/hr) & & 28 & & & 20.4 & & & & 27 & \\
\hline VSS $(m \underline{a} / 1)$ & 2605 & 2890 & 2737 & 2860 & 2590 & 2334 & 2329 & 2299 & 2440 & 3228 \\
\hline IVSS (PAC correc:Ed) & 2605 & 2890 & 2797 & 2860 & 2590 & 8334 & 8389 & 2899 & 8440 & 3Eट̇ \\
\hline$v 99 / 99$ & 0.87 & 0.07 & 0.87 & 0.87 & 0.85 & 0.85 & 0.85 & 0.83 & v. $y y$ & U. $8 y$ \\
\hline tPAC Added (mg) & 0 & 8 & 0 & $\theta$ & 0 & 0 & 0 & $\theta$ & 8 & 0 \\
\hline Inventory (mg/l basin) & 8 & 8 & 8 & 6 & 8 & 8 & 0 & 8 & 0 & 8 \\
\hline \multicolumn{11}{|l|}{ EFRUENT PARAMETERS } \\
\hline Voluase (liters) & 4.05 & 3.95 & 3.85 & 4.26 & 4.35 & 3.95 & 4.49 & 4.85 & 4.18 & 3.85 \\
\hline COD $(a g / 1)$ & 192 & 193 & 170 & 170 & 103 & 197 & 193 & 204 & 208 & 188 \\
\hline ss $(\mathrm{og} / 1)$ & 84 & 38 & 68 & 58 & 46 & 46 & 130 & 32 & 54 & 46 \\
\hline APAC corrected & 108 & 22 & & & & & & & & \\
\hline pH (std units) & 7.3 & 7.9 & 8.1 & 8.1 & 8.1 & 7.4 & 7.6 & 8 & 8.4 & 8.3 \\
\hline VSS $(m g / 1)$ & 40 & 38 & & & 46 & & & & 54 & \\
\hline *PAC corrected & 38 & 20 & & & & & & & & \\
\hline NH3N (ng/l) & 15.8 & 15.8 & 4.3 & 4.3 & 4.3 & 14.0 & 14.0 & 14.0 & $<4.0$ & 14.0 \\
\hline$C N(m g / 1)$ & & & 1.01 & 1.01 & 1.81 & 1.01 & 1.01 & 1.01 & 1.01 & \\
\hline $\operatorname{SCN}(n g / 1)$ & & & 4.1 & 4.1 & 4.1 & 4.1 & 4.1 & 4.1 & 4.1 & \\
\hline NO2 $(0 \mathrm{~g} / 1)$ & & & 4.39 & 4.39 & 4.39 & 4.39 & 4.39 & 4.39 & 4.39 & \\
\hline $\mathrm{NOO3}(\mathrm{mg} / 1)$ & & & 177 & 177 & 177 & 177 & 177 & 177 & 177 & \\
\hline Phenolics $(\mathrm{mg} / \mathrm{l})$ & & & 1. 8.25 & 1.025 & 1.025 & <.825 & $<.025$ & 1.025 & 1.025 & 1.025 \\
\hline$P 04-P(m g / 1)$ & 8.3 & 8.3 & 2.3 & 2.3 & 2.3 & 3.5 & 3.5 & 3.5 & 3.5 & 4.4 \\
\hline $\begin{array}{l}\text { Org-N }(m g / 1) \\
\text { TDS }(m g / 1)\end{array}$ & & & 4.8 & 4.8 & 4.8 & 4.8 & $\begin{array}{l}4.8 \\
.\end{array}$ & 4.8 & 4.8 & \\
\hline Color (APHA units) & 875 & 875 & 750 & 750 & 758 & 750 & 758 & 750 & 750 & 750 \\
\hline TOC (⿴囗口 $/ 1)$ & 53 & 53 & 56 & 56 & 56 & 56 & 56 & 56 & 56 & 53 \\
\hline BOD 5-day (mg/l) & 3.3 & 3.3 & 2.3 & 2.3 & 2.3 & 2.8 & 2.8 & 2.8 & 2.8 & 1.6 \\
\hline
\end{tabular}




\begin{tabular}{|c|c|c|c|c|c|c|c|c|c|c|}
\hline MONTH & NOV & NOV & NOV & NOV & NOV & NOV & NOV & NOV & JEC & $D E C$ \\
\hline DAY & 23 & 24 & 25 & 26 & 27 & 28 & 29 & 30 & 1 & 2 \\
\hline DAY : $: 0$. & 120 & 121 & 122 & 123 & 124 & 125 & 126 & 127 & 128 & 123 \\
\hline \multicolumn{11}{|l|}{ SYSTEM FARAYETERS } \\
\hline *Sludg̣e Ag̣e & 20.11 & 20.81 & 20.20 & 19.99 & 19.39 & 20.81 & 19.39 & 19.38 & 20.81 & 20.20 \\
\hline$*(F / M) C O D$ & 0.31 & 0.25 & 0.28 & 0.25 & 0.29 & 0.29 & 0.30 & 0.29 & 0.23 & 0.31 \\
\hline *HRT & 2.20 & 1.92 & 2.10 & 2.27 & 1.99 & 2.13 & 2.04 & 2.16 & 2.58 & 2.02 \\
\hline \multicolumn{11}{|l|}{ FEED PARAMETERS (BATCH } \\
\hline Volume (liters) & 4.23 & 4.85 & 4.43 & 4.1 & 4.675 & 4.36 & 4.55 & 4.3 & 3.6 & 4.6 \\
\hline ph (std units) & 11 & 12 & 11.85 & 12 & 11.9 & 11.9 & 11.9 & 11.8 & 11.2 & 11.7 \\
\hline $\operatorname{coD}(\mathrm{mg} / 1)$ & 2959 & 2059 & 2098 & 2098 & 2898 & 2098 & 2027 & 2027 & 2027 & 2246 \\
\hline NH3N $(\operatorname{mg} / 1)$ & 134 & 134 & 147 & 147 & 147 & 147 & 147 & .147 & 147 & 142 \\
\hline SS (mg/1) & 795 & 795 & 795 & 795 & 795 & 795 & 395 & 395 & 395 & 395 \\
\hline USS $(\pi g / 1)$ & 170 & 170 & 170 & 170 & 170 & 170 & 85 & 85 & 85 & 85 \\
\hline CN (mg/1) & 1.24 & 1.24 & 1.24 & 1.24 & 1.24 & 1.24 & 1.24 & 1.24 & 1.24 & 1.34 \\
\hline $\operatorname{SCN}(\operatorname{mg} / 1)$ & 188 & 188 & 188 & 188 & 188 & 188 & 188 & 198 & 188 & 188 \\
\hline 800 5-day $(\operatorname{mg} / 1)$ & 1248 & 1240 & 1220 & 1220 & 1220 & 1220 & 1200 & 1200 & 1300 & 1250 \\
\hline TOC $(\operatorname{sg} / 1)$ & 650 & 650 & 660 & 660 & 660 & 660 & 647 & 647 & $E 47$ & 638 \\
\hline Phenolics (mg/1) & 5.11 & 5.11 & 5.11 & 5.11 & 5.11 & 5.11 & 5.09 & 5.09 & 5.29 & 5.09 \\
\hline \multicolumn{11}{|l|}{ PO4-P (mg/1) } \\
\hline Org-N (mg/1) & 73 & 73 & 73 & 73 & 73 & 73 & 73 & 73 & 73 & 73 \\
\hline \multicolumn{11}{|l|}{$.02(m g / 1)$} \\
\hline \multirow{2}{*}{\multicolumn{11}{|c|}{$\mathrm{NOJ}(\mathrm{mg} / \mathrm{ll})$}} \\
\hline $\operatorname{TDS}(m g / 1)$ & & & & & & & & & & \\
\hline Color (APHA Units) & 1500 & 1508 & 1580 & 1580 & 1580 & 1508 & 1580 & 1508 & 1590 & 1508 \\
\hline \multicolumn{11}{|l|}{ BASIN PARAMETERS } \\
\hline Vol Wasted (ml) & 520 & 470 & -230 & 239 & 328 & 478 & 415 & 485 & 511 & 516 \\
\hline D.0. (ag/l) & 7.4 & 7.8 & 7.8 & 6.4 & 6.6 & 6.5 & 6.7 & 6.7 & 6.8 & 7 \\
\hline SS (mg/l) & 2695 & 3730 & 3160 & 3215 & 3170 & $3 \hat{205}$ & 3200 & 3145 & 3220 & 3190 \\
\hline *SS (PAC corrected) & 2695 & 3730 & 3168 & 3215 & 3178 & 3205 & 3200 & 3145 & 3220 & 3190 \\
\hline Temp (C) & 20 & 28 & 20 & 19 & 20 & 20 & 20 & 20 & 19 & 18 \\
\hline $\begin{array}{l}\text { pH (std units) } \\
\text { OUR (agg/l/hr) }\end{array}$ & 7.4 & 7.7 & 7.55 & 7.8 & 7.1 & $\begin{array}{r}7.8 \\
24\end{array}$ & 8 & 8.1 & $\begin{array}{r}7.6 \\
24\end{array}$ & 7.6 \\
\hline VSS (mg/1) & 2375 & 3287 & 2785 & 2833 & 2794 & 2585 & 2581 & 2537 & 2595 & 2571 \\
\hline *USS (PAC corrected) & 2375 & 3287 & 2785 & 2833 & 2794 & 2585 & 2581 & 2537 & 2595 & 2571 \\
\hline USS/SS & 0.88 & 0.88 & 8.88 & 0.88 & 0.88 & 0.81 & Q.81 & 0.81 & 0.81 & 0.81 \\
\hline *PAC Added (mg) & 0 & 0 & 0 & 0 & 0 & 0 & 0 & 0 & 0 & 0 \\
\hline IInventory (ag/l basin) & 0 & 8 & 0 & 0 & 0 & 0 & 0 & 8 & 0 & 8 \\
\hline \multicolumn{11}{|l|}{ EFFLUENT PARAMETERS } \\
\hline Volume (Iiters) & 4.14 & 4.48 & 4.57 & 3.95 & 4.59 & 3.9 & 4.12 & 3.95 & 3.1 & 4.18 \\
\hline $\cos (\operatorname{mg} / 1)$ & .176 & 187 & 185 & 168 & 153 & 157 & 146 & 142 & 143 & 137 \\
\hline SS $(m g / 1)$ & 50 & 108 & 574 & 294 & 188 & 100 & 144 & 92 & $\$$ & 64 \\
\hline \multicolumn{11}{|l|}{ FAC corrected } \\
\hline oH (std units) & 8.1 & 7.7 & 7.8 & 7.8 & 7.6 & 7.7 & 8 & 8 & 7.9 & 7.8 \\
\hline VSS (mg/1) & 44 & 38 & & & & 58 & & & 60 & \\
\hline \multicolumn{11}{|l|}{ PAC corrected } \\
\hline NH3N $(n g / 1)$ & $\langle 4.0$ & 14.0 & 14.0 & $\langle 4.8$ & 14.8 & 44.0 & $\langle 4.0$ & 44.0 & 44.0 & $<4.0$ \\
\hline CN $(\operatorname{mg} / 1)$ & & & & & & & 1.33 & 1.33 & 1.33 & 1.33 \\
\hline $\operatorname{SCN}$ (ag/l) & & & & & & & 2.8 & 2.8 & 2.8 & 2.8 \\
\hline NO2 (ng/1) & & & & & & & 2.0 & 2.0 & 2.0 & 2.0 \\
\hline $\mathrm{NO3}(\operatorname{lag} / 1)$ & & & & & & & 170 & 170 & 170 & 170 \\
\hline Phenolics (mg/l) & $<.025$ & 1.025 & 1.025 & <. 825 & ४.825 & 1.825 & $<.825$ & <.825 & $<.825$ & 1.005 \\
\hline$P 04-P(m g / 1)$ & 4.4 & 4.4 & 8.4 & 8.4 & 8.4 & 8.4 & 5.4 & 5.4 & 5.4 & 8.4 \\
\hline $\operatorname{Org}-N(m g / 1)$ & & & & & & & 14 & 14 & 14 & 14 \\
\hline TDS (mg/1) & & & & & & & 4660 & 4660 & 4660 & 4660 \\
\hline Color (GPHA units) & 758 & 750 & 750 & 758 & 750 & 750 & 750 & 750 & 750 & 750 \\
\hline $\operatorname{TOC}(\operatorname{mg} / 1)$ & 53 & 53 & 42 & 42 & 42 & 42 & 38 & 38 & 38 & 43 \\
\hline BOD 5-day $($ gnop/1) & 1.5 & 1.6 & 12 & 12 & 12 & 12 & 1.5 & 1.5 & 1.5 & 12 \\
\hline
\end{tabular}


UNIT $2 A$ TABLE A3

\begin{tabular}{|c|c|c|c|c|c|c|c|c|c|c|}
\hline MONTH & DEC & $D E C$ & DEC & DEC & DEC & $D \equiv C$ & UEC & DEC & $D E C$ & $D E C$ \\
\hline DAY & 3 & 4 & 5 & 6 & 7 & 8 & 9 & 10 & $1:$ & 12 \\
\hline DAY NO. & 130 & 131 & 132 & 133 & 134 & 135 & 136 & 137 & 138 & 139 \\
\hline \multicolumn{11}{|l|}{ SYSTEY FARAMETERS } \\
\hline *Slưgge Age & 20.00 & 20.01 & 20.80 & 29.98 & 51.47 & 49.92 & 58.85 & 50.01 & 50.09 & 50.08 \\
\hline$*(F / M) C O D$ & 0.32 & 8.26 & 0.23 & $0 . \hat{\varepsilon} 5$ & 0.28 & 0.25 & 0.27 & 0.29 & 0.24 & 0.24 \\
\hline \#HRT & 1.80 & 2.21 & 2.84 & 2.42 & 1.96 & 2.16 & 1.96 & $1.8 !$ & 2.21 & 2.02 \\
\hline \multicolumn{11}{|l|}{ FEED PARAMETERS (BATCH \&) } \\
\hline Volume (liters) & 5.18 & 4.2 & 4.55 & 3.85 & 4.74 & 4.3 & 4.75 & 5.15 & 4.2 & 4.6 \\
\hline of (std units) & 11.7 & 11.8 & 11.5 & 10.5 & 11.6 & 11.6 & 11.7 & 11.8 & 11.4 & 11.5 \\
\hline $\operatorname{COD}(m g / 1)$ & 2046 & 2046 & 2046 & 2050 & 2050 & 2060 & 2122 & 2122 & 2122 & 2122 \\
\hline$N H i J N($ mg/l) & 142 & 142 & 142 & 131 & 131 & 131 & 140 & 140 & 140 & 140 \\
\hline ss (ong/1) & 395 & 395 & 395 & 522 & 522 & 522 & 522 & 522 & 522 & 522 \\
\hline VSS $(m g / 1)$ & 85 & 85 & 85 & 114 & 114 & 114 & $1 ! 4$ & 114 & 114 & 114 \\
\hline$C N(m g / 1)$ & 1.24 & 1.24 & 1.24 & & & & & & & \\
\hline $\operatorname{SCN}(\operatorname{mg} / 1)$ & 188 & 188 & 188 & & & & & & & \\
\hline BOD 5-day (mọ/l) & 1250 & 1250 & 1250 & 1210 & 1210 & 1210 & 1260 & 1252 & 1268 & 1260 \\
\hline TOC $(m g / 1)$ & 638 & 638 & 638 & 637 & 637 & 637 & 664 & 664 & 664 & 664 \\
\hline Phenolics $(\mathrm{mg} / 1)$ & 5.09 & 5.89 & 5.09 & 5.51 & 5.61 & 5.61 & 5.51 & 5.61 & 5.61 & 5.61 \\
\hline Org-N (mg/l) & 73 & 73 & 73 & & & & & & & \\
\hline $\mathrm{NOC}(\mathrm{mg} / \mathrm{I})$ & & & & & & & & & & \\
\hline $\mathrm{NO3}$ (mg/1) & & & & & & & & & & \\
\hline $\operatorname{TDS}(\mathrm{mg} / 1)$ & $\cdot$ & & & & & & & & & \\
\hline Color (APHA Lnits) & 1500 & 1500 & 1500 & 1580 & 1500 & 1500 & 1500 & 1580 & 1500 & 1530 \\
\hline \multicolumn{11}{|l|}{ BASIN PARAMETERS } \\
\hline Vol Wasted (nl) & 558 & 437 & 545 & 348 & 198 & 182 & 165 & 170 & 187 & 208 \\
\hline $0.0 .(\mathrm{gg} / 1)$ & 7 & 6.8 & 6.8 & 6.9 & 8.8 & 8.8 & 8.5 & 8.4 & 8.4 & 8.4 \\
\hline SS (mg/l) & 3415 & 3395 & 3430 & 3355 & 3655 & 3885 & 3980 & 4065 & 4855 & 4250 \\
\hline *SS (PAC corrected) & 3415 & 3395 & 3430 & 3355 & 3655 & 3805 & 3980 & 4065 & 4255 & 4250 \\
\hline Tewo (C) & 19 & 19 & 19 & 19 & 18 & 18 & 19 & 19 & 18 & 19 \\
\hline $\mathrm{pH}$ (std units) & 7.4 & 7.8 & 7.5 & 7.7 & 7.2 & 8.1 & 8 & 7.8 & 7.7 & 7.4 \\
\hline OUR $(m g / 1 / h r)$ & & & & 36 & & & & & & 24 \\
\hline VSS $(m g / 1)$ & 2752 & 2736 & 2683 & 2690 & 29.31 & 8355 & 3091 & 3157 & 3149 & 3380 \\
\hline IVSS (PAC corrected) & 2752 & 2736 & 2683 & 2690 & 2931 & 2955 & 2091 & 3157 & $3 ! 49$ & 3380 \\
\hline VSS/SS & 0.81 & 0.81 & 0.78 & 0.80 & 0.80 & 0.78 & 0.78 & 0.78 & 0.78 & 0.80 \\
\hline *FAC Added (mg) & 0 & 8 & 0 & 0 & 0 & 0 & 8 & a & $a$ & 0 \\
\hline Inventory (mg/l basin) & 8 & 0 & 8 & 0 & 0 & 0 & 0 & 0 & 0 & 0 \\
\hline \multicolumn{11}{|l|}{ EFFLUENT PARAMETERS } \\
\hline Volume (1iters) & 4.74 & 4 & 4.1 & 3.25 & 4.14 & 4.05 & 4.25 & 4.74 & 4.1 & 4.2 \\
\hline $\operatorname{coD}(\operatorname{mg} / 1)$ & 154 & 157 & 155 & 146 & 144 & 155 & 161 & 156 & 154 & 153 \\
\hline S5 $(m g / 1)$ & 36 & 138 & 46 & 54 & 38 & 130 & 70 & 68 & 52 & 32 \\
\hline \multicolumn{11}{|l|}{ PAC corrected } \\
\hline oH (std units) & 7.6 & 7.9 & 7.8 & 8 & 8 & 7.9 & 7.9 & 7.8 & 7.8 & 7.7 \\
\hline USS (mo/1) & & & 34 & 24 & & 78 & & & & 22 \\
\hline \multicolumn{11}{|l|}{ IPAC coirrected } \\
\hline NH3N (mด/1) & 14.0 & 14.8 & 14.8 & 12 & 12 & 12 & 3 & 2 & 2 & 2 \\
\hline CN $(a g / 1)$ & 1.33 & 1.33 & 1.33 & & & & & & & \\
\hline $\operatorname{SCN}(\operatorname{mg} / 1)$ & 2.8 & 2.8 & 2.8 & & & & & & & \\
\hline NO2 $(\mathrm{gg} / 1)$ & 2.0 & 2.8 & 2.0 & & & & & & & \\
\hline $\mathrm{NO3}(\mathrm{og} / 1)$ & 170 & 170 & 170 & & & & & & & \\
\hline Phenolics (mg/l) & 1.025 & 1.025 & 1.825 & 1.025 & 1.025 & 1.825 & 1.025 & 1.825 & 1.025 & C.025 \\
\hline FO4-D (mg/1) & 8.4 & 8.4 & 8.4 & 8.8 & 8.8 & 8.8 & 6.5 & 6.5 & 6.5 & 6.5 \\
\hline Org-N (mg/l) & 14 & 14 & $<4$ & & & & & & & \\
\hline TDS (man/1) & 4668 & 4560 & 4660 & 4685 & 4685 & 4685 & 4685 & 4605 & 4685 & 4605 \\
\hline Color (APHA units) & 750 & 750 & 758 & 758 & 758 & 758 & 758 & 750 & 750 & 758 \\
\hline $\operatorname{TOC}(m g / 1)$ & 43 & 43 & 43 & 45 & 45 & 45 & 41 & 41 & 41 & 41 \\
\hline BOD 5-day (mg/1) & 〈2 & 《2 & $<2$ & 2 & 2 & 2 & 2 & 2 & 2 & 2 \\
\hline
\end{tabular}




\begin{tabular}{|c|c|c|c|c|c|c|c|c|c|c|}
\hline MONTH & $D E C$ & DEC & DEC & DEC & $D E C$ & DEC & DEC & DEC & IEEC & $D E C$ \\
\hline DAY & 13 & 14 & 15 & 16 & 17 & 18 & 19 & 20 & $\hat{c l}$ & 22 \\
\hline DAY NO. & 140 & 141 & 142 & 143 & 144 & 145 & 146 & 147 & 148 & 149 \\
\hline \multicolumn{11}{|l|}{ SYSTEM PARAMETERS } \\
\hline +Sludge Age & 49.91 & 25.20 & 50.89 & 43.93 & 49. 39 & 49.89 & 50.02 & 49.38 & 58.03 & 49.91 \\
\hline$\because(F / M) C O D$ & 0.26 & 0.22 & 0.20 & 0.23 & 0.22 & 0.20 & 0.17 & 0.23 & 8. 22 & 0.22 \\
\hline \#HRT & 1.79 & 2.02 & 2.04 & 1.90 & 1.75 & 2.09 & 2.29 & 1.94 & 1.98 & 1.79 \\
\hline \multicolumn{11}{|l|}{ FEED PARAMETERS (BATCH \#) } \\
\hline Volure (liters) & 5.2 & 4.6 & 4.55 & 4.9 & $5.3 !$ & 4.45 & 4.07 & 4.8 & 4.9 & 5.2 \\
\hline$D H$ (std units) & 11.6 & 11.5 & 11.5 & 11.3 & 11.6 & 11.6 & 10.9 & 11.3 & 10.9 & 11.2 \\
\hline $\operatorname{CCD}(m g / 1)$ & 2011 & 2011 & 2011 & 2016 & 2016 & 2016 & 2016 & 2937 & 2037 & 2037 \\
\hline NHIN $(m g / 1)$ & 136 & 136 & 136 & 136 & 136 & 136 & 136 & 123 & 123 & 123 \\
\hline SS $(\operatorname{mog} / 1)$ & 482 & 482 & 482 & 482 & 482 & 482 & 482 & 0 & 0 & 0 \\
\hline VSS (mg/l) & 94 & 94 & $94^{\circ}$ & 34 & 94 & 94 & 94 & $\theta$ & 8 & 0 \\
\hline CN $(m g / 1)$ & 1.88 & 1.08 & 1.88 & 1.88 & 1.08 & 1.88 & 1.08 & 0 & 0 & 0 \\
\hline $\operatorname{SCN}(\operatorname{sg} / 1)$ & 184 & 184 & 184 & 184 & 184 & 184 & 184 & 0 & 8 & 0 \\
\hline 300 5-day $(\mathrm{mo} / 1)$ & 1280 & 1200 & 1200 & 1220 & 1220 & $: 220$ & 1220 & 1168 & 1160 & 1160 \\
\hline TaC (mg/1) & 648 & 648 & 640 & 651 & 651 & 651 & 651 & 650 & 658 & 650 \\
\hline \multicolumn{10}{|l|}{$P 04-P(m g / 1)$} & 6.96 \\
\hline $\operatorname{Org}-\mathrm{N}(\mathrm{mg} / 1)$ & 78 & 78 & 78 & 78 & 78 & 78 & 78 & & & \\
\hline \multicolumn{11}{|l|}{ NO2 (mo/l) } \\
\hline \multicolumn{11}{|l|}{$\mathrm{NO3}$ (nig/1) } \\
\hline \multicolumn{11}{|l|}{ TDS (mg/1) } \\
\hline Color (APHA Linits) & $\$ 520$ & 1520 & 1580 & 1580 & 1580 & 1508 & 1508 & 2580 & 2509 & 2500 \\
\hline \multicolumn{11}{|l|}{ ERSIN PRRAMETERS } \\
\hline Vol Wasted (al) & 190 & 28 & $: 76$ & 187 & 234 & 188 & 282 & 197 & 213 & 287 \\
\hline D.0. (go/l) & 8.2 & 8.4 & 8.5 & 8.6 & 8.6 & 8.8 & 8.8 & 8.4 & 8.4 & 8.4 \\
\hline SS (mg/1) & 4275 & 4335 & 4645 & 4365 & 4960 & 4625 & 5405 & 4655 & 5825 & 5170 \\
\hline ISS (PAC corrected) & 4275 & 4335 & 4645 & 4365 & 4968 & $46 \div 5$ & 5485 & 4655 & 5825 & 5170 \\
\hline Teפo (C) & 19 & 19 & 19 & 19 & 17 & 18 & 18 & 18 & 17 & 18 \\
\hline gh (std units) & 7.4 & 7.6 & 7.5 & 7.5 & 8 & 8 & 8.1 & $\theta$ & 8 & 7.8 \\
\hline OUR (mg/l/hr) & & & & & & & 24 & & & 24 \\
\hline VSS (mo/1) & 3400 & 3448 & 3740 & 3515 & 3994 & 3724 & 4115 & 3544 & 3825 & 3980 \\
\hline *VSS (PAC corrected) & 3400 & 3448 & 3740 & 3515 & 3994 & 3724 & $4 ! 15$ & 3544 & 3825 & 3980 \\
\hline USS/SS & 8.80 & 0.80 & 8.81 & 0.81 & 0.81 & 0.81 & 0.76 & 8.76 & 0.76 & 0.77 \\
\hline tPAC Added (mg) & 0 & 0 & 0 & 0 & 8 & 0 & 0 & 0 & 0 & 0 \\
\hline Inventory (mg/l basin) & 0 & 8 & 0 & 0 & 8 & 0 & 0 & 0 & 0 & 8 \\
\hline \multicolumn{11}{|l|}{ EFFLUENT PARAMETERS } \\
\hline Volune (liters) & 4.9 & 4.35 & 4.1 & 4.85 & 4.99 & 4.5 & 4.1 & 4.4 & 4.5 & 4.8 \\
\hline $\operatorname{coD}(m g / 1)$ & 158 & 150 & 150 & $2 \varepsilon^{4}$ & 161 & 163 & 163 & 171 & 148 & 168 \\
\hline SS (ag/1) & 44 & 448 & 72 & 48 & 6 & 54 & 50 & 46 & 30 & 36 \\
\hline \multicolumn{11}{|l|}{ YPAC corrected } \\
\hline of (std units) & 7.6 & 7.6 & 7.6 & 7.4 & 8 & 8 & 8 & 8.1 & 7.9 & 7.8 \\
\hline USS (mg/1) & & 383 & 42 & 28 & 4 & 32 & 26 & 24 & 16 & 26 \\
\hline \multicolumn{11}{|l|}{ *PAC corrected } \\
\hline NH3N (mg/l) & 4.8 & 4.8 & 4.8 & 27.4 & 27.4 & 27.4 & 27.4 & 47.1 & 47.1 & 47.1 \\
\hline CN $(\mathrm{mg} / \mathrm{l})$ & 0.814 & 0.814 & 0.814 & 0.814 & 8.814 & 0.814 & 0.814 & & & \\
\hline $\operatorname{SCN}(\mathrm{mg} / 1)$ & 3.7 & 3.7 & 3.7 & 3.7 & 3.7 & 3.7 & 3.7 & & & \\
\hline NO2 (ag/1) & 0.66 & 0.66 & 0.66 & 0.66 & 0.66 & 8.66 & 0.66 & & & \\
\hline NO3 (ng/l) & 126 & 126 & 126 & 126 & 126 & 126 & 126 & & & \\
\hline Phenolics (mg/h) & 6.025 & 1.025 & 1.025 & 1.825 & 1.025 & 1.025 & 1.025 & 1.025 & 1.025 & 1.025 \\
\hline$P 04-P(g g / 1)$ & 8.3 & 8.3 & 8.3 & 8.7 & 8.7 & 8.7 & 8.7 & 6.2 & 6.2 & 6.2 \\
\hline Org-N (*g/1) & 12 & 12 & 12 & 12 & 12 & 12 & 12 & & & \\
\hline TDS $(\mathrm{mg} / \mathrm{ll})$ & 4495 & 4495 & 4495 & 4495 & 4495 & 4495 & 4495 & 4375 & 4375 & 4375 \\
\hline Color (APHA units) & 873 & 875 & 875 & 875 & 875 & 875 & 875 & 875 & 875 & 875 \\
\hline $\operatorname{TOC}(\operatorname{mg} / 1)$ & 46 & 46 & 46 & 46 & 46 & 46 & 46 & 46 & 46 & 46 \\
\hline EOD 5-day $(\operatorname{mg} / 1)$ & 2.2 & 2.2 & 2.2 & 2 & 2 & 2 & 2 & 4 & 4 & 4 \\
\hline & & & & -115 & & & & & & \\
\hline
\end{tabular}




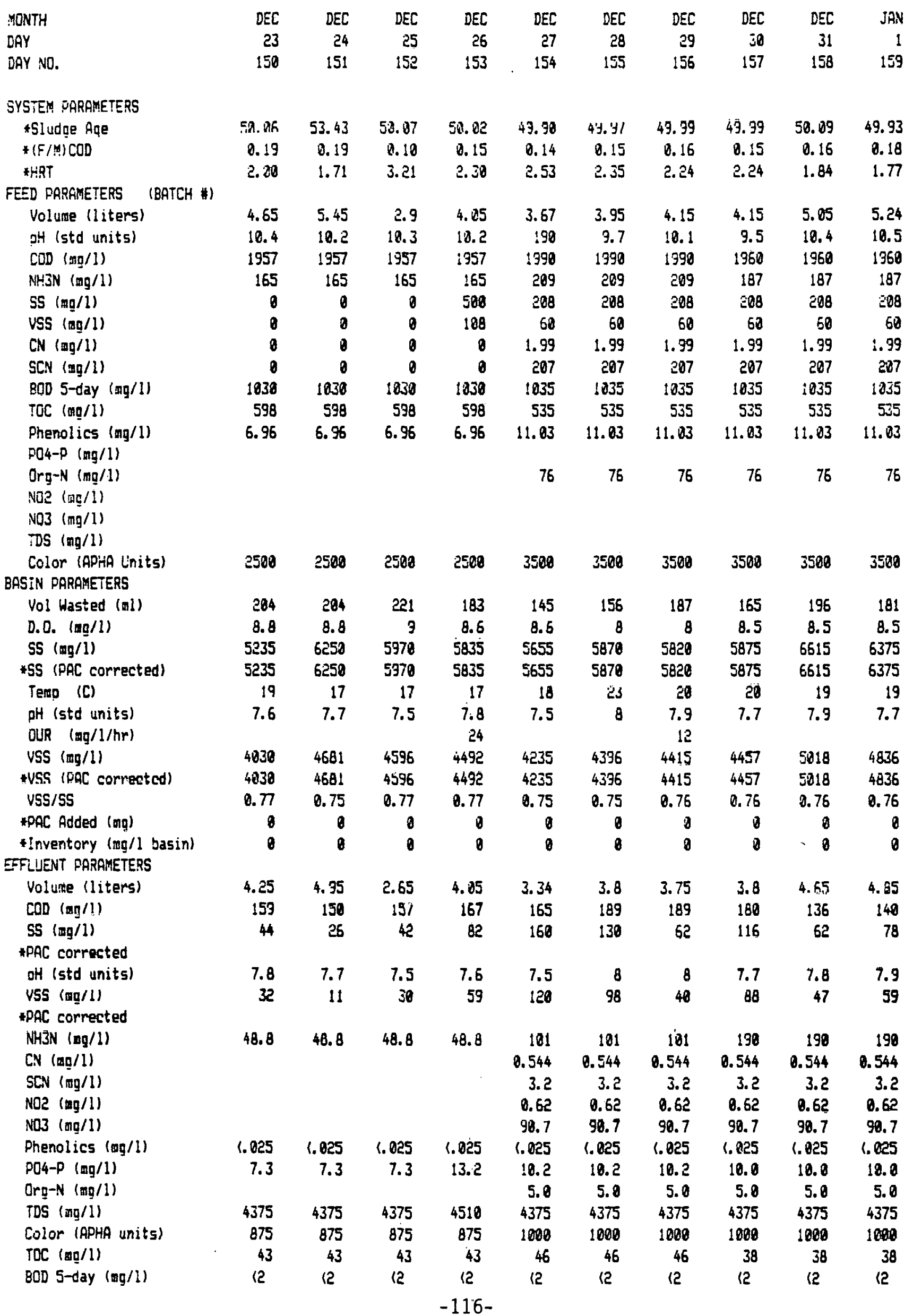


UNIT CA TABLE A3

MONTH

DAY NO.

SYSTEM PARA:HETERS

*Sludge Age

*(F/M) COD

*HRT

FEED PARAMETERS (BATCH \#)

Volune (liters)

pH (std units)

COD (mo/I)

NHH $3 N(\mathrm{mg} / 1)$

SS (बog/l)

USS (mo/l)

CN $(m g / 1)$

SCN (mg/1)

BOD 5-day (mg/1)

TOC (mo/l)

Phenolics (mg/l)

PO4-P (ag/l)

Org-N (mg/l)

NGe (mg/l)

$\mathrm{NO3}$ (mg/l)

TDS (mg/l)

Color (APHA Units)

BRSIN PARAKETERS

Vol Hasted (m1)

D.0. $(\mathrm{ag} / 1)$

SS (mg/l)

*SS (PAC corrected)

Temp (C)

ph (std units)

OUR (ng/l/hr)

USS (mg/l)

*USS (PAC corrected)

VSS/SS

*PAC Added (ing)

* Inventory (ag/l basin)

EFFLUENT PARAMETERS

Volume (liters)
COD (mg/1)
SS (mg/1)
tPAC corrected
OH (std units)
VSS (mg/1)
PPAC corrected
NH3N (mg/1)
CN (mg/1)
SCN (mg/1)
NO2 (mg/1)
NO3 (mg/1)
Phenolics (mg/1)
PO4-P (mg/1)
Org-N (mg/1)
TDS (mg/1)
Color (APHA units)
TOC (mg/1)
BOD 5-day (mg/1)

$\begin{array}{rrrrrrrrrr}\text { JAN } & \text { JAN } & \text { JAN } & \text { JAN } & \text { JAN } & \text { JAN } & \text { JAN } & \text { JAN } & \text { JAN } & \text { JAN } \\ 2 & 3 & 4 & 5 & 6 & 7 & 8 & 7 & 18 & 11 \\ 160 & 161 & 162 & 163 & 164 & 165 & 165 & 167 & 168 & 169\end{array}$

$\begin{array}{llllllllll}49.95 & 49.35 & 50.89 & 49.93 & 50.86 & 49.96 & 49.93 & 43.31 & 50.87 & 75.31\end{array}$

$\begin{array}{llllllllll}0.14 & 0.13 & 0.14 & 0.16 & 0.13 & 0.14 & 0.12 & 0.39 & 0.16 & 0.13\end{array}$

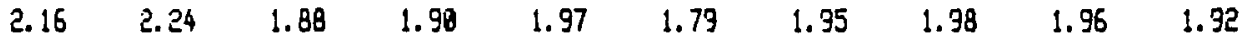

$\begin{array}{llllllllll}4.3 & 4.15 & 4.95 & 4.5 & 4.72 & 5.19 & 4.78 & 4.7 & 4.75 & 4.84\end{array}$

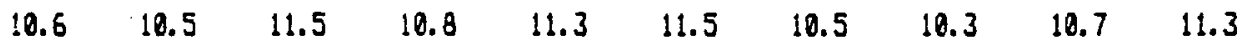

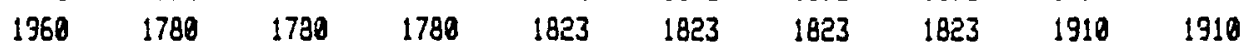

$\begin{array}{lllllllllll}187 & 185 & 185 & 185 & 176 & 176 & 176 & 176 & 195 & 195\end{array}$

$\begin{array}{llllllllll}288 & 474 & 474 & 474 & 474 & 474 & 474 & 474 & 344 & 344\end{array}$

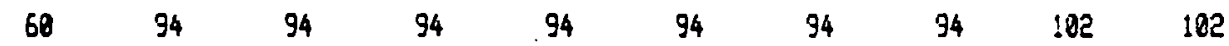

1.99

207

1035

535

11.03

$1050 \quad 1050$

1050
560

$10.3 \quad 10.3$

$10.3 \quad 10.3$

1058
568
10.3

1830

1030

1230
560

1830

560

10.3

10.3

10.3

0.503

0.203

155

155

$1020 \quad 1020$

1038

$603 \quad 603$

76

10.3

3.3

9.3

$66 \quad 66$

350

$1750 \quad 1750$

1750

1750

1758

1758

1750

2500

2500

$$
127
$$

8.5

6560

129

98

70

165

198

211

218

225

8.5

6815

6815

5945

8

7650

$\begin{array}{rr}8 & 7.1 \\ 9150 & 11810\end{array}$

7.

5945

7205

7660

8150

11810

19.20

$8.4 \quad 8.3$

8.2

18

20

18

17

17
7.6

18

7275

7275

3455

3455

4976

4976

0.76

4565

5233

36

4475

5423

7.8

20

20

5233

4475

5423

5766

613

24

$7.8 \quad 7.8$

0.75

0.75

5766

6135

7520

4632

6020

อ. 75

0.75

7528

4632

6020

8

0

0.64

0.64

0.64

4. 22

142

176

$3.8 \quad 4.55$

3.85

4. 34

4.93

4.43

4.27

4.45

4. 48

151

8.3

8. 1

224

332

129

162

158

106

185

134

8.3

8.2

8.1

B. 1

190

0.544 134 172

250

7.7

$\begin{array}{ll}7.6 & 7.7\end{array}$

3.2

0.62

90.7

1.025

10.0
5.0

5.0
4375

4375
1808

38

12

$$
202 \quad 202
$$$$
202
$$$$
211
$$$$
211
$$

$$
211
$$

211

$\begin{array}{rr}210 & 210 \\ 0.521 & 0.521 \\ 1.3 & 1.3 \\ 10.25 & 10.25 \\ 11.25 & 11.25 \\ 1.025 & 1.025 \\ 7.4 & 7.4 \\ 76 & 76 \\ 5280 & 5288 \\ 258 & 250 \\ 18 & 18 \\ 12 & 12\end{array}$




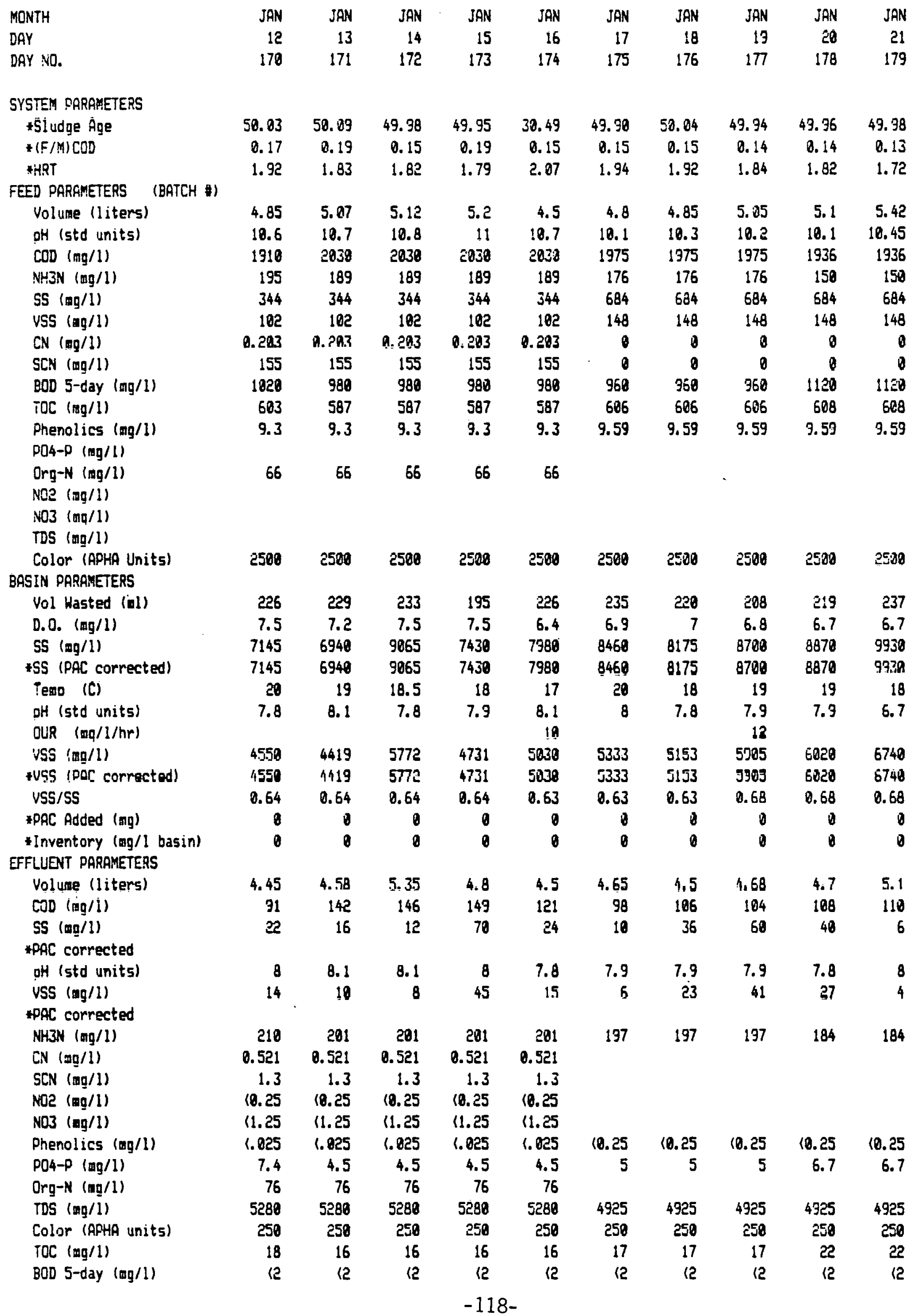




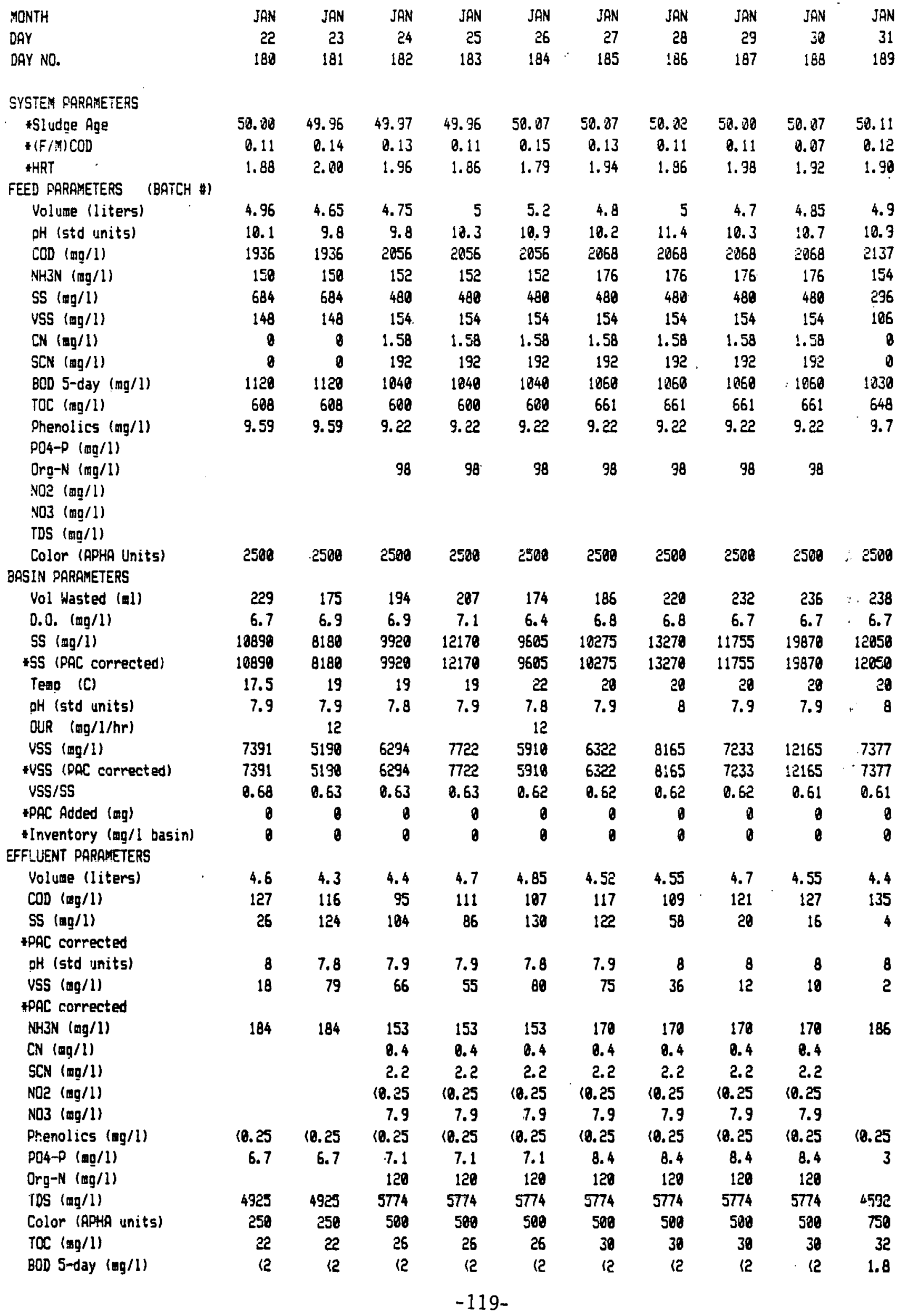


UNIT $2 A$ TABLE A3

\begin{tabular}{|c|c|c|c|c|c|c|c|c|c|c|}
\hline MONTH & FEB & FEB & $F \equiv B$ & FEB & FEB & FEB & FEB & FEB & FEB & FEB \\
\hline DAY & 1 & 2 & 3 & 4 & 5 & 6 & 7 & 8 & 9 & 10 \\
\hline DAY NO. & 190 & 191 & 132 & 193 & 194 & 135 & 196 & 197 & 198 & 199 \\
\hline \multicolumn{11}{|l|}{ SYSTEM CARRAMETERS } \\
\hline *Sludgoe Age & 40.26 & 39.94 & 39.34 & 40.24 & 39.96 & 39.37 & 40.81 & 39.38 & 39.95 & 39.95 \\
\hline$*(F / M) C O D$ & 0.11 & 0.13 & 0.13 & 0.12 & 0.89 & 0.13 & 0.14 & 0.13 & 0.12 & 0.11 \\
\hline AHRT & 1.93 & 1.36 & 1.84 & 1.36 & 2.27 & 1.78 & 1.69 & 1.63 & 2.19 & 2.19 \\
\hline \multicolumn{11}{|l|}{ FEED PARAMETERS (BATCH \#) } \\
\hline Volure (liters) & 4.83 & 5 & 5.05 & 4.99 & 4.1 & 5.22 & 5.5 & 5.5 & 4.25 & 4.25 \\
\hline oh (std units) & 10.4 & 10.6 & 10.7 & 10.3 & 10.9 & 11.5 & 11.5 & 11.2 & 10.4 & 10.4 \\
\hline $\operatorname{coD}(\operatorname{mog} / 1)$ & 2137 & 2137 & 2118 & 2118 & 2118 & 2118 & 2108 & 2120 & $2: 20$ & 2076 \\
\hline NH3N (mg/l) & 154 & 154 & 163 & 163 & 163 & 163 & 182 & $! 82$ & 182 & 183 \\
\hline SS $(\operatorname{mg} / 1)$ & 296 & 296 & 236 & 296 & 296 & 296 & 336 & 336 & 336 & 336 \\
\hline VSS (agg/1) & 106 & 186 & 106 & 106 & 106 & 106 & 154 & 154 & 154 & 154 \\
\hline $\mathrm{CN}(\operatorname{mog} / 1)$ & 0 & & & & & & 9.59 & 9.59 & 9.59 & 9.59 \\
\hline $\operatorname{SCN}(m g / 1)$ & 0 & & & & & & 184 & 184 & 194 & 184 \\
\hline BOD S=day $(\mathrm{mg} / \mathrm{l})$ & 1030 & 1030 & 1060 & 1250 & 1060 & ibse & 930 & 930 & 930 & $1: 30$ \\
\hline TOC $(\operatorname{mgg} / 1)$ & 648 & 648 & 610 & 610 & 610 & 610 & 645 & 645 & 645 & 711 \\
\hline $\begin{array}{l}\text { Phenolics (mg/1) } \\
\text { pn4-p (ang/1) }\end{array}$ & & 8.5 \\
\hline $\operatorname{Org}-\mathrm{N}(\boldsymbol{m o} / 1)$ & & & & & & & 80 & 88 & 80 & 80 \\
\hline \multicolumn{11}{|l|}{ NO2 (mg /1) } \\
\hline \multirow{2}{*}{\multicolumn{11}{|c|}{$\begin{array}{l}\text { NDJ (mg/1) } \\
\text { TDS (mo/l) }\end{array}$}} \\
\hline & & & & & & & & & & \\
\hline Color (APHA Units) & 2580 & 25200 & 2580 & 2500 & 2500 & 2500 & 2500 & 2530 & 2520 & 2500 \\
\hline \multicolumn{11}{|l|}{ BASIN PARAMETERS } \\
\hline Vol Wasted (al) & 284 & 269 & 278 & 270 & 259 & 259 & 266 & 227 & 242 & 242 \\
\hline D.0. (mo/1) & 6.8 & 6.8 & 6.4 & 6.4 & 6 & 6.9 & 6.8 & 7 & 7 & 6.7 \\
\hline SS $(\operatorname{mg} / 1)$ & 12300 & 11770 & 11880 & 12835 & 13218 & 11620 & 11880 & 12230 & 12825 & 12020 \\
\hline tSS (PAC corrected) & 12300 & 11770 & 11880 & 12035 & 13210 & 11620 & 11880 & 12230 & 12025 & 12020 \\
\hline Tomp (C) & 20 & 20 & 20 & 20 & y & ' d d & 20 & 20 & 20 & 20 \\
\hline $\mathrm{pH}$ (std units) & 8 & 8.2 & 8 & 8.1 & 8.1 & 8. 1 & 8.2 & 8.3 & 8.2 & 8.2 \\
\hline OUR $(\mathrm{mg} / 1 / \mathrm{hr})$ & & 12 & & & & i? & & & 12 & 12 \\
\hline VS9 (⿴囗十口贝 /1) & על & 6985 & 7850 & 7142 & 7840 & 6840 & 6993 & 7139 & 6429 & 6420 \\
\hline +USS (PAC corrected) & 7530 & 6985 & 7850 & 7142 & 7840 & 6848 & $699 ?$ & 7199 & 5420 & $\sigma^{\prime} 12 \theta$ \\
\hline VS5/5S & 0.61 & 0.59 & 9.59 & 0.59 & 0.59 & 0.59 & 0.59 & 0.59 & 0.53 & 0.53 \\
\hline APAC Added (mg) & 0 & 0 & 0 & 0 & 0 & 0 & 0 & 0 & 0 & a \\
\hline +Inventory (ug/1 basin) & 8 & 0 & 8 & 0 & 0 & 0 & 0 & 0 & 0 & 0 \\
\hline \multicolumn{11}{|l|}{ EFFLUENT PARAMETERS } \\
\hline Volume (liters) & 4.35 & 5 & 4.3 & 4.83 & 4.55 & 4.7 & 5.3 & 5.2 & 3.9 & 3.9 \\
\hline $\operatorname{coD}(\pi g / 1)$ & 162 & 164 & 161 & 165 & 165 & 187 & 210 & 151 & 173 & 228 \\
\hline S5 (ng/1) & 44 & 74 & 62 & 74 & 12h & 102 & 76 & 172 & 180 & 212 \\
\hline \multicolumn{11}{|l|}{ PAC corrected } \\
\hline DH (std units) & 8.1 & 8.2 & 8.1 & 8.3 & 8.3 & 8.3 & 8.3 & ค. 3 & 8.4 & 8.4 \\
\hline Vss (mg/1) & 27 & 44 & 37 & 44 & 71 & 60 & 45 & 101 & 96 & 112 \\
\hline \multicolumn{11}{|l|}{ tPAC corrected } \\
\hline NHIN $(m g / 1)$ & 186 & 186 & 174 & 174 & 174 & 174 & 164 & 164 & 164 & 158 \\
\hline CN $(\operatorname{sog} / 1)$ & & & & & & & 1.14 & 1.14 & 1.14 & 1.14 \\
\hline $\operatorname{SCN}$ (ngg/l) & & & & & & & 3.2 & 3.2 & 3.2 & 3.2 \\
\hline NO2 $(\mathrm{ng} / 1)$ & & & & & & & 6.41 & 6.41 & 6.41 & 6.41 \\
\hline N03 (mg/l) & & & & & & & 25.9 & 25.9 & 25.9 & 25.9 \\
\hline Phenolics (mg/1) & 10.025 & 10.025 & 10.025 & 10.825 & 10.025 & (0.025 & (0.025 & 10.025 & 10.025 & 10.025 \\
\hline$P O 4-P(m g / 1)$ & 3 & 3 & 2.5 & 2.5 & 2.5 & 2.5 & 2.2 & 2.2 & 2.2 & 2.8 \\
\hline $0 \mathrm{rg}-\mathrm{N}(\pi g / \mathrm{l})$ & & & & & & & 14 & 14 & 14 & 14 \\
\hline TDS (sg/1) & 5822 & 5822 & 5822 & 5822 & 5822 & 5822 & 4800 & 4800 & 4800 & 4800 \\
\hline Color (APHA units) & 750 & 750 & 750 & 750 & 758 & 758 & 1000 & 1680 & 1080 & 1000 \\
\hline $\operatorname{TOC}(a g / 1)$ & 32 & 32 & 34 & 34 & 34 & 34 & 39 & 39 & 39 & 47 \\
\hline BOD S-day $(\mathrm{mg} / \mathrm{l})$ & 1.8 & 1.8 & 2 & 2 & 2 & 2 & 12 & 12 & (2 & 3 \\
\hline
\end{tabular}


UNIT JA TAELE A3

\begin{tabular}{|c|c|c|c|c|c|c|c|c|c|c|}
\hline MONTH & FEB & FEB & FEB & FEB & $F E B$ & $\mathrm{FEB}$ & FEB & FEB & TEB & $F \Xi B$ \\
\hline DAY & 11 & 12 & 13 & 14 & 15 & 16 & 17 & 18 & 19 & 20 \\
\hline DAY :NO. & 280 & 201 & 202 & 203 & 204 & 205 & 206 & 207 & 208 & 209 \\
\hline \multicolumn{11}{|l|}{ SYSTEM PARAMETERS } \\
\hline *Sludoge Age & 48.82 & 39.99 & 35.59 & 39.84 & 40.04 & 40.80 & 40.02 & 40.01 & 33.96 & 39.97 \\
\hline$*(F / M) C O D$ & 0.13 & 0.15 & 0.13 & 8.13 & 0.14 & 0.12 & 0.68 & 0.10 & 0.11 & 0.09 \\
\hline *HRT & 1.88 & 1.69 & 1.86 & 1.98 & 1.94 & 2.00 & 1.94 & 1.83 & 1.76 & 2.25 \\
\hline \multicolumn{11}{|l|}{ FEED PARAMETERS (BATCH \#) } \\
\hline Volume (liters) & 4.95 & 5.5 & 5 & 4.7 & 4.8 & 4.65 & 4.8 & 5.08 & 5.27 & 4.14 \\
\hline oh (std units) & 10.4 & 10.8 & 10.3 & 9.7 & 9.7 & 9.6 & 11.3 & 11.2 & 11 & 11 \\
\hline $\operatorname{coD}(\operatorname{mg} / 1)$ & 2076 & 2076 & 2076 & 2124 & 2124 & 2134 & 1649 & 1649 & 1649 & 1649 \\
\hline NH3N (mg/l) & 183 & 183 & 183 & 166 & 166 & 166 & 166 & 166 & 166 & 166 \\
\hline SS $(m g / 1)$ & 336 & 336 & 336 & 254 & 254 & 254 & 254 & 254 & 254 & 254 \\
\hline VSS $(m 0 / 1)$ & 154 & 154 & 154 & 124 & 124 & 124 & 124 & 124 & 124 & 124 \\
\hline$C N(m \underline{D} / 1)$ & 9.59 & 9.59 & 9.59 & & & & & & & \\
\hline $\operatorname{SCN}(m g / 1)$ & 184 & 184 & 194 & & & & & & & \\
\hline BOD S-day $(m g / 1)$ & 1130 & 1130 & 1130 & 1080 & 1080 & 1080 & 1030 & 1030 & 1038 & 1030 \\
\hline TOC (mg/1) & 711 & 711 & 711 & 698 & 698 & 698 & 575 & 575 & 575 & 575 \\
\hline Phenolics (ag/1) & 8.5 & 8.5 & 8.5 & 9.8 & 9.8 & 9.8 & 3.8 & 9.8 & 9.8 & 9.8 \\
\hline \multicolumn{11}{|l|}{$P 04-P(D g / 1)$} \\
\hline Ora-N (mg/1) & 80 & 80 & 80 & & & & & & & \\
\hline \multicolumn{11}{|l|}{$\mathrm{NOC}(\mathrm{Og} / \mathrm{l})$} \\
\hline \multicolumn{11}{|l|}{$\mathrm{NO3}$ (ag/l) } \\
\hline \multicolumn{11}{|l|}{ TDS (mg/1) } \\
\hline Color (APHA Units) & 2589 & 2500 & 2500 & 2580 & 2500 & 2580 & 2500 & 2500 & $25 e 8$ & $\because 2500$ \\
\hline \multicolumn{11}{|l|}{ BASIN PARAMETERS } \\
\hline . Vol Wasted (al) & 242 & 126 & 20 & 189 & 231 & 252 & 218 & 222 & 160 & 237 \\
\hline D.0. $(m g / 1)$ & 6.7 & 6.7 & 6.9 & 6.9 & 6.7 & 6.8 & 6.8 & 6.8 & 6.8 & .6 .8 \\
\hline SS (mg/1) & 11898 & 12010 & 11685 & 11768 & 11110 & 10760 & 12688 & 10670 & 18690 & 10300 \\
\hline ÆSS (PAC corrected) & 11890 & 12010 & 11685 & 11760 & 11110 & 10760 & 12680 & 10670 & 10698 & 10300 \\
\hline Teapo (C) & 20 & 20 & 20 & 20 & 20 & 20 & 20 & 19 & 19 & 19 \\
\hline pH (std units) & 8.2 & 8.1 & 7.9 & 7.9 & 7.6 & 7.3 & 6.8 & 6.6 & 7 & 6.8 \\
\hline OUR $(\mathrm{gg} / 1 / \mathrm{hr})$ & & & 12 & & & 12 & & 12 & & \\
\hline VSS (mg/1) & 6348 & 6412 & 6535 & 6577 & 6213 & 6815 & 8031 & 6758 & 6771 & .6495 \\
\hline WSS (PAC corrected) & 6348 & 6412 & 6535 & 6577 & 6213 & 6815 & 8031 & 6758 & 6771 & 6485 \\
\hline USS/SS & 0.53 & 0.53 & 0.56 & 0.56 & 0.56 & 0.63 & 0.63 & 0.63 & 0.63 & 0.63 \\
\hline FPAC Added (mg) & 0 & 8 & 8 & 0 & 8 & 0 & 0 & 0 & 0 & 0 \\
\hline * inventory (四/l basin) & 8 & 8 & 0 & 8 & 0 & 0 & 0 & 0 & 0 & 0 \\
\hline \multicolumn{11}{|l|}{ EFFLUENT PARAMETERS } \\
\hline Voluwe (liters) & 4.65 & 5.1 & 5.05 & 4.55 & 4.6 & 4.45 & 4.55 & 4.95 & 5 & 4.4 \\
\hline COD (mo/l) & 218 & २२२ & 214 & 203 & 209 & 201 & 199 & 197 & 184 & 180 \\
\hline $55(m g / 1)$ & 148 & 410 & 734 & 290 & 166 & 116 & 228 & 168 & 380 & 148 \\
\hline \multicolumn{11}{|l|}{ APAC corrected } \\
\hline oh (std units) & 8.3 & 8.2 & 8 & 7.9 & 7.6 & 7.1 & 6.6 & 7.1 & 7.4 & 6.9 \\
\hline VSS $(m g / 1)$ & 79 & 219 & 410 & 162 & 93 & 73 & 144 & 106 & 190 & 93 \\
\hline \multicolumn{11}{|l|}{ *PAC corrected } \\
\hline NH3N $(\mathrm{sg} / 1)$ & 158 & 158 & 158 & 107 & 187 & 107 & 11 & 11 & 11 & 11 \\
\hline$C N(m g / 1)$ & 1.14 & 1.14 & 1.14 & & & & & & & \\
\hline SCN $(\mathrm{mg} / 1)$ & 3.2 & 3.2 & 3.2 & & & & & & & \\
\hline NO2 (ag/l) & 6.41 & 6.41 & 6.41 & & & & & & & \\
\hline $\mathrm{NO3}$ (ag/1) & 25.9 & 25.9 & 25.9 & & & & & & & \\
\hline Phenolics (ag/l) & 10.025 & 10.825 & 18.025 & 10.825 & 10.025 & 10.025 & 10.025 & 10.025 & 10.025 & 10.025 \\
\hline$P 04-P(B g / 1)$ & 2.8 & 2.8 & 2.8 & 10.9 & 10.9 & 10.9 & 47.2 & 47.2 & 47.2 & 47.2 \\
\hline Org-N (mg/1) & 14 & 14 & 14 & & & & & & & \\
\hline $\operatorname{TDS}(\mathrm{mg} / \mathrm{l})$ & 4800 & 4800 & 4800 & 5644 & 5644 & 5644 & 5644 & 5644 & 5644 & 5644 \\
\hline Color (APHA units) & 1000 & 1000 & 1000 & 1000 & 1800 & 1800 & 1000 & 1000 & 1088 & 1000 \\
\hline TOC $(\operatorname{mg} / 1)$ & 47 & 47 & 47 & $5 !$ & 51 & 51 & 55 & 55 & 55 & 55 \\
\hline BOD 5 -day $(\operatorname{mg} / 1)$ & 3 & 3 & 3 & 2 & 2 & 2 & 2 & 2 & 2 & 2 \\
\hline
\end{tabular}




\begin{tabular}{|c|c|c|c|c|c|c|c|c|c|c|}
\hline MONTH & FEB & FEB & FẸB & FEB & FEB & FEB & FEB & FEB & FEB & MAR \\
\hline DAY & 21 & 22 & 23 & 24 & 25 & 26 & 27 & 28 & 29 & 1 \\
\hline DAY :OO. & 210 & 211 & $21 ?$ & 213 & 214 & 215 & 216 & 217 & 218 & 219 \\
\hline \multicolumn{11}{|l|}{ SYSTEM PARAMETERS } \\
\hline *sludode noge & 40.23 & 39.96 & 33.48 & 40.85 & 40.88 & 39.98 & 40.84 & 29.98 & 39.97 & 34.37 \\
\hline$*(F / M) C O D$ & 0.10 & 0.10 & 0.10 & 0.11 & 0.12 & 0.11 & 0.10 & 0.12 & 0.10 & 0.11 \\
\hline KHRT & 1.98 & 2.02 & 2.11 & 2.04 & 1.82 & 1.89 & 2.21 & 1.83 & 2.11 & 2.11 \\
\hline \multicolumn{11}{|l|}{ FEED PARAMETERS (BATCH } \\
\hline Volume (1iters) & 4.7 & 4.6 & 4.4 & 4.56 & 5.1 & 4.93 & 4.2 & 5.48 & 4.4 & 4.4 \\
\hline pH (std units) & 11.3 & 11 & 11 & 10.7 & 11.2 & 10.5 & 10.6 & 11.1 & 10.6 & 10.3 \\
\hline $\operatorname{COD}(\mathrm{mg} / 1)$ & 1630 & 1630 & 1630 & 1766 & 1766 & 1766 & 1766 & 1883 & 1983 & 1803 \\
\hline NH3N (ag/1) & 176 & 176 & 176 & 174 & 174 & 174 & 174 & 149 & 149 & 149 \\
\hline SS (mg/1) & & & & & & & & 188 & 188 & 188 \\
\hline USS (ng/1) & & & & & & & & 88 & 80 & 80 \\
\hline CN (ag/1). & 2.55 & 2.55 & 2.55 & 2.55 & 2.55 & 2.55 & 2.55 & & & \\
\hline $\mathrm{SCN}$ (ng/1) & 174 & 174 & 174 & 174 & 174 & 174 & 174 & & & \\
\hline 800 5-day (mg/l) & 980 & 990 & 900 & 985 & 985 & 98.5 & 985 & 1898 & 1890 & 1090 \\
\hline TOC (mg/l) & 566 & 566 & 566 & 564 & 564 & 564 & 564 & 569 & 569 & 569 \\
\hline Phenolics (ng/1) & 6.6 & 6.6 & 6.6 & 6.6 & 6.6 & 6.6 & 6.6 & & & \\
\hline \multicolumn{11}{|l|}{$P \cap .4-P(m g / 1)$} \\
\hline \multicolumn{11}{|l|}{ Org-N $(\mathrm{mg} / \mathrm{l})$} \\
\hline \multicolumn{11}{|l|}{ NOP (mg/l) } \\
\hline \multirow{2}{*}{\multicolumn{11}{|c|}{$\begin{array}{l}\text { ND3 (mg/1) } \\
\text { TDS (mg/1) }\end{array}$}} \\
\hline & & & & & & & & & & \\
\hline Color (APHA Units) & 3000 & 3000 & 3000 & 3000 & 3000 & 3820 & 3000 & & & \\
\hline \multicolumn{11}{|l|}{ BRSIN PARAMETERS } \\
\hline Vol Wasted ([0]) & 236 & 277 & 283 & 288 & 300 & 257 & 127 & 35 & 24 & 24 \\
\hline D.0. (mg/l) & 6.9 & 6.8 & 6.8 & 6.8 & 6.8 & 6.8 & 6 & 6 & 6 & 6 \\
\hline SS ing/l) & 10160 & 10095 & 9805 & 9572 & 9975 & 18760 & 9875 & 9655 & 9525 & 9135 \\
\hline +SS (PAC corrected) & 10160 & 10895 & 9805 & 9572 & 9975 & 10768 & 9875 & 9655 & 3525 & 91,35 \\
\hline Tosp (C) & 19 & نे & 19 & 19 & 19 & 19 & 18 & 19 & 19 & 19 \\
\hline pH (std units) & 7.2 & 7.5 & 7.4 & 7.5 & 7.3 & 6.7 & $\begin{array}{r}6.8 \\
.9\end{array}$ & 7.3 & 6.9 & 7.1 \\
\hline USS (mg/l) & $\begin{array}{r}56 \\
6397\end{array}$ & 6356 & 6165 & 6018 & 6272 & 6765 & 6000 & 6.383 & 6798 & 6079 \\
\hline 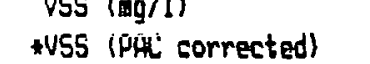 & 6397 & 6356 & 5165 & ลค18 & 6272 & 6765 & 6000 & 0502 & oc & 0.612 \\
\hline $\begin{array}{l}\text { W55/SS } \\
\text { V5S corrected }\end{array}$ & 0.63 & 0.63 & 0.63 & 0.63 & 0.63 & 0.63 & 0.66 & 8003 & 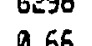 & 9.67 \\
\hline $\begin{array}{l}\text { VSS/SS } \\
\text { ^PAC Added (⿴囗十) }\end{array}$ & 0 & 0 & 0 & 0 & 0 & 8 & $\begin{array}{r}0.00 \\
8\end{array}$ & 0.00 & 0.66 & 0.01 \\
\hline $\begin{array}{l}\text { \#PAC Added (⿴囗十) } \\
\text { +inventory (mo / basin) }\end{array}$ & & & & 8 & & 8 & & 0 & a & 6 \\
\hline tinventory (wg/1 basin) & 8 & 8 & $\theta$ & $y$ & 0 & 0 & 8 & 8 & 8 & 0 \\
\hline \multicolumn{11}{|l|}{ EFFLUENT PARAMETERS } \\
\hline Volume (1iters) & 4.38 & 4.35 & 4 & 4.65 & 5.25 & 4.55 & 4.33 & 5 & 4.4 & 4.79 \\
\hline COD (ag/1) & 218 & 128 & 233 & 124 & 138 & 248 & 153 & 155 & 167 & 148 \\
\hline SS (mg/1) & 148 & 54 & 42 & 24 & 0 & 102 & 362 & 512 & 598 & 620 \\
\hline \multicolumn{11}{|l|}{ \#PAC corrected } \\
\hline pH (std units) & 7.2 & 7.3 & 7.6 & 7.8 & 7.5 & 7 & 7.2 & 7.8 & 7.1 & 7.1 \\
\hline USS (ag/1) & 93 & 34 & 26 & 15 & 0 & 64 & 239 & 339 & 395 & 412 \\
\hline \multicolumn{11}{|l|}{ APAC corrected } \\
\hline NK3N $\log / 11)$ & <Q & 18 & 18 & lद & 12 & 纪 & <2 & 2.8 & 2.8 & 2.8 \\
\hline CN (agg/1) & 0.489 & 0.489 & 8.489 & 0.489 & 0.489 & 0.489 & 8.489 & & & \\
\hline SCN (mg/1) & 2.9 & 2.9 & 2.9 & 2.3 & 2.9 & 2.9 & 2.9 & & & \\
\hline NO2 (ag/l) & 1.5 & 1.5 & 1.5 & 1.5 & 1.5 & 1.5 & 1.5 & & & \\
\hline $\mathrm{ND3}(\mathrm{gg} / 1)$ & 178 & 178 & 178 & 178 & 178 & 178 & 178 & & & \\
\hline Phenolics $(\mathrm{g} / 1)$ & 10.025 & (0.825 & 10.025 & $<0.025$ & 10.025 & (9.025 & 10.025 & & & \\
\hline P04-P (mq/1) & 15.6 & 15.6 & 15.6 & 25.1 & 25.1 & 25.1 & 25.1 & & & \\
\hline Org-N (mg/l) & $<8$ & 18 & 18 & 18 & 18 & 18 & $<8$ & & & \\
\hline TDS (mg/1) & 4964 & 4964 & 4964 & 4964 & 4964 & 4964 & 4964 & & & \\
\hline Color (APHA units) & 1000 & 12080 & 1680 & 1860 & 1800 & 1000 & 1000 & & & \\
\hline TOC $(\pi g / 1)$ & 45 & 45 & 45 & 45.2 & 45.2 & 45.2 & 45.2 & 53.2 & 53.2 & 53.2 \\
\hline \multirow[t]{2}{*}{ BOD 5-day $(\lg / 1)$} & 12 & 12 & (2 & 《2 & 12 & 12 & 12 & 12 & 12 & 12 \\
\hline & \multicolumn{10}{|c|}{$-122-$} \\
\hline
\end{tabular}


UNIT $2 A$ TABLE A3

\begin{tabular}{|c|c|c|c|c|c|c|c|c|c|c|}
\hline MONTH & MAR & MAR & MAR & MAR & MAR & MAR & MAR & MAR & YAR & MAR \\
\hline DAY & 2 & 3 & 4 & 5 & 6 & 7 & 8 & 9 & 10 & 11 \\
\hline DAY NO. & 220 & 221 & 222 & 223 & 224 & 225 & 226 & 227 & 228 & 229 \\
\hline \multicolumn{11}{|l|}{ SYSTEX F'ARAMETERS } \\
\hline tSludge Aoge & 19.58 & 39.96 & 37.18 & 47.14 & 45.53 & 45.49 & 51.59 & 82.15 & 106.70 & 84.89 \\
\hline$*(F / M) C O D$ & 0.12 & 0.13 & 0.14 & $0 .: 10$ & 2.13 & 0.14 & 0.14 & 0.12 & 0.13 & 0.13 \\
\hline$\#$ \#RT & 2.80 & 1.69 & 1.73 & 2. 14 & 1.88 & 1.71 & 1.71 & 1.96 & 1.84 & 1.91 \\
\hline \multicolumn{11}{|l|}{ FEED PARAMETERS (BATCH \#) } \\
\hline Volume (jiters) & 4.66 & 5.5 & 5.38 & 4.35 & 4.95 & 5.45 & 5.45 & 4.75 & 5.05 & 4.87 \\
\hline $\mathrm{pH}$ (std units) & 11.8 & 11.7 & 12.25 & 11.3 & 11.7 & 11.7 & 11.8 & 11.7 & 11.8 & 11.8 \\
\hline $\operatorname{coD}(\mathrm{mg} / 1)$ & 1803 & 1803 & 1803 & 1803 & 1834 & 1934 & 1834 & 1928 & 1828 & 1828 \\
\hline NH3N (ag/1) & 177 & 177 & 177 & 177 & 162 & 162 & 162 & 186 & 186 & 186 \\
\hline SS $(m g / 1)$ & 188 & 188 & 188 & 198 & & & & 143 & 143 & 143 \\
\hline USS $(\operatorname{mg} / 1)$ & 88 & 80 & 88 & 88 & & & & 66 & 66 & 66 \\
\hline \multicolumn{11}{|l|}{ CN $(m g / 1)$} \\
\hline \multicolumn{11}{|l|}{$\operatorname{SCN}(\pi 0 / 1)$} \\
\hline BOD 5-day (mg/1) & 1090 & 1890 & 1098 & 1090 & 1208 & 1200 & 1200 & 1170 & 1170 & 1170 \\
\hline TOC $(\mathrm{mg} / 1)$ & 569 & 569 & 569 & 569 & 570 & 570 & 570 & 578 & 578 & 578 \\
\hline \multicolumn{11}{|l|}{ Phenolics (mg/l) } \\
\hline \multicolumn{11}{|l|}{$p 04-p$ (ag/l) } \\
\hline \multicolumn{11}{|l|}{ Org-N $(\mathrm{mg} / \mathrm{l})$} \\
\hline \multicolumn{11}{|l|}{$\mathrm{N03}(\mathrm{mg} / 1)$} \\
\hline \multicolumn{11}{|l|}{$\operatorname{TDS}(\mathrm{mg} / 1)$} \\
\hline \multicolumn{11}{|l|}{ Color (APHA Units) } \\
\hline \multicolumn{11}{|l|}{ BRSIN PARAMETERS } \\
\hline Vol Wasted (al) & 20 & 267 & 20 & 20 & 20 & 25 & 40 & 48 & 20 & 40 \\
\hline D.0. $(\operatorname{gg} / 1)$ & 6 & 6.1 & 6.1 & 6.1 & 5 & 6.7 & 6.8 & 6.4 & 6.5 & 6.4 \\
\hline S5 (ag/1) & 8910 & 9910 & 8895 & 8775 & 8385 & 8710 & 8613 & 8520 & 8425 & 8250 \\
\hline *SS (PAC corrected) & 8910 & 9910 & 8895 & 8775 & 8385 & 8710 & 8613 & 8520 & 8425 & 9260 \\
\hline Temp (C) & 18 & 18 & ;3 & 19 & 19 & 18.5 & 18 & 18 & 18 & 19 \\
\hline $\mathrm{pH}$ (std units) & 7.3 & 7.2 & 7.1 & 7.1 & 6.4 & 6.7 & 7 & 7.1 & 7.2 & 7.2 \\
\hline OUR (mg/1/hr) & & & & 56 & & & 48 & & & \\
\hline VSS (mg/1) & 5925 & 6590 & 5915 & 6259 & 5857 & 5944 & 5988 & 5923 & 5857 & 5743 \\
\hline WSS (PAC corrected) & 5925 & 6530 & 5915 & 6250 & 5857 & 5944 & 5988 & 5923 & 5857 & 5743 \\
\hline VSS/SS & 0.67 & 0.67 & 0.67 & 0.71 & 0.71 & 0.68 & 0.70 & 8.78 & 0.70 & 0.70 \\
\hline *PAC Added (䍏) & 8 & 0 & 0 & 0 & 8 & 0 & 0 & 0 & 0 & 0 \\
\hline +Inventory (ug/l basin) & 8 & 0 & 0 & 0 & $\theta$ & 8 & 8 & 0 & $\theta$ & 0 \\
\hline \multicolumn{11}{|l|}{ EFFLUENT PARRAETERS } \\
\hline Volume (liters) & 4.65 & 5.5 & 4.98 & 4.15 & 4.75 & 5.2 & 5.25 & 4.43 & 4.75 & 4.55 \\
\hline $\operatorname{COD}(m / 1)$ & 148 & 158 & 160 & 168 & 176 & 181 & 183 & 150 & 185 & 175 \\
\hline SS $(m g / 1)$ & 1136 & 60 & 549 & 496 & 430 & 400 & 316 & 204 & 164 & 184 \\
\hline \multicolumn{11}{|l|}{ YPAC corrected } \\
\hline oH (std units) & 7.5 & 7 & 7.35 & 6.3 & 7.4 & 6.6 & 6.9 & 7.1 & 7.4 & 7.3 \\
\hline VSS $(n g / 1)$ & 755 & 40 & 360 & 353 & 305 & 273 & 220 & 142 & 114 & 128 \\
\hline \multicolumn{11}{|l|}{ tPAC corrected } \\
\hline NH3N $( \pm g / 1)$ & 2.1 & 2.1 & 2.1 & 2.1 & 1.4 & 1.4 & 1.4 & 1 & 1 & 1 \\
\hline \multicolumn{11}{|l|}{$C N(\mathrm{mg} / \mathrm{l})$} \\
\hline $\operatorname{scN}(\operatorname{mg} / 1)$ & & & & & & & & & & \\
\hline $\begin{array}{l}\text { NOC }(a g / 1) \\
\text { Non }(a 0 / 1)\end{array}$ & & & & & & & & & & \\
\hline Phenolics (ag/1) & & & & & & & & & & \\
\hline $004-P(\mathrm{mg} / 1)$ & & & & & & & & & & \\
\hline Org-N (mg/1) & & & & & & & & & & \\
\hline $\operatorname{TDS}(\operatorname{mg} / 1)$ & & & & & & & & & & \\
\hline Color (APHA units) & & & & & & & & & & \\
\hline $\operatorname{TOC}(\pi g / 1)$ & 54.3 & $54 . \ddot{3}$ & 54.3 & 54.3 & 51.2 & 51.2 & 51.2 & 49.6 & 49.6 & 49.6 \\
\hline BOD 5-day (mg/1) & (2) & 12 & ใ? & 1? & ใ? & 12 & 12 & 《弓 & 12 & 12 \\
\hline
\end{tabular}


UNIT 2A TABLE A3

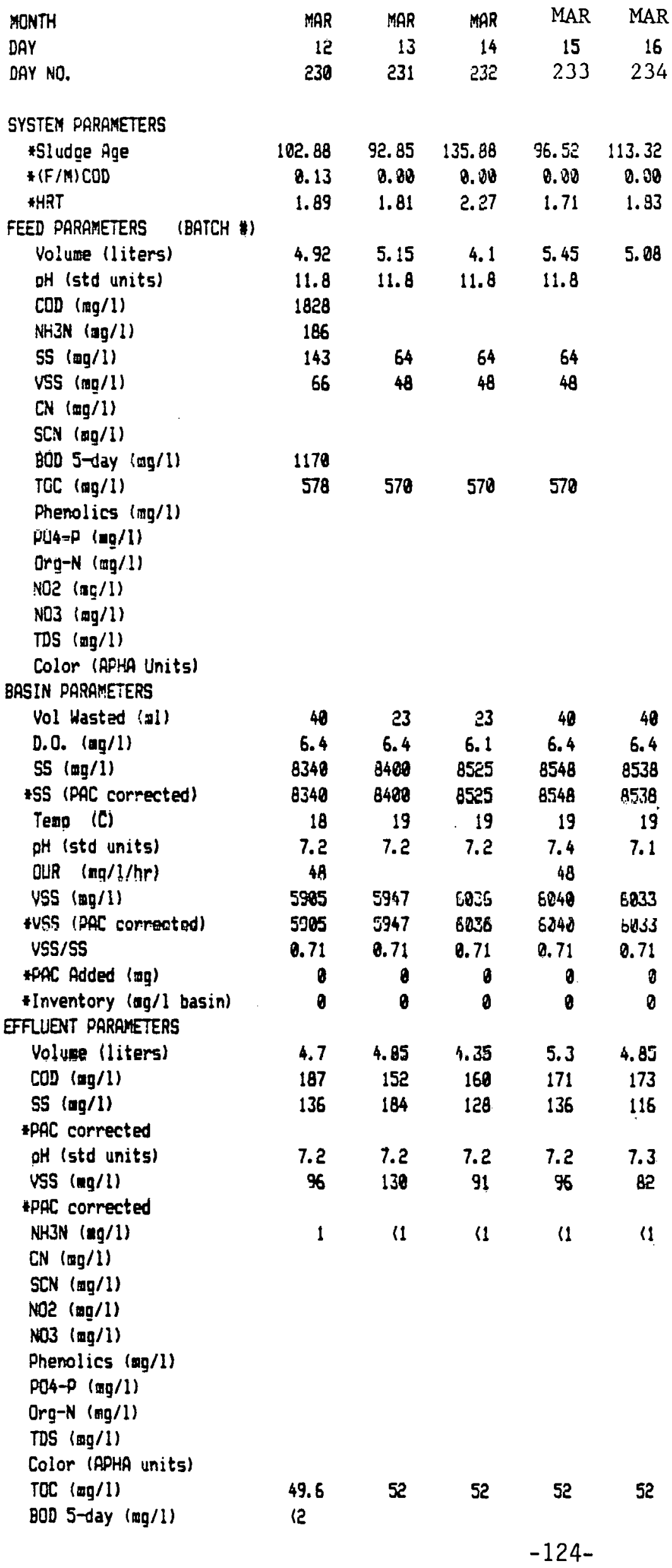


UNIT $2 B$ TABLE A4

\begin{tabular}{|c|c|c|c|c|c|c|c|c|c|c|}
\hline MONTH & JULY & JULY & JLY & JULY & JULY & JULY & RUG & AUG & ALG & RUS \\
\hline DAY & 26 & 27 & 28 & 29 & 30 & 31 & 1 & 2 & 3 & 4 \\
\hline DAY NO. & 0 & 1 & 2 & 3 & 4 & 5 & 6 & 7 & 8 & 9 \\
\hline \multicolumn{11}{|l|}{ SYSTEM PARAMETERS } \\
\hline *Sludẹe Age & 32.6 & 30 & 30 & 30 & 30 & 30 & 38 & 30 & 38 & 30 \\
\hline$*(F / M) C O D$ & 0.08 & 0.10 & 0.18 & 0.25 & 0.11 & 0.06 & 0.09 & 0.08 & 0.89 & 0.18 \\
\hline *HRT & 2.0 & 2.5 & 2.0 & 1.9 & 2.8 & 2.1 & 2.0 & 2.0 & 2.1 & 2.0 \\
\hline FEED PARAMETERS (BATCH \$) & 27 & 27 & 27 & 27 & 27 & 27 & 27 & 27 & 27 & 27 \\
\hline Volume (liters) & 3.48 & 2.85 & 3.52 & 3.70 & 3.55 & 3.39 & 3.54 & 3.55 & 3.30 & 3.50 \\
\hline $\mathrm{pH}$ (std units) & $7.5 / 9.6$ & $7.4 / 8.8$ & 7.5 & 7.4 & $1 / 8.8$ & $7.3 / 9.2$ & 7.4 & 7.4 & 7.6 & 7.4 \\
\hline $\operatorname{COD}(\pi q / 1)$ & 329 & 323 & 308 & 303 & 290 & 301 & 293 & 251 & 262 & 212 \\
\hline NH3N $(\mathrm{Bg} / 1)$ & 24 & 15 & 15 & 15 & 8 & 8 & 8 & 8 & 12.8 & 12.8 \\
\hline \multicolumn{11}{|l|}{$\begin{array}{l}\text { SS }(m g / 1) \\
\text { VSS }(n g / 1)\end{array}$} \\
\hline CN $(\log / 1)$ & 2.6 & 6.1 & 6.1 & 6.1 & 6.1 & 6.1 & 6.1 & 6.1 & 2.23 & 2.23 \\
\hline $\operatorname{SCN}(\operatorname{mog} / 1)$ & 6.4 & 1.38 & 1.38 & 1.38 & 1.38 & 1.38 & 1.38 & 1. 38 & 7.8 & 7.0 \\
\hline \multicolumn{11}{|l|}{ BOD 5-day (ng/1) } \\
\hline TOC (mg/l) & & 58 & 58 & 58 & 58 & 58 & 58 & 58 & & \\
\hline Phenolics (ag/1) & 1.025 & & & & & & & & 1.025 & 1.025 \\
\hline $\mathrm{PO4}-\mathrm{P}(\mathrm{mg} / 1)$ & 4.7 & & & & & & & & 2.5 & 2.5 \\
\hline Org-N (mg/1) & 21 & & & & 11 & 11 & 11 & 11 & & \\
\hline NOS $(\mathrm{ng} / 1)$ & 137 & 101 & 181 & 101 & 181 & 101 & 101 & 101 & 67.4 & 87.4 \\
\hline$: 03(\mathrm{mg} / \mathrm{l})$ & 48 & 73 & 73 & 73 & 73 & 73 & 73 & 73 & 96.6 & 36.6 \\
\hline \multicolumn{11}{|l|}{ TDS (ma/l) } \\
\hline Color (APHA Units) & & 500 & 500 & 500 & 500 & 508 & 500 & 500 & & \\
\hline BASIN PARAMETERS & & & & & & & & & & $\cdot$ \\
\hline Vol Wasted (nl) & 84 & 115 & 122 & 93 & 117 & 237 & 220 & 227 & 234 & 152 \\
\hline $0.0 . \quad(m g / 1)$ & 8.1 & 8.1 & 8.0 & 8.0 & B.1 & 7.4 & 7.4 & 7.7 & 7.2 & .7 .6 \\
\hline $5 S(\operatorname{mg} / 1)$ & 2648 & 1970 & 2060 & 1220 & 1950 & 2788 & 2178 & 2240 & 1910 & 1060 \\
\hline *SS (PAC corrected) & 2140 & 1470 & 1568 & 728 & 1450 & 2280 & 1670 & 1740 & 1410 & 560 \\
\hline Tewp (C) & 25 & 25.5 & 26 & 26 & 26 & 26 & 26 & 26 & 25.5 & 26 \\
\hline $\mathrm{pH}$ (std units) & 7.1 & 7.7 & 7.8 & 7.3 & 7.3 & 7.9 & 7.9 & 7.9 & 7.8 & 7.4 \\
\hline OUR (mg/1/hr) & & & 4 & & & & 4 & & & 3 \\
\hline USS $(\operatorname{mg} / 1)$ & 2101 & 1568 & 1730 & 1025 & 1638 & 2335 & 1820 & 1879 & 1602 & 380 \\
\hline USS (PAC corrected) & 1681 & 1068 & 1230 & 525 & 1138 & 1835 & $13 \hat{c} 0$ & 1379 & 1102 & 480 \\
\hline VSS/SS & & & 8.84 & & & & 0.84 & & & 0.92 \\
\hline APAC Added (mg) & 132 & 143 & 143 & 143 & 143 & 144 & 143 & 143 & 143 & 143 \\
\hline +Inventory (mg/l basin) & 580 & 500 & 580 & 500 & 580 & 500 & 580 & 500 & 508 & 580 \\
\hline \multicolumn{11}{|l|}{ EFFLUENT PARAMETERS } \\
\hline Volume (liters) & 3.30 & 2.38 & 3.20 & 3.38 & 3.18 & 3.03 & 3.00 & 3.05 & 2.90 & 2.98 \\
\hline COD $(\operatorname{sg} / 1)$ & 297 & 289 & 299 & 275 & 278 & 270 & 214 & 193 & 184 & 188 \\
\hline SS $(m g / 1)$ & 144 & 142 & 186 & 70 & 104 & 46 & 48 & 44 & 36 & 48 \\
\hline \multicolumn{11}{|l|}{ IPAC corrected } \\
\hline ôH (std units) & 7.2 & 7.6 & 7.8 & 7.4 & 7.2 & 7.6 & 7.8 & 8.0 & 7.8 & 7.6 \\
\hline VSS $(m g / 1)$ & & & 80 & & & & 38 & & & 40 \\
\hline \multicolumn{11}{|l|}{ APAC corrected } \\
\hline NH3N $(\pi g / 1)$ & 8 & 8 & 8 & 3 & 3 & 3 & 3 & 3.5 & 3.5 & 3.5 \\
\hline CN (mg/l) & 1.38 & 1.38 & 1,38 & 1.38 & 1.38 & 1.38 & 1.38 & 2.08 & 2.08 & 2.08 \\
\hline $\operatorname{SCN}(\mathrm{mg} / 1)$ & 6.1 & 6.1 & 6.1 & 6.1 & 6.1 & 6.1 & 6.1 & 6.7 & 6.7 & 6.7 \\
\hline No2 (mg/l) & 107 & 107 & 107 & 187 & 107 & 107 & 107 & 28 & 28 & 28 \\
\hline N03 $(\operatorname{sg} / 1)$ & 91 & 91 & 91 & 91 & 91 & 91 & 91 & 160 & 160 & 160 \\
\hline Phenolics (ag/l) & & & & & & & & 1.825 & 1.025 & 1.025 \\
\hline $904-P(\mathrm{gg} / 1)$ & & & & & & & & 3 & 3 & 3 \\
\hline $\begin{array}{l}\text { Org-N (mg/l) } \\
\operatorname{TDS}(m g / 1)\end{array}$ & & & & 9 & 9 & 9 & 9 & & & \\
\hline Color (APHA units) & 750 & 750 & 750 & 758 & 750 & 750 & 750 & & & \\
\hline TOC $(\mathrm{mg} / 1)$ & 46 & 46 & 46 & 46 & 46 & 46 & 46 & & & \\
\hline BOD 5-day (my/l) & & & & & & & & & & \\
\hline
\end{tabular}




\begin{tabular}{|c|c|c|c|c|c|c|c|c|c|c|}
\hline MONTH & AUG & AUG & AúG & AUG & ALIG & AUG & AUG & AUG & AU6 & AUG \\
\hline DAY & 5 & 6 & 7 & 8 & 9 & 10 & 11 & 12 & 13 & 14 \\
\hline DAY NO. & 10 & 11 & 12 & 13 & 14 & 15 & 16 & 17 & 18 & 19 \\
\hline \multicolumn{11}{|l|}{ SYSTLM PARRMETERS } \\
\hline Fludge Age & 30 & 30 & 30 & 30 & 30 & 30 & 13.8 & 38.5 & 37.1 & 38 \\
\hline$+(F / M) C O D$ & 0.18 & 0.09 & 0.87 & 0.08 & 0.08 & 0.06 & 0.21 & 0.72 & 0.07 & 0.07 \\
\hline *HRT & 2.1 & 2.1 & 1.3 & 2.0 & 2.1 & 2.1 & 2.0 & 2.1 & 2.1 & 2.0 \\
\hline FEED PARAMETERS (BATCH & 27 & 27 & 27 & 27 & 27 & 27 & 27 & 28 & 28 & 28 \\
\hline Volume (liters) & 3.40 & 3.35 & 3.60 & 3.45 & 3.38 & 3.40 & 3.45 & 3.39 & 3.40 & 3.48 \\
\hline pH (std units) & 7.2 & 7.6 & 7.5 & 7.2 & 7.2 & 7.0 & $3 / 8.8$ & $7.5 / 3.0$ & 7.5 & 7.6 \\
\hline $\operatorname{COD}(\mathrm{mg} / 1)$ & 272 & 272 & 272 & 228 & 220 & 223 & 223 & 184 & 190 & 239 \\
\hline 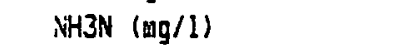 & 12.8 & 5.9 & 5.9 & 5.9 & 5.9 & 9.7 & 9.7 & 9.7 & 18.8 & 18.8 \\
\hline \multicolumn{11}{|l|}{ SS (mg/1) } \\
\hline \multicolumn{11}{|l|}{ USS (mg/1) } \\
\hline CN (mg/l) & 2.23 & 2.23 & 2.23 & 2.23 & 2.23 & 1.806 & <.2066 & $<.086$ & $<.006$ & $<.006$ \\
\hline $\mathrm{SCN}(\mathrm{mg} / 1)$ & 7.0 & 7.0 & 7.0 & 7.8 & 7 & 7.5 & 7.5 & 7.5 & 7.5 & 7.5 \\
\hline \multicolumn{11}{|l|}{800 5-day (ag/1) } \\
\hline TOC (mg/1) & & & & & & 58 & 58 & 58 & 58 & 58 \\
\hline Phenolics (ag/l) & $\{.025$ & 1.025 & 1.825 & 1.025 & $1.08 \mathrm{~g}$ & & & & & \\
\hline$P 04-p(m g / 1)$ & 2.5 & 2.5 & 2.5 & 2.5 & 2.5 & & & & & \\
\hline Org-N (afg/1) & & 14.4 & $! 4.4$ & 14.4 & 14.4 & & & & 18.7 & 18.7 \\
\hline NOP $(\mathrm{mg} / \mathrm{l})$ & 67.4 & 67.4 & 67.4 & 67.4 & 67.4 & 43.8 & 43.9 & 43.8 & 43.8 & 43.8 \\
\hline$N 03(\mathrm{mg} / 1)$ & 96.6 & 96.6 & 36.6 & 36.6 & 96.6 & $1: 6$ & 116 & 116 & 116 & 116 \\
\hline \multicolumn{11}{|l|}{ TDS $(m g / 1)$} \\
\hline Color (APHA Units) & & & & & & 500 & 500 & 500 & 500 & 500 \\
\hline \multicolumn{11}{|l|}{ BASIN PARAMETERS } \\
\hline Vol Wasted (all) & 142 & 248 & 206 & 191 & 205 & 287 & 10 & 10 & 163 & 230 \\
\hline D.0. $(\mathrm{ag} / 1)$ & 7.6 & 7.5 & 7.0 & 7.5 & 7.5 & 7.8 & 8.2 & 7.9 & 8.4 & 8.6 \\
\hline SS (mg/1) & 1200 & 1840 & 2340 & 1948 & 1830 & 2230 & 1000 & 620 & 1870 & 2120 \\
\hline WEE (PAC corrected) & 700 & 1340 & 1040 & 1440 & 1350 & $1 / 30$ & 500 & 120 & 1370 & $16 \geq 0$ \\
\hline Temp (C) & 26 & 26 & 26 & 27 & 28 & 26 & 25 & 25 & 24 & 23 \\
\hline $\mathrm{pH}$ (std units) & 7.1 & 7.9 & 7.9 & 7.8 & 7.7 & 7.5 & 7.1 & 7.7 & 8.8 & 7.8 \\
\hline OUR (agil/nr) & & & & 4 & & & 2 & & & \\
\hline vSS $(m p / 1)$ & 1109 & 1701 & 2163 & 1660 & 1566 & 1908 & 849 & .TP1 & 1,571 & 1781 \\
\hline * VSIS (PAC corrected) & 609 & 1201 & 1663 & 1160 & 1138 & 1480 & 420 & 101 & $\lfloor 15\rfloor$ & 1361 \\
\hline USS/SS & & & & 0.86 & & & 0.84 & & & \\
\hline \#PAC Added (mg) & 143 & 144 & 143 & 143 & 143 & 143 & 312 & 112 & 114 & 143 \\
\hline Inventory (mg/l basin) & 500 & 500 & 500 & 500 & 500 & 500 & 580 & 500 & 500 & 500 \\
\hline \multicolumn{11}{|l|}{ EFFLUENT PARAMETERS } \\
\hline Voluane (1iters) & 3.00 & 3.00 & 3.15 & 3.00 & 3.00 & 3.85 & 2.65 & 3.15 & 3.20 & 3.14 \\
\hline $\operatorname{coD}(\mathrm{mg} / 1)$ & 190 & 197 & 170 & 170 & 177 & 1?? & 125 & 148 & 171 & 179 \\
\hline SS $(\operatorname{mg} / 1)$ & 58 & 24 & 60 & 62 & 50 & 58 & 232 & 42 & 38 & 38 \\
\hline \multicolumn{11}{|l|}{ PPAC corrected } \\
\hline oH (std units) & 7.2 & 7.8 & 7.8 & 7.8 & 7.8 & 7.4 & 7.1 & 7.5 & 7.8 & 7.8 \\
\hline VSS (mg/1) & & & & 52 & & & 180 & & & \\
\hline \multicolumn{11}{|l|}{ PAC corrected } \\
\hline NH3N (mg/1) & 2.4 & 2.4 & 2.4 & 2.4 & 2 & 2 & 2 & 6 & 6 & 6 \\
\hline$C N(\pi g / 1)$ & 2.08 & 2.08 & 2.08 & 2.08 & 1.19 & 1.19 & 1.19 & 1.19 & 1.19 & 1.19 \\
\hline $\operatorname{SCN}(\mathrm{mg} / 1)$ & 6.7 & 6.7 & 6.7 & $6: 7$ & 5.2 & 5.2 & 5.2 & 5.2 & 5.2 & 5.2 \\
\hline NO2 $(\mathrm{mg} / 1)$ & 28 & 28 & 280 & 28 & 13.5 & 13.5 & 13.5 & 13.5 & 13.5 & 13.5 \\
\hline $\mathrm{NOO3}(\mathrm{gg} / 1)$ & 168 & 160 & 160 & 160 & 174 & 174 & 174 & 174 & 174 & 174 \\
\hline Phenolics (mg/1) & 1.825 & 1.025 & 1.025 & 1.025 & & & & & & \\
\hline$P 04-P(a g / 1)$ & 3 & 3 & 3 & 3 & & & & & & \\
\hline \multicolumn{11}{|l|}{$\operatorname{TDS}(\mathrm{ag} / \bar{l})$} \\
\hline Color (APHA units) & & & & & 1000 & 1880 & 1000 & 1000 & 1000 & 1880 \\
\hline $\mathrm{TDC}(\mathrm{mg} / \mathrm{l})$ & & & & & 53 & 53 & 53 & 53 & 53 & 53 \\
\hline BOD 5-day $(\mathrm{mg} / 1)$ & & & & -12 & & & & & & \\
\hline
\end{tabular}




\begin{tabular}{|c|c|c|c|c|c|c|c|c|c|c|}
\hline MONTH & RIJG & RUG & AUS & AUG & RUS & AUG & AUG & AUG & AUG & AUG \\
\hline JAY & 15 & 16 & 17 & 18 & 19 & 20 & 21 & 22 & 23 & 24 \\
\hline DAY NO. & 20 & 21 & 22 & 23 & 24 & 25 & 26 & 27 & 28 & 29 \\
\hline \multicolumn{11}{|l|}{ SYSTEM PARAMETERS } \\
\hline *Sludge Age & 30 & 30 & 30 & 30 & 35.4 & 30 & 30 & 30 & 38 & 30 \\
\hline$*(F / M) C O D$ & 0.07 & 0.09 & 0.13 & 0.12 & 0.80 & 0.13 & 0.16 & 0.20 & 0.17 & 0.18 \\
\hline *HRT & 2.1 & 2.8 & 2.1 & 2.1 & 1.9 & 1.9 & 1.9 & 2.1 & 2.8 & 2.1 \\
\hline FEED PRRAMETERS (BATCH \#) & 28 & 28 & 28 & 28 & 28 & 28 & 28 & 28 & 28 & 28 \\
\hline Voluae (liters) & 3.29 & 3.57 & 3.37 & 3.37 & 3.70 & 3.70 & 3.70 & 3.39 & 3.50 & 3.40 \\
\hline ph (std units) & 7.5 & 7.8 & 7.3 & 7.7 & 7.7 & 7.7 & \multicolumn{2}{|c|}{$7.67 .5 / 8.9$} & $7.3 / 9.0$ & $7.4 / 8.9$ \\
\hline $\operatorname{COD}(\operatorname{mg} / 1)$ & 250 & 265 & 276 & 258 & 287 & 298 & 230 & 289 & 383 & 320 \\
\hline $\mathrm{NH} J \mathbf{N}(\mathrm{mg} / 1)$ & 18.8 & 18.8 & 34.8 & 34.8 & 34.8 & 32.2 & 32.2 & 32.2 & 32.2 & 34.2 \\
\hline \multirow{2}{*}{\multicolumn{11}{|c|}{$\begin{array}{l}55(\mathrm{mg} / 1) \\
\text { USS }(\mathrm{mg} / 1)\end{array}$}} \\
\hline & & & & & & & & & & \\
\hline CN (mg/1) & 1.086 & 1.006 & 3.28 & 3.28 & 3.28 & 3.28 & 3.28 & 3.28 & 3.28 & 2.37 \\
\hline $\operatorname{SCN}(m g / 1)$ & 7.5 & 7.5 & - 4.9 & 4.9 & 4.9 & 4.9 & 4.9 & 4.9 & 4.9 & \\
\hline \multicolumn{11}{|l|}{ BOD 5-day (mg/l) } \\
\hline TOC $(\mathrm{mg} / 1)$ & 58 & 58 & & & & & & & & 69.6 \\
\hline Phenolics (og/l) & & & 1.025 & 1.025 & 1.025 & 1.025 & 1.025 & 1.025 & 1.025 & \\
\hline PC4-P (mg/1) & & & 4.2 & 4.2 & 4.2 & 4.2 & 4.2 & 4.2 & 4.2 & \\
\hline Org-N (mg/1) & 18.7 & 18.7 & & & & 17.2 & 17.2 & 17.2 & 17.2 & \\
\hline$N 02(\mathrm{mg} / 1)$ & 43.8 & 43.8 & 85.1 & 85.1 & 85.1 & 85.1 & 85.1 & 85.1 & 85.1 & 97.1 \\
\hline $\mathrm{N03}(\mathrm{ag} / \mathrm{l})$ & 116 & 116 & 36.9 & 96.9 & 96.9 & 36.9 & 96.9 & 96.7 & 36.9 & 83 \\
\hline \multicolumn{11}{|l|}{$\operatorname{TDS}(\mathrm{xg} / 1)$} \\
\hline Color (APHA Units) & 580 & 500 & & & & & & & & 750 \\
\hline \multicolumn{11}{|l|}{ BASIN PGRAMETERS } \\
\hline Vol Wasted (al) & 206 & 196 & 228 & 175 & 10 & 229 & 123 & 185 & 200 & 158 \\
\hline D.0. (四/1) & 8.4 & 8.3 & 8.4 & 7.8 & 8.8 & 7.8 & 7.7 & 8.1 & 8.2 & 8.0 \\
\hline SS $(\operatorname{mg} / 1)$ & 2410 & 2188 & 1640 & 1750 & 730 & 1920 & 1680 & 1290 & 1510 & 1470 \\
\hline \#SS (PAC corrected) & 1910 & 1680 & 1140 & 1250 & 230 & 1420 & 1180 & 790 & 1010 & 970 \\
\hline Te鲜 $\quad(C)$ & 23 & 24 & 23.5 & 25 & 24 & 25 & 25 & 24 & 25 & 24 \\
\hline $\mathrm{pH}$ (std units) & 7.6 & 7.6 & 7.8 & 7.8 & 7.6 & 7.7 & 7.6 & 7.5 & 7.7 & 7.7 \\
\hline DUR (ag/1/hr) & 2 & & & 4 & & & & 3 & & \\
\hline VSS (mg/1) & 1770 & 1601 & 1204 & 1180 & 492 & $1 \tilde{2} 35$ & 1133 & 920 & 1077 & 1048 \\
\hline USS (PAC corrected) & 1403 & 1234 & 837 & 843 & 155 & 957 & 796 & 563 & 720 & 532 \\
\hline VSS/SS & 0.73 & & & 0.67 & & & & 0.71 & & \\
\hline FAC Added (mg) & 143 & 144 & 143 & 143 & 121 & 143 & 143 & 143 & 143 & 143 \\
\hline *inventory (ug/l basin) & 500 & 508 & 500 & 500 & 500 & 500 & 500 & 500 & 500 & 500 \\
\hline \multicolumn{11}{|l|}{ EFFLUENIT PARAPETERS } \\
\hline Volume (liters) & 2.85 & 3.28 & 3.20 & 3.05 & 3.40 & 3.45 & 3.20 & 2.98 & 3.12 & 2.35 \\
\hline $\operatorname{COD}(\operatorname{sg} / 1)$ & 183 & 198 & 212 & 216 & 227 & 244 & 240 & 271 & 271 & 262 \\
\hline SS $(\mathrm{mg} / \mathrm{l})$ & 68 & 62 & 30 & 64 & 50 & 32 & 86 & 44 & 42 & 64 \\
\hline \multicolumn{11}{|l|}{ \&PAC corrected } \\
\hline oH (std units) & 7.6 & 7.6 & 7.8 & 7.8 & 7.6 & 7.6 & 7.6 & 7.5 & 7.5 & 7.6 \\
\hline VSS (mg/1) & 38 & & & & 48 & & & 28 & & \\
\hline \multicolumn{11}{|l|}{ *PAC corrected } \\
\hline NH3N $(5 g / 1)$ & 6 & 15.2 & 15.2 & 15.2 & 21.9 & 21.9 & 21.9 & 21.9 & 19.8 & 19.8 \\
\hline CN (mg/l) & 1.19 & 2.58 & 2.58 & 2.58 & 2.58 & 2.58 & 2.58 & 2.58 & 1.11 & 1.11 \\
\hline $\operatorname{SCN}(m g / 1)$ & 5.2 & 5.1 & 5.1 & 5.1 & 5.1 & 5.1 & 5.1 & 5.1 & 3.7 & 3.7 \\
\hline ND2 $(\operatorname{sg} / 1)$ & 13.5 & 73.7 & 73.7 & 73.7 & 73.7 & 73.7 & 73.7 & 73.7 & 96.1 & 96.1 \\
\hline $\mathrm{NO3}$ (ag/1) & 174 & 94.3 & 94.3 & 94.3 & 94.3 & 94.3 & 94.3 & 94.3 & 98.9 & 98.9 \\
\hline Phenolics (ng/l) & & 1.025 & 1.825 & 1.025 & 1.025 & 1.025 & 1.025 & 1.025 & & \\
\hline$P 04-P \cdot(m g / 1)$ & & 2.4 & 2.4 & 2.4 & 2.4 & 2.4 & 2.4 & 2.4 & & \\
\hline $\begin{array}{l}\text { Org-N }(\mathrm{mg} / 1) \\
\operatorname{TDS}(\mathrm{mg} / 1)\end{array}$ & 7.4 & & & & 18.3 & 18.3 & 18.3 & 18.3 & & \\
\hline Color (APHA units) & 1800 & & & & & & & & 758 & 758 \\
\hline $\begin{array}{l}\text { TOC }(\mathrm{mg} / 1) \\
800 \text { 5-day }(\mathrm{mg} / 1)\end{array}$ & 53 & & & $-127-$ & & & & & 59.6 & 59.6 \\
\hline
\end{tabular}


UNIT 2B TABLE R4

\begin{tabular}{|c|c|c|c|c|c|c|c|c|c|c|}
\hline MONTH & AUE & AUG & AUG & AUG & AUG & AUG & RUG & SEPT & SEPT & SEPT \\
\hline DAY & 25 & 26 & 27 & 28 & 29 & 30 & 31 & 1 & 2 & 3 \\
\hline DAY NO. & 30 & 31 & 32 & 33 & 34 & 35 & 36 & 37 & 38 & 39 \\
\hline \multicolumn{11}{|l|}{ SYSTEM PARAMETERS } \\
\hline tSludge Age & 30 & 30.1 & 30 & 24.5 & 30.1 & 30 & 30 & 30.1 & 30 & 38 \\
\hline$*(F / m) C O D$ & 0.10 & 0.09 & 0.12 & 0.08 & 0.25 & 0.06 & 0.28 & 0.05 & 0.85 & 0.86 \\
\hline ^HRT & 1.9 & 2.1 & 1.9 & 2.0 & 2.0 & 1.9 & 2.8 & 1.9 & 2.0 & 2.0 \\
\hline FEED PARAMETERS (BATCH & 28 & 28 & 28 & 29 & 29 & 29 & 29 & 29 & 29 & 29 \\
\hline Volume (liters) & 3.66 & 3.40 & 3.60 & 3.56 & 3.44 & 3.7 & 3.50 & 3.60 & 3.54 & 3.55 \\
\hline $\mathrm{pH}$ (std units) & $7.4 / 9.3$ & $7.4 / 8.9$ & 7.5 & $: / 9.0$ & 7.3 & 8.4 & $5 / 9.5$ & 7.5 & 7.4 & $4 / 8.9$ \\
\hline $\operatorname{coD}(\pi g / 1)$ & 329 & 316 & 322 & 279 & 291 & 260 & 281 & 213 & 170 & 193 \\
\hline NH3N $(\operatorname{mg} / 1)$ & 34.2 & 34.2 & 21.6 & 21.6 & 21.6 & 21.6 & 9.3 & 9.9 & 9.3 & 9.2 \\
\hline \multicolumn{11}{|l|}{$5 S(\mathrm{mg} / 1)$} \\
\hline \multicolumn{11}{|l|}{ USS (ng/1) } \\
\hline$C N(\pi q / 1)$ & 2.37 & 2.37 & 2.37 & 2.37 & 2.37 & 2.37 & 1.96 & ¿.96 & 1.96 & 1.96 \\
\hline \multicolumn{11}{|l|}{$\operatorname{SCN}(\operatorname{gg} / 1)$} \\
\hline \multicolumn{11}{|l|}{800 5-day (mg/1) } \\
\hline $\operatorname{TOC}(\operatorname{mg} / 1)$ & 69.6 & 69.6 & 69.6 & 69.6 & 69.6 & 69.6 & & & & \\
\hline Phenolics (ng/l) & & & & & & & 1.025 & 1.925 & 1.925 & 1.025 \\
\hline PO4-P (ag/l) & & & & & & & 3.9 & 3.9 & 3.9 & 3.9 \\
\hline Org-N (ng/1) & & & 10.7 & 10.7 & 10.7 & 10.7 & 3.5 & 3.5 & 3.5 & 3.5 \\
\hline NOâ $(m g / l)$ & 97.1 & 97.1 & 97.1 & 97.1 & 97.1 & 97.1 & 22.9 & 22.9 & 22.9 & 22.9 \\
\hline $\mathrm{NO} 3(\mathrm{mg} / \mathrm{l})$ & 83 & 83 & $8 \overline{3}$ & 83 & 83 & 83 & 182 & 182 & 182 & 182 \\
\hline \multicolumn{11}{|l|}{$\operatorname{TDS}(\mathrm{ng} / 1)$} \\
\hline Color (APHA Units) & 750 & 750 & 750 & 750 & 758 & 750 & & & & \\
\hline \multicolumn{11}{|l|}{ GASIN PARAMETERS } \\
\hline Vol Wasted (al) & 201 & 185 & 152 & 264 & 205 & 225 & 209 & 218 & 206 & 216 \\
\hline D.0. $(\mathrm{mg} / 1)$ & 8.0 & 7.8 & 7.4 & 7.3 & 7.8 & 8.0 & 8.0 & 8.0 & 8.3 & 8.1 \\
\hline $5 S(m g / 1)$ & 2340 & 2350 & 1958 & 2340 & 1028 & 2640 & 2050 & 2718 & 2240 & 2170 \\
\hline *SS (PAC corrected) & 1840 & 1850 & 1450 & 1840 & 520 & 2149 & 1550 & 8210 & 1710 & 1670 \\
\hline Teap (C) & 23 & 24 & 25 & 24 & 25 & 24 & 24 & 23.5 & 24 & 25 \\
\hline oH (std units) & 7.3 & 7.3 & 7.7 & 7.2 & 7.7 & 7.5 & 7.4 & 7.5 & 7.3 & 7.4 \\
\hline OUR (mg/l/hr) & 5 & & & & 4 & & & 4 & & \\
\hline VSS $(m g / h)$ & 1750 & 1757 & 1458 & 1750 & 920 & 2381 & 1849 & 2120 & 1752 & $169 \overline{8}$ \\
\hline \#USS (PAC carrected) & 1376 & 1384 & 1004 & 1376 & 489 & 1930 & 1394 & 1729 & 1361 & 1386 \\
\hline USS/SS & 0.75 & & & & 0.90 & & & 0.78 & & \\
\hline APAC Added (mg) & 143 & 143 & 143 & 176 & 143 & 144 & 143 & 143 & 143 & 143 \\
\hline $\begin{array}{l}\text { +lnventory (mg/l basin) } \\
\text { EFFLUENT PARAMETERS }\end{array}$ & 500 & 500 & 500 & 590 & 580 & 500 & 500 & 500 & 580 & 500 \\
\hline Volune (iters) & 3.34 & 2.90 & 3.20 & 3.88 & c. 92 & 3.25 & 2.99 & 3.42 & 3.16 & 3.20 \\
\hline $\operatorname{COD}(\operatorname{ag} / 1)$ & 280 & 283 & 264 & 234 & 243 & 228 & 219 & 167 & 145 & 138 \\
\hline SS $(\mathrm{mg} / 1)$ & 60 & 82 & 82 & 68 & 28 & 26 & 54 & 54 & 58 & 48 \\
\hline \multicolumn{11}{|l|}{ FPAC corrected } \\
\hline ọ (std units) & 7.3 & 7.6 & 7.7 & 7.5 & 7.5 & 7.2 & 7.4 & 7.1 & 7.4 & 7.4 \\
\hline VSS (mg/l) & 52 & & & & 26 & & & 48 & & \\
\hline \multicolumn{11}{|l|}{ $P A C$ corrected } \\
\hline NH3N (ng/1) & 19.8 & 6.2 & 6.2 & 6.2 & 6.2 & 12.1 & 12.1 & 12.1 & 8.8 & 0.8 \\
\hline$C N(0 g / 1)$ & 1.11 & 1.11 & 1.11 & 1.11 & 1.11 & 1.91 & 1.91 & 1.91 & 1.91 & 1.91 \\
\hline $\operatorname{SCN} \log / 1)$ & 3.7 & 3.7 & 3.7 & 3.7 & 3.7 & 3.7 & 3.7 & 3.7 & 3.7 & 3.7 \\
\hline NO2 $(\mathrm{mg} / 1)$ & 96.1 & 96.1 & 96.1 & 9.. 1 & 96.1 & 49.2 & 49.2 & 49.2 & 49.2 & 49.2 \\
\hline $\mathrm{Nat3}(\operatorname{ag} / 1)$ & 98.9 & 98.9 & 98.9 & 98.9 & 98.9 & 170 & 170 & 170 & 170 & 170 \\
\hline Phenolics (ng/1) & & & & & & 1.025 & 1.025 & 1.025 & 1.025 & 1.025 \\
\hline$P D 4-P(\operatorname{mg} / 1)^{-2}$ & & & & & & 4.2 & 4.2 & 4.2 & 4.2 & 4.2 \\
\hline $\begin{array}{l}\text { Org-N (ng/1) } \\
\text { TDS (ng/1) }\end{array}$ & & 10.4 & 10.4 & 10.4 & 10.4 & & & & 10.1 & $<0.1$ \\
\hline Color (APHA units) & 750 & 750 & 758 & 758 & 758 & & & & & \\
\hline TOC (ig/l) & 59.6 & 59.6 & 59.6 & 59.6 & 59.6 & & & & & \\
\hline BOD 5-day (ng/1) & & & & & & & & & & \\
\hline
\end{tabular}




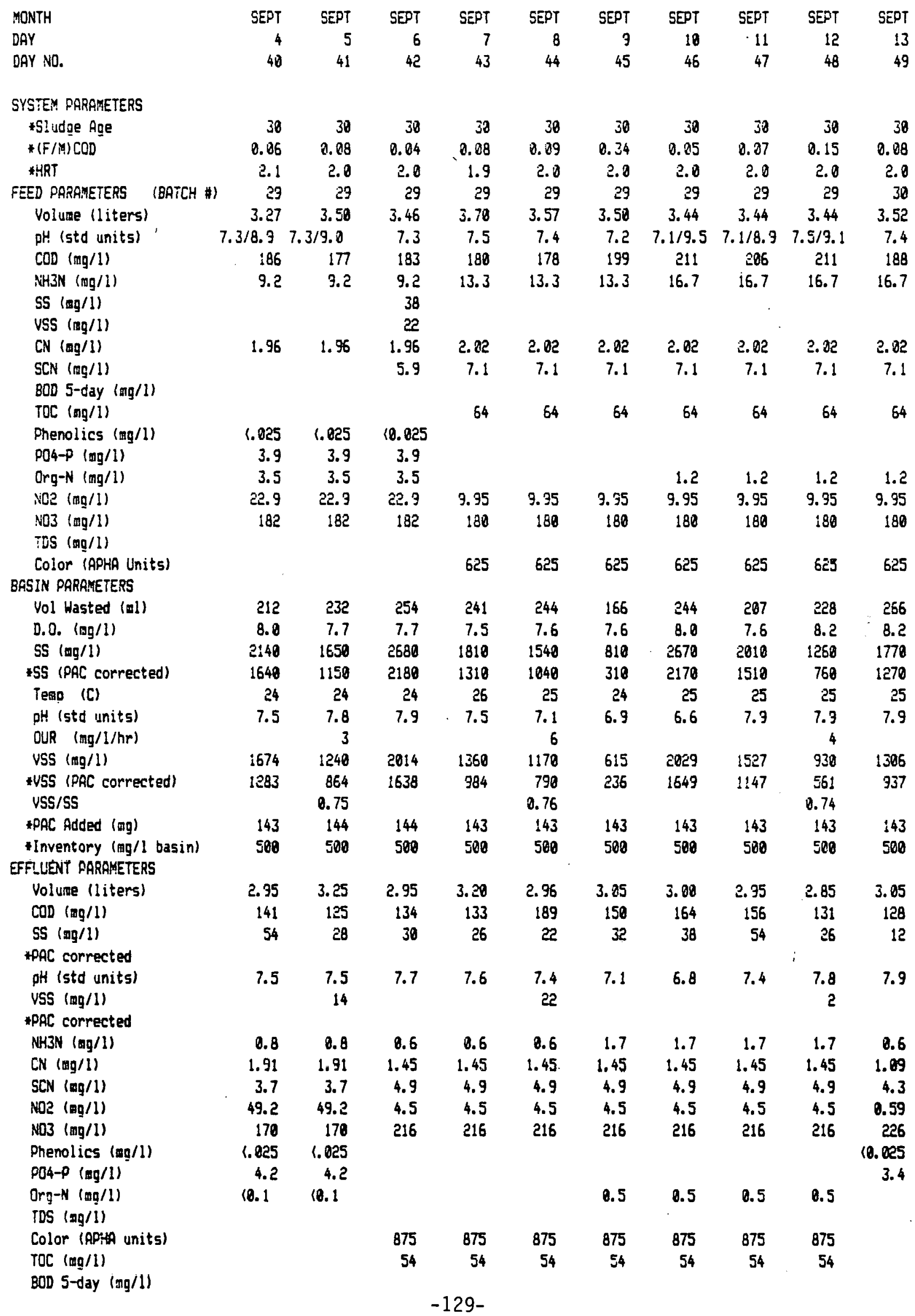


UNIT $2 B$ TABLE A4

\begin{tabular}{|c|c|c|c|c|c|c|c|c|c|c|}
\hline HONTH & SEPT & SEPT & SEPT & SEPT & SEPT & SEPT & SEPT & SEPT & SEPT & SEPT \\
\hline DAY & 14 & 15 & 16 & 17 & 18 & 19 & 20 & 21 & 22 & 23 \\
\hline DAY NO. & 50 & 51 & 52 & 53 & 54 & 55 & 56 & 57 & 58 & 59 \\
\hline \multicolumn{11}{|l|}{ SYSTEM PARAMETERS } \\
\hline *Sludge Age & 38 & 38 & 30 & 30 & 30 & 30 & 30 & 30 & 30 & 30 \\
\hline$+(F / M) C O D$ & 0.07 & 0.86 & 0.88 & 0.85 & 0.85 & 8.88 & 0.04 & 0.05 & 8.03 & 0.03 \\
\hline FHRT & 2.8 & 2.3 & 2.1 & 2.1 & 2.0 & 2.4 & 2.0 & 2.0 & 2.1 & 2.1 \\
\hline FEED PARAMETERS (BATCH \#) & 30 & 38 & 30 & 30 & 30 & 30 & 30 & 30 & 30 & 30 \\
\hline Volume (liters) & 3.57 & 3.10 & 3.48 & 3.38 & 3.50 & 2.98 & 3.60 & 3.68 & 3.40 & 3.30 \\
\hline pH (std units) & 7.4 & $6.9 / 8.9$ & $7.2 / 9.6$ & 7.4 & 7.4 & 7.8 & 6.9 & 7.0 & $7.1 / 8.8$ & 7.3 \\
\hline $\cos (\operatorname{agg} / 1)$ & 183 & 179 & 188 & 179 & 179 & 174 & 171 & 148 & 151 & 119 \\
\hline NHSN (ag/1) & 5.5 & 5.5 & 5.5 & 5.5 & 5.5 & 5.5 & 5.5 & 4.3 & 4.3 & 4.3 \\
\hline SS (ng/1) & & & & & & & 32 & & & \\
\hline V5S (mg/1) & & & & & & & 26 & & & \\
\hline CN (agg/l) & & & & & & & 1.62 & & & \\
\hline $\operatorname{SCN}(m g / 1)$ & & & & & & & 6.5 & $(10$ & $(10$ & $\$ 10$ \\
\hline \multicolumn{11}{|l|}{ BOD 5-day $(\mathrm{mg} / \mathrm{d})$} \\
\hline TOC (mg/1) & & & & & & & & 58 & 58 & 58 \\
\hline Phenolics (mg/l) & & & & & & & 10.025 & & & \\
\hline PO4-P (mg/l) & & & & & & & 4.3 & & & \\
\hline Org-N (mg/l) & & & & & & & 3.6 & & & \\
\hline NO2 (mg/1) & & & & & & & 4.7 & & & \\
\hline N03 (mog/1) & & & & & & & 196 & & & \\
\hline \multicolumn{11}{|l|}{ TDS (mg/1) } \\
\hline Color (APHA Units) & & & & & & & & 500 & 500 & 508 \\
\hline \multicolumn{11}{|l|}{ BASIN PARAMETERS } \\
\hline Vol Hasted (al) & 243 & 163 & 234 & 244 & 237 & 192 & 218 & 188 & 238 & 246 \\
\hline D.0. $(\mathrm{mg} / 1)$ & 9.2 & 9.0 & 8.9 & 8.8 & 8.4 & 7.9 & 8.0 & 7.8 & 8.8 & 8.0 \\
\hline SS $(\operatorname{mg} / 1)$ & 1890 & 1848 & 1680 & 2130 & 2580 & 1480 & 2770 & 2128 & 3080 & 2558 \\
\hline +SS (PAC corrected) & 1.390 & 1340 & 1180 & 1630 & 2080 & 200 & $\varepsilon \varepsilon 70$ & 1620 & 2380 & 2800 \\
\hline Tewo (C) & 23 & 21 & 21 & 22 & 22 & 22 & 23 & 24 & 23 & 22 \\
\hline $\mathrm{pH}$ (std units) & 7.9 & 7.5 & 8.1 & 8.2 & 8.2 & 8.8 & 7.6 & 7.2 & 7.1 & 7.8 \\
\hline OUR $(\mathrm{ng} / \mathrm{l} / \mathrm{hr})$ & & 4 & & & & 2 & & & 2 & \\
\hline VSS (ng/l) & 1393 & 1420 & 1297 & 1644 & 1991 & 1150 & 2152 & 1632 & 2898 & 1730 \\
\hline +VSS (PAC corrected) & 1026 & 1034 & 911 & 1258 & 1605 & 761 & 1764 & 1243 & 1751 & 1391 \\
\hline VSS/SS & & 0.77 & & & & 0.78 & & & 0.68 & \\
\hline \#PAC Added (mg) & 143 & 143 & 143 & 143 & 143 & 143 & 143 & 143 & 144 & 143 \\
\hline Inventory (ag/l basin) & 580 & 500 & 500 & 508 & 580 & 500 & 500 & 500 & 500 & 500 \\
\hline \multicolumn{11}{|l|}{ EFFLLENT PARAMETERS } \\
\hline Volume (liters) & 2.97 & 3.15 & 2.90 & 3.10 & 2. 90 & 3.50 & 3.30 & 3.40 & 3.20 & 3.08 \\
\hline $\operatorname{cod}(\operatorname{mg} / 1)$ & 126 & 133 & 145 & 142 & 140 & 143 & 184 & 186 & 97 & 36 \\
\hline SS (og/1) & 28 & 72 & 30 & 30 & 44 & 40 & 58 & 62 & 48 & 34 \\
\hline \multicolumn{11}{|l|}{ «PAC corrected } \\
\hline $\mathrm{gH}$ (std units) & 8.1 & 7.6 & 7.8 & 8.1 & 8.1 & 8.1 & 7.6 & 7.4 & 7.1 & 7.4 \\
\hline USS (mog/1) & & 6? & & & & 32 & & & 42 & \\
\hline \multicolumn{11}{|l|}{ *PAC corrected } \\
\hline NH3N $(m g / 1)$ & 0.6 & 8.6 & 18.1 & 10.1 & (0.1 & (8.1 & 1.2 & 1.2 & 1.2 & 1.2 \\
\hline CN $(\pi g / 1)$ & 1.09 & 1.09 & 1.09 & 1.09 & 1.89 & 1.89 & & & & \\
\hline $\operatorname{SCN}(m g / 1)$ & 4.3 & 4.3 & 4.3 & 4.3 & 4.3 & 4.3 & & & & \\
\hline Nก? $(n g / 1)$ & 0.59 & 0.59 & 0.53 & 0.59 & 0.59 & 0.59 & & & & \\
\hline$N 03$ (mg/l) & 226 & 226 & 226 & 226 & 226 & 226 & & & & \\
\hline Phenolics $(a g / l)$ & 10.025 & 10.025 & 10.025 & 10.025 & 10.825 & 10.025 & & & & \\
\hline$P O 4-P(19 / 1)$ & 3.4 & 3.4 & 3.4 & 3.4 & 3.4 & 3.4 & & & & \\
\hline $0 r g-N(m g / 1)$ & & & $\ll 1$ & 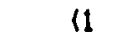 & 11 & $\$ 1$ & & & & \\
\hline \multicolumn{11}{|l|}{ TDS (mg/l) } \\
\hline Color (APHA units) & & & & & & & 1000 & 1080 & 1000 & 1000 \\
\hline TOC (mg/1) & & & & & & & 52 & 52 & 52 & 52 \\
\hline BOD 5-day (mg/1) & & & & & & & 110 & $(18$ & $(18$ & 110 \\
\hline
\end{tabular}


UNIT $3 B$ TABLE A4

\begin{tabular}{|c|c|c|c|c|c|c|c|c|c|c|}
\hline MONTH & SEPT & SEPT & SEPT & SEPT & SEPT & SEPT & SEPT & OCT & OCT & OCT \\
\hline DAY & 24 & 25 & 26 & 27 & 28 & 29 & 30 & 1 & 2 & 3 \\
\hline DAY NO. & 60 & 61 & 62 & 63 & 64 & 65 & 66 & .67 & 68 & 69 \\
\hline \multicolumn{11}{|l|}{ SYSTEM PGRFMETERS } \\
\hline *Sludge fage & 30 & 38 & 30 & 30 & 30 & 30 & 30 & 30 & 30 & 30 \\
\hline$*(F / M) C O D$ & ERR & 0.17 & 0.84 & 0.08 & 0.05 & 0.06 & 0.13 & 0.07 & 0.05 & 8.08 \\
\hline$\star H R T$ & 2.1 & 1.9 & 2.2 & 1.9 & 1.9 & 1.9 & 1.9 & 1.9 & 1.9 & 1.9 \\
\hline FEED PARAMEIERS (BATCH *) & 30 & 30 & 30 & 30 & 31 & 31 & 31 & 31 & 31 & 31 \\
\hline Volune (1iters) & 3.40 & 3.70 & 3.20 & 3.4 & 3.7 & 3.6 & 3.7 & 3.1 & 3.7 & 3.7 \\
\hline pH (std units) & 7.4 & 6.7 & 7.2 & $7.0 / 8.9$ & 7.4 & 7.3 & 7.4 & 7.5 & 7.8 & 7.8 \\
\hline $\cos (\operatorname{sig} / 1)$ & เลิ2 & 176 & 176 & 162 & 172 & 176 & 177 & $\$ 88$ & 172 & 180 \\
\hline NH.3N (ng/1) & 6.1 & 6.1 & 6.1 & 6.1 & 4.3 & 4.9 & 4.9 & & & \\
\hline SS $(m g / 1)$ & & & & 294 & & & & & & \\
\hline VSS $(m g / 1)$ & & & & 290 & & & & & & \\
\hline \multicolumn{11}{|l|}{ CN (mg/l) } \\
\hline $\mathrm{SCN}(\mathrm{mg} / 1)$ & 110 & 110 & $\$ 10$ & 110 & & & & & & \\
\hline \multicolumn{11}{|l|}{ BOD 5-day (ng/l) } \\
\hline $\operatorname{TOC}(\mathrm{gg} / 1)$ & 58 & 58 & 58 & 58 & & & & & & \\
\hline \multicolumn{11}{|l|}{ Phenolics $(a g / 1)$} \\
\hline \multicolumn{11}{|l|}{$P O 4-P(\mathrm{mg} / 1)$} \\
\hline \multicolumn{11}{|l|}{ Orno-N (mg/1) } \\
\hline \multicolumn{11}{|l|}{ :02 (mg/1) } \\
\hline \multicolumn{11}{|l|}{$\mathrm{N} 03(\mathrm{mo} / 1)$} \\
\hline \multicolumn{11}{|l|}{$\operatorname{TDS}(\mathrm{mg} / \mathrm{l})$} \\
\hline Color (AFHA Units) & 500 & 500 & 580 & 500 & & & & & & \\
\hline \multicolumn{11}{|l|}{ BRSIN PARAKETERS } \\
\hline Vol Wasted (nl) & 10 & 168 & 235 & 200 & 244 & 227 & 108 & 209 & 222 & 176 \\
\hline 0.0. $(\pi 0 / 1)$ & 8.3 & 8.0 & 8.2 & 8.8 & 8.6 & 8.9 & 8.9 & 9.0 & 8.5 & 8.6 \\
\hline SS $(m \underline{0} / 1)$ & & 1160 & 2448 & 1630 & 2490 & 2400 & 1060 & 1920 & 2550 & 1938 \\
\hline *SS (PAC corrected) & & 660 & 1340 & 1130 & 1990 & 1900 & 560 & 1420 & 2050 & 1430 \\
\hline Temo (C) & 21 & 20 & 20 & 28 & 21 & 20 & 20 & 20 & 21 & 21 \\
\hline $\mathrm{pH}$ (std units) & 7.8 & 7.4 & 7.1 & 7.0 & 7.6 & 7.5 & 7.4 & 7.5 & 7.6 & 7.9 \\
\hline OUR (ng/l/hr) & & & & & & 2 & & & & 2 \\
\hline USS $(n g / 1)$ & & 787 & 1840 & 1229 & 1878 & 1690 & 746 & 1352 & 1796 & 1340 \\
\hline *USS (PAC corrected) & & 448 & 1463 & 852 & 1501 & 1338 & 394 & 1000 & 1440 & 993 \\
\hline USS/SS & & & 0.75 & & & 0.70 & & & & 0.69 \\
\hline „PAC Added (mg) & 143 & 143 & 143 & 143 & 143 & 143 & 143 & 143 & 143 & 143 \\
\hline tinventory (ag/l basin) & 500 & 500 & 500 & 500 & 500 & 500 & 500 & 580 & 580 & 580 \\
\hline \multicolumn{11}{|l|}{ EFFLUENT PARAMETERS } \\
\hline Volume (11ters) & 3.18 & 3.58 & 2.85 & 3.20 & 3.35 & 3.42 & 3.50 & 3.49 & 3.40 & 3.00 \\
\hline $\operatorname{COD}(\operatorname{mg} / 1)$ & 150 & 153 & 155 & 146 & 150 & 155 & 153 & 150 & 152 & 152 \\
\hline SS $(\mathrm{mg} / 1)$ & 58 & 40 & 44 & 44 & 32 & 42 & 54 & 44 & 48 & 72 \\
\hline \multicolumn{11}{|l|}{ PAC corrected } \\
\hline of (std units) & 7.7 & 7.5 & 7.3 & 6.9 & 7.2 & 7.5 & 7.4 & 7.3 & 7.4 & 7.7 \\
\hline USS (mg/l) & & & 30 & & & 38 & & & & 52 \\
\hline \multicolumn{11}{|l|}{ APAC corrected } \\
\hline NH3N $(m g / 1)$ & 1.2 & 1.2 & 1.2 & 0.3 & 0.3 & 0.3 & 1.5 & 1.5 & 1.5 & 1.5 \\
\hline \multicolumn{11}{|l|}{ CN $(\operatorname{tog} / 1)$} \\
\hline \multicolumn{11}{|l|}{$\mathrm{SCN}(\mathrm{ag} / 1)$} \\
\hline \multicolumn{11}{|l|}{ NO2 $(m g / 1)$} \\
\hline Phenolics (mg/l) & & & & 10.825 & 10.025 & 10.025 & 10.025 & 10.025 & 10.025 & 10.025 \\
\hline PO4-P (ag/1) & & & & 14.6 & 14.6 & 14.6 & 14.6 & 14.6 & 14.6 & 14.6 \\
\hline $\begin{array}{l}\text { Org-N (mg/l) } \\
\text { TDS (mg/1) }\end{array}$ & & & & & & & & & & \\
\hline Color (APHA units) & 1280 & 1080 & 1090 & & & & & & & \\
\hline $\operatorname{TOC}(\operatorname{\theta g} / 1)$ & 52 & 52 & 52 & 46 & 46 & 46 & 46 & 46 & 46 & 46 \\
\hline BOD 5-day (mg/l) & 110 & 110 & 110 & 15.4 & 15.4 & 15.4 & 15.4 & 15.4 & 15.4 & 15.4 \\
\hline
\end{tabular}


UNIT 2B TABLE A4

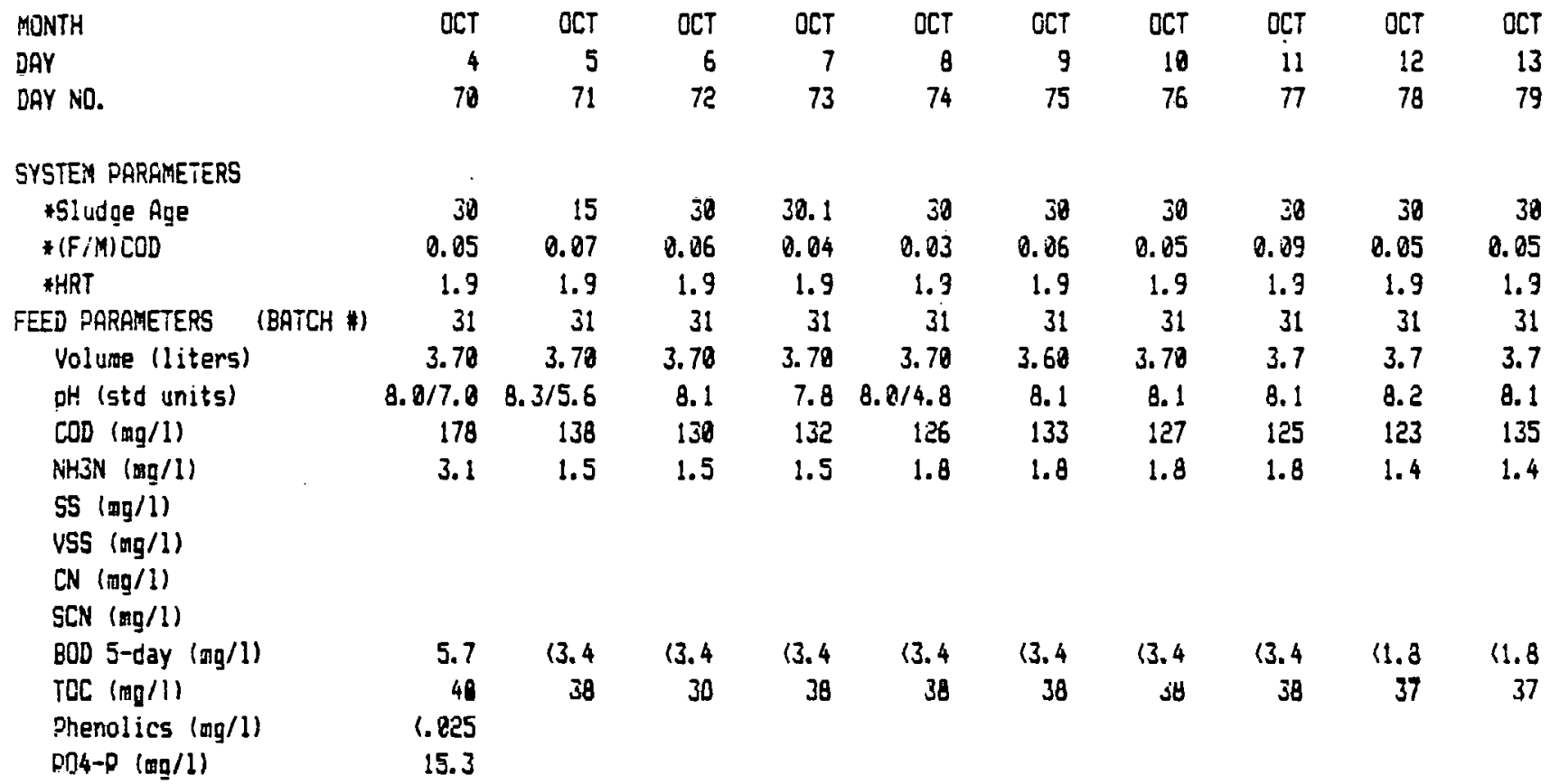

Örg-N (mg/l)

NOS (mg/!)

$\mathrm{NO3}(\mathrm{mg} / 1)$

TDS (nc/l)

Color (APHA Units)

BASIN PARAMETERS

Vol Wasted (⿴囗十)

D.0. (ng/1)

$55(\mathrm{mg} / 1)$

*SS (PAC corrected)

Teno (C)

at (std units)

our (ng/1/hr)

VBS (mo/l)

IVSS (FAC corrected)

VSS/SS

tPAC Added (mg)

+Inventory (rog/1 basin)

15.3

EFFLUENT PARAMETERS

Volune (liters)

COD (ng/l)

S5 (ag/1)

*PPC corrected

oH (std units)

VSS $(\mathrm{sg} / 1)$

+PAC corrected

NH3N (ag/1)

CN (mg/l)

SCN $(\mathrm{ag} / 1)$

NO2 (mg/l)

$\mathrm{N03}$ (8g/1)

Phenolics (ng/l)

P04-P (mg/l)

Drg-N (mg/l)

$\begin{array}{lllllll}625 & 625 & 625 & 625 & 625 & 625 & 625\end{array}$

229

8. 2

2848

2340

8.1

197

162

143

508

3.40$$
134
$$

13

125

10

196

$22 \theta \quad 231$

$177 \quad 234$

$8.8 \quad 7.8$

$2390 \quad 2758$

$8.0 \quad 8.1$

$1660 \quad 2120$

$1160 \quad 1620$

1270

1720

1890

टç50

22

7.8

8.87 .8

8.2

8.8

7.8

$1806 \quad 3078$

$1424 \quad 1701$

1255

8. 1

8.1
$?$

1300

922

0.76

$286 \quad 143$

143

500

500

144

500

7.98

11
$3.50 \quad 3.50 \quad 3.45$

3.45
125

3.15

120

115

117

8.2 7.9

7.9
38

7.9

$8.0 \quad 7.9$

3.12
118
36
8.1
34

1.3

1.3

1.3

TDS (mg/1)

Color (APHA units)

TOC (mg/1)

BOD 5-day (mg/l)

75

33
$<2.6$
33

750
33

12.6

$\begin{array}{r}750 \\ 33 \\ \hline\end{array}$

12.6
0.6

0.6

0.6
75

12. 6 $\begin{array}{rrr}212 & 246 & 221 \\ 8.2 & 8.0 & 8.0 \\ 1340 & 2870 & 2220 \\ 840 & 1570 & 1720 \\ 21 & 22 & 22\end{array}$

$\begin{array}{lll}8.2 & 8.3 & 8.3\end{array}$

$\begin{array}{lll}986 & 1523 \quad 1660\end{array}$

$618 \quad 1155 \quad 1286$

0.75

$143 \quad 143 \quad 143$

$580 \quad 580 \quad 580$

$3.32 \quad 3.50 \quad 3.62$

$\begin{array}{rrr}127 & 116 & 118 \\ 38 & 24 & 48\end{array}$

B.1 B.2 8.3

$2.4 \quad 2.4 \quad 2.4$ 
UNIT $2 B$ TABLE A4

\begin{tabular}{|c|c|c|c|c|c|c|c|c|c|c|}
\hline MONTH & OCT & OCT & OCT & OCT & OCT & OCT & OCT & OCT & BST & OCT \\
\hline DAY & 14 & 15 & 16 & 17 & 18 & 19 & 20 & 21 & 22 & 23 \\
\hline DAY :NO. & 80 & 81 & 82 & 83 & 84 & 85 & 86 & 87 & 88 & 89 \\
\hline \multicolumn{11}{|l|}{ SYSTEM PARAMETERS } \\
\hline †SIudg̣e Age & 30 & 30 & 30 & 30 & 30.01 & 29.98 & 32.96 & 30.00 & 30.00 & 30.90 \\
\hline$\because(F / M) C O D$ & 0.05 & 0.04 & 0.03 & 0.85 & 0.00 & 0.07 & 0.05 & 0.85 & 0.04 & 0.05 \\
\hline IHRT & 1.9 & 1.9 & 2.2 & 2.0 & 2.21 & 1.98 & 1.89 & 1.89 & 1.89 & 1.89 \\
\hline FEED PARAPETERS (BATCH \#) & 31 & 31 & 31 & 31 & & & & & & \\
\hline Volune (1iters) & 3.7 & 3.7 & 3.25 & 3.45 & 3.17 & 3.53 & 3.7 & 3.7 & 3.7 & 3.7 \\
\hline $\mathrm{pH}$ (std units) & 8.1 & 8.1 & 8.2 & $8.4 / 5.6$ & 8.3 & 8 & 8.1 & 8.4 & 8.1 & 8.1 \\
\hline $\operatorname{COD}(\mathrm{mg} / 1)$ & 146 & 146 & 136 & 142 & & 131 & 141 & 126 & 114 & 138 \\
\hline NH.JN (酉/l) & 1.4 & 1.2 & 1.2 & 1.2 & & 1.4 & 1.4 & 1.4 & 2 & 2 \\
\hline SS $(\operatorname{mg} / 1)$ & & & & & & 64 & 36 & & & \\
\hline \multicolumn{11}{|l|}{ USS $(\operatorname{mo} / i)$} \\
\hline \multicolumn{11}{|l|}{ CN (mg/1) } \\
\hline \multicolumn{11}{|l|}{$\operatorname{SCN}(\operatorname{mg} / 1)$} \\
\hline BOD 5-day $(m g / 1)$ & 11.8 & 11.8 & 11.8 & 11.8 & & 8.9 & 3. 9 & 8. 9 & 0.9 & 0.9 \\
\hline iOC $(m g / 1)$ & 37 & 37 & 37 & 37 & & 40 & 40 & 40 & 40 & 40 \\
\hline Phenolics (mg/l) & & & & & & & & & & \\
\hline \multicolumn{11}{|l|}{ PO4-P (wg/1) } \\
\hline \multicolumn{11}{|l|}{ Org-N (mg/1) } \\
\hline NO2 (mg/l) & & & & & & & & - & & \\
\hline \multicolumn{11}{|l|}{$\mathrm{NO}$ (mg/l) } \\
\hline \multicolumn{11}{|l|}{ TDS (mg/l) } \\
\hline Color (APHA linits) & & & & & & 625 & 625 & 625 & 625 & 625 \\
\hline \multicolumn{11}{|l|}{ BRSIN PARAMETERS } \\
\hline Vol Wasted (nl) & 249 & 241 & 238 & 218 & 206 & 222 & 248 & 184 & 175 & 159 \\
\hline $0.0 .(\mathrm{mg} / 1)$ & 8.2 & 8.2 & 8.0 & 8.0 & 8 & 8.4 & 8.6 & 8.6 & 8.6 & 8.5 \\
\hline $5 S(m g / 1)$ & 2030 & 2530 & 2860 & $: 910$ & 2010 & 1420 & 2200 & 2130 & 2630 & 2660 \\
\hline *SS (PAC corrected) & 1530 & 2030 & 2360 & 1410 & $15: 0$ & 920 & 1700 & 1629 & 2129 & 2159 \\
\hline Temo (C) & 21 & 21 & 21 & 21 & 21 & 21 & 19 & 19 & 19 & 20 \\
\hline oH (std units) & 8.4 & 8.4 & 8.4 & 8.4 & 8.3 & 8.2 & 8.3 & 8.4 & 8.2 & 7.8 \\
\hline OUR (mg/l/hr) & & & & 2 & & & 2 & & & \\
\hline VSS (mg /1) & 1518 & 1892 & 2139 & 1410 & 1300 & 1300 & 1630 & 1630 & $\$ 630$ & $i 690$ \\
\hline +VSS (PAC corrected) & 1144 & 1518 & 1765 & .1041 & 808 & 808 & 1130 & 1199 & 1189 & $\therefore 1189$ \\
\hline VSS/SS & & & & 0.74 & 0.76 & 0.76 & 0.77 & 0.77 & 0.77 & 0.77 \\
\hline ¥PAC Added (mg) & 143 & 143 & 143 & 143 & 143 & 143 & 143 & 143 & 143 & 143 \\
\hline *Inventory (mg/l basin) & 500 & 500 & 500 & 580 & 500 & 500 & 580 & 501 & 501 & 501 \\
\hline \multicolumn{11}{|l|}{ EFFLUENT PARAYETERS } \\
\hline Volune (liters) & 3.44 & 3.60 & 3.00 & 3.30 & 3 & 3. 29 & 3.57 & 3.43 & 3.4 & 3.6 \\
\hline $\operatorname{coD}(m g / 1)$ & 130 & 132 & 130 & 124 & 120 & 114 & 110 & 108 & 107 & 99 \\
\hline SS $(a q / 1)$ & 22 & 32 & 46 & 40 & 54 & 28 & 24 & 64 & 86 & 94 \\
\hline \multicolumn{11}{|l|}{ *PAC corrected } \\
\hline oH (sto units) & 8.3 & 8.4 & 8.1 & 8.2 & 8.3 & 8.1 & 8.3 & 8.4 & 8.2 & 8 \\
\hline VSS $(m g / 1)$ & & & & 26 & 38 & 38 & 20 & 20 & 20 & 20 \\
\hline \multicolumn{11}{|l|}{ \#AQC corrected } \\
\hline NHFN $(\mathrm{gg} / 1)$ & 0.6 & 0.6 & a. 6 & 0.6 & 1.1 & 1.1 & 1.1 & 0.9 & 0.9 & 0.9 \\
\hline \multicolumn{11}{|l|}{ CN $(m g / 1)$} \\
\hline \multicolumn{11}{|l|}{ SCN (ng/1) } \\
\hline \multicolumn{11}{|l|}{$\begin{array}{l}\text { NO2 (mg/1) } \\
\text { NO3 (mg/1) }\end{array}$} \\
\hline $\mathrm{NO3}$ (mg/1) & & & & & & & & & & \\
\hline Phenolics (mg/l) & & & & & & & & & & \\
\hline$P 04-P(n g / 1)$ & 2.4 & 2.4 & 2.4 & 2.4 & & & & & & \\
\hline $\begin{array}{l}O r g-N(m g / 1) \\
\operatorname{TDS}(m g / 1)\end{array}$ & & & & & & & & & & \\
\hline Color (APHA units) & & & & & 500 & 500 & 500 & 500 & 588 & 580 \\
\hline TOC $(m g / 1)$ & 31 & 31 & 31 & 31 & 32 & 32 & 32 & 32 & 32 & 32 \\
\hline BOD 5-day (mg/1) & 1.8 & 1.8 & 1.8 & 1.8 & 10.9 & 10.9 & 10.9 & 10.9 & 18.9 & 10.9 \\
\hline
\end{tabular}




\begin{tabular}{|c|c|c|c|c|c|c|c|c|c|c|}
\hline MONTH & OCT & OCT & OCT & OCT & $\mathrm{OCT}$ & OCT & OCT & OCT & NOV & NOV \\
\hline DAY & 24 & 25 & 26 & 27 & 28 & 29 & 30 & 31 & 1 & 2 \\
\hline DAY NO. & 90 & 91 & 92 & 93 & 94 & 95 & 96 & 97 & 38 & 99 \\
\hline \multicolumn{11}{|l|}{ SYSTEY FARAMETERS } \\
\hline *5ludģe Age & 36.66 & 30.01 & 30.01 & 38.03 & 30.03 & 29.96 & 29.37 & 29.97 & 30.25 & 30.84 \\
\hline$\mapsto(F / M) C O D$ & 0.85 & 0.06 & 0.06 & 0.03 & 0.04 & 0.04 & 0.03 & 0.05 & 0.05 & 0.05 \\
\hline \#HRT & 1.89 & 1.89 & 1.99 & 2.69 & 2.33 & 1.99 & 2.35 & 1.94 & 2. 82 & 1.89 \\
\hline \multicolumn{11}{|l|}{ FEED PARAMETERS (BRTCH \#) } \\
\hline Volume (liters) & 3.7 & 3.7 & 3.7 & 2.6 & 3.01 & 3.7 & 2.98 & 3.6 & 3.47 & 3.7 \\
\hline $\mathrm{pH}$ (std units) & 8.2 & 8.1 & 8 & 8.2 & 8.3 & 7.9 & 8.2 & 7.4 & 7.2 & 7.1 \\
\hline $\operatorname{COD}(\operatorname{mg} / 1)$ & 134 & 138 & 139 & 142 & 130 & 132 & 130 & 140 & 134 & 148 \\
\hline NHJNN $(\operatorname{mg} / 1)$ & 2 & 2 & 2.3 & 2.3 & 2.3 & 3 & 3 & 3 & & 10.0 \\
\hline SS (mg/1) & & & & & & & & & & 54 \\
\hline USS (mg/1) & & & & & & & & & & 30 \\
\hline CN $(m g / 1)$ & & & & & & & & & & 1.51 \\
\hline $\operatorname{SCN}(\mathrm{mg} / 1)$ & & & & & & & & & & 3.1 \\
\hline BOD 5-day $(m g / 1)$ & 0.9 & 0.9 & 0.9 & 1.7 & 1.7 & 1.7 & 1.7 & 1.7 & & 1.3 \\
\hline TOC (mg/l) & 40 & 48 & 40 & 40 & 40 & 40 & 40 & 40 & & 45 \\
\hline Phenolics (mg/1) & & & 1.025 & 1.025 & 1.025 & 1.025 & 1.025 & 1.025 & & 10.025 \\
\hline$P 04-P(\lg / 1)$ & & & & & & & & & & 34.6 \\
\hline Org-N (mg/1) & & & & & & & & & & $<4.0$ \\
\hline $\mathrm{NO}$ (mg/1) & & & & & & & & & & 3.81 \\
\hline $\mathrm{N03}$ (mq/1) & & & & & & & & & & 148 \\
\hline \multicolumn{11}{|l|}{ TDS $(m g / 1)$} \\
\hline Color (APHA Units) & 625 & 625 & & & & & & & & 875 \\
\hline \multicolumn{11}{|l|}{ EASIN PARRMETERS } \\
\hline Vol Wasted (al) & 160 & 215 & 171 & 236 & 216 & 178 & 225 & 246 & 172 & 141 \\
\hline D.0. $(\mathrm{mg} / 1)$ & 8.6 & 8.6 & 8.6 & 8.8 & 8.8 & 8.8 & 8.8 & 8.9 & 8.4 & 8.8 \\
\hline SS (mg/l) & 2120 & 2530 & 2030 & 2410 & 2189 & 2150 & 2500 & 2160 & 1960 & 1520 \\
\hline ISS (PAC corrected) & 1619 & 2029 & 1529 & 1909 & 1673 & 1659 & 2099 & 1659 & 1459 & 1019 \\
\hline Tewy (C) & .19 & 19 & 18.5 & 18 & 18 & 18.5 & 18 & 18 & 18 & 18 \\
\hline pH (std units) & 8.2 & 8.5 & 8.2 & 8.3 & 8.2 & 8.3 & 8.3 & 8 & 8.1 & 7.8 \\
\hline OUR $(m g / 1 / h r)$ & 4 & & & 2 & & & & 2 & & \\
\hline USS (mg/l) & 1550 & 1550 & 1550 & 1790 & 1790 & 1790 & 1790 & 1678 & 1678 & 1670 \\
\hline IVSS (PAC corrected) & 1049 & 1049 & 1049 & 1289 & 1239 & 1289 & 1289 & 1169 & 1169 & 1169 \\
\hline VSS/SS & 0.73 & 0.73 & 0.73 & 0.74 & 8.74 & 0.74 & 0.74 & 0.77 & 0.77 & 0.77 \\
\hline *PAC Added (田) & 143 & 143 & 143 & 143 & 143 & 143 & 143 & 143 & 143 & 143 \\
\hline +Inventory (ng/l basin) & 501 & 501 & 501 & 501 & 501 & 501 & 501 & 581 & 501 & 501 \\
\hline \multicolumn{11}{|l|}{ EFFLLLENT PARAMETERS } \\
\hline Volube (liters) & 3.45 & 3.48 & 3.45 & 2.25 & 2.74 & 3.1 & 2.6 & 3.4 & 3.5 & 3.45 \\
\hline $\operatorname{cod}(\mathrm{eg} / 1)$ & 113 & 115 & 115 & 111 & 111 & 117 & 107 & 105 & 110 & 131 \\
\hline SS (sg/l) & 78 & 52 & 68 & 54 & 56 & 76 & 62 & 26 & 64 & 62 \\
\hline \multicolumn{11}{|l|}{ APAC corrected } \\
\hline oH (std units) & 8.2 & 8.6 & 8.2 & 8.4 & 8.2 & 8.2 & 0.2 & 9.2 & 8.1 & 7.9 \\
\hline VSS $(\log / 1)$ & 54 & 54 & 54 & 38 & 38 & 38 & 38 & 24 & 24 & 24 \\
\hline \multicolumn{11}{|l|}{ wALi corrected } \\
\hline NKËN $(n g / 1)$ & 0.9 & 0.3 & 0.3 & 0.3 & 0.6 & 0.6 & 9.6 & 0.6 & $<4.0$ & 14.0 \\
\hline$C N(m g / l)$ & & & & & & & & & 1.14 & 1.14 \\
\hline SCN (mg/l) & & & & & & & & & 2.3 & 2.3 \\
\hline No2 (mg/1) & & & & & & & & & 2.2 & 2.2 \\
\hline $\mathrm{NO3}(\mathrm{ag} / 1)$ & & & & & & & & & 208 & 200 \\
\hline Phenolics $(\mathrm{mg} / \mathrm{l})$ & & 10.025 & (0.825 & $(0.025$ & 10.025 & 10.025 & 10.025 & $<0.025$ & 10.025 & 10.825 \\
\hline$P 04-P(\pi g / 1)$ & & 2.8 & 2.8 & 2.8 & 2.8 & 2.8 & 2.8 & 2.8 & 12.2 & 12.2 \\
\hline $\begin{array}{l}\text { Org-N (mg/l) } \\
\operatorname{TDS}(\mathrm{mg} / 1)\end{array}$ & & & & & & & & & $(4.8$ & 14.0 \\
\hline Color (APHA units) & 500 & & & & & & & & 625 & 625 \\
\hline TOC $(n g / 1)$ & 32 & 31 & 31 & 31 & 31 & 31 & 31 & 31 & 33 & 33 \\
\hline BOD 5-day (mg/l) & 18.9 & 0.9 & 8.9 & 8.9 & 0.9 & 0.9 & 0.9 & 0.9 & 1.1 & 1.1 \\
\hline
\end{tabular}


UNIT 2B TABLE A4

\begin{tabular}{|c|c|c|c|c|c|c|c|c|c|c|}
\hline MONTH & NOV & NOV & NOV & NOV & $\mathrm{NOV}$ & NOV & NOV & NOV & AUV & NOV \\
\hline DAY & 3 & 4 & 5 & 6 & 7 & 8 & 3 & 18 & 11 & 12 \\
\hline DAY NO. & 100 & 101 & 102 & 103 & 184 & 185 & 106 & 107 & 108 & 109 \\
\hline \multicolumn{11}{|l|}{ SYSTEM PARAMETERS } \\
\hline * Sludge Poge & 29.97 & 29.89 & 30.02 & 30.83 & 29.38 & 38.02 & 29.98 & 30.35 & 30.01 & 23.97 \\
\hline$\pm(F / M) C O D$ & 0.18 & 0.11 & 0.11 & 0.07 & 0.85 & 0.87 & 0.87 & 0.06 & 0.05 & 0.29 \\
\hline$\star H R T$ & 1.89 & 1.89 & 1.89 & 1.89 & 1.89 & 1.75 & 2.86 & 2.13 & 2.14 & 2.02 \\
\hline \multicolumn{11}{|l|}{ FEED PARAMETERS (BATCH H) } \\
\hline Volume (liters) & 3.7 & 3.7 & 3.7 & 3.7 & 3.7 & 4 & 3.39 & 3.29 & 3.27 & 3.46 \\
\hline oH (std inits) & 6.9 & 6.6 & 6.7 & 8.3 & 8.3 & 8. 1 & 7.4 & 7.1 & 7.3 & 7.5 \\
\hline $\operatorname{COD}($ mg $/ 1)$ & 144 & 152 & 163 & 163 & 163 & 197 & 273 & 237 & 226 & $\hat{c} 05$ \\
\hline $\mathrm{NH} 3 \mathrm{~N}(\mathrm{mg} / \mathrm{l})$ & 10.0 & 10.0 & 37.5 & 37.5 & 37.5 & 37.5 & 34.2 & 34.2 & 34.2 & 15.8 \\
\hline $55(\operatorname{mg} / 1)$ & 52 & 252 & 24 & 70 & 124 & 62 & 58 & 64 & 52 & 28 \\
\hline USS (mg/!) & 30 & 206 & 206 & 286 & 206 & 40 & 40 & 40 & 40 & 40 \\
\hline CN (mg/1) & 1.51 & 1.51 & 1.51 & 1.51 & 1.51 & 1.51 & & & & \\
\hline $\operatorname{SCN}($ ㅁg $/ 1)$ & 3.1 & 3.1 & 3.1 & 3.1 & 3.1 & 3.1 & & & & \\
\hline 8005 -day $(\mathrm{mg} / 1)$ & 1.9 & 1.9 & 2.3 & 2.3 & 2.3 & 2.3 & 1.6 & 1.6 & 1.6 & 3.3 \\
\hline TOC (mg/1) & 45 & 45 & 47 & 47 & 47 & 47 & 51 & 51 & 51 & 53 \\
\hline Phenolics (ng/1) & 18.025 & 10.025 & 10.025 & 10.025 & 10.025 & 10.825 & & & & \\
\hline $\mathrm{PO4-D}(\operatorname{mg} / 1)$ & 34.6 & 34.6 & 23.3 & 23.3 & 23.3 & 23.3 & 12.3 & 12.3 & 12.7 & 8.3 \\
\hline Org-N (mg/1) & 14.0 & 14.0 & 14.0 & (4. 1 & 14.8 & 14.8 & & & & \\
\hline $\mathrm{NO2}(\mathrm{mg} / 1)$ & 3.81 & 3.81 & 3.81 & 3.81 & 3.81 & 3.81 & & & & \\
\hline : 03 (mg/1) & 148 & 148 & i48 & 148 & 148 & $\vdots 48$ & & & & \\
\hline \multicolumn{11}{|l|}{$\operatorname{TDS}(\mathrm{mg} / 1)$} \\
\hline Color (APHA Units) & 875 & 875 & 875 & 875 & 875 & 875 & 875 & 875 & 875 & 875 \\
\hline \multicolumn{11}{|l|}{ BASIN PARAMETERS } \\
\hline Vol Wasted (nl) & 196 & 189 & 203 & 181 & 215 & 218 & 219 & 173 & 257 & 239 \\
\hline $0.0 .(\mathrm{mg} / \mathrm{l})$ & 8.4 & 9.4 & 9.2 & 8.9 & 9.7 & 9.5 & 9.5 & 9.3 & 9.2 & 9.2 \\
\hline SS (ag/1) & 1500 & 1290 & 1760 & 2830 & 2630 & 2500 & $2 \in 60$ & 2680 & 2890 & 1800 \\
\hline *SS (PAC corrected) & 999 & 789 & 1259 & 1529 & 2129 & 1999 & 2159 & 2180 & 2389 & 1299 \\
\hline Tegp $(C)$ & 18.5 & 18 & 18 & 18 & 17.5 & 17.5 & 18 & 18 & 18 & 18 \\
\hline ph (std units) & 7.6 & 7 & 6.7 & 6.9 & 7.8 & 8.3 & 8 & 7.9 & 7.1 & 6.7 \\
\hline OUR (mo/1/hr) & 0.25 & 0.25 & & & 5 & & & 3.6 & & \\
\hline VSS $(m g / 1)$ & 1120 & 1120 & 1120 & 1516 & 1950 & 1854 & 1972 & 2260 & 2221 & 1384 \\
\hline *USS (PAC corrected) & 619 & 619 & 619 & 1015 & 1449 & 1353 & 1472 & 1560 & 1721 & 883 \\
\hline VSS/SS & 0.75 & 0.75 & 0.75 & 0.75 & 0.74 & 0.74 & 0.74 & 0.77 & 0.77 & 0.77 \\
\hline FPAC Added (mg) & 143 & 143 & 143 & 143 & 143 & 143 & 143 & 143 & 143 & 143 \\
\hline Inventory (rgo/l basin) & 501 & 501 & 501 & 501 & 501 & 501 & 501 & 580 & 501 & 501 \\
\hline \multicolumn{11}{|l|}{ EFFLLLENT PARAMETERS } \\
\hline Volume (liters) & 3.44 & 3.5 & 3.5 & 3.45 & $\overline{3} . \overline{5}$ & 3.72 & 3.1 & 3.1 & 3.05 & 3.15 \\
\hline $\operatorname{COD}(\operatorname{mg} / 1)$ & 115 & 115 & 125 & 134 & 153 & 140 & 163 & 174 & 163 & 161 \\
\hline $5 S(m g / 1)$ & 44 & 36 & 42 & 62 & 54 & 46 & 58 & 98 & 28 & 28 \\
\hline \multicolumn{11}{|l|}{ \#PAC corrected } \\
\hline oH (std units) & 7.6 & 7.3 & 6.9 & 6.6 & 7.3 & 7.9 & 8.4 & 7.4 & 7.5 & 6.7 \\
\hline VSS $(m g / 1)$ & 28 & 28 & 28 & 28 & 44 & 44 & 44 & 44 & 44 & 44 \\
\hline \multicolumn{11}{|l|}{ *PAC corrected } \\
\hline NH3N (mg/1) & $\langle 4.0$ & 4.7 & 4.7 & 4.7 & 4.7 & 16.1 & 16.1 & 16.1 & 13.5 & 13.5 \\
\hline CN (mg/1) & 1.14 & 1.14 & 1.14 & 1.14 & 1.14 & & & & & \\
\hline SCN (mg/1) & 2.3 & 2.3 & 2.3 & 2.3 & 2.3 & & & & & \\
\hline NO2 (mg/l) & 2.2 & 2.2 & 2.2 & 2.2 & 2.2 & & & & . & $?$ \\
\hline N03 (mg/1) & 200 & 200 & 200 & 208 & 200 & & & & & \\
\hline Phenolics (ag/1) & 10.025 & 10.825 & 10.025 & 10.025 & 10.025 & & & & & \\
\hline$P 04-P$ ( $6 g / 1)$ & 12.2 & 24.6 & 24.6 & 24.6 & 24.6 & 11.4 & 11.4 & 11.4 & 17.7 & 17.7 \\
\hline $\begin{array}{l}\text { Org-N }(m g / 1) \\
\text { iDS }(m g / 1)\end{array}$ & 14.8 & 14.0 & $<4.0$ & 14.8 & 14.8 & & . & & & \\
\hline Color (APHA units) & 625 & 625 & 625 & 625 & 625 & 1000 & 1008 & $: 880$ & 1000 & 1080 \\
\hline TOC $(\operatorname{mg} / 1)$ & 33 & 37 & 37 & 37 & 37 & 40 & 40 & 48 & 44 & 44 \\
\hline BO0 5-diay (mg/ $/ 1)$ & 1.1 & 2.1 & 2.1 & 2.1 & 2.1 & 2.8 & 2.8 & 2.8 & 3.8 & $3: 0$ \\
\hline
\end{tabular}


IJNIT $2 B$ TABLE A4

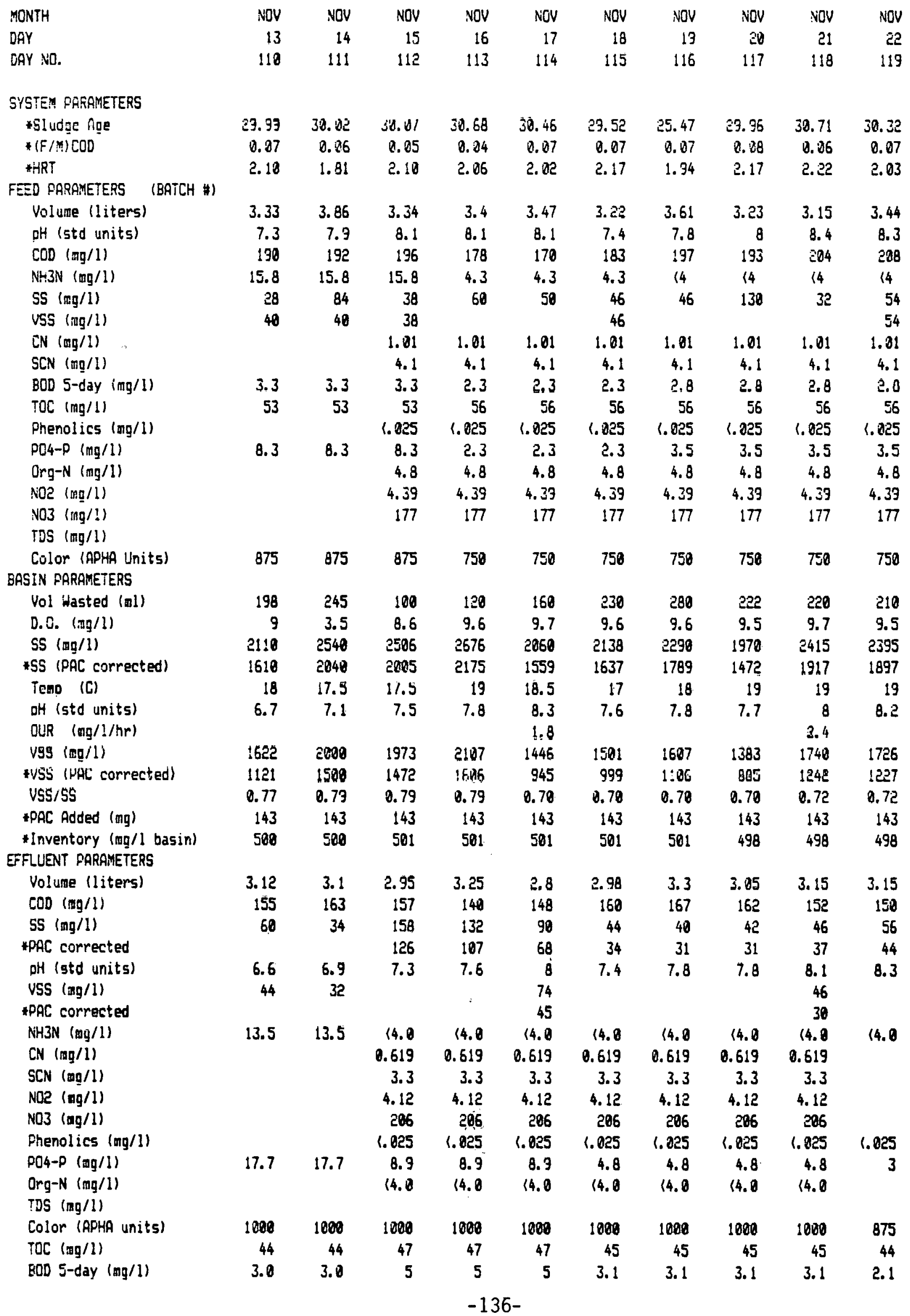




\begin{tabular}{|c|c|c|c|c|c|c|c|c|c|c|}
\hline MONTH & NOV & NOV & NOV & NOV & NOV & NOV & VOV & NOV & $D E C$ & $D E C$ \\
\hline DAY & 23 & 24 & 25 & 26 & 27 & 28 & 29 & 30 & 1 & 2 \\
\hline DAY NO. & 120 & 121 & 122 & 123 & 124 & 125 & 126 & 127 & 138 & 129 \\
\hline \multicolumn{11}{|l|}{ SYSTEYY PARAMETERS } \\
\hline *Sludge Gque & 30.27 & 30.15 & 29.92 & 30.24 & 21.71 & 29.95 & 38.00 & 30.03 & 29.35 & 23.98 \\
\hline$+(F / M) C C D$ & 0.83 & 0.86 & 0.08 & 0.07 & 0.89 & 0.85 & 0.03 & 8.86 & 0.05 & 0.85 \\
\hline *HRT & 3.95 & 1.97 & 1.95 & 2.23 & 1.93 & 2.11 & 1.99 & 1.98 & 1.79 & 2.00 \\
\hline \multicolumn{11}{|l|}{ FEED FARAMETERS (BATCH \#) } \\
\hline Voluwe (1iters) & 1.92 & 3.55 & 3.59 & 3.14 & 3. 63 & 3.32 & 3.52 & 3.53 & 3.92 & 3.5 \\
\hline DH (std units) & 8.1 & 7.7 & 7.8 & 7.7 & 7.6 & 7.8 & 8 & 8 & 7.9 & 7.8 \\
\hline $\operatorname{COD}(\operatorname{mo} / 1)$ & 188 & 176 & 187 & ¿85 & 168 & 153 & 157 & $\$ 46$ & 142 & 143 \\
\hline 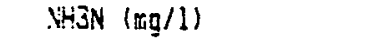 & 14 & 14 & $\ll 4$ & 14 & $\ll$ & 14 & 14 & 14 & 14 & $\ll 4$ \\
\hline S5 (mg/1) & 46 & 50 & 108 & 574 & 294 & 188 & 108 & 144 & 92 & \\
\hline VSS (mg/1) & & 44 & & & & & 58 & & & \\
\hline CN (mgg/1) & & & & & & & 1.33 & 1.33 & 1.33 & 1.33 \\
\hline $\operatorname{SCN}($ mg $/ 1$ ) & & & & & & & 2.8 & 2.8 & 2.8 & 2.8 \\
\hline 800 5-day (mg/l) & 1.6 & 1.6 & 1.6 & 12 & 12 & 12 & 12 & 1.5 & 1.5 & 1.5 \\
\hline TOC (mg/1) & 53 & 53 & .53 & 42 & 42 & 42 & 42 & 38 & 38 & 38 \\
\hline Phenolics (mg/l) & 4.825 & 1.025 & 1.825 & 1.025 & 1.025 & 1.825 & 1.025 & 1.025 & 1.025 & 1.025 \\
\hline P04-P (mgn/1) & 4.4 & 4.4 & 4.4 & 8.4 & 8.4 & 8.4 & 8.4 & 5.4 & 5.4 & 5.4 \\
\hline $\operatorname{Drg} \underline{\mathrm{N}}-\mathrm{N}(\mathrm{mg} / \mathrm{l})$ & & & & & & & 14 & $<4$ & 14 & $<4$ \\
\hline NO2 (mo/l) & & & & & & & 2 & 2 & 2 & \\
\hline NO3 (ng / $/ 1)$ & & & & & & & 178 & 170 & 178 & 170 \\
\hline TDS (mqg/1) & & & & & & & & 4660 & 4660 & 4650 \\
\hline Color (APHA Units) & 750 & 750 & 750 & 758 & 750 & 750 & 750 & 750 & 750 & 750 \\
\hline \multicolumn{11}{|l|}{ BASIN PARAMETERS } \\
\hline Yol Wasted (m!) & 250 & 230 & 220 & 65 & 58 & 165 & 197 & 148 & 68 & 184 \\
\hline D.0. (mg/l) & 9.2 & 9.2 & 9.2 & 9.4 & 9.1 & 9 & 9 & 9.9 & 8.8 & 8.8 \\
\hline SS $(m g / 1)$ & 2380 & 2550 & 2895 & 2088 & 1878 & 2252 & 3682 & 1932 & 2564 & 2472 \\
\hline *SS (PRC corrected) & 1881 & 2851 & 1596 & 1589 & 1379 & 1759 & 3189 & 1439 & 2071 & 1978 \\
\hline Tead (C) & 19 & .19 & 20 & 18 & 20 & 20 & 20 & 19 & 19 & 18 \\
\hline pH (std units) & 8.2 & 8 & 7.7 & 7.7 & 7.8 & 7.6 & 8 & 8.1 & 8 & 0 \\
\hline QUR (ng/l/hr) & & & & & & 1.2 & & & 1.2 & \\
\hline VSS img/1) & 1675 & 1795 & 1474 & 1469 & 1322 & 1674 & 2737 & 1436 & 1796 & 1732 \\
\hline \&VSS (PAC corrected) & 1176 & 1296 & 976 & 971 & 823 & 1181 & 2244 & 943 & 1303 & 1238 \\
\hline VSS/SS & 0.78 & 0.70 & 0.70 & 8.70 & 0.70 & 0.74 & 0.74 & 0.74 & 0.70 & 0.78 \\
\hline ¥PAC Added (mg) & 143 & 143 & 143 & 143 & 143 & 143 & 143 & 143 & 143 & 143 \\
\hline Inventory (ng/l basin) & 499 & 499 & 499 & 499 & 499 & 493 & 493 & 493 & 493 & 494 \\
\hline \multicolumn{11}{|l|}{ FFFI LIENT PARAMETERS } \\
\hline Volume (liters) & 1.45 & 3.2 & 3.72 & 3 & 3.25 & 3.125 & 3.3 & 3.26 & $3 . \ddot{2}$ & 3.1 \\
\hline $\operatorname{coD}(\pi g / 1)$ & 151 & 149 & 153 & 151 & 149 & 151 & 132 & 125 & 132 & 126 \\
\hline SS (触 $/ 1$ ) & 56 & 44 & 38 & 154 & 200 & 88 & 100 & 82 & 182 & 82 \\
\hline *PAC corrected & 44 & 35 & 29 & 117 & 147 & 69 & 87 & 61 & 147 & 66 \\
\hline $\mathrm{OH}$ (std units) & 8.3 & 8 & 7.9 & 7.6 & 7.6 & 7.8 & 7.8 & 7.9 & 8 & 8 \\
\hline VSS (mg/l) & 42 & & & & & 66 & & & 92 & \\
\hline tPAC corrected & 26 & & & & & 44 & & & 58 & \\
\hline$N H 3 N($ mg/1) & 14.8 & $\ll 4.0$ & 14.8 & 44.8 & $\langle 4.0$ & 14.8 & $(4.0$ & $\langle 4.8$ & $(4.8$ & $(4.8$ \\
\hline CN (mg/l) & & & & & & & 0.965 & 0.905 & 0.985 & 0.985 \\
\hline $\operatorname{SCN}(\operatorname{mg} / 1)$ & & & & & & & 2.4 & 2.4 & 8.4 & 2.4 \\
\hline NO2 (mg/1) & & & & & & & 0.53 & 0.53 & 0.53 & 0.53 \\
\hline $\mathrm{N} 03$ (mg/l) & & & & & & & 178 & 178 & 178 & 178 \\
\hline Phenolics (mg/1) & 4.025 & 1.025 & 1.025 & 1.025 & 1.825 & (.025 & 1.025 & 1.025 & 1.025 & 1.025 \\
\hline$P 04-P(\operatorname{mg} / 1)$ & 3 & 3 & 9,4 & 9.4 & 9.4 & 9.4 & 7.1 & 7.1 & 7.1 & 5.5 \\
\hline $\operatorname{Org}-\mathrm{N}(\mathrm{mg} / \mathrm{l})$ & & & & & & & 14.8 & 14.8 & 14.8 & 14.0 \\
\hline TDS (mg/1) & & & & & & & 4970 & 4970 & 4970 & 4970 \\
\hline Color (APHA units) & 875 & 875 & 875 & 875 & 875 & 875 & 625 & 625 & 625 & 625 \\
\hline TOC (mg/1) & 44 & 44 & 42 & 42 & 42 & 42 & 36 & 36 & 36 & 35 \\
\hline 80D 5-day $(\mathrm{mg} / 11)$ & 2.1 & 2.1 & 13 & $\langle 3$ & 13 & 13 & 1.8 & 1.8 & 1.8 & 12 \\
\hline
\end{tabular}




\begin{tabular}{|c|c|c|c|c|c|c|c|c|c|c|}
\hline MONTH & DEC & DEC & DEC & DEC & DEC & DEC & $D E C$ & DEC & DEC & DEC \\
\hline DAY & 3 & 4 & 5 & 6 & 7 & 8 & 9 & 10 & 11 & 12 \\
\hline DAY NO. & 130 & 131 & 132 & 133 & 134 & 135 & 136 & 137 & 138 & 139 \\
\hline \multicolumn{11}{|l|}{ SYSTEM FARAMETERS } \\
\hline tSludge Age & 89.96 & 39.95 & 29.97 & 33.03 & $39.8 E$ & 30.04 & 30.04 & 89.70 & 29.38 & 29.99 \\
\hline$\#(F / M) C O D$ & 0.05 & 0.04 & 0.87 & 0.06 & 0.07 & 0.88 & 0.05 & 0.35 & 0.07 & 0.05 \\
\hline \#HRT & 1.92 & 1.32 & 1.71 & 2.03 & 1.84 & 1.94 & 1.97 & 1.99 & 2.85 & 2.86 \\
\hline \multicolumn{11}{|l|}{ FEED PARAMETERS (BATCH-\#) } \\
\hline Volume (1 iters) & 3.65 & 3.64 & 4.1 & 3.45 & 3.8 & 3.6 & 3.55 & 3.7 & 3.42 & 3.4 \\
\hline pH (std units) & 7.6 & 7.9 & 7.8 & 8.1 & 8 & 7.9 & 7.3 & 7.9 & 7.8 & 7.7 \\
\hline $\operatorname{COD}(\mathrm{mg} / \mathrm{l})$ & 137 & 154 & 157 & 155 & 146 & 144 & 155 & 161 & 156 & 154 \\
\hline NHEN (mg/1) & 14 & 14 & 14 & 14 & 12 & 12 & 12 & 12 & 12 & 12 \\
\hline SS (mo/l) & 64 & 36 & 138 & 46 & 54 & 38 & 130 & 78 & 60 & 52 \\
\hline VSS $(\operatorname{mg} / 1)$ & & & & 34 & 24 & & 78 & & & \\
\hline CN $(m g / 1)$ & 1.33 & 1.33 & 1.33 & 1.33 & & & & & & \\
\hline $\operatorname{SCN}(\mathrm{mg} / 1)$ & 2.8 & 2.8 & 2.8 & 2.8 & & & & & & \\
\hline 800 5-day (mg/1) & 12 & 12 & 12 & (2 & 2 & 2 & 2 & 3 & 2 & 2 \\
\hline TOC $(\mathrm{mg} / \mathrm{l})$ & 43 & 43 & 43 & 43 & 45 & 45 & 45 & 41 & 41 & 41 \\
\hline Phenolics (ng/l) & 1.025 & 1.825 & 1.025 & 1.025 & 1.025 & 1.025 & 1.025 & 1.025 & 1.825 & 1.025 \\
\hline PO4-P (mg/1) & 8.4 & 8.4 & 6.4 & 8.4 & 8.8 & 8.8 & 8.8 & 6.5 & 6.5 & 6.5 \\
\hline $\operatorname{Org}-\mathrm{N}(\mathrm{mg} / 1)$ & 14 & $<4$ & 14 & 14 & & & & & & \\
\hline $\mathrm{NO2}(\mathrm{mg} / \mathrm{l})$ & 2 & 2 & 2 & 2 & & & & & & \\
\hline N03 $(\mathrm{ng} / 1)$ & 170 & 170 & 170 & 170 & & & & & & \\
\hline TDS $(3 y / 1)$ & 4660 & 4660 & 4660 & 4666 & 4605 & 4605 & 4605 & 4605 & 4605 & 4605 \\
\hline Color (APHA Units) & 758 & 750 & 750 & 750 & 750 & 750 & 750 & 750 & 750 & 750 \\
\hline \multicolumn{11}{|l|}{ BASIN PARAMETERS } \\
\hline Vol Wasted (al) & 137 & 197 & 164 & 133 & 108 & 3 & 134 & 170 & $\vdots 61$ & 215 \\
\hline 0.0. $(\mathrm{mg} / 1)$ & 9 & 3 & 8.8 & 8.8 & 9.2 & 9 & 9.1 & 3 & 8.8 & 8.8 \\
\hline SS $(\operatorname{mg} / 1)$ & 2456 & 3034 & 2164 & 2136 & 2010 & 1762 & 2518 & 2772 & 1872 & 2614 \\
\hline ÆSS (PAC corrected) & 1962 & 2540 & 1670 & 1642 & 1516 & 1267 & 2823 & 2277 & 1377 & 2119 \\
\hline Temp (C) & 19 & 19 & 17 & 13 & 18.5 & 17 & 18 & 18 & 18 & 18 \\
\hline oh (std units) & 8.2 & 7.9 & $B$ & 8.2 & 8.4 & 6.9 & 7.6 & 7.7 & 7.9 & 8 \\
\hline OUR (mg/1/hr) & & & & 1.2 & & & & & & 1.2 \\
\hline VSS $(\mathrm{mg} / 1)$ & 1720 & 2123 & 1514 & 1452 & 1366 & 1256 & $\$ 795$ & 1976 & 1334 & 1688 \\
\hline IUSS (PAC corrected) & 1227 & 1631 & 1020 & 958 & 872 & 761 & 1300 & 1481 & 840 & 1193 \\
\hline USS/SS & 0.70 & 0.70 & 0.70 & 0.68 & 0.68 & 0.71 & 0.71 & 0.71 & 0.71 & 0.65 \\
\hline «PAC Added (mg) & 143 & 143 & 143 & 143 & 143 & 143 & 143 & 143 & 143 & 143 \\
\hline Inventory (og/l basin) & 494 & 494 & 494 & 494 & 494 & 495 & 495 & 495 & 495 & 495 \\
\hline \multicolumn{11}{|l|}{ EFFLUENT PARAMETERS } \\
\hline Volume (liters) & 3.35 & 3.6 & 3.5 & 2.6 & 3.5 & 3.2 & 3.25 & 3.25 & 3.1 & 3.35 \\
\hline $\cos (\pi g / 1)$ & 121 & Iटl & 132 & 130 & 128 & 132 & 147 & 135 & 129 & 133 \\
\hline SS $(m g / 1)$ & 110 & 76 & 76 & 126 & 106 & 156 & 118 & 102 & 75 & 56 \\
\hline \#PAC corrected & 88 & 64 & 59 & 97 & 88 & 112 & 95 & 84 & 56 & 45 \\
\hline oH (std units) & 8.1 & 7.9 & 7.9 & 8.1 & 8 & 7.4 & 7.2 & 7.4 & 7.9 & 8 \\
\hline USS (mg/l) & & & 56 & 68 & & 106 & & & & 44 \\
\hline por. corrected & & & 34 & 40 & & & & & & 25 \\
\hline NH3N (agg/l) & 14.8 & 14.8 & 14.0 & 12 & (2) & (2 & 12 & 12 & (2) & 12 \\
\hline$C N(m g / 1)$ & 0.985 & 0.385 & 0.905 & & & & & & & \\
\hline $\mathrm{SCN}(\mathrm{mg} / \mathrm{l})$ & 2.4 & 2.4 & 2.4 & & & & & & & \\
\hline NDe $(a g / 1)$ & 0.53 & 0.53 & 0.53 & & & & & & & \\
\hline $\mathrm{NO3}(\mathrm{ag} / 1)$ & 178 & 178 & 178 & & & & & & & \\
\hline Phenolics $(\mathrm{mg} / \mathrm{l})$ & 1.025 & 1.025 & l. 025 & 1.025 & ४.025 & 1.025 & 1.025 & ८.025 & 1.025 & 1.025 \\
\hline$P O 4-P(m g / 1)$ & 5.5 & 5.5 & 5.5 & 8.2 & 8.2 & 8.2 & 9.5 & 9.5 & 9.5 & 9.5 \\
\hline $\operatorname{Org} g-N(m g / 1)$ & 14.8 & 14.8 & 14.0 & & & & & & & \\
\hline $\operatorname{TDS}(\operatorname{mg} / 1)$ & 4970 & 4970 & 4978 & 4898. & 4898 & 4890 & 4890 & 4898 & 4890 & 4890 \\
\hline Color (APHA units) & 625 & 625 & 625 & 750 & 750 & 750 & 750 & 750 & 758 & 750 \\
\hline TOC $(\Phi q / 1)$ & 35 & 35 & 35 & 38 & 38 & 38 & 40 & 40 & 48 & 40 \\
\hline BOD 5-day (ag/1) & 12 & 12 & 12 & 4 & 4 & 4 & 2.4 & 2.4 & 2.4 & 2.4 \\
\hline & & & & -13 & & & & & & \\
\hline
\end{tabular}




\begin{tabular}{|c|c|c|c|c|c|c|c|c|c|c|}
\hline MONTH & DEC & DEC & $D E C$ & DEC & DEC & DEC & DEC & DEC & DEC & DEC \\
\hline DAY & 13 & 14 & 15 & 16 & 17 & 18 & 19 & 20 & 21 & 22 \\
\hline DAY NO. & 140 & 141 & 142 & 143 & 144 & 145 & 146 & 147 & 148 & 149 \\
\hline \multicolumn{11}{|l|}{ SYSTEM PARAMETERS } \\
\hline *Sludghe Age & 30.00 & 25.81 & 25.84 & 24.98 & 24.99 & 25.84 & 25.98 & 25.00 & 25.80 & 25.02 \\
\hline$\triangle(F / M) C O D$ & 0.25 & 0.87 & 0.05 & 0.06 & 0.10 & 0.32 & 9.86 & 0.18 & .0 .69 & 0.10 \\
\hline HiRT & 1.32 & 1.34 & 2.85 & 1.88 & 1.75 & 1.30 & 2.89 & 1.91 & 1.87 & 1.77 \\
\hline \multicolumn{11}{|l|}{ FEED PARAMETERS (BATCH \#) } \\
\hline Volune (liters) & 3.64 & 3.61 & 3.42 & 3.73 & 4 & 3.69 & 3.35 & 3.67 & 3.75 & 3.96 \\
\hline aH (std units) & 7.7 & 7.6 & 7.6 & 7.4 & 8 & 8 & 8 & 8.1 & 7.9 & 7.8 \\
\hline[] $0(\mathrm{mg} / \mathrm{ll})$ & 153 & 158 & 158 & 150 & 224 & 161 & 163 & 163 & 171 & 148 \\
\hline NH:SN (mg/1) & 12 & 4.8 & 4.8 & 4.8 & 27.4 & 27.4 & 27.4 & 27.4 & 47.1 & 47.1 \\
\hline SS $(a g / 1)$ & 32 & 44 & 440 & 72 & 48 & 6 & 54 & 50 & 46 & 38 \\
\hline VSS (mg/l) & 22 & 0 & 383 & 42 & 28 & 4 & 32 & 26 & 24 & 16 \\
\hline CN $(a g / 1)$ & 0 & 0.814 & 0.814 & 0.814 & 0.814 & 0.814 & 0.814 & 0.814 & $\theta$ & \\
\hline $\operatorname{SCN}(\operatorname{mg} / 1)$ & 8 & 3.7 & 3.7 & 3.7 & 3.7 & 3.7 & 3.7 & 3.7 & 0 & \\
\hline 800 5-day $(\mathrm{mg} / \mathrm{l})$ & 2 & 2.2 & 2.2 & 2.2 & 2 & 2 & 2 & 2 & 4 & \\
\hline TOC (ng/1) & 41 & 46 & 46 & 46 & 46 & 46 & 46 & 46 & 46 & \\
\hline Phenolics (ag/l) & 1.825 & 1.825 & 1.025 & 1.025 & 1.025 & 1.025 & 1.025 & 1.025 & <.825 & 1.025 \\
\hline$P C 4-P$ (mg/1) & 6.5 & 8.3 & 8.3 & B.3 & 8.7 & 8.7 & 8.7 & 8.7 & 0 & \\
\hline \multicolumn{11}{|l|}{$\operatorname{Org}-\mathrm{N}(\mathrm{mg} / \mathrm{l})$} \\
\hline No2 (iag/1) & 0 & 0.56 & 8.66 & 0.86 & 0.65 & 0. 66 & 0.65 & 0.66 & 0 & \\
\hline $\mathrm{NO3}$ (ag/1) & 8 & 125 & 126 & 126 & 126 & 126 & 126 & 126 & 8 & \\
\hline TDS img/1) & 4685 & 4495 & 4495 & 4495 & 4495 & 4495 & 4495 & 4495 & $\theta$ & \\
\hline Color (APHA Units) & 750 & 875 & 875 & 875 & 875 & 875 & 875 & 875 & 875 & 875 \\
\hline \multicolumn{11}{|l|}{ BASIN PARAMETERS } \\
\hline Vol Wasted (ml) & 196 & 292 & 153 & 269 & 140 & 156 & 288 & $\lfloor 88$ & 224 & 218 \\
\hline D.D. $(\operatorname{mg} / 1)$ & 9 & 9 & 9 & 8.8 & 8.8 & 9 & 9 & 3 & 8.7 & 8.8 \\
\hline SS $(n g / 1)$ & 2808 & 2194 & 2508 & 2358 & 2462 & 1098 & 2410 & 1846 & 2040 & 1742 \\
\hline *SS (PAC corrected) & 2313 & 1699 & 2116 & 1869 & 1976 & 615 & 1929 & 1368 & $\$ 564$ & 1269 \\
\hline Temo (C) & 19 & 19 & 19 & 19 & 18 & 17 & 18 & 18 & 18 & 18 \\
\hline pH (std units) & 7.8 & 8 & 7.8 & 7.7 & 7.6 & 7.6 & 7.9 & 7.9 & 7.7 & 7.8 \\
\hline OUR (mg/l/hr) & & & & & & & 2.4 & & & \\
\hline VSS (mg/1) & 1813 & 1417 & $\$ 666$ & 1506 & 1573 & 701 & 1562 & 1196 & 1322 & 1156 \\
\hline *USS (PAC corrected) & 1318 & 922 & 1174 & 1017 & 1087 & 218 & 1081 & 718 & 847 & 683 \\
\hline USs/SS & 0.65 & 0.65 & 0.64 & 8.64 & 0.64 & 0.64 & 0.65 & 0.65 & 0.65 & 0.66 \\
\hline †PAC Added (ng) & 143 & 143 & 143 & 143 & 143 & 143 & 143 & 143 & 143 & 143 \\
\hline IInventory (mg/l basin) & 495 & 495 & 492 & 489 & 486 & 483 & 481 & 478 & 476 & 473 \\
\hline \multicolumn{11}{|l|}{ EFFLUENT PARAMETERS } \\
\hline Volume (liters) & 3.35 & 3.35 & 3.45 & 3.55 & 3.75 & 3.32 & 3.15 & 3.35 & 3.4 & 3.65 \\
\hline $\operatorname{cod}(\mathrm{mg} / \mathrm{l})$ & 137 & 141 & 133 & 140 & 133 & 140 & 138 & 137 & 142 & 153 \\
\hline SS $(m g / 1)$ & 76 & 34 & 144 & 58 & 134 & 62 & 104 & 86 & 72 & 6 \\
\hline APAC corrected & 63 & & & & & & & & & \\
\hline QH (std units) & 8 & 8 & 7.9 & 7.8 & 7.8 & 7.7 & 7.9 & 8.1 & 7.9 & 7.6 \\
\hline USS $(m g / 1)$ & & 27 & 86 & 30 & 90 & 37 & 60 & 50 & 42 & \\
\hline \multicolumn{11}{|l|}{ APAC corrected } \\
\hline NH3N (mg/1) & 12 & $\ll 2$ & 《2 & 2.8 & 2.8 & 2.8 & 2.8 & 13.4 & 13.4 & 13. \\
\hline CN (mg/l) & 3.17 & 3.17 & 3.17 & 3.17 & 3.17 & 3.17 & 3.17 & & & \\
\hline $\operatorname{SCN}(\mathrm{mg} / 1)$ & 2.8 & 2.8 & 2.8 & 2.8 & 2.8 & 2.8 & 2.8 & & & \\
\hline NO2 (mg/h) & 1.5 & 1.5 & 1.5 & 1.5 & 1.5 & 1.5 & 1.5 & & & \\
\hline $\mathrm{NO3}(\mathrm{mg} / 1)$ & 163 & 163 & 163 & 163 & 163 & 163 & 163 & & & \\
\hline Phenolics (ag/l) & 1.025 & 1.025 & 1.825 & 1.825 & 1.025 & 1.825 & (.825 & (.025 & 1.025 & (.825 \\
\hline$P O 4-P(a g / 1)$ & 6.5 & 6.5 & 6.5 & 7.7 & 7.7 & 7.7 & 7.7 & & & \\
\hline \multicolumn{11}{|l|}{$O r g-N(\operatorname{sg} / 1)$} \\
\hline TDS $(m g / 1)$ & 4730 & 4730 & 4730 & $4730^{\circ}$ & 4730 & 4730 & 4730 & & & \\
\hline Color (APHA units) & 875 & 875 & 875 & 875 & 875 & 875 & 875 & 875 & 875 & 875 \\
\hline $\operatorname{TDC}(m q / 1)$ & 40 & 40 & 40 & 42 & 42 & 42 & 42 & 40 & 40 & 40 \\
\hline DOD 5 day $(\mathrm{mg} / \mathrm{l})$ & 2.3 & 2.3 & 2.3 & le & 12 & 12 & 12 & 2.5 & 2.5 & 8.5 \\
\hline
\end{tabular}




\begin{tabular}{|c|c|c|c|c|c|c|c|c|c|c|}
\hline MONTH & DEC & DEC & DEC & DEC & DEC & DEC & DEC & DEC & DEC & JAN \\
\hline DAY & 23 & 24 & 25 & 26 & 27 & 28 & 29 & 30 & 31 & 1 \\
\hline DAY NO. & 150 & 151 & 152 & 153 & 154 & 155 & 156 & 157 & 158 & 159 \\
\hline \multicolumn{11}{|l|}{ SYSTEM ORRAMETERS } \\
\hline *Sludge Age & 34.98 & 14.65 & 24.99 & 25.03 & 24.98 & 35.01 & $24.9 \overline{8}$ & 24.97 & 24.97 & 25.03 \\
\hline$*(F / A) C O D$ & 0.09 & -0.36 & 0.05 & 0.32 & 0.01 & 2.03 & 0.07 & 0.11 & 0.08 & 0.87 \\
\hline$\forall H R T$ & 1.92 & 1.72 & 1.81 & 1.89 & 2.10 & 1.87 & 1.98 & 1.88 & 1.85 & 1.53 \\
\hline \multicolumn{11}{|l|}{ FEED FRRAMETERS (BATCH \#) } \\
\hline Volume (liters) & 3.64 & 4.28 & 3.87 & 3.7 & 3.33 & 3.75 & 3.73 & 3.73 & 3.79 & 4.3 \\
\hline oH (std units) & 7.8 & 7.7 & 7.4 & 7.6 & 7.5 & 8 & 7.9 & 7.7 & 7.8 & 7.9 \\
\hline COD（g $/ \mathrm{i})$ & 168 & 159 & 150 & 157 & 167 & 165 & 189 & 189 & 180 & 136 \\
\hline $\mathrm{NH} 3 \mathrm{~N}(\mathrm{mg} / 1)$ & 47.1 & 48.8 & 48.8 & 48.8 & 48.8 & 101 & 101 & 101 & 190 & 190 \\
\hline SS $(\mathrm{mg} / 1)$ & 36 & 44 & 26 & 42 & 82 & 160 & 130 & 62 & 116 & 62 \\
\hline VSS $(m g / 1)$ & 26 & 32 & 11 & 30 & 59 & 128 & 98 & 40 & 88 & 47 \\
\hline CN $(a g / 1)$ & 0 & 0 & 0 & 0 & 0 & 0.544 & 0.544 & 0.544 & 0.544 & 0.544 \\
\hline $\operatorname{SCN}(a g / 1)$ & 0 & 0 & 0 & 0 & 0 & 3.2 & 3.2 & 3.2 & 3.2 & 3.2 \\
\hline BOD 5-day (mg/1) & 4 & 12 & 《2 & 12 & 12 & <2 & 《2 & 12 & 12 & <2 \\
\hline TOC $(\mathrm{ng} / 1)$ & 46 & 43 & 43 & 43 & 43 & 46 & 46 & 46 & 38 & 38 \\
\hline Phenolics (mg/1) & <.025 & 1.825 & 1.025 & 1.825 & 1.225 & 1.025 & 1.025 & (. . 225 & 1.025 & <.225 \\
\hline$P 04-P(m g / 1)$ & 0 & 0 & 0 & 0 & 13.2 & 10.2 & 10.2 & 10.2 & 10 & 10 \\
\hline Org-N $(\mathrm{mg} / 1)$ & & & & & 0 & 5 & $\mathbf{5}$ & 5 & 5 & 5 \\
\hline NOe (mg/l) & 0 & 8 & 0 & 0 & 8 & 0.62 & 0.62 & 0.62 & 0.62 & 0.62 \\
\hline $\mathrm{N03}$ (ng/1) & 0 & 8 & 0 & 0 & 0 & 90.7 & 90.7 & 30.7 & 90.7 & 98.7 \\
\hline TDS $(\mathrm{mg} / 1)$ & 0 & 0 & 0 & 0 & 4510 & 4375 & 4375 & 4375 & 4375 & 4375 \\
\hline Color (APHA Units) & 875 & 875 & 875 & 875 & 875 & 1000 & 1080 & 1080 & 1000 & 1080 \\
\hline \multicolumn{11}{|l|}{ BASIN PARAMETERS } \\
\hline Vol Hasted (al) & 261 & 261 & 298 & 199 & 338 & 273 & 192 & 174 & 174 & 189 \\
\hline D.0. (mg/1) & 9 & 9 & 9 & 8.8 & 8.8 & 3 & 3.8 & 1.2 & 1.2 & 1.2 \\
\hline $55(\mathrm{mg} / 1)$ & 1878 & 458 & 2544 & 1002 & 14020 & 4574 & 1840 & 1376 & 1525 & 1720 \\
\hline *SS (PAC corrected) & 1407 & -19 & 2091 & 550 & 13570 & 4125 & 1392 & 930 & $1: 90$ & 1276 \\
\hline Temp (C) & 19 & 17 & 17 & 17 & 17 & $2 \dot{4}$ & 20 & 20 & 20 & 28 \\
\hline pH (std units) & 7.7 & 7.55 & 7.5 & 7.3 & 7.3 & 7 & 7 & 7 & 6.7 & 6.3 \\
\hline QUR (mg/l/hr) & & & & 6 & & & 6 & & & \\
\hline VSS (mg/1) & 1246 & 256 & 16AR & F. 5.5 & 7980 & 2603 & 1508 & 1128 & 1332 & 1410 \\
\hline +USS (PAC corrected) & 775 & -212 & 1235 & 213 & 7724 & 2348 & 1141 & 762 & 967 & 1046 \\
\hline VSS/SS & 0.66 & 0.57 & 0.66 & 0.66 & 0.57 & 0.57 & 0.82 & 0.82 & 0.82 & 0.82 \\
\hline tPAC Added (ag) & 143 & 143 & 143 & 143 & 143 & 143 & 143 & 143 & 143 & 143 \\
\hline Inventory (mg/l basin) & 471 & 469 & 453 & 452 & 458 & 449 & 448 & 446 & 445 & 444 \\
\hline \multicolumn{11}{|l|}{ EFFLUENT PARAMETERS } \\
\hline Volume (liters) & 3.4 & 3.67 & 3.45 & 3.45 & 2.94 & 3.45 & 3.4 & 3.45 & 3.55 & 3.8 \\
\hline COD $\{(9 \mathrm{~g} / \mathrm{l})$ & 161 & 148 & 142 & 140 & 153 & $: 65$ & 165 & 161 & 142 & 136 \\
\hline SS (mg/l) & 46 & 40 & 34 & 42 & 38 & 94 & 68 & 78 & 70 & 70 \\
\hline IPAC corrected & & & & & 29 & 85 & 51 & 53 & 51 & $\$ 2$ \\
\hline of (std units) & 7.6 & 7.5 & 7.4 & 7.2 & 7.3 & 6.9 & 7 & 7 & 7 & 6.5 \\
\hline VSS (ng/1) & 37 & 35 & 27 & 34 & 26 & 81 & 58 & 56 & 64 & 57 \\
\hline *PAC corrected & & & & & 15 & 46 & 48 & 46 & b' & 41 \\
\hline NHJ̈N (mg/1) & 14.8 & 14.8 & 14.8 & 14.8 & 21 & 21 & 21 & 69.3 & 69.3 & 69.3 \\
\hline$C N(\mathrm{gg} / 1)$ & & & & & 1.22 & 1.22 & 1.22 & 1.22 & 1.22 & 1.22 \\
\hline $\operatorname{SCN}(m g / 1)$ & & & & & 3.4 & 3.4 & 3.4 & 3.4 & 3.4 & 3.4 \\
\hline No2 (ng/1) & & & & & 0.46 & 0.46 & 0.46 & 0.46 & 0.46 & 0.46 \\
\hline $\mathrm{NO3}(\mathrm{mg} / 1)$ & & & & & 58 & 58 & 58 & 58 & 58 & 58 \\
\hline Phenolics $(\mathrm{ng} / 1)$ & $<.025$ & ২.025 & 1.025 & 1.025 & 1.025 & 1.825 & 1.025 & 1.025 & 1.025 & 1.025 \\
\hline$P 04-P(m g / 1)$ & & & & 12.0 & 21.4 & 21.4 & 21.4 & 44.5 & 44.5 & 44.5 \\
\hline $\operatorname{Org}-\mathrm{N}(\operatorname{mg} / 1)$ & & & & & 14 & 14 & 14 & 14 & 14 & 14 \\
\hline TDS $(\Delta g / 1)$ & & & & 4778 & 4805 & 4805 & 4885 & 4805 & 4885 & 4885 \\
\hline Color (APHA units) & 875 & 875 & 975 & 875 & 1000 & 1000 & 1000 & 1000 & 1080 & 1000 \\
\hline TOC $(m g / 1)$ & 43 & 43 & 43 & 43 & 44 & 44 & 44 & 48 & 40 & 40 \\
\hline BOD 5-day (mg/l) & 2 & 2 & 2 & 2 & 3 & 3 & 3 & 12 & 12 & 12 \\
\hline
\end{tabular}


INIT ZB TARLE A4

\begin{tabular}{|c|c|c|c|c|c|c|c|c|c|c|}
\hline MONTH & JAN & JAN & JAN & JAN & JAN & JAN & JAN & JAN & JAN & JAN \\
\hline DAY & 2 & 3 & 4 & 5 & 6 & 7 & $\theta$ & 9 & 10 & 11 \\
\hline DAY NO. & 168 & 161 & 162 & 163 & 164 & 165 & 166 & 167 & 168 & 169 \\
\hline \multicolumn{11}{|l|}{ SYSTEM PGRAMETERS } \\
\hline *Sludạe Age & 25.02 & 25.20 & 17.76 & 18.40 & 19.81 & 14.43 & 25.20 & 20.68 & 25.01 & 33.88 \\
\hline$*(F / M) C O D$ & 0.10 & 0.85 & 0.21 & 0.20 & 0.19 & 0.11 & 0.89 & 0.18 & 8.88 & 0.83 \\
\hline *HRT & 1.87 & 2. 12 & 1.77 & 1.79 & 2.33 & 1.82 & 1.84 & 1.89 & 1.89 & 1.89 \\
\hline \multicolumn{11}{|l|}{ FEED PAFGMETERS (BATCH \#) } \\
\hline Voluwe (liters) & 3.75 & 3.3 & 3.96 & 3.91 & 3 & 3.85 & 3.8 & 3.7 & 3.7 & 3.7 \\
\hline pH (std units) & 8.2 & 8.1 & 8.3 & 8.2 & 8.1 & 8.1 & 8 & 7.7 & 7.6 & 7.7 \\
\hline$C O D(-\operatorname{qg} / 1)$ & 140 & 142 & $15 !$ & 188 & 150 & 129 & 162 & 158 & 106 & 71 \\
\hline$N H 3 N(\operatorname{mg} / \mathrm{l})$ & 190 & 190 & 202 & 202 & 202 & 211 & 211 & 211 & 211 & 210 \\
\hline SS (mg/l) & 78 & 176 & 174 & 224 & 332 & 124 & 78 & 54 & 62 & 24 \\
\hline VSS (rag/1) & 59 & 134 & 134 & 172 & 250 & 93 & 59 & 41 & 56 & 15 \\
\hline CN (ag/l) & 0.544 & 8.544 & 0 & 0 & 0 & 0 & 0 & 0 & 0 & 0.521 \\
\hline SCN (nqg/1) & 3.2 & 3.2 & 0 & $\theta$ & 0 & 0 & 0 & 0 & $\theta$ & 1.3 \\
\hline BOD 5-day (mg/l) & 12 & $\ll 2$ & 12 & 12 & 12 & 12 & 12 & 12 & 12 & 12 \\
\hline $\operatorname{TOC}(\operatorname{mg} / \mathrm{ll})$ & 38 & 38 & 40 & 40 & 40 & 27 & 27 & 27 & 27 & 18 \\
\hline Phenolics (mg/l) & 1.025 & 1.825 & <.825 & 1.025 & 1.025 & 1. 825 & 1. .225 & 1.825 & 1.025 & <. 825 \\
\hline PO4-P $(m g / 1)$ & 10 & 10 & 4.1 & 4.1 & 4.1 & 7.9 & 7.9 & 7.9 & 7.9 & 7.4 \\
\hline Org-N (mg/l) & 5 & 5 & 0 & 0 & 8 & 0 & 0 & 0 & 0 & 76 \\
\hline NOD $(m g / i)$ & 0.62 & 0.62 & 8 & 0 & 8 & 0 & 0 & 8 & 10.25 & 10.25 \\
\hline $\mathrm{N} 03(\mathrm{mg} / \mathrm{l})$ & 90.7 & 30.7 & 0 & 0 & 0 & 8 & 0 & 0 & 11.25 & 11.25 \\
\hline TDS (mag/i) & 4375 & 4375 & 5072 & 5072 & 5072 & 5072 & 5072 & 5072 & 5072 & 5280 \\
\hline Color (APHA Units) & 1000 & 1000 & 750 & 750 & 750 & 750 & 758 & 750 & 750 & 250 \\
\hline \multicolumn{11}{|l|}{ BASIN PARAMETERS } \\
\hline Vol Wasted (al) & 140 & 288 & 50 & 58 & 58 & 28 & 84 & 58 & 94 & 50 \\
\hline D.0. $(\mathrm{mg} / \mathrm{l})$ & 1.2 & 3.2 & 3 & 3.2 & 3.2 & 3.2 & 3.2 & 2.9 & 6.8 & 6.9 \\
\hline SS (mg/l) & 1185 & 1946 & 920 & 962 & 764 & 1100 & 1420 & 692 & 1108 & 1702 \\
\hline HSS (PAC corrected) & 742 & 1504 & 487 & 535 & 343 & 692 & 1012 & 487 & 702 & .1291 \\
\hline Temo (C) & 20 & 20 & 20 & 19 & 21 & 19 & 18 & 18 & 20 & 20 \\
\hline $\mathrm{OH}$ (std units) & 7.1 & 7.9 & 7.3 & 7.8 & 7.8 & 7.8 & 7.5 & 7.5 & 7.1 & 7.1 \\
\hline Uี่ก $(\mathrm{mg} / 1 / \mathrm{hr})$ & & 6 & & 6 & & & & 3 & & \\
\hline VSS (mg/h) & 971 & 1346 & 636 & 758 & 602 & 867 & 1113 & 696 & 865 & 1328 \\
\hline IVSS (PAC corrected) & 688 & 1041 & 337 & 422 & 271 & 545 & 797 & $380^{\circ}$ & 548 & 1008 \\
\hline USS/SS & 0.82 & 0.69 & 0.69 & 0.79 & 0.79 & 0.79 & 0.79 & 0.78 & 0.78 & 0.78 \\
\hline EPAC Added (mg) & 143 & 143 & 143 & 143 & 143 & 143 & 143 & 143 & 143 & 143 \\
\hline Inventory (ag/l basin) & 443 & 442 & 433 & 427 & 421 & 408 & 488 & 405 & 486 & 411 \\
\hline \multicolumn{11}{|l|}{ EFFLUENT PARAMETERS } \\
\hline Volume (liters) & 3.55 & 3.15 & 3.7 & 3.65 & 2.9 & 3.6 & 3.55 & 3.4 & 3.55 & 3.55 \\
\hline $\operatorname{COD}($ fig/l) & 132 & 138 & 145 & 143 & 154 & 139 & 152 & 186 & 93 & 82 \\
\hline SS $(\mathrm{mg} / \mathrm{l})$ & 68 & 84 & 108 & 110 & 106 & 176 & 184 & 96 & 78 & 98 \\
\hline *PAC corrected & 43 & 65 & 57 & 61 & 48 & 111 & 74 & 52 & 49 & 74 \\
\hline ọH (std units) & 6.9 & 7.3 & 7.9 & 7.7 & 7.8 & 7.9 & 7.7 & 7.5 & 7.3 & 7.1 \\
\hline USS $(m g / 1)$ & 56 & 60 & 77 & 70 & 68 & 120 & 71 & 84 & 68 & 85 \\
\hline PPAC corrected & 46 & 42 & 53 & 55 & 54 & 95 & 56 & 66 & 53 & 66 \\
\hline NHZ̈N (mg/1) & 69.3 & 147 & 147 & 147 & 188 & 188 & 188 & 188 & 201 & 201 \\
\hline CN $(* g / 1)$ & 1.22 & & & & & & & & 0.787 & 0.787 \\
\hline $\operatorname{SCN}(\mathbb{m g} / 1)$ & 3.4 & & & & & & & & 1.6 & 1.6 \\
\hline NO2 (mg/1) & 0.46 & & & & & & & & 0.49 & 8.49 \\
\hline $\mathrm{NO3}(\mathrm{mg} / \mathrm{l})$ & 58 & & & & & & & & 21 & 21 \\
\hline Phenolics (mg/l) & 1.825 & 1.025 & 1.025 & 1.025 & 1.025 & 1.025 & 1.025 & 1.825 & 1.025 & 1.825 \\
\hline P04-D (ag/1) & 44.5 & 16 & 16 & 16 & 15 & 15 & 15 & 15 & 16 & 16 \\
\hline$O \mathrm{rg}-\mathrm{N}(\mathrm{mg} / \mathrm{l})$ & $\ll 4$ & & & & & & & & $(4$ & \\
\hline TDS $(m g / 1)$ & 4805 & 4946 & 4946 & 4946 & 4946 & 4946 & 4946 & 4946 & 5814 & 5814 \\
\hline Color (APHA units) & 1080 & 875 & 875 & 875 & 875 & 875 & 875 & 875 & 350 & 350 \\
\hline TOC $(\mathrm{mg} / \mathrm{l})$ & 40 & 37 & 37 & 37 & 29 & 29 & 29 & 29 & 24 & 24 \\
\hline BOD 5-day (mg/1) & 12 & $\ll 2$ & 12 & $\ll 2$ & 12 & i2 & 12 & $<2$ & «? & 12 \\
\hline & & & & & & & & & & \\
\hline
\end{tabular}




\begin{tabular}{|c|c|c|c|c|c|c|c|c|c|c|}
\hline FONTH & JAN & JAN & JAN & JAN & JAN & JAN & JAN & JAN & JAN & JAN \\
\hline DAY & 12 & 13 & 14 & 15 & 16 & 17 & 18 & 19 & 20 & 21 \\
\hline DAY ND. & 170 & 171 & 172 & 173 & 174 & 175 & 176 & 177 & 178 & 179 \\
\hline \multicolumn{11}{|l|}{ SYSTEM PARAXETERS } \\
\hline $\begin{array}{l}\text { *SIudge Age } \\
\text { (F/M)COD } \\
\text { FHRT }\end{array}$ & $\begin{array}{r}17.82 \\
0.87 \\
1.84\end{array}$ & $\begin{array}{r}50.78 \\
8.82 \\
1.59\end{array}$ & $\begin{array}{l}51.76 \\
0.05 \\
1.74\end{array}$ & $\begin{array}{l}3 y .86 \\
0.07 \\
1.74\end{array}$ & $\begin{array}{r}24.158 \\
0.08 \\
1.77\end{array}$ & $\begin{array}{l}25.00 \\
0.05 \\
1.77\end{array}$ & $\begin{array}{r}24.99 \\
0.05 \\
1.87\end{array}$ & $\begin{array}{l}24.97 \\
0.26 \\
1.79\end{array}$ & $\begin{array}{r}25.80 \\
0.05 \\
1.79\end{array}$ & $\begin{array}{r}11.81 \\
-0.44 \\
1.75\end{array}$ \\
\hline \multicolumn{11}{|l|}{ FEED PARAMETERS (BATCH ) } \\
\hline Volume (liters) & 3.8 & 4.4 & 4.03 & 4.03 & 3.95 & 3.95 & 3.75 & 3.92 & 3.91 & \\
\hline pH (std units) & 8 & 8.1 & 8.1 & 8 & 7.7 & 7.9 & 7.3 & 7.9 & 7.8 & \\
\hline $\operatorname{COD}(m g / 1)$ & 105 & 91 & 142 & 146 & 149 & 121 & 98 & 106 & 104 & 108 \\
\hline NH3N $(m g / 1)$ & 210 & 218 & 201 & 201 & 201 & 201 & 197 & 197 & 197 & 184 \\
\hline SS $(m g / 1)$ & 20 & 22 & 16 & 12 & 78 & 24 & 10 & 36 & 60 & 40 \\
\hline VSS (ng/1) & 13 & 14 & 10 & 8 & 45 & 15 & 6 & 23 & 41 & 27 \\
\hline CN (ag/l) & 0.521 & 0.521 & 0.521 & 0.521 & 0.521 & 0.521 & 0 & 0 & 0 & 0 \\
\hline $\mathrm{SCN}$ (ag/l) & 1.3 & 1.3 & 1.3 & 1.3 & 1.3 & 1.3 & 0 & 0 & 0 & 0 \\
\hline BOD 5-day (mg/1) & (2 & «2 & $\ll 2$ & $(2$ & 12 & $\{2$ & 12 & 12 & 12 & \2 \\
\hline $\operatorname{TOC}(\operatorname{mg} / \mathrm{l})$ & IR & 18 & 16 & 16 & 16 & 16 & 17 & 17 & 17 & 22 \\
\hline Phenolics (agg/1) & <.825 & 1.025 & 1.025 & 1.825 & 1.025 & 1.025 & 4.025 & <.825 & 1.825 & 1.025 \\
\hline PO4-p (ng/1) & 7.4 & 7.4 & 4.5 & 4.5 & 4.5 & 4.5 & 5 & 5 & 5 & 6.7 \\
\hline $\operatorname{Org}-\mathrm{N}($ agg/l) & 76 & 76 & 76 & 76 & 76 & 76 & 8 & 0 & 0 & 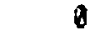 \\
\hline NO2 (mg/1) & <0.25 & (8.25 & 18.25 & 10.25 & 10.25 & D & 0 & 0 & 0 & 0 \\
\hline $\mathrm{N03}$ (ag/1) & 11.25 & 11.25 & 11.25 & 11.25 & 11.25 & 0 & 0 & 0 & 0 & 0 \\
\hline TDS (ag/l) & $\operatorname{Seg0}$ & 5280 & 5288 & 5280 & 5280 & 5280 & 4925 & 4925 & 4925 & 4225 \\
\hline Color (APHA Units) & 250 & 250 & 250 & 250 & 250 & 250 & 250 & 250 & 250 & 250 \\
\hline \multicolumn{11}{|l|}{ BASIN PARRMETERS } \\
\hline Vol Hasted (nl) & 50 & 224 & 28 & 50 & 116 & 294 & 207 & 169 & 215 & 28 \\
\hline D.0. (ng/l) & 6.4 & 6.8 & 6.4 & 6.4 & 7.9 & 7.5 & 7.5 & 7.4 & 7.2 & 7.2 \\
\hline SS $(m g / 1)$ & 1280 & 2999 & 2278 & 1696 & 1736 & 2158 & 1760 & 1706 & 1954 & 236 \\
\hline ISS (PAC corrected) & 876 & 2577 & 1848 & 1268 & 1309 & 1731 & 1334 & 1280 & 1529 & -170 \\
\hline reato (C) & 80 & 20 & 19.5 & 19 & 18 & P.? & 18 & 19 & 19 & 18 \\
\hline pH (std units) & 7.3 & 7.4 & 7.2 & 7.6 & 7.6 & 7.8 & 7.7 & 7.7 & 7.8 & 7.2 \\
\hline OUR $(\mathrm{mg} / 1 / \mathrm{hr})$ & & & & & 2 & & & 2 & & \\
\hline VSS (mp / 1$)$ & 999 & 2333 & 1771 & 1323 & 1100 & 1467 & 1196 & $\$ 134$ & 1299 & 1.57 \\
\hline *YSS (PAC corrected) & 683 & 2011 & 1442 & 990 & 890 & 1177 & 307 & $85 !$ & 1016 & -113 \\
\hline VSS/SS & 0.78 & 0.78 & 0.78 & 0.78 & 0.68 & 0.68 & 0.68 & 0.66 & 0.66 & $0: 66$ \\
\hline tPAC Added (mg) & 143 & 143 & 143 & 143 & 143 & 143 & 143 & 143 & 143 & 143 \\
\hline tinventory (og/l basin) & 404 & 413 & 422 & 428 & 427 & 427 & 426 & 426 & 425 & 406 \\
\hline \multicolumn{11}{|l|}{ EFFLUENT PARAMETERS } \\
\hline Volume (liters) & 3.55 & 3.65 & 3.95 & 3.8 & 3.67 & 3.6 & 3.55 & 3.65 & 3.6 & 3.8 \\
\hline $\operatorname{cod}(\mathrm{mg} / 1)$ & 89 & 127 & 136 & 102 & 123 & 100 & 120 & 97 & 108 & 116 \\
\hline SS (mg/1) & 96 & 98 & 84 & 74 & 108 & 39 & 58 & 82 & 70 & \\
\hline *PAC corrected & 66 & 84 & 68 & 55 & 81 & 24 & 52 & 62 & 55 & $-3 \hat{c}$ \\
\hline pH (std units) & 7.1 & 7.6 & 7.3 & 7.7 & 7.6 & 7.6 & 7.6 & 7.7 & 7.8 & 7. \\
\hline USS (mg/1) & 82 & 85 & 72 & 68 & 74 & 20 & 46 & 55 & 47 & $x$ \\
\hline SPAC corrected & 64 & 66 & .56 & 53 & 50 & 14 & 31 & 37 & $3 t$ & 1 \\
\hline $\begin{array}{l}\text { NH3N (ag/1) } \\
\text { CN }(m / 1)\end{array}$ & $\begin{array}{r}201 \\
787\end{array}$ & $\begin{array}{r}192 \\
0787\end{array}$ & $\begin{array}{r}132 \\
0.787\end{array}$ & $\begin{array}{r}192 \\
0.787\end{array}$ & $\begin{array}{r}192 \\
9787\end{array}$ & 183 & 183 & 183 & 172 & 176 \\
\hline CN (mg/1) & 0.787 & 0.787 & 0.787 & 0.787 & 0.787 & & & & & \\
\hline $\begin{array}{l}\text { SCN }(m g / 1) \\
\text { NO2 }(m g / 1) \\
\text { NO3 }(m g / 1)\end{array}$ & $\begin{array}{r}1.6 \\
0.49 \\
21\end{array}$ & $\begin{array}{r}1.6 \\
0.49 \\
21\end{array}$ & $\begin{array}{r}1.6 \\
0.49 \\
21\end{array}$ & $\begin{array}{r}1.6 \\
0.49 \\
21\end{array}$ & $\begin{array}{r}1.6 \\
0.49 \\
21\end{array}$ & & & & & \\
\hline Phenolics (ng/1) & (.025 & 6.825 & 1.025 & 1.025 & 1.825 & 1.025 & 1.025 & 1.025 & 1.825 & 1.025 \\
\hline $\begin{array}{l}\mathrm{PO4}-\mathrm{P}(\mathrm{mg} / \mathrm{l}) \\
\text { Org-N (mg/1) }\end{array}$ & $\begin{array}{l}16 \\
14\end{array}$ & $\begin{array}{r}9.76 \\
14\end{array}$ & $\begin{array}{r}9.76 \\
.44\end{array}$ & $\begin{array}{r}9.76 \\
14\end{array}$ & $\begin{array}{r}9.76 \\
14\end{array}$ & 6.2 & 6.2 & 6.2 & 11.7 & 11.7 \\
\hline $\operatorname{TDS}(m g / 1)$ & 5814 & 5814 & 5814 & 5814 & 5814 & 4585 & 4585 & 4585 & 4585 & 4585 \\
\hline Color (APHA units) & 350 & 350 & 358 & 350 & 358 & 300 & 380 & 300 & 388 & 30 \\
\hline $\begin{array}{l}\text { TOC (mg/l) } \\
\text { BOD } 5-d a y \text { (mg/l) }\end{array}$ & $\begin{array}{l}24 \\
12\end{array}$ & $\begin{array}{l}18 \\
12\end{array}$ & 18 & $\frac{18}{12}$ & 18 & $\begin{array}{l}16 \\
12\end{array}$ & $\begin{array}{l}16 \\
12\end{array}$ & 16 & 16 & 1 \\
\hline & & & & & & & IE & & 6 & \\
\hline
\end{tabular}




\begin{tabular}{|c|c|c|c|c|c|c|c|c|c|c|}
\hline MONTH & JAN & JAN & JAN & JAN & JAN & JAN & JAN & JAN & JAN & JAN \\
\hline DAY & 22 & 23 & 24 & 25 & 26 & 27 & 28 & 29 & 30 & 31 \\
\hline DAY NO. & 180 & 181 & 192 & 183 & 184 & 185 & 186 & 197 & 188 & 189 \\
\hline \multicolumn{11}{|l|}{ SYSTEY PARAMETERS } \\
\hline *Sludg̣e Aọe & 24.96 & 24.36 & 24.99 & 24.99 & 24.96 & 25.82 & 24.98 & 25.83 & 25.82 & 24.98 \\
\hline *(5/M)COD & 0.89 & 0.11 & 0.05 & 0.25 & 0.86 & 0.06 & 0.10 & 0.09 & 0.08 & 0.10 \\
\hline FHT & 1.76 & 1.32 & 1.89 & 1.79 & 1.77 & 1.84 & 1.75 & 1.75 & 1.83 & 1.75 \\
\hline \multicolumn{11}{|l|}{ FEED PARAMETERS (BATCH \#) } \\
\hline Voluse (liters) & 3.38 & 3.65 & 3.7 & 3.9 & 3.35 & 3.8 & 4 & 4 & 3.83 & 4 \\
\hline ph (std units) & 8 & 7.8 & 7.9 & 7.3 & 7.8 & 7.9 & 8 & 8 & 8 & $\dot{\theta}$ \\
\hline $\operatorname{COD}(\mathrm{mg} / 1)$ & 110 & 127 & 116 & 95 & 111 & 107 & 117 & 189 & 121 & 127 \\
\hline NH3N ( $(\mathrm{gg} / 1)$ & 184 & 184 & 184 & 153 & 153 & 153 & 170 & 170 & 178 & 178 \\
\hline SS $(m g / 1)$ & 6 & 26 & 124 & 184 & 86 & 130 & 122 & 58 & 20 & 16 \\
\hline VSS (mg/1) & 4 & 18 & 79 & 66 & 55 & 80 & 75 & 36 & 12 & 10 \\
\hline CN (mag/h) & 0 & 0 & 0 & 0.4 & 0.4 & 0.4 & 0.4 & 0.4 & 0.4 & 0.4 \\
\hline $\operatorname{SCN}(\mathrm{mg} / 1)$ & 0 & 0 & 0 & 2.2 & 2.2 & 2.2 & 2.2 & 2.2 & 2.2 & 2.2 \\
\hline BOD 5 -day $(\mathrm{mg} / \mathrm{l})$ & «2 & 12 & 12 & 12 & 12 & 12 & 12 & 12 & 12 & 12 \\
\hline $\operatorname{TOC}(\operatorname{tag} / 1)$ & 22 & 22 & 22 & 26 & 26 & 26 & 30 & 30 & 30 & 50 \\
\hline Phenolics (mg/l) & 1.825 & 1.025 & 1.825 & 1.025 & 1.025 & 1.025 & 1.825 & 1.025 & 1.025 & 1.025 \\
\hline F04-P (mg/1) & 6.7 & 6.7 & 6.7 & 7.1 & 7.1 & 7.1 & 8.4 & 8.4 & 8.4 & 8.4 \\
\hline $\operatorname{Org}-\mathrm{N}(\mathrm{mg} / \mathrm{l})$ & 0 & 8 & 0 & 120 & 120 & 128 & 120 & 128 & 120 & 120 \\
\hline$: 02(\pi g ̣ / 1)$ & 8 & 0 & $\theta$ & 10.25 & 10.25 & 10.25 & (0. 25 & 10.25 & 18. 25 & 10.25 \\
\hline :N93 (mo/1) & 0 & $\theta$ & 0 & 7.9 & 7.9 & 7.9 & 7.9 & 7.9 & 7.9 & 7.9 \\
\hline TDS (tog/l) & 4925 & 4925 & 4325 & 5774 & 5774 & 5774 & 5774 & 5774 & 5774 & 5774 \\
\hline Color (APHA Units) & 250 & 250 & 258 & 500 & 508 & 580 & 580 & 500 & 500 & 500 \\
\hline \multicolumn{11}{|l|}{ BASIN PARAYETERS } \\
\hline Yol Hasted (ml) & 216 & 222 & 227 & 275 & 273 & 255 & 74 & 247 & 243 & 219 \\
\hline $0.0 . \quad(\mathrm{mg} / 1)$ & 7.2 & 7.1 & 6.9 & 7 & 6.2 & 6.5 & 6.5 & 6.3 & 6.3 & 6.2 \\
\hline SS $(m g / 1)$ & ¿2225 & 1894 & 1844 & 1606 & 1782 & 1578 & 1252 & 1320 & 1430 & 1264 \\
\hline $\begin{array}{l}\text { *SS (PAC corrected) } \\
\text { Temo (C) }\end{array}$ & $\begin{array}{r}819 \\
18\end{array}$ & $\begin{array}{r}687 \\
19\end{array}$ & $\begin{array}{r}1437 \\
18\end{array}$ & $\begin{array}{r}1199 \\
19\end{array}$ & $\begin{array}{r}1374 \\
21\end{array}$ & $\begin{array}{r}1170 \\
20\end{array}$ & $\begin{array}{r}844 \\
20\end{array}$ & $\begin{array}{r}911 \\
20\end{array}$ & $\begin{array}{r}1021 \\
20\end{array}$ & $\begin{array}{r}855 \\
28\end{array}$ \\
\hline $\mathrm{pH}$ (std units) & 7.75 & 7.7 & 7.6 & 7.6 & 7.2 & 7 & 7.1 & 7.2 & 6.9 & 7.2 \\
\hline OUR (mg/1/hr) & & 2 & & & 2 & & & & & \\
\hline VSS (mg/l) & 814 & 784 & 1321 & 1151 & 1148 & 1009 & 801 & 844 & 960 & 849 \\
\hline *USS (PAC corrected) & 544 & 493 & 1830 & 859 & 879 & 748 & 540 & 583 & 685 & 574 \\
\hline VSS/SS & 0.66 & 0.72 & 0.72 & 0.72 & 0.64 & 0.64 & 0.64 & 8.64 & 0.67 & 0.67 \\
\hline APAC Added (ag) & 143 & 143 & 143 & 143 & 143 & 143 & 143 & 143 & 143 & 143 \\
\hline IInventory (mgl/ basin) & 486 & 407 & 487 & 407 & 408 & 488 & 408 & 409 & 409 & 409 \\
\hline \multicolumn{11}{|l|}{ EFFLUENT ORRAMETERS } \\
\hline Volume (liters) & 3.72 & 3.35 & 3.6 & 3.7 & 3.75 & 3.5 & 3.6 & 3.75 & 3.6 & 3.6 \\
\hline $\operatorname{coD}(\operatorname{mg} / 1)$ & 99 & 96 & 80 & 89 & 76 & 86 & 36 & 102 & 100 & 188 \\
\hline SS $(\pi g / 1)$ & 42 & 40 & 60 & 30 & 34 & 40 & 94 & 34 & 40 & 44 \\
\hline \#PAC corrected & 28 & 25 & 47 & 22 & 26 & 30 & 63 & 23 & 29 . & 30 \\
\hline $\mathrm{OH}$ (std units) & 7.85 & 7.6 & 7.5 & 7.3 & 7 & 6.9 & 7.5 & 6.7 & 6.6 & 6.7 \\
\hline VSS $(\operatorname{tog} / 1)$ & 28 & 29 & 43 & 21 & 22 & 26 & 60 & 22 & 27 & 30 \\
\hline FAC corrected & 19 & 21 & 31 & 15 & 14 & 16 & 38 & 14 & 18 & 20 \\
\hline NH3N (ng/1) & 172 & 172 & 153 & 153 & 153 & 123 & 123 & 123 & 123 & 119 \\
\hline CN $(m g / 1)$ & & & 0.486 & 0.486 & 0.486 & 0.486 & 0.486 & 0.486 & 0.486 & \\
\hline $\operatorname{SCN}(a g / 1)$ & & & 1.9 & 1.9 & 1.9 & 1.9 & 1.9 & 1.9 & 1.9 & . \\
\hline NO2 (mg/1) & & & 2.3 & 2.3 & 2.3 & 2.3 & 2.3 & 2.3 & 2.3 & \\
\hline $\mathrm{NOZ}(\operatorname{mg} / \mathrm{i})$ & & & 29.7 & 29.7 & 29.7 & 29.7 & 29.7 & 29.7 & 29.7 & \\
\hline Phenolics (mg/l) & 1.025 & 1.025 & 1.825 & 6.025 & 1.825 & 1.025 & 1.025 & 1.825 & 1.025 & 4.025 \\
\hline $\mathrm{PO4}-\mathrm{P}(\mathrm{mg} / 1)$ & $11.7^{\circ}$ & 11.7 & 7.5 & 7.5 & 7.5 & 9.8 & 9.8 & 9.8 & 9.8 & 10.8 \\
\hline Drg-N $(m g / 1)$ & & & 60 & 68 & 60 & 60 & 60 & 60 & 68 & \\
\hline TDS (mg/l) & 4585 & 4585 & 5184 & 5184 & 5184 & 5184 & 5184 & 5184 & 5184 & \\
\hline Color (APHA units) & 300 & 300 & 350 & 358 & 350 & 350 & 350 & 358 & 350 & 600 \\
\hline TOC (mo/1) & 16 & 16 & 20 & 20 & 20 & 24 & 24 & 24 & 24 & 29 \\
\hline \multirow[t]{2}{*}{800 3-day $(m g / 1)$} & 6 & 6 & 1 & 4 & 4 & 12 & 12 & 12 & 12 & 12.7 \\
\hline & & & & -143 & & & & & & \\
\hline
\end{tabular}


UNIT 28 TABLE A4

\begin{tabular}{|c|c|c|c|c|c|c|c|c|c|c|}
\hline BONTH & FEB & FEB & FEB & FEB & FEB & FEB & FEB & FEB & FEB & FEB \\
\hline DAY & 1 & 2 & 3 & 4 & 5 & 6 & 7 & 8 & 9 & 10 \\
\hline JAY NO]. & 190 & 191 & 192 & 193 & 194 & 135 & 196 & 197 & 138 & 199 \\
\hline \multicolumn{11}{|l|}{ SYSTEN PRRAYETERS } \\
\hline *Sludg̣e Ag̣e & 21.47 & 24.97 & 25.02 & 10.11 & 25.81 & 25.80 & 24.93 & 21.49 & 19.23 & 19.23 \\
\hline$*(F / M) C O D$ & 0.13 & 0.12 & 0.15 & 0.16 & 0.15 & 0.12 & 0.19 & 0.21 & 0.18 & 0.18 \\
\hline *HRT & 1.85 & 1.71 & 1.79 & 1.80 & 1.86 & 1.97 & 1.77 & 1.73 & 1.75 & 1.75 \\
\hline \multicolumn{11}{|l|}{ FEED PARAMETERS (BATCH \#) } \\
\hline Volume (liters) & 3.78 & 4.09 & 3.92 & 3.88 & 3.77 & 3.55 & 3.96 & 4.04 & 4 & 4 \\
\hline DH (std units) & 8.2 & 8.2 & 8.1 & 8.3 & 8.3 & 8.3 & 8.3 & 8.3 & 8. 4 & B.4 \\
\hline COD $(m g / 1)$ & 135 & 162 & 154 & 161 & 165 & 165 & 187 & 210 & 151 & 173 \\
\hline $\mathrm{NH} 3 \mathrm{~N}(\mathrm{mg} / 1)$ & 186 & 186 & 186 & 174 & 174 & 174 & 174 & 164 & 164 & 164 \\
\hline SS (mo/1) & 4 & 44 & 74 & 62 & 74 & 120 & 182 & 76 & 172 & 180 \\
\hline VSS (mg/1) & 2 & 27 & 44 & 37 & 44 & 71 & 60 & 45 & 101 & 96 \\
\hline$C N(\operatorname{mg} / 1)$ & 0 & 0 & $n$ & $a$ & 0 & 0 & 8 & 1.14 & 1,14 & 1.14 \\
\hline $\operatorname{SCN}$ (mg/1) & 0 & 8 & 0 & 0 & 0 & 0 & 0 & 3.2 & 3.2 & 3.2 \\
\hline BOD 5-day (mg/l) & 1.8 & 1.8 & 1.8 & 2 & 2 & 2 & 2 & 12 & 12 & 《2 \\
\hline $\operatorname{TOC}(\mathbf{n g} / \mathrm{i})$ & 32 & 32 & 32 & 34 & 34 & 34 & 34 & 39 & 39 & 39 \\
\hline Phenolics $(\mathrm{mg} / \mathrm{l})$ & 1.025 & 1.825 & 1.025 & 1.025 & <.825 & <.025 & <.025 & <.025 & 1.025 & 1.025 \\
\hline$P O 4=P(a n / 1)$ & 3 & 3 & 3 & c..5 & 2.5 & 2.5 & 2.5 & 2.2 & 2.2 & 2.2 \\
\hline $\operatorname{Org}-\mathrm{N}(\mathrm{mg} / 1)$ & 8 & 3 & 3 & 2.5 & 2.5 & 2.5 & 2.5 & 2.2 & 2.2 & 2.2 \\
\hline $\mathrm{NO} 2(\mathrm{mg} / \mathrm{l})$ & & & & & & & & 6.41 & 6.41 & 6.41 \\
\hline $\mathrm{NO3}$ (ng/1) & & & & & & & & 25.9 & 25.9 & 25.9 \\
\hline $\operatorname{TLS}(\mathrm{mg} / 1)$ & 0 & ร922 & 5822 & 5822 & 5822 & 5822 & 5822 & 4800 & 4880 & 4800 \\
\hline Color (APHA Units) & 750 & 750 & 750 & 750 & 750 & 750 & 758 & 1000 & 1080 & 1000 \\
\hline \multicolumn{11}{|l|}{ BASIN PARAMETERS } \\
\hline Vol Wasted (ml) & 58 & 259 & 12 & 58 & 114 & 250 & 116 & 50 & 58 & 50 \\
\hline $0.0 .(m 0 / 1)$ & 6.2 & 6.6 & 6.8 & 6.8 & 6.8 & 6.6 & 6.6 & 6.3 & 6.7 & 6.4 \\
\hline SS $(m g / 1)$ & 1098 & 1386 & 1160 & 1852 & 1096 & 1254 & 1052 & 1092 & 950 & 1098 \\
\hline *SS (PAC corrected) & 691 & 979 & 753 & 668 & 711 & 868 & 66.5 & 706 & 567 & 711 \\
\hline léà (U) & 20 & 20 & 20 & 20 & 20 & 20 & 20 & 20 & 20 & 19 \\
\hline pH (std units) & 6.7 & 6.7 & 6.8 & 6.5 & 6.6 & 6.7 & 6.9 & 7.1 & 7.3 & 7 \\
\hline OUR (mg/l/hr) & & 2 & & & & 2 & & & 2 & \\
\hline VSS $(m g / 1)$ & 737 & 928 & 777 & 704 & 734 & 834 & 700 & 726 & 670 & 670 \\
\hline *USS (PAC corrected) & 464 & 655 & 504 & 447 & 476 & 577 & 442 & 470 & 400 & 437 \\
\hline VSS/SS & 0.67 & 0.67 & 0.67 & 0.67 & 0.67 & 0.67 & 0.67 & 0.67 & 0.71 & 0.71 \\
\hline IPAC Added (mg) & 143 & 143 & 143 & 143 & 143 & 143 & 143 & 143 & 143 & 143 \\
\hline +lriventory (ma/l basin) & 407 & 407 & 407 & 384 & 385 & 386 & 387 & 386 & 383 & 379 \\
\hline \multicolumn{11}{|l|}{ EF:LLENT OARAMETERS } \\
\hline Vo!:ue (1'iters) & 3.5 & 3.7 & 3.7 & 3.9 & 3.6 & 3.1 & 3.75 & 3.75 & 3.7 & 3.7 \\
\hline $\operatorname{coD}(m 0 /\rfloor)$ & 155 & 111 & 159 & 150 & 156 & 166 & 173 & 131 & 159 & 170 \\
\hline SS $(\pi g / 1)$ & 110 & 32 & 104 & 216 & 70 & 38 & 64 & 102 & 182 & 44 \\
\hline +FEC =srrected & 69 & 23 & 67 & 137 & 45 & 26 & 40 & 66 & 61 & 29 \\
\hline :t (std ani:s) & 6.8 & 6 & 6.5 & 6.7 & 6.8 & 6.3 & 6.1 & 6.6 & 7.1 & 6.7 \\
\hline VSS $: \mathrm{mg} / 1\}$ & 74 & 21 & 70 & 145 & 47 & 25 & 43 & 68 & 72 & 72 \\
\hline *AC currocted & 50 & 14 & 47 & 97 & 31 & 17 & 28 & 45 & 51 & 44 \\
\hline NhīN (mg/1) & 119 & $\$ 19$ & 110 & 110 & 110 & 110 & 86 & 86 & 86 & 66.2 \\
\hline C. $(\mathrm{mg} / \mathrm{l})$ & & & & & & & 1.31 & 1.31 & 1.31 & 1.31 \\
\hline $\operatorname{SCN}(\mathrm{mg} / 1)$ & & & & & & & 3.1 & 3.1 & 3.1 & 3.1 \\
\hline :02 $(\mathrm{mg} / 1)$ & & & & & & & 22.3 & 22.3 & 22.3 & 22.3 \\
\hline N03 $(90 / 1)$ & & & & & & & 92.7 & 92.7 & 92.7 & 92.7 \\
\hline Chenolics (ms/l) & 1.825 & 1.025 & 1.025 & 1.025 & $<.025$ & 1.025 & 1.025 & 1.025 & 1.025 & 1.825 \\
\hline$P 04-P(\mathrm{mg} / 1)$ & 10.8 & 10.8 & 7.1 & 7.1 & 7.1 & 7.1 & 5.6 & 5.6 & 5.6 & 8 \\
\hline $\operatorname{Crg}-\mathrm{N}(\mathrm{mg} / \mathrm{l})$ & & & & & & & 14 & 14 & 14 & $<4$ \\
\hline TDS (TE/1) & 5164 & 5164 & 5164 & 5164 & $5 ! 64$ & 5164 & 7524 & 7524 & 7524 & 7524 \\
\hline Cojor (APHA units) & 608 & 500 & 600 & 600 & 680 & 628 & 625 & 625 & 625 & 625 \\
\hline TDC (ng/1) & 29 & 29 & 29 & 29 & 27 & 29 & 34 & 34 & 34 & 43 \\
\hline 800 5-day (mg/l) & $\{2.7$ & 12.7 & 12 & (2) & 12 & 12 & 12 & 12 & 12 & 3 \\
\hline & & & & & -144 & & & & & \\
\hline
\end{tabular}




\begin{tabular}{|c|c|c|c|c|c|c|c|c|c|c|}
\hline MONTH & 潧B & TEB & FEB & $F E B$ & $F \equiv B$ & FEB & FEB & $F E B$ & $F E B$ & $F \subseteq B$ \\
\hline OAY & 11 & 12 & 13 & 14 & 15 & 16 & 17 & 18 & 19 & 20 \\
\hline OAY NO. & 200 & 201 & 202 & 203 & 2014 & 205 & 206 & 207 & 208 & 203 \\
\hline \multicolumn{11}{|l|}{ SYGTEM FARRMETERS } \\
\hline \$Sludge 0.je & 2.81 & 14.14 & !3.5! & 15.18 & 9.35 & 14.84 & $2 ! .73$ & 12.82 & 8.81 & 25.20 \\
\hline$f(F /, Y) S B D$ & $0.2 !$ & 0.26 & 0.20 & 0.16 & 0.38 & 0.20 & 0.13 & 0.07 & 0.21 & 0.34 \\
\hline HHRT & 1.82 & 1.75 & 1.75 & 2.06 & 1.65 & 1.75 & 2.58 & 1.75 & 1.75 & 6.36 \\
\hline \multicolumn{11}{|l|}{ FEED FARAYE:ERS (EATCH \#) } \\
\hline Volume iliters) & 3.85 & 4 & 4 & 3.4 & 4.25 & 4 & 2.8 & 4 & 4 & 1.1 \\
\hline pH (ste urits) & 8.3 & 8.2 & 8 & 7.9 & 7.6 & 7.1 & 7.8 & 7.1 & 7.4 & 7 \\
\hline CoD $(a g / 1)$ & 228 & 218 & 222 & 214 & 203 & 209 & 201 & 139 & 197 & $18 \dot{4}$ \\
\hline NHSN (ne/!) & 158 & 158 & 158 & 158 & 107 & 187 & 107 & 11 & 11 & 11 \\
\hline SS $(m g / 1)$ & 212 & 148 & 410 & 734 & 290 & $: 66$ & 115 & 228 & 168 & 300 \\
\hline VSS $(\mathrm{mg} / !)$ & 112 & 73 & 219 & 410 & 162 & 93 & 73 & 144 & 106 & 190 \\
\hline CN $(m g / 1)$ & 1.14 & 1.14 & 1.14 & 1.14 & 0 & 0 & 0 & 0 & 0 & 0 \\
\hline SCN (nฐ $/ 1)$ & 3.2 & 3.2 & 3.2 & $3 . ?$ & 8 & 0 & 0 & 0 & 8 & 0 \\
\hline 590 5-cay $(m g / 1)$ & 3 & 3 & 3 & 3 & 2 & 2 & 2 & 2 & 2 & 2 \\
\hline isc $(\mathrm{mg} / 1)$ & 47 & 47 & 47 & 47 & 51 & 51 & $5 !$ & 55 & 55 & 55 \\
\hline Fhanolics $(\mathrm{mg} / 1)$ & 1. 2025 & 1.225 & 1.025 & 1.825 & 1.825 & 1.025 & <. 225 & 1.025 & 1.025 & 1.025 \\
\hline $\mathrm{PC} 4-\mathrm{P}(\mathrm{mg} / \mathrm{l})$ & 2.8 & 2.8 & 2.8 & 2.8 & 10.9 & 10.9 & 18.9 & 47.2 & 47.2 & 47.2 \\
\hline $\operatorname{Crc}-N(m o / 1)$ & 2.8 & 2.8 & 2.8 & 2.9 & 18.9 & 10.9 & 10.9 & 47.2 & 47.2 & 47.2 \\
\hline UD? $(m g / !)$ & 6.41 & 5.41 & 5.41 & 6.41 & & & & & & \\
\hline $912(\operatorname{mg} / !)$ & 25.9 & 25.9 & E5. 9 & 25.9 & & & & & & \\
\hline WS $(m g / \vdots)$ & 4880 & 4820 & 48280 & $4 \overline{800}$ & 5644 & 5644 & 5644 & 5644 & 5644 & 5644 \\
\hline Color (AFHA Units) & 1020 & 12000 & 1000 & $\vdots 000$ & 1200 & 1000 & 1000 & 1308 & 1820 & 1800 \\
\hline \multicolumn{11}{|l|}{ ERSIN PARGXETERS } \\
\hline Vo! Nasted (ml) & 117 & 50 & 50 & 50 & 50 & 50 & 50 & 50 & 50 & 83 \\
\hline D.0. (mg/1) & 6.7 & 6.7 & 6.4 & 6.4 & 6.3 & 6.4 & 6.4 & 6.4 & 6.4 & 6.6 \\
\hline $5 S(n g / \bar{l})$ & 1062 & 920 & 1116 & 1120 & 1062 & 1018 & 1266 & 314 & 318. & 1044 \\
\hline *SS (PAC corrected) & 681 & 550 & 756 & 768 & 731 & 692 & 739 & 597 & 621 & 742 \\
\hline Texpo $\quad(C)$ & 20 & 20 & 20 & 20 & 20 & 20 & 20 & 19 & 19 & 19 \\
\hline oid (std units) & 7 & 7.3 & 7.3 & 7.3 & 7 & 6.2 & 6.5 & 7 & 6.1 & 6.6 \\
\hline SIR (mg/1/hr) & & & 2 & & & 2 & & 2 & & \\
\hline VSS (ma/1) & 749 & 549 & 758 & $7 \in !$ & 721 & 720 & 754 & 2176 & 649 & 766 \\
\hline USS (PAC corrected) & 488 & 388 & 514 & 521 & 496 & 490 & 522 & $! 4 \mathrm{e} 2$ & 439 & 544 \\
\hline VSS/SS & 0.71 & 8.71 & 0.68 & 0.68 & 0.68 & 0.71 & 0.71 & 0.71 & 0.71 & 0.73 \\
\hline AFAC Aoded (mg) & 143 & 143 & 143 & 143 & 143 & 143 & 143 & 143 & 143 & 143 \\
\hline - lrivertory (mo/i basin) & 381 & 370 & 360 & 352 & 331 & 326 & 327 & 317 & 297 & 302 \\
\hline \multicolumn{11}{|l|}{ EFEL:JENT FARAYETERS } \\
\hline Volure ('iters) & 3.65 & 3.95 & 3.85 & 3 & 4. 89 & 3.89 & 2. 75 & 4.8 & 3.9 & 0.8 \\
\hline COD (mc/l) & 209 & 205 & 201 & $19 !$ & 166 & 184 & 188 & 184 & 160 & 166 \\
\hline $55(\operatorname{mg} / 1)$ & 66 & 130 & 170 & 194 & 228 & 148 & 134 & 160 & 218 & 184 \\
\hline *PAC corrected & 42 & 78 & 115 & 133 & 157 & 95 & 93 & 105 & 147 & 131 \\
\hline 0.4 (std units) & 6.1 & 7.4 & 7.2 & 7.2 & 6.9 & 6 & 6.4 & 7.2 & 6.5 & 5.8 \\
\hline VSS (mg/!) & 47 & 92 & 115 & 132 & 155 & 99 & 95 & 18 & 154 & 135 \\
\hline XFAC corrected & 33 & 65 & 78 & 89 & 105 & 78 & 67 & 42 & 109 & 99 \\
\hline :HHIN $(\mathrm{mg} / 1)$ & 66.2 & 66.2 & 66.2 & 65 & 65 & 65 & 59 & 59 & 59 & 59 \\
\hline CN $(m g / 1)$ & 1.31 & 1.31 & 1.31 & & & & & & & \\
\hline $3 C N(\operatorname{mo} / 1)$ & 3.1 & 3.1 & 3.1 & & & & & & & \\
\hline VDS (mg/l) & 22.3 & 22. 3 & 22.3 & & & & & & & \\
\hline Noj $(9 g / 1)$ & 92.7 & 92.7 & 92.7 & & & & & & & \\
\hline Thenolics (ng/l) & 1.025 & 1.025 & 1.825 & 1.025 & 1.025 & 1.025 & 1.825 & 1.025 & 1.825 & 1.025 \\
\hline$f: 04-P(x \underline{n} / 1)$ & 8 & 8 & 8 & 12.1 & 12.1 & 12.1 & 29.2 & 29.2 & 29.2 & 29.2 \\
\hline Srg-N (ning/l) & $<4$ & 14 & $<4$ & & & & & & & \\
\hline TuS $\operatorname{lng} / 1)$ & 7524 & 7524 & 7524 & 5980 & 5900 & 5300 & 5900 & 5900 & 5300 & 5980 \\
\hline Solor (APLA units) & 625 & 625 & 625 & 875 & 875 & 875 & 875 & 875 & 875 & 875 \\
\hline ac $(\operatorname{agg} / 1)$ & 43 & 43 & 43 & 49 & 49 & 49 & 51 & $5 !$ & $5 !$ & $5 !$ \\
\hline a.jo 5-riay (mp/l) & 3 & 3 & 3 & 《2 & 12 & 12 & 2 & 2 & 2 & 2 \\
\hline
\end{tabular}


rentid

sey :0.

EVETEM TARAYETERS

* Shurne tne

(FFICJD

that

EEED PRATARETESS (BATCH \#)

Vol cine (liters)

or? (ste :nits)

$\operatorname{cod}(m g / n)$

in: $3 \mathrm{~N}(\operatorname{mog} / \mathrm{h})$

Ss (ing/:)

USS (mo/l)

¿N (mọ/l)

SCN $(m g / 2)$

god 5-day (mg/!)

TOC !mg/l)

Theriolics (mg/l)

PC4-P (as/i)

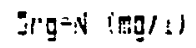

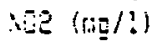

: $35(\mathrm{ag} / \mathrm{i})$

TJS (me/1)

Color (AFtA Units)

BAETN PARGYETERS

Vol Wastad (mI)

j.0. $(\mathrm{mg} / \mathrm{i})$

SS (mg/1)

*SS (PAAC corrected)

Tera (C)

g.4 (std units)

Cis ing/l/thi

iss (mo $/ 1$ )

* iSO (JAC corretted)

VSg/55

HipC Added (ng)

* Eiveritory (aro/l basin)

EF:LEVT MARAYETERS

Voi une (liters)

CCD (moger)

SS (mg/1)

*PAC corrected

of (std units)

VSS (soo/1)

*FAE currected

(iH 4 N (mg/1)

CN $(m g / 1)$

SCN (mo/l)

ㄴo $(\mathrm{mg} / \mathrm{ll})$

: 103 (zn/1)

Phenolics (mg/1)

Fo4-p (mg/l)

Drg-N (mg/l)

TLS (mg/l)

Coior (APHA units)

TCC (xo/1)

SCD 5-ciay (ing/1) $\begin{array}{rrrrrrrrrr}\text { FEB } & \text { FEB } & \text { FEB } & \text { FEB } & \text { FEB } & \text { FEB } & \text { FEB } & \text { FEB } & \text { FEB } & \text { FAR } \\ 31 & 22 & 23 & 24 & 25 & 26 & 27 & 28 & 29 & 1 \\ 2 ! 1 & 211 & 212 & 2 ! 3 & 214 & 245 & 216 & 217 & 218 & 219\end{array}$

$\begin{array}{rrrrrrrrrr}13.01 & 11.29 & 12.35 & 1.34 & 19.47 & 20.92 & 23.80 & 15.23 & 24.56 & 12.41 \\ 0.13 & 2.17 & 0.20 & 0.16 & 0.11 & 0.12 & 0.12 & 0.11 & 0.10 & 0.11 \\ 1.75 & 1.75 & 1.85 & 1.75 & 1.75 & 1.91 & 1.38 & 1.75 & 1.97 & 1.93\end{array}$

$\begin{array}{rrrrrrrrrr}4 & 4 & 3.79 & 4 & 4 & 3.66 & 3.53 & 4 & 3.56 & 3.83\end{array}$

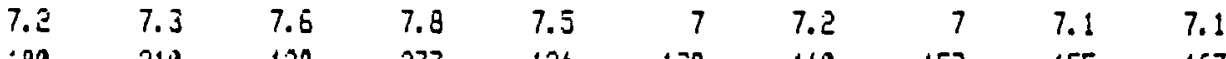

$\begin{array}{llllllllll}: 80 & 218 & 128 & 233 & 124 & 138 & 148 & 153 & 155 & 167\end{array}$

$\begin{array}{llllllllll}11 & 18 & 18 & 19 & 12 & 12 & 12 & 12 & 2.9 & 2.8\end{array}$

$\begin{array}{rrrrrrrrrr}148 & 148 & 54 & 42 & 24 & 0 & 102 & 362 & 512 & 598 \\ 93 & 93 & 34 & 25 & 15 & 0 & 64 & 239 & 359 & 595\end{array}$

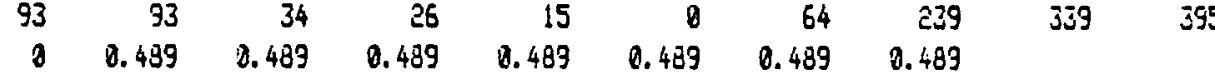

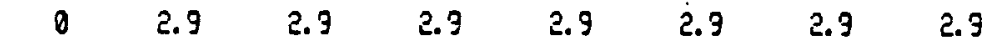

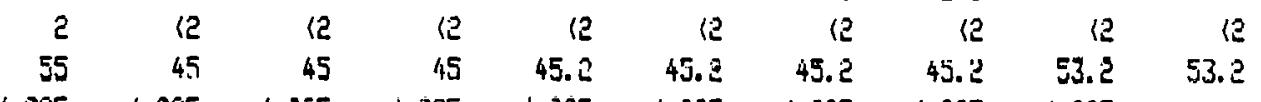

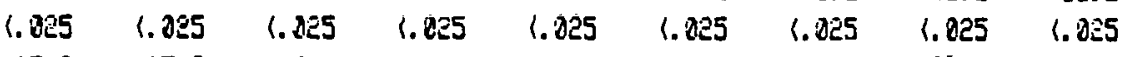

$\begin{array}{llllllll}47.2 & 15.6 & 15.6 & 15.6 & 5.9 .1 & 25.1 & 35.1 & 25.1\end{array}$

$\begin{array}{llllllll}47.2 & 15.6 & 15.6 & : 5.6 & 25.1 & 25.1 & 25.1 & 25.1\end{array}$

$\begin{array}{lllllll}1.5 & 1.5 & 1.5 & 1.5 & 1.5 & 1.5 & 1.5\end{array}$

$\begin{array}{lllllll}178 & \quad 178 & : 78 & 178 & 178 & 178 & 178\end{array}$

$\begin{array}{llllllll}5544 & 4954 & 4964 & 4964 & 4964 & 4964 & 4964 & 4964\end{array}$

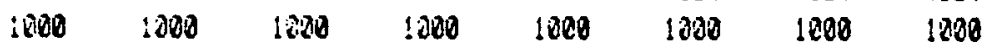

$\begin{array}{rrrrrrrrrr}50 & 50 & 50 & 50 & 50 & 50 & 50 & 50 & 50 & 50 \\ 6.3 & 6.5 & 6.5 & 6.5 & 6.5 & 6.5 & 9.2 & 7 & 9 & 9 \\ 1182 & 1054 & 1358 & 1208 & 1020 & 950 & 954 & 1182 & 1178 & 1324 \\ 886 & 768 & 783 & 336 & 746 & 673 & 672 & 902 & 893 & 1045 \\ 19 & 19 & 19 & 19 & 19 & 19 & 18 & 19 & 19 & 19\end{array}$

$\begin{array}{llllllllll}6.5 & 6.4 & 6.4 & 6.9 & 7.3 & 7.5 & 7.2 & 6.8 & 6.9 & 7.2\end{array}$

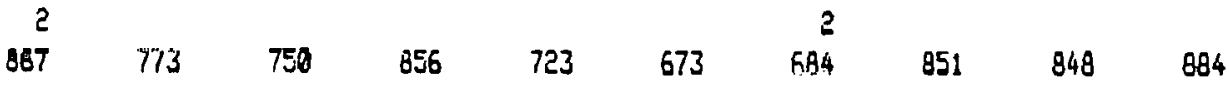

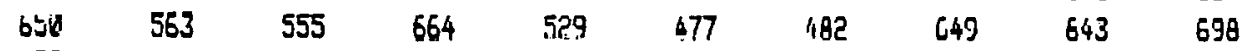

$\begin{array}{llllllllll}0.73 & 0.73 & 0.71 & 0.71 & 0.71 & 0.71 & 0.72 & 0.72 & 0.72 & 0.67\end{array}$

$\begin{array}{llllllllll}143 & 143 & 143 & 143 & 143 & 143 & 143 & 143 & 143 & 143\end{array}$

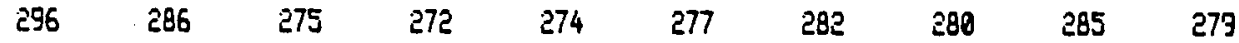

$\begin{array}{llllllllll}3.9 & 3.75 & 3.6 & 3.75 & 4 & 3.45 & 3.3 & 5.1 & 3.4 & 3.7\end{array}$

$\begin{array}{llllllllll}197 & 150 & 123 & 142 & 132 & 126 & 124 & 121 & 130 & 122\end{array}$

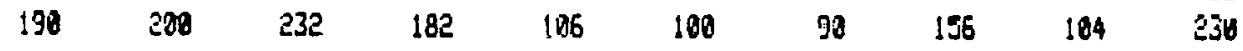

$\begin{array}{llllllllll}142 & 146 & 172 & 141 & 78 & 71 & 63 & 119 & 79 & 182\end{array}$

$\begin{array}{llllllllll}5.7 & 5.8 & 6.6 & 7 & 7.1 & 7.7 & 7.7 & 7.2 & 7.1 & 7.3\end{array}$

$\begin{array}{llllllllll}139 & 147 & 164 & 129 & 75 & 71 & 80 & 229 & 75 & 154\end{array}$

$\begin{array}{llllllllll}102 & 108 & 117 & 91 & 53 & 50 & 57 & 165 & 54 & 103\end{array}$

$\begin{array}{llllllllll}15 & 15 & 15 & 14 & 14 & 14 & 14 & 0.36 & 0.36 & 0.36\end{array}$

$\begin{array}{lllllll}0.872 & 0.872 & 0.872 & 0.872 & 0.872 & 0.872 & 0.872\end{array}$

$\begin{array}{lllllll}3.4 & 3.4 & 3.4 & 3.4 & 3.4 & 3.4 & 3.4\end{array}$

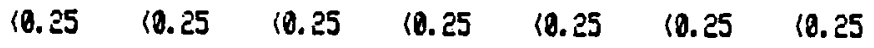

$202 \quad 202 \quad 202 \quad 202 \quad 202 \quad 202 \quad 202$

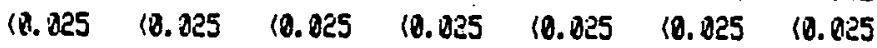

$\begin{array}{lllllll}43.5 & 43.5 & 43.5 & 21.3 & 21.3 & 21.3 & 21.3\end{array}$

$\begin{array}{ccccccc}18 & 18 & 18 & 18 & 18 & 18 & 18 \\ 5449 & 5449 & 5449 & 5449 & 5449 & 5449 & 5449\end{array}$

$\begin{array}{lllllll}750 & 758 & 750 & 750 & 750 & 750 & 750\end{array}$

$\begin{array}{lllllll}47.5 & 47.5 & 47.5 & 41.9 & 41.9 & 41.9 & 41.9\end{array}$

$12 \quad 12 \quad 12$

$12 \quad 12 \quad 12 \quad 12$

$\begin{array}{lll}43.7 & 43.7 & 43.7\end{array}$ 
YidT:H

DAY

DAY 10.

\section{GYSED SARTYETERS}

* Sludie fue

$*(F / P)=000$

NGST

SEED PARAMETERS (ERTCH \#)

voluine (liters)

oH (std units)

col (ng/!)

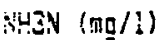

55 (mg/1)

USS (mg/1)

C.V (mg/1)

SCN (mg/1)

SoD 5-day $(m g / 1)$

TCC (mo/l)

Pheno:ics (ng/1)

pD4-p (mo/l)

Ong-N $(n g / 1)$

$\sqrt{202}(x g / 1)$

(4) $(\mathrm{mg} / \mathrm{l})$

TDS $(\mathrm{me} / \mathrm{d})$

Solor (APHA Units)

SAGSN DAFA.YETERS

Vo: dasted (nl)

J.c. (mo/l)

55 $(\mathrm{mg} / \mathrm{ll})$

*SS (FAC corrected)

Teno (C)

j:- (std units)

S. $(\mathrm{me} / \mathrm{h} / \mathrm{hr})$

VSS (ac/l)

*USS (FAC corrected)

USS/SS

*PAC Aided (mg)

* invertory (mo/l jasin)

EFELUENT PARAMEIERS

Voluse (liters)

$\cos (\mathrm{ma} / 1)$

SS (mg/1)

*PAC corrected

sid (std units)

USS (mo/i)

*PAll corrected

NHIN (ang/1)

CN (rag/1)

SCN (ng/1)

NO2 $\mathrm{ing} / \mathrm{ll}$

$\mathrm{NOJ}$ (ng/l)

Phenolics (mg/l)

P04-D (mo/I)

$\operatorname{rin}-V(\pi g / 1)$

ins (mg/1)

Eior (AJHA units)

-cingli

Bod 5-tay (mo/1)

$\begin{array}{rrrrrrrrrr}\text { YAR } & \text { KAR } & \text { MAR } & \text { MAR } & \text { MAR } & \text { MAR } & \text { MAR } & \text { MAR } & \text { MAR } & \text { MAR } \\ 2 & 3 & 4 & 5 & 6 & 7 & 8 & 9 & 10 & 11 \\ 220 & 221 & 222 & 223 & 224 & 225 & 226 & 227 & 228 & 239\end{array}$

\section{$5.72 \quad 25.81$}

S. 33

10.06

8.65

9.19
0.12

11.92

13.91

11.51

8. 58

$0.22 \quad 0.85$

1. 82

2. 15

1.92

1.82

1.33

0.11

0.11

0.14

$1.84 \quad 3.12$

$\begin{array}{lllll}3.31 \quad 1.7 & 3.84 & 3.25\end{array}$

3.65

3.85

3. 62

3.53

3.77

1.96

$\begin{array}{lll}7.5 & 7.2 & 7.4\end{array}$

6.6

6.5

6.7

6.3

7.1

7.4

3.58

$148 \quad 148$

158

160

168

176

181

183

150

7.3

$2.8 \quad 2.1$

2.12 .1

2.1

1.4

1.4

430

480

1.4

436

305

273

316

1
204

1

$412 \quad 755$

$40 \quad 360$

353

$\begin{array}{rrrr}12 & 12 & 12 & 12\end{array}$

12
4.3

12
54.3

12
51.2

51.2
12

12
51.3

12
49.6

12

49.6

\begin{tabular}{|c|c|c|c|c|c|c|c|c|c|}
\hline 58 & 351 & 50 & 50 & 50 & 50 & 58 & 50 & 50 & 50 \\
\hline 9 & 9.2 & 9.2 & 3.2 & 8.5 & 8.6 & 8.8 & 8.8 & 8.8 & 8.6 \\
\hline 698 & 1138 & 1128 & 1182 & 1184 & 1236 & 1888 & 1192 & 1180 & 1042 \\
\hline 451 & 984 & 888 & 949 & 362 & 1021 & 875 & 977 & 887 & 237 \\
\hline 18 & 18 & 18.5 & 18.5 & 19 & 18 & 18 & 18 & 18 & 19 \\
\hline 7.4 & 6.9 & 6.95 & $\begin{array}{r}6.35 \\
2\end{array}$ & 7.1 & 7.1 & $\begin{array}{r}7.5 \\
5\end{array}$ & 7.8 & 7.3 & 7.8 \\
\hline 466 & 760 & 753 & 782 & 781 & $8 ! 8$ & 730 & 800 & 738 & 699 \\
\hline 301 & 590 & 593 & 629 & 634 & 676 & 587 & 656 & 595 & 562 \\
\hline 0.67 & 0.67 & 0.67 & 0.66 & 0.66 & 0.66 & 0.67 & 0.67 & 0.67 & 0.67 \\
\hline 143 & 143 & 143 & 143 & 143 & 143 & 143 & 143 & 143 & 143 \\
\hline 247 & 254 & 248 & 233 & 222 & 215 & 213 & 215 & 213 & 205 \\
\hline 3.55 & 1.6 & 3.62 & 3.05 & 3.45 & 3.75 & 3.35 & 3.32 & 3.55 & 3.4 \\
\hline 175 & 136 & 141 & 152 & 163 & 143 & 152 & 124 & 132 & 140 \\
\hline 286 & 66 & 386 & 312 & 324 & 292 & 218 & 284 & 214 & 292 \\
\hline 185 & 51 & 241 & 251 & 263 & 241 & 175 & 167 & 173 & 235 \\
\hline 7.5 & 6.9 & 7.05 & 6.7 & 7.1 & 7.1 & 7.3 & 7.6 & 7.7 & 7.9 \\
\hline 191 & 44 & 284 & 206 & 214 & 193 & 146 & 137 & 144 & 196 \\
\hline 127 & 29 & 136 & 137 & $14 !$ & 138 & $3 B$ & 92 & 36 & 131 \\
\hline 0.29 & 0.29 & 0.29 & 0.29 & (1 & (1) & 11 & 54.6 & 54.6 & 54.6 \\
\hline
\end{tabular}

\section{6.}

$\begin{array}{llllllllll}46.9 & 46.9 & 46.9 & 46.9 & 45.8 & 45.8 & 45.8 & 40.9 & 40.9 & 40.9\end{array}$ 
LNIT AB TABLE AQ

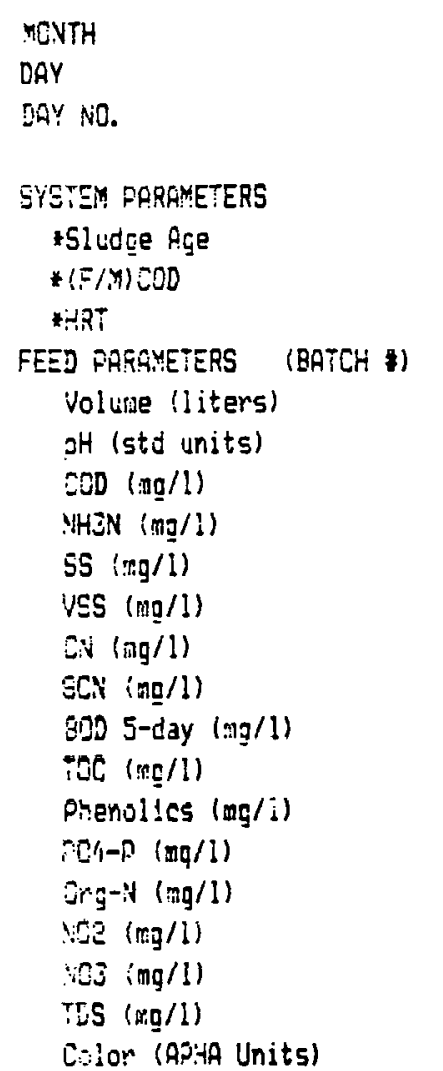

$\begin{array}{rrrrr}\text { MAR } & \text { MAR } & \text { MAR } & \text { MAR } & \text { MAR } \\ 12 & 13 & 14 & 15 & 16 \\ 230 & 231 & 232 & 233 & 234\end{array}$

$\begin{array}{lllll}17.90 & 13.20 & 15.56 & 10.75 & 11.90\end{array}$

$\begin{array}{lllll}0.12 & 0.15 & 0.18 & 0.12 & 0.12\end{array}$

$\begin{array}{lllll}1.30 & 1.85 & 2.85 & 1.86 & 2.10\end{array}$

$\begin{array}{lllll}3.69 & 3.78 & 3.42 & 3.77 & 3.34\end{array}$

$\begin{array}{lllll}7.2 & 7.1 & 7.2 & 7.2 & \\ 175 & 187 & 152 & 160 & 171\end{array}$

$\begin{array}{rrrrr}175 & 187 & 152 & 160 & 171 \\ 1 & 1 & 11 & 11 & 11\end{array}$

$\begin{array}{lllll}134 & 136 & 184 & 128 & 136\end{array}$

$\begin{array}{lllll}128 & 96 & 130 & 91 & 96\end{array}$

$\begin{array}{rrrrr}12 & 12 & 12 & & \\ 49.6 & 49.6 & 52 & 52 & 52\end{array}$

SAGIV DR TAMETERS

Vol viasted (al)

3.0. (no/l)

汭 (mg/1)

*SE (PAC corrected)

Tamp (C)

cit (std units)

CUR (mg/i/hr)

vis img/l)

+VES (FAC corrected)

USS/SS

* FAC Added (mq)

*Inventory (mo/1 basin)

EFFLLENT PRRAMETERS

Volune (liters)

CDD (ng/l)

SS $(\mathrm{mg} / 1)$

*PAC corrected

oH (std inits)

VSS (ma/!)

AFTC corrected

lit:3N (mg/l)

C.V (mg/1)

SCN (mog/7)

NOE $(\mathrm{mg} / 1)$

No3 ( $\pi \mathrm{g} / \mathrm{l})$

Pherolics (mg/l)

F04-P (mo/l)

Org-N $(\mathrm{mg} / \mathrm{l})$

ĩs (mo/l)

Coior (APHA units)

- IC $(\mathrm{mg} / 1)$

BCD 5-day (mo/l)

$\begin{array}{rrrrr}58 & 58 & 50 & 58 & 58 \\ 8.8 & 8.8 & 8.8 & 8.8 & 8.5 \\ 1138 & 1022 & 1074 & 1038 & 1018 \\ 328 & 812 & 868 & 828 & 809 \\ 18 & 14 & 19 & 19 & 19 \\ 7.7 & 7.5 & 7.5 & 7.4 & 7.4 \\ 3 & & & 3 & \\ 768 & 690 & 725 & 724 & 710 \\ 626 & 548 & 581 & 577 & 564 \\ 0.67 & 0.67 & 0.67 & 0.70 & 0.70 \\ 143 & 143 & 143 & 143 & 143 \\ 210 & 210 & 214 & 210 & 209 \\ & & & & \\ 3.45 & 3.55 & 3.27 & 3.6 & 3.2 \\ 138 & 121 & 109 & 158 & 127 \\ 142 & 175 & 164 & 216 & 216 \\ 116 & 140 & 131 & 172 & 172 \\ 7.7 & 7.7 & 7.7 & 7.5 & 7.5 \\ 96 & 119 & 111 & 151 & 151 \\ 65 & 80 & 75 & 165 & 105 \\ 54.6 & 11 & 11 & 11 & 11\end{array}$

$\begin{array}{lllll}40.9 & 38 & 38 & 38 & 38\end{array}$ 


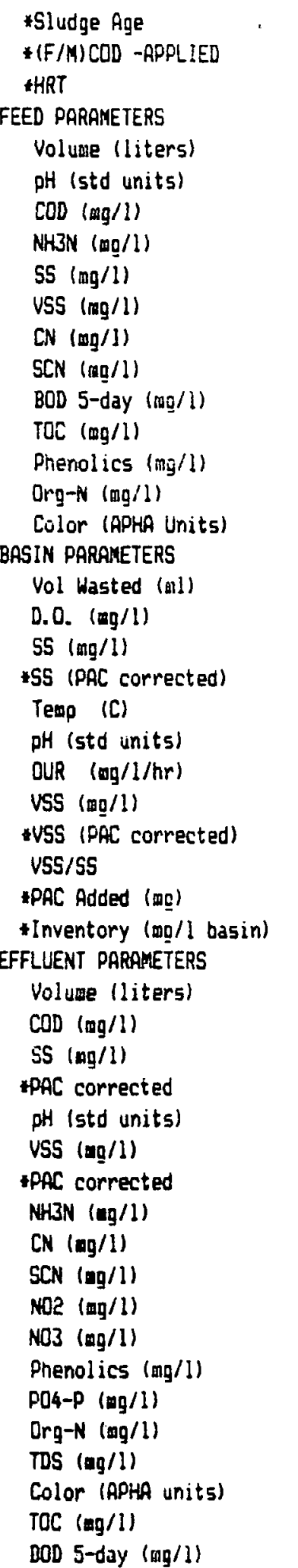

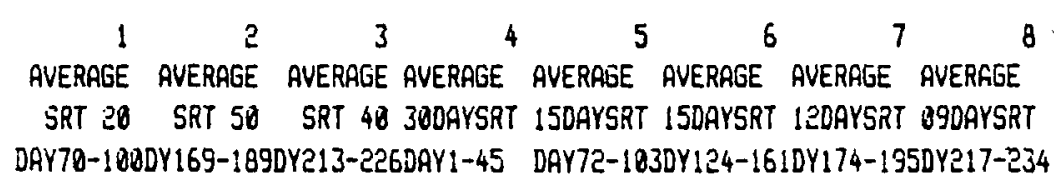

\begin{tabular}{|c|c|c|c|c|c|c|c|}
\hline 19.62 & 50.29 & 40.06 & 30.00 & 14.38 & 14.95 & 12.80 & 8.99 \\
\hline 0.29 & 0.14 & 0.12 & 0.25 & 0.34 & 0.36 & 0.42 & 0.38 \\
\hline 1.98 & 1.89 & 1.92 & 2.08 & 2.03 & 1.99 & 1.90 & 1.90 \\
\hline 4.75 & 4.93 & 4.89 & 4.62 & 4.72 & 4.71 & 4.93 & 4.93 \\
\hline 9.51 & 10.51 & 11.23 & 4.68 & 10.28 & 11.88 & 18.54 & 11.54 \\
\hline 1989 & 2009 & 1799 & 1982 & 1989 & 2020 & 2046 & 1788 \\
\hline 162.94 & 170.86 & 166.93 & 182.83 & 162.94 & 152.55 & 163.23 & 170.36 \\
\hline 273 & 500 & 188 & 53 & 263 & 442 & 480 & 149 \\
\hline 68 & 135 & 80 & 27 & 69 & 96 & 134 & 69 \\
\hline 4.10 & 0.58 & 2.55 & 0.43 & 4.10 & 1.42 & 1.41 & \\
\hline 173 & 108 & 174 & 132 & 173 & 193 & 187 & \\
\hline 1249 & 1034 & 1084 & 1420 & 1247 & 1158 & 1047 & 1136 \\
\hline 633 & 614 & 568 & 630 & 632 & 624 & 621 & 572 \\
\hline 0.97 & 9.39 & 6.60 & 1.52 & 1. 27 & 6.83 & 9.49 & \\
\hline 58.80 & 83.23 & & & 58.00 & 75.43 & 94.00 & \\
\hline 1000 & 2508 & 3000 & & 1000 & 2059 & 2500 & \\
\hline 468 & 217 & 105 & 359 & 695 & 671 & 847 & 776 \\
\hline 6.8 & 6.9 & 6.2 & 6.8 & 6.5 & 7.5 & 6.7 & 8.4 \\
\hline 3242 & 10006 & 9278 & 3398 & 2746 & 3182 & 3226 & 2239 \\
\hline 3242 & 10006 & 9278 & 3579 & 2746 & 3182 & 3226 & 2239 \\
\hline 21.2 & 19.2 & 18.7 & 24.4 & 21.8 & 19.1 & 19.6 & 18.6 \\
\hline 7.7 & 7.8 & 7.0 & 7.0 & 7.7 & 8.1 & 8.2 & 7.5 \\
\hline 15.6 & 2.2 & 11.9 & 46.3 & 23.3 & 6.5 & 3.4 & 10.0 \\
\hline 2687 & 6354 & 6163 & 3026 & 2338 & २ว२9 & 2100 & 1493 \\
\hline 2687 & 6354 & 6163 & 3026 & 2338 & 22२9 & 2100 & 1493 \\
\hline 0.81 & 0.64 & 0.67 & 0.89 & 8.85 & 0.70 & 0.66 & 0.67 \\
\hline 0.08 & B. 00 & 0.00 & 0.00 & 0.00 & 0.00 & 0.00 & 0.00 \\
\hline 0.80 & 0.00 & 0.00 & 0.80 & 8.00 & 0.00 & 0.80 & 0.00 \\
\hline
\end{tabular}

$\begin{array}{rrrrrrrr}4.51 & 4.63 & 4.82 & 4.88 & 4.46 & 4.37 & 4.67 & 4.74 \\ 136 & 116 & 157 & 188 & 138 & 186 & 204 & 238 \\ 143 & 48 & 400 & 32 & 69 & 95 & 102 & 224\end{array}$

$\begin{array}{llllllll}7.9 & 7.9 & 7.2 & 7.1 & 7.9 & 8.1 & 8.2 & 7.6\end{array}$

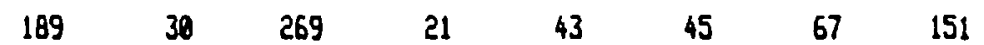

$\begin{array}{rrrrrrrr}2.7 & 184.6 & 1.5 & 10.9 & 8.7 & 79.4 & 177.8 & 198.9\end{array}$

$\begin{array}{lllllll}3.10 & 1.78 & 2.90 & 7.62 & 6.30 & 3.80 & 4.99\end{array}$

$\begin{array}{lllllll}3.81 & 0.00 & 1.50 & 12.62 & 3.26 & 7.66 & 0.77\end{array}$

$\begin{array}{lllllll}148.0 & 4.3 & 178.8 & 184.2 & 146.0 & 69.7 & 1.9\end{array}$

$\begin{array}{lllllll}9.3 & 6.3 & 25.1 & 4.4 & 9.4 & 5.1 & 6.6\end{array}$

$\begin{array}{lllllll}0.80 & 99.69 & 0.00 & 4.42 & 0.00 & 8.67 & 33.00\end{array}$

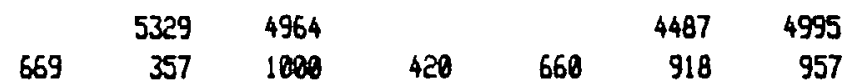

$\begin{array}{llllllll}39 & 22 & 51 & 56 & 39 & 58 & 55 & 69\end{array}$

$\begin{array}{lllllll}1.2 & 0.1 & 0.0 & 2.4 & 2.8 & 3.7 & 2.9\end{array}$ 
FIGURE A-1

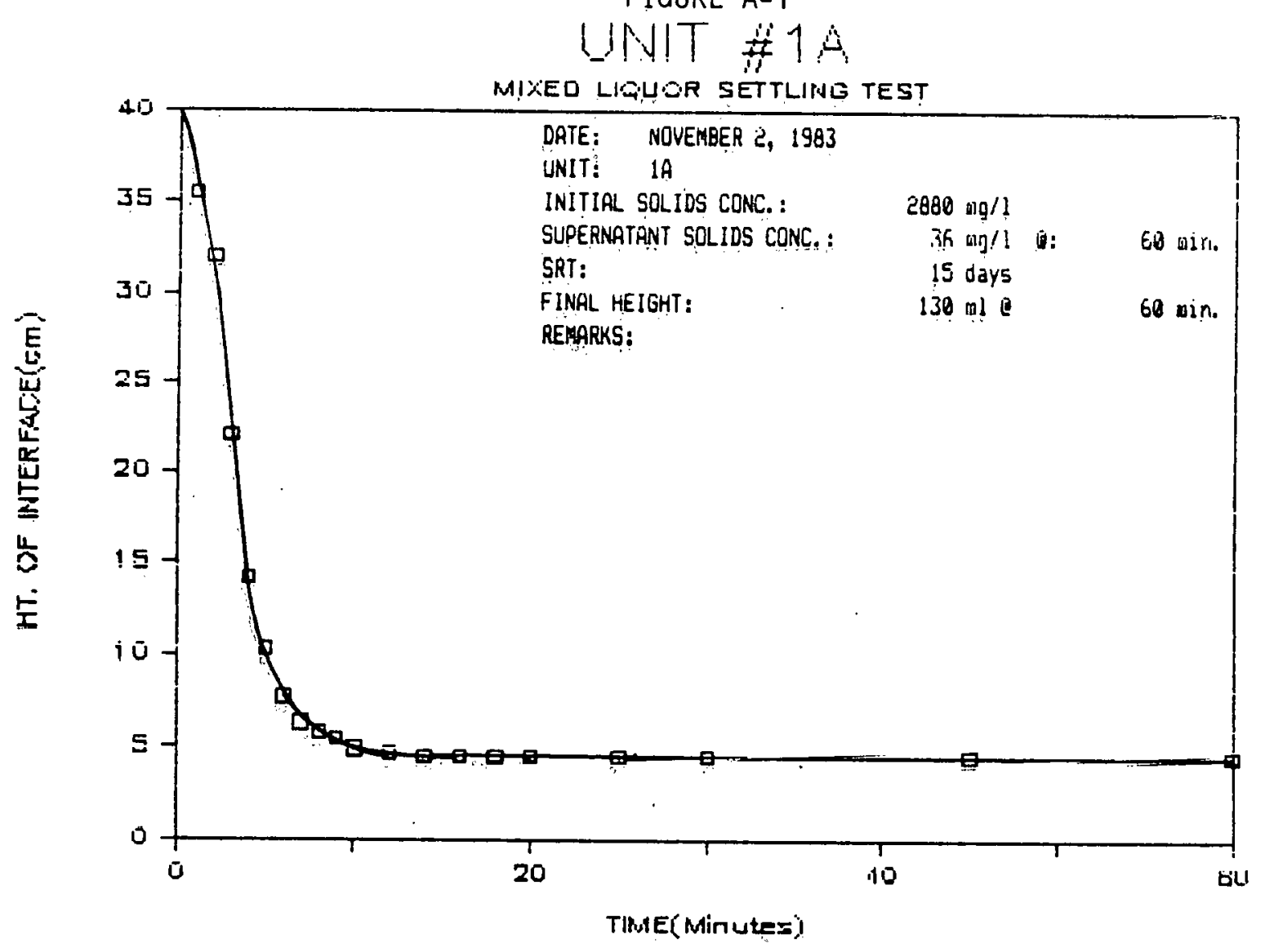

FIGURE A-2

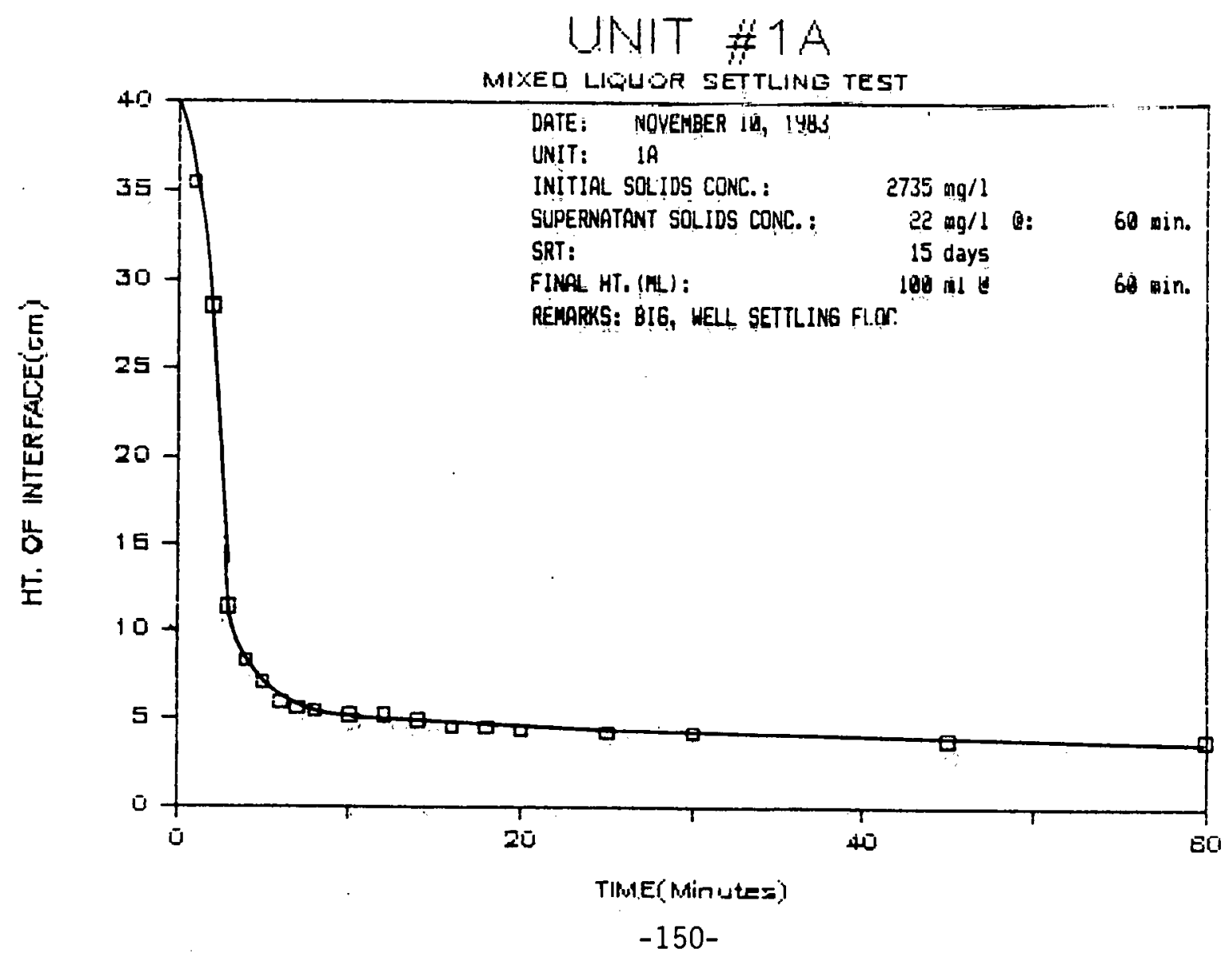


FIGURE $A-3$

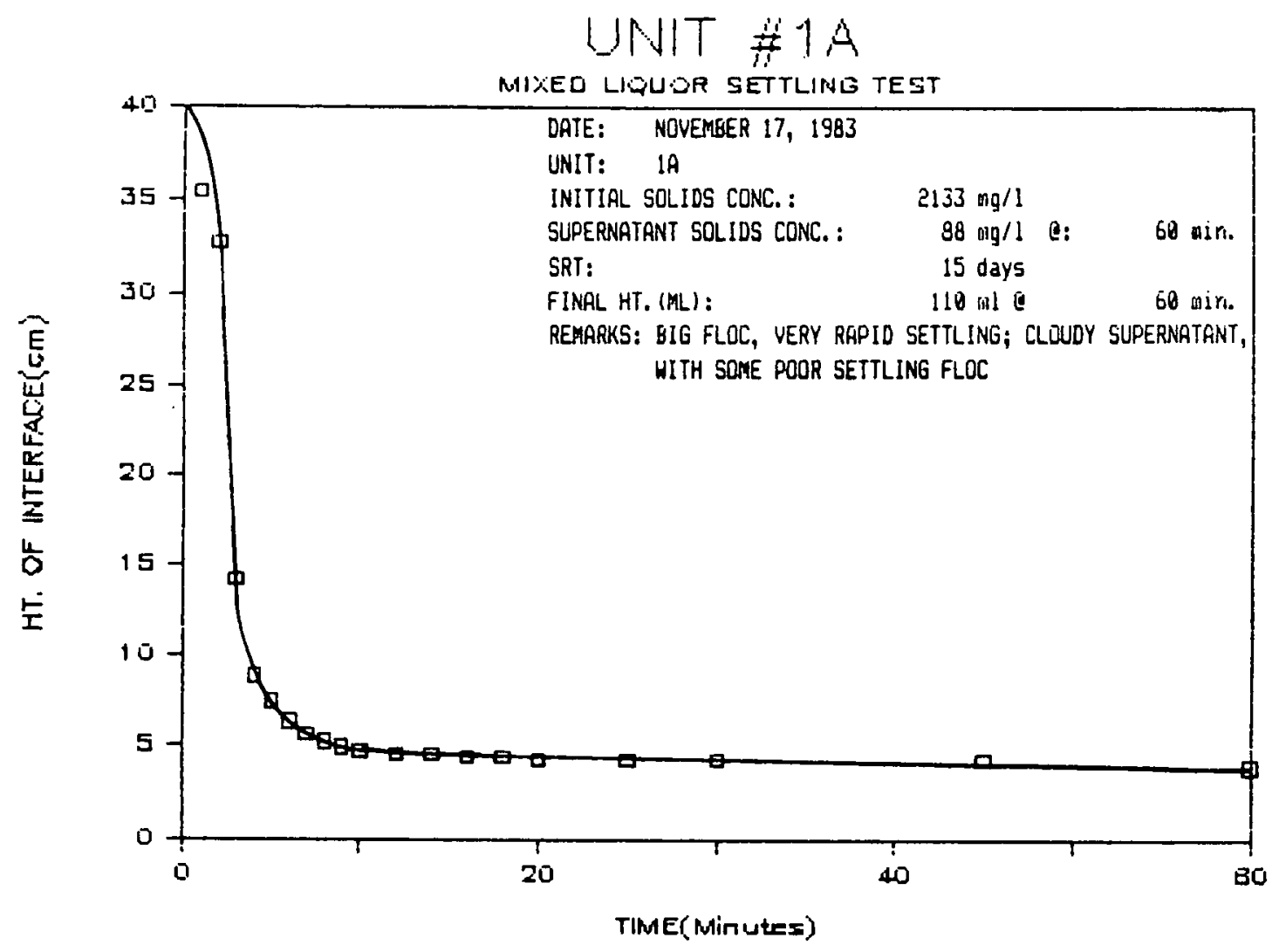

FIGURE A-4

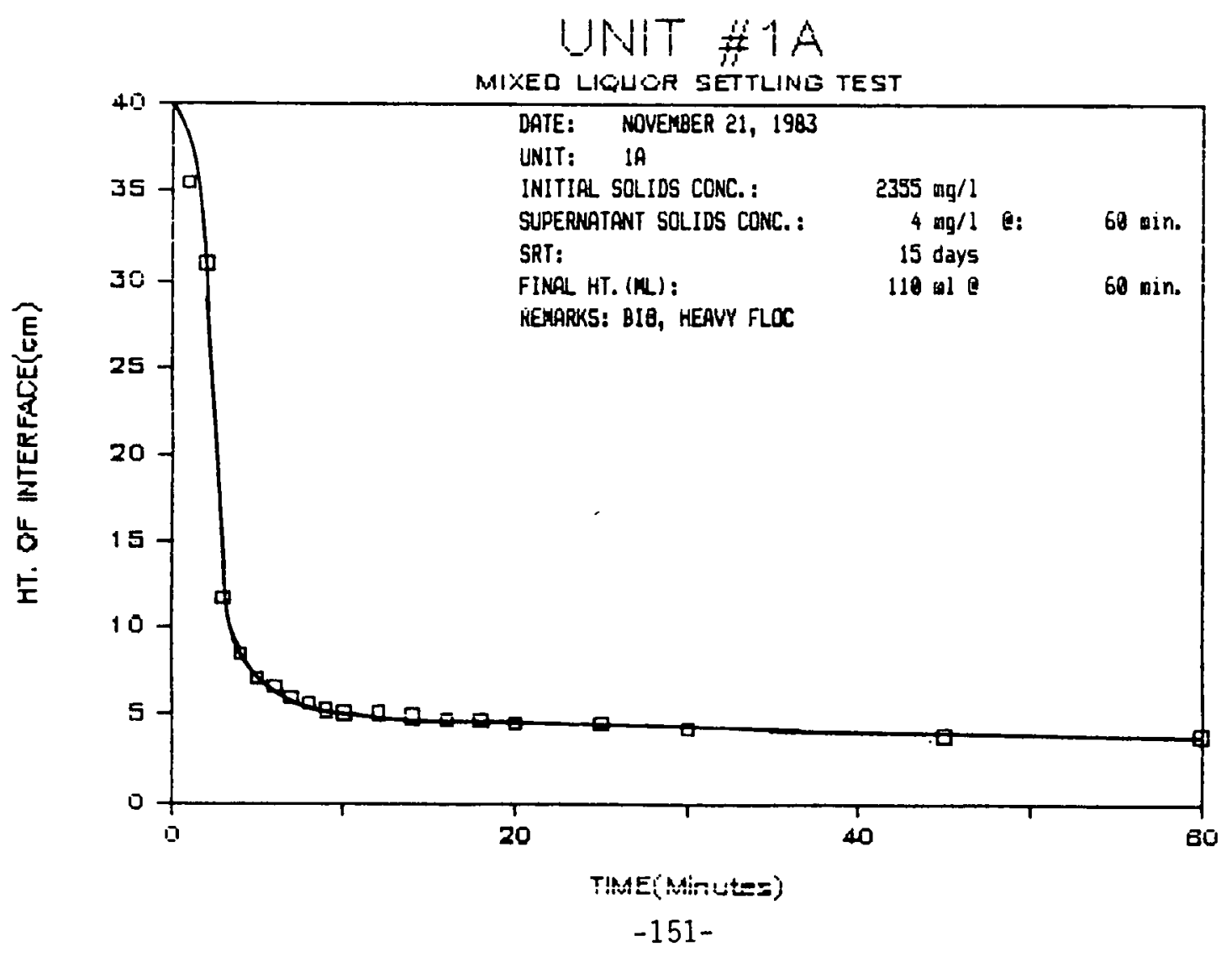




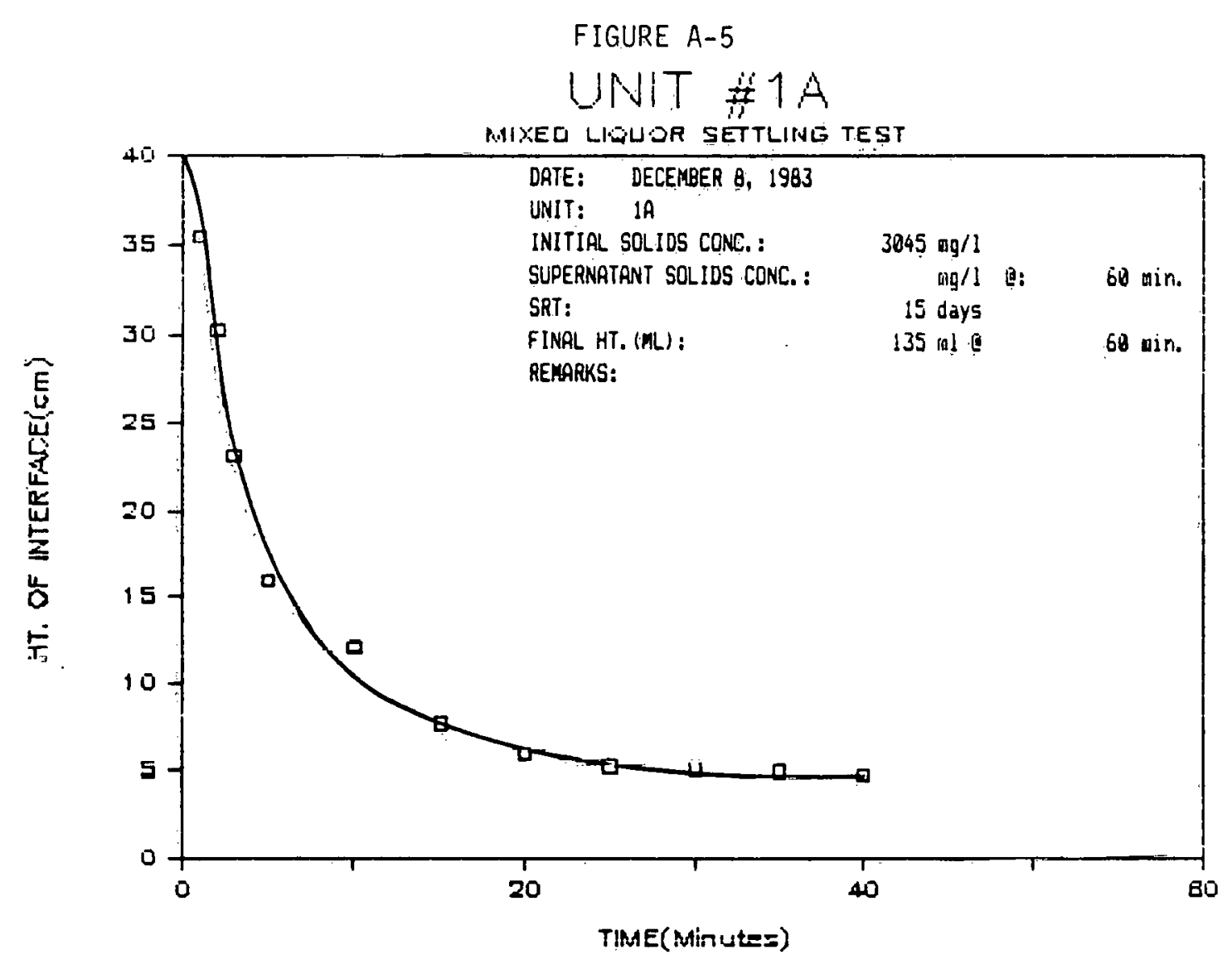

FIGURE A-6

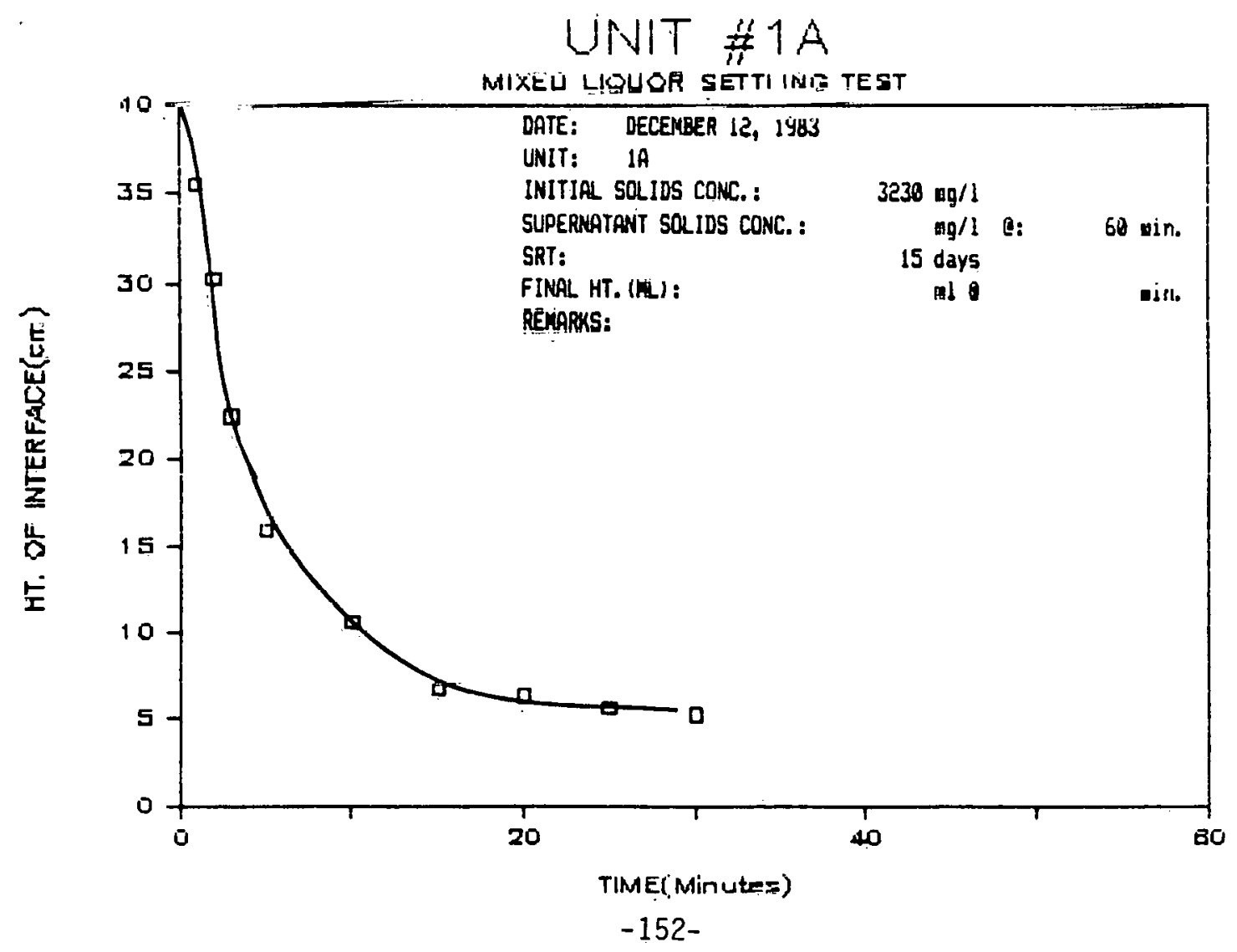


FIGURE A-7
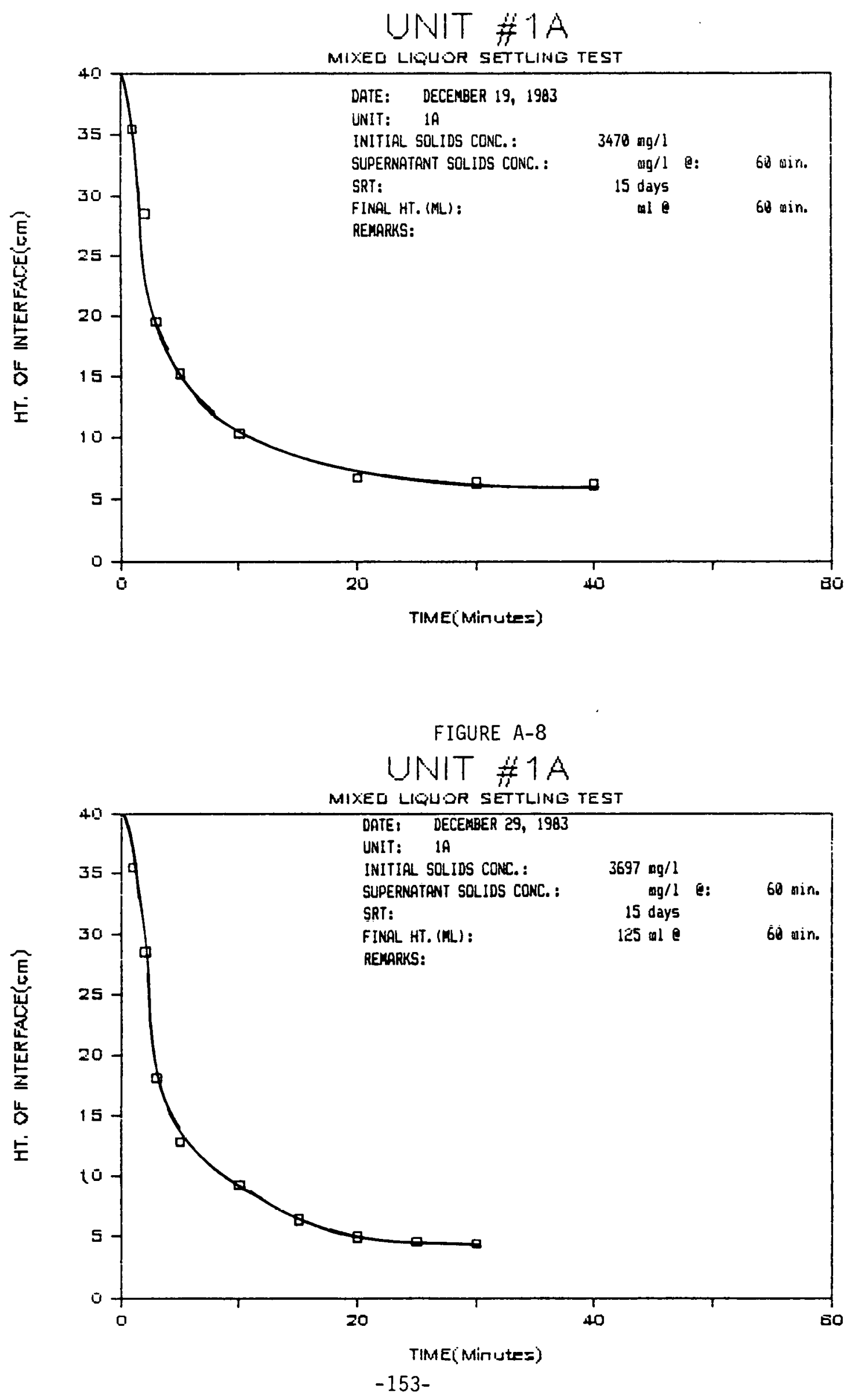
FIGURE A-9

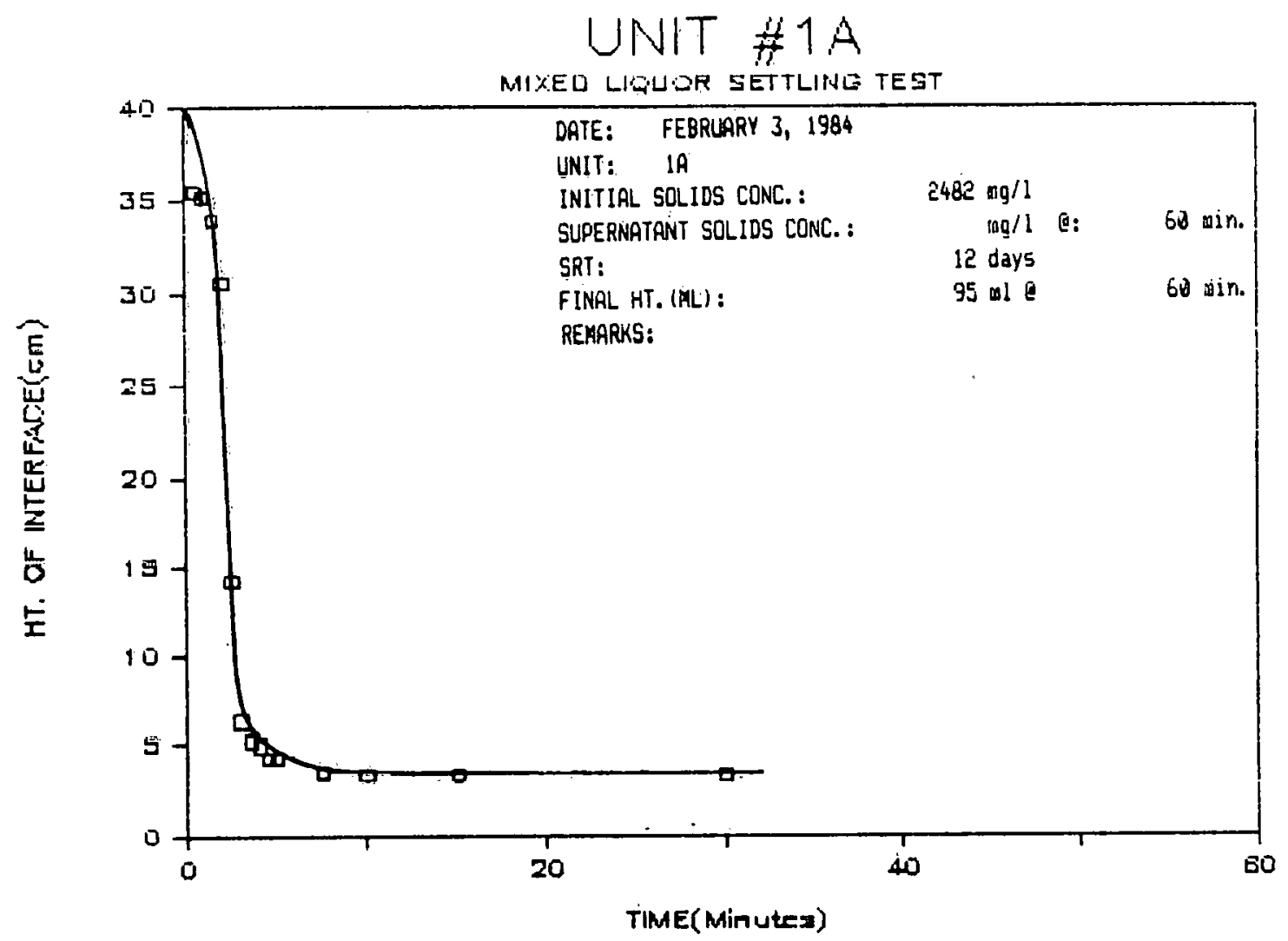

FIGURE $A-10$

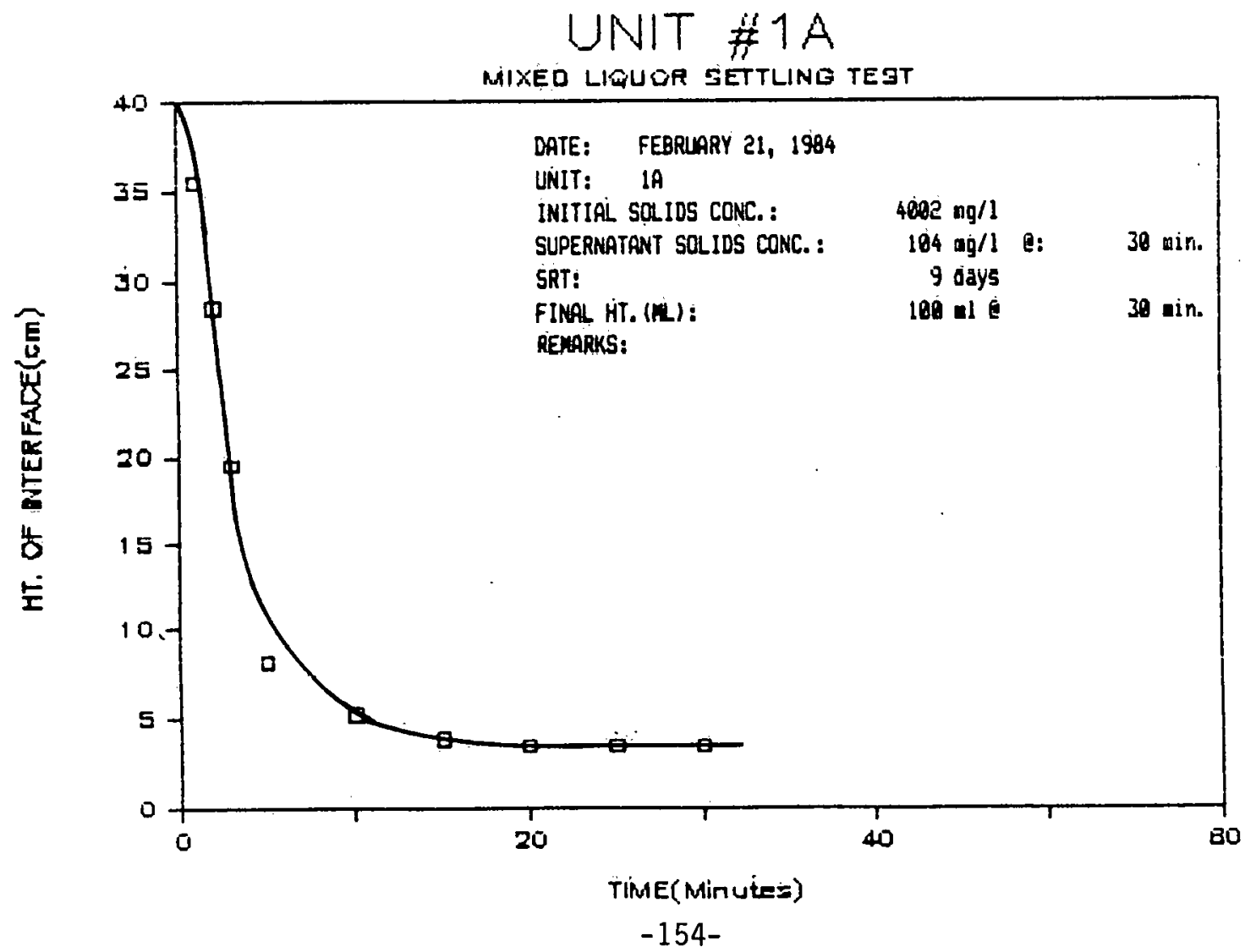




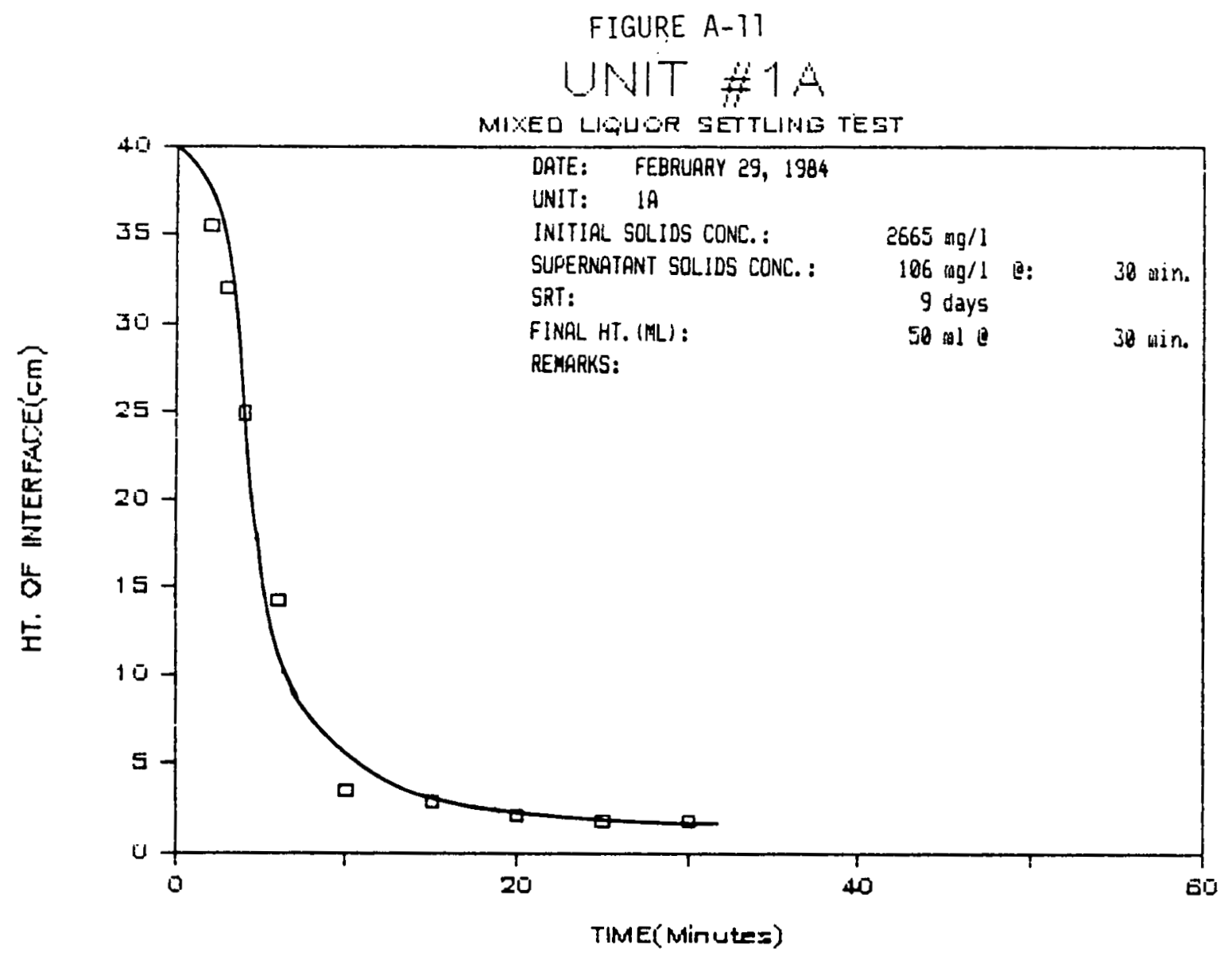


FIGURE $A-12$

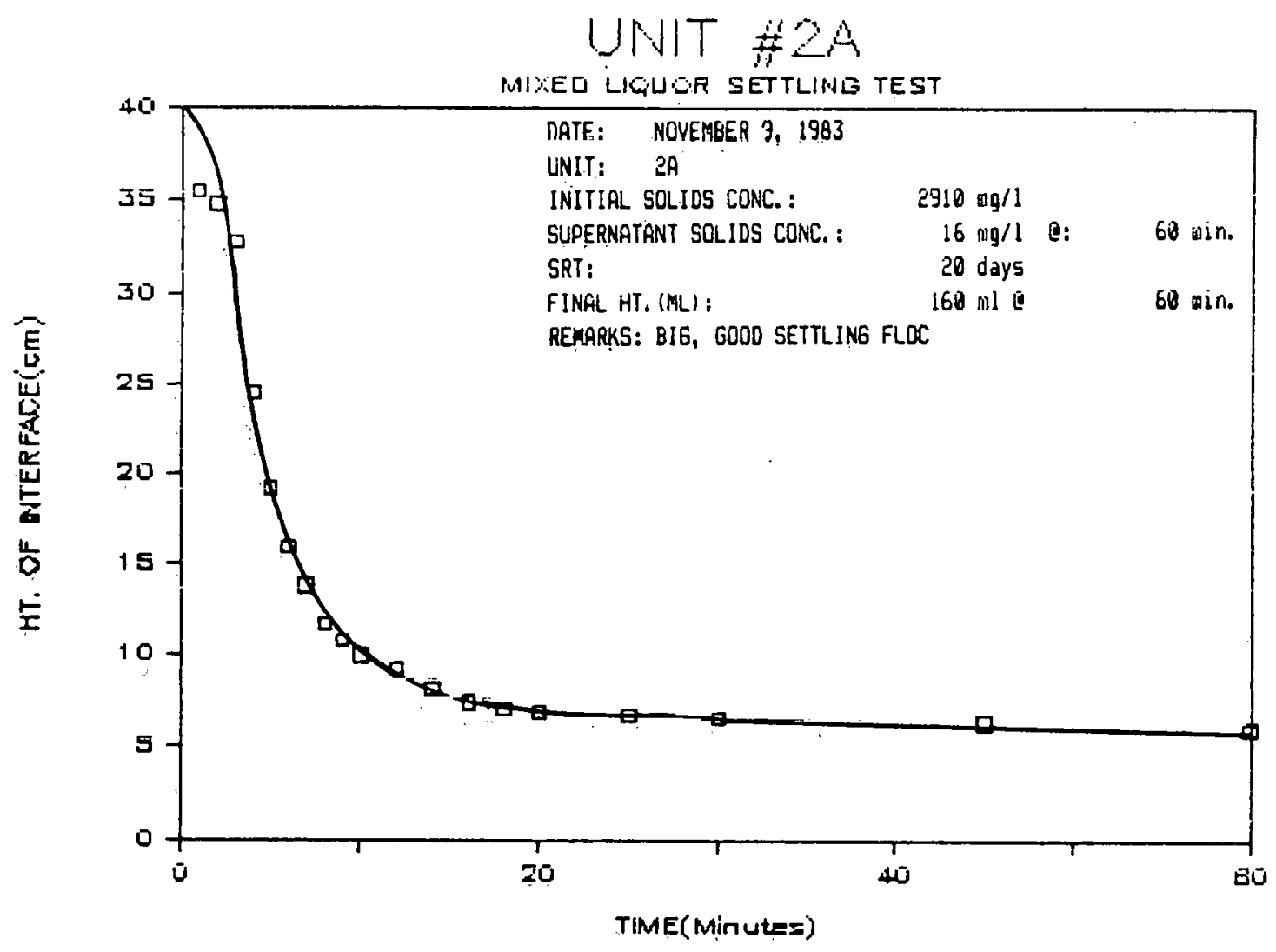

FIGURE A-13

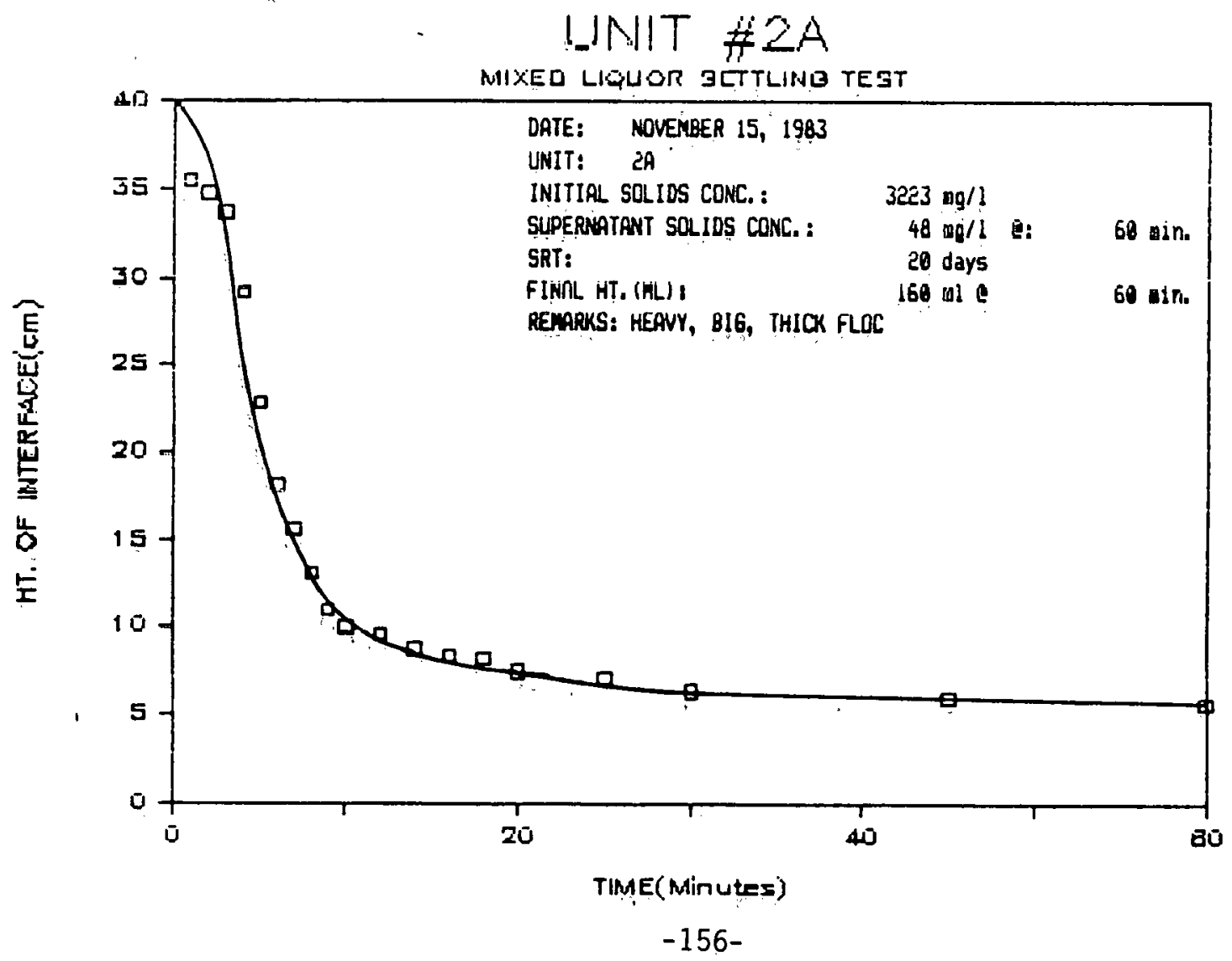


FIGURE A-14

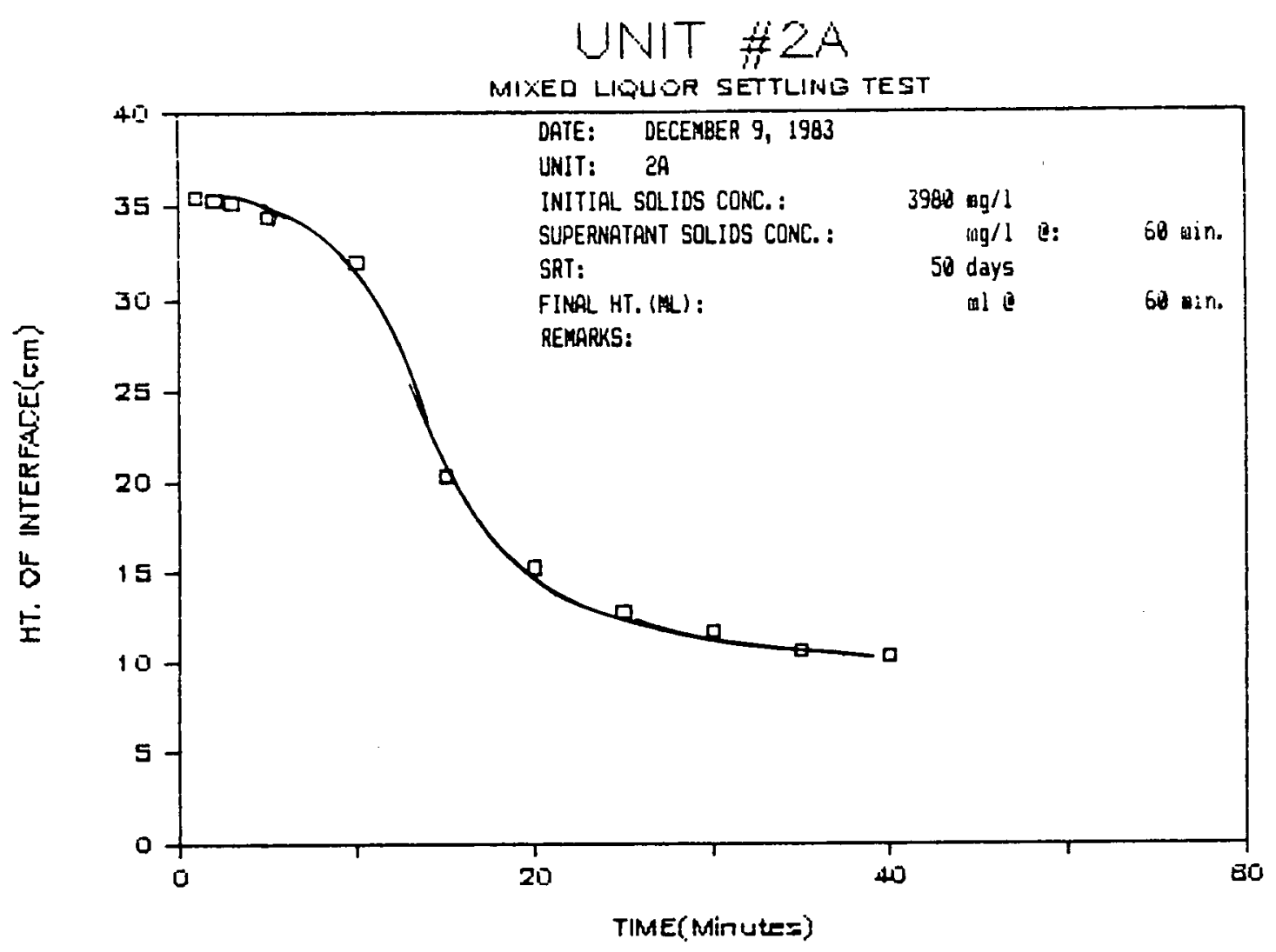

FIGURE $A-15$

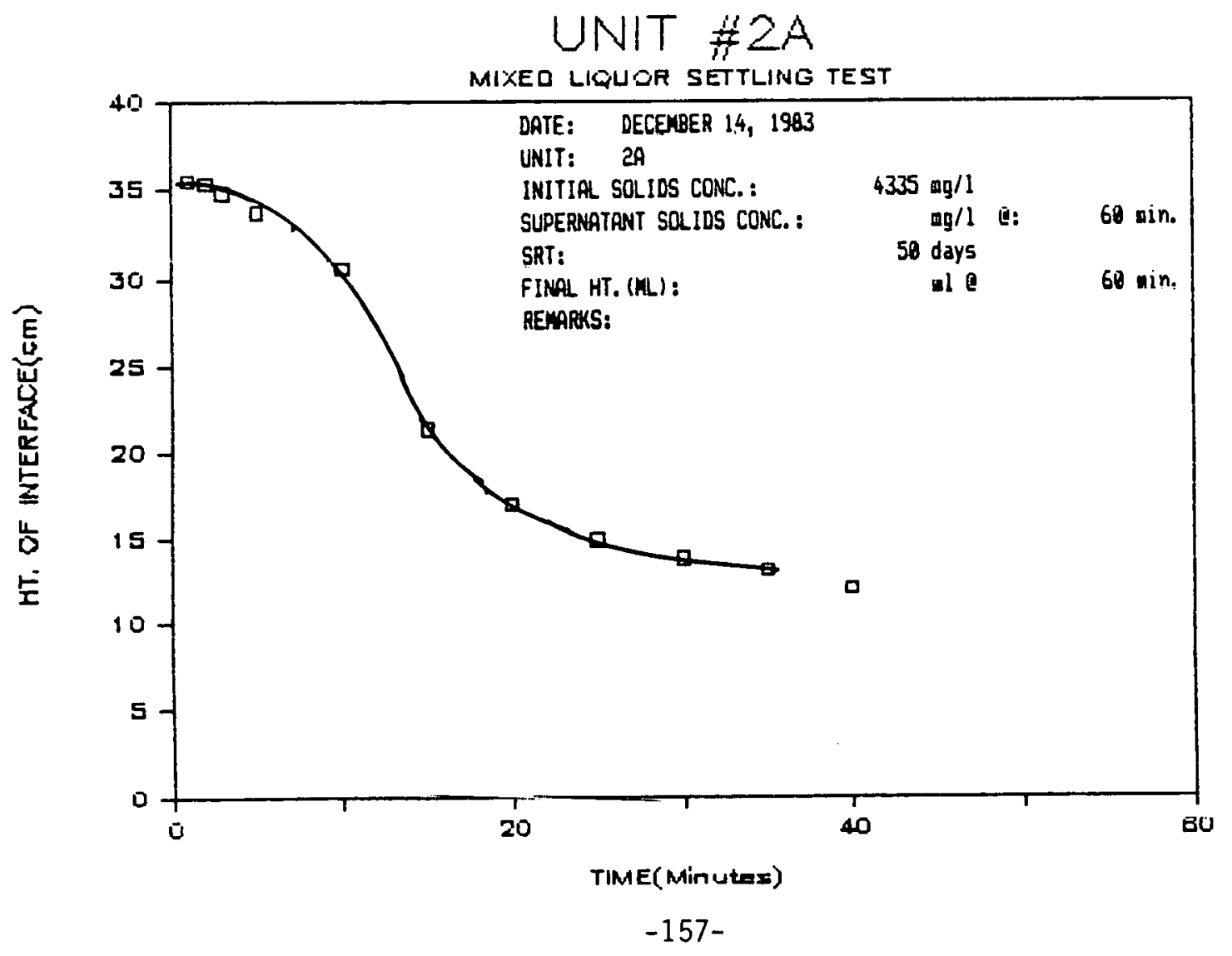


FIGURE A-16

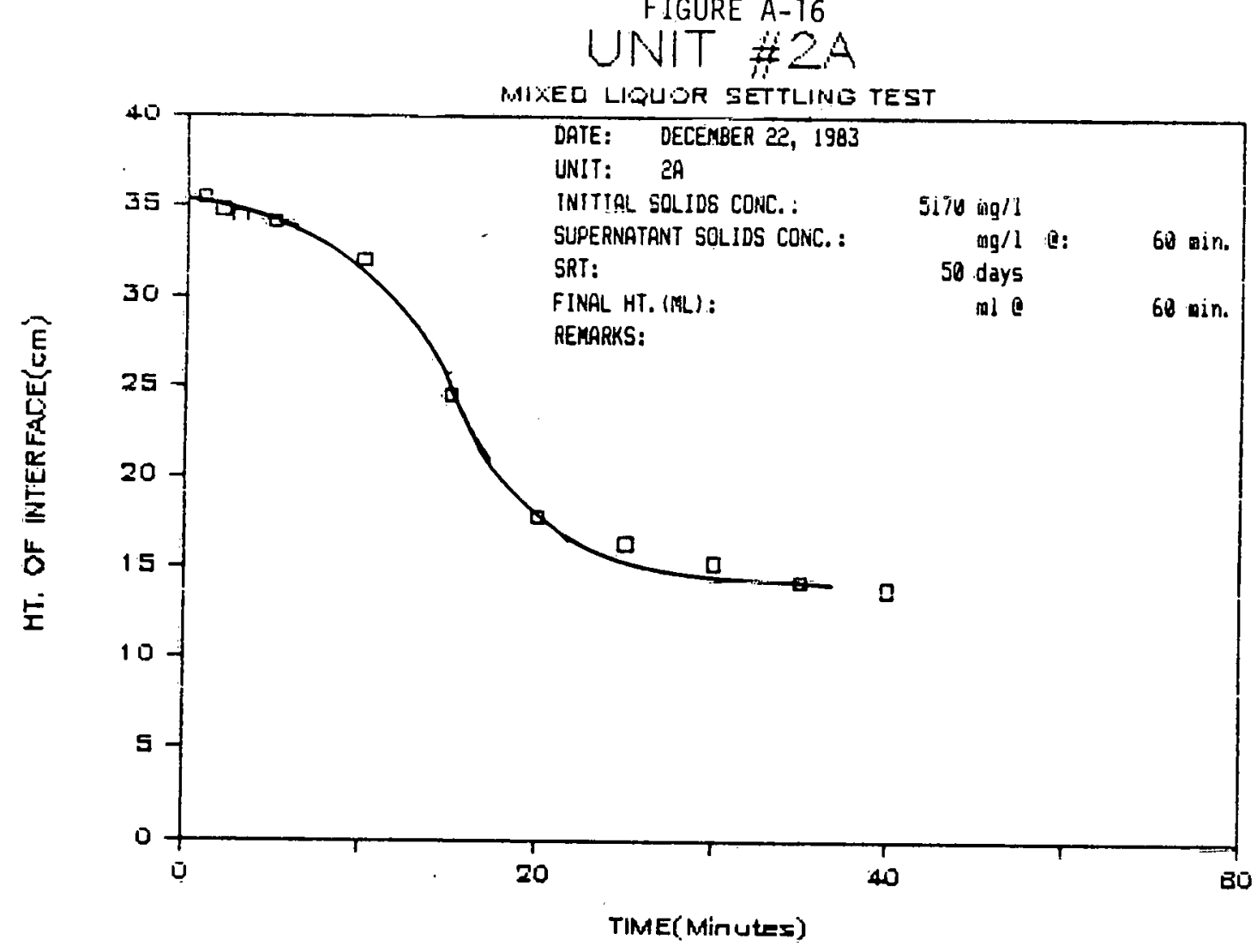

FIGURE A-17

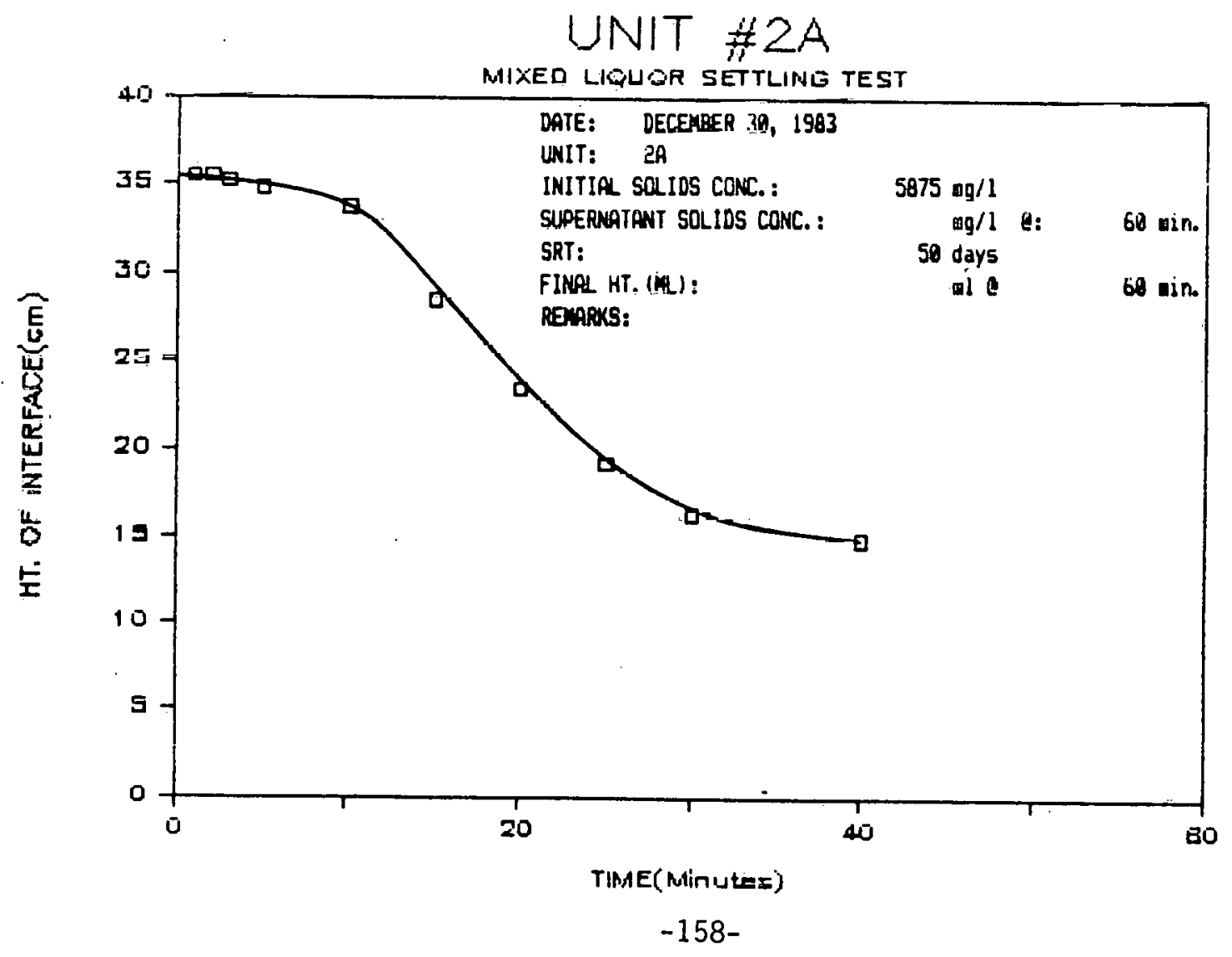


FIGURE A-18

UNIT \#2A

PUIXER LIOUOR SETTLINE TEST

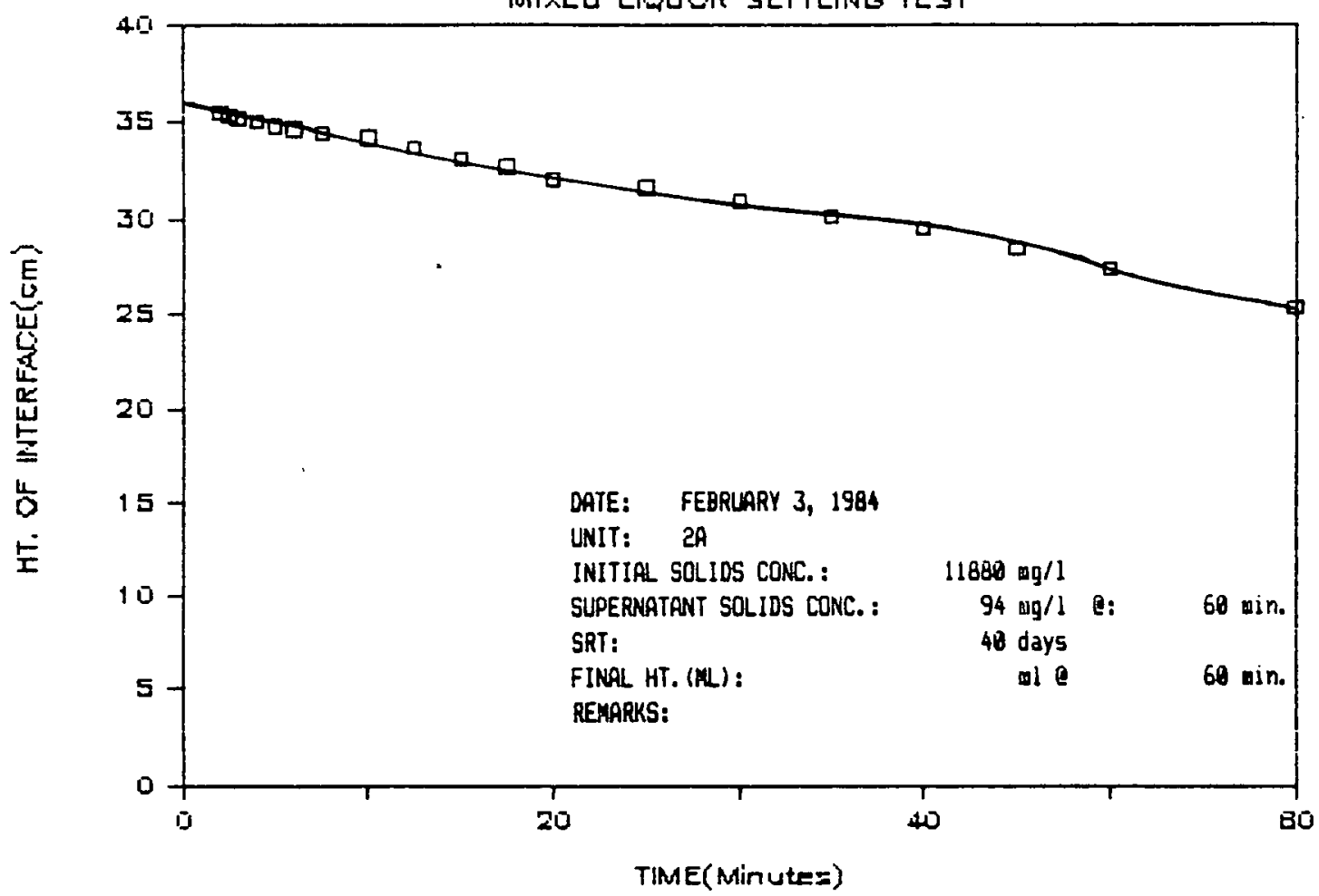

FIGURE A-19

UNIT \#2A

MIIXED LIQUOR SETTLINE TEST

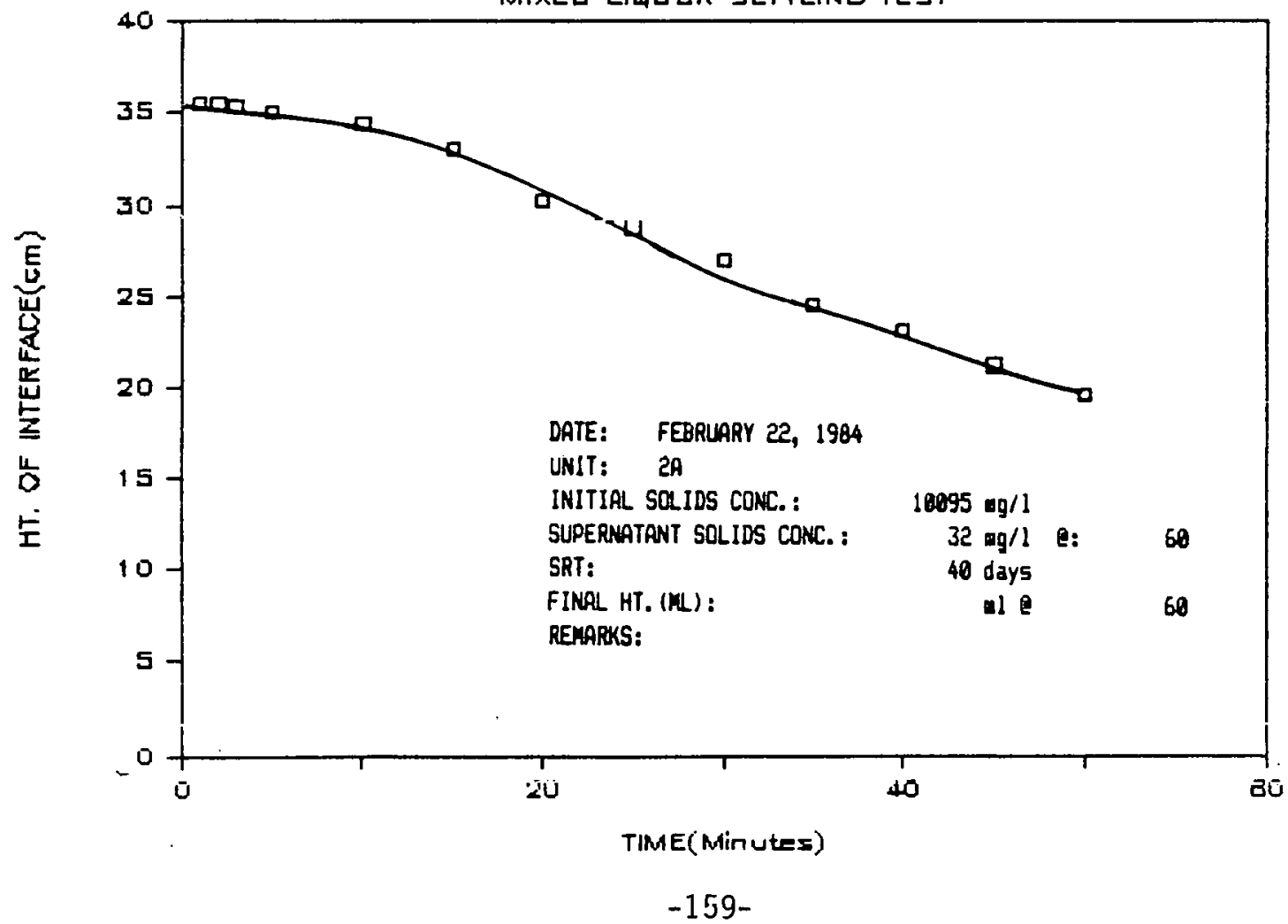




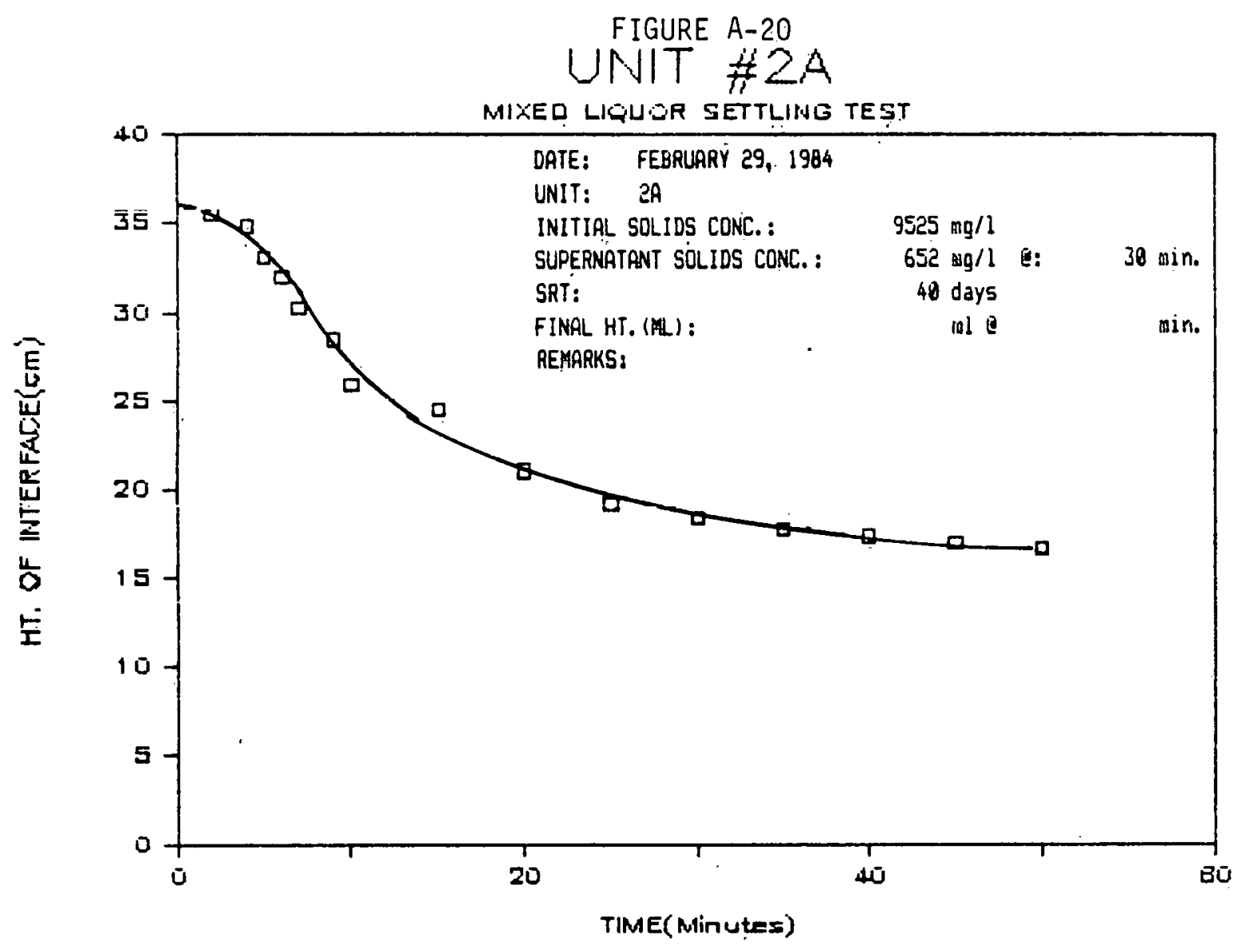


APPENDIX B

ANALYTICAL METHODS 


\section{ÄPPENDIX B}

ANALYTICAL METHODS

\section{QUALITY CONTROL PROCEDURES}

The routine quality control procedures described in Quality Assurance Program for Environmental Systems Division Laboratory Facility, Catalytic, Inc., were strictly followed. To monitor "accuracy" of analyses, that program requires spike recovery determination on distililed water and samples. For distilled water spiking, the following equation is used to calculate the recovery:

$$
P=100(\mathrm{C} / \mathrm{T})
$$

where $P$ is percent recovery of a standard, $C$ is the measured concentration, and $T$ is the true concentration. For each analysis, $P$ must fall within the acceptable range for that analys is to be considered "in control."

For sample spiking, the percent recovery $P$ is:

$$
P=100(\mathrm{dC} / \mathrm{sp} \text { ike added) }
$$

where $\mathrm{dC}$ is the difference between the concentrations measured for the spiked and unspiked samples. Spike added is the concentration increase of the analyte if the recovery were 100\%. Again, P must fall within the acceptable range for a given analyte to be considered "in control."

The quality assurance program also monitored "precision" of the analyses. Precision was controlled by analysis of replicate pairs; the difference between the two analyses was compared to the expected precision of 
the method. This is expressed mathematically as:

$$
R=|A-B|
$$

where $A$ and $B$ are observed concentrations of the replicate analyses, and $R$ is the absolute value of the difference. $R$ must fall within the acceptable range for each analytical procedure to be considered "in control" and is concentration dependent.

A frequency of $10 \%$ of sample load was used for analyzing sample spikes or water spikes. Also, 10\% of the sample load was used for replicate sample analysis. Where replicates were run, the average value was reported.

Figure B-1 shows the reporting form for quality control data collected under this program. In addition to spiked-sample, distilled-water spike, and replicate sample data, the analyst was required to report the standard curve. In this way, the analyst could tell if the standard curve had changed. By comparing spike and replication data to previous QC data obtained with the method, the analyst could detect out-of-control situations.

Gas chromatography/mass spectrometry (GC/MS) analyses of SRC wastewaters were performed by Mead CompuChem, Research Triangle Park, North Carolina, or Radiation Management Corporation, Phoenixville, Pennsylvania. These laboratories used the following methods to analyze the wastewaters: Purgeable Halocarbons, Method 624 (EPA, 1982), and Base/Neutral and Acid Compounds, Method 625 (EPA, 1982).

These analyses are designed to detect, identify, and quantitate EPA, organic priority pollutants. Other compounds present in the wastewater are not reported by these methods. 


\section{Laboratory Analysis Quality Control Data}

Tout for.

Mninod:

Laboratory Non Antyred:

Typs Container.

Standard Cunv:

8TANDARD CUAVE:

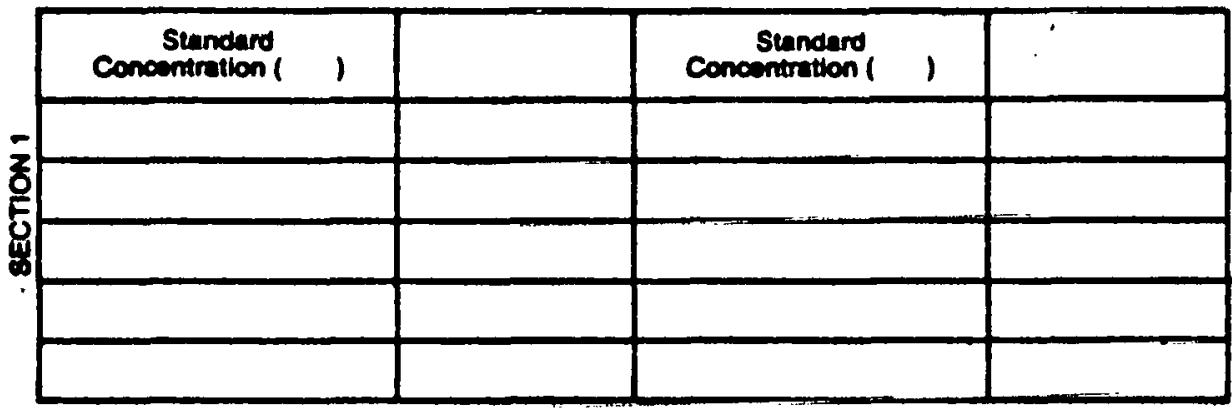

Lab. Notebook No.

Stock Solution Rot. No.

Analyst

Reviewer

ACCURACY CHECK (Sundarda):

\begin{tabular}{|l|l|l|l|l|}
\hline $\begin{array}{c}\text { Standard 1 } \\
\text { Concontration ( I }\end{array}$ & & $\begin{array}{c}\text { Calculated Concontration ( ) } \\
\text { Recovery }\end{array}$ \\
\hline & & & & \\
\hline & & & & \\
\hline & & & & \\
\hline & & & & \\
\hline & & & & \\
\hline
\end{tabular}

PAtciston CHECK (Dupltatis Smpin)!

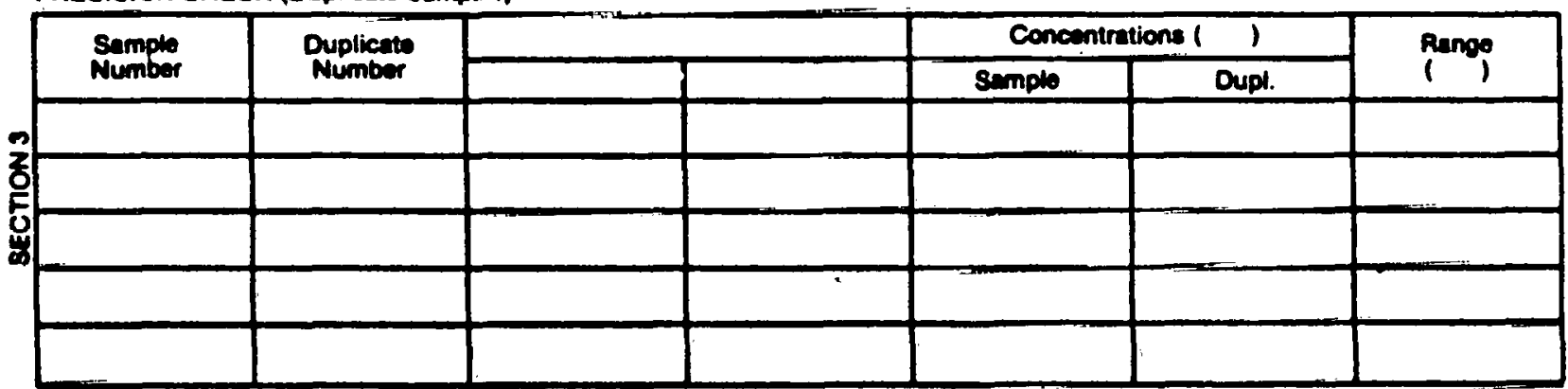

ACCUAACY CHECK (Spithed Sampla):

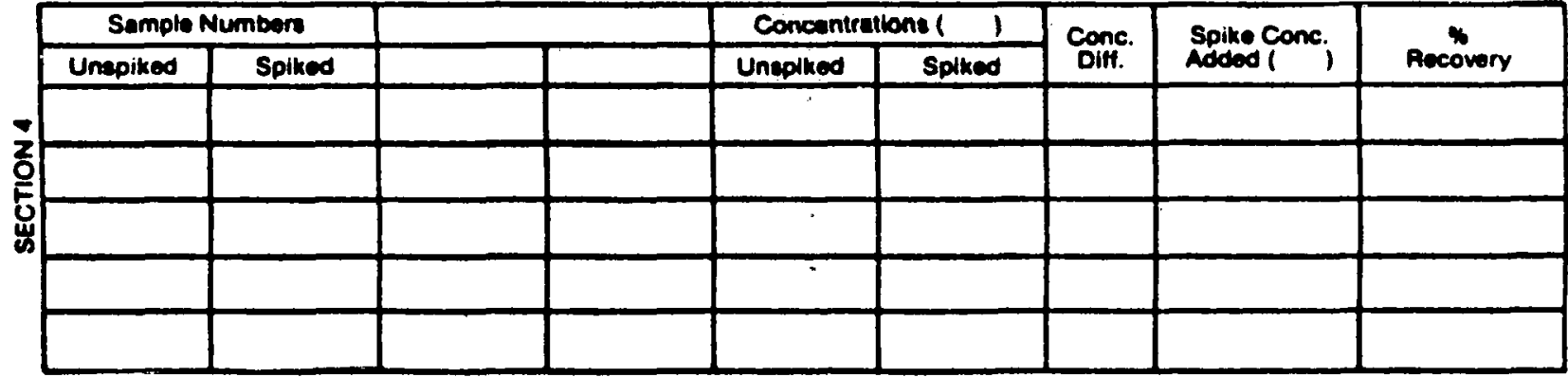


Quality control measures included with these GC/MS methods included daily calibration of the GC/MS with decafluorotriphenylphosphine (DFTPP) or parafluorobromobenzene (PFBB), depending upon whether base/neutrals/acids or volatiles are being run, respectively. In addition, method water blanks were run to ensure that the system was interference-free. Where possible, internal standards were used to calibrate the method. Otherwise, calibration curves were prepared using the external standard method.

Those quality control procedures that are specific to the analytical method are presented with the following method descriptions.

\section{TOTAL CYANIDE}

Total cyanide was stripped from the samples by distillation and digestion according to EPA Method 335.2 (1979). The distillates were treated with approximately $0.2 \mathrm{~g}$ of cadmium carbonate to precipitate sulfide, according to the procedure proposed by Barton et al. (1978), to remove interferences from thiocyanate breakdown during distillation.

The cyanide trapped in the distillates was measured by the pyridine/ barbituric acid method (EPA Method 335.2), with either a 1 or $5 \mathrm{~cm}$ cell on a Spectronic 710 spectrophotometer. For a $500 \mathrm{ml}$ sample distilled for tota 1 cyanide, limits of detection of 0.02 and $0.004 \mathrm{mg} / 1$ were estimated for the 1 and $5 \mathrm{~cm}$ cells, respectively.

Quality control procedures included analyzing spiked distilled water solutions to check recovery of cyanide in the distillation/digestion step. analyzing spiked samples, and analyzing in replicate. Several of the sample types showed low recoveries of sodium cyanide spikes, due to reactions of the cyanide with sample components. The nature of these reactions was not investigated. Both distilled water and sample spikes were tabulated to indicate the method's performance. Replicate analyses were recorded on precision control tables. 


\section{THIOCYANATE}

Thiocyanate $\left(\mathrm{SCN}^{-}\right)$was determined with a color blank/standard additions procedure to correct for sample color and response variations due to sample type. The samples were diluted into four $50 \mathrm{ml}$ volumetric flasks (minimum dilution $40 / 50 \mathrm{ml}$ ), and then acidified to $\mathrm{pH} \mathrm{5-7}$ with ? + $1 \mathrm{HNO}_{3}$. To one of the four volumetric flasks in each set was added $5 \mathrm{~m}$ ! of $2.5 \%(\mathrm{v} / \mathrm{v})$ nitric acid only, to act as a color blank while the other flasks were treated with $5 \mathrm{ml}$ of color reagent containing iron and nitric acid, as specified in Standard Methods (1981), Method 412-K. One of the flasks in each set was spiked with $2 \mathrm{mg} / 1$ and another with $4 \mathrm{mg} / 1$ of thiocyanate from a $100 \mathrm{mg} / 1$ standard solution. The concentration of thiocyanate in the unspiked sample was found by the method of standard additions, using readings made on a Spectronic 710 spectrophotometer ( $1 \mathrm{~cm}$ cell), after subtracting the color blank absorbance reading from that of each of the other sample dilutions in each set.

With a sample dilution of $40 / 50 \mathrm{ml}$ and a $1 \mathrm{~cm}$ spectrophotometer ce11, the detection limit of the method was estimațed to be $0.25 \mathrm{mg} / 1$.

Quality control steps included titration of the standard stock thiocyanate solution by the Volhard mẹthods, using a silver/sulfiḍe (Orion Model 94-16) electrode to indicate the end point, as described by Burroughs and Attia (1968). To $75 \mathrm{ml}$ of water was added $10 \mathrm{ml}$ of. $0.0142 \mathrm{~N} \mathrm{AgNO}_{3}$, followed by, $10 \mathrm{ml}$ of $20 \%$ $(\mathrm{w} / \mathrm{V}) \mathrm{Fe}\left(\mathrm{NO}_{3}\right)_{3} \cdot 9 \mathrm{H}_{2} \mathrm{O}$, solution and $5 \mathrm{ml}$ of $\mathrm{I}+1 \mathrm{HNO}_{3}$ (boiled to remove $\mathrm{NO}_{2}$ ). The solution was then titrated with the thiocyanate solution to the electrode inflection end point. The stock thiocyanate solution used to make standard addition spikes was checked daily by developing a standard curve in distilled water with the referenced method (Standard Methods, 1981), and by checking the response of the spectrophotometer (absorbance/concentration ratio). Replicate analyses carried through the entire standard addition, method were performed and recorded on precision control tables. 


\section{CHEMICAL OXYGEN DEMAND (COO)}

COD was determined on unfiltered samples by EPA Method 410.4 (1979). using sealed-ampule digestion in an oven with subsequent colorimetric analysis on a Spectronic 710 spectrophotometer. Prefilled ampules containing digestion reagents were purchased from Oceanographics International Corporation. A volume of $2.5 \mathrm{ml}$ sample was added to each ampule.

Interferences in this procedure, due mainly to chlorides, were overcome by sample dilution and by the complexation of chloride by mercury ion present in the catalyst solution.

The lower limit of detection for this method was approximately $9 \mathrm{mg} / 1$.

Quality control measurements were made on accuracy check standards and sample spikes, as well as on replicate samples. Standards were prepared with potassium acid phthalate.

\section{NITRATE-NITROGEN}

Nitrate $N$ was determined on filtered samples, using the cadmium reduction method, EPA Method 353.3 (1979), to determine (nitrate + nitrite) $N$. Nitrate-N was then calculated by subtracting the nitrite-N concentration.

Because of low recovery in the cadmium reduction step, a minimum dilution of $2 \mathrm{ml} / 100 \mathrm{ml}$ of water followed by $10 \mathrm{ml} / 25 \mathrm{ml}$ of water and then dilution of this $25 \mathrm{ml}$ of solution to $100 \mathrm{ml}$ with ammonium chloride buffer was required. The nitrate plus nitrite color following cadmium reduction was read on a spectronic 710 spectrophotometer with a $1 \mathrm{~cm}$ cell.

With a sample dilution of $2 \mathrm{ml} / 100 \mathrm{ml}$ and then $10 \mathrm{ml} / 25 \mathrm{ml}$, the lower limit of detection for the method was $1.25 \mathrm{mg} / 1$. 
Quality control analys is included determination of the cadmium column efficiency before each use and analysis of accuracy check standards or spiked samples, as well as replicate analyses.

\section{NITRITE-NITROGEN}

Nitrite-N was determined on filtered samples, also with EPA Method 353.3 (1979). The recoveries were poor unless the samples were diluted prior to analysis. The samples were first diluted $1 \mathrm{ml} / 25 \mathrm{ml}$ with water, and then diluted again by $25 / 100$ with ammonium chloride buffer before colorimetric analysis on a Spectronic 710 spectrophotometer with a $1 \mathrm{~cm}, \mathrm{cell}$. Interferences due to colored organic matter were avoided or reduced by dilution or by running color blanks made up with color reagent that did not contain $\mathrm{N}-1-(1-$ naphthy 1$)$ ethylenediamine reagent.

With a $1 / 25$ dilution and the method as practiced, a lower limit of detection of $0.25 \mathrm{mg} / 1$ was estimated.

Quality control for this method consisted of frequent standardization of the stock nitrite solution with standardized (vs. primary standard grade sodium oxalate) potassium permanganate. Sample spikes and: sample replicates were also run.

\section{TOTAL ORGANIC CARBON (TOC)}

TOC was determined on a DC-80 (Dohrmann) TOC analyzer. The liquid samples were first acidified with concentrated phosphoric acid and sparged with oxygen to remove inorganic carbon. The treated solutions were then injected into a reactor containing potassium persulfate., phosphoric acid, and an ultraviolet lamp. The carbon dioxide released by the oxidation reactions was detected with an infrared detector. Interferences with this instrument, mainly due to high chloride concentrations, were not a problem with the bio-unit effluent or feed samples. 
The estimated limit of detection for the liquid samples was $0.1 \mathrm{mg} / \mathrm{l}$.

Quality control measurements included reinjection of a potassium acid phthalate quality control standard to verify the instrument calibration and replicate injections to check method precision.

\section{BIOCHEMICAL OXYGEN DEMAND (BOD)}

BOD was determined on unfiltered, unpreserved samples, using Method 507 in Standard Methods. Nitrification was inhibited by adding 2-chloro6-trichoromethylpyridine for all determinations. Seed was derived from bench-scale biological reactor mixed liquor, composited from reactors $1 A$ and $1 B$ and $2 A$ and $2 B$. A lower 1 imit of detection of $2 \mathrm{mg} / 1$ was expected for a $300 \mathrm{ml}$ sample volume, assuming a depletion of $2 \mathrm{mg} / \mathrm{l}$ dissolved oxygen and a zero blank value.

Quality control procedures for BOD included replicate sample analyses, seed curves for each sample set, dilution water checks, and a glucose/glutamic acid standard with an expected value of $200 \pm 37 \mathrm{mg} / 1$.

\section{PHENOLICS (COLORIMETRIC)}

Phenolics were determined on unfiltered samples. These samples were distilled and analyzed with or without solvent extraction, using the 4-aminoantipyrene colorimetric procedure of EPA Method 420.1 (EPA, 1979). A $1 \mathrm{~cm}$ cell was used with the Spectronic 760 spectrophotometer.

No interferences were found with this procedure, although the feed samples required extra dilution (usually $2 \mathrm{ml} / 100 \mathrm{ml}$ ) in order to obtain a sample response within the calibrated scale of phenol concentrations in the colorimetric step of the procedure. 
Using the extraction procedure, the detection limit with a $100 \mathrm{ml}$ effluent sample was $25 \mathrm{\mu g} / \mathrm{l}$. For a $10 \mathrm{ml}$ sample (used for analys is of feed samples) analyzed without extraction, a detection limit of $2.5 \mathrm{mg} / 1$ was estimated.

Quality control analysis included sample replicates and analyses of a standard carried through the distillation procedure. In addition, sample spikes were processed.

\section{TOTAL DISSOLVED SOLIOS (TOS)}

TOS was measured by filtering unpreserved samples and evaporating the filtrates in an oven set at $180^{\circ} \mathrm{C}$, according to EPA Method 160.1. The practical range of the determination is 10 to $20,000 \mathrm{mg} / 1$; the method's lower detection limit practiced with a sample volume of $50 \mathrm{ml}$ was estimated to be $20 \mathrm{mg} / 1$.

Quality control measures involved prewashing the glass fiber filters used to separate solids from the samples, as well as performing replicate analyses.

\section{TOTAL SUSPENDED SOLIDS (TSS) AND VOLATILE SUSPENDED SOLIOS (VSS)}

TSS and VSS in unpreserved samples were measured according to Methods 209.D and 209.E in Standard Methods (1981). In these procedures, the residue on the filter from the ToS determination is dried first at $1.03-105^{\circ} \mathrm{C}$ and then at $550^{\circ} \mathrm{C}$ to find the TSS and VSS components of the wastewater.

The detection limit for a $50 \mathrm{ml}$ sample volume was estimated to be $20 \mathrm{mg} / 1$, for either TSS or VSṢ. 
Quality control measures involved prewashing the glass fiber filters and passing distilled water rather than sample through the filters as a check. The weight change was recorded on quality control sheets. Sample replicate data were also recorded for TSS. The results of the TSS showed a high variability (low precision) for samples with very high suspended solids levels, due to the difficulty of sampling these suspensions.

pH

pH was measured with a combination glass electrode standardized against commercially available buffer solutions, as referenced in EPA Method 150.1. Temperature compensation, if required, was provided by manually adjusting the meter control, after measuring the sample temperature.

\section{PHOSPHATE (ORTHO)}

Ortho phosphate was determined by EPA Method 365.2 (ascorbic acid, single reagent colorimetric method). The lower detection limit of this method was approximately $0.01 \mathrm{mg} / 1$. Generaliy, sample dilutions were required to remain within the range of $0-0.5 \mathrm{mg} / 1$ for the colorimetric step. A Spectronic 710 spectrophotometer with a $1 \mathrm{~cm}$ cell was used for this analys is.

Quality control procedures included spiked samples and replicate sample analyses, and analyses of an accuracy check standard.

\section{COLOR}

Color was measured in $50 \mathrm{ml}$ Nessler tubes by visual comparison against a standard made up with potassium chloroplatinate, as described in Standard Methods; Method 204A. The samples were diluted if necessary to bring the color into the range of $0-70$ color units.

The practical detection limit is 5 color units. 
AMMNNTA

Ammonia-N analyses were performed on unfiltered samples using EPA Method 350.2 (1979), which employed distillation and titrimetric analysis of the ammonia trapped in boric acid, using $0.02 \mathrm{~N}$ sulfuric acid. With a $400 \mathrm{~m} 1$ sample taken for distillation, the estimated limit of detection was $1 \mathrm{mg} / 1$.

Any volatile amines present interfered with the method, by titrating as ammonia in the boric acid collection medium. Such interferences could not be eliminated.

Quality control measures included analys is of distilled water and sample spikes and an accuracy check standard. Replicate analyses were also run to check the method's precision. The sulfuric acid used for titration was standardized, via standard $\mathrm{NaOH}$ solution, with a potassium acid phthalate acidimetric standard.

\section{ORGANIC NITROGEN}

Organic-N was analyzed on unfiltered samples using EPA Method 351.3 (1979) for total Kjeldahl-N (TKN). Organic-N was found by subtracting ammonia-N from TKN. As in the ammonia analysis, the boric acid solutions of distilled ammonia were titrated with $0.02 \mathrm{~N}$ sulfuric acld. The estimated limit of detection for this method with a $500 \mathrm{ml}$ sample was $1 \mathrm{mg} / 1$.

Quality rontrol measures included analysis of an ammonium chloride accuracy check standard and replicate sample analyses. The same standardized sulfuric acid (via potassium acid phthalate) was used for both TKN and ammonia-N analyses. 
METALS (IRON, CALCIUM, SODIUM, MAGNESIUM)

All metals analyses were performed on unfiltered samples. The samples were digested according to the EPA Manual's nitric acid digestion procedure in the Metals Section of the Manual, Paragraph 4.1.3. The digested samples were diluted to volume with water and analyzed by aspiration into the air/acetylene flame of an Instrumentation Laboratories Mode 1457 atomic absorption spectrophotometer. Background correction with a deuterium lamp was used for analytical wavelengths below $300 \mathrm{~nm}$.

Quality control steps included measuring at least four standards and a blank with each analysis, plus a digestion blank containing those reagents used for the digestion. Spikes were added to samples before and after digestion, and replicate samples were processed through the digestion and analys is steps. 